\title{
Geochemical Study of Grab Samples Collected From BX Tank Farm Borehole C5989
}

Michael Lindberg

March 2009

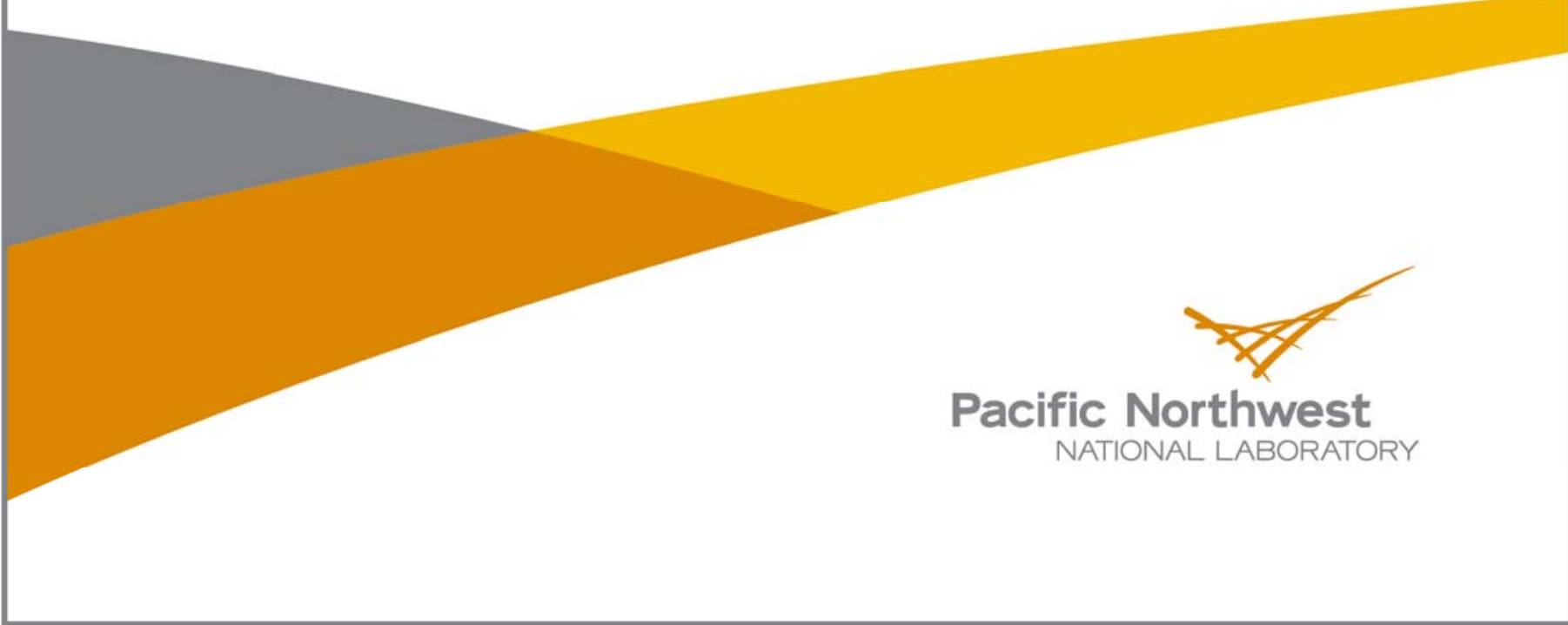




\title{
DISCLAIMER
}

This report was prepared as an account of work sponsored by an agency of the United States Government. Neither the United States Government nor any agency thereof, nor Battelle Memorial Institute, nor any of their employees, makes any warranty, express or implied, or assumes any legal liability or responsibility for the accuracy, completeness, or usefulness of any information, apparatus, product, or process disclosed, or represents that its use would not infringe privately owned rights. Reference herein to any specific commercial product, process, or service by trade name, trademark, manufacturer, or otherwise does not necessarily constitute or imply its endorsement, recommendation, or favoring by the United States Government or any agency thereof, or Battelle Memorial Institute. The views and opinions of authors expressed herein do not necessarily state or reflect those of the United States Government or any agency thereof.

\author{
PACIFIC NORTHWEST NATIONAL LABORATORY \\ operated by \\ BATTELLE \\ for the \\ UNITED STATES DEPARTMENT OF ENERGY \\ under Contract DE-AC05-76RL01830
}

Printed in the United States of America
Available to DOE and DOE contractors from the Office of Scientific and Technical Information,
P.O. Box 62, Oak Ridge, TN 37831-0062;
ph: (865) 576-8401
fax: $(865)$ 576-5728
email: reports@adonis.osti.gov

\begin{abstract}
Available to the public from the National Technical Information Service, U.S. Department of Commerce, 5285 Port Royal Rd., Springfield, VA 22161 ph: (800) 553-6847 fax: $(703) 605-6900$ email: orders@ntis.fedworld.gov online ordering: http://www.ntis.gov/ordering.htm
\end{abstract}

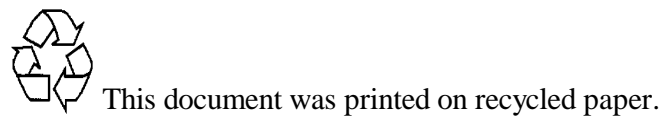


03/19/09 10:08

To: Frederick Mann

From: Michael J. Lindberg

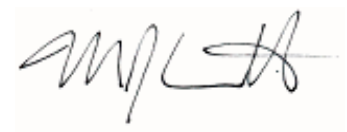

Environmental Sciences Laboratory

Energy and Environment Directorate, Pacific Northwest National Laboratory

Subject: Analytical Data Report for Sediment Samples Collected from BX Tank Farm Borehole C5989, Sample Delivery Groups ESL080020 and ESL080021, SAF Number F08-102 and V08-005

This is a comprehensive report combining the data from PNNL report PNNL- SA-62559 with analytical data from follow on analyses of sediment samples collected from BX Tank Farm Borehole C5989.

This letter contains the following information for sample delivery groups ESL080020 and ESL080021

- Cover Sheet

- Narrative

- Analytical Results

- Quality Control

- Geologic Logs

- Geologic Photos

- Chain of Custodies 


\section{Introduction}

Between May 6, 2008 and July 24, 2008 sediment samples were received from BX Tank Farm Borehole C5989 for geochemical studies.

\section{Analytical Results/Methodology}

The analyses for this project were performed at the 325 building located in the 300 Area of the Hanford Site. The analyses were performed according to Pacific Northwest National Laboratory (PNNL) approved procedures and/or nationally recognized test procedures. The data sets include the sample identification numbers, analytical results, estimated quantification limits (EQL), and quality control data.

ICP-MS data is reported as total element concentration in terms of the specific isotope measured. The instrument software converts the concentration of an isotope of an element to the total concentration of the element based on the distribution of isotopes in the natural environment. For example, the total $\mathrm{Cr}$ concentration is reported from the raw count rates for both ${ }^{52} \mathrm{Cr}$ and ${ }^{53} \mathrm{Cr}$ based on taking the raw counts and dividing by the fraction of ${ }^{52} \mathrm{Cr}$ and ${ }^{53} \mathrm{Cr}$ found in nature to yield estimates of total $\mathrm{Cr}$ in the sample.

\section{Quality Control}

The preparatory and analytical quality control requirements, calibration requirements, acceptance criteria, and failure actions are defined in the on-line QA plan "Conducting Analytical Work in Support of Regulatory Programs" (CAW). This QA plan implements the Hanford Analytical Services Quality Assurance Requirements Documents (HASQARD) for PNNL.

\section{Definitions}

$\begin{array}{ll}\text { Dup } & \text { Duplicate } \\ \text { RPD } & \text { Relative Percent Difference } \\ \text { NR } & \text { No Recovery (percent recovery less than zero) } \\ \text { ND } & \text { Non-Detectable } \\ \text { \%REC } & \text { Percent Recovery }\end{array}$

\section{Sample Receipt}

Samples were received with a chain of custody (COC) and were analyzed according to the sample identification numbers supplied by the client. All Samples were refrigerated upon receipt until prepared for analysis.

All samples were received with custody seals intact unless noted in the Case Narrative.

\section{Holding Times}

Holding time is defined as the time from sample preparation to the time of analyses. The prescribed holding times were met for all analytes unless noted in the Case Narrative.

\section{Analytical Results}

All reported analytical results meet the requirements of the CAW or client specified SOW unless noted in the case narrative. 


\section{Case Narrative Report}

\section{Hold Time:}

No discrepancies noted

\section{Preparation Blank (PB):}

QC Sample 8I15002-BLK1 failed criteria for Barium in ICP-OES Vadose-WE.

$\mathrm{MDL}=0.00879 \mathrm{ug} / \mathrm{g}$

$\mathrm{MRL}=0.00879 \mathrm{ug} / \mathrm{g}$

Result $=0.0129 \mathrm{ug} / \mathrm{g}$

Criterion $=1 \times$ MRL

Blank Concentration was greater than $5 \%$ of the measured concentrations of the samples. Barium will not be reported for samples associated with this preparation batch.

QC Sample 8L15001-BLK1 failed criteria for Chromium 53 in ICPMS-RCRA-AE.

$\mathrm{MDL}=0.161 \mathrm{ug} / \mathrm{g}$

$\mathrm{MRL}=0.161 \mathrm{ug} / \mathrm{g}$

Result $=0.575 \mathrm{ug} / \mathrm{g}$

Criterion $=1 \times$ MRL

Blank Concentration was greater than $5 \%$ of the measured concentrations of the samples. Chromium 53 will not be reported for samples associated with this preparation batch.

\section{Duplicate (DUP):}

Duplicate RPD for Arsenic 75 (55.9\%) was above the acceptance limit (35) in 8L15001-DUP1 for ICPMS-RCRA-AE. All other duplicates and QC associated with the batch were within limits. Duplicate failure may be due to sample heterogeneity. There should be no impact to sample data as reported.

Duplicate RPD for Lead 206 (71.4\%) was above the acceptance limit (35) in 8L15001-DUP1 for ICPMS-RCRA-AE. All other duplicates and QC associated with the batch were within limits. Duplicate failure may be due to sample heterogeneity. There should be no impact to sample data as reported.

Duplicate RPD for Lead 208 (73.6\%) was above the acceptance limit (35) in 8L15001-DUP1 for ICPMS-RCRA-AE. All other duplicates and QC associated with the batch were within limits. Duplicate failure may be due to sample heterogeneity. There should be no impact to sample data as reported.

\section{Laboratory Control Samples (LCS):}

LCS Recovery for Silicon (124\%) was outside acceptance limits (80-120) in 8I15003-BS1 for ICP-OES Vadose-WE. Silicon will not be reported for samples associated with this preparation batch.

\section{Post spike (PS) and post spike duplicate (PSD):}

Post-Spike Recovery for Chromium 53 (65.7\%) was outside acceptance limits (75-125) in 8L15001-PS1 for ICPMS-RCRA-AE. Chromium 53 will not be reported for samples associated with this preparation batch. 


\section{Case Narrative Report}

\section{Post spike (PS) and post spike duplicate (PSD):}

Post-Spike Recovery for Aluminum (207\%) was outside acceptance limits (75-125) in 8L12002-PS1 for ICP-OES Vadose-AE. The native sample concentration of the sample was greater than 5 times the spike concentration. There should be not impact to data as reported.

Post-Spike Recovery for Calcium (203\%) was outside acceptance limits (75-125) in 8L12002-PS1 for ICP-OES Vadose-AE. The native sample concentration of the sample was greater than 5 times the spike concentration. There should be not impact to data as reported.

Post-Spike Recovery for Magnesium (148\%) was outside acceptance limits (75-125) in 8L12002-PS1 for ICP-OES Vadose-AE. The native sample concentration of the sample was greater than 5 times the spike concentration. There should be not impact to data as reported.

Post-Spike Recovery for Iron (251\%) was outside acceptance limits (75-125) in 8L12002-PS1 for ICP-OES Vadose-AE. The native sample concentration of the sample was greater than 5 times the spike concentration. There should be not impact to data as reported.

\section{Matrix Spike (MS):}

Not applicable.

\section{Other QC Criteria:}

No discrepancies noted.

\section{DISCLAIMER}

This report was prepared as an account of work sponsored by an agency of the United States Government. Neither the United States Government nor any agency thereof, nor Battelle Memorial Institute, nor any of their employees, makes any warranty, express or implied, or assumes any legal liability or responsibility for the accuracy, completeness, or usefulness of any information, apparatus, product, or process disclosed, or represents that its use would not infringe privately owned rights. Reference herein to any specific commercial product, process, or service by trade name, trademark, manufacturer, or otherwise does not necessarily constitute or imply its endorsement, recommendation, or favoring by the United States Government or any agency thereof, or Battelle Memorial Institute. The views and opinions of authors expressed herein do not necessarily state or reflect those of the United States Government or any agency thereof. 


\section{BX Farm C5989}

$\begin{array}{lll}\text { HEIS No. } & \text { Laboratory ID } & \text { Matrix } \\ \text { B1VDW5 } & 0805001-01 & \text { SOIL } \\ \text { B1VDW6 } & 0805001-02 & \text { SOIL } \\ \text { B1VDW7 } & 0805001-03 & \text { SOIL } \\ \text { B1VDW8 } & 0805001-04 & \text { SOIL } \\ \text { B1VDW9 } & 0805001-05 & \text { SOIL } \\ \text { B1VDX0 } & 0805001-06 & \text { SOIL } \\ \text { B1V991 } & 0805003-01 & \text { SOIL } \\ \text { B1V992 } & 0805003-02 & \text { SOIL } \\ \text { B1V993 } & 0805003-03 & \text { SOIL } \\ \text { B1V994 } & 0805003-04 & \text { SOIL } \\ \text { B1V995 } & 0805003-05 & \text { SOIL } \\ \text { B1V996 } & 0805003-06 & \text { SOIL } \\ \text { B1V997 } & 0805003-07 & \text { SOIL } \\ \text { B1V998 } & 0805003-08 & \text { SOIL } \\ \text { B1V999 } & 0805003-09 & \text { SOIL } \\ \text { B1V9B0 } & 0805003-10 & \text { SOIL } \\ \text { B1V9B1 } & 0805003-11 & \text { SOIL } \\ \text { B1V9B2 } & 0805003-12 & \text { SOIL } \\ \text { B1V9B3 } & 0805003-13 & \text { SOIL } \\ \text { B1V9B4 } & 0805003-14 & \text { SOIL } \\ \text { B1V9B5 } & 0805003-15 & \text { SOIL } \\ \text { B1V9B6 } & 0805003-16 & \text { SOIL } \\ \text { B1V9B7 } & 0805003-17 & \text { SOIL } \\ \text { B1V9B8 } & 0805003-18 & \text { SOIL } \\ \text { B1V9B9 } & 0805003-19 & \text { SOIL } \\ \text { B1V9C0 } & 0805003-20 & \text { SOIL } \\ \text { B1V9C1 } & 0805003-21 & \text { SOIL } \\ \text { B1V9C2 } & 0805003-22 & \text { SOIL } \\ \text { B1V9C3 } & 0805003-23 & \text { SOIL } \\ \text { B1V9C4 } & 0805003-24 & \text { SOIL } \\ \text { B1V2V5 } & 0805003-25 & \text { SOIL }\end{array}$

$\begin{array}{llll}\text { Date Collected } & \text { Date Received } \\ \text { 4/30/08 } & 09: 30 & 5 / 6 / 08 & 14: 30 \\ 5 / 1 / 08 & 11: 00 & 5 / 6 / 08 & 14: 30 \\ 5 / 5 / 08 & 09: 15 & 5 / 6 / 08 & 14: 30 \\ 5 / 5 / 08 & 09: 15 & 5 / 6 / 08 & 14: 30 \\ 5 / 5 / 08 & 09: 15 & 5 / 6 / 08 & 14: 30 \\ 5 / 5 / 08 & 09: 15 & 5 / 6 / 08 & 14: 30 \\ \text { 4/30/08 } & 09: 30 & 5 / 9 / 08 & 14: 00 \\ 5 / 5 / 08 & 09: 15 & 5 / 9 / 08 & 14: 00 \\ 5 / 5 / 08 & 09: 40 & 5 / 9 / 08 & 14: 00 \\ 5 / 5 / 08 & 10: 00 & 5 / 9 / 08 & 14: 00 \\ 5 / 5 / 08 & 10: 25 & 5 / 9 / 08 & 14: 00 \\ 5 / 5 / 08 & 10: 45 & 5 / 9 / 08 & 14: 00 \\ 5 / 5 / 08 & 11: 00 & 5 / 9 / 08 & 14: 00 \\ 5 / 5 / 08 & 13: 20 & 5 / 9 / 08 & 14: 00 \\ 5 / 6 / 08 & 08: 30 & 5 / 13 / 08 & 13: 30 \\ 5 / 6 / 08 & 10: 00 & 5 / 13 / 08 & 13: 30 \\ 5 / 6 / 08 & 10: 06 & 5 / 13 / 08 & 13: 30 \\ 5 / 6 / 08 & 13: 15 & 5 / 13 / 08 & 13: 30 \\ 5 / 6 / 08 & 13: 27 & 5 / 13 / 08 & 13: 30 \\ 5 / 7 / 08 & 14: 35 & 5 / 13 / 08 & 13: 30 \\ 5 / 8 / 08 & 09: 14 & 5 / 13 / 08 & 13: 30 \\ 5 / 8 / 08 & 09: 45 & 5 / 13 / 08 & 13: 30 \\ 5 / 8 / 08 & 10: 04 & 5 / 14 / 08 & 09: 30 \\ 5 / 8 / 08 & 10: 50 & 5 / 14 / 08 & 09: 30 \\ 5 / 8 / 08 & 13: 00 & 5 / 14 / 08 & 09: 30 \\ 5 / 8 / 08 & 13: 40 & 5 / 14 / 08 & 09: 30 \\ 5 / 8 / 08 & 14: 20 & 5 / 14 / 08 & 09: 30 \\ 5 / 8 / 08 & 14: 40 & 5 / 14 / 08 & 09: 30 \\ 5 / 12 / 08 & 09: 20 & 5 / 14 / 08 & 09: 30 \\ 5 / 12 / 08 & 10: 05 & 5 / 14 / 08 & 09: 30 \\ 5 / 12 / 08 & 13: 10 & 5 / 16 / 08 & 11: 00 \\ & & & \\ & 140\end{array}$


The following analyses were performed on the following samples included in this report:

Metals 1:1 DI Water Extract by ICPMS

Metals Acid Extract by ICPMS

1:1 DI Water Extract

Carbon, Total, Combustion or Oxidation

Inorganic Carbon, Total, Combustion or Oxidation

Anions By Ion Chromatography

Alkalinity, Titrimetic ( $\mathrm{pH} 4.5)$

GEA No Preparation

Metals 1:1 Water Extract by ICPOES

Metals Acid Extract by ICPOES

Moisture Content

Nitric Acid Digestion

$\mathrm{pH}$ of Waters By Electrode

Specific Conductance

Tc_U Acid Extract by ICPMS

Tc_U 1:1 DI Water Extract by ICPMS

Total Alpha Total Beta 1:1 DI Water Extract By LSC

Total Alpha Total Beta Acid Extract By LSC 


$\begin{array}{lll}\text { HEIS No. } & \text { Laboratory ID } & \text { Matrix } \\ \text { B1VDW5 } & 0805001-01 & \text { SOIL } \\ \text { B1VDW6 } & 0805001-02 & \text { SOIL } \\ \text { B1VDW7 } & 0805001-03 & \text { SOIL } \\ \text { B1VDW8 } & 0805001-04 & \text { SOIL } \\ \text { B1VDW9 } & 0805001-05 & \text { SOIL } \\ \text { B1VDX0 } & 0805001-06 & \text { SOIL } \\ \text { B1V991 } & 0805003-01 & \text { SOIL } \\ \text { B1V992 } & 0805003-02 & \text { SOIL } \\ \text { B1V993 } & 0805003-03 & \text { SOIL } \\ \text { B1V994 } & 0805003-04 & \text { SOIL } \\ \text { B1V995 } & 0805003-05 & \text { SOIL } \\ \text { B1V996 } & 0805003-06 & \text { SOIL } \\ \text { B1V997 } & 0805003-07 & \text { SOIL } \\ \text { B1V998 } & 0805003-08 & \text { SOIL } \\ \text { B1V999 } & 0805003-09 & \text { SOIL } \\ \text { B1V9B0 } & 0805003-10 & \text { SOIL } \\ \text { B1V9B1 } & 0805003-11 & \text { SOIL } \\ \text { B1V9B2 } & 0805003-12 & \text { SOIL } \\ \text { B1V9B3 } & 0805003-13 & \text { SOIL } \\ \text { B1V9B4 } & 0805003-14 & \text { SOIL } \\ \text { B1V9B5 } & 0805003-15 & \text { SOIL } \\ \text { B1V9B6 } & 0805003-16 & \text { SOIL } \\ \text { B1V9B7 } & 0805003-17 & \text { SOIL } \\ \text { B1V9B8 } & 0805003-18 & \text { SOIL } \\ \text { B1V9B9 } & 0805003-19 & \text { SOIL } \\ \text { B1V9C0 } & 0805003-20 & \text { SOIL } \\ \text { B1V9C1 } & 0805003-21 & \text { SOIL } \\ \text { B1V9C2 } & 0805003-22 & \text { SOIL } \\ \text { B1V9C3 } & 0805003-23 & \text { SOIL } \\ \text { B1V9C4 } & 0805003-24 & \text { SOIL } \\ \text { B1V2V5 } & 0805003-25 & \text { SOIL }\end{array}$

$\begin{array}{llll}\text { Date Collected } & \text { Date Received } \\ \text { 4/30/08 } & 09: 30 & 5 / 6 / 08 & 14: 30 \\ 5 / 1 / 08 & 11: 00 & 5 / 6 / 08 & 14: 30 \\ 5 / 5 / 08 & 09: 15 & 5 / 6 / 08 & 14: 30 \\ 5 / 5 / 08 & 09: 15 & 5 / 6 / 08 & 14: 30 \\ 5 / 5 / 08 & 09: 15 & 5 / 6 / 08 & 14: 30 \\ 5 / 5 / 08 & 09: 15 & 5 / 6 / 08 & 14: 30 \\ \text { 4/30/08 } & 09: 30 & 5 / 9 / 08 & 14: 00 \\ 5 / 5 / 08 & 09: 15 & 5 / 9 / 08 & 14: 00 \\ 5 / 5 / 08 & 09: 40 & 5 / 9 / 08 & 14: 00 \\ 5 / 5 / 08 & 10: 00 & 5 / 9 / 08 & 14: 00 \\ 5 / 5 / 08 & 10: 25 & 5 / 9 / 08 & 14: 00 \\ 5 / 5 / 08 & 10: 45 & 5 / 9 / 08 & 14: 00 \\ 5 / 5 / 08 & 11: 00 & 5 / 9 / 08 & 14: 00 \\ 5 / 5 / 08 & 13: 20 & 5 / 9 / 08 & 14: 00 \\ 5 / 6 / 08 & 08: 30 & 5 / 13 / 08 & 13: 30 \\ 5 / 6 / 08 & 10: 00 & 5 / 13 / 08 & 13: 30 \\ 5 / 6 / 08 & 10: 06 & 5 / 13 / 08 & 13: 30 \\ 5 / 6 / 08 & 13: 15 & 5 / 13 / 08 & 13: 30 \\ 5 / 6 / 08 & 13: 27 & 5 / 13 / 08 & 13: 30 \\ 5 / 7 / 08 & 14: 35 & 5 / 13 / 08 & 13: 30 \\ 5 / 8 / 08 & 09: 14 & 5 / 13 / 08 & 13: 30 \\ 5 / 8 / 08 & 09: 45 & 5 / 13 / 08 & 13: 30 \\ 5 / 8 / 08 & 10: 04 & 5 / 14 / 08 & 09: 30 \\ 5 / 8 / 08 & 10: 50 & 5 / 14 / 08 & 09: 30 \\ 5 / 8 / 08 & 13: 00 & 5 / 14 / 08 & 09: 30 \\ 5 / 8 / 08 & 13: 40 & 5 / 14 / 08 & 09: 30 \\ 5 / 8 / 08 & 14: 20 & 5 / 14 / 08 & 09: 30 \\ 5 / 8 / 08 & 14: 40 & 5 / 14 / 08 & 09: 30 \\ 5 / 12 / 08 & 09: 20 & 5 / 14 / 08 & 09: 30 \\ 5 / 12 / 08 & 10: 05 & 5 / 14 / 08 & 09: 30 \\ 5 / 12 / 08 & 13: 10 & 5 / 16 / 08 & 11: 00 \\ & & & \\ & & & \end{array}$




\section{Wet Chemistry}

\begin{tabular}{|c|c|c|c|c|c|}
\hline $\begin{array}{l}\text { Alkalinity } \\
\text { Lab ID }\end{array}$ & $\begin{array}{c}\text { CaCO3 ( } \\
\text { HEIS No. }\end{array}$ & $\begin{array}{l}\text { Method } \\
\text { Results }\end{array}$ & $\begin{array}{c}\text { 20B } \\
\text { EQL }\end{array}$ & Analyzed & Batch \\
\hline 0805001-01 & B1VDW5 & $3.86 \mathrm{E} 1$ & 2.34E1 & $8 / 06 / 08$ & $8 \mathrm{H} 06003$ \\
\hline 0805001-02 & B1VDW6 & $3.78 \mathrm{E} 1$ & $2.29 \mathrm{E} 1$ & 8/06/08 & $8 \mathrm{H} 06003$ \\
\hline 0805001-03 & B1VDW7 & $4.38 \mathrm{E} 1$ & $2.34 \mathrm{E} 1$ & 8/06/08 & $8 \mathrm{H} 06003$ \\
\hline 0805001-04 & B1VDW8 & 4.79E1 & $2.31 \mathrm{E} 1$ & 8/06/08 & $8 \mathrm{H} 06003$ \\
\hline 0805001-05 & B1VDW9 & 4.62E1 & $2.31 \mathrm{E} 1$ & 8/06/08 & $8 \mathrm{H} 06003$ \\
\hline 0805001-06 & B1VDX0 & $5.90 \mathrm{E} 1$ & $2.53 \mathrm{E} 1$ & 8/06/08 & $8 \mathrm{H} 06003$ \\
\hline 0805003-01 & B1V991 & $3.65 \mathrm{E} 1$ & $2.35 \mathrm{E} 1$ & 8/06/08 & $8 \mathrm{H} 06003$ \\
\hline 0805003-02 & B1V992 & $4.85 \mathrm{E} 1$ & $2.34 \mathrm{E} 1$ & 8/06/08 & $8 \mathrm{H} 06003$ \\
\hline 0805003-03 & B1V993 & 4.37E1 & $2.33 \mathrm{E} 1$ & 8/06/08 & $8 \mathrm{H} 06003$ \\
\hline 0805003-04 & B1V994 & $5.83 \mathrm{E} 1$ & $2.34 \mathrm{E} 1$ & 8/06/08 & $8 \mathrm{H} 06003$ \\
\hline 0805003-05 & B1V995 & $5.40 \mathrm{E} 1$ & $2.35 \mathrm{E} 1$ & 8/06/08 & $8 \mathrm{H} 06003$ \\
\hline 0805003-06 & B1V996 & $7.98 \mathrm{E} 1$ & $2.35 \mathrm{E} 1$ & 8/06/08 & $8 \mathrm{H} 06002$ \\
\hline 0805003-07 & B1V997 & $6.91 \mathrm{E} 1$ & $2.35 \mathrm{E} 1$ & $8 / 06 / 08$ & $8 \mathrm{H} 06002$ \\
\hline 0805003-08 & B1V998 & $5.78 \mathrm{E} 1$ & $2.35 \mathrm{E} 1$ & 8/06/08 & $8 \mathrm{H} 06002$ \\
\hline 0805003-09 & B1V999 & $5.61 \mathrm{E} 1$ & $2.34 \mathrm{E} 1$ & 8/06/08 & $8 \mathrm{H} 06002$ \\
\hline 0805003-10 & B1V9B0 & $5.68 \mathrm{E} 1$ & $2.34 \mathrm{E} 1$ & 8/06/08 & $8 \mathrm{H} 06002$ \\
\hline 0805003-11 & B1V9B1 & $5.00 \mathrm{E} 1$ & $2.34 \mathrm{E} 1$ & 8/06/08 & $8 \mathrm{H} 06002$ \\
\hline 0805003-12 & B1V9B2 & $4.41 \mathrm{E} 1$ & $2.35 \mathrm{E} 1$ & 8/06/08 & $8 \mathrm{H} 06002$ \\
\hline 0805003-13 & B1V9B3 & $6.24 \mathrm{E} 1$ & $2.35 \mathrm{E} 1$ & 8/06/08 & $8 \mathrm{H} 06002$ \\
\hline 0805003-14 & B1V9B4 & 5.39E1 & $2.35 \mathrm{E} 1$ & $8 / 06 / 08$ & $8 \mathrm{H} 06002$ \\
\hline 0805003-15 & B1V9B5 & $3.57 \mathrm{E} 1$ & $2.35 \mathrm{E} 1$ & 8/06/08 & $8 \mathrm{H} 06002$ \\
\hline 0805003-16 & B1V9B6 & $4.01 \mathrm{E} 1$ & $2.34 \mathrm{E} 1$ & $8 / 06 / 08$ & $8 \mathrm{H} 06002$ \\
\hline 0805003-17 & B1V9B7 & $3.65 \mathrm{E} 1$ & $2.35 \mathrm{E} 1$ & 8/06/08 & $8 \mathrm{H} 06002$ \\
\hline 0805003-18 & B1V9B8 & $3.44 \mathrm{E} 1$ & $2.36 \mathrm{E} 1$ & 8/06/08 & $8 \mathrm{H} 06002$ \\
\hline 0805003-19 & B1V9B9 & 3.63E1 & $2.34 \mathrm{E} 1$ & 8/06/08 & $8 \mathrm{H} 06002$ \\
\hline 0805003-20 & $\mathrm{B} 1 \mathrm{~V} 9 \mathrm{C} 0$ & $3.43 \mathrm{E} 1$ & $2.36 \mathrm{E} 1$ & 8/06/08 & $8 \mathrm{H} 06002$ \\
\hline 0805003-21 & $\mathrm{B} 1 \mathrm{~V} 9 \mathrm{C} 1$ & $3.19 \mathrm{E} 1$ & $2.35 \mathrm{E} 1$ & $8 / 06 / 08$ & $8 \mathrm{H} 06002$ \\
\hline 0805003-22 & $\mathrm{B} 1 \mathrm{~V} 9 \mathrm{C} 2$ & $3.26 \mathrm{E} 1$ & $2.35 \mathrm{E} 1$ & 8/06/08 & $8 \mathrm{H} 06002$ \\
\hline 0805003-23 & B1V9C3 & $3.56 \mathrm{E} 1$ & $2.34 \mathrm{E} 1$ & 8/06/08 & $8 \mathrm{H} 06002$ \\
\hline 0805003-24 & B1V9C4 & $3.73 \mathrm{E} 1$ & $2.35 \mathrm{E} 1$ & 8/06/08 & $8 \mathrm{H} 06002$ \\
\hline 0805003-25 & $\mathrm{B} 1 \mathrm{~V} 2 \mathrm{~V} 5$ & 4.09E1 & $2.34 \mathrm{E} 1$ & 8/06/08 & $8 \mathrm{H} 06002$ \\
\hline
\end{tabular}


Wet Chemistry

\begin{tabular}{|c|c|c|c|c|c|}
\hline \multicolumn{6}{|c|}{ Specific Conductance (EC) (mS/cm) by EPA 120.1} \\
\hline Lab ID & HEIS No. & Results & EQL & Analyzed & Batch \\
\hline 0805001-01 & B1VDW5 & $6.90 \mathrm{E}-2$ & $1.00 \mathrm{E}-2$ & $8 / 05 / 08$ & $8 \mathrm{H} 04007$ \\
\hline 0805001-02 & B1VDW6 & $6.80 \mathrm{E}-2$ & $1.00 \mathrm{E}-2$ & $8 / 05 / 08$ & $8 \mathrm{H} 04007$ \\
\hline 0805001-03 & B1VDW7 & $8.30 \mathrm{E}-2$ & $1.00 \mathrm{E}-2$ & $8 / 05 / 08$ & $8 \mathrm{H} 04007$ \\
\hline 0805001-04 & B1VDW8 & $8.70 \mathrm{E}-2$ & $1.00 \mathrm{E}-2$ & $8 / 05 / 08$ & $8 \mathrm{H} 04007$ \\
\hline 0805001-05 & B1VDW9 & $7.70 \mathrm{E}-2$ & $1.00 \mathrm{E}-2$ & $8 / 05 / 08$ & $8 \mathrm{H} 04007$ \\
\hline 0805001-06 & B1VDX0 & $6.50 \mathrm{E}-2$ & $1.00 \mathrm{E}-2$ & $8 / 05 / 08$ & $8 \mathrm{H} 04007$ \\
\hline 0805003-01 & B1V991 & $6.00 \mathrm{E}-2$ & $1.00 \mathrm{E}-2$ & $8 / 05 / 08$ & $8 \mathrm{H} 04007$ \\
\hline 0805003-02 & B1V992 & $8.00 \mathrm{E}-2$ & $1.00 \mathrm{E}-2$ & $8 / 05 / 08$ & $8 \mathrm{H} 04007$ \\
\hline 0805003-03 & B1V993 & $7.30 \mathrm{E}-2$ & $1.00 \mathrm{E}-2$ & $8 / 05 / 08$ & $8 \mathrm{H} 04007$ \\
\hline 0805003-04 & B1V994 & $9.20 \mathrm{E}-2$ & $1.00 \mathrm{E}-2$ & $8 / 05 / 08$ & $8 \mathrm{H} 04007$ \\
\hline 0805003-05 & B1V995 & $9.10 \mathrm{E}-2$ & $1.00 \mathrm{E}-2$ & $8 / 05 / 08$ & $8 \mathrm{H} 04007$ \\
\hline 0805003-06 & B1V996 & $1.51 \mathrm{E}-1$ & $1.00 \mathrm{E}-2$ & 8/01/08 & $8 \mathrm{G} 31008$ \\
\hline 0805003-07 & B1V997 & $1.47 \mathrm{E}-1$ & $1.00 \mathrm{E}-2$ & 8/01/08 & $8 \mathrm{G} 31008$ \\
\hline 0805003-08 & B1V998 & $1.37 \mathrm{E}-1$ & $1.00 \mathrm{E}-2$ & 8/01/08 & $8 \mathrm{G} 31008$ \\
\hline 0805003-09 & B1V999 & $1.39 \mathrm{E}-1$ & $1.00 \mathrm{E}-2$ & 8/01/08 & $8 \mathrm{G} 31008$ \\
\hline 0805003-10 & B1V9B0 & $1.33 \mathrm{E}-1$ & $1.00 \mathrm{E}-2$ & 8/01/08 & $8 \mathrm{G} 31008$ \\
\hline 0805003-11 & B1V9B1 & $1.32 \mathrm{E}-1$ & $1.00 \mathrm{E}-2$ & 8/01/08 & $8 \mathrm{G} 31008$ \\
\hline 0805003-12 & B1V9B2 & $1.33 \mathrm{E}-1$ & $1.00 \mathrm{E}-2$ & 8/01/08 & $8 \mathrm{G} 31008$ \\
\hline 0805003-13 & B1V9B3 & $1.74 \mathrm{E}-1$ & $1.00 \mathrm{E}-2$ & 8/01/08 & $8 \mathrm{G} 31008$ \\
\hline 0805003-14 & B1V9B4 & $1.26 \mathrm{E}-1$ & $1.00 \mathrm{E}-2$ & 8/01/08 & $8 \mathrm{G} 31008$ \\
\hline 0805003-15 & B1V9B5 & $9.00 \mathrm{E}-2$ & $1.00 \mathrm{E}-2$ & 8/01/08 & $8 \mathrm{G} 31008$ \\
\hline 0805003-16 & B1V9B6 & $1.19 \mathrm{E}-1$ & $1.00 \mathrm{E}-2$ & 8/01/08 & $8 \mathrm{G} 31008$ \\
\hline 0805003-17 & B1V9B7 & $1.44 \mathrm{E}-1$ & $1.00 \mathrm{E}-2$ & $8 / 01 / 08$ & $8 \mathrm{G} 31008$ \\
\hline 0805003-18 & B1V9B8 & $1.36 \mathrm{E}-1$ & $1.00 \mathrm{E}-2$ & 8/01/08 & $8 \mathrm{G} 31008$ \\
\hline 0805003-19 & B1V9B9 & $1.59 \mathrm{E}-1$ & $1.00 \mathrm{E}-2$ & 8/01/08 & $8 \mathrm{G} 31008$ \\
\hline 0805003-20 & B1V9C0 & $1.63 \mathrm{E}-1$ & $1.00 \mathrm{E}-2$ & 8/01/08 & $8 \mathrm{G} 31008$ \\
\hline $0805003-21$ & B1V9C1 & $1.74 \mathrm{E}-1$ & $1.00 \mathrm{E}-2$ & $8 / 01 / 08$ & $8 G 31008$ \\
\hline 0805003-22 & B1V9C2 & $1.79 \mathrm{E}-1$ & $1.00 \mathrm{E}-2$ & 8/01/08 & $8 \mathrm{G} 31008$ \\
\hline 0805003-23 & B1V9C3 & $1.37 \mathrm{E}-1$ & $1.00 \mathrm{E}-2$ & $8 / 01 / 08$ & $8 \mathrm{G} 31008$ \\
\hline 0805003-24 & B1V9C4 & $1.38 \mathrm{E}-1$ & $1.00 \mathrm{E}-2$ & 8/01/08 & $8 \mathrm{G} 31008$ \\
\hline 0805003-25 & B1V2V5 & $1.00 \mathrm{E}-1$ & $1.00 \mathrm{E}-2$ & $8 / 01 / 08$ & $8 G 31008$ \\
\hline
\end{tabular}




\section{Wet Chemistry}

\begin{tabular}{|c|c|c|c|c|c|}
\hline $\begin{array}{l}\text { Moisture } \\
\text { Lab ID }\end{array}$ & $\begin{array}{l}\text { htent (\% b } \\
\text { HEIS No. }\end{array}$ & $\begin{array}{l}\text { C-001 } \\
\text { Results }\end{array}$ & EQL & Analyzed & Batch \\
\hline 0805001-01 & B1VDW5 & $1.82 \mathrm{E} 0$ & $\mathrm{~N} / \mathrm{A}$ & $7 / 30 / 08$ & $8 \mathrm{G} 21008$ \\
\hline 0805001-02 & B1VDW6 & 1.99E0 & N/A & $7 / 30 / 08$ & $8 \mathrm{G} 21008$ \\
\hline 0805001-03 & B1VDW7 & $3.23 \mathrm{E} 0$ & $\mathrm{~N} / \mathrm{A}$ & $7 / 30 / 08$ & $8 \mathrm{G} 21008$ \\
\hline 0805001-04 & B1VDW8 & $3.65 \mathrm{E} 0$ & N/A & $7 / 30 / 08$ & $8 \mathrm{G} 21008$ \\
\hline 0805001-05 & B1VDW9 & 4.45E0 & N/A & $7 / 30 / 08$ & $8 \mathrm{G} 21008$ \\
\hline 0805001-06 & B1VDX0 & 5.67E0 & N/A & $7 / 30 / 08$ & $8 \mathrm{G} 21008$ \\
\hline 0805003-01 & B1V991 & $1.78 \mathrm{E} 0$ & $\mathrm{~N} / \mathrm{A}$ & $7 / 30 / 08$ & $8 \mathrm{G} 21008$ \\
\hline 0805003-02 & B1V992 & $5.00 \mathrm{E} 0$ & N/A & $7 / 30 / 08$ & $8 \mathrm{G} 21008$ \\
\hline 0805003-03 & B1V993 & $2.98 \mathrm{E} 0$ & N/A & $7 / 30 / 08$ & $8 \mathrm{G} 21008$ \\
\hline 0805003-04 & B1V994 & 4.73E0 & N/A & $7 / 30 / 08$ & $8 \mathrm{G} 21008$ \\
\hline 0805003-05 & B1V995 & $5.39 \mathrm{E} 0$ & N/A & $7 / 30 / 08$ & $8 \mathrm{G} 21008$ \\
\hline 0805003-06 & B1V996 & $8.32 \mathrm{E} 0$ & N/A & $7 / 30 / 08$ & $8 \mathrm{G} 21008$ \\
\hline 0805003-07 & B1V997 & $6.98 \mathrm{E} 0$ & N/A & $7 / 30 / 08$ & $8 \mathrm{G} 21008$ \\
\hline 0805003-08 & B1V998 & 4.84E0 & N/A & $7 / 30 / 08$ & $8 \mathrm{G} 21008$ \\
\hline 0805003-09 & B1V999 & $5.28 \mathrm{E} 0$ & N/A & $7 / 30 / 08$ & $8 \mathrm{G} 21008$ \\
\hline 0805003-10 & B1V9B0 & $5.06 \mathrm{E} 0$ & N/A & $7 / 30 / 08$ & $8 \mathrm{G} 21008$ \\
\hline 0805003-11 & B1V9B1 & $5.95 \mathrm{E} 0$ & N/A & $7 / 30 / 08$ & $8 \mathrm{G} 21008$ \\
\hline 0805003-12 & B1V9B2 & 4.15E0 & N/A & $7 / 30 / 08$ & $8 \mathrm{G} 21008$ \\
\hline 0805003-13 & B1V9B3 & 4.37E0 & N/A & $7 / 30 / 08$ & $8 \mathrm{G} 21008$ \\
\hline 0805003-14 & B1V9B4 & $6.34 \mathrm{E} 0$ & N/A & $7 / 30 / 08$ & $8 \mathrm{G} 21008$ \\
\hline 0805003-15 & B1V9B5 & $2.23 \mathrm{E} 0$ & N/A & $7 / 30 / 08$ & $8 \mathrm{G} 21008$ \\
\hline 0805003-16 & B1V9B6 & $2.23 \mathrm{E} 0$ & N/A & $7 / 30 / 08$ & $8 \mathrm{G} 21008$ \\
\hline 0805003-17 & B1V9B7 & $2.46 \mathrm{E} 0$ & N/A & $7 / 30 / 08$ & $8 \mathrm{G} 21008$ \\
\hline 0805003-18 & B1V9B8 & $2.50 \mathrm{E} 0$ & N/A & $7 / 30 / 08$ & $8 \mathrm{G} 21008$ \\
\hline 0805003-19 & B1V9B9 & $2.59 \mathrm{E} 0$ & N/A & $7 / 30 / 08$ & $8 \mathrm{G} 21008$ \\
\hline 0805003-20 & $\mathrm{B} 1 \mathrm{~V} 9 \mathrm{C} 0$ & $2.57 \mathrm{E} 0$ & N/A & $7 / 30 / 08$ & $8 \mathrm{G} 21008$ \\
\hline 0805003-21 & $\mathrm{B} 1 \mathrm{~V} 9 \mathrm{C} 1$ & 2.49E0 & N/A & $7 / 30 / 08$ & $8 \mathrm{G} 21008$ \\
\hline $0805003-22$ & $\mathrm{~B} 1 \mathrm{~V} 9 \mathrm{C} 2$ & $4.40 \mathrm{E} 0$ & N/A & $7 / 30 / 08$ & $8 \mathrm{G} 21008$ \\
\hline 0805003-23 & B1V9C3 & $4.86 \mathrm{E} 0$ & N/A & $7 / 30 / 08$ & $8 \mathrm{G} 21008$ \\
\hline 0805003-24 & B1V9C4 & $5.44 \mathrm{E} 0$ & N/A & $7 / 30 / 08$ & $8 \mathrm{G} 21008$ \\
\hline 0805003-25 & B1V2V5 & 4.30E0 & N/A & 7/30/08 & $8 \mathrm{G} 21008$ \\
\hline
\end{tabular}




\section{Wet Chemistry}

pH (pH Units) by AGG-pH-001

\begin{tabular}{|c|c|c|c|c|c|}
\hline Lab ID & HEIS No. & Results & EQL & Analyzed & Batch \\
\hline 0805001-01 & B1VDW5 & $9.35 \mathrm{E} 0$ & $\mathrm{~N} / \mathrm{A}$ & $8 / 05 / 08$ & $8 \mathrm{H} 04008$ \\
\hline 0805001-02 & B1VDW6 & $8.46 \mathrm{E} 0$ & N/A & $8 / 05 / 08$ & $8 \mathrm{H} 04008$ \\
\hline 0805001-03 & B1VDW7 & $8.57 \mathrm{E} 0$ & N/A & $8 / 05 / 08$ & $8 \mathrm{H} 04008$ \\
\hline 0805001-04 & B1VDW8 & $8.31 \mathrm{E} 0$ & N/A & $8 / 05 / 08$ & 8H04008 \\
\hline 0805001-05 & B1VDW9 & $8.57 \mathrm{E} 0$ & $\mathrm{~N} / \mathrm{A}$ & $8 / 05 / 08$ & $8 \mathrm{H} 04008$ \\
\hline 0805001-06 & B1VDX0 & $8.66 \mathrm{E} 0$ & $\mathrm{~N} / \mathrm{A}$ & $8 / 05 / 08$ & $8 \mathrm{H} 04008$ \\
\hline 0805003-01 & B1V991 & $8.39 \mathrm{E} 0$ & $\mathrm{~N} / \mathrm{A}$ & $8 / 05 / 08$ & $8 \mathrm{H} 04008$ \\
\hline 0805003-02 & B1V992 & $8.56 \mathrm{E} 0$ & $\mathrm{~N} / \mathrm{A}$ & $8 / 05 / 08$ & $8 \mathrm{H} 04008$ \\
\hline 0805003-03 & B1V993 & $8.65 \mathrm{E} 0$ & $\mathrm{~N} / \mathrm{A}$ & $8 / 05 / 08$ & $8 \mathrm{H} 04008$ \\
\hline 0805003-04 & B1V994 & $8.64 \mathrm{E} 0$ & $\mathrm{~N} / \mathrm{A}$ & $8 / 05 / 08$ & $8 \mathrm{H} 04008$ \\
\hline 0805003-05 & B1V995 & 8.67E0 & $\mathrm{N} / \mathrm{A}$ & $8 / 05 / 08$ & $8 \mathrm{H} 04008$ \\
\hline 0805003-06 & B1V996 & $8.94 \mathrm{E} 0$ & $\mathrm{~N} / \mathrm{A}$ & $8 / 01 / 08$ & $8 \mathrm{H} 01005$ \\
\hline 0805003-07 & B1V997 & $8.99 \mathrm{E} 0$ & $\mathrm{~N} / \mathrm{A}$ & $8 / 01 / 08$ & $8 \mathrm{H} 01005$ \\
\hline 0805003-08 & B1V998 & $9.09 \mathrm{E} 0$ & $\mathrm{~N} / \mathrm{A}$ & $8 / 01 / 08$ & $8 \mathrm{H} 01005$ \\
\hline 0805003-09 & B1V999 & $8.85 \mathrm{E} 0$ & $\mathrm{~N} / \mathrm{A}$ & 8/01/08 & $8 \mathrm{H} 01005$ \\
\hline 0805003-10 & B1V9B0 & $8.88 \mathrm{E} 0$ & $\mathrm{~N} / \mathrm{A}$ & $8 / 01 / 08$ & $8 \mathrm{H} 01005$ \\
\hline 0805003-11 & B1V9B1 & $8.65 \mathrm{E} 0$ & $\mathrm{~N} / \mathrm{A}$ & 8/01/08 & $8 \mathrm{H} 01005$ \\
\hline 0805003-12 & B1V9B2 & $8.51 \mathrm{E} 0$ & $\mathrm{~N} / \mathrm{A}$ & 8/01/08 & $8 \mathrm{H} 01005$ \\
\hline 0805003-13 & B1V9B3 & $8.59 \mathrm{E} 0$ & $\mathrm{~N} / \mathrm{A}$ & $8 / 01 / 08$ & $8 \mathrm{H} 01005$ \\
\hline 0805003-14 & B1V9B4 & $8.34 \mathrm{E} 0$ & $\mathrm{~N} / \mathrm{A}$ & $8 / 01 / 08$ & $8 \mathrm{H} 01005$ \\
\hline 0805003-15 & B1V9B5 & $8.84 \mathrm{E} 0$ & $\mathrm{~N} / \mathrm{A}$ & 8/01/08 & $8 \mathrm{H} 01005$ \\
\hline 0805003-16 & B1V9B6 & $8.65 \mathrm{E} 0$ & $\mathrm{~N} / \mathrm{A}$ & 8/01/08 & $8 \mathrm{H} 01005$ \\
\hline 0805003-17 & B1V9B7 & $8.56 \mathrm{E} 0$ & $\mathrm{~N} / \mathrm{A}$ & $8 / 01 / 08$ & $8 \mathrm{H} 01005$ \\
\hline 0805003-18 & B1V9B8 & $8.38 \mathrm{E} 0$ & $\mathrm{~N} / \mathrm{A}$ & 8/01/08 & $8 \mathrm{H} 01005$ \\
\hline 0805003-19 & B1V9B9 & $8.36 \mathrm{E} 0$ & $\mathrm{~N} / \mathrm{A}$ & $8 / 01 / 08$ & $8 \mathrm{H} 01005$ \\
\hline 0805003-20 & $\mathrm{B} 1 \mathrm{~V} 9 \mathrm{C} 0$ & $8.25 \mathrm{E} 0$ & $\mathrm{~N} / \mathrm{A}$ & $8 / 01 / 08$ & $8 \mathrm{H} 01005$ \\
\hline 0805003-21 & $\mathrm{B} 1 \mathrm{~V} 9 \mathrm{C} 1$ & $8.13 \mathrm{E} 0$ & N/A & $8 / 01 / 08$ & $8 \mathrm{H} 01005$ \\
\hline 0805003-22 & B1V9C2 & $8.02 \mathrm{E} 0$ & $\mathrm{~N} / \mathrm{A}$ & 8/01/08 & $8 \mathrm{H} 01005$ \\
\hline $0805003-23$ & B1V9C3 & $8.30 \mathrm{E} 0$ & $\mathrm{~N} / \mathrm{A}$ & 8/01/08 & $8 \mathrm{H} 01005$ \\
\hline 0805003-24 & B1V9C4 & $8.48 \mathrm{E} 0$ & $\mathrm{~N} / \mathrm{A}$ & $8 / 01 / 08$ & $8 \mathrm{H} 01005$ \\
\hline 0805003-25 & B1V2V5 & $8.60 \mathrm{E} 0$ & N/A & $8 / 01 / 08$ & $8 \mathrm{H} 01005$ \\
\hline
\end{tabular}




\section{Anions by Ion Chromatography}

\begin{tabular}{|c|c|c|c|c|c|c|c|}
\hline CAS \# & Analyte & Results & Units & EQL & Analyzed & Batch & Method \\
\hline HEIS No. & B1VDW5 & \multicolumn{3}{|c|}{ Lab ID: $\quad$ 0805001-01 } & & & \\
\hline $16984-48-8$ & Fluoride & $2.37 \mathrm{E}-1$ & ug/g dry & $1.99 \mathrm{E}-1$ & $8 / 06 / 08$ & $8 \mathrm{H} 05004$ & AGG-IC-001 \\
\hline $16887-00-6$ & Chloride & $6.21 \mathrm{E}-1$ & ug/g dry & $4.98 \mathrm{E}-1$ & $8 / 06 / 08$ & 8H05004 & AGG-IC-001 \\
\hline $14797-65-0$ & Nitrite & $<9.96 \mathrm{E}-1$ & ug/g dry & $9.96 \mathrm{E}-1$ & $8 / 06 / 08$ & 8H05004 & AGG-IC-001 \\
\hline 24959-67-9 & Bromide & $<9.96 \mathrm{E}-1$ & ug/g dry & $9.96 \mathrm{E}-1$ & $8 / 06 / 08$ & 8H05004 & AGG-IC-001 \\
\hline $14797-55-8$ & Nitrate & $2.74 \mathrm{E} 0$ & ug/g dry & $9.96 \mathrm{E}-1$ & $8 / 06 / 08$ & $8 \mathrm{H} 05004$ & AGG-IC-001 \\
\hline $14808-79-8$ & Sulfate & $<1.49 \mathrm{E} 0$ & ug/g dry & $1.49 \mathrm{E} 0$ & $8 / 06 / 08$ & 8H05004 & AGG-IC-001 \\
\hline $14265-44-2$ & Phosphate & $<1.49 \mathrm{E} 0$ & ug/g dry & 1.49E0 & $8 / 06 / 08$ & 8H05004 & AGG-IC-001 \\
\hline HEIS No. & B1VDW6 & \multicolumn{3}{|c|}{ Lab ID: $\quad$ 0805001-02 } & & & \\
\hline $16984-48-8$ & Fluoride & $6.08 \mathrm{E}-1$ & ug/g dry & $1.95 \mathrm{E}-1$ & $8 / 06 / 08$ & $8 \mathrm{H} 05004$ & AGG-IC-001 \\
\hline $16887-00-6$ & Chloride & $5.20 \mathrm{E}-1$ & ug/g dry & $4.88 \mathrm{E}-1$ & $8 / 06 / 08$ & 8H05004 & AGG-IC-001 \\
\hline $14797-65-0$ & Nitrite & $<9.76 \mathrm{E}-1$ & ug/g dry & $9.76 \mathrm{E}-1$ & $8 / 06 / 08$ & 8H05004 & AGG-IC-001 \\
\hline 24959-67-9 & Bromide & $<9.76 \mathrm{E}-1$ & ug/g dry & $9.76 \mathrm{E}-1$ & $8 / 06 / 08$ & 8H05004 & AGG-IC-001 \\
\hline $14797-55-8$ & Nitrate & $2.45 \mathrm{E} 0$ & ug/g dry & $9.76 \mathrm{E}-1$ & $8 / 06 / 08$ & $8 \mathrm{H} 05004$ & AGG-IC-001 \\
\hline $14808-79-8$ & Sulfate & $<1.46 \mathrm{E} 0$ & ug/g dry & $1.46 \mathrm{E} 0$ & $8 / 06 / 08$ & $8 \mathrm{H} 05004$ & AGG-IC-001 \\
\hline $14265-44-2$ & Phosphate & $<1.46 \mathrm{E} 0$ & ug/g dry & $1.46 \mathrm{E} 0$ & $8 / 06 / 08$ & $8 \mathrm{H} 05004$ & AGG-IC-001 \\
\hline HEIS No. & B1VDW7 & \multicolumn{3}{|c|}{ Lab ID: $\quad$ 0805001-03 } & & & \\
\hline $16984-48-8$ & Fluoride & $7.20 \mathrm{E}-1$ & ug/g dry & $1.99 \mathrm{E}-1$ & $8 / 06 / 08$ & $8 \mathrm{H} 05004$ & AGG-IC-001 \\
\hline $16887-00-6$ & Chloride & $8.08 \mathrm{E}-1$ & ug/g dry & $4.97 \mathrm{E}-1$ & $8 / 06 / 08$ & $8 \mathrm{H} 05004$ & AGG-IC-001 \\
\hline $14797-65-0$ & Nitrite & $<9.93 \mathrm{E}-1$ & ug/g dry & $9.93 \mathrm{E}-1$ & $8 / 06 / 08$ & 8H05004 & AGG-IC-001 \\
\hline $24959-67-9$ & Bromide & $<9.93 \mathrm{E}-1$ & ug/g dry & $9.93 \mathrm{E}-1$ & $8 / 06 / 08$ & $8 \mathrm{H} 05004$ & AGG-IC-001 \\
\hline $14797-55-8$ & Nitrate & $2.37 \mathrm{E} 0$ & ug/g dry & $9.93 \mathrm{E}-1$ & $8 / 06 / 08$ & $8 \mathrm{H} 05004$ & AGG-IC-001 \\
\hline $14808-79-8$ & Sulfate & $1.87 \mathrm{E} 0$ & ug/g dry & $1.49 \mathrm{E} 0$ & $8 / 06 / 08$ & 8H05004 & AGG-IC-001 \\
\hline $14265-44-2$ & Phosphate & $<1.49 \mathrm{E} 0$ & ug/g dry & $1.49 \mathrm{E} 0$ & $8 / 06 / 08$ & 8H05004 & AGG-IC-001 \\
\hline HEIS No. & B1VDW8 & \multicolumn{3}{|c|}{ Lab ID: $\quad$ 0805001-04 } & & & \\
\hline $16984-48-8$ & Fluoride & $9.32 \mathrm{E}-1$ & ug/g dry & $1.97 \mathrm{E}-1$ & $8 / 06 / 08$ & $8 \mathrm{H} 05004$ & AGG-IC-001 \\
\hline $16887-00-6$ & Chloride & $5.34 \mathrm{E}-1$ & ug/g dry & $4.92 \mathrm{E}-1$ & $8 / 06 / 08$ & $8 \mathrm{H} 05004$ & AGG-IC-001 \\
\hline $14797-65-0$ & Nitrite & $<9.85 \mathrm{E}-1$ & ug/g dry & $9.85 \mathrm{E}-1$ & $8 / 06 / 08$ & 8H05004 & AGG-IC-001 \\
\hline 24959-67-9 & Bromide & $<9.85 \mathrm{E}-1$ & ug/g dry & $9.85 \mathrm{E}-1$ & $8 / 06 / 08$ & $8 \mathrm{H} 05004$ & AGG-IC-001 \\
\hline $14797-55-8$ & Nitrate & $1.55 \mathrm{E} 0$ & ug/g dry & $9.85 \mathrm{E}-1$ & $8 / 06 / 08$ & $8 \mathrm{H} 05004$ & AGG-IC-001 \\
\hline $14808-79-8$ & Sulfate & $1.99 \mathrm{E} 0$ & ug/g dry & $1.48 \mathrm{E} 0$ & $8 / 06 / 08$ & $8 \mathrm{H} 05004$ & AGG-IC-001 \\
\hline $14265-44-2$ & Phosphate & $<1.48 \mathrm{E} 0$ & ug/g dry & $1.48 \mathrm{E} 0$ & $8 / 06 / 08$ & 8H05004 & AGG-IC-001 \\
\hline HEIS No. & B1VDW9 & \multicolumn{3}{|c|}{ Lab ID: $\quad$ 0805001-05 } & & & \\
\hline $16984-48-8$ & Fluoride & $5.95 \mathrm{E}-1$ & ug/g dry & $1.97 \mathrm{E}-1$ & $8 / 06 / 08$ & $8 \mathrm{H} 05004$ & AGG-IC-001 \\
\hline $16887-00-6$ & Chloride & $<4.93 \mathrm{E}-1$ & ug/g dry & $4.93 \mathrm{E}-1$ & $8 / 06 / 08$ & $8 \mathrm{H} 05004$ & AGG-IC-001 \\
\hline $14797-65-0$ & Nitrite & $<9.86 \mathrm{E}-1$ & ug/g dry & $9.86 \mathrm{E}-1$ & $8 / 06 / 08$ & $8 \mathrm{H} 05004$ & AGG-IC-001 \\
\hline $24959-67-9$ & Bromide & $<9.86 \mathrm{E}-1$ & ug/g dry & $9.86 \mathrm{E}-1$ & $8 / 06 / 08$ & 8H05004 & AGG-IC-001 \\
\hline $14797-55-8$ & Nitrate & $<9.86 \mathrm{E}-1$ & ug/g dry & $9.86 \mathrm{E}-1$ & $8 / 06 / 08$ & 8H05004 & AGG-IC-001 \\
\hline $14808-79-8$ & Sulfate & $<1.48 \mathrm{E} 0$ & ug/g dry & $1.48 \mathrm{E} 0$ & $8 / 06 / 08$ & $8 \mathrm{H} 05004$ & AGG-IC-001 \\
\hline $14265-44-2$ & Phosphate & $<1.48 \mathrm{E} 0$ & ug/g dry & $1.48 \mathrm{E} 0$ & $8 / 06 / 08$ & 8H05004 & AGG-IC-001 \\
\hline HEIS No. & B1VDX0 & \multicolumn{3}{|c|}{ Lab ID: $\quad$ 0805001-06 } & & & \\
\hline $16984-48-8$ & Fluoride & $9.93 \mathrm{E}-1$ & ug/g dry & $2.16 \mathrm{E}-1$ & $8 / 06 / 08$ & $8 \mathrm{H} 05004$ & AGG-IC-001 \\
\hline $16887-00-6$ & Chloride & $<5.39 \mathrm{E}-1$ & ug/g dry & $5.39 \mathrm{E}-1$ & $8 / 06 / 08$ & $8 \mathrm{H} 05004$ & AGG-IC-001 \\
\hline $14797-65-0$ & Nitrite & $<1.08 \mathrm{E} 0$ & ug/g dry & $1.08 \mathrm{E} 0$ & $8 / 06 / 08$ & $8 \mathrm{H} 05004$ & AGG-IC-001 \\
\hline $24959-67-9$ & Bromide & $<1.08 \mathrm{E} 0$ & ug/g dry & $1.08 \mathrm{E} 0$ & $8 / 06 / 08$ & 8H05004 & AGG-IC-001 \\
\hline $14797-55-8$ & Nitrate & $<1.08 \mathrm{E} 0$ & ug/g dry & $1.08 \mathrm{E} 0$ & $8 / 06 / 08$ & $8 \mathrm{H} 05004$ & AGG-IC-001 \\
\hline $14808-79-8$ & Sulfate & $1.66 \mathrm{E} 0$ & ug/g dry & $1.62 \mathrm{E} 0$ & $8 / 06 / 08$ & $8 \mathrm{H} 05004$ & AGG-IC-001 \\
\hline $14265-44-2$ & Phosphate & $<1.62 \mathrm{E} 0$ & ug/g dry & $1.62 \mathrm{E} 0$ & 8/06/08 & $8 \mathrm{H} 05004$ & AGG-IC-001 \\
\hline
\end{tabular}




\section{Anions by Ion Chromatography}

\begin{tabular}{|c|c|c|c|c|c|c|c|}
\hline CAS \# & Analyte & Results & Units & EQL & Analyzed & Batch & Method \\
\hline HEIS No. & B1V991 & \multicolumn{3}{|c|}{ Lab ID: $\quad$ 0805003-01 } & & & \\
\hline $16984-48-8$ & Fluoride & $2.43 \mathrm{E}-1$ & ug/g dry & $2.00 \mathrm{E}-1$ & $8 / 06 / 08$ & $8 \mathrm{H} 05004$ & AGG-IC-001 \\
\hline $16887-00-6$ & Chloride & $<5.00 \mathrm{E}-1$ & ug/g dry & $5.00 \mathrm{E}-1$ & $8 / 06 / 08$ & 8H05004 & AGG-IC-001 \\
\hline $14797-65-0$ & Nitrite & $<1.00 \mathrm{E} 0$ & ug/g dry & $1.00 \mathrm{E} 0$ & $8 / 06 / 08$ & 8H05004 & AGG-IC-001 \\
\hline 24959-67-9 & Bromide & $<1.00 \mathrm{E} 0$ & ug/g dry & $1.00 \mathrm{E} 0$ & $8 / 06 / 08$ & 8H05004 & AGG-IC-001 \\
\hline $14797-55-8$ & Nitrate & $2.40 \mathrm{E} 0$ & ug/g dry & $1.00 \mathrm{E} 0$ & $8 / 06 / 08$ & $8 \mathrm{H} 05004$ & AGG-IC-001 \\
\hline $14808-79-8$ & Sulfate & $<1.50 \mathrm{E} 0$ & ug/g dry & $1.50 \mathrm{E} 0$ & $8 / 06 / 08$ & 8H05004 & AGG-IC-001 \\
\hline $14265-44-2$ & Phosphate & $<1.50 \mathrm{E} 0$ & ug/g dry & $1.50 \mathrm{E} 0$ & $8 / 06 / 08$ & 8H05004 & AGG-IC-001 \\
\hline HEIS No. & B1V992 & \multicolumn{3}{|c|}{ Lab ID: $\quad$ 0805003-02 } & & & \\
\hline $16984-48-8$ & Fluoride & $1.37 \mathrm{E} 0$ & ug/g dry & $1.99 \mathrm{E}-1$ & $8 / 06 / 08$ & $8 \mathrm{H} 05004$ & AGG-IC-001 \\
\hline $16887-00-6$ & Chloride & $<4.99 \mathrm{E}-1$ & ug/g dry & $4.99 \mathrm{E}-1$ & $8 / 06 / 08$ & 8H05004 & AGG-IC-001 \\
\hline $14797-65-0$ & Nitrite & $<9.97 \mathrm{E}-1$ & ug/g dry & $9.97 \mathrm{E}-1$ & $8 / 06 / 08$ & 8H05004 & AGG-IC-001 \\
\hline 24959-67-9 & Bromide & $<9.97 \mathrm{E}-1$ & ug/g dry & $9.97 \mathrm{E}-1$ & $8 / 06 / 08$ & 8H05004 & AGG-IC-001 \\
\hline $14797-55-8$ & Nitrate & $<9.97 \mathrm{E}-1$ & ug/g dry & $9.97 \mathrm{E}-1$ & $8 / 06 / 08$ & $8 \mathrm{H} 05004$ & AGG-IC-001 \\
\hline $14808-79-8$ & Sulfate & $1.68 \mathrm{E} 0$ & ug/g dry & $1.50 \mathrm{E} 0$ & $8 / 06 / 08$ & $8 \mathrm{H} 05004$ & AGG-IC-001 \\
\hline $14265-44-2$ & Phosphate & $<1.50 \mathrm{E} 0$ & ug/g dry & $1.50 \mathrm{E} 0$ & $8 / 06 / 08$ & $8 \mathrm{H} 05004$ & AGG-IC-001 \\
\hline HEIS No. & B1V993 & \multicolumn{3}{|c|}{ Lab ID: $\quad$ 0805003-03 } & & & \\
\hline $16984-48-8$ & Fluoride & $4.00 \mathrm{E}-1$ & ug/g dry & $1.98 \mathrm{E}-1$ & $8 / 06 / 08$ & $8 \mathrm{H} 05004$ & AGG-IC-001 \\
\hline $16887-00-6$ & Chloride & $9.46 \mathrm{E}-1$ & ug/g dry & $4.96 \mathrm{E}-1$ & $8 / 06 / 08$ & $8 \mathrm{H} 05004$ & AGG-IC-001 \\
\hline $14797-65-0$ & Nitrite & $<9.92 \mathrm{E}-1$ & ug/g dry & $9.92 \mathrm{E}-1$ & $8 / 06 / 08$ & 8H05004 & AGG-IC-001 \\
\hline 24959-67-9 & Bromide & $<9.92 \mathrm{E}-1$ & ug/g dry & $9.92 \mathrm{E}-1$ & $8 / 06 / 08$ & $8 \mathrm{H} 05004$ & AGG-IC-001 \\
\hline $14797-55-8$ & Nitrate & $1.98 \mathrm{E} 0$ & ug/g dry & $9.92 \mathrm{E}-1$ & $8 / 06 / 08$ & $8 \mathrm{H} 05004$ & AGG-IC-001 \\
\hline $14808-79-8$ & Sulfate & $<1.49 \mathrm{E} 0$ & ug/g dry & $1.49 \mathrm{E} 0$ & $8 / 06 / 08$ & 8H05004 & AGG-IC-001 \\
\hline $14265-44-2$ & Phosphate & $<1.49 \mathrm{E} 0$ & ug/g dry & $1.49 \mathrm{E} 0$ & $8 / 06 / 08$ & 8H05004 & AGG-IC-001 \\
\hline HEIS No. & B1V994 & \multicolumn{3}{|c|}{ Lab ID: $\quad$ 0805003-04 } & & & \\
\hline $16984-48-8$ & Fluoride & $1.05 \mathrm{E} 0$ & ug/g dry & $1.99 \mathrm{E}-1$ & $8 / 06 / 08$ & $8 \mathrm{H} 05004$ & AGG-IC-001 \\
\hline $16887-00-6$ & Chloride & $5.80 \mathrm{E}-1$ & ug/g dry & $4.98 \mathrm{E}-1$ & $8 / 06 / 08$ & $8 \mathrm{H} 05004$ & AGG-IC-001 \\
\hline $14797-65-0$ & Nitrite & $<9.96 \mathrm{E}-1$ & ug/g dry & $9.96 \mathrm{E}-1$ & $8 / 06 / 08$ & 8H05004 & AGG-IC-001 \\
\hline 24959-67-9 & Bromide & $<9.96 \mathrm{E}-1$ & ug/g dry & $9.96 \mathrm{E}-1$ & $8 / 06 / 08$ & $8 \mathrm{H} 05004$ & AGG-IC-001 \\
\hline $14797-55-8$ & Nitrate & $1.53 \mathrm{E} 0$ & ug/g dry & $9.96 \mathrm{E}-1$ & $8 / 06 / 08$ & $8 \mathrm{H} 05004$ & AGG-IC-001 \\
\hline $14808-79-8$ & Sulfate & $2.72 \mathrm{E} 0$ & ug/g dry & $1.49 \mathrm{E} 0$ & $8 / 06 / 08$ & $8 \mathrm{H} 05004$ & AGG-IC-001 \\
\hline $14265-44-2$ & Phosphate & $<1.49 \mathrm{E} 0$ & ug/g dry & $1.49 \mathrm{E} 0$ & $8 / 06 / 08$ & 8H05004 & AGG-IC-001 \\
\hline HEIS No. & B1V995 & \multicolumn{3}{|c|}{ Lab ID: $\quad$ 0805003-05 } & & & \\
\hline $16984-48-8$ & Fluoride & $1.01 \mathrm{E} 0$ & ug/g dry & $2.00 \mathrm{E}-1$ & $8 / 06 / 08$ & $8 \mathrm{H} 05004$ & AGG-IC-001 \\
\hline $16887-00-6$ & Chloride & $<5.00 \mathrm{E}-1$ & ug/g dry & $5.00 \mathrm{E}-1$ & $8 / 06 / 08$ & $8 \mathrm{H} 05004$ & AGG-IC-001 \\
\hline $14797-65-0$ & Nitrite & $<1.00 \mathrm{E} 0$ & ug/g dry & $1.00 \mathrm{E} 0$ & $8 / 06 / 08$ & $8 \mathrm{H} 05004$ & AGG-IC-001 \\
\hline $24959-67-9$ & Bromide & $<1.00 \mathrm{E} 0$ & ug/g dry & $1.00 \mathrm{E} 0$ & $8 / 06 / 08$ & 8H05004 & AGG-IC-001 \\
\hline $14797-55-8$ & Nitrate & $1.44 \mathrm{E} 0$ & ug/g dry & $1.00 \mathrm{E} 0$ & $8 / 06 / 08$ & 8H05004 & AGG-IC-001 \\
\hline $14808-79-8$ & Sulfate & $2.02 \mathrm{E} 0$ & ug/g dry & $1.50 \mathrm{E} 0$ & $8 / 06 / 08$ & $8 \mathrm{H} 05004$ & AGG-IC-001 \\
\hline $14265-44-2$ & Phosphate & $<1.50 \mathrm{E} 0$ & ug/g dry & $1.50 \mathrm{E} 0$ & $8 / 06 / 08$ & 8H05004 & AGG-IC-001 \\
\hline HEIS No. & B1V996 & \multicolumn{3}{|c|}{ Lab ID: $\quad$ 0805003-06 } & & & \\
\hline $16984-48-8$ & Fluoride & $1.14 \mathrm{E} 0$ & ug/g dry & $2.00 \mathrm{E}-1$ & $8 / 01 / 08$ & $8 \mathrm{H} 01004$ & AGG-IC-001 \\
\hline $16887-00-6$ & Chloride & $<5.00 \mathrm{E}-1$ & ug/g dry & $5.00 \mathrm{E}-1$ & $8 / 01 / 08$ & $8 \mathrm{H} 01004$ & AGG-IC-001 \\
\hline $14797-65-0$ & Nitrite & $<1.00 \mathrm{E} 0$ & ug/g dry & $1.00 \mathrm{E} 0$ & $8 / 01 / 08$ & 8H01004 & AGG-IC-001 \\
\hline $24959-67-9$ & Bromide & $<1.00 \mathrm{E} 0$ & ug/g dry & $1.00 \mathrm{E} 0$ & $8 / 01 / 08$ & 8H01004 & AGG-IC-001 \\
\hline $14797-55-8$ & Nitrate & 4.60E0 & ug/g dry & $1.00 \mathrm{E} 0$ & 8/01/08 & 8H01004 & AGG-IC-001 \\
\hline $14808-79-8$ & Sulfate & $2.68 \mathrm{E} 0$ & ug/g dry & $1.50 \mathrm{E} 0$ & $8 / 01 / 08$ & $8 \mathrm{H} 01004$ & AGG-IC-001 \\
\hline $14265-44-2$ & Phosphate & $<1.50 \mathrm{E} 0$ & ug/g dry & $1.50 \mathrm{E} 0$ & 8/01/08 & 8H01004 & AGG-IC-001 \\
\hline
\end{tabular}




\section{Anions by Ion Chromatography}

\begin{tabular}{|c|c|c|c|c|c|c|c|}
\hline CAS \# & Analyte & Results & Units & EQL & Analyzed & Batch & Method \\
\hline HEIS No. & B1V997 & \multicolumn{3}{|c|}{ Lab ID: $\quad$ 0805003-07 } & & & \\
\hline $16984-48-8$ & Fluoride & $1.01 \mathrm{E} 0$ & ug/g dry & $2.00 \mathrm{E}-1$ & $8 / 01 / 08$ & $8 \mathrm{H} 01004$ & AGG-IC-001 \\
\hline $16887-00-6$ & Chloride & $<5.00 \mathrm{E}-1$ & ug/g dry & $5.00 \mathrm{E}-1$ & $8 / 01 / 08$ & 8H01004 & AGG-IC-001 \\
\hline $14797-65-0$ & Nitrite & $<9.99 \mathrm{E}-1$ & ug/g dry & $9.99 \mathrm{E}-1$ & $8 / 01 / 08$ & 8H01004 & AGG-IC-001 \\
\hline 24959-67-9 & Bromide & $<9.99 \mathrm{E}-1$ & ug/g dry & $9.99 \mathrm{E}-1$ & $8 / 01 / 08$ & 8H01004 & AGG-IC-001 \\
\hline $14797-55-8$ & Nitrate & $4.16 \mathrm{E} 0$ & ug/g dry & $9.99 \mathrm{E}-1$ & $8 / 01 / 08$ & $8 \mathrm{H} 01004$ & AGG-IC-001 \\
\hline $14808-79-8$ & Sulfate & $4.78 \mathrm{E} 0$ & ug/g dry & $1.50 \mathrm{E} 0$ & $8 / 01 / 08$ & 8H01004 & AGG-IC-001 \\
\hline $14265-44-2$ & Phosphate & $<1.50 \mathrm{E} 0$ & ug/g dry & $1.50 \mathrm{E} 0$ & $8 / 01 / 08$ & 8H01004 & AGG-IC-001 \\
\hline HEIS No. & B1V998 & \multicolumn{3}{|c|}{ Lab ID: $\quad$ 0805003-08 } & & & \\
\hline $16984-48-8$ & Fluoride & $7.16 \mathrm{E}-1$ & ug/g dry & $2.00 \mathrm{E}-1$ & $8 / 01 / 08$ & $8 \mathrm{H} 01004$ & AGG-IC-001 \\
\hline $16887-00-6$ & Chloride & $<5.00 \mathrm{E}-1$ & ug/g dry & $5.00 \mathrm{E}-1$ & $8 / 01 / 08$ & 8H01004 & AGG-IC-001 \\
\hline $14797-65-0$ & Nitrite & $<1.00 \mathrm{E} 0$ & ug/g dry & $1.00 \mathrm{E} 0$ & $8 / 01 / 08$ & 8H01004 & AGG-IC-001 \\
\hline 24959-67-9 & Bromide & $<1.00 \mathrm{E} 0$ & ug/g dry & $1.00 \mathrm{E} 0$ & $8 / 01 / 08$ & $8 \mathrm{H} 01004$ & AGG-IC-001 \\
\hline $14797-55-8$ & Nitrate & $3.59 \mathrm{E} 0$ & ug/g dry & $1.00 \mathrm{E} 0$ & $8 / 01 / 08$ & $8 \mathrm{H} 01004$ & AGG-IC-001 \\
\hline $14808-79-8$ & Sulfate & $8.36 \mathrm{E} 0$ & ug/g dry & $1.50 \mathrm{E} 0$ & $8 / 01 / 08$ & 8H01004 & AGG-IC-001 \\
\hline $14265-44-2$ & Phosphate & $<1.50 \mathrm{E} 0$ & ug/g dry & $1.50 \mathrm{E} 0$ & $8 / 01 / 08$ & $8 \mathrm{H} 01004$ & AGG-IC-001 \\
\hline HEIS No. & B1V999 & \multicolumn{3}{|c|}{ Lab ID: $\quad$ 0805003-09 } & & & \\
\hline $16984-48-8$ & Fluoride & $6.47 \mathrm{E}-1$ & ug/g dry & $1.99 \mathrm{E}-1$ & $8 / 01 / 08$ & $8 \mathrm{H} 01004$ & AGG-IC-001 \\
\hline $16887-00-6$ & Chloride & $5.70 \mathrm{E}-1$ & ug/g dry & $4.99 \mathrm{E}-1$ & $8 / 01 / 08$ & 8H01004 & AGG-IC-001 \\
\hline $14797-65-0$ & Nitrite & $<9.97 \mathrm{E}-1$ & ug/g dry & $9.97 \mathrm{E}-1$ & $8 / 01 / 08$ & 8H01004 & AGG-IC-001 \\
\hline 24959-67-9 & Bromide & $<9.97 \mathrm{E}-1$ & ug/g dry & $9.97 \mathrm{E}-1$ & $8 / 01 / 08$ & $8 \mathrm{H} 01004$ & AGG-IC-001 \\
\hline $14797-55-8$ & Nitrate & $4.26 \mathrm{E} 0$ & ug/g dry & $9.97 \mathrm{E}-1$ & $8 / 01 / 08$ & $8 \mathrm{H} 01004$ & AGG-IC-001 \\
\hline $14808-79-8$ & Sulfate & $1.09 \mathrm{E} 1$ & ug/g dry & $1.50 \mathrm{E} 0$ & $8 / 01 / 08$ & 8H01004 & AGG-IC-001 \\
\hline $14265-44-2$ & Phosphate & $<1.50 \mathrm{E} 0$ & ug/g dry & $1.50 \mathrm{E} 0$ & $8 / 01 / 08$ & 8H01004 & AGG-IC-001 \\
\hline HEIS No. & B1V9B0 & \multicolumn{3}{|c|}{ Lab ID: $\quad$ 0805003-10 } & & & \\
\hline $16984-48-8$ & Fluoride & $3.74 \mathrm{E}-1$ & ug/g dry & $1.99 \mathrm{E}-1$ & $8 / 01 / 08$ & 8H01004 & AGG-IC-001 \\
\hline $16887-00-6$ & Chloride & $6.72 \mathrm{E}-1$ & ug/g dry & $4.98 \mathrm{E}-1$ & $8 / 01 / 08$ & 8H01004 & AGG-IC-001 \\
\hline $14797-65-0$ & Nitrite & $<9.97 \mathrm{E}-1$ & ug/g dry & $9.97 \mathrm{E}-1$ & $8 / 01 / 08$ & 8H01004 & AGG-IC-001 \\
\hline 24959-67-9 & Bromide & $<9.97 \mathrm{E}-1$ & ug/g dry & $9.97 \mathrm{E}-1$ & $8 / 01 / 08$ & $8 \mathrm{H} 01004$ & AGG-IC-001 \\
\hline $14797-55-8$ & Nitrate & $4.81 \mathrm{E} 0$ & ug/g dry & $9.97 \mathrm{E}-1$ & $8 / 01 / 08$ & $8 \mathrm{H} 01004$ & AGG-IC-001 \\
\hline $14808-79-8$ & Sulfate & $1.19 \mathrm{E} 1$ & ug/g dry & $1.49 \mathrm{E} 0$ & $8 / 01 / 08$ & $8 \mathrm{H} 01004$ & AGG-IC-001 \\
\hline 14265-44-2 & Phosphate & $<1.49 \mathrm{E} 0$ & ug/g dry & $1.49 \mathrm{E} 0$ & 8/01/08 & 8H01004 & AGG-IC-001 \\
\hline HEIS No. & B1V9B1 & \multicolumn{3}{|c|}{ Lab ID: $\quad$ 0805003-11 } & & & \\
\hline $16984-48-8$ & Fluoride & $4.30 \mathrm{E}-1$ & ug/g dry & $2.00 \mathrm{E}-1$ & $8 / 01 / 08$ & $8 \mathrm{H} 01004$ & AGG-IC-001 \\
\hline $16887-00-6$ & Chloride & $6.51 \mathrm{E}-1$ & ug/g dry & $4.99 \mathrm{E}-1$ & $8 / 01 / 08$ & $8 \mathrm{H} 01004$ & AGG-IC-001 \\
\hline $14797-65-0$ & Nitrite & $<9.98 \mathrm{E}-1$ & ug/g dry & $9.98 \mathrm{E}-1$ & $8 / 01 / 08$ & 8H01004 & AGG-IC-001 \\
\hline 24959-67-9 & Bromide & $<9.98 \mathrm{E}-1$ & ug/g dry & $9.98 \mathrm{E}-1$ & $8 / 01 / 08$ & 8H01004 & AGG-IC-001 \\
\hline $14797-55-8$ & Nitrate & $5.54 \mathrm{E} 0$ & ug/g dry & $9.98 \mathrm{E}-1$ & $8 / 01 / 08$ & 8H01004 & AGG-IC-001 \\
\hline $14808-79-8$ & Sulfate & $1.08 \mathrm{E} 1$ & ug/g dry & $1.50 \mathrm{E} 0$ & 8/01/08 & 8H01004 & AGG-IC-001 \\
\hline $14265-44-2$ & Phosphate & $<1.50 \mathrm{E} 0$ & ug/g dry & $1.50 \mathrm{E} 0$ & $8 / 01 / 08$ & 8H01004 & AGG-IC-001 \\
\hline HEIS No. & B1V9B2 & \multicolumn{3}{|c|}{ Lab ID: $\quad$ 0805003-12 } & & & \\
\hline $16984-48-8$ & Fluoride & $4.67 \mathrm{E}-1$ & ug/g dry & $2.00 \mathrm{E}-1$ & $8 / 01 / 08$ & $8 \mathrm{H} 01004$ & AGG-IC-001 \\
\hline $16887-00-6$ & Chloride & $1.01 \mathrm{E} 0$ & ug/g dry & $5.00 \mathrm{E}-1$ & $8 / 01 / 08$ & $8 \mathrm{H} 01004$ & AGG-IC-001 \\
\hline $14797-65-0$ & Nitrite & $<1.00 \mathrm{E} 0$ & ug/g dry & $1.00 \mathrm{E} 0$ & $8 / 01 / 08$ & 8H01004 & AGG-IC-001 \\
\hline 24959-67-9 & Bromide & $<1.00 \mathrm{E} 0$ & ug/g dry & $1.00 \mathrm{E} 0$ & $8 / 01 / 08$ & 8H01004 & AGG-IC-001 \\
\hline $14797-55-8$ & Nitrate & 4.42E0 & ug/g dry & $1.00 \mathrm{E} 0$ & 8/01/08 & 8H01004 & AGG-IC-001 \\
\hline $14808-79-8$ & Sulfate & $1.23 \mathrm{E} 1$ & ug/g dry & $1.50 \mathrm{E} 0$ & $8 / 01 / 08$ & $8 \mathrm{H} 01004$ & AGG-IC-001 \\
\hline $14265-44-2$ & Phosphate & $<1.50 \mathrm{E} 0$ & ug/g dry & $1.50 \mathrm{E} 0$ & 8/01/08 & 8H01004 & AGG-IC-001 \\
\hline
\end{tabular}




\section{Anions by Ion Chromatography}

\begin{tabular}{|c|c|c|c|c|c|c|c|}
\hline CAS \# & Analyte & Results & Units & EQL & Analyzed & Batch & Method \\
\hline HEIS No. & B1V9B3 & \multicolumn{3}{|c|}{ Lab ID: $\quad$ 0805003-13 } & & & \\
\hline $16984-48-8$ & Fluoride & $1.02 \mathrm{E} 0$ & ug/g dry & $2.00 \mathrm{E}-1$ & $8 / 01 / 08$ & $8 \mathrm{H} 01004$ & AGG-IC-001 \\
\hline $16887-00-6$ & Chloride & $1.48 \mathrm{E} 0$ & ug/g dry & $5.00 \mathrm{E}-1$ & $8 / 01 / 08$ & 8H01004 & AGG-IC-001 \\
\hline $14797-65-0$ & Nitrite & $<1.00 \mathrm{E} 0$ & ug/g dry & $1.00 \mathrm{E} 0$ & $8 / 01 / 08$ & 8H01004 & AGG-IC-001 \\
\hline 24959-67-9 & Bromide & $<1.00 \mathrm{E} 0$ & ug/g dry & $1.00 \mathrm{E} 0$ & $8 / 01 / 08$ & 8H01004 & AGG-IC-001 \\
\hline $14797-55-8$ & Nitrate & $4.00 \mathrm{E} 0$ & ug/g dry & $1.00 \mathrm{E} 0$ & $8 / 01 / 08$ & $8 \mathrm{H} 01004$ & AGG-IC-001 \\
\hline $14808-79-8$ & Sulfate & $1.81 \mathrm{E} 1$ & ug/g dry & $1.50 \mathrm{E} 0$ & $8 / 01 / 08$ & 8H01004 & AGG-IC-001 \\
\hline $14265-44-2$ & Phosphate & $<1.50 \mathrm{E} 0$ & ug/g dry & $1.50 \mathrm{E} 0$ & $8 / 01 / 08$ & 8H01004 & AGG-IC-001 \\
\hline HEIS No. & B1V9B4 & \multicolumn{3}{|c|}{ Lab ID: $\quad 0805003-14$} & & & \\
\hline $16984-48-8$ & Fluoride & $4.33 \mathrm{E}-1$ & ug/g dry & $2.00 \mathrm{E}-1$ & $8 / 01 / 08$ & $8 \mathrm{H} 01004$ & AGG-IC-001 \\
\hline $16887-00-6$ & Chloride & 7.17E-1 & ug/g dry & $4.99 \mathrm{E}-1$ & $8 / 01 / 08$ & 8H01004 & AGG-IC-001 \\
\hline $14797-65-0$ & Nitrite & $<9.98 \mathrm{E}-1$ & ug/g dry & $9.98 \mathrm{E}-1$ & $8 / 01 / 08$ & 8H01004 & AGG-IC-001 \\
\hline 24959-67-9 & Bromide & $<9.98 \mathrm{E}-1$ & ug/g dry & $9.98 \mathrm{E}-1$ & $8 / 01 / 08$ & 8H01004 & AGG-IC-001 \\
\hline $14797-55-8$ & Nitrate & 7.43E0 & ug/g dry & $9.98 \mathrm{E}-1$ & $8 / 01 / 08$ & $8 \mathrm{H} 01004$ & AGG-IC-001 \\
\hline $14808-79-8$ & Sulfate & $8.54 \mathrm{E} 0$ & ug/g dry & $1.50 \mathrm{E} 0$ & $8 / 01 / 08$ & 8H01004 & AGG-IC-001 \\
\hline $14265-44-2$ & Phosphate & $<1.50 \mathrm{E} 0$ & ug/g dry & $1.50 \mathrm{E} 0$ & $8 / 01 / 08$ & $8 \mathrm{H} 01004$ & AGG-IC-001 \\
\hline HEIS No. & B1V9B5 & \multicolumn{3}{|c|}{ Lab ID: $\quad$ 0805003-15 } & & & \\
\hline $16984-48-8$ & Fluoride & $2.34 \mathrm{E}-1$ & ug/g dry & $2.00 \mathrm{E}-1$ & $8 / 01 / 08$ & $8 \mathrm{H} 01004$ & AGG-IC-001 \\
\hline $16887-00-6$ & Chloride & $<5.00 \mathrm{E}-1$ & ug/g dry & $5.00 \mathrm{E}-1$ & $8 / 01 / 08$ & $8 \mathrm{H} 01004$ & AGG-IC-001 \\
\hline $14797-65-0$ & Nitrite & $<9.99 \mathrm{E}-1$ & ug/g dry & $9.99 \mathrm{E}-1$ & $8 / 01 / 08$ & 8H01004 & AGG-IC-001 \\
\hline 24959-67-9 & Bromide & $<9.99 \mathrm{E}-1$ & ug/g dry & $9.99 \mathrm{E}-1$ & $8 / 01 / 08$ & $8 \mathrm{H} 01004$ & AGG-IC-001 \\
\hline $14797-55-8$ & Nitrate & $2.46 \mathrm{E} 0$ & ug/g dry & $9.99 \mathrm{E}-1$ & $8 / 01 / 08$ & $8 \mathrm{H} 01004$ & AGG-IC-001 \\
\hline $14808-79-8$ & Sulfate & 7.27E0 & ug/g dry & $1.50 \mathrm{E} 0$ & $8 / 01 / 08$ & 8H01004 & AGG-IC-001 \\
\hline $14265-44-2$ & Phosphate & $<1.50 \mathrm{E} 0$ & ug/g dry & $1.50 \mathrm{E} 0$ & $8 / 01 / 08$ & 8H01004 & AGG-IC-001 \\
\hline HEIS No. & B1V9B6 & \multicolumn{3}{|c|}{ Lab ID: $\quad$ 0805003-16 } & & & \\
\hline $16984-48-8$ & Fluoride & $2.40 \mathrm{E}-1$ & ug/g dry & $1.99 \mathrm{E}-1$ & $8 / 01 / 08$ & 8H01004 & AGG-IC-001 \\
\hline $16887-00-6$ & Chloride & $<4.98 \mathrm{E}-1$ & ug/g dry & $4.98 \mathrm{E}-1$ & $8 / 01 / 08$ & 8H01004 & AGG-IC-001 \\
\hline $14797-65-0$ & Nitrite & $<9.97 \mathrm{E}-1$ & ug/g dry & $9.97 \mathrm{E}-1$ & $8 / 01 / 08$ & 8H01004 & AGG-IC-001 \\
\hline 24959-67-9 & Bromide & $<9.97 \mathrm{E}-1$ & ug/g dry & $9.97 \mathrm{E}-1$ & $8 / 01 / 08$ & $8 \mathrm{H} 01004$ & AGG-IC-001 \\
\hline $14797-55-8$ & Nitrate & $5.69 \mathrm{E} 0$ & ug/g dry & $9.97 \mathrm{E}-1$ & $8 / 01 / 08$ & $8 \mathrm{H} 01004$ & AGG-IC-001 \\
\hline $14808-79-8$ & Sulfate & $1.44 \mathrm{E} 1$ & ug/g dry & $1.49 \mathrm{E} 0$ & $8 / 01 / 08$ & $8 \mathrm{H} 01004$ & AGG-IC-001 \\
\hline $14265-44-2$ & Phosphate & $<1.49 \mathrm{E} 0$ & ug/g dry & $1.49 \mathrm{E} 0$ & 8/01/08 & 8H01004 & AGG-IC-001 \\
\hline HEIS No. & B1V9B7 & \multicolumn{3}{|c|}{ Lab ID: $\quad$ 0805003-17 } & & & \\
\hline $16984-48-8$ & Fluoride & $<2.00 \mathrm{E}-1$ & ug/g dry & $2.00 \mathrm{E}-1$ & $8 / 01 / 08$ & $8 \mathrm{H} 01004$ & AGG-IC-001 \\
\hline $16887-00-6$ & Chloride & 4.77E0 & ug/g dry & $5.00 \mathrm{E}-1$ & $8 / 01 / 08$ & $8 \mathrm{H} 01004$ & AGG-IC-001 \\
\hline $14797-65-0$ & Nitrite & $<9.99 \mathrm{E}-1$ & ug/g dry & $9.99 \mathrm{E}-1$ & $8 / 01 / 08$ & 8H01004 & AGG-IC-001 \\
\hline $24959-67-9$ & Bromide & $<9.99 \mathrm{E}-1$ & ug/g dry & $9.99 \mathrm{E}-1$ & $8 / 01 / 08$ & 8H01004 & AGG-IC-001 \\
\hline $14797-55-8$ & Nitrate & 7.43E0 & ug/g dry & $9.99 \mathrm{E}-1$ & $8 / 01 / 08$ & 8H01004 & AGG-IC-001 \\
\hline $14808-79-8$ & Sulfate & $1.89 \mathrm{E} 1$ & ug/g dry & $1.50 \mathrm{E} 0$ & 8/01/08 & 8H01004 & AGG-IC-001 \\
\hline $14265-44-2$ & Phosphate & $<1.50 \mathrm{E} 0$ & ug/g dry & $1.50 \mathrm{E} 0$ & $8 / 01 / 08$ & 8H01004 & AGG-IC-001 \\
\hline HEIS No. & B1V9B8 & \multicolumn{3}{|c|}{ Lab ID: $\quad$ 0805003-18 } & & & \\
\hline $16984-48-8$ & Fluoride & $2.12 \mathrm{E}-1$ & ug/g dry & $2.01 \mathrm{E}-1$ & $8 / 01 / 08$ & $8 \mathrm{H} 01004$ & AGG-IC-001 \\
\hline $16887-00-6$ & Chloride & $6.68 \mathrm{E} 0$ & ug/g dry & $5.03 \mathrm{E}-1$ & $8 / 01 / 08$ & $8 \mathrm{H} 01004$ & AGG-IC-001 \\
\hline $14797-65-0$ & Nitrite & $<1.01 \mathrm{E} 0$ & ug/g dry & $1.01 \mathrm{E} 0$ & $8 / 01 / 08$ & 8H01004 & AGG-IC-001 \\
\hline $24959-67-9$ & Bromide & $<1.01 \mathrm{E} 0$ & ug/g dry & $1.01 \mathrm{E} 0$ & $8 / 01 / 08$ & 8H01004 & AGG-IC-001 \\
\hline $14797-55-8$ & Nitrate & $5.42 \mathrm{E} 0$ & ug/g dry & $1.01 \mathrm{E} 0$ & 8/01/08 & 8H01004 & AGG-IC-001 \\
\hline $14808-79-8$ & Sulfate & $1.45 \mathrm{E} 1$ & ug/g dry & $1.51 \mathrm{E} 0$ & $8 / 01 / 08$ & $8 \mathrm{H} 01004$ & AGG-IC-001 \\
\hline $14265-44-2$ & Phosphate & $<1.51 \mathrm{E} 0$ & ug/g dry & $1.51 \mathrm{E} 0$ & 8/01/08 & 8H01004 & AGG-IC-001 \\
\hline
\end{tabular}




\section{Anions by Ion Chromatography}

\begin{tabular}{|c|c|c|c|c|c|c|c|}
\hline CAS \# & Analyte & Results & Units & EQL & Analyzed & Batch & Method \\
\hline HEIS No. & B1V9B9 & \multicolumn{3}{|c|}{ Lab ID: $\quad$ 0805003-19 } & & & \\
\hline $16984-48-8$ & Fluoride & $2.63 \mathrm{E}-1$ & ug/g dry & $1.99 \mathrm{E}-1$ & $8 / 01 / 08$ & $8 \mathrm{H} 01004$ & AGG-IC-001 \\
\hline $16887-00-6$ & Chloride & $7.23 \mathrm{E} 0$ & ug/g dry & $4.98 \mathrm{E}-1$ & $8 / 01 / 08$ & 8H01004 & AGG-IC-001 \\
\hline $14797-65-0$ & Nitrite & $<9.95 \mathrm{E}-1$ & ug/g dry & $9.95 \mathrm{E}-1$ & $8 / 01 / 08$ & 8H01004 & AGG-IC-001 \\
\hline 24959-67-9 & Bromide & $<9.95 \mathrm{E}-1$ & ug/g dry & $9.95 \mathrm{E}-1$ & $8 / 01 / 08$ & 8H01004 & AGG-IC-001 \\
\hline $14797-55-8$ & Nitrate & $3.88 \mathrm{E} 0$ & ug/g dry & $9.95 \mathrm{E}-1$ & $8 / 01 / 08$ & $8 \mathrm{H} 01004$ & AGG-IC-001 \\
\hline $14808-79-8$ & Sulfate & $2.19 \mathrm{E} 1$ & ug/g dry & $1.49 \mathrm{E} 0$ & $8 / 01 / 08$ & 8H01004 & AGG-IC-001 \\
\hline $14265-44-2$ & Phosphate & $<1.49 \mathrm{E} 0$ & ug/g dry & 1.49E0 & $8 / 01 / 08$ & 8H01004 & AGG-IC-001 \\
\hline HEIS No. & B1V9C0 & \multicolumn{3}{|c|}{ Lab ID: $\quad 0805003-20$} & & & \\
\hline $16984-48-8$ & Fluoride & $2.19 \mathrm{E}-1$ & ug/g dry & $2.01 \mathrm{E}-1$ & $8 / 01 / 08$ & $8 \mathrm{H} 01004$ & AGG-IC-001 \\
\hline $16887-00-6$ & Chloride & $6.83 \mathrm{E} 0$ & ug/g dry & $5.01 \mathrm{E}-1$ & $8 / 01 / 08$ & 8H01004 & AGG-IC-001 \\
\hline $14797-65-0$ & Nitrite & $<1.00 \mathrm{E} 0$ & ug/g dry & $1.00 \mathrm{E} 0$ & $8 / 01 / 08$ & 8H01004 & AGG-IC-001 \\
\hline 24959-67-9 & Bromide & $<1.00 \mathrm{E} 0$ & ug/g dry & $1.00 \mathrm{E} 0$ & $8 / 01 / 08$ & 8H01004 & AGG-IC-001 \\
\hline $14797-55-8$ & Nitrate & $3.52 \mathrm{E} 0$ & ug/g dry & $1.00 \mathrm{E} 0$ & $8 / 01 / 08$ & $8 \mathrm{H} 01004$ & AGG-IC-001 \\
\hline $14808-79-8$ & Sulfate & $2.76 \mathrm{E} 1$ & ug/g dry & $1.50 \mathrm{E} 0$ & $8 / 01 / 08$ & 8H01004 & AGG-IC-001 \\
\hline $14265-44-2$ & Phosphate & $<1.50 \mathrm{E} 0$ & ug/g dry & $1.50 \mathrm{E} 0$ & $8 / 01 / 08$ & $8 \mathrm{H} 01004$ & AGG-IC-001 \\
\hline HEIS No. & B1V9C1 & \multicolumn{3}{|c|}{ Lab ID: $\quad$ 0805003-21 } & & & \\
\hline $16984-48-8$ & Fluoride & $2.29 \mathrm{E}-1$ & ug/g dry & $2.00 \mathrm{E}-1$ & $8 / 01 / 08$ & $8 \mathrm{H} 01004$ & AGG-IC-001 \\
\hline $16887-00-6$ & Chloride & 5.57E0 & ug/g dry & $4.99 \mathrm{E}-1$ & $8 / 01 / 08$ & $8 \mathrm{H} 01004$ & AGG-IC-001 \\
\hline $14797-65-0$ & Nitrite & $<9.99 \mathrm{E}-1$ & ug/g dry & $9.99 \mathrm{E}-1$ & $8 / 01 / 08$ & 8H01004 & AGG-IC-001 \\
\hline 24959-67-9 & Bromide & $<9.99 \mathrm{E}-1$ & ug/g dry & $9.99 \mathrm{E}-1$ & $8 / 01 / 08$ & $8 \mathrm{H} 01004$ & AGG-IC-001 \\
\hline $14797-55-8$ & Nitrate & $3.87 \mathrm{E} 0$ & ug/g dry & $9.99 \mathrm{E}-1$ & $8 / 01 / 08$ & $8 \mathrm{H} 01004$ & AGG-IC-001 \\
\hline $14808-79-8$ & Sulfate & $3.41 \mathrm{E} 1$ & ug/g dry & $1.50 \mathrm{E} 0$ & $8 / 01 / 08$ & 8H01004 & AGG-IC-001 \\
\hline $14265-44-2$ & Phosphate & $<1.50 \mathrm{E} 0$ & ug/g dry & $1.50 \mathrm{E} 0$ & $8 / 01 / 08$ & 8H01004 & AGG-IC-001 \\
\hline HEIS No. & B1V9C2 & \multicolumn{3}{|c|}{ Lab ID: $\quad 0805003-22$} & & & \\
\hline $16984-48-8$ & Fluoride & $2.53 \mathrm{E}-1$ & ug/g dry & $2.00 \mathrm{E}-1$ & $8 / 01 / 08$ & 8H01004 & AGG-IC-001 \\
\hline $16887-00-6$ & Chloride & $4.08 \mathrm{E} 0$ & ug/g dry & $4.99 \mathrm{E}-1$ & $8 / 01 / 08$ & 8H01004 & AGG-IC-001 \\
\hline $14797-65-0$ & Nitrite & $<9.99 \mathrm{E}-1$ & ug/g dry & $9.99 \mathrm{E}-1$ & $8 / 01 / 08$ & 8H01004 & AGG-IC-001 \\
\hline 24959-67-9 & Bromide & $<9.99 \mathrm{E}-1$ & ug/g dry & $9.99 \mathrm{E}-1$ & $8 / 01 / 08$ & $8 \mathrm{H} 01004$ & AGG-IC-001 \\
\hline $14797-55-8$ & Nitrate & $8.18 \mathrm{E} 0$ & ug/g dry & $9.99 \mathrm{E}-1$ & $8 / 01 / 08$ & $8 \mathrm{H} 01004$ & AGG-IC-001 \\
\hline $14808-79-8$ & Sulfate & $3.42 \mathrm{E} 1$ & ug/g dry & $1.50 \mathrm{E} 0$ & $8 / 01 / 08$ & $8 \mathrm{H} 01004$ & AGG-IC-001 \\
\hline 14265-44-2 & Phosphate & $<1.50 \mathrm{E} 0$ & ug/g dry & $1.50 \mathrm{E} 0$ & 8/01/08 & 8H01004 & AGG-IC-001 \\
\hline HEIS No. & B1V9C3 & \multicolumn{3}{|c|}{ Lab ID: $\quad$ 0805003-23 } & & & \\
\hline $16984-48-8$ & Fluoride & $2.33 \mathrm{E}-1$ & ug/g dry & $1.99 \mathrm{E}-1$ & $8 / 01 / 08$ & $8 \mathrm{H} 01004$ & AGG-IC-001 \\
\hline $16887-00-6$ & Chloride & $2.36 \mathrm{E} 0$ & ug/g dry & $4.98 \mathrm{E}-1$ & $8 / 01 / 08$ & $8 \mathrm{H} 01004$ & AGG-IC-001 \\
\hline $14797-65-0$ & Nitrite & $<9.96 \mathrm{E}-1$ & ug/g dry & $9.96 \mathrm{E}-1$ & $8 / 01 / 08$ & 8H01004 & AGG-IC-001 \\
\hline 24959-67-9 & Bromide & $<9.96 \mathrm{E}-1$ & ug/g dry & $9.96 \mathrm{E}-1$ & $8 / 01 / 08$ & 8H01004 & AGG-IC-001 \\
\hline $14797-55-8$ & Nitrate & $6.37 \mathrm{E} 0$ & ug/g dry & $9.96 \mathrm{E}-1$ & $8 / 01 / 08$ & 8H01004 & AGG-IC-001 \\
\hline $14808-79-8$ & Sulfate & $1.90 \mathrm{E} 1$ & ug/g dry & $1.49 \mathrm{E} 0$ & 8/01/08 & 8H01004 & AGG-IC-001 \\
\hline $14265-44-2$ & Phosphate & $<1.49 \mathrm{E} 0$ & ug/g dry & $1.49 \mathrm{E} 0$ & $8 / 01 / 08$ & 8H01004 & AGG-IC-001 \\
\hline HEIS No. & B1V9C4 & \multicolumn{3}{|c|}{ Lab ID: $\quad$ 0805003-24 } & & & \\
\hline $16984-48-8$ & Fluoride & $2.78 \mathrm{E}-1$ & ug/g dry & $2.00 \mathrm{E}-1$ & $8 / 01 / 08$ & $8 \mathrm{H} 01004$ & AGG-IC-001 \\
\hline $16887-00-6$ & Chloride & $1.50 \mathrm{E} 0$ & ug/g dry & $5.01 \mathrm{E}-1$ & $8 / 01 / 08$ & $8 \mathrm{H} 01004$ & AGG-IC-001 \\
\hline $14797-65-0$ & Nitrite & $<1.00 \mathrm{E} 0$ & ug/g dry & $1.00 \mathrm{E} 0$ & $8 / 01 / 08$ & 8H01004 & AGG-IC-001 \\
\hline 24959-67-9 & Bromide & $<1.00 \mathrm{E} 0$ & ug/g dry & $1.00 \mathrm{E} 0$ & $8 / 01 / 08$ & 8H01004 & AGG-IC-001 \\
\hline $14797-55-8$ & Nitrate & $5.15 \mathrm{E} 0$ & ug/g dry & $1.00 \mathrm{E} 0$ & 8/01/08 & 8H01004 & AGG-IC-001 \\
\hline $14808-79-8$ & Sulfate & $1.74 \mathrm{E} 1$ & ug/g dry & $1.50 \mathrm{E} 0$ & $8 / 01 / 08$ & $8 \mathrm{H} 01004$ & AGG-IC-001 \\
\hline $14265-44-2$ & Phosphate & $<1.50 \mathrm{E} 0$ & ug/g dry & $1.50 \mathrm{E} 0$ & 8/01/08 & 8H01004 & AGG-IC-001 \\
\hline
\end{tabular}




\section{Anions by Ion Chromatography}

\begin{tabular}{|c|c|c|c|c|c|c|c|}
\hline CAS \# & Analyte & Results & Units & EQL & Analyzed & Batch & Method \\
\hline HEIS No. & B1V2V5 & & b ID: & $05003-25$ & & & \\
\hline $16984-48-8$ & Fluoride & $2.08 \mathrm{E}-1$ & ug/g dry & $1.99 \mathrm{E}-1$ & $8 / 01 / 08$ & 8H01004 & AGG-IC-001 \\
\hline $16887-00-6$ & Chloride & $<4.98 \mathrm{E}-1$ & ug/g dry & $4.98 \mathrm{E}-1$ & $8 / 01 / 08$ & $8 \mathrm{H} 01004$ & AGG-IC-001 \\
\hline $14797-65-0$ & Nitrite & $<9.96 \mathrm{E}-1$ & ug/g dry & $9.96 \mathrm{E}-1$ & $8 / 01 / 08$ & 8H01004 & AGG-IC-001 \\
\hline $24959-67-9$ & Bromide & $<9.96 \mathrm{E}-1$ & ug/g dry & $9.96 \mathrm{E}-1$ & $8 / 01 / 08$ & 8H01004 & AGG-IC-001 \\
\hline $14797-55-8$ & Nitrate & $3.26 \mathrm{E} 0$ & ug/g dry & $9.96 \mathrm{E}-1$ & $8 / 01 / 08$ & $8 \mathrm{H} 01004$ & AGG-IC-001 \\
\hline $14808-79-8$ & Sulfate & 8.90E0 & ug/g dry & $1.49 \mathrm{E} 0$ & $8 / 01 / 08$ & 8H01004 & AGG-IC-001 \\
\hline $14265-44-2$ & Phosphate & $<1.49 \mathrm{E} 0$ & ug/g dry & $1.49 \mathrm{E} 0$ & $8 / 01 / 08$ & 8H01004 & AGG-IC-001 \\
\hline
\end{tabular}


Total Metals by PNNL-AGG-ICP-AES/Water Extract

\begin{tabular}{|c|c|c|c|c|c|c|c|}
\hline CAS \# & Analyte & Results & Units & EQL & Analyzed & Batch & Method \\
\hline HEIS No. & B1VDW5 & & b ID: & 5001-01 & & & \\
\hline $7429-90-5$ & Aluminum & $2.30 \mathrm{E}-1$ & ug/g dry & $8.55 \mathrm{E}-2$ & $9 / 16 / 08$ & $8 \mathrm{I} 15002$ & PNNL-AGG-ICP-AES \\
\hline $7440-38-2$ & Arsenic & $<3.59 \mathrm{E}-1$ & ug/g dry & $3.59 \mathrm{E}-1$ & $9 / 16 / 08$ & $8 \mathrm{I} 15002$ & PNNL-AGG-ICP-AES \\
\hline $7440-42-8$ & Boron & $<1.93 \mathrm{E} 0$ & ug/g dry & $1.93 \mathrm{E} 0$ & $9 / 16 / 08$ & $8 \mathrm{I} 15002$ & PNNL-AGG-ICP-AES \\
\hline $7440-41-7$ & Beryllium & $<2.83 \mathrm{E}-2$ & ug/g dry & $2.83 \mathrm{E}-2$ & $9 / 16 / 08$ & $8 \mathrm{I} 15002$ & PNNL-AGG-ICP-AES \\
\hline $7440-69-9$ & Bismuth & $<1.87 \mathrm{E}-1$ & ug/g dry & $1.87 \mathrm{E}-1$ & $9 / 16 / 08$ & $8 \mathrm{I} 15002$ & PNNL-AGG-ICP-AES \\
\hline $7440-70-2$ & Calcium & $9.67 \mathrm{E} 0$ & ug/g dry & $3.86 \mathrm{E}-1$ & $9 / 16 / 08$ & $8 \mathrm{I} 15002$ & PNNL-AGG-ICP-AES \\
\hline $7440-43-9$ & Cadmium & $<2.68 \mathrm{E}-2$ & ug/g dry & $2.68 \mathrm{E}-2$ & $9 / 16 / 08$ & $8 \mathrm{I} 15002$ & PNNL-AGG-ICP-AES \\
\hline $7440-48-4$ & Cobalt & $<9.57 \mathrm{E}-2$ & ug/g dry & $9.57 \mathrm{E}-2$ & $9 / 16 / 08$ & $8 \mathrm{II} 15002$ & PNNL-AGG-ICP-AES \\
\hline $7440-47-3$ & Chromium & $<3.32 \mathrm{E}-2$ & ug/g dry & $3.32 \mathrm{E}-2$ & $9 / 16 / 08$ & $8 \mathrm{I} 15002$ & PNNL-AGG-ICP-AES \\
\hline $7440-50-8$ & Copper & $<8.01 \mathrm{E}-2$ & ug/g dry & $8.01 \mathrm{E}-2$ & $9 / 16 / 08$ & $8 \mathrm{I} 15002$ & PNNL-AGG-ICP-AES \\
\hline $7439-89-6$ & Iron & $2.13 \mathrm{E}-1$ & ug/g dry & $1.42 \mathrm{E}-1$ & $9 / 16 / 08$ & $8 \mathrm{I} 15002$ & PNNL-AGG-ICP-AES \\
\hline $7440-09-7$ & Potassium & $<2.32 \mathrm{E} 0$ & ug/g dry & $2.32 \mathrm{E} 0$ & $9 / 16 / 08$ & $8 \mathrm{II} 15002$ & PNNL-AGG-ICP-AES \\
\hline $7439-93-2$ & Lithium & $<5.38 \mathrm{E}-1$ & ug/g dry & $5.38 \mathrm{E}-1$ & $9 / 16 / 08$ & $8 \mathrm{I} 15002$ & PNNL-AGG-ICP-AES \\
\hline $7439-95-4$ & Magnesium & $1.26 \mathrm{E} 0$ & ug/g dry & $8.31 \mathrm{E}-2$ & $9 / 16 / 08$ & $8 \mathrm{I} 15002$ & PNNL-AGG-ICP-AES \\
\hline $7439-96-5$ & Manganese & $<1.71 \mathrm{E}-2$ & ug/g dry & $1.71 \mathrm{E}-2$ & $9 / 16 / 08$ & $8 \mathrm{I} 15002$ & PNNL-AGG-ICP-AES \\
\hline $7439-98-7$ & Molybdenum & $<1.39 \mathrm{E}-1$ & ug/g dry & $1.39 \mathrm{E}-1$ & $9 / 16 / 08$ & $8 \mathrm{I} 15002$ & PNNL-AGG-ICP-AES \\
\hline $7440-02-0$ & Nickel & $<9.30 \mathrm{E}-2$ & ug/g dry & $9.30 \mathrm{E}-2$ & $9 / 16 / 08$ & $8 \mathrm{I} 15002$ & PNNL-AGG-ICP-AES \\
\hline $7723-14-0$ & Phosphorus & $<1.03 \mathrm{E} 0$ & ug/g dry & $1.03 \mathrm{E} 0$ & $9 / 16 / 08$ & $8 \mathrm{I} 15002$ & PNNL-AGG-ICP-AES \\
\hline $7439-92-1$ & Lead & $<4.30 \mathrm{E}-1$ & ug/g dry & $4.30 \mathrm{E}-1$ & $9 / 16 / 08$ & $8 \mathrm{I} 15002$ & PNNL-AGG-ICP-AES \\
\hline $7782-49-2$ & Selenium & $<1.94 \mathrm{E} 0$ & ug/g dry & $1.94 \mathrm{E} 0$ & $9 / 16 / 08$ & $8 \mathrm{II} 15002$ & PNNL-AGG-ICP-AES \\
\hline $7440-24-6$ & Strontium & $<5.20 \mathrm{E}-2$ & ug/g dry & $5.20 \mathrm{E}-2$ & $9 / 16 / 08$ & $8 \mathrm{I} 15002$ & PNNL-AGG-ICP-AES \\
\hline $7440-28-0$ & Thallium & $<1.02 \mathrm{E} 0$ & ug/g dry & $1.02 \mathrm{E} 0$ & $9 / 16 / 08$ & $8 \mathrm{I} 15002$ & PNNL-AGG-ICP-AES \\
\hline $7440-62-2$ & Vanadium & $<4.51 \mathrm{E}-2$ & ug/g dry & $4.51 \mathrm{E}-2$ & $9 / 16 / 08$ & 8115002 & PNNL-AGG-ICP-AES \\
\hline $7440-66-6$ & Zinc & $<9.24 \mathrm{E}-2$ & ug/g dry & $9.24 \mathrm{E}-2$ & $9 / 16 / 08$ & $8 \mathrm{I} 15002$ & PNNL-AGG-ICP-AES \\
\hline $7440-23-5$ & Sodium & $1.39 \mathrm{E} 0$ & ug/g dry & $6.67 \mathrm{E}-1$ & $9 / 16 / 08$ & $8 \mathrm{I} 15002$ & PNNL-AGG-ICP-AES \\
\hline $7704-34-9$ & Sulfur & $<3.06 \mathrm{E} 0$ & ug/g dry & $3.06 \mathrm{E} 0$ & $9 / 16 / 08$ & $8 \mathrm{I} 15002$ & PNNL-AGG-ICP-AES \\
\hline $7440-32-6$ & Titanium & $1.17 \mathrm{E}-2$ & ug/g dry & $8.73 \mathrm{E}-3$ & $9 / 16 / 08$ & $8 \mathrm{I} 15002$ & PNNL-AGG-ICP-AES \\
\hline $7440-67-7$ & Zirconium & $<1.49 \mathrm{E}-1$ & ug/g dry & $1.49 \mathrm{E}-1$ & $9 / 16 / 08$ & $8 \mathrm{I} 15002$ & PNNL-AGG-ICP-AES \\
\hline $7440-22-4$ & Silver & $<7.68 \mathrm{E}-2$ & ug/g dry & $7.68 \mathrm{E}-2$ & $9 / 16 / 08$ & $8 \mathrm{I} 15002$ & PNNL-AGG-ICP-AES \\
\hline $7440-15-5$ & Rhenium & $<1.51 \mathrm{E}-1$ & ug/g dry & $1.51 \mathrm{E}-1$ & $9 / 16 / 08$ & $8 \mathrm{I} 15002$ & PNNL-AGG-ICP-AES \\
\hline $7440-36-0$ & Antimony & $<6.31 \mathrm{E}-1$ & ug/g dry & $6.31 \mathrm{E}-1$ & $9 / 16 / 08$ & $8 \mathrm{I} 15002$ & PNNL-AGG-ICP-AES \\
\hline HEIS No. & B1VDW6 & & b ID: & 5001-02 & & & \\
\hline $7429-90-5$ & Aluminum & $2.18 \mathrm{E}-1$ & ug/g dry & $8.38 \mathrm{E}-2$ & 9/16/08 & $8 \mathrm{I} 15002$ & PNNL-AGG-ICP-AES \\
\hline $7440-38-2$ & Arsenic & $<3.51 \mathrm{E}-1$ & ug/g dry & $3.51 \mathrm{E}-1$ & $9 / 16 / 08$ & $8 \mathrm{I} 15002$ & PNNL-AGG-ICP-AES \\
\hline $7440-42-8$ & Boron & $<1.89 \mathrm{E} 0$ & ug/g dry & $1.89 \mathrm{E} 0$ & $9 / 16 / 08$ & $8 \mathrm{II} 15002$ & PNNL-AGG-ICP-AES \\
\hline $7440-41-7$ & Beryllium & $<2.77 \mathrm{E}-2$ & ug/g dry & $2.77 \mathrm{E}-2$ & $9 / 16 / 08$ & $8 \mathrm{I} 15002$ & PNNL-AGG-ICP-AES \\
\hline $7440-69-9$ & Bismuth & $<1.84 \mathrm{E}-1$ & ug/g dry & $1.84 \mathrm{E}-1$ & $9 / 16 / 08$ & $8 \mathrm{I} 15002$ & PNNL-AGG-ICP-AES \\
\hline $7440-70-2$ & Calcium & $9.03 \mathrm{E} 0$ & ug/g dry & $3.78 \mathrm{E}-1$ & $9 / 16 / 08$ & $8 \mathrm{I} 15002$ & PNNL-AGG-ICP-AES \\
\hline $7440-43-9$ & Cadmium & $<2.62 \mathrm{E}-2$ & ug/g dry & $2.62 \mathrm{E}-2$ & $9 / 16 / 08$ & $8 \mathrm{I} 15002$ & PNNL-AGG-ICP-AES \\
\hline $7440-48-4$ & Cobalt & $<9.37 \mathrm{E}-2$ & ug/g dry & $9.37 \mathrm{E}-2$ & $9 / 16 / 08$ & $8 \mathrm{I} 15002$ & PNNL-AGG-ICP-AES \\
\hline $7440-47-3$ & Chromium & $<3.25 \mathrm{E}-2$ & ug/g dry & $3.25 \mathrm{E}-2$ & $9 / 16 / 08$ & $8 \mathrm{I} 15002$ & PNNL-AGG-ICP-AES \\
\hline $7440-50-8$ & Copper & $<7.85 \mathrm{E}-2$ & ug/g dry & $7.85 \mathrm{E}-2$ & $9 / 16 / 08$ & $8 \mathrm{I} 15002$ & PNNL-AGG-ICP-AES \\
\hline $7439-89-6$ & Iron & $1.88 \mathrm{E}-1$ & ug/g dry & $1.39 \mathrm{E}-1$ & $9 / 16 / 08$ & $8 \mathrm{II} 15002$ & PNNL-AGG-ICP-AES \\
\hline $7440-09-7$ & Potassium & $2.28 \mathrm{E} 0$ & ug/g dry & $2.27 \mathrm{E} 0$ & $9 / 16 / 08$ & $8 \mathrm{I} 15002$ & PNNL-AGG-ICP-AES \\
\hline $7439-93-2$ & Lithium & $<5.27 \mathrm{E}-1$ & ug/g dry & $5.27 \mathrm{E}-1$ & $9 / 16 / 08$ & $8 \mathrm{I} 15002$ & PNNL-AGG-ICP-AES \\
\hline $7439-95-4$ & Magnesium & $1.27 \mathrm{E} 0$ & ug/g dry & $8.14 \mathrm{E}-2$ & $9 / 16 / 08$ & $8 \mathrm{II} 15002$ & PNNL-AGG-ICP-AES \\
\hline $7439-96-5$ & Manganese & $<1.67 \mathrm{E}-2$ & ug/g dry & $1.67 \mathrm{E}-2$ & $9 / 16 / 08$ & $8 \mathrm{I} 15002$ & PNNL-AGG-ICP-AES \\
\hline $7439-98-7$ & Molybdenum & $<1.36 \mathrm{E}-1$ & ug/g dry & $1.36 \mathrm{E}-1$ & $9 / 16 / 08$ & $8 \mathrm{I} 15002$ & PNNL-AGG-ICP-AES \\
\hline
\end{tabular}


Total Metals by PNNL-AGG-ICP-AES/Water Extract

\begin{tabular}{|c|c|c|c|c|c|c|c|}
\hline CAS \# & Analyte & Results & Units & EQL & Analyzed & Batch & Method \\
\hline HEIS No. & B1VDW6 & \multicolumn{3}{|c|}{ Lab ID: $\quad$ 0805001-02 } & & & \\
\hline $7440-02-0$ & Nickel & $<9.11 \mathrm{E}-2$ & ug/g dry & $9.11 \mathrm{E}-2$ & $9 / 16 / 08$ & $8 \mathrm{II} 15002$ & PNNL-AGG-ICP-AES \\
\hline $7723-14-0$ & Phosphorus & $<1.01 \mathrm{E} 0$ & ug/g dry & $1.01 \mathrm{E} 0$ & $9 / 16 / 08$ & $8 \mathrm{I} 15002$ & PNNL-AGG-ICP-AES \\
\hline $7439-92-1$ & Lead & $<4.22 \mathrm{E}-1$ & ug/g dry & $4.22 \mathrm{E}-1$ & $9 / 16 / 08$ & $8 \mathrm{I} 15002$ & PNNL-AGG-ICP-AES \\
\hline $7782-49-2$ & Selenium & $<1.90 \mathrm{E} 0$ & ug/g dry & $1.90 \mathrm{E} 0$ & $9 / 16 / 08$ & $8 \mathrm{II} 15002$ & PNNL-AGG-ICP-AES \\
\hline $7440-24-6$ & Strontium & $<5.10 \mathrm{E}-2$ & ug/g dry & $5.10 \mathrm{E}-2$ & $9 / 16 / 08$ & $8 \mathrm{I} 15002$ & PNNL-AGG-ICP-AES \\
\hline $7440-28-0$ & Thallium & $<1.00 \mathrm{E} 0$ & ug/g dry & $1.00 \mathrm{E} 0$ & $9 / 16 / 08$ & $8 \mathrm{II} 15002$ & PNNL-AGG-ICP-AES \\
\hline $7440-62-2$ & Vanadium & $<4.42 \mathrm{E}-2$ & ug/g dry & $4.42 \mathrm{E}-2$ & $9 / 16 / 08$ & $8 \mathrm{II} 15002$ & PNNL-AGG-ICP-AES \\
\hline $7440-66-6$ & Zinc & $<9.05 \mathrm{E}-2$ & ug/g dry & $9.05 \mathrm{E}-2$ & $9 / 16 / 08$ & $8 \mathrm{II} 15002$ & PNNL-AGG-ICP-AES \\
\hline $7440-23-5$ & Sodium & $1.59 \mathrm{E} 0$ & ug/g dry & $6.53 \mathrm{E}-1$ & $9 / 16 / 08$ & $8 \mathrm{II} 15002$ & PNNL-AGG-ICP-AES \\
\hline 7704-34-9 & Sulfur & $<3.00 \mathrm{E} 0$ & ug/g dry & $3.00 \mathrm{E} 0$ & $9 / 16 / 08$ & $8 \mathrm{II} 15002$ & PNNL-AGG-ICP-AES \\
\hline $7440-32-6$ & Titanium & $9.64 \mathrm{E}-3$ & ug/g dry & $8.55 \mathrm{E}-3$ & $9 / 16 / 08$ & $8 \mathrm{I} 15002$ & PNNL-AGG-ICP-AES \\
\hline $7440-67-7$ & Zirconium & $<1.46 \mathrm{E}-1$ & ug/g dry & $1.46 \mathrm{E}-1$ & $9 / 16 / 08$ & $8 \mathrm{I} 15002$ & PNNL-AGG-ICP-AES \\
\hline $7440-22-4$ & Silver & $<7.53 \mathrm{E}-2$ & ug/g dry & 7.53E-2 & $9 / 16 / 08$ & $8 \mathrm{I} 15002$ & PNNL-AGG-ICP-AES \\
\hline $7440-15-5$ & Rhenium & $<1.48 \mathrm{E}-1$ & ug/g dry & $1.48 \mathrm{E}-1$ & $9 / 16 / 08$ & $8 \mathrm{I} 15002$ & PNNL-AGG-ICP-AES \\
\hline $7440-36-0$ & Antimony & $<6.18 \mathrm{E}-1$ & ug/g dry & $6.18 \mathrm{E}-1$ & $9 / 16 / 08$ & $8 \mathrm{II} 15002$ & PNNL-AGG-ICP-AES \\
\hline HEIS No. & B1VDW7 & \multicolumn{3}{|c|}{ Lab ID: $\quad$ 0805001-03 } & & & \\
\hline $7429-90-5$ & Aluminum & $2.31 \mathrm{E}-1$ & ug/g dry & $8.52 \mathrm{E}-2$ & $9 / 16 / 08$ & $8 \mathrm{II} 15002$ & PNNL-AGG-ICP-AES \\
\hline $7440-38-2$ & Arsenic & $<3.58 \mathrm{E}-1$ & ug/g dry & $3.58 \mathrm{E}-1$ & $9 / 16 / 08$ & $8 \mathrm{II} 15002$ & PNNL-AGG-ICP-AES \\
\hline $7440-42-8$ & Boron & $<1.93 \mathrm{E} 0$ & ug/g dry & $1.93 \mathrm{E} 0$ & $9 / 16 / 08$ & $8 \mathrm{II} 15002$ & PNNL-AGG-ICP-AES \\
\hline $7440-41-7$ & Beryllium & $<2.82 \mathrm{E}-2$ & ug/g dry & $2.82 \mathrm{E}-2$ & $9 / 16 / 08$ & $8 \mathrm{II} 15002$ & PNNL-AGG-ICP-AES \\
\hline $7440-69-9$ & Bismuth & $<1.87 \mathrm{E}-1$ & ug/g dry & $1.87 \mathrm{E}-1$ & $9 / 16 / 08$ & $8 \mathrm{II} 15002$ & PNNL-AGG-ICP-AES \\
\hline $7440-70-2$ & Calcium & $9.77 \mathrm{E} 0$ & ug/g dry & $3.84 \mathrm{E}-1$ & $9 / 16 / 08$ & $8 \mathrm{I} 15002$ & PNNL-AGG-ICP-AES \\
\hline $7440-43-9$ & Cadmium & $<2.67 \mathrm{E}-2$ & ug/g dry & $2.67 \mathrm{E}-2$ & $9 / 16 / 08$ & $8 \mathrm{I} 15002$ & PNNL-AGG-ICP-AES \\
\hline $7440-48-4$ & Cobalt & $<9.54 \mathrm{E}-2$ & ug/g dry & $9.54 \mathrm{E}-2$ & $9 / 16 / 08$ & $8 \mathrm{II} 15002$ & PNNL-AGG-ICP-AES \\
\hline $7440-47-3$ & Chromium & $<3.31 \mathrm{E}-2$ & ug/g dry & $3.31 \mathrm{E}-2$ & $9 / 16 / 08$ & $8 \mathrm{I} 15002$ & PNNL-AGG-ICP-AES \\
\hline $7440-50-8$ & Copper & $<7.99 \mathrm{E}-2$ & ug/g dry & $7.99 \mathrm{E}-2$ & $9 / 16 / 08$ & $8 \mathrm{I} 15002$ & PNNL-AGG-ICP-AES \\
\hline $7439-89-6$ & Iron & $2.17 \mathrm{E}-1$ & ug/g dry & $1.41 \mathrm{E}-1$ & $9 / 16 / 08$ & $8 \mathrm{II} 15002$ & PNNL-AGG-ICP-AES \\
\hline $7440-09-7$ & Potassium & $2.85 \mathrm{E} 0$ & ug/g dry & $2.31 \mathrm{E} 0$ & $9 / 16 / 08$ & $8 \mathrm{II} 15002$ & PNNL-AGG-ICP-AES \\
\hline $7439-93-2$ & Lithium & $<5.36 \mathrm{E}-1$ & ug/g dry & $5.36 \mathrm{E}-1$ & $9 / 16 / 08$ & $8 \mathrm{II} 15002$ & PNNL-AGG-ICP-AES \\
\hline $7439-95-4$ & Magnesium & $1.68 \mathrm{E} 0$ & ug/g dry & $8.29 \mathrm{E}-2$ & $9 / 16 / 08$ & $8 \mathrm{I} 15002$ & PNNL-AGG-ICP-AES \\
\hline $7439-96-5$ & Manganese & $<1.70 \mathrm{E}-2$ & ug/g dry & $1.70 \mathrm{E}-2$ & $9 / 16 / 08$ & $8 \mathrm{II} 15002$ & PNNL-AGG-ICP-AES \\
\hline $7439-98-7$ & Molybdenum & $<1.38 \mathrm{E}-1$ & ug/g dry & $1.38 \mathrm{E}-1$ & $9 / 16 / 08$ & $8 \mathrm{II} 15002$ & PNNL-AGG-ICP-AES \\
\hline $7440-02-0$ & Nickel & $<9.27 \mathrm{E}-2$ & ug/g dry & $9.27 \mathrm{E}-2$ & $9 / 16 / 08$ & $8 \mathrm{I} 15002$ & PNNL-AGG-ICP-AES \\
\hline $7723-14-0$ & Phosphorus & $<1.03 \mathrm{E} 0$ & ug/g dry & $1.03 \mathrm{E} 0$ & $9 / 16 / 08$ & $8 \mathrm{I} 15002$ & PNNL-AGG-ICP-AES \\
\hline $7439-92-1$ & Lead & $<4.29 \mathrm{E}-1$ & ug/g dry & $4.29 \mathrm{E}-1$ & $9 / 16 / 08$ & $8 \mathrm{I} 15002$ & PNNL-AGG-ICP-AES \\
\hline $7782-49-2$ & Selenium & $<1.94 \mathrm{E} 0$ & ug/g dry & $1.94 \mathrm{E} 0$ & $9 / 16 / 08$ & $8 \mathrm{II} 15002$ & PNNL-AGG-ICP-AES \\
\hline $7440-24-6$ & Strontium & $<5.19 \mathrm{E}-2$ & ug/g dry & $5.19 \mathrm{E}-2$ & $9 / 16 / 08$ & $8 \mathrm{I} 15002$ & PNNL-AGG-ICP-AES \\
\hline $7440-28-0$ & Thallium & $<1.02 \mathrm{E} 0$ & ug/g dry & $1.02 \mathrm{E} 0$ & $9 / 16 / 08$ & $8 \mathrm{II} 15002$ & PNNL-AGG-ICP-AES \\
\hline $7440-62-2$ & Vanadium & $<4.50 \mathrm{E}-2$ & ug/g dry & $4.50 \mathrm{E}-2$ & $9 / 16 / 08$ & $8 \mathrm{II} 15002$ & PNNL-AGG-ICP-AES \\
\hline $7440-66-6$ & Zinc & $<9.21 \mathrm{E}-2$ & ug/g dry & $9.21 \mathrm{E}-2$ & $9 / 16 / 08$ & $8 \mathrm{II} 15002$ & PNNL-AGG-ICP-AES \\
\hline $7440-23-5$ & Sodium & $3.30 \mathrm{E} 0$ & ug/g dry & $6.65 \mathrm{E}-1$ & $9 / 16 / 08$ & $8 \mathrm{I} 15002$ & PNNL-AGG-ICP-AES \\
\hline $7704-34-9$ & Sulfur & $<3.05 \mathrm{E} 0$ & ug/g dry & 3.05E0 & $9 / 16 / 08$ & $8 \mathrm{I} 15002$ & PNNL-AGG-ICP-AES \\
\hline $7440-32-6$ & Titanium & $1.08 \mathrm{E}-2$ & ug/g dry & $8.70 \mathrm{E}-3$ & $9 / 16 / 08$ & $8 \mathrm{II} 15002$ & PNNL-AGG-ICP-AES \\
\hline $7440-67-7$ & Zirconium & $<1.49 \mathrm{E}-1$ & ug/g dry & $1.49 \mathrm{E}-1$ & $9 / 16 / 08$ & $8 \mathrm{I} 15002$ & PNNL-AGG-ICP-AES \\
\hline $7440-22-4$ & Silver & $<7.66 \mathrm{E}-2$ & ug/g dry & $7.66 \mathrm{E}-2$ & $9 / 16 / 08$ & $8 \mathrm{I} 15002$ & PNNL-AGG-ICP-AES \\
\hline $7440-15-5$ & Rhenium & $<1.51 \mathrm{E}-1$ & ug/g dry & $1.51 \mathrm{E}-1$ & $9 / 16 / 08$ & $8 \mathrm{II} 15002$ & PNNL-AGG-ICP-AES \\
\hline $7440-36-0$ & Antimony & $<6.29 \mathrm{E}-1$ & ug/g dry & $6.29 \mathrm{E}-1$ & $9 / 16 / 08$ & $8 \mathrm{II} 15002$ & PNNL-AGG-ICP-AES \\
\hline
\end{tabular}


Total Metals by PNNL-AGG-ICP-AES/Water Extract

\begin{tabular}{|c|c|c|c|c|c|c|c|}
\hline CAS \# & Analyte & Results & Units & EQL & Analyzed & Batch & Method \\
\hline HEIS No. & B1VDW8 & & b ID: & 5001-04 & & & \\
\hline $7429-90-5$ & Aluminum & $2.49 \mathrm{E}-1$ & ug/g dry & $8.45 \mathrm{E}-2$ & $9 / 16 / 08$ & $8 \mathrm{I} 15002$ & PNNL-AGG-ICP-AES \\
\hline $7440-38-2$ & Arsenic & $<3.54 \mathrm{E}-1$ & ug/g dry & $3.54 \mathrm{E}-1$ & $9 / 16 / 08$ & $8 \mathrm{I} 15002$ & PNNL-AGG-ICP-AES \\
\hline $7440-42-8$ & Boron & $<1.91 \mathrm{E} 0$ & ug/g dry & $1.91 \mathrm{E} 0$ & $9 / 16 / 08$ & $8 \mathrm{I} 15002$ & PNNL-AGG-ICP-AES \\
\hline $7440-41-7$ & Beryllium & $<2.80 \mathrm{E}-2$ & ug/g dry & $2.80 \mathrm{E}-2$ & $9 / 16 / 08$ & $8 \mathrm{I} 15002$ & PNNL-AGG-ICP-AES \\
\hline $7440-69-9$ & Bismuth & $<1.85 \mathrm{E}-1$ & ug/g dry & $1.85 \mathrm{E}-1$ & $9 / 16 / 08$ & $8 \mathrm{I} 15002$ & PNNL-AGG-ICP-AES \\
\hline $7440-70-2$ & Calcium & $9.86 \mathrm{E} 0$ & ug/g dry & $3.81 \mathrm{E}-1$ & $9 / 16 / 08$ & $8 \mathrm{I} 15002$ & PNNL-AGG-ICP-AES \\
\hline $7440-43-9$ & Cadmium & $<2.65 \mathrm{E}-2$ & ug/g dry & $2.65 \mathrm{E}-2$ & $9 / 16 / 08$ & $8 \mathrm{I} 15002$ & PNNL-AGG-ICP-AES \\
\hline $7440-48-4$ & Cobalt & $<9.45 \mathrm{E}-2$ & ug/g dry & $9.45 \mathrm{E}-2$ & $9 / 16 / 08$ & $8 \mathrm{II} 15002$ & PNNL-AGG-ICP-AES \\
\hline $7440-47-3$ & Chromium & $<3.28 \mathrm{E}-2$ & ug/g dry & $3.28 \mathrm{E}-2$ & $9 / 16 / 08$ & $8 \mathrm{I} 15002$ & PNNL-AGG-ICP-AES \\
\hline $7440-50-8$ & Copper & $<7.92 \mathrm{E}-2$ & ug/g dry & $7.92 \mathrm{E}-2$ & $9 / 16 / 08$ & $8 \mathrm{I} 15002$ & PNNL-AGG-ICP-AES \\
\hline $7439-89-6$ & Iron & $2.53 \mathrm{E}-1$ & ug/g dry & $1.40 \mathrm{E}-1$ & $9 / 16 / 08$ & $8 \mathrm{I} 15002$ & PNNL-AGG-ICP-AES \\
\hline $7440-09-7$ & Potassium & $3.16 \mathrm{E} 0$ & ug/g dry & $2.29 \mathrm{E} 0$ & $9 / 16 / 08$ & $8 \mathrm{II} 15002$ & PNNL-AGG-ICP-AES \\
\hline $7439-93-2$ & Lithium & $<5.32 \mathrm{E}-1$ & ug/g dry & $5.32 \mathrm{E}-1$ & $9 / 16 / 08$ & $8 \mathrm{I} 15002$ & PNNL-AGG-ICP-AES \\
\hline $7439-95-4$ & Magnesium & $1.50 \mathrm{E} 0$ & ug/g dry & $8.21 \mathrm{E}-2$ & $9 / 16 / 08$ & $8 \mathrm{I} 15002$ & PNNL-AGG-ICP-AES \\
\hline $7439-96-5$ & Manganese & $<1.69 \mathrm{E}-2$ & ug/g dry & $1.69 \mathrm{E}-2$ & $9 / 16 / 08$ & $8 \mathrm{I} 15002$ & PNNL-AGG-ICP-AES \\
\hline $7439-98-7$ & Molybdenum & $<1.37 \mathrm{E}-1$ & ug/g dry & $1.37 \mathrm{E}-1$ & $9 / 16 / 08$ & $8 \mathrm{I} 15002$ & PNNL-AGG-ICP-AES \\
\hline $7440-02-0$ & Nickel & $<9.19 \mathrm{E}-2$ & ug/g dry & $9.19 \mathrm{E}-2$ & $9 / 16 / 08$ & $8 \mathrm{I} 15002$ & PNNL-AGG-ICP-AES \\
\hline $7723-14-0$ & Phosphorus & $<1.02 \mathrm{E} 0$ & ug/g dry & $1.02 \mathrm{E} 0$ & $9 / 16 / 08$ & $8 \mathrm{I} 15002$ & PNNL-AGG-ICP-AES \\
\hline $7439-92-1$ & Lead & $<4.25 \mathrm{E}-1$ & ug/g dry & $4.25 \mathrm{E}-1$ & $9 / 16 / 08$ & $8 \mathrm{I} 15002$ & PNNL-AGG-ICP-AES \\
\hline $7782-49-2$ & Selenium & $<1.92 \mathrm{E} 0$ & ug/g dry & $1.92 \mathrm{E} 0$ & $9 / 16 / 08$ & $8 \mathrm{II} 15002$ & PNNL-AGG-ICP-AES \\
\hline $7440-24-6$ & Strontium & $<5.14 \mathrm{E}-2$ & ug/g dry & $5.14 \mathrm{E}-2$ & $9 / 16 / 08$ & $8 \mathrm{I} 15002$ & PNNL-AGG-ICP-AES \\
\hline $7440-28-0$ & Thallium & $<1.01 \mathrm{E} 0$ & ug/g dry & $1.01 \mathrm{E} 0$ & $9 / 16 / 08$ & $8 \mathrm{I} 15002$ & PNNL-AGG-ICP-AES \\
\hline $7440-62-2$ & Vanadium & $<4.46 \mathrm{E}-2$ & ug/g dry & $4.46 \mathrm{E}-2$ & $9 / 16 / 08$ & 8115002 & PNNL-AGG-ICP-AES \\
\hline $7440-66-6$ & Zinc & $<9.13 \mathrm{E}-2$ & ug/g dry & $9.13 \mathrm{E}-2$ & $9 / 16 / 08$ & $8 \mathrm{I} 15002$ & PNNL-AGG-ICP-AES \\
\hline $7440-23-5$ & Sodium & 4.62E0 & ug/g dry & $6.59 \mathrm{E}-1$ & $9 / 16 / 08$ & $8 \mathrm{I} 15002$ & PNNL-AGG-ICP-AES \\
\hline $7704-34-9$ & Sulfur & $<3.03 \mathrm{E} 0$ & ug/g dry & 3.03E0 & $9 / 16 / 08$ & $8 \mathrm{I} 15002$ & PNNL-AGG-ICP-AES \\
\hline $7440-32-6$ & Titanium & $1.18 \mathrm{E}-2$ & ug/g dry & $8.62 \mathrm{E}-3$ & $9 / 16 / 08$ & $8 \mathrm{I} 15002$ & PNNL-AGG-ICP-AES \\
\hline $7440-67-7$ & Zirconium & $<1.48 \mathrm{E}-1$ & ug/g dry & $1.48 \mathrm{E}-1$ & $9 / 16 / 08$ & $8 \mathrm{I} 15002$ & PNNL-AGG-ICP-AES \\
\hline $7440-22-4$ & Silver & $<7.59 \mathrm{E}-2$ & ug/g dry & $7.59 \mathrm{E}-2$ & $9 / 16 / 08$ & $8 \mathrm{I} 15002$ & PNNL-AGG-ICP-AES \\
\hline $7440-15-5$ & Rhenium & $<1.49 \mathrm{E}-1$ & ug/g dry & $1.49 \mathrm{E}-1$ & $9 / 16 / 08$ & $8 \mathrm{I} 15002$ & PNNL-AGG-ICP-AES \\
\hline $7440-36-0$ & Antimony & $<6.23 \mathrm{E}-1$ & ug/g dry & $6.23 \mathrm{E}-1$ & $9 / 16 / 08$ & $8 \mathrm{I} 15002$ & PNNL-AGG-ICP-AES \\
\hline HEIS No. & B1VDW9 & & b ID: & 5001-05 & & & \\
\hline $7429-90-5$ & Aluminum & $4.42 \mathrm{E}-1$ & ug/g dry & $8.46 \mathrm{E}-2$ & 9/16/08 & $8 \mathrm{I} 15002$ & PNNL-AGG-ICP-AES \\
\hline $7440-38-2$ & Arsenic & $<3.55 \mathrm{E}-1$ & ug/g dry & $3.55 \mathrm{E}-1$ & $9 / 16 / 08$ & $8 \mathrm{I} 15002$ & PNNL-AGG-ICP-AES \\
\hline $7440-42-8$ & Boron & $<1.91 \mathrm{E} 0$ & ug/g dry & $1.91 \mathrm{E} 0$ & $9 / 16 / 08$ & $8 \mathrm{II} 15002$ & PNNL-AGG-ICP-AES \\
\hline $7440-41-7$ & Beryllium & $<2.80 \mathrm{E}-2$ & ug/g dry & $2.80 \mathrm{E}-2$ & $9 / 16 / 08$ & $8 \mathrm{I} 15002$ & PNNL-AGG-ICP-AES \\
\hline $7440-69-9$ & Bismuth & $<1.86 \mathrm{E}-1$ & ug/g dry & $1.86 \mathrm{E}-1$ & $9 / 16 / 08$ & $8 \mathrm{I} 15002$ & PNNL-AGG-ICP-AES \\
\hline $7440-70-2$ & Calcium & 7.19E0 & ug/g dry & $3.82 \mathrm{E}-1$ & $9 / 16 / 08$ & $8 \mathrm{I} 15002$ & PNNL-AGG-ICP-AES \\
\hline $7440-43-9$ & Cadmium & $<2.65 \mathrm{E}-2$ & ug/g dry & $2.65 \mathrm{E}-2$ & $9 / 16 / 08$ & $8 \mathrm{I} 15002$ & PNNL-AGG-ICP-AES \\
\hline $7440-48-4$ & Cobalt & $<9.47 \mathrm{E}-2$ & ug/g dry & $9.47 \mathrm{E}-2$ & $9 / 16 / 08$ & $8 \mathrm{I} 15002$ & PNNL-AGG-ICP-AES \\
\hline $7440-47-3$ & Chromium & $<3.29 \mathrm{E}-2$ & ug/g dry & $3.29 \mathrm{E}-2$ & $9 / 16 / 08$ & $8 \mathrm{I} 15002$ & PNNL-AGG-ICP-AES \\
\hline $7440-50-8$ & Copper & $<7.93 \mathrm{E}-2$ & ug/g dry & $7.93 \mathrm{E}-2$ & $9 / 16 / 08$ & $8 \mathrm{I} 15002$ & PNNL-AGG-ICP-AES \\
\hline $7439-89-6$ & Iron & $4.86 \mathrm{E}-1$ & ug/g dry & $1.40 \mathrm{E}-1$ & $9 / 16 / 08$ & $8 \mathrm{II} 15002$ & PNNL-AGG-ICP-AES \\
\hline 7440-09-7 & Potassium & $2.94 \mathrm{E} 0$ & ug/g dry & $2.30 \mathrm{E} 0$ & $9 / 16 / 08$ & $8 \mathrm{II} 15002$ & PNNL-AGG-ICP-AES \\
\hline $7439-93-2$ & Lithium & $<5.33 \mathrm{E}-1$ & ug/g dry & $5.33 \mathrm{E}-1$ & $9 / 16 / 08$ & $8 \mathrm{I} 15002$ & PNNL-AGG-ICP-AES \\
\hline $7439-95-4$ & Magnesium & $1.15 \mathrm{E} 0$ & ug/g dry & $8.23 \mathrm{E}-2$ & $9 / 16 / 08$ & $8 \mathrm{II} 15002$ & PNNL-AGG-ICP-AES \\
\hline $7439-96-5$ & Manganese & $<1.69 \mathrm{E}-2$ & ug/g dry & $1.69 \mathrm{E}-2$ & $9 / 16 / 08$ & $8 \mathrm{I} 15002$ & PNNL-AGG-ICP-AES \\
\hline $7439-98-7$ & Molybdenum & $<1.37 \mathrm{E}-1$ & ug/g dry & $1.37 \mathrm{E}-1$ & $9 / 16 / 08$ & $8 \mathrm{I} 15002$ & PNNL-AGG-ICP-AES \\
\hline
\end{tabular}


Total Metals by PNNL-AGG-ICP-AES/Water Extract

\begin{tabular}{|c|c|c|c|c|c|c|c|}
\hline CAS \# & Analyte & Results & Units & EQL & Analyzed & Batch & Method \\
\hline HEIS No. & B1VDW9 & \multicolumn{3}{|c|}{ Lab ID: $\quad$ 0805001-05 } & & & \\
\hline $7440-02-0$ & Nickel & $<9.20 \mathrm{E}-2$ & ug/g dry & $9.20 \mathrm{E}-2$ & $9 / 16 / 08$ & $8 \mathrm{II} 15002$ & PNNL-AGG-ICP-AES \\
\hline $7723-14-0$ & Phosphorus & $<1.02 \mathrm{E} 0$ & ug/g dry & $1.02 \mathrm{E} 0$ & $9 / 16 / 08$ & $8 \mathrm{I} 15002$ & PNNL-AGG-ICP-AES \\
\hline $7439-92-1$ & Lead & $<4.26 \mathrm{E}-1$ & ug/g dry & $4.26 \mathrm{E}-1$ & $9 / 16 / 08$ & $8 \mathrm{I} 15002$ & PNNL-AGG-ICP-AES \\
\hline $7782-49-2$ & Selenium & $<1.92 \mathrm{E} 0$ & ug/g dry & $1.92 \mathrm{E} 0$ & $9 / 16 / 08$ & $8 \mathrm{II} 15002$ & PNNL-AGG-ICP-AES \\
\hline $7440-24-6$ & Strontium & $<5.15 \mathrm{E}-2$ & ug/g dry & $5.15 \mathrm{E}-2$ & $9 / 16 / 08$ & $8 \mathrm{I} 15002$ & PNNL-AGG-ICP-AES \\
\hline $7440-28-0$ & Thallium & $<1.01 \mathrm{E} 0$ & ug/g dry & $1.01 \mathrm{E} 0$ & $9 / 16 / 08$ & $8 \mathrm{II} 15002$ & PNNL-AGG-ICP-AES \\
\hline $7440-62-2$ & Vanadium & $<4.47 \mathrm{E}-2$ & ug/g dry & 4.47E-2 & $9 / 16 / 08$ & $8 \mathrm{II} 15002$ & PNNL-AGG-ICP-AES \\
\hline $7440-66-6$ & Zinc & $<9.14 \mathrm{E}-2$ & ug/g dry & $9.14 \mathrm{E}-2$ & $9 / 16 / 08$ & $8 \mathrm{II} 15002$ & PNNL-AGG-ICP-AES \\
\hline $7440-23-5$ & Sodium & $6.60 \mathrm{E} 0$ & ug/g dry & $6.60 \mathrm{E}-1$ & $9 / 16 / 08$ & $8 \mathrm{II} 15002$ & PNNL-AGG-ICP-AES \\
\hline 7704-34-9 & Sulfur & $<3.03 \mathrm{E} 0$ & ug/g dry & 3.03E0 & $9 / 16 / 08$ & $8 \mathrm{II} 15002$ & PNNL-AGG-ICP-AES \\
\hline $7440-32-6$ & Titanium & $2.32 \mathrm{E}-2$ & ug/g dry & $8.64 \mathrm{E}-3$ & $9 / 16 / 08$ & $8 \mathrm{I} 15002$ & PNNL-AGG-ICP-AES \\
\hline $7440-67-7$ & Zirconium & $<1.48 \mathrm{E}-1$ & ug/g dry & $1.48 \mathrm{E}-1$ & $9 / 16 / 08$ & $8 \mathrm{I} 15002$ & PNNL-AGG-ICP-AES \\
\hline $7440-22-4$ & Silver & $<7.61 \mathrm{E}-2$ & ug/g dry & $7.61 \mathrm{E}-2$ & $9 / 16 / 08$ & $8 \mathrm{I} 15002$ & PNNL-AGG-ICP-AES \\
\hline $7440-15-5$ & Rhenium & $<1.50 \mathrm{E}-1$ & ug/g dry & $1.50 \mathrm{E}-1$ & $9 / 16 / 08$ & $8 \mathrm{I} 15002$ & PNNL-AGG-ICP-AES \\
\hline $7440-36-0$ & Antimony & $<6.24 \mathrm{E}-1$ & ug/g dry & $6.24 \mathrm{E}-1$ & $9 / 16 / 08$ & $8 \mathrm{II} 15002$ & PNNL-AGG-ICP-AES \\
\hline HEIS No. & B1VDX0 & \multicolumn{3}{|c|}{ Lab ID: $\quad$ 0805001-06 } & & & \\
\hline $7429-90-5$ & Aluminum & $2.44 \mathrm{E}-1$ & ug/g dry & $9.25 \mathrm{E}-2$ & $9 / 16 / 08$ & $8 \mathrm{I} 15002$ & PNNL-AGG-ICP-AES \\
\hline $7440-38-2$ & Arsenic & $<3.88 \mathrm{E}-1$ & ug/g dry & $3.88 \mathrm{E}-1$ & $9 / 16 / 08$ & $8 \mathrm{II} 15002$ & PNNL-AGG-ICP-AES \\
\hline $7440-42-8$ & Boron & $<2.09 \mathrm{E} 0$ & ug/g dry & $2.09 \mathrm{E} 0$ & $9 / 16 / 08$ & $8 \mathrm{II} 15002$ & PNNL-AGG-ICP-AES \\
\hline $7440-41-7$ & Beryllium & $<3.06 \mathrm{E}-2$ & ug/g dry & $3.06 \mathrm{E}-2$ & $9 / 16 / 08$ & $8 \mathrm{II} 15002$ & PNNL-AGG-ICP-AES \\
\hline $7440-69-9$ & Bismuth & $<2.03 \mathrm{E}-1$ & ug/g dry & $2.03 \mathrm{E}-1$ & $9 / 16 / 08$ & $8 \mathrm{II} 15002$ & PNNL-AGG-ICP-AES \\
\hline $7440-70-2$ & Calcium & $1.06 \mathrm{E} 1$ & ug/g dry & $4.17 \mathrm{E}-1$ & $9 / 16 / 08$ & $8 \mathrm{I} 15002$ & PNNL-AGG-ICP-AES \\
\hline $7440-43-9$ & Cadmium & $<2.90 \mathrm{E}-2$ & ug/g dry & $2.90 \mathrm{E}-2$ & $9 / 16 / 08$ & $8 \mathrm{I} 15002$ & PNNL-AGG-ICP-AES \\
\hline $7440-48-4$ & Cobalt & $<1.04 \mathrm{E}-1$ & ug/g dry & $1.04 \mathrm{E}-1$ & $9 / 16 / 08$ & $8 \mathrm{II} 15002$ & PNNL-AGG-ICP-AES \\
\hline $7440-47-3$ & Chromium & $<3.59 \mathrm{E}-2$ & ug/g dry & $3.59 \mathrm{E}-2$ & $9 / 16 / 08$ & $8 \mathrm{I} 15002$ & PNNL-AGG-ICP-AES \\
\hline $7440-50-8$ & Copper & $<8.67 \mathrm{E}-2$ & ug/g dry & $8.67 \mathrm{E}-2$ & $9 / 16 / 08$ & $8 \mathrm{I} 15002$ & PNNL-AGG-ICP-AES \\
\hline $7439-89-6$ & Iron & $2.34 \mathrm{E}-1$ & ug/g dry & $1.53 \mathrm{E}-1$ & $9 / 16 / 08$ & $8 \mathrm{II} 15002$ & PNNL-AGG-ICP-AES \\
\hline $7440-09-7$ & Potassium & $3.50 \mathrm{E} 0$ & ug/g dry & $2.51 \mathrm{E} 0$ & $9 / 16 / 08$ & $8 \mathrm{II} 15002$ & PNNL-AGG-ICP-AES \\
\hline $7439-93-2$ & Lithium & $<5.82 \mathrm{E}-1$ & ug/g dry & $5.82 \mathrm{E}-1$ & $9 / 16 / 08$ & $8 \mathrm{II} 15002$ & PNNL-AGG-ICP-AES \\
\hline $7439-95-4$ & Magnesium & $1.85 \mathrm{E} 0$ & ug/g dry & $8.99 \mathrm{E}-2$ & $9 / 16 / 08$ & $8 \mathrm{I} 15002$ & PNNL-AGG-ICP-AES \\
\hline $7439-96-5$ & Manganese & $<1.85 \mathrm{E}-2$ & ug/g dry & $1.85 \mathrm{E}-2$ & $9 / 16 / 08$ & $8 \mathrm{II} 15002$ & PNNL-AGG-ICP-AES \\
\hline $7439-98-7$ & Molybdenum & $<1.50 \mathrm{E}-1$ & ug/g dry & $1.50 \mathrm{E}-1$ & $9 / 16 / 08$ & $8 \mathrm{II} 15002$ & PNNL-AGG-ICP-AES \\
\hline $7440-02-0$ & Nickel & $<1.01 \mathrm{E}-1$ & ug/g dry & $1.01 \mathrm{E}-1$ & $9 / 16 / 08$ & $8 \mathrm{II} 15002$ & PNNL-AGG-ICP-AES \\
\hline $7723-14-0$ & Phosphorus & $<1.11 \mathrm{E} 0$ & ug/g dry & $1.11 \mathrm{E} 0$ & $9 / 16 / 08$ & $8 \mathrm{I} 15002$ & PNNL-AGG-ICP-AES \\
\hline $7439-92-1$ & Lead & $<4.66 \mathrm{E}-1$ & ug/g dry & $4.66 \mathrm{E}-1$ & $9 / 16 / 08$ & $8 \mathrm{I} 15002$ & PNNL-AGG-ICP-AES \\
\hline $7782-49-2$ & Selenium & $<2.10 \mathrm{E} 0$ & ug/g dry & $2.10 \mathrm{E} 0$ & $9 / 16 / 08$ & $8 \mathrm{II} 15002$ & PNNL-AGG-ICP-AES \\
\hline $7440-24-6$ & Strontium & $<5.63 \mathrm{E}-2$ & ug/g dry & $5.63 \mathrm{E}-2$ & $9 / 16 / 08$ & $8 \mathrm{I} 15002$ & PNNL-AGG-ICP-AES \\
\hline $7440-28-0$ & Thallium & $<1.11 \mathrm{E} 0$ & ug/g dry & $1.11 \mathrm{E} 0$ & $9 / 16 / 08$ & $8 \mathrm{II} 15002$ & PNNL-AGG-ICP-AES \\
\hline $7440-62-2$ & Vanadium & $<4.88 \mathrm{E}-2$ & ug/g dry & $4.88 \mathrm{E}-2$ & $9 / 16 / 08$ & $8 \mathrm{II} 15002$ & PNNL-AGG-ICP-AES \\
\hline $7440-66-6$ & Zinc & $<1.00 \mathrm{E}-1$ & ug/g dry & $1.00 \mathrm{E}-1$ & $9 / 16 / 08$ & $8 \mathrm{II} 15002$ & PNNL-AGG-ICP-AES \\
\hline $7440-23-5$ & Sodium & $6.22 \mathrm{E} 0$ & ug/g dry & $7.21 \mathrm{E}-1$ & $9 / 16 / 08$ & $8 \mathrm{I} 15002$ & PNNL-AGG-ICP-AES \\
\hline $7704-34-9$ & Sulfur & $<3.32 \mathrm{E} 0$ & ug/g dry & 3.32E0 & $9 / 16 / 08$ & $8 \mathrm{I} 15002$ & PNNL-AGG-ICP-AES \\
\hline $7440-32-6$ & Titanium & $1.11 \mathrm{E}-2$ & ug/g dry & $9.45 \mathrm{E}-3$ & $9 / 16 / 08$ & $8 \mathrm{II} 15002$ & PNNL-AGG-ICP-AES \\
\hline $7440-67-7$ & Zirconium & $<1.62 \mathrm{E}-1$ & ug/g dry & $1.62 \mathrm{E}-1$ & $9 / 16 / 08$ & $8 \mathrm{I} 15002$ & PNNL-AGG-ICP-AES \\
\hline $7440-22-4$ & Silver & $<8.31 \mathrm{E}-2$ & ug/g dry & $8.31 \mathrm{E}-2$ & $9 / 16 / 08$ & $8 \mathrm{I} 15002$ & PNNL-AGG-ICP-AES \\
\hline $7440-15-5$ & Rhenium & $<1.64 \mathrm{E}-1$ & ug/g dry & $1.64 \mathrm{E}-1$ & $9 / 16 / 08$ & $8 \mathrm{I} 15002$ & PNNL-AGG-ICP-AES \\
\hline $7440-36-0$ & Antimony & $<6.83 \mathrm{E}-1$ & ug/g dry & $6.83 \mathrm{E}-1$ & $9 / 16 / 08$ & $8 \mathrm{II} 15002$ & PNNL-AGG-ICP-AES \\
\hline
\end{tabular}


Total Metals by PNNL-AGG-ICP-AES/Water Extract

\begin{tabular}{|c|c|c|c|c|c|c|c|}
\hline CAS \# & Analyte & Results & Units & EQL & Analyzed & Batch & Method \\
\hline HEIS No. & B1V991 & & b ID: & 5003-01 & & & \\
\hline $7429-90-5$ & Aluminum & $2.80 \mathrm{E}-1$ & $\mathrm{ug} / \mathrm{g}$ dry & $8.58 \mathrm{E}-2$ & $9 / 16 / 08$ & $8 \mathrm{I} 15002$ & PNNL-AGG-ICP-AES \\
\hline $7440-38-2$ & Arsenic & $<3.60 \mathrm{E}-1$ & ug/g dry & $3.60 \mathrm{E}-1$ & $9 / 16 / 08$ & $8 \mathrm{I} 15002$ & PNNL-AGG-ICP-AES \\
\hline $7440-42-8$ & Boron & $<1.94 \mathrm{E} 0$ & $\mathrm{ug} / \mathrm{g}$ dry & $1.94 \mathrm{E} 0$ & $9 / 16 / 08$ & $8 \mathrm{I} 15002$ & PNNL-AGG-ICP-AES \\
\hline $7440-41-7$ & Beryllium & $<2.84 \mathrm{E}-2$ & ug/g dry & $2.84 \mathrm{E}-2$ & $9 / 16 / 08$ & $8 \mathrm{I} 15002$ & PNNL-AGG-ICP-AES \\
\hline $7440-69-9$ & Bismuth & $<1.88 \mathrm{E}-1$ & ug/g dry & $1.88 \mathrm{E}-1$ & $9 / 16 / 08$ & 8115002 & PNNL-AGG-ICP-AES \\
\hline $7440-70-2$ & Calcium & $8.76 \mathrm{E} 0$ & ug/g dry & $3.87 \mathrm{E}-1$ & $9 / 16 / 08$ & $8 \mathrm{I} 15002$ & PNNL-AGG-ICP-AES \\
\hline $7440-43-9$ & Cadmium & $<2.69 \mathrm{E}-2$ & ug/g dry & $2.69 \mathrm{E}-2$ & $9 / 16 / 08$ & $8 \mathrm{I} 15002$ & PNNL-AGG-ICP-AES \\
\hline $7440-48-4$ & Cobalt & $<9.60 \mathrm{E}-2$ & ug/g dry & $9.60 \mathrm{E}-2$ & $9 / 16 / 08$ & 8115002 & PNNL-AGG-ICP-AES \\
\hline $7440-47-3$ & Chromium & $<3.33 \mathrm{E}-2$ & ug/g dry & $3.33 \mathrm{E}-2$ & $9 / 16 / 08$ & $8 \mathrm{I} 15002$ & PNNL-AGG-ICP-AES \\
\hline $7440-50-8$ & Copper & $<8.04 \mathrm{E}-2$ & $\mathrm{ug} / \mathrm{g}$ dry & $8.04 \mathrm{E}-2$ & $9 / 16 / 08$ & $8 \mathrm{I} 15002$ & PNNL-AGG-ICP-AES \\
\hline $7439-89-6$ & Iron & $2.72 \mathrm{E}-1$ & ug/g dry & $1.42 \mathrm{E}-1$ & $9 / 16 / 08$ & $8 \mathrm{I} 15002$ & PNNL-AGG-ICP-AES \\
\hline $7440-09-7$ & Potassium & $<2.33 \mathrm{E} 0$ & ug/g dry & $2.33 \mathrm{E} 0$ & $9 / 16 / 08$ & $8 \mathrm{I} 15002$ & PNNL-AGG-ICP-AES \\
\hline $7439-93-2$ & Lithium & $<5.40 \mathrm{E}-1$ & ug/g dry & $5.40 \mathrm{E}-1$ & $9 / 16 / 08$ & $8 \mathrm{I} 15002$ & PNNL-AGG-ICP-AES \\
\hline $7439-95-4$ & Magnesium & $1.18 \mathrm{E} 0$ & $\mathrm{ug} / \mathrm{g}$ dry & $8.34 \mathrm{E}-2$ & $9 / 16 / 08$ & $8 \mathrm{I} 15002$ & PNNL-AGG-ICP-AES \\
\hline $7439-96-5$ & Manganese & $<1.71 \mathrm{E}-2$ & ug/g dry & $1.71 \mathrm{E}-2$ & $9 / 16 / 08$ & $8 \mathrm{I} 15002$ & PNNL-AGG-ICP-AES \\
\hline $7439-98-7$ & Molybdenum & $<1.39 \mathrm{E}-1$ & ug/g dry & $1.39 \mathrm{E}-1$ & $9 / 16 / 08$ & $8 \mathrm{I} 15002$ & PNNL-AGG-ICP-AES \\
\hline $7440-02-0$ & Nickel & $<9.33 \mathrm{E}-2$ & ug/g dry & $9.33 \mathrm{E}-2$ & $9 / 16 / 08$ & $8 \mathrm{I} 15002$ & PNNL-AGG-ICP-AES \\
\hline $7723-14-0$ & Phosphorus & $<1.03 \mathrm{E} 0$ & $\mathrm{ug} / \mathrm{g}$ dry & $1.03 \mathrm{E} 0$ & $9 / 16 / 08$ & $8 \mathrm{I} 15002$ & PNNL-AGG-ICP-AES \\
\hline $7439-92-1$ & Lead & $<4.32 \mathrm{E}-1$ & ug/g dry & $4.32 \mathrm{E}-1$ & $9 / 16 / 08$ & $8 \mathrm{I} 15002$ & PNNL-AGG-ICP-AES \\
\hline $7782-49-2$ & Selenium & $<1.95 \mathrm{E} 0$ & ug/g dry & $1.95 \mathrm{E} 0$ & $9 / 16 / 08$ & $8 \mathrm{I} 15002$ & PNNL-AGG-ICP-AES \\
\hline $7440-24-6$ & Strontium & $<5.22 \mathrm{E}-2$ & ug/g dry & $5.22 \mathrm{E}-2$ & $9 / 16 / 08$ & $8 \mathrm{I} 15002$ & PNNL-AGG-ICP-AES \\
\hline $7440-28-0$ & Thallium & $<1.03 \mathrm{E} 0$ & ug/g dry & $1.03 \mathrm{E} 0$ & $9 / 16 / 08$ & $8 \mathrm{I} 15002$ & PNNL-AGG-ICP-AES \\
\hline $7440-62-2$ & Vanadium & $<4.53 \mathrm{E}-2$ & $\mathrm{ug} / \mathrm{g}$ dry & $4.53 \mathrm{E}-2$ & $9 / 16 / 08$ & $8 \mathrm{I} 15002$ & PNNL-AGG-ICP-AES \\
\hline $7440-66-6$ & Zinc & $<9.27 \mathrm{E}-2$ & ug/g dry & $9.27 \mathrm{E}-2$ & $9 / 16 / 08$ & $8 \mathrm{I} 15002$ & PNNL-AGG-ICP-AES \\
\hline $7440-23-5$ & Sodium & $1.33 \mathrm{E} 0$ & $\mathrm{ug} / \mathrm{g}$ dry & $6.69 \mathrm{E}-1$ & $9 / 16 / 08$ & $8 \mathrm{II} 15002$ & PNNL-AGG-ICP-AES \\
\hline $7440-21-3$ & Silicon & $8.56 \mathrm{E} 0$ & ug/g dry & $1.50 \mathrm{E} 0$ & $9 / 16 / 08$ & $8 \mathrm{I} 15002$ & PNNL-AGG-ICP-AES \\
\hline $7704-34-9$ & Sulfur & $<3.08 \mathrm{E} 0$ & ug/g dry & $3.08 \mathrm{E} 0$ & $9 / 16 / 08$ & $8 \mathrm{II} 15002$ & PNNL-AGG-ICP-AES \\
\hline $7440-32-6$ & Titanium & $1.32 \mathrm{E}-2$ & ug/g dry & $8.76 \mathrm{E}-3$ & $9 / 16 / 08$ & $8 \mathrm{I} 15002$ & PNNL-AGG-ICP-AES \\
\hline $7440-67-7$ & Zirconium & $<1.50 \mathrm{E}-1$ & $\mathrm{ug} / \mathrm{g}$ dry & $1.50 \mathrm{E}-1$ & $9 / 16 / 08$ & $8 \mathrm{II} 15002$ & PNNL-AGG-ICP-AES \\
\hline $7440-22-4$ & Silver & $<7.71 \mathrm{E}-2$ & ug/g dry & $7.71 \mathrm{E}-2$ & $9 / 16 / 08$ & $8 \mathrm{I} 15002$ & PNNL-AGG-ICP-AES \\
\hline $7440-15-5$ & Rhenium & $<1.52 \mathrm{E}-1$ & ug/g dry & $1.52 \mathrm{E}-1$ & $9 / 16 / 08$ & $8 \mathrm{I} 15002$ & PNNL-AGG-ICP-AES \\
\hline $7440-36-0$ & Antimony & $<6.33 \mathrm{E}-1$ & ug/g dry & $6.33 \mathrm{E}-1$ & $9 / 16 / 08$ & $8 \mathrm{I} 15002$ & PNNL-AGG-ICP-AES \\
\hline HEIS No. & B1V992 & & b ID: & 5003-02 & & & \\
\hline $7429-90-5$ & Aluminum & $3.23 \mathrm{E}-1$ & ug/g dry & $8.55 \mathrm{E}-2$ & $9 / 16 / 08$ & $8 \mathrm{I} 15002$ & PNNL-AGG-ICP-AES \\
\hline $7440-38-2$ & Arsenic & $<3.59 \mathrm{E}-1$ & ug/g dry & $3.59 \mathrm{E}-1$ & $9 / 16 / 08$ & $8 \mathrm{I} 15002$ & PNNL-AGG-ICP-AES \\
\hline $7440-42-8$ & Boron & $<1.94 \mathrm{E} 0$ & ug/g dry & $1.94 \mathrm{E} 0$ & $9 / 16 / 08$ & $8 \mathrm{II} 15002$ & PNNL-AGG-ICP-AES \\
\hline $7440-41-7$ & Beryllium & $<2.83 \mathrm{E}-2$ & ug/g dry & $2.83 \mathrm{E}-2$ & $9 / 16 / 08$ & $8 \mathrm{I} 15002$ & PNNL-AGG-ICP-AES \\
\hline $7440-69-9$ & Bismuth & $<1.88 \mathrm{E}-1$ & $\mathrm{ug} / \mathrm{g}$ dry & $1.88 \mathrm{E}-1$ & $9 / 16 / 08$ & $8 \mathrm{I} 15002$ & PNNL-AGG-ICP-AES \\
\hline $7440-70-2$ & Calcium & $8.48 \mathrm{E} 0$ & $\mathrm{ug} / \mathrm{g}$ dry & $3.86 \mathrm{E}-1$ & $9 / 16 / 08$ & $8 \mathrm{I} 15002$ & PNNL-AGG-ICP-AES \\
\hline $7440-43-9$ & Cadmium & $<2.68 \mathrm{E}-2$ & ug/g dry & $2.68 \mathrm{E}-2$ & $9 / 16 / 08$ & $8 \mathrm{I} 15002$ & PNNL-AGG-ICP-AES \\
\hline $7440-48-4$ & Cobalt & $<9.57 \mathrm{E}-2$ & $\mathrm{ug} / \mathrm{g}$ dry & $9.57 \mathrm{E}-2$ & $9 / 16 / 08$ & $8 \mathrm{I} 15002$ & PNNL-AGG-ICP-AES \\
\hline $7440-47-3$ & Chromium & $<3.32 \mathrm{E}-2$ & ug/g dry & $3.32 \mathrm{E}-2$ & $9 / 16 / 08$ & $8 \mathrm{I} 15002$ & PNNL-AGG-ICP-AES \\
\hline $7440-50-8$ & Copper & $<8.02 \mathrm{E}-2$ & ug/g dry & $8.02 \mathrm{E}-2$ & $9 / 16 / 08$ & $8 \mathrm{I} 15002$ & PNNL-AGG-ICP-AES \\
\hline $7439-89-6$ & Iron & $3.41 \mathrm{E}-1$ & ug/g dry & $1.42 \mathrm{E}-1$ & $9 / 16 / 08$ & $8 \mathrm{I} 15002$ & PNNL-AGG-ICP-AES \\
\hline $7440-09-7$ & Potassium & 3.01E0 & ug/g dry & $2.32 \mathrm{E} 0$ & $9 / 16 / 08$ & $8 \mathrm{I} 15002$ & PNNL-AGG-ICP-AES \\
\hline $7439-93-2$ & Lithium & $<5.38 \mathrm{E}-1$ & ug/g dry & $5.38 \mathrm{E}-1$ & $9 / 16 / 08$ & $8 \mathrm{I} 15002$ & PNNL-AGG-ICP-AES \\
\hline $7439-95-4$ & Magnesium & $1.29 \mathrm{E} 0$ & ug/g dry & $8.32 \mathrm{E}-2$ & $9 / 16 / 08$ & $8 \mathrm{I} 15002$ & PNNL-AGG-ICP-AES \\
\hline $7439-96-5$ & Manganese & $<1.71 \mathrm{E}-2$ & ug/g dry & $1.71 \mathrm{E}-2$ & $9 / 16 / 08$ & $8 \mathrm{I} 15002$ & PNNL-AGG-ICP-AES \\
\hline
\end{tabular}


Total Metals by PNNL-AGG-ICP-AES/Water Extract

\begin{tabular}{|c|c|c|c|c|c|c|c|}
\hline CAS \# & Analyte & Results & Units & EQL & Analyzed & Batch & Method \\
\hline HEIS No. & B1V992 & & b ID: & 5003-02 & & & \\
\hline $7439-98-7$ & Molybdenum & $<1.39 \mathrm{E}-1$ & ug/g dry & $1.39 \mathrm{E}-1$ & $9 / 16 / 08$ & $8 \mathrm{II} 15002$ & PNNL-AGG-ICP-AES \\
\hline $7440-02-0$ & Nickel & $<9.30 \mathrm{E}-2$ & ug/g dry & $9.30 \mathrm{E}-2$ & $9 / 16 / 08$ & $8 \mathrm{II} 15002$ & PNNL-AGG-ICP-AES \\
\hline $7723-14-0$ & Phosphorus & $<1.03 \mathrm{E} 0$ & ug/g dry & $1.03 \mathrm{E} 0$ & $9 / 16 / 08$ & $8 \mathrm{I} 15002$ & PNNL-AGG-ICP-AES \\
\hline $7439-92-1$ & Lead & $<4.31 \mathrm{E}-1$ & ug/g dry & $4.31 \mathrm{E}-1$ & $9 / 16 / 08$ & $8 \mathrm{II} 15002$ & PNNL-AGG-ICP-AES \\
\hline $7782-49-2$ & Selenium & $<1.94 \mathrm{E} 0$ & ug/g dry & $1.94 \mathrm{E} 0$ & $9 / 16 / 08$ & $8 \mathrm{I} 15002$ & PNNL-AGG-ICP-AES \\
\hline $7440-24-6$ & Strontium & $<5.20 \mathrm{E}-2$ & ug/g dry & $5.20 \mathrm{E}-2$ & $9 / 16 / 08$ & $8 \mathrm{II} 15002$ & PNNL-AGG-ICP-AES \\
\hline $7440-28-0$ & Thallium & $<1.02 \mathrm{E} 0$ & ug/g dry & $1.02 \mathrm{E} 0$ & $9 / 16 / 08$ & $8 \mathrm{I} 15002$ & PNNL-AGG-ICP-AES \\
\hline $7440-62-2$ & Vanadium & $<4.52 \mathrm{E}-2$ & ug/g dry & $4.52 \mathrm{E}-2$ & $9 / 16 / 08$ & $8 \mathrm{II} 15002$ & PNNL-AGG-ICP-AES \\
\hline $7440-66-6$ & Zinc & $<9.24 \mathrm{E}-2$ & ug/g dry & $9.24 \mathrm{E}-2$ & $9 / 16 / 08$ & $8 \mathrm{II} 15002$ & PNNL-AGG-ICP-AES \\
\hline $7440-23-5$ & Sodium & $6.38 \mathrm{E} 0$ & ug/g dry & $6.67 \mathrm{E}-1$ & $9 / 16 / 08$ & $8 \mathrm{II} 15002$ & PNNL-AGG-ICP-AES \\
\hline $7440-21-3$ & Silicon & $1.17 \mathrm{E} 1$ & ug/g dry & $1.50 \mathrm{E} 0$ & $9 / 16 / 08$ & $8 \mathrm{II} 15002$ & PNNL-AGG-ICP-AES \\
\hline $7704-34-9$ & Sulfur & $<3.07 \mathrm{E} 0$ & ug/g dry & 3.07E0 & $9 / 16 / 08$ & $8 \mathrm{II} 15002$ & PNNL-AGG-ICP-AES \\
\hline $7440-32-6$ & Titanium & $1.54 \mathrm{E}-2$ & ug/g dry & $8.73 \mathrm{E}-3$ & $9 / 16 / 08$ & $8 \mathrm{II} 15002$ & PNNL-AGG-ICP-AES \\
\hline $7440-67-7$ & Zirconium & $<1.50 \mathrm{E}-1$ & ug/g dry & $1.50 \mathrm{E}-1$ & $9 / 16 / 08$ & $8 \mathrm{I} 15002$ & PNNL-AGG-ICP-AES \\
\hline $7440-22-4$ & Silver & $<7.69 \mathrm{E}-2$ & ug/g dry & $7.69 \mathrm{E}-2$ & $9 / 16 / 08$ & $8 \mathrm{II} 15002$ & PNNL-AGG-ICP-AES \\
\hline $7440-15-5$ & Rhenium & $<1.51 \mathrm{E}-1$ & ug/g dry & $1.51 \mathrm{E}-1$ & $9 / 16 / 08$ & $8 \mathrm{II} 15002$ & PNNL-AGG-ICP-AES \\
\hline $7440-36-0$ & Antimony & $<6.31 \mathrm{E}-1$ & ug/g dry & $6.31 \mathrm{E}-1$ & $9 / 16 / 08$ & $8 \mathrm{II} 15002$ & PNNL-AGG-ICP-AES \\
\hline HEIS No. & B1V993 & & b ID: & $5003-03$ & & & \\
\hline $7429-90-5$ & Aluminum & $2.62 \mathrm{E}-1$ & ug/g dry & $8.51 \mathrm{E}-2$ & $9 / 16 / 08$ & $8 \mathrm{I} 15002$ & PNNL-AGG-ICP-AES \\
\hline $7440-38-2$ & Arsenic & $<3.57 \mathrm{E}-1$ & ug/g dry & $3.57 \mathrm{E}-1$ & $9 / 16 / 08$ & $8 \mathrm{II} 15002$ & PNNL-AGG-ICP-AES \\
\hline $7440-42-8$ & Boron & $<1.93 \mathrm{E} 0$ & ug/g dry & $1.93 \mathrm{E} 0$ & $9 / 16 / 08$ & $8 \mathrm{II} 15002$ & PNNL-AGG-ICP-AES \\
\hline $7440-41-7$ & Beryllium & $<2.82 \mathrm{E}-2$ & ug/g dry & $2.82 \mathrm{E}-2$ & $9 / 16 / 08$ & $8 \mathrm{I} 15002$ & PNNL-AGG-ICP-AES \\
\hline $7440-69-9$ & Bismuth & $<1.87 \mathrm{E}-1$ & ug/g dry & $1.87 \mathrm{E}-1$ & $9 / 16 / 08$ & $8 \mathrm{II} 15002$ & PNNL-AGG-ICP-AES \\
\hline $7440-70-2$ & Calcium & $9.06 \mathrm{E} 0$ & ug/g dry & $3.84 \mathrm{E}-1$ & $9 / 16 / 08$ & $8 \mathrm{II} 15002$ & PNNL-AGG-ICP-AES \\
\hline $7440-43-9$ & Cadmium & $<2.67 \mathrm{E}-2$ & ug/g dry & $2.67 \mathrm{E}-2$ & $9 / 16 / 08$ & $8 \mathrm{I} 15002$ & PNNL-AGG-ICP-AES \\
\hline $7440-48-4$ & Cobalt & $<9.52 \mathrm{E}-2$ & ug/g dry & $9.52 \mathrm{E}-2$ & $9 / 16 / 08$ & $8 \mathrm{I} 15002$ & PNNL-AGG-ICP-AES \\
\hline $7440-47-3$ & Chromium & $<3.30 \mathrm{E}-2$ & ug/g dry & $3.30 \mathrm{E}-2$ & $9 / 16 / 08$ & $8 \mathrm{I} 15002$ & PNNL-AGG-ICP-AES \\
\hline $7440-50-8$ & Copper & $<7.98 \mathrm{E}-2$ & ug/g dry & $7.98 \mathrm{E}-2$ & $9 / 16 / 08$ & $8 \mathrm{II} 15002$ & PNNL-AGG-ICP-AES \\
\hline $7439-89-6$ & Iron & $2.63 \mathrm{E}-1$ & ug/g dry & $1.41 \mathrm{E}-1$ & $9 / 16 / 08$ & $8 \mathrm{II} 15002$ & PNNL-AGG-ICP-AES \\
\hline $7440-09-7$ & Potassium & $2.64 \mathrm{E} 0$ & ug/g dry & $2.31 \mathrm{E} 0$ & $9 / 16 / 08$ & $8 \mathrm{I} 15002$ & PNNL-AGG-ICP-AES \\
\hline $7439-93-2$ & Lithium & $<5.36 \mathrm{E}-1$ & ug/g dry & $5.36 \mathrm{E}-1$ & $9 / 16 / 08$ & $8 \mathrm{II} 15002$ & PNNL-AGG-ICP-AES \\
\hline $7439-95-4$ & Magnesium & $1.45 \mathrm{E} 0$ & ug/g dry & $8.27 \mathrm{E}-2$ & $9 / 16 / 08$ & $8 \mathrm{II} 15002$ & PNNL-AGG-ICP-AES \\
\hline $7439-96-5$ & Manganese & $<1.70 \mathrm{E}-2$ & ug/g dry & $1.70 \mathrm{E}-2$ & $9 / 16 / 08$ & $8 \mathrm{I} 15002$ & PNNL-AGG-ICP-AES \\
\hline $7439-98-7$ & Molybdenum & $<1.38 \mathrm{E}-1$ & ug/g dry & $1.38 \mathrm{E}-1$ & $9 / 16 / 08$ & $8 \mathrm{II} 15002$ & PNNL-AGG-ICP-AES \\
\hline $7440-02-0$ & Nickel & $<9.26 \mathrm{E}-2$ & ug/g dry & $9.26 \mathrm{E}-2$ & $9 / 16 / 08$ & $8 \mathrm{II} 15002$ & PNNL-AGG-ICP-AES \\
\hline $7723-14-0$ & Phosphorus & $<1.02 \mathrm{E} 0$ & ug/g dry & $1.02 \mathrm{E} 0$ & $9 / 16 / 08$ & $8 \mathrm{II} 15002$ & PNNL-AGG-ICP-AES \\
\hline $7439-92-1$ & Lead & $<4.29 \mathrm{E}-1$ & ug/g dry & $4.29 \mathrm{E}-1$ & $9 / 16 / 08$ & $8 \mathrm{I} 15002$ & PNNL-AGG-ICP-AES \\
\hline $7782-49-2$ & Selenium & $<1.93 \mathrm{E} 0$ & ug/g dry & $1.93 \mathrm{E} 0$ & $9 / 16 / 08$ & $8 \mathrm{I} 15002$ & PNNL-AGG-ICP-AES \\
\hline $7440-24-6$ & Strontium & $<5.18 \mathrm{E}-2$ & ug/g dry & $5.18 \mathrm{E}-2$ & $9 / 16 / 08$ & $8 \mathrm{II} 15002$ & PNNL-AGG-ICP-AES \\
\hline $7440-28-0$ & Thallium & $<1.02 \mathrm{E} 0$ & ug/g dry & $1.02 \mathrm{E} 0$ & $9 / 16 / 08$ & $8 \mathrm{II} 15002$ & PNNL-AGG-ICP-AES \\
\hline $7440-62-2$ & Vanadium & $<4.49 \mathrm{E}-2$ & ug/g dry & $4.49 \mathrm{E}-2$ & $9 / 16 / 08$ & $8 \mathrm{I} 15002$ & PNNL-AGG-ICP-AES \\
\hline $7440-66-6$ & Zinc & $<9.20 \mathrm{E}-2$ & ug/g dry & $9.20 \mathrm{E}-2$ & $9 / 16 / 08$ & $8 \mathrm{II} 15002$ & PNNL-AGG-ICP-AES \\
\hline $7440-23-5$ & Sodium & $3.12 \mathrm{E} 0$ & ug/g dry & $6.64 \mathrm{E}-1$ & $9 / 16 / 08$ & $8 \mathrm{II} 15002$ & PNNL-AGG-ICP-AES \\
\hline $7440-21-3$ & Silicon & $1.04 \mathrm{E} 1$ & ug/g dry & $1.49 \mathrm{E} 0$ & $9 / 16 / 08$ & $8 \mathrm{I} 15002$ & PNNL-AGG-ICP-AES \\
\hline $7704-34-9$ & Sulfur & $<3.05 \mathrm{E} 0$ & ug/g dry & 3.05E0 & $9 / 16 / 08$ & $8 \mathrm{I} 15002$ & PNNL-AGG-ICP-AES \\
\hline $7440-32-6$ & Titanium & $1.22 \mathrm{E}-2$ & ug/g dry & $8.69 \mathrm{E}-3$ & $9 / 16 / 08$ & $8 \mathrm{I} 15002$ & PNNL-AGG-ICP-AES \\
\hline $7440-67-7$ & Zirconium & $<1.49 \mathrm{E}-1$ & ug/g dry & $1.49 \mathrm{E}-1$ & $9 / 16 / 08$ & $8 \mathrm{II} 15002$ & PNNL-AGG-ICP-AES \\
\hline $7440-22-4$ & Silver & $<7.65 \mathrm{E}-2$ & ug/g dry & $7.65 \mathrm{E}-2$ & $9 / 16 / 08$ & $8 \mathrm{I} 15002$ & PNNL-AGG-ICP-AES \\
\hline
\end{tabular}


Total Metals by PNNL-AGG-ICP-AES/Water Extract

\begin{tabular}{|c|c|c|c|c|c|c|c|}
\hline CAS \# & Analyte & Results & Units & EQL & Analyzed & Batch & Method \\
\hline HEIS No. & B1V993 & \multicolumn{3}{|c|}{ Lab ID: $\quad$ 0805003-03 } & & & \\
\hline $7440-15-5$ & Rhenium & $<1.51 \mathrm{E}-1$ & ug/g dry & $1.51 \mathrm{E}-1$ & $9 / 16 / 08$ & $8 \mathrm{I} 15002$ & PNNL-AGG-ICP-AES \\
\hline $7440-36-0$ & Antimony & $<6.28 \mathrm{E}-1$ & ug/g dry & $6.28 \mathrm{E}-1$ & $9 / 16 / 08$ & $8 \mathrm{I} 15002$ & PNNL-AGG-ICP-AES \\
\hline HEIS No. & B1V994 & \multicolumn{3}{|c|}{ Lab ID: $\quad$ 0805003-04 } & & & \\
\hline $7429-90-5$ & Aluminum & $1.71 \mathrm{E}-1$ & ug/g dry & $8.55 \mathrm{E}-2$ & $9 / 16 / 08$ & $8 \mathrm{I} 15002$ & PNNL-AGG-ICP-AES \\
\hline $7440-38-2$ & Arsenic & $<3.59 \mathrm{E}-1$ & ug/g dry & $3.59 \mathrm{E}-1$ & $9 / 16 / 08$ & $8 \mathrm{I} 15002$ & PNNL-AGG-ICP-AES \\
\hline $7440-42-8$ & Boron & $<1.93 \mathrm{E} 0$ & ug/g dry & $1.93 \mathrm{E} 0$ & $9 / 16 / 08$ & $8 \mathrm{I} 15002$ & PNNL-AGG-ICP-AES \\
\hline $7440-41-7$ & Beryllium & $<2.83 \mathrm{E}-2$ & ug/g dry & $2.83 \mathrm{E}-2$ & $9 / 16 / 08$ & $8 \mathrm{I} 15002$ & PNNL-AGG-ICP-AES \\
\hline $7440-69-9$ & Bismuth & $<1.87 \mathrm{E}-1$ & ug/g dry & $1.87 \mathrm{E}-1$ & $9 / 16 / 08$ & $8 \mathrm{I} 15002$ & PNNL-AGG-ICP-AES \\
\hline $7440-70-2$ & Calcium & $9.84 \mathrm{E} 0$ & ug/g dry & $3.85 \mathrm{E}-1$ & $9 / 16 / 08$ & $8 \mathrm{I} 15002$ & PNNL-AGG-ICP-AES \\
\hline $7440-43-9$ & Cadmium & $<2.68 \mathrm{E}-2$ & ug/g dry & $2.68 \mathrm{E}-2$ & $9 / 16 / 08$ & $8 \mathrm{II} 15002$ & PNNL-AGG-ICP-AES \\
\hline $7440-48-4$ & Cobalt & $<9.56 \mathrm{E}-2$ & ug/g dry & $9.56 \mathrm{E}-2$ & $9 / 16 / 08$ & $8 \mathrm{I} 15002$ & PNNL-AGG-ICP-AES \\
\hline $7440-47-3$ & Chromium & $<3.32 \mathrm{E}-2$ & ug/g dry & $3.32 \mathrm{E}-2$ & $9 / 16 / 08$ & $8 \mathrm{I} 15002$ & PNNL-AGG-ICP-AES \\
\hline $7440-50-8$ & Copper & $<8.01 \mathrm{E}-2$ & ug/g dry & $8.01 \mathrm{E}-2$ & $9 / 16 / 08$ & $8 \mathrm{I} 15002$ & PNNL-AGG-ICP-AES \\
\hline $7439-89-6$ & Iron & $1.52 \mathrm{E}-1$ & ug/g dry & $1.42 \mathrm{E}-1$ & $9 / 16 / 08$ & $8 \mathrm{I} 15002$ & PNNL-AGG-ICP-AES \\
\hline $7440-09-7$ & Potassium & $3.55 \mathrm{E} 0$ & ug/g dry & $2.32 \mathrm{E} 0$ & $9 / 16 / 08$ & $8 \mathrm{I} 15002$ & PNNL-AGG-ICP-AES \\
\hline $7439-93-2$ & Lithium & $<5.38 \mathrm{E}-1$ & ug/g dry & $5.38 \mathrm{E}-1$ & $9 / 16 / 08$ & $8 \mathrm{I} 15002$ & PNNL-AGG-ICP-AES \\
\hline $7439-95-4$ & Magnesium & $1.85 \mathrm{E} 0$ & ug/g dry & $8.31 \mathrm{E}-2$ & $9 / 16 / 08$ & $8 \mathrm{I} 15002$ & PNNL-AGG-ICP-AES \\
\hline $7439-96-5$ & Manganese & $<1.71 \mathrm{E}-2$ & ug/g dry & $1.71 \mathrm{E}-2$ & $9 / 16 / 08$ & $8 \mathrm{I} 15002$ & PNNL-AGG-ICP-AES \\
\hline $7439-98-7$ & Molybdenum & $<1.39 \mathrm{E}-1$ & ug/g dry & $1.39 \mathrm{E}-1$ & $9 / 16 / 08$ & $8 \mathrm{I} 15002$ & PNNL-AGG-ICP-AES \\
\hline $7440-02-0$ & Nickel & $<9.29 \mathrm{E}-2$ & ug/g dry & $9.29 \mathrm{E}-2$ & $9 / 16 / 08$ & $8 \mathrm{I} 15002$ & PNNL-AGG-ICP-AES \\
\hline $7723-14-0$ & Phosphorus & $<1.03 \mathrm{E} 0$ & ug/g dry & $1.03 \mathrm{E} 0$ & $9 / 16 / 08$ & $8 \mathrm{I} 15002$ & PNNL-AGG-ICP-AES \\
\hline $7439-92-1$ & Lead & $<4.30 \mathrm{E}-1$ & ug/g dry & $4.30 \mathrm{E}-1$ & $9 / 16 / 08$ & $8 \mathrm{I} 15002$ & PNNL-AGG-ICP-AES \\
\hline $7782-49-2$ & Selenium & $<1.94 \mathrm{E} 0$ & ug/g dry & $1.94 \mathrm{E} 0$ & $9 / 16 / 08$ & $8 \mathrm{I} 15002$ & PNNL-AGG-ICP-AES \\
\hline $7440-24-6$ & Strontium & $<5.20 \mathrm{E}-2$ & ug/g dry & $5.20 \mathrm{E}-2$ & $9 / 16 / 08$ & $8 \mathrm{I} 15002$ & PNNL-AGG-ICP-AES \\
\hline $7440-28-0$ & Thallium & $<1.02 \mathrm{E} 0$ & ug/g dry & $1.02 \mathrm{E} 0$ & $9 / 16 / 08$ & $8 \mathrm{I} 15002$ & PNNL-AGG-ICP-AES \\
\hline $7440-62-2$ & Vanadium & $<4.51 \mathrm{E}-2$ & ug/g dry & $4.51 \mathrm{E}-2$ & $9 / 16 / 08$ & $8 \mathrm{I} 15002$ & PNNL-AGG-ICP-AES \\
\hline $7440-66-6$ & Zinc & $3.32 \mathrm{E}-1$ & ug/g dry & $9.23 \mathrm{E}-2$ & $9 / 16 / 08$ & $8 \mathrm{I} 15002$ & PNNL-AGG-ICP-AES \\
\hline $7440-23-5$ & Sodium & $6.36 \mathrm{E} 0$ & ug/g dry & $6.66 \mathrm{E}-1$ & $9 / 16 / 08$ & $8 \mathrm{I} 15002$ & PNNL-AGG-ICP-AES \\
\hline $7440-21-3$ & Silicon & $1.16 \mathrm{E} 1$ & ug/g dry & $1.49 \mathrm{E} 0$ & $9 / 16 / 08$ & $8 \mathrm{I} 15002$ & PNNL-AGG-ICP-AES \\
\hline 7704-34-9 & Sulfur & $<3.06 \mathrm{E} 0$ & ug/g dry & $3.06 \mathrm{E} 0$ & $9 / 16 / 08$ & $8 \mathrm{I} 15002$ & PNNL-AGG-ICP-AES \\
\hline $7440-32-6$ & Titanium & $<8.73 \mathrm{E}-3$ & ug/g dry & 8.73E-3 & $9 / 16 / 08$ & $8 \mathrm{I} 15002$ & PNNL-AGG-ICP-AES \\
\hline $7440-67-7$ & Zirconium & $<1.49 \mathrm{E}-1$ & ug/g dry & $1.49 \mathrm{E}-1$ & $9 / 16 / 08$ & $8 \mathrm{I} 15002$ & PNNL-AGG-ICP-AES \\
\hline $7440-22-4$ & Silver & $<7.68 \mathrm{E}-2$ & ug/g dry & $7.68 \mathrm{E}-2$ & $9 / 16 / 08$ & $8 \mathrm{I} 15002$ & PNNL-AGG-ICP-AES \\
\hline $7440-15-5$ & Rhenium & $<1.51 \mathrm{E}-1$ & ug/g dry & $1.51 \mathrm{E}-1$ & $9 / 16 / 08$ & $8 \mathrm{I} 15002$ & PNNL-AGG-ICP-AES \\
\hline $7440-36-0$ & Antimony & $<6.31 \mathrm{E}-1$ & ug/g dry & $6.31 \mathrm{E}-1$ & $9 / 16 / 08$ & $8 \mathrm{I} 15002$ & PNNL-AGG-ICP-AES \\
\hline HEIS No. & B1V995 & & b ID: & 5003-05 & & & \\
\hline $7429-90-5$ & Aluminum & $2.47 \mathrm{E}-1$ & ug/g dry & $8.58 \mathrm{E}-2$ & $9 / 16 / 08$ & $8 \mathrm{I} 15002$ & PNNL-AGG-ICP-AES \\
\hline $7440-38-2$ & Arsenic & $<3.60 \mathrm{E}-1$ & ug/g dry & $3.60 \mathrm{E}-1$ & $9 / 16 / 08$ & $8 \mathrm{I} 15002$ & PNNL-AGG-ICP-AES \\
\hline $7440-42-8$ & Boron & $<1.94 \mathrm{E} 0$ & ug/g dry & $1.94 \mathrm{E} 0$ & $9 / 16 / 08$ & $8 \mathrm{I} 15002$ & PNNL-AGG-ICP-AES \\
\hline $7440-41-7$ & Beryllium & $<2.84 \mathrm{E}-2$ & ug/g dry & $2.84 \mathrm{E}-2$ & $9 / 16 / 08$ & $8 \mathrm{I} 15002$ & PNNL-AGG-ICP-AES \\
\hline $7440-69-9$ & Bismuth & $<1.88 \mathrm{E}-1$ & ug/g dry & $1.88 \mathrm{E}-1$ & $9 / 16 / 08$ & $8 \mathrm{I} 15002$ & PNNL-AGG-ICP-AES \\
\hline $7440-70-2$ & Calcium & 8.99E0 & ug/g dry & $3.87 \mathrm{E}-1$ & $9 / 16 / 08$ & $8 \mathrm{I} 15002$ & PNNL-AGG-ICP-AES \\
\hline $7440-43-9$ & Cadmium & $<2.69 \mathrm{E}-2$ & ug/g dry & $2.69 \mathrm{E}-2$ & $9 / 16 / 08$ & $8 \mathrm{I} 15002$ & PNNL-AGG-ICP-AES \\
\hline $7440-48-4$ & Cobalt & $<9.60 \mathrm{E}-2$ & ug/g dry & $9.60 \mathrm{E}-2$ & $9 / 16 / 08$ & $8 \mathrm{I} 15002$ & PNNL-AGG-ICP-AES \\
\hline $7440-47-3$ & Chromium & $<3.33 \mathrm{E}-2$ & ug/g dry & $3.33 \mathrm{E}-2$ & $9 / 16 / 08$ & $8 \mathrm{I} 15002$ & PNNL-AGG-ICP-AES \\
\hline $7440-50-8$ & Copper & $<8.04 \mathrm{E}-2$ & ug/g dry & $8.04 \mathrm{E}-2$ & $9 / 16 / 08$ & $8 \mathrm{I} 15002$ & PNNL-AGG-ICP-AES \\
\hline $7439-89-6$ & Iron & $2.50 \mathrm{E}-1$ & ug/g dry & $1.42 \mathrm{E}-1$ & $9 / 16 / 08$ & $8 \mathrm{I} 15002$ & PNNL-AGG-ICP-AES \\
\hline $7440-09-7$ & Potassium & 3.44E0 & ug/g dry & 2.33E0 & $9 / 16 / 08$ & $8 \mathrm{II} 15002$ & PNNL-AGG-ICP-AES \\
\hline
\end{tabular}


Total Metals by PNNL-AGG-ICP-AES/Water Extract

\begin{tabular}{|c|c|c|c|c|c|c|c|}
\hline CAS \# & Analyte & Results & Units & EQL & Analyzed & Batch & Method \\
\hline HEIS No. & B1V995 & & b ID: & 05003-05 & & & \\
\hline $7439-93-2$ & Lithium & $<5.40 \mathrm{E}-1$ & $\mathrm{ug} / \mathrm{g}$ dry & $5.40 \mathrm{E}-1$ & $9 / 16 / 08$ & $8 \mathrm{I} 15002$ & PNNL-AGG-ICP-AES \\
\hline $7439-95-4$ & Magnesium & $1.58 \mathrm{E} 0$ & ug/g dry & $8.34 \mathrm{E}-2$ & $9 / 16 / 08$ & $8 \mathrm{I} 15002$ & PNNL-AGG-ICP-AES \\
\hline $7439-96-5$ & Manganese & $<1.71 \mathrm{E}-2$ & $\mathrm{ug} / \mathrm{g}$ dry & $1.71 \mathrm{E}-2$ & $9 / 16 / 08$ & $8 \mathrm{I} 15002$ & PNNL-AGG-ICP-AES \\
\hline $7439-98-7$ & Molybdenum & $<1.39 \mathrm{E}-1$ & ug/g dry & $1.39 \mathrm{E}-1$ & $9 / 16 / 08$ & $8 \mathrm{I} 15002$ & PNNL-AGG-ICP-AES \\
\hline $7440-02-0$ & Nickel & $<9.33 \mathrm{E}-2$ & ug/g dry & $9.33 \mathrm{E}-2$ & $9 / 16 / 08$ & 8115002 & PNNL-AGG-ICP-AES \\
\hline $7723-14-0$ & Phosphorus & $<1.03 \mathrm{E} 0$ & ug/g dry & $1.03 \mathrm{E} 0$ & $9 / 16 / 08$ & $8 \mathrm{I} 15002$ & PNNL-AGG-ICP-AES \\
\hline $7439-92-1$ & Lead & $<4.32 \mathrm{E}-1$ & ug/g dry & 4.32E-1 & $9 / 16 / 08$ & $8 \mathrm{I} 15002$ & PNNL-AGG-ICP-AES \\
\hline $7782-49-2$ & Selenium & $<1.95 \mathrm{E} 0$ & ug/g dry & $1.95 \mathrm{E} 0$ & $9 / 16 / 08$ & $8 \mathrm{I} 15002$ & PNNL-AGG-ICP-AES \\
\hline $7440-24-6$ & Strontium & $<5.22 \mathrm{E}-2$ & ug/g dry & $5.22 \mathrm{E}-2$ & $9 / 16 / 08$ & $8 \mathrm{I} 15002$ & PNNL-AGG-ICP-AES \\
\hline $7440-28-0$ & Thallium & $<1.03 \mathrm{E} 0$ & $\mathrm{ug} / \mathrm{g}$ dry & $1.03 \mathrm{E} 0$ & $9 / 16 / 08$ & $8 \mathrm{I} 15002$ & PNNL-AGG-ICP-AES \\
\hline $7440-62-2$ & Vanadium & $<4.53 \mathrm{E}-2$ & ug/g dry & $4.53 \mathrm{E}-2$ & $9 / 16 / 08$ & $8 \mathrm{I} 15002$ & PNNL-AGG-ICP-AES \\
\hline $7440-66-6$ & Zinc & $<9.27 \mathrm{E}-2$ & ug/g dry & $9.27 \mathrm{E}-2$ & $9 / 16 / 08$ & $8 \mathrm{I} 15002$ & PNNL-AGG-ICP-AES \\
\hline $7440-23-5$ & Sodium & $8.03 \mathrm{E} 0$ & ug/g dry & $6.69 \mathrm{E}-1$ & $9 / 16 / 08$ & $8 \mathrm{I} 15002$ & PNNL-AGG-ICP-AES \\
\hline $7440-21-3$ & Silicon & $1.17 \mathrm{E} 1$ & ug/g dry & $1.50 \mathrm{E} 0$ & $9 / 16 / 08$ & $8 \mathrm{I} 15002$ & PNNL-AGG-ICP-AES \\
\hline $7704-34-9$ & Sulfur & $<3.07 \mathrm{E} 0$ & ug/g dry & $3.07 \mathrm{E} 0$ & $9 / 16 / 08$ & $8 \mathrm{I} 15002$ & PNNL-AGG-ICP-AES \\
\hline $7440-32-6$ & Titanium & $1.09 \mathrm{E}-2$ & ug/g dry & $8.76 \mathrm{E}-3$ & $9 / 16 / 08$ & $8 \mathrm{I} 15002$ & PNNL-AGG-ICP-AES \\
\hline $7440-67-7$ & Zirconium & $<1.50 \mathrm{E}-1$ & ug/g dry & $1.50 \mathrm{E}-1$ & $9 / 16 / 08$ & $8 \mathrm{I} 15002$ & PNNL-AGG-ICP-AES \\
\hline $7440-22-4$ & Silver & $<7.71 \mathrm{E}-2$ & $\mathrm{ug} / \mathrm{g}$ dry & 7.71E-2 & $9 / 16 / 08$ & $8 \mathrm{I} 15002$ & PNNL-AGG-ICP-AES \\
\hline $7440-15-5$ & Rhenium & $<1.52 \mathrm{E}-1$ & ug/g dry & $1.52 \mathrm{E}-1$ & $9 / 16 / 08$ & $8 \mathrm{I} 15002$ & PNNL-AGG-ICP-AES \\
\hline $7440-36-0$ & Antimony & $<6.33 \mathrm{E}-1$ & ug/g dry & $6.33 \mathrm{E}-1$ & $9 / 16 / 08$ & $8 \mathrm{II} 15002$ & PNNL-AGG-ICP-AES \\
\hline HEIS No. & B1V996 & & b ID: & 5003-06 & & & \\
\hline $7429-90-5$ & Aluminum & $4.07 \mathrm{E}-1$ & ug/g dry & $8.58 \mathrm{E}-2$ & $9 / 16 / 08$ & $8 \mathrm{I} 15003$ & PNNL-AGG-ICP-AES \\
\hline $7440-38-2$ & Arsenic & $<3.60 \mathrm{E}-1$ & ug/g dry & $3.60 \mathrm{E}-1$ & $9 / 16 / 08$ & $8 \mathrm{I} 15003$ & PNNL-AGG-ICP-AES \\
\hline $7440-42-8$ & Boron & $<1.94 \mathrm{E} 0$ & $\mathrm{ug} / \mathrm{g}$ dry & $1.94 \mathrm{E} 0$ & $9 / 16 / 08$ & $8 \mathrm{I} 15003$ & PNNL-AGG-ICP-AES \\
\hline $7440-41-7$ & Beryllium & $<2.84 \mathrm{E}-2$ & ug/g dry & $2.84 \mathrm{E}-2$ & $9 / 16 / 08$ & $8 \mathrm{I} 15003$ & PNNL-AGG-ICP-AES \\
\hline $7440-69-9$ & Bismuth & $<1.88 \mathrm{E}-1$ & $\mathrm{ug} / \mathrm{g}$ dry & $1.88 \mathrm{E}-1$ & $9 / 16 / 08$ & $8 \mathrm{I} 15003$ & PNNL-AGG-ICP-AES \\
\hline $7440-70-2$ & Calcium & $6.13 \mathrm{E} 0$ & ug/g dry & $3.87 \mathrm{E}-1$ & $9 / 16 / 08$ & $8 \mathrm{I} 15003$ & PNNL-AGG-ICP-AES \\
\hline $7440-43-9$ & Cadmium & $<2.69 \mathrm{E}-2$ & ug/g dry & $2.69 \mathrm{E}-2$ & $9 / 16 / 08$ & $8 \mathrm{I} 15003$ & PNNL-AGG-ICP-AES \\
\hline $7440-48-4$ & Cobalt & $<9.60 \mathrm{E}-2$ & ug/g dry & $9.60 \mathrm{E}-2$ & $9 / 16 / 08$ & $8 \mathrm{I} 15003$ & PNNL-AGG-ICP-AES \\
\hline $7440-47-3$ & Chromium & $<3.33 \mathrm{E}-2$ & ug/g dry & 3.33E-2 & $9 / 16 / 08$ & $8 \mathrm{I} 15003$ & PNNL-AGG-ICP-AES \\
\hline $7440-50-8$ & Copper & $<8.04 \mathrm{E}-2$ & ug/g dry & $8.04 \mathrm{E}-2$ & $9 / 16 / 08$ & $8 \mathrm{I} 15003$ & PNNL-AGG-ICP-AES \\
\hline $7439-89-6$ & Iron & $4.35 \mathrm{E}-1$ & ug/g dry & $1.42 \mathrm{E}-1$ & $9 / 16 / 08$ & $8 \mathrm{I} 15003$ & PNNL-AGG-ICP-AES \\
\hline $7440-09-7$ & Potassium & $2.97 \mathrm{E} 0$ & $\mathrm{ug} / \mathrm{g}$ dry & $2.33 \mathrm{E} 0$ & $9 / 16 / 08$ & $8 \mathrm{I} 15003$ & PNNL-AGG-ICP-AES \\
\hline $7439-93-2$ & Lithium & $<5.40 \mathrm{E}-1$ & ug/g dry & $5.40 \mathrm{E}-1$ & $9 / 16 / 08$ & $8 \mathrm{I} 15003$ & PNNL-AGG-ICP-AES \\
\hline $7439-95-4$ & Magnesium & $1.06 \mathrm{E} 0$ & ug/g dry & $8.34 \mathrm{E}-2$ & $9 / 16 / 08$ & $8 \mathrm{I} 15003$ & PNNL-AGG-ICP-AES \\
\hline $7439-96-5$ & Manganese & $<1.71 \mathrm{E}-2$ & ug/g dry & $1.71 \mathrm{E}-2$ & $9 / 16 / 08$ & $8 \mathrm{II} 15003$ & PNNL-AGG-ICP-AES \\
\hline $7439-98-7$ & Molybdenum & $<1.39 \mathrm{E}-1$ & ug/g dry & $1.39 \mathrm{E}-1$ & $9 / 16 / 08$ & $8 \mathrm{I} 15003$ & PNNL-AGG-ICP-AES \\
\hline $7440-02-0$ & Nickel & $<9.33 \mathrm{E}-2$ & $\mathrm{ug} / \mathrm{g}$ dry & $9.33 \mathrm{E}-2$ & $9 / 16 / 08$ & $8 \mathrm{I} 15003$ & PNNL-AGG-ICP-AES \\
\hline $7723-14-0$ & Phosphorus & $<1.03 \mathrm{E} 0$ & $\mathrm{ug} / \mathrm{g}$ dry & $1.03 \mathrm{E} 0$ & $9 / 16 / 08$ & $8 \mathrm{I} 15003$ & PNNL-AGG-ICP-AES \\
\hline $7439-92-1$ & Lead & $<4.32 \mathrm{E}-1$ & ug/g dry & $4.32 \mathrm{E}-1$ & $9 / 16 / 08$ & $8 \mathrm{I} 15003$ & PNNL-AGG-ICP-AES \\
\hline $7782-49-2$ & Selenium & $<1.95 \mathrm{E} 0$ & $\mathrm{ug} / \mathrm{g}$ dry & $1.95 \mathrm{E} 0$ & $9 / 16 / 08$ & $8 \mathrm{I} 15003$ & PNNL-AGG-ICP-AES \\
\hline $7440-24-6$ & Strontium & $<5.22 \mathrm{E}-2$ & ug/g dry & $5.22 \mathrm{E}-2$ & $9 / 16 / 08$ & $8 \mathrm{I} 15003$ & PNNL-AGG-ICP-AES \\
\hline $7440-28-0$ & Thallium & $<1.03 \mathrm{E} 0$ & ug/g dry & $1.03 \mathrm{E} 0$ & $9 / 16 / 08$ & $8 \mathrm{I} 15003$ & PNNL-AGG-ICP-AES \\
\hline $7440-62-2$ & Vanadium & $5.96 \mathrm{E}-2$ & ug/g dry & $4.53 \mathrm{E}-2$ & $9 / 16 / 08$ & $8 \mathrm{I} 15003$ & PNNL-AGG-ICP-AES \\
\hline $7440-66-6$ & Zinc & $<9.27 \mathrm{E}-2$ & ug/g dry & $9.27 \mathrm{E}-2$ & $9 / 16 / 08$ & $8 \mathrm{I} 15003$ & PNNL-AGG-ICP-AES \\
\hline $7440-23-5$ & Sodium & $2.40 \mathrm{E} 1$ & ug/g dry & $6.69 \mathrm{E}-1$ & $9 / 16 / 08$ & $8 \mathrm{I} 15003$ & PNNL-AGG-ICP-AES \\
\hline $7704-34-9$ & Sulfur & $<3.08 \mathrm{E} 0$ & ug/g dry & $3.08 \mathrm{E} 0$ & $9 / 16 / 08$ & $8 \mathrm{I} 15003$ & PNNL-AGG-ICP-AES \\
\hline $7440-32-6$ & Titanium & $2.04 \mathrm{E}-2$ & ug/g dry & $8.76 \mathrm{E}-3$ & $9 / 16 / 08$ & $8 \mathrm{I} 15003$ & PNNL-AGG-ICP-AES \\
\hline
\end{tabular}


Total Metals by PNNL-AGG-ICP-AES/Water Extract

\begin{tabular}{|c|c|c|c|c|c|c|c|}
\hline CAS \# & Analyte & Results & Units & EQL & Analyzed & Batch & Method \\
\hline HEIS No. & B1V996 & \multicolumn{3}{|c|}{ Lab ID: $\quad$ 0805003-06 } & & & \\
\hline $7440-67-7$ & Zirconium & $<1.50 \mathrm{E}-1$ & ug/g dry & $1.50 \mathrm{E}-1$ & $9 / 16 / 08$ & $8 \mathrm{I} 15003$ & PNNL-AGG-ICP-AES \\
\hline $7440-22-4$ & Silver & $<7.71 \mathrm{E}-2$ & ug/g dry & $7.71 \mathrm{E}-2$ & $9 / 16 / 08$ & $8 \mathrm{I} 15003$ & PNNL-AGG-ICP-AES \\
\hline $7440-15-5$ & Rhenium & $<1.52 \mathrm{E}-1$ & ug/g dry & $1.52 \mathrm{E}-1$ & $9 / 16 / 08$ & $8 \mathrm{I} 15003$ & PNNL-AGG-ICP-AES \\
\hline $7440-36-0$ & Antimony & $<6.33 \mathrm{E}-1$ & ug/g dry & $6.33 \mathrm{E}-1$ & $9 / 16 / 08$ & $8 \mathrm{I} 15003$ & PNNL-AGG-ICP-AES \\
\hline HEIS No. & B1V997 & \multicolumn{3}{|c|}{ Lab ID: $\quad$ 0805003-07 } & & & \\
\hline $7429-90-5$ & Aluminum & $4.62 \mathrm{E}-1$ & ug/g dry & $8.57 \mathrm{E}-2$ & $9 / 16 / 08$ & $8 \mathrm{I} 15003$ & PNNL-AGG-ICP-AES \\
\hline $7440-38-2$ & Arsenic & $<3.60 \mathrm{E}-1$ & ug/g dry & $3.60 \mathrm{E}-1$ & $9 / 16 / 08$ & $8 \mathrm{I} 15003$ & PNNL-AGG-ICP-AES \\
\hline $7440-42-8$ & Boron & $<1.94 \mathrm{E} 0$ & ug/g dry & $1.94 \mathrm{E} 0$ & $9 / 16 / 08$ & $8 \mathrm{I} 15003$ & PNNL-AGG-ICP-AES \\
\hline $7440-41-7$ & Beryllium & $<2.84 \mathrm{E}-2$ & ug/g dry & $2.84 \mathrm{E}-2$ & $9 / 16 / 08$ & $8 \mathrm{I} 15003$ & PNNL-AGG-ICP-AES \\
\hline $7440-69-9$ & Bismuth & $<1.88 \mathrm{E}-1$ & ug/g dry & $1.88 \mathrm{E}-1$ & $9 / 16 / 08$ & $8 \mathrm{I} 15003$ & PNNL-AGG-ICP-AES \\
\hline $7440-70-2$ & Calcium & $5.68 \mathrm{E} 0$ & ug/g dry & $3.87 \mathrm{E}-1$ & $9 / 16 / 08$ & $8 \mathrm{I} 15003$ & PNNL-AGG-ICP-AES \\
\hline $7440-43-9$ & Cadmium & $<2.69 \mathrm{E}-2$ & ug/g dry & $2.69 \mathrm{E}-2$ & $9 / 16 / 08$ & $8 \mathrm{I} 15003$ & PNNL-AGG-ICP-AES \\
\hline $7440-48-4$ & Cobalt & $<9.59 \mathrm{E}-2$ & ug/g dry & $9.59 \mathrm{E}-2$ & $9 / 16 / 08$ & $8 \mathrm{I} 15003$ & PNNL-AGG-ICP-AES \\
\hline $7440-47-3$ & Chromium & $<3.33 \mathrm{E}-2$ & ug/g dry & $3.33 \mathrm{E}-2$ & $9 / 16 / 08$ & $8 \mathrm{I} 15003$ & PNNL-AGG-ICP-AES \\
\hline $7440-50-8$ & Copper & $<8.03 \mathrm{E}-2$ & ug/g dry & $8.03 \mathrm{E}-2$ & $9 / 16 / 08$ & $8 \mathrm{I} 15003$ & PNNL-AGG-ICP-AES \\
\hline $7439-89-6$ & Iron & $5.35 \mathrm{E}-1$ & ug/g dry & $1.42 \mathrm{E}-1$ & $9 / 16 / 08$ & $8 \mathrm{I} 15003$ & PNNL-AGG-ICP-AES \\
\hline $7440-09-7$ & Potassium & $2.82 \mathrm{E} 0$ & ug/g dry & 2.33E0 & $9 / 16 / 08$ & $8 \mathrm{I} 15003$ & PNNL-AGG-ICP-AES \\
\hline $7439-93-2$ & Lithium & $<5.40 \mathrm{E}-1$ & ug/g dry & $5.40 \mathrm{E}-1$ & $9 / 16 / 08$ & $8 \mathrm{I} 15003$ & PNNL-AGG-ICP-AES \\
\hline $7439-95-4$ & Magnesium & $1.01 \mathrm{E} 0$ & ug/g dry & $8.33 \mathrm{E}-2$ & $9 / 16 / 08$ & $8 \mathrm{I} 15003$ & PNNL-AGG-ICP-AES \\
\hline $7439-96-5$ & Manganese & $<1.71 \mathrm{E}-2$ & ug/g dry & $1.71 \mathrm{E}-2$ & $9 / 16 / 08$ & $8 \mathrm{I} 15003$ & PNNL-AGG-ICP-AES \\
\hline $7439-98-7$ & Molybdenum & $<1.39 \mathrm{E}-1$ & ug/g dry & $1.39 \mathrm{E}-1$ & $9 / 16 / 08$ & $8 \mathrm{I} 15003$ & PNNL-AGG-ICP-AES \\
\hline $7440-02-0$ & Nickel & $<9.32 \mathrm{E}-2$ & ug/g dry & $9.32 \mathrm{E}-2$ & $9 / 16 / 08$ & $8 \mathrm{I} 15003$ & PNNL-AGG-ICP-AES \\
\hline $7723-14-0$ & Phosphorus & $<1.03 \mathrm{E} 0$ & ug/g dry & $1.03 \mathrm{E} 0$ & $9 / 16 / 08$ & $8 \mathrm{I} 15003$ & PNNL-AGG-ICP-AES \\
\hline $7439-92-1$ & Lead & $<4.32 \mathrm{E}-1$ & ug/g dry & $4.32 \mathrm{E}-1$ & $9 / 16 / 08$ & $8 \mathrm{I} 15003$ & PNNL-AGG-ICP-AES \\
\hline $7782-49-2$ & Selenium & $<1.95 \mathrm{E} 0$ & ug/g dry & $1.95 \mathrm{E} 0$ & $9 / 16 / 08$ & $8 \mathrm{I} 15003$ & PNNL-AGG-ICP-AES \\
\hline $7440-24-6$ & Strontium & $<5.22 \mathrm{E}-2$ & ug/g dry & $5.22 \mathrm{E}-2$ & $9 / 16 / 08$ & $8 \mathrm{I} 15003$ & PNNL-AGG-ICP-AES \\
\hline $7440-28-0$ & Thallium & $<1.03 \mathrm{E} 0$ & ug/g dry & $1.03 \mathrm{E} 0$ & $9 / 16 / 08$ & $8 \mathrm{I} 15003$ & PNNL-AGG-ICP-AES \\
\hline $7440-62-2$ & Vanadium & $5.02 \mathrm{E}-2$ & ug/g dry & $4.53 \mathrm{E}-2$ & $9 / 16 / 08$ & $8 \mathrm{I} 15003$ & PNNL-AGG-ICP-AES \\
\hline $7440-66-6$ & Zinc & $<9.26 \mathrm{E}-2$ & ug/g dry & $9.26 \mathrm{E}-2$ & $9 / 16 / 08$ & $8 \mathrm{I} 15003$ & PNNL-AGG-ICP-AES \\
\hline $7440-23-5$ & Sodium & $2.29 \mathrm{E} 1$ & ug/g dry & $6.68 \mathrm{E}-1$ & $9 / 16 / 08$ & $8 \mathrm{I} 15003$ & PNNL-AGG-ICP-AES \\
\hline 7704-34-9 & Sulfur & $<3.07 \mathrm{E} 0$ & ug/g dry & $3.07 \mathrm{E} 0$ & $9 / 16 / 08$ & $8 \mathrm{I} 15003$ & PNNL-AGG-ICP-AES \\
\hline $7440-32-6$ & Titanium & $2.20 \mathrm{E}-2$ & ug/g dry & $8.75 \mathrm{E}-3$ & $9 / 16 / 08$ & $8 \mathrm{I} 15003$ & PNNL-AGG-ICP-AES \\
\hline $7440-67-7$ & Zirconium & $<1.50 \mathrm{E}-1$ & ug/g dry & $1.50 \mathrm{E}-1$ & $9 / 16 / 08$ & $8 \mathrm{I} 15003$ & PNNL-AGG-ICP-AES \\
\hline $7440-22-4$ & Silver & $<7.70 \mathrm{E}-2$ & ug/g dry & $7.70 \mathrm{E}-2$ & $9 / 16 / 08$ & $8 \mathrm{I} 15003$ & PNNL-AGG-ICP-AES \\
\hline $7440-15-5$ & Rhenium & $<1.52 \mathrm{E}-1$ & ug/g dry & $1.52 \mathrm{E}-1$ & $9 / 16 / 08$ & $8 \mathrm{I} 15003$ & PNNL-AGG-ICP-AES \\
\hline $7440-36-0$ & Antimony & $<6.32 \mathrm{E}-1$ & ug/g dry & $6.32 \mathrm{E}-1$ & 9/16/08 & $8 \mathrm{I} 15003$ & PNNL-AGG-ICP-AES \\
\hline HEIS No. & B1V998 & & b ID: & $5003-08$ & & & \\
\hline $7429-90-5$ & Aluminum & $5.70 \mathrm{E}-1$ & ug/g dry & $8.59 \mathrm{E}-2$ & $9 / 16 / 08$ & $8 \mathrm{I} 15003$ & PNNL-AGG-ICP-AES \\
\hline $7440-38-2$ & Arsenic & $<3.60 \mathrm{E}-1$ & ug/g dry & $3.60 \mathrm{E}-1$ & $9 / 16 / 08$ & $8 \mathrm{I} 15003$ & PNNL-AGG-ICP-AES \\
\hline $7440-42-8$ & Boron & $<1.94 \mathrm{E} 0$ & ug/g dry & $1.94 \mathrm{E} 0$ & $9 / 16 / 08$ & $8 \mathrm{I} 15003$ & PNNL-AGG-ICP-AES \\
\hline $7440-41-7$ & Beryllium & $<2.84 \mathrm{E}-2$ & ug/g dry & $2.84 \mathrm{E}-2$ & $9 / 16 / 08$ & $8 \mathrm{I} 15003$ & PNNL-AGG-ICP-AES \\
\hline $7440-69-9$ & Bismuth & $<1.88 \mathrm{E}-1$ & ug/g dry & $1.88 \mathrm{E}-1$ & $9 / 16 / 08$ & $8 \mathrm{I} 15003$ & PNNL-AGG-ICP-AES \\
\hline $7440-70-2$ & Calcium & $3.40 \mathrm{E} 0$ & ug/g dry & $3.87 \mathrm{E}-1$ & $9 / 16 / 08$ & $8 \mathrm{I} 15003$ & PNNL-AGG-ICP-AES \\
\hline $7440-43-9$ & Cadmium & $<2.69 \mathrm{E}-2$ & ug/g dry & $2.69 \mathrm{E}-2$ & 9/16/08 & $8 \mathrm{I} 15003$ & PNNL-AGG-ICP-AES \\
\hline $7440-48-4$ & Cobalt & $<9.61 \mathrm{E}-2$ & ug/g dry & $9.61 \mathrm{E}-2$ & $9 / 16 / 08$ & $8 \mathrm{I} 15003$ & PNNL-AGG-ICP-AES \\
\hline $7440-47-3$ & Chromium & $<3.33 \mathrm{E}-2$ & ug/g dry & $3.33 \mathrm{E}-2$ & $9 / 16 / 08$ & $8 \mathrm{I} 15003$ & PNNL-AGG-ICP-AES \\
\hline $7440-50-8$ & Copper & $<8.05 \mathrm{E}-2$ & ug/g dry & $8.05 \mathrm{E}-2$ & $9 / 16 / 08$ & $8 \mathrm{I} 15003$ & PNNL-AGG-ICP-AES \\
\hline $7439-89-6$ & Iron & $6.84 \mathrm{E}-1$ & ug/g dry & $1.42 \mathrm{E}-1$ & 9/16/08 & $8 \mathrm{I} 15003$ & PNNL-AGG-ICP-AES \\
\hline
\end{tabular}


Total Metals by PNNL-AGG-ICP-AES/Water Extract

\begin{tabular}{|c|c|c|c|c|c|c|c|}
\hline CAS \# & Analyte & Results & Units & EQL & Analyzed & Batch & Method \\
\hline HEIS No. & B1V998 & & b ID: & 55003-08 & & & \\
\hline 7440-09-7 & Potassium & $2.82 \mathrm{E} 0$ & $\mathrm{ug} / \mathrm{g}$ dry & $2.33 \mathrm{E} 0$ & $9 / 16 / 08$ & $8 \mathrm{I} 15003$ & PNNL-AGG-ICP-AES \\
\hline $7439-93-2$ & Lithium & $<5.40 \mathrm{E}-1$ & ug/g dry & $5.40 \mathrm{E}-1$ & $9 / 16 / 08$ & $8 \mathrm{I} 15003$ & PNNL-AGG-ICP-AES \\
\hline $7439-95-4$ & Magnesium & 8.47E-1 & $\mathrm{ug} / \mathrm{g}$ dry & $8.35 \mathrm{E}-2$ & $9 / 16 / 08$ & $8 \mathrm{I} 15003$ & PNNL-AGG-ICP-AES \\
\hline $7439-96-5$ & Manganese & $<1.71 \mathrm{E}-2$ & ug/g dry & $1.71 \mathrm{E}-2$ & $9 / 16 / 08$ & $8 \mathrm{I} 15003$ & PNNL-AGG-ICP-AES \\
\hline $7439-98-7$ & Molybdenum & $<1.39 \mathrm{E}-1$ & ug/g dry & $1.39 \mathrm{E}-1$ & $9 / 16 / 08$ & $8 \mathrm{I} 15003$ & PNNL-AGG-ICP-AES \\
\hline $7440-02-0$ & Nickel & $<9.34 \mathrm{E}-2$ & ug/g dry & $9.34 \mathrm{E}-2$ & $9 / 16 / 08$ & $8 \mathrm{I} 15003$ & PNNL-AGG-ICP-AES \\
\hline $7723-14-0$ & Phosphorus & $<1.03 \mathrm{E} 0$ & ug/g dry & $1.03 \mathrm{E} 0$ & $9 / 16 / 08$ & $8 \mathrm{I} 15003$ & PNNL-AGG-ICP-AES \\
\hline $7439-92-1$ & Lead & $<4.32 \mathrm{E}-1$ & ug/g dry & $4.32 \mathrm{E}-1$ & $9 / 16 / 08$ & $8 \mathrm{I} 15003$ & PNNL-AGG-ICP-AES \\
\hline $7782-49-2$ & Selenium & $<1.95 \mathrm{E} 0$ & ug/g dry & $1.95 \mathrm{E} 0$ & $9 / 16 / 08$ & $8 \mathrm{I} 15003$ & PNNL-AGG-ICP-AES \\
\hline $7440-24-6$ & Strontium & $<5.22 \mathrm{E}-2$ & $\mathrm{ug} / \mathrm{g}$ dry & $5.22 \mathrm{E}-2$ & $9 / 16 / 08$ & $8 \mathrm{I} 15003$ & PNNL-AGG-ICP-AES \\
\hline $7440-28-0$ & Thallium & $<1.03 \mathrm{E} 0$ & ug/g dry & $1.03 \mathrm{E} 0$ & $9 / 16 / 08$ & $8 \mathrm{I} 15003$ & PNNL-AGG-ICP-AES \\
\hline $7440-62-2$ & Vanadium & $5.17 \mathrm{E}-2$ & ug/g dry & $4.53 \mathrm{E}-2$ & $9 / 16 / 08$ & $8 \mathrm{I} 15003$ & PNNL-AGG-ICP-AES \\
\hline $7440-66-6$ & Zinc & $1.69 \mathrm{E}-1$ & ug/g dry & $9.28 \mathrm{E}-2$ & $9 / 16 / 08$ & $8 \mathrm{I} 15003$ & PNNL-AGG-ICP-AES \\
\hline $7440-23-5$ & Sodium & $2.18 \mathrm{E} 1$ & ug/g dry & $6.70 \mathrm{E}-1$ & $9 / 16 / 08$ & $8 \mathrm{I} 15003$ & PNNL-AGG-ICP-AES \\
\hline $7704-34-9$ & Sulfur & $3.23 \mathrm{E} 0$ & $\mathrm{ug} / \mathrm{g}$ dry & $3.08 \mathrm{E} 0$ & $9 / 16 / 08$ & $8 \mathrm{I} 15003$ & PNNL-AGG-ICP-AES \\
\hline $7440-32-6$ & Titanium & $2.94 \mathrm{E}-2$ & ug/g dry & $8.77 \mathrm{E}-3$ & $9 / 16 / 08$ & $8 \mathrm{I} 15003$ & PNNL-AGG-ICP-AES \\
\hline $7440-67-7$ & Zirconium & $<1.50 \mathrm{E}-1$ & ug/g dry & $1.50 \mathrm{E}-1$ & $9 / 16 / 08$ & $8 \mathrm{I} 15003$ & PNNL-AGG-ICP-AES \\
\hline $7440-22-4$ & Silver & $<7.72 \mathrm{E}-2$ & $\mathrm{ug} / \mathrm{g}$ dry & $7.72 \mathrm{E}-2$ & $9 / 16 / 08$ & $8 \mathrm{I} 15003$ & PNNL-AGG-ICP-AES \\
\hline $7440-15-5$ & Rhenium & $<1.52 \mathrm{E}-1$ & ug/g dry & $1.52 \mathrm{E}-1$ & $9 / 16 / 08$ & $8 \mathrm{I} 15003$ & PNNL-AGG-ICP-AES \\
\hline $7440-36-0$ & Antimony & $<6.34 \mathrm{E}-1$ & ug/g dry & $6.34 \mathrm{E}-1$ & $9 / 16 / 08$ & $8 \mathrm{I} 15003$ & PNNL-AGG-ICP-AES \\
\hline HEIS No. & B1V999 & & b ID: & 5003-09 & & & \\
\hline $7429-90-5$ & Aluminum & $3.68 \mathrm{E}-1$ & ug/g dry & $8.56 \mathrm{E}-2$ & $9 / 16 / 08$ & $8 \mathrm{I} 15003$ & PNNL-AGG-ICP-AES \\
\hline $7440-38-2$ & Arsenic & $<3.59 \mathrm{E}-1$ & ug/g dry & $3.59 \mathrm{E}-1$ & $9 / 16 / 08$ & $8 \mathrm{I} 15003$ & PNNL-AGG-ICP-AES \\
\hline $7440-42-8$ & Boron & $<1.94 \mathrm{E} 0$ & $\mathrm{ug} / \mathrm{g}$ dry & $1.94 \mathrm{E} 0$ & $9 / 16 / 08$ & $8 \mathrm{I} 15003$ & PNNL-AGG-ICP-AES \\
\hline $7440-41-7$ & Beryllium & $<2.83 \mathrm{E}-2$ & ug/g dry & $2.83 \mathrm{E}-2$ & $9 / 16 / 08$ & $8 \mathrm{I} 15003$ & PNNL-AGG-ICP-AES \\
\hline $7440-69-9$ & Bismuth & $<1.88 \mathrm{E}-1$ & $\mathrm{ug} / \mathrm{g}$ dry & $1.88 \mathrm{E}-1$ & $9 / 16 / 08$ & $8 \mathrm{I} 15003$ & PNNL-AGG-ICP-AES \\
\hline $7440-70-2$ & Calcium & 4.13E0 & ug/g dry & $3.86 \mathrm{E}-1$ & $9 / 16 / 08$ & $8 \mathrm{I} 15003$ & PNNL-AGG-ICP-AES \\
\hline $7440-43-9$ & Cadmium & $<2.68 \mathrm{E}-2$ & ug/g dry & $2.68 \mathrm{E}-2$ & $9 / 16 / 08$ & $8 \mathrm{I} 15003$ & PNNL-AGG-ICP-AES \\
\hline $7440-48-4$ & Cobalt & $<9.57 \mathrm{E}-2$ & ug/g dry & $9.57 \mathrm{E}-2$ & $9 / 16 / 08$ & $8 \mathrm{I} 15003$ & PNNL-AGG-ICP-AES \\
\hline $7440-47-3$ & Chromium & $<3.32 \mathrm{E}-2$ & ug/g dry & $3.32 \mathrm{E}-2$ & $9 / 16 / 08$ & $8 \mathrm{I} 15003$ & PNNL-AGG-ICP-AES \\
\hline $7440-50-8$ & Copper & $<8.02 \mathrm{E}-2$ & ug/g dry & $8.02 \mathrm{E}-2$ & $9 / 16 / 08$ & $8 \mathrm{I} 15003$ & PNNL-AGG-ICP-AES \\
\hline $7439-89-6$ & Iron & $4.46 \mathrm{E}-1$ & ug/g dry & $1.42 \mathrm{E}-1$ & $9 / 16 / 08$ & $8 \mathrm{I} 15003$ & PNNL-AGG-ICP-AES \\
\hline $7440-09-7$ & Potassium & $2.90 \mathrm{E} 0$ & $\mathrm{ug} / \mathrm{g}$ dry & $2.32 \mathrm{E} 0$ & $9 / 16 / 08$ & $8 \mathrm{I} 15003$ & PNNL-AGG-ICP-AES \\
\hline $7439-93-2$ & Lithium & $<5.38 \mathrm{E}-1$ & ug/g dry & $5.38 \mathrm{E}-1$ & $9 / 16 / 08$ & $8 \mathrm{I} 15003$ & PNNL-AGG-ICP-AES \\
\hline $7439-95-4$ & Magnesium & $8.08 \mathrm{E}-1$ & ug/g dry & $8.32 \mathrm{E}-2$ & $9 / 16 / 08$ & $8 \mathrm{I} 15003$ & PNNL-AGG-ICP-AES \\
\hline $7439-96-5$ & Manganese & $<1.71 \mathrm{E}-2$ & ug/g dry & $1.71 \mathrm{E}-2$ & $9 / 16 / 08$ & $8 \mathrm{I} 15003$ & PNNL-AGG-ICP-AES \\
\hline $7439-98-7$ & Molybdenum & $<1.39 \mathrm{E}-1$ & ug/g dry & $1.39 \mathrm{E}-1$ & $9 / 16 / 08$ & $8 \mathrm{I} 15003$ & PNNL-AGG-ICP-AES \\
\hline $7440-02-0$ & Nickel & $<9.30 \mathrm{E}-2$ & $\mathrm{ug} / \mathrm{g}$ dry & $9.30 \mathrm{E}-2$ & $9 / 16 / 08$ & $8 \mathrm{I} 15003$ & PNNL-AGG-ICP-AES \\
\hline $7723-14-0$ & Phosphorus & $<1.03 \mathrm{E} 0$ & $\mathrm{ug} / \mathrm{g}$ dry & $1.03 \mathrm{E} 0$ & $9 / 16 / 08$ & $8 \mathrm{I} 15003$ & PNNL-AGG-ICP-AES \\
\hline $7439-92-1$ & Lead & $<4.31 \mathrm{E}-1$ & ug/g dry & $4.31 \mathrm{E}-1$ & $9 / 16 / 08$ & $8 \mathrm{I} 15003$ & PNNL-AGG-ICP-AES \\
\hline $7782-49-2$ & Selenium & $<1.94 \mathrm{E} 0$ & $\mathrm{ug} / \mathrm{g}$ dry & $1.94 \mathrm{E} 0$ & $9 / 16 / 08$ & $8 \mathrm{I} 15003$ & PNNL-AGG-ICP-AES \\
\hline $7440-24-6$ & Strontium & $<5.20 \mathrm{E}-2$ & ug/g dry & $5.20 \mathrm{E}-2$ & $9 / 16 / 08$ & $8 \mathrm{I} 15003$ & PNNL-AGG-ICP-AES \\
\hline $7440-28-0$ & Thallium & $<1.02 \mathrm{E} 0$ & ug/g dry & $1.02 \mathrm{E} 0$ & $9 / 16 / 08$ & $8 \mathrm{I} 15003$ & PNNL-AGG-ICP-AES \\
\hline $7440-62-2$ & Vanadium & $<4.52 \mathrm{E}-2$ & ug/g dry & $4.52 \mathrm{E}-2$ & $9 / 16 / 08$ & $8 \mathrm{I} 15003$ & PNNL-AGG-ICP-AES \\
\hline $7440-66-6$ & Zinc & $<9.24 \mathrm{E}-2$ & ug/g dry & $9.24 \mathrm{E}-2$ & $9 / 16 / 08$ & $8 \mathrm{I} 15003$ & PNNL-AGG-ICP-AES \\
\hline $7440-23-5$ & Sodium & $2.02 \mathrm{E} 1$ & ug/g dry & $6.67 \mathrm{E}-1$ & $9 / 16 / 08$ & $8 \mathrm{I} 15003$ & PNNL-AGG-ICP-AES \\
\hline $7704-34-9$ & Sulfur & $4.32 \mathrm{E} 0$ & ug/g dry & 3.07E0 & $9 / 16 / 08$ & $8 \mathrm{I} 15003$ & PNNL-AGG-ICP-AES \\
\hline $7440-32-6$ & Titanium & $1.92 \mathrm{E}-2$ & ug/g dry & $8.73 \mathrm{E}-3$ & $9 / 16 / 08$ & $8 \mathrm{I} 15003$ & PNNL-AGG-ICP-AES \\
\hline
\end{tabular}


Total Metals by PNNL-AGG-ICP-AES/Water Extract

\begin{tabular}{|c|c|c|c|c|c|c|c|}
\hline CAS \# & Analyte & Results & Units & EQL & Analyzed & Batch & Method \\
\hline HEIS No. & B1V999 & \multicolumn{3}{|c|}{ Lab ID: $\quad$ 0805003-09 } & & & \\
\hline $7440-67-7$ & Zirconium & $<1.50 \mathrm{E}-1$ & ug/g dry & $1.50 \mathrm{E}-1$ & $9 / 16 / 08$ & $8 \mathrm{I} 15003$ & PNNL-AGG-ICP-AES \\
\hline $7440-22-4$ & Silver & $<7.69 \mathrm{E}-2$ & ug/g dry & $7.69 \mathrm{E}-2$ & $9 / 16 / 08$ & $8 \mathrm{I} 15003$ & PNNL-AGG-ICP-AES \\
\hline $7440-15-5$ & Rhenium & $<1.51 \mathrm{E}-1$ & ug/g dry & $1.51 \mathrm{E}-1$ & $9 / 16 / 08$ & $8 \mathrm{I} 15003$ & PNNL-AGG-ICP-AES \\
\hline $7440-36-0$ & Antimony & $<6.31 \mathrm{E}-1$ & ug/g dry & $6.31 \mathrm{E}-1$ & $9 / 16 / 08$ & $8 \mathrm{I} 15003$ & PNNL-AGG-ICP-AES \\
\hline HEIS No. & B1V9B0 & \multicolumn{3}{|c|}{ Lab ID: $\quad$ 0805003-10 } & & & \\
\hline $7429-90-5$ & Aluminum & $2.95 \mathrm{E}-1$ & ug/g dry & $8.55 \mathrm{E}-2$ & $9 / 16 / 08$ & $8 \mathrm{I} 15003$ & PNNL-AGG-ICP-AES \\
\hline $7440-38-2$ & Arsenic & $<3.59 \mathrm{E}-1$ & ug/g dry & $3.59 \mathrm{E}-1$ & $9 / 16 / 08$ & $8 \mathrm{I} 15003$ & PNNL-AGG-ICP-AES \\
\hline $7440-42-8$ & Boron & $<1.93 \mathrm{E} 0$ & ug/g dry & $1.93 \mathrm{E} 0$ & $9 / 16 / 08$ & $8 \mathrm{I} 15003$ & PNNL-AGG-ICP-AES \\
\hline $7440-41-7$ & Beryllium & $<2.83 \mathrm{E}-2$ & ug/g dry & $2.83 \mathrm{E}-2$ & $9 / 16 / 08$ & $8 \mathrm{I} 15003$ & PNNL-AGG-ICP-AES \\
\hline $7440-69-9$ & Bismuth & $<1.87 \mathrm{E}-1$ & ug/g dry & $1.87 \mathrm{E}-1$ & $9 / 16 / 08$ & $8 \mathrm{I} 15003$ & PNNL-AGG-ICP-AES \\
\hline $7440-70-2$ & Calcium & $5.08 \mathrm{E} 0$ & ug/g dry & $3.86 \mathrm{E}-1$ & $9 / 16 / 08$ & $8 \mathrm{I} 15003$ & PNNL-AGG-ICP-AES \\
\hline $7440-43-9$ & Cadmium & $<2.68 \mathrm{E}-2$ & ug/g dry & $2.68 \mathrm{E}-2$ & $9 / 16 / 08$ & $8 \mathrm{I} 15003$ & PNNL-AGG-ICP-AES \\
\hline $7440-48-4$ & Cobalt & $<9.57 \mathrm{E}-2$ & ug/g dry & $9.57 \mathrm{E}-2$ & $9 / 16 / 08$ & $8 \mathrm{I} 15003$ & PNNL-AGG-ICP-AES \\
\hline $7440-47-3$ & Chromium & $<3.32 \mathrm{E}-2$ & ug/g dry & $3.32 \mathrm{E}-2$ & $9 / 16 / 08$ & $8 \mathrm{I} 15003$ & PNNL-AGG-ICP-AES \\
\hline $7440-50-8$ & Copper & $<8.01 \mathrm{E}-2$ & ug/g dry & $8.01 \mathrm{E}-2$ & $9 / 16 / 08$ & $8 \mathrm{I} 15003$ & PNNL-AGG-ICP-AES \\
\hline $7439-89-6$ & Iron & $3.60 \mathrm{E}-1$ & ug/g dry & $1.42 \mathrm{E}-1$ & $9 / 16 / 08$ & $8 \mathrm{I} 15003$ & PNNL-AGG-ICP-AES \\
\hline $7440-09-7$ & Potassium & $2.91 \mathrm{E} 0$ & ug/g dry & $2.32 \mathrm{E} 0$ & $9 / 16 / 08$ & $8 \mathrm{I} 15003$ & PNNL-AGG-ICP-AES \\
\hline $7439-93-2$ & Lithium & $<5.38 \mathrm{E}-1$ & ug/g dry & $5.38 \mathrm{E}-1$ & $9 / 16 / 08$ & $8 \mathrm{I} 15003$ & PNNL-AGG-ICP-AES \\
\hline $7439-95-4$ & Magnesium & $1.06 \mathrm{E} 0$ & ug/g dry & $8.31 \mathrm{E}-2$ & $9 / 16 / 08$ & $8 \mathrm{I} 15003$ & PNNL-AGG-ICP-AES \\
\hline $7439-96-5$ & Manganese & $<1.71 \mathrm{E}-2$ & ug/g dry & $1.71 \mathrm{E}-2$ & $9 / 16 / 08$ & $8 \mathrm{I} 15003$ & PNNL-AGG-ICP-AES \\
\hline 7439-98-7 & Molybdenum & $<1.39 \mathrm{E}-1$ & ug/g dry & $1.39 \mathrm{E}-1$ & $9 / 16 / 08$ & $8 \mathrm{I} 15003$ & PNNL-AGG-ICP-AES \\
\hline $7440-02-0$ & Nickel & $<9.30 \mathrm{E}-2$ & ug/g dry & $9.30 \mathrm{E}-2$ & $9 / 16 / 08$ & $8 \mathrm{I} 15003$ & PNNL-AGG-ICP-AES \\
\hline $7723-14-0$ & Phosphorus & $<1.03 \mathrm{E} 0$ & ug/g dry & $1.03 \mathrm{E} 0$ & $9 / 16 / 08$ & $8 \mathrm{I} 15003$ & PNNL-AGG-ICP-AES \\
\hline $7439-92-1$ & Lead & $<4.31 \mathrm{E}-1$ & ug/g dry & $4.31 \mathrm{E}-1$ & $9 / 16 / 08$ & $8 \mathrm{I} 15003$ & PNNL-AGG-ICP-AES \\
\hline $7782-49-2$ & Selenium & $<1.94 \mathrm{E} 0$ & ug/g dry & $1.94 \mathrm{E} 0$ & $9 / 16 / 08$ & $8 \mathrm{I} 15003$ & PNNL-AGG-ICP-AES \\
\hline $7440-24-6$ & Strontium & $<5.20 \mathrm{E}-2$ & ug/g dry & $5.20 \mathrm{E}-2$ & $9 / 16 / 08$ & $8 \mathrm{I} 15003$ & PNNL-AGG-ICP-AES \\
\hline $7440-28-0$ & Thallium & $<1.02 \mathrm{E} 0$ & ug/g dry & $1.02 \mathrm{E} 0$ & $9 / 16 / 08$ & $8 \mathrm{I} 15003$ & PNNL-AGG-ICP-AES \\
\hline $7440-62-2$ & Vanadium & $<4.51 \mathrm{E}-2$ & ug/g dry & $4.51 \mathrm{E}-2$ & $9 / 16 / 08$ & $8 \mathrm{I} 15003$ & PNNL-AGG-ICP-AES \\
\hline $7440-66-6$ & Zinc & $<9.24 \mathrm{E}-2$ & ug/g dry & $9.24 \mathrm{E}-2$ & $9 / 16 / 08$ & $8 \mathrm{I} 15003$ & PNNL-AGG-ICP-AES \\
\hline $7440-23-5$ & Sodium & $1.74 \mathrm{E} 1$ & ug/g dry & $6.67 \mathrm{E}-1$ & $9 / 16 / 08$ & $8 \mathrm{I} 15003$ & PNNL-AGG-ICP-AES \\
\hline 7704-34-9 & Sulfur & 4.60E0 & ug/g dry & $3.06 \mathrm{E} 0$ & $9 / 16 / 08$ & $8 \mathrm{I} 15003$ & PNNL-AGG-ICP-AES \\
\hline $7440-32-6$ & Titanium & $1.45 \mathrm{E}-2$ & ug/g dry & $8.73 \mathrm{E}-3$ & $9 / 16 / 08$ & $8 \mathrm{I} 15003$ & PNNL-AGG-ICP-AES \\
\hline $7440-67-7$ & Zirconium & $<1.49 \mathrm{E}-1$ & ug/g dry & $1.49 \mathrm{E}-1$ & $9 / 16 / 08$ & $8 \mathrm{I} 15003$ & PNNL-AGG-ICP-AES \\
\hline $7440-22-4$ & Silver & $<7.68 \mathrm{E}-2$ & ug/g dry & $7.68 \mathrm{E}-2$ & $9 / 16 / 08$ & $8 \mathrm{I} 15003$ & PNNL-AGG-ICP-AES \\
\hline $7440-15-5$ & Rhenium & $<1.51 \mathrm{E}-1$ & ug/g dry & $1.51 \mathrm{E}-1$ & $9 / 16 / 08$ & $8 \mathrm{I} 15003$ & PNNL-AGG-ICP-AES \\
\hline $7440-36-0$ & Antimony & $<6.31 \mathrm{E}-1$ & ug/g dry & $6.31 \mathrm{E}-1$ & 9/16/08 & $8 \mathrm{I} 15003$ & PNNL-AGG-ICP-AES \\
\hline HEIS No. & B1V9B1 & & b ID: & $5003-11$ & & & \\
\hline $7429-90-5$ & Aluminum & $2.05 \mathrm{E}-1$ & ug/g dry & $8.56 \mathrm{E}-2$ & $9 / 16 / 08$ & $8 \mathrm{I} 15003$ & PNNL-AGG-ICP-AES \\
\hline $7440-38-2$ & Arsenic & $<3.59 \mathrm{E}-1$ & ug/g dry & $3.59 \mathrm{E}-1$ & $9 / 16 / 08$ & $8 \mathrm{I} 15003$ & PNNL-AGG-ICP-AES \\
\hline $7440-42-8$ & Boron & $<1.94 \mathrm{E} 0$ & ug/g dry & $1.94 \mathrm{E} 0$ & $9 / 16 / 08$ & $8 \mathrm{I} 15003$ & PNNL-AGG-ICP-AES \\
\hline $7440-41-7$ & Beryllium & $<2.83 \mathrm{E}-2$ & ug/g dry & $2.83 \mathrm{E}-2$ & $9 / 16 / 08$ & $8 \mathrm{I} 15003$ & PNNL-AGG-ICP-AES \\
\hline $7440-69-9$ & Bismuth & $<1.88 \mathrm{E}-1$ & ug/g dry & $1.88 \mathrm{E}-1$ & $9 / 16 / 08$ & $8 \mathrm{I} 15003$ & PNNL-AGG-ICP-AES \\
\hline $7440-70-2$ & Calcium & $5.94 \mathrm{E} 0$ & ug/g dry & $3.86 \mathrm{E}-1$ & 9/16/08 & $8 \mathrm{I} 15003$ & PNNL-AGG-ICP-AES \\
\hline $7440-43-9$ & Cadmium & $<2.68 \mathrm{E}-2$ & ug/g dry & $2.68 \mathrm{E}-2$ & $9 / 16 / 08$ & $8 \mathrm{I} 15003$ & PNNL-AGG-ICP-AES \\
\hline $7440-48-4$ & Cobalt & $<9.58 \mathrm{E}-2$ & ug/g dry & $9.58 \mathrm{E}-2$ & $9 / 16 / 08$ & $8 \mathrm{I} 15003$ & PNNL-AGG-ICP-AES \\
\hline $7440-47-3$ & Chromium & $<3.32 \mathrm{E}-2$ & ug/g dry & $3.32 \mathrm{E}-2$ & $9 / 16 / 08$ & $8 \mathrm{I} 15003$ & PNNL-AGG-ICP-AES \\
\hline $7440-50-8$ & Copper & $<8.02 \mathrm{E}-2$ & ug/g dry & $8.02 \mathrm{E}-2$ & $9 / 16 / 08$ & $8 \mathrm{I} 15003$ & PNNL-AGG-ICP-AES \\
\hline $7439-89-6$ & Iron & $1.81 \mathrm{E}-1$ & ug/g dry & $1.42 \mathrm{E}-1$ & $9 / 16 / 08$ & $8 \mathrm{I} 15003$ & PNNL-AGG-ICP-AES \\
\hline
\end{tabular}


Total Metals by PNNL-AGG-ICP-AES/Water Extract

\begin{tabular}{|c|c|c|c|c|c|c|c|}
\hline CAS \# & Analyte & Results & Units & EQL & Analyzed & Batch & Method \\
\hline HEIS No. & B1V9B1 & & b ID: & 55003-11 & & & \\
\hline 7440-09-7 & Potassium & $2.92 \mathrm{E} 0$ & $\mathrm{ug} / \mathrm{g}$ dry & $2.32 \mathrm{E} 0$ & $9 / 16 / 08$ & $8 \mathrm{I} 15003$ & PNNL-AGG-ICP-AES \\
\hline $7439-93-2$ & Lithium & $<5.39 \mathrm{E}-1$ & ug/g dry & $5.39 \mathrm{E}-1$ & $9 / 16 / 08$ & $8 \mathrm{I} 15003$ & PNNL-AGG-ICP-AES \\
\hline $7439-95-4$ & Magnesium & $1.26 \mathrm{E} 0$ & ug/g dry & $8.32 \mathrm{E}-2$ & $9 / 16 / 08$ & $8 \mathrm{I} 15003$ & PNNL-AGG-ICP-AES \\
\hline $7439-96-5$ & Manganese & $<1.71 \mathrm{E}-2$ & ug/g dry & $1.71 \mathrm{E}-2$ & $9 / 16 / 08$ & $8 \mathrm{I} 15003$ & PNNL-AGG-ICP-AES \\
\hline $7439-98-7$ & Molybdenum & $<1.39 \mathrm{E}-1$ & ug/g dry & $1.39 \mathrm{E}-1$ & $9 / 16 / 08$ & $8 \mathrm{I} 15003$ & PNNL-AGG-ICP-AES \\
\hline $7440-02-0$ & Nickel & $<9.31 \mathrm{E}-2$ & ug/g dry & $9.31 \mathrm{E}-2$ & $9 / 16 / 08$ & $8 \mathrm{I} 15003$ & PNNL-AGG-ICP-AES \\
\hline $7723-14-0$ & Phosphorus & $<1.03 \mathrm{E} 0$ & ug/g dry & $1.03 \mathrm{E} 0$ & $9 / 16 / 08$ & $8 \mathrm{I} 15003$ & PNNL-AGG-ICP-AES \\
\hline $7439-92-1$ & Lead & $<4.31 \mathrm{E}-1$ & ug/g dry & $4.31 \mathrm{E}-1$ & $9 / 16 / 08$ & $8 \mathrm{I} 15003$ & PNNL-AGG-ICP-AES \\
\hline $7782-49-2$ & Selenium & $<1.95 \mathrm{E} 0$ & ug/g dry & $1.95 \mathrm{E} 0$ & $9 / 16 / 08$ & $8 \mathrm{I} 15003$ & PNNL-AGG-ICP-AES \\
\hline $7440-24-6$ & Strontium & $<5.21 \mathrm{E}-2$ & $\mathrm{ug} / \mathrm{g}$ dry & $5.21 \mathrm{E}-2$ & $9 / 16 / 08$ & $8 \mathrm{II} 15003$ & PNNL-AGG-ICP-AES \\
\hline $7440-28-0$ & Thallium & $<1.02 \mathrm{E} 0$ & ug/g dry & $1.02 \mathrm{E} 0$ & $9 / 16 / 08$ & $8 \mathrm{I} 15003$ & PNNL-AGG-ICP-AES \\
\hline $7440-62-2$ & Vanadium & $<4.52 \mathrm{E}-2$ & ug/g dry & $4.52 \mathrm{E}-2$ & $9 / 16 / 08$ & $8 \mathrm{I} 15003$ & PNNL-AGG-ICP-AES \\
\hline $7440-66-6$ & Zinc & $<9.25 \mathrm{E}-2$ & ug/g dry & $9.25 \mathrm{E}-2$ & $9 / 16 / 08$ & $8 \mathrm{I} 15003$ & PNNL-AGG-ICP-AES \\
\hline $7440-23-5$ & Sodium & $1.57 \mathrm{E} 1$ & ug/g dry & $6.67 \mathrm{E}-1$ & $9 / 16 / 08$ & $8 \mathrm{I} 15003$ & PNNL-AGG-ICP-AES \\
\hline 7704-34-9 & Sulfur & $4.00 \mathrm{E} 0$ & ug/g dry & 3.07E0 & $9 / 16 / 08$ & $8 \mathrm{I} 15003$ & PNNL-AGG-ICP-AES \\
\hline $7440-32-6$ & Titanium & $<8.74 \mathrm{E}-3$ & ug/g dry & $8.74 \mathrm{E}-3$ & $9 / 16 / 08$ & $8 \mathrm{I} 15003$ & PNNL-AGG-ICP-AES \\
\hline $7440-67-7$ & Zirconium & $<1.50 \mathrm{E}-1$ & ug/g dry & $1.50 \mathrm{E}-1$ & $9 / 16 / 08$ & $8 \mathrm{I} 15003$ & PNNL-AGG-ICP-AES \\
\hline $7440-22-4$ & Silver & $<7.69 \mathrm{E}-2$ & $\mathrm{ug} / \mathrm{g}$ dry & $7.69 \mathrm{E}-2$ & $9 / 16 / 08$ & $8 \mathrm{I} 15003$ & PNNL-AGG-ICP-AES \\
\hline $7440-15-5$ & Rhenium & $<1.51 \mathrm{E}-1$ & ug/g dry & $1.51 \mathrm{E}-1$ & $9 / 16 / 08$ & $8 \mathrm{I} 15003$ & PNNL-AGG-ICP-AES \\
\hline $7440-36-0$ & Antimony & $<6.31 \mathrm{E}-1$ & ug/g dry & $6.31 \mathrm{E}-1$ & $9 / 16 / 08$ & $8 \mathrm{II} 15003$ & PNNL-AGG-ICP-AES \\
\hline HEIS No. & B1V9B2 & & b ID: & 5003-12 & & & \\
\hline $7429-90-5$ & Aluminum & $2.70 \mathrm{E}-1$ & ug/g dry & $8.58 \mathrm{E}-2$ & $9 / 16 / 08$ & $8 \mathrm{I} 15003$ & PNNL-AGG-ICP-AES \\
\hline $7440-38-2$ & Arsenic & $<3.60 \mathrm{E}-1$ & ug/g dry & $3.60 \mathrm{E}-1$ & $9 / 16 / 08$ & $8 \mathrm{I} 15003$ & PNNL-AGG-ICP-AES \\
\hline $7440-42-8$ & Boron & $<1.94 \mathrm{E} 0$ & $\mathrm{ug} / \mathrm{g}$ dry & $1.94 \mathrm{E} 0$ & $9 / 16 / 08$ & $8 \mathrm{I} 15003$ & PNNL-AGG-ICP-AES \\
\hline $7440-41-7$ & Beryllium & $<2.84 \mathrm{E}-2$ & ug/g dry & $2.84 \mathrm{E}-2$ & $9 / 16 / 08$ & $8 \mathrm{I} 15003$ & PNNL-AGG-ICP-AES \\
\hline $7440-69-9$ & Bismuth & $<1.88 \mathrm{E}-1$ & $\mathrm{ug} / \mathrm{g}$ dry & $1.88 \mathrm{E}-1$ & $9 / 16 / 08$ & $8 \mathrm{I} 15003$ & PNNL-AGG-ICP-AES \\
\hline $7440-70-2$ & Calcium & $6.86 \mathrm{E} 0$ & ug/g dry & $3.87 \mathrm{E}-1$ & $9 / 16 / 08$ & $8 \mathrm{I} 15003$ & PNNL-AGG-ICP-AES \\
\hline $7440-43-9$ & Cadmium & $<2.69 \mathrm{E}-2$ & ug/g dry & $2.69 \mathrm{E}-2$ & $9 / 16 / 08$ & $8 \mathrm{I} 15003$ & PNNL-AGG-ICP-AES \\
\hline $7440-48-4$ & Cobalt & $<9.60 \mathrm{E}-2$ & ug/g dry & $9.60 \mathrm{E}-2$ & $9 / 16 / 08$ & $8 \mathrm{I} 15003$ & PNNL-AGG-ICP-AES \\
\hline $7440-47-3$ & Chromium & $<3.33 \mathrm{E}-2$ & ug/g dry & 3.33E-2 & $9 / 16 / 08$ & $8 \mathrm{I} 15003$ & PNNL-AGG-ICP-AES \\
\hline $7440-50-8$ & Copper & $<8.04 \mathrm{E}-2$ & ug/g dry & $8.04 \mathrm{E}-2$ & $9 / 16 / 08$ & $8 \mathrm{I} 15003$ & PNNL-AGG-ICP-AES \\
\hline $7439-89-6$ & Iron & $2.58 \mathrm{E}-1$ & ug/g dry & $1.42 \mathrm{E}-1$ & $9 / 16 / 08$ & $8 \mathrm{I} 15003$ & PNNL-AGG-ICP-AES \\
\hline $7440-09-7$ & Potassium & $3.45 \mathrm{E} 0$ & $\mathrm{ug} / \mathrm{g}$ dry & $2.33 \mathrm{E} 0$ & $9 / 16 / 08$ & $8 \mathrm{I} 15003$ & PNNL-AGG-ICP-AES \\
\hline $7439-93-2$ & Lithium & $<5.40 \mathrm{E}-1$ & ug/g dry & $5.40 \mathrm{E}-1$ & $9 / 16 / 08$ & $8 \mathrm{I} 15003$ & PNNL-AGG-ICP-AES \\
\hline $7439-95-4$ & Magnesium & $1.56 \mathrm{E} 0$ & ug/g dry & $8.34 \mathrm{E}-2$ & $9 / 16 / 08$ & $8 \mathrm{I} 15003$ & PNNL-AGG-ICP-AES \\
\hline $7439-96-5$ & Manganese & $<1.71 \mathrm{E}-2$ & ug/g dry & $1.71 \mathrm{E}-2$ & $9 / 16 / 08$ & $8 \mathrm{I} 15003$ & PNNL-AGG-ICP-AES \\
\hline $7439-98-7$ & Molybdenum & $<1.39 \mathrm{E}-1$ & ug/g dry & $1.39 \mathrm{E}-1$ & $9 / 16 / 08$ & $8 \mathrm{I} 15003$ & PNNL-AGG-ICP-AES \\
\hline $7440-02-0$ & Nickel & $<9.33 \mathrm{E}-2$ & $\mathrm{ug} / \mathrm{g}$ dry & $9.33 \mathrm{E}-2$ & $9 / 16 / 08$ & $8 \mathrm{I} 15003$ & PNNL-AGG-ICP-AES \\
\hline $7723-14-0$ & Phosphorus & $<1.03 \mathrm{E} 0$ & $\mathrm{ug} / \mathrm{g}$ dry & $1.03 \mathrm{E} 0$ & $9 / 16 / 08$ & $8 \mathrm{I} 15003$ & PNNL-AGG-ICP-AES \\
\hline $7439-92-1$ & Lead & $<4.32 \mathrm{E}-1$ & ug/g dry & $4.32 \mathrm{E}-1$ & $9 / 16 / 08$ & $8 \mathrm{I} 15003$ & PNNL-AGG-ICP-AES \\
\hline $7782-49-2$ & Selenium & $<1.95 \mathrm{E} 0$ & $\mathrm{ug} / \mathrm{g}$ dry & $1.95 \mathrm{E} 0$ & $9 / 16 / 08$ & $8 \mathrm{I} 15003$ & PNNL-AGG-ICP-AES \\
\hline $7440-24-6$ & Strontium & $<5.22 \mathrm{E}-2$ & ug/g dry & $5.22 \mathrm{E}-2$ & $9 / 16 / 08$ & $8 \mathrm{I} 15003$ & PNNL-AGG-ICP-AES \\
\hline $7440-28-0$ & Thallium & $<1.03 \mathrm{E} 0$ & ug/g dry & $1.03 \mathrm{E} 0$ & $9 / 16 / 08$ & $8 \mathrm{I} 15003$ & PNNL-AGG-ICP-AES \\
\hline $7440-62-2$ & Vanadium & $<4.53 \mathrm{E}-2$ & ug/g dry & $4.53 \mathrm{E}-2$ & $9 / 16 / 08$ & $8 \mathrm{I} 15003$ & PNNL-AGG-ICP-AES \\
\hline $7440-66-6$ & Zinc & $<9.27 \mathrm{E}-2$ & ug/g dry & $9.27 \mathrm{E}-2$ & $9 / 16 / 08$ & $8 \mathrm{I} 15003$ & PNNL-AGG-ICP-AES \\
\hline $7440-23-5$ & Sodium & $1.40 \mathrm{E} 1$ & ug/g dry & $6.69 \mathrm{E}-1$ & $9 / 16 / 08$ & $8 \mathrm{I} 15003$ & PNNL-AGG-ICP-AES \\
\hline $7704-34-9$ & Sulfur & $4.75 \mathrm{E} 0$ & ug/g dry & $3.08 \mathrm{E} 0$ & $9 / 16 / 08$ & $8 \mathrm{I} 15003$ & PNNL-AGG-ICP-AES \\
\hline $7440-32-6$ & Titanium & $1.11 \mathrm{E}-2$ & ug/g dry & $8.76 \mathrm{E}-3$ & $9 / 16 / 08$ & $8 \mathrm{I} 15003$ & PNNL-AGG-ICP-AES \\
\hline
\end{tabular}


Total Metals by PNNL-AGG-ICP-AES/Water Extract

\begin{tabular}{|c|c|c|c|c|c|c|c|}
\hline CAS \# & Analyte & Results & Units & EQL & Analyzed & Batch & Method \\
\hline HEIS No. & B1V9B2 & \multicolumn{3}{|c|}{ Lab ID: $\quad$ 0805003-12 } & & & \\
\hline $7440-67-7$ & Zirconium & $<1.50 \mathrm{E}-1$ & ug/g dry & $1.50 \mathrm{E}-1$ & $9 / 16 / 08$ & $8 \mathrm{I} 15003$ & PNNL-AGG-ICP-AES \\
\hline $7440-22-4$ & Silver & $<7.71 \mathrm{E}-2$ & ug/g dry & $7.71 \mathrm{E}-2$ & $9 / 16 / 08$ & $8 \mathrm{I} 15003$ & PNNL-AGG-ICP-AES \\
\hline $7440-15-5$ & Rhenium & $<1.52 \mathrm{E}-1$ & ug/g dry & $1.52 \mathrm{E}-1$ & $9 / 16 / 08$ & $8 \mathrm{I} 15003$ & PNNL-AGG-ICP-AES \\
\hline $7440-36-0$ & Antimony & $<6.33 \mathrm{E}-1$ & ug/g dry & $6.33 \mathrm{E}-1$ & $9 / 16 / 08$ & $8 \mathrm{I} 15003$ & PNNL-AGG-ICP-AES \\
\hline HEIS No. & B1V9B3 & \multicolumn{3}{|c|}{ Lab ID: $\quad$ 0805003-13 } & & & \\
\hline $7429-90-5$ & Aluminum & $3.26 \mathrm{E}-1$ & ug/g dry & $8.58 \mathrm{E}-2$ & $9 / 16 / 08$ & $8 \mathrm{I} 15003$ & PNNL-AGG-ICP-AES \\
\hline $7440-38-2$ & Arsenic & $<3.60 \mathrm{E}-1$ & ug/g dry & $3.60 \mathrm{E}-1$ & $9 / 16 / 08$ & $8 \mathrm{I} 15003$ & PNNL-AGG-ICP-AES \\
\hline $7440-42-8$ & Boron & $<1.94 \mathrm{E} 0$ & ug/g dry & $1.94 \mathrm{E} 0$ & $9 / 16 / 08$ & $8 \mathrm{I} 15003$ & PNNL-AGG-ICP-AES \\
\hline $7440-41-7$ & Beryllium & $<2.84 \mathrm{E}-2$ & ug/g dry & $2.84 \mathrm{E}-2$ & $9 / 16 / 08$ & $8 \mathrm{I} 15003$ & PNNL-AGG-ICP-AES \\
\hline $7440-69-9$ & Bismuth & $<1.88 \mathrm{E}-1$ & ug/g dry & $1.88 \mathrm{E}-1$ & $9 / 16 / 08$ & $8 \mathrm{I} 15003$ & PNNL-AGG-ICP-AES \\
\hline $7440-70-2$ & Calcium & $6.44 \mathrm{E} 0$ & ug/g dry & $3.87 \mathrm{E}-1$ & $9 / 16 / 08$ & $8 \mathrm{I} 15003$ & PNNL-AGG-ICP-AES \\
\hline $7440-43-9$ & Cadmium & $<2.69 \mathrm{E}-2$ & ug/g dry & $2.69 \mathrm{E}-2$ & $9 / 16 / 08$ & $8 \mathrm{I} 15003$ & PNNL-AGG-ICP-AES \\
\hline $7440-48-4$ & Cobalt & $<9.61 \mathrm{E}-2$ & ug/g dry & $9.61 \mathrm{E}-2$ & $9 / 16 / 08$ & $8 \mathrm{I} 15003$ & PNNL-AGG-ICP-AES \\
\hline $7440-47-3$ & Chromium & $<3.33 \mathrm{E}-2$ & ug/g dry & $3.33 \mathrm{E}-2$ & $9 / 16 / 08$ & $8 \mathrm{I} 15003$ & PNNL-AGG-ICP-AES \\
\hline $7440-50-8$ & Copper & $<8.04 \mathrm{E}-2$ & ug/g dry & $8.04 \mathrm{E}-2$ & $9 / 16 / 08$ & $8 \mathrm{I} 15003$ & PNNL-AGG-ICP-AES \\
\hline $7439-89-6$ & Iron & $3.25 \mathrm{E}-1$ & ug/g dry & $1.42 \mathrm{E}-1$ & $9 / 16 / 08$ & $8 \mathrm{I} 15003$ & PNNL-AGG-ICP-AES \\
\hline $7440-09-7$ & Potassium & $4.81 \mathrm{E} 0$ & ug/g dry & 2.33E0 & $9 / 16 / 08$ & $8 \mathrm{I} 15003$ & PNNL-AGG-ICP-AES \\
\hline $7439-93-2$ & Lithium & $<5.40 \mathrm{E}-1$ & ug/g dry & $5.40 \mathrm{E}-1$ & $9 / 16 / 08$ & $8 \mathrm{I} 15003$ & PNNL-AGG-ICP-AES \\
\hline $7439-95-4$ & Magnesium & $1.61 \mathrm{E} 0$ & ug/g dry & $8.34 \mathrm{E}-2$ & $9 / 16 / 08$ & $8 \mathrm{I} 15003$ & PNNL-AGG-ICP-AES \\
\hline $7439-96-5$ & Manganese & $<1.71 \mathrm{E}-2$ & ug/g dry & $1.71 \mathrm{E}-2$ & $9 / 16 / 08$ & $8 \mathrm{I} 15003$ & PNNL-AGG-ICP-AES \\
\hline $7439-98-7$ & Molybdenum & $<1.39 \mathrm{E}-1$ & ug/g dry & $1.39 \mathrm{E}-1$ & $9 / 16 / 08$ & $8 \mathrm{I} 15003$ & PNNL-AGG-ICP-AES \\
\hline $7440-02-0$ & Nickel & $<9.34 \mathrm{E}-2$ & ug/g dry & $9.34 \mathrm{E}-2$ & $9 / 16 / 08$ & $8 \mathrm{I} 15003$ & PNNL-AGG-ICP-AES \\
\hline $7723-14-0$ & Phosphorus & $<1.03 \mathrm{E} 0$ & ug/g dry & $1.03 \mathrm{E} 0$ & $9 / 16 / 08$ & $8 \mathrm{I} 15003$ & PNNL-AGG-ICP-AES \\
\hline $7439-92-1$ & Lead & $<4.32 \mathrm{E}-1$ & ug/g dry & $4.32 \mathrm{E}-1$ & $9 / 16 / 08$ & $8 \mathrm{I} 15003$ & PNNL-AGG-ICP-AES \\
\hline $7782-49-2$ & Selenium & $<1.95 \mathrm{E} 0$ & ug/g dry & $1.95 \mathrm{E} 0$ & $9 / 16 / 08$ & $8 \mathrm{I} 15003$ & PNNL-AGG-ICP-AES \\
\hline $7440-24-6$ & Strontium & $<5.22 \mathrm{E}-2$ & ug/g dry & $5.22 \mathrm{E}-2$ & 9/16/08 & $8 \mathrm{I} 15003$ & PNNL-AGG-ICP-AES \\
\hline $7440-28-0$ & Thallium & $<1.03 \mathrm{E} 0$ & ug/g dry & $1.03 \mathrm{E} 0$ & $9 / 16 / 08$ & $8 \mathrm{I} 15003$ & PNNL-AGG-ICP-AES \\
\hline $7440-62-2$ & Vanadium & $<4.53 \mathrm{E}-2$ & ug/g dry & $4.53 \mathrm{E}-2$ & $9 / 16 / 08$ & $8 \mathrm{I} 15003$ & PNNL-AGG-ICP-AES \\
\hline $7440-66-6$ & Zinc & $<9.28 \mathrm{E}-2$ & ug/g dry & $9.28 \mathrm{E}-2$ & $9 / 16 / 08$ & $8 \mathrm{I} 15003$ & PNNL-AGG-ICP-AES \\
\hline $7440-23-5$ & Sodium & $2.30 \mathrm{E} 1$ & ug/g dry & $6.69 \mathrm{E}-1$ & $9 / 16 / 08$ & $8 \mathrm{I} 15003$ & PNNL-AGG-ICP-AES \\
\hline 7704-34-9 & Sulfur & $6.86 \mathrm{E} 0$ & ug/g dry & $3.08 \mathrm{E} 0$ & $9 / 16 / 08$ & $8 \mathrm{I} 15003$ & PNNL-AGG-ICP-AES \\
\hline $7440-32-6$ & Titanium & $1.27 \mathrm{E}-2$ & ug/g dry & $8.76 \mathrm{E}-3$ & $9 / 16 / 08$ & $8 \mathrm{I} 15003$ & PNNL-AGG-ICP-AES \\
\hline $7440-67-7$ & Zirconium & $<1.50 \mathrm{E}-1$ & ug/g dry & $1.50 \mathrm{E}-1$ & $9 / 16 / 08$ & $8 \mathrm{I} 15003$ & PNNL-AGG-ICP-AES \\
\hline $7440-22-4$ & Silver & $<7.71 \mathrm{E}-2$ & ug/g dry & $7.71 \mathrm{E}-2$ & $9 / 16 / 08$ & $8 \mathrm{I} 15003$ & PNNL-AGG-ICP-AES \\
\hline $7440-15-5$ & Rhenium & $<1.52 \mathrm{E}-1$ & ug/g dry & $1.52 \mathrm{E}-1$ & $9 / 16 / 08$ & $8 \mathrm{I} 15003$ & PNNL-AGG-ICP-AES \\
\hline $7440-36-0$ & Antimony & $<6.33 \mathrm{E}-1$ & ug/g dry & $6.33 \mathrm{E}-1$ & 9/16/08 & $8 \mathrm{I} 15003$ & PNNL-AGG-ICP-AES \\
\hline HEIS No. & B1V9B4 & & b ID: & $5003-14$ & & & \\
\hline $7429-90-5$ & Aluminum & $2.25 \mathrm{E}-1$ & ug/g dry & $8.56 \mathrm{E}-2$ & $9 / 16 / 08$ & $8 \mathrm{I} 15003$ & PNNL-AGG-ICP-AES \\
\hline $7440-38-2$ & Arsenic & $<3.59 \mathrm{E}-1$ & ug/g dry & $3.59 \mathrm{E}-1$ & $9 / 16 / 08$ & $8 \mathrm{I} 15003$ & PNNL-AGG-ICP-AES \\
\hline $7440-42-8$ & Boron & $<1.94 \mathrm{E} 0$ & ug/g dry & $1.94 \mathrm{E} 0$ & $9 / 16 / 08$ & $8 \mathrm{I} 15003$ & PNNL-AGG-ICP-AES \\
\hline $7440-41-7$ & Beryllium & $<2.84 \mathrm{E}-2$ & ug/g dry & $2.84 \mathrm{E}-2$ & $9 / 16 / 08$ & $8 \mathrm{I} 15003$ & PNNL-AGG-ICP-AES \\
\hline $7440-69-9$ & Bismuth & $<1.88 \mathrm{E}-1$ & ug/g dry & $1.88 \mathrm{E}-1$ & $9 / 16 / 08$ & $8 \mathrm{I} 15003$ & PNNL-AGG-ICP-AES \\
\hline $7440-70-2$ & Calcium & 7.64E0 & ug/g dry & $3.86 \mathrm{E}-1$ & $9 / 16 / 08$ & $8 \mathrm{I} 15003$ & PNNL-AGG-ICP-AES \\
\hline $7440-43-9$ & Cadmium & $<2.68 \mathrm{E}-2$ & ug/g dry & $2.68 \mathrm{E}-2$ & 9/16/08 & $8 \mathrm{I} 15003$ & PNNL-AGG-ICP-AES \\
\hline $7440-48-4$ & Cobalt & $<9.58 \mathrm{E}-2$ & ug/g dry & $9.58 \mathrm{E}-2$ & $9 / 16 / 08$ & $8 \mathrm{I} 15003$ & PNNL-AGG-ICP-AES \\
\hline $7440-47-3$ & Chromium & $<3.32 \mathrm{E}-2$ & ug/g dry & $3.32 \mathrm{E}-2$ & $9 / 16 / 08$ & $8 \mathrm{I} 15003$ & PNNL-AGG-ICP-AES \\
\hline $7440-50-8$ & Copper & $<8.03 \mathrm{E}-2$ & ug/g dry & $8.03 \mathrm{E}-2$ & $9 / 16 / 08$ & $8 \mathrm{I} 15003$ & PNNL-AGG-ICP-AES \\
\hline $7439-89-6$ & Iron & $<1.42 \mathrm{E}-1$ & ug/g dry & $1.42 \mathrm{E}-1$ & 9/16/08 & $8 \mathrm{I} 15003$ & PNNL-AGG-ICP-AES \\
\hline
\end{tabular}


Total Metals by PNNL-AGG-ICP-AES/Water Extract

\begin{tabular}{|c|c|c|c|c|c|c|c|}
\hline CAS \# & Analyte & Results & Units & EQL & Analyzed & Batch & Method \\
\hline HEIS No. & B1V9B4 & & b ID: & $05003-14$ & & & \\
\hline 7440-09-7 & Potassium & $3.79 \mathrm{E} 0$ & ug/g dry & $2.32 \mathrm{E} 0$ & $9 / 16 / 08$ & $8 \mathrm{I} 15003$ & PNNL-AGG-ICP-AES \\
\hline $7439-93-2$ & Lithium & $<5.39 \mathrm{E}-1$ & ug/g dry & $5.39 \mathrm{E}-1$ & $9 / 16 / 08$ & $8 \mathrm{I} 15003$ & PNNL-AGG-ICP-AES \\
\hline $7439-95-4$ & Magnesium & $1.87 \mathrm{E} 0$ & ug/g dry & $8.32 \mathrm{E}-2$ & $9 / 16 / 08$ & $8 \mathrm{I} 15003$ & PNNL-AGG-ICP-AES \\
\hline $7439-96-5$ & Manganese & $<1.71 \mathrm{E}-2$ & ug/g dry & $1.71 \mathrm{E}-2$ & $9 / 16 / 08$ & $8 \mathrm{I} 15003$ & PNNL-AGG-ICP-AES \\
\hline $7439-98-7$ & Molybdenum & $<1.39 \mathrm{E}-1$ & ug/g dry & $1.39 \mathrm{E}-1$ & $9 / 16 / 08$ & $8 \mathrm{I} 15003$ & PNNL-AGG-ICP-AES \\
\hline $7440-02-0$ & Nickel & $<9.31 \mathrm{E}-2$ & ug/g dry & $9.31 \mathrm{E}-2$ & $9 / 16 / 08$ & $8 \mathrm{I} 15003$ & PNNL-AGG-ICP-AES \\
\hline $7723-14-0$ & Phosphorus & $<1.03 \mathrm{E} 0$ & ug/g dry & $1.03 \mathrm{E} 0$ & $9 / 16 / 08$ & $8 \mathrm{I} 15003$ & PNNL-AGG-ICP-AES \\
\hline $7439-92-1$ & Lead & $<4.31 \mathrm{E}-1$ & ug/g dry & 4.31E-1 & $9 / 16 / 08$ & $8 \mathrm{I} 15003$ & PNNL-AGG-ICP-AES \\
\hline $7782-49-2$ & Selenium & $<1.95 \mathrm{E} 0$ & ug/g dry & $1.95 \mathrm{E} 0$ & $9 / 16 / 08$ & $8 \mathrm{I} 15003$ & PNNL-AGG-ICP-AES \\
\hline $7440-24-6$ & Strontium & $<5.21 \mathrm{E}-2$ & ug/g dry & $5.21 \mathrm{E}-2$ & $9 / 16 / 08$ & $8 \mathrm{I} 15003$ & PNNL-AGG-ICP-AES \\
\hline $7440-28-0$ & Thallium & $<1.02 \mathrm{E} 0$ & ug/g dry & $1.02 \mathrm{E} 0$ & $9 / 16 / 08$ & $8 \mathrm{I} 15003$ & PNNL-AGG-ICP-AES \\
\hline $7440-62-2$ & Vanadium & $<4.52 \mathrm{E}-2$ & ug/g dry & $4.52 \mathrm{E}-2$ & $9 / 16 / 08$ & $8 \mathrm{I} 15003$ & PNNL-AGG-ICP-AES \\
\hline $7440-66-6$ & Zinc & $<9.25 \mathrm{E}-2$ & ug/g dry & $9.25 \mathrm{E}-2$ & $9 / 16 / 08$ & $8 \mathrm{I} 15003$ & PNNL-AGG-ICP-AES \\
\hline $7440-23-5$ & Sodium & $1.08 \mathrm{E} 1$ & ug/g dry & $6.68 \mathrm{E}-1$ & $9 / 16 / 08$ & $8 \mathrm{I} 15003$ & PNNL-AGG-ICP-AES \\
\hline 7704-34-9 & Sulfur & $3.13 \mathrm{E} 0$ & ug/g dry & 3.07E0 & $9 / 16 / 08$ & $8 \mathrm{I} 15003$ & PNNL-AGG-ICP-AES \\
\hline $7440-32-6$ & Titanium & $<8.74 \mathrm{E}-3$ & ug/g dry & $8.74 \mathrm{E}-3$ & $9 / 16 / 08$ & $8 \mathrm{I} 15003$ & PNNL-AGG-ICP-AES \\
\hline $7440-67-7$ & Zirconium & $<1.50 \mathrm{E}-1$ & ug/g dry & $1.50 \mathrm{E}-1$ & $9 / 16 / 08$ & $8 \mathrm{I} 15003$ & PNNL-AGG-ICP-AES \\
\hline $7440-22-4$ & Silver & $<7.70 \mathrm{E}-2$ & ug/g dry & $7.70 \mathrm{E}-2$ & $9 / 16 / 08$ & $8 \mathrm{I} 15003$ & PNNL-AGG-ICP-AES \\
\hline $7440-15-5$ & Rhenium & $<1.52 \mathrm{E}-1$ & ug/g dry & $1.52 \mathrm{E}-1$ & $9 / 16 / 08$ & $8 \mathrm{I} 15003$ & PNNL-AGG-ICP-AES \\
\hline $7440-36-0$ & Antimony & $<6.32 \mathrm{E}-1$ & ug/g dry & $6.32 \mathrm{E}-1$ & $9 / 16 / 08$ & $8 \mathrm{I} 15003$ & PNNL-AGG-ICP-AES \\
\hline HEIS No. & B1V9B5 & & b ID: & 5003-15 & & & \\
\hline $7429-90-5$ & Aluminum & $2.07 \mathrm{E}-1$ & ug/g dry & $8.57 \mathrm{E}-2$ & $9 / 16 / 08$ & $8 \mathrm{I} 15003$ & PNNL-AGG-ICP-AES \\
\hline $7440-38-2$ & Arsenic & $<3.60 \mathrm{E}-1$ & ug/g dry & $3.60 \mathrm{E}-1$ & $9 / 16 / 08$ & $8 \mathrm{I} 15003$ & PNNL-AGG-ICP-AES \\
\hline $7440-42-8$ & Boron & $<1.94 \mathrm{E} 0$ & ug/g dry & $1.94 \mathrm{E} 0$ & $9 / 16 / 08$ & $8 \mathrm{I} 15003$ & PNNL-AGG-ICP-AES \\
\hline $7440-41-7$ & Beryllium & $<2.84 \mathrm{E}-2$ & ug/g dry & $2.84 \mathrm{E}-2$ & $9 / 16 / 08$ & $8 \mathrm{I} 15003$ & PNNL-AGG-ICP-AES \\
\hline $7440-69-9$ & Bismuth & $<1.88 \mathrm{E}-1$ & ug/g dry & $1.88 \mathrm{E}-1$ & $9 / 16 / 08$ & $8 \mathrm{I} 15003$ & PNNL-AGG-ICP-AES \\
\hline $7440-70-2$ & Calcium & $5.26 \mathrm{E} 0$ & ug/g dry & $3.87 \mathrm{E}-1$ & $9 / 16 / 08$ & $8 \mathrm{I} 15003$ & PNNL-AGG-ICP-AES \\
\hline $7440-43-9$ & Cadmium & $<2.69 \mathrm{E}-2$ & ug/g dry & $2.69 \mathrm{E}-2$ & $9 / 16 / 08$ & $8 \mathrm{I} 15003$ & PNNL-AGG-ICP-AES \\
\hline $7440-48-4$ & Cobalt & $<9.59 \mathrm{E}-2$ & ug/g dry & $9.59 \mathrm{E}-2$ & $9 / 16 / 08$ & $8 \mathrm{I} 15003$ & PNNL-AGG-ICP-AES \\
\hline $7440-47-3$ & Chromium & $<3.33 \mathrm{E}-2$ & ug/g dry & 3.33E-2 & $9 / 16 / 08$ & $8 \mathrm{I} 15003$ & PNNL-AGG-ICP-AES \\
\hline $7440-50-8$ & Copper & $<8.03 \mathrm{E}-2$ & ug/g dry & $8.03 \mathrm{E}-2$ & $9 / 16 / 08$ & $8 \mathrm{I} 15003$ & PNNL-AGG-ICP-AES \\
\hline $7439-89-6$ & Iron & $1.42 \mathrm{E}-1$ & ug/g dry & $1.42 \mathrm{E}-1$ & $9 / 16 / 08$ & $8 \mathrm{I} 15003$ & PNNL-AGG-ICP-AES \\
\hline $7440-09-7$ & Potassium & $2.88 \mathrm{E} 0$ & ug/g dry & $2.33 \mathrm{E} 0$ & $9 / 16 / 08$ & $8 \mathrm{I} 15003$ & PNNL-AGG-ICP-AES \\
\hline $7439-93-2$ & Lithium & $<5.40 \mathrm{E}-1$ & ug/g dry & $5.40 \mathrm{E}-1$ & $9 / 16 / 08$ & $8 \mathrm{I} 15003$ & PNNL-AGG-ICP-AES \\
\hline $7439-95-4$ & Magnesium & $1.96 \mathrm{E} 0$ & ug/g dry & $8.33 \mathrm{E}-2$ & $9 / 16 / 08$ & $8 \mathrm{I} 15003$ & PNNL-AGG-ICP-AES \\
\hline $7439-96-5$ & Manganese & $<1.71 \mathrm{E}-2$ & ug/g dry & $1.71 \mathrm{E}-2$ & $9 / 16 / 08$ & $8 \mathrm{I} 15003$ & PNNL-AGG-ICP-AES \\
\hline $7439-98-7$ & Molybdenum & $<1.39 \mathrm{E}-1$ & ug/g dry & $1.39 \mathrm{E}-1$ & $9 / 16 / 08$ & $8 \mathrm{I} 15003$ & PNNL-AGG-ICP-AES \\
\hline $7440-02-0$ & Nickel & $<9.32 \mathrm{E}-2$ & ug/g dry & $9.32 \mathrm{E}-2$ & $9 / 16 / 08$ & $8 \mathrm{I} 15003$ & PNNL-AGG-ICP-AES \\
\hline $7723-14-0$ & Phosphorus & $<1.03 \mathrm{E} 0$ & ug/g dry & $1.03 \mathrm{E} 0$ & $9 / 16 / 08$ & $8 \mathrm{I} 15003$ & PNNL-AGG-ICP-AES \\
\hline $7439-92-1$ & Lead & $<4.32 \mathrm{E}-1$ & ug/g dry & $4.32 \mathrm{E}-1$ & $9 / 16 / 08$ & $8 \mathrm{I} 15003$ & PNNL-AGG-ICP-AES \\
\hline $7782-49-2$ & Selenium & $<1.95 \mathrm{E} 0$ & ug/g dry & $1.95 \mathrm{E} 0$ & $9 / 16 / 08$ & $8 \mathrm{I} 15003$ & PNNL-AGG-ICP-AES \\
\hline $7440-24-6$ & Strontium & $<5.22 \mathrm{E}-2$ & ug/g dry & $5.22 \mathrm{E}-2$ & $9 / 16 / 08$ & $8 \mathrm{I} 15003$ & PNNL-AGG-ICP-AES \\
\hline $7440-28-0$ & Thallium & $<1.03 \mathrm{E} 0$ & ug/g dry & $1.03 \mathrm{E} 0$ & $9 / 16 / 08$ & $8 \mathrm{I} 15003$ & PNNL-AGG-ICP-AES \\
\hline $7440-62-2$ & Vanadium & $<4.53 \mathrm{E}-2$ & ug/g dry & $4.53 \mathrm{E}-2$ & $9 / 16 / 08$ & $8 \mathrm{I} 15003$ & PNNL-AGG-ICP-AES \\
\hline $7440-66-6$ & Zinc & $<9.26 \mathrm{E}-2$ & ug/g dry & $9.26 \mathrm{E}-2$ & $9 / 16 / 08$ & $8 \mathrm{I} 15003$ & PNNL-AGG-ICP-AES \\
\hline $7440-23-5$ & Sodium & 5.94E0 & ug/g dry & $6.69 \mathrm{E}-1$ & $9 / 16 / 08$ & $8 \mathrm{I} 15003$ & PNNL-AGG-ICP-AES \\
\hline $7704-34-9$ & Sulfur & $<3.07 \mathrm{E} 0$ & ug/g dry & $3.07 \mathrm{E} 0$ & $9 / 16 / 08$ & $8 \mathrm{I} 15003$ & PNNL-AGG-ICP-AES \\
\hline $7440-32-6$ & Titanium & $<8.75 \mathrm{E}-3$ & ug/g dry & $8.75 \mathrm{E}-3$ & $9 / 16 / 08$ & $8 \mathrm{I} 15003$ & PNNL-AGG-ICP-AES \\
\hline
\end{tabular}


Total Metals by PNNL-AGG-ICP-AES/Water Extract

\begin{tabular}{|c|c|c|c|c|c|c|c|}
\hline CAS \# & Analyte & Results & Units & EQL & Analyzed & Batch & Method \\
\hline HEIS No. & B1V9B5 & \multicolumn{3}{|c|}{ Lab ID: $\quad$ 0805003-15 } & & & \\
\hline $7440-67-7$ & Zirconium & $<1.50 \mathrm{E}-1$ & ug/g dry & $1.50 \mathrm{E}-1$ & $9 / 16 / 08$ & $8 \mathrm{I} 15003$ & PNNL-AGG-ICP-AES \\
\hline $7440-22-4$ & Silver & $<7.70 \mathrm{E}-2$ & ug/g dry & $7.70 \mathrm{E}-2$ & $9 / 16 / 08$ & $8 \mathrm{I} 15003$ & PNNL-AGG-ICP-AES \\
\hline $7440-15-5$ & Rhenium & $<1.52 \mathrm{E}-1$ & ug/g dry & $1.52 \mathrm{E}-1$ & $9 / 16 / 08$ & $8 \mathrm{I} 15003$ & PNNL-AGG-ICP-AES \\
\hline $7440-36-0$ & Antimony & $<6.33 \mathrm{E}-1$ & ug/g dry & $6.33 \mathrm{E}-1$ & $9 / 16 / 08$ & $8 \mathrm{I} 15003$ & PNNL-AGG-ICP-AES \\
\hline HEIS No. & B1V9B6 & \multicolumn{3}{|c|}{ Lab ID: $\quad$ 0805003-16 } & & & \\
\hline $7429-90-5$ & Aluminum & $1.93 \mathrm{E}-1$ & ug/g dry & $8.55 \mathrm{E}-2$ & $9 / 16 / 08$ & $8 \mathrm{I} 15003$ & PNNL-AGG-ICP-AES \\
\hline $7440-38-2$ & Arsenic & $<3.59 \mathrm{E}-1$ & ug/g dry & $3.59 \mathrm{E}-1$ & $9 / 16 / 08$ & $8 \mathrm{I} 15003$ & PNNL-AGG-ICP-AES \\
\hline $7440-42-8$ & Boron & $<1.93 \mathrm{E} 0$ & ug/g dry & $1.93 \mathrm{E} 0$ & $9 / 16 / 08$ & $8 \mathrm{I} 15003$ & PNNL-AGG-ICP-AES \\
\hline $7440-41-7$ & Beryllium & $<2.83 \mathrm{E}-2$ & ug/g dry & $2.83 \mathrm{E}-2$ & $9 / 16 / 08$ & $8 \mathrm{I} 15003$ & PNNL-AGG-ICP-AES \\
\hline $7440-69-9$ & Bismuth & $<1.87 \mathrm{E}-1$ & ug/g dry & $1.87 \mathrm{E}-1$ & $9 / 16 / 08$ & $8 \mathrm{I} 15003$ & PNNL-AGG-ICP-AES \\
\hline $7440-70-2$ & Calcium & $6.42 \mathrm{E} 0$ & ug/g dry & $3.86 \mathrm{E}-1$ & $9 / 16 / 08$ & $8 \mathrm{I} 15003$ & PNNL-AGG-ICP-AES \\
\hline $7440-43-9$ & Cadmium & $<2.68 \mathrm{E}-2$ & ug/g dry & $2.68 \mathrm{E}-2$ & $9 / 16 / 08$ & $8 \mathrm{I} 15003$ & PNNL-AGG-ICP-AES \\
\hline $7440-48-4$ & Cobalt & $<9.57 \mathrm{E}-2$ & ug/g dry & $9.57 \mathrm{E}-2$ & $9 / 16 / 08$ & $8 \mathrm{I} 15003$ & PNNL-AGG-ICP-AES \\
\hline $7440-47-3$ & Chromium & $<3.32 \mathrm{E}-2$ & ug/g dry & $3.32 \mathrm{E}-2$ & $9 / 16 / 08$ & $8 \mathrm{I} 15003$ & PNNL-AGG-ICP-AES \\
\hline $7440-50-8$ & Copper & $<8.01 \mathrm{E}-2$ & ug/g dry & $8.01 \mathrm{E}-2$ & $9 / 16 / 08$ & $8 \mathrm{I} 15003$ & PNNL-AGG-ICP-AES \\
\hline $7439-89-6$ & Iron & $<1.42 \mathrm{E}-1$ & ug/g dry & $1.42 \mathrm{E}-1$ & $9 / 16 / 08$ & $8 \mathrm{I} 15003$ & PNNL-AGG-ICP-AES \\
\hline $7440-09-7$ & Potassium & $4.01 \mathrm{E} 0$ & ug/g dry & $2.32 \mathrm{E} 0$ & $9 / 16 / 08$ & $8 \mathrm{I} 15003$ & PNNL-AGG-ICP-AES \\
\hline $7439-93-2$ & Lithium & $<5.38 \mathrm{E}-1$ & ug/g dry & $5.38 \mathrm{E}-1$ & $9 / 16 / 08$ & $8 \mathrm{I} 15003$ & PNNL-AGG-ICP-AES \\
\hline $7439-95-4$ & Magnesium & $2.54 \mathrm{E} 0$ & ug/g dry & $8.31 \mathrm{E}-2$ & $9 / 16 / 08$ & $8 \mathrm{I} 15003$ & PNNL-AGG-ICP-AES \\
\hline $7439-96-5$ & Manganese & $<1.71 \mathrm{E}-2$ & ug/g dry & $1.71 \mathrm{E}-2$ & $9 / 16 / 08$ & $8 \mathrm{I} 15003$ & PNNL-AGG-ICP-AES \\
\hline 7439-98-7 & Molybdenum & $<1.39 \mathrm{E}-1$ & ug/g dry & $1.39 \mathrm{E}-1$ & $9 / 16 / 08$ & $8 \mathrm{I} 15003$ & PNNL-AGG-ICP-AES \\
\hline $7440-02-0$ & Nickel & $<9.30 \mathrm{E}-2$ & ug/g dry & $9.30 \mathrm{E}-2$ & $9 / 16 / 08$ & $8 \mathrm{I} 15003$ & PNNL-AGG-ICP-AES \\
\hline $7723-14-0$ & Phosphorus & $<1.03 \mathrm{E} 0$ & ug/g dry & $1.03 \mathrm{E} 0$ & $9 / 16 / 08$ & $8 \mathrm{I} 15003$ & PNNL-AGG-ICP-AES \\
\hline $7439-92-1$ & Lead & $<4.31 \mathrm{E}-1$ & ug/g dry & $4.31 \mathrm{E}-1$ & $9 / 16 / 08$ & $8 \mathrm{I} 15003$ & PNNL-AGG-ICP-AES \\
\hline $7782-49-2$ & Selenium & $<1.94 \mathrm{E} 0$ & ug/g dry & $1.94 \mathrm{E} 0$ & $9 / 16 / 08$ & $8 \mathrm{I} 15003$ & PNNL-AGG-ICP-AES \\
\hline $7440-24-6$ & Strontium & $<5.20 \mathrm{E}-2$ & ug/g dry & $5.20 \mathrm{E}-2$ & $9 / 16 / 08$ & $8 \mathrm{I} 15003$ & PNNL-AGG-ICP-AES \\
\hline $7440-28-0$ & Thallium & $<1.02 \mathrm{E} 0$ & ug/g dry & $1.02 \mathrm{E} 0$ & $9 / 16 / 08$ & $8 \mathrm{I} 15003$ & PNNL-AGG-ICP-AES \\
\hline $7440-62-2$ & Vanadium & $<4.51 \mathrm{E}-2$ & ug/g dry & $4.51 \mathrm{E}-2$ & $9 / 16 / 08$ & $8 \mathrm{I} 15003$ & PNNL-AGG-ICP-AES \\
\hline $7440-66-6$ & Zinc & $<9.24 \mathrm{E}-2$ & ug/g dry & $9.24 \mathrm{E}-2$ & $9 / 16 / 08$ & $8 \mathrm{I} 15003$ & PNNL-AGG-ICP-AES \\
\hline $7440-23-5$ & Sodium & 7.99E0 & ug/g dry & $6.67 \mathrm{E}-1$ & $9 / 16 / 08$ & $8 \mathrm{I} 15003$ & PNNL-AGG-ICP-AES \\
\hline 7704-34-9 & Sulfur & 5.33E0 & ug/g dry & $3.06 \mathrm{E} 0$ & $9 / 16 / 08$ & $8 \mathrm{I} 15003$ & PNNL-AGG-ICP-AES \\
\hline $7440-32-6$ & Titanium & $<8.73 \mathrm{E}-3$ & ug/g dry & $8.73 \mathrm{E}-3$ & $9 / 16 / 08$ & $8 \mathrm{I} 15003$ & PNNL-AGG-ICP-AES \\
\hline $7440-67-7$ & Zirconium & $<1.49 \mathrm{E}-1$ & ug/g dry & $1.49 \mathrm{E}-1$ & $9 / 16 / 08$ & $8 \mathrm{I} 15003$ & PNNL-AGG-ICP-AES \\
\hline $7440-22-4$ & Silver & $<7.68 \mathrm{E}-2$ & ug/g dry & $7.68 \mathrm{E}-2$ & $9 / 16 / 08$ & $8 \mathrm{I} 15003$ & PNNL-AGG-ICP-AES \\
\hline $7440-15-5$ & Rhenium & $<1.51 \mathrm{E}-1$ & ug/g dry & $1.51 \mathrm{E}-1$ & $9 / 16 / 08$ & $8 \mathrm{I} 15003$ & PNNL-AGG-ICP-AES \\
\hline $7440-36-0$ & Antimony & $<6.31 \mathrm{E}-1$ & ug/g dry & $6.31 \mathrm{E}-1$ & 9/16/08 & $8 \mathrm{I} 15003$ & PNNL-AGG-ICP-AES \\
\hline HEIS No. & B1V9B7 & & b ID: & $5003-17$ & & & \\
\hline $7429-90-5$ & Aluminum & $1.91 \mathrm{E}-1$ & ug/g dry & $8.58 \mathrm{E}-2$ & $9 / 16 / 08$ & $8 \mathrm{I} 15003$ & PNNL-AGG-ICP-AES \\
\hline $7440-38-2$ & Arsenic & $<3.60 \mathrm{E}-1$ & ug/g dry & $3.60 \mathrm{E}-1$ & $9 / 16 / 08$ & $8 \mathrm{I} 15003$ & PNNL-AGG-ICP-AES \\
\hline $7440-42-8$ & Boron & $<1.94 \mathrm{E} 0$ & ug/g dry & $1.94 \mathrm{E} 0$ & $9 / 16 / 08$ & $8 \mathrm{I} 15003$ & PNNL-AGG-ICP-AES \\
\hline $7440-41-7$ & Beryllium & $<2.84 \mathrm{E}-2$ & ug/g dry & $2.84 \mathrm{E}-2$ & $9 / 16 / 08$ & $8 \mathrm{I} 15003$ & PNNL-AGG-ICP-AES \\
\hline $7440-69-9$ & Bismuth & $<1.88 \mathrm{E}-1$ & ug/g dry & $1.88 \mathrm{E}-1$ & $9 / 16 / 08$ & $8 \mathrm{I} 15003$ & PNNL-AGG-ICP-AES \\
\hline $7440-70-2$ & Calcium & $7.84 \mathrm{E} 0$ & ug/g dry & $3.87 \mathrm{E}-1$ & 9/16/08 & $8 \mathrm{I} 15003$ & PNNL-AGG-ICP-AES \\
\hline $7440-43-9$ & Cadmium & $<2.69 \mathrm{E}-2$ & ug/g dry & $2.69 \mathrm{E}-2$ & $9 / 16 / 08$ & $8 \mathrm{I} 15003$ & PNNL-AGG-ICP-AES \\
\hline $7440-48-4$ & Cobalt & $<9.59 \mathrm{E}-2$ & ug/g dry & $9.59 \mathrm{E}-2$ & $9 / 16 / 08$ & $8 \mathrm{I} 15003$ & PNNL-AGG-ICP-AES \\
\hline $7440-47-3$ & Chromium & $<3.33 \mathrm{E}-2$ & ug/g dry & $3.33 \mathrm{E}-2$ & $9 / 16 / 08$ & $8 \mathrm{I} 15003$ & PNNL-AGG-ICP-AES \\
\hline $7440-50-8$ & Copper & $<8.04 \mathrm{E}-2$ & ug/g dry & $8.04 \mathrm{E}-2$ & $9 / 16 / 08$ & $8 \mathrm{I} 15003$ & PNNL-AGG-ICP-AES \\
\hline $7439-89-6$ & Iron & $<1.42 \mathrm{E}-1$ & ug/g dry & $1.42 \mathrm{E}-1$ & $9 / 16 / 08$ & $8 \mathrm{I} 15003$ & PNNL-AGG-ICP-AES \\
\hline
\end{tabular}


Total Metals by PNNL-AGG-ICP-AES/Water Extract

\begin{tabular}{|c|c|c|c|c|c|c|c|}
\hline CAS \# & Analyte & Results & Units & EQL & Analyzed & Batch & Method \\
\hline HEIS No. & B1V9B7 & \multicolumn{3}{|c|}{ Lab ID: $\quad$ 0805003-17 } & & & \\
\hline 7440-09-7 & Potassium & $4.07 \mathrm{E} 0$ & $\mathrm{ug} / \mathrm{g}$ dry & $2.33 \mathrm{E} 0$ & $9 / 16 / 08$ & $8 \mathrm{I} 15003$ & PNNL-AGG-ICP-AES \\
\hline $7439-93-2$ & Lithium & $<5.40 \mathrm{E}-1$ & ug/g dry & $5.40 \mathrm{E}-1$ & $9 / 16 / 08$ & $8 \mathrm{I} 15003$ & PNNL-AGG-ICP-AES \\
\hline $7439-95-4$ & Magnesium & $3.18 \mathrm{E} 0$ & ug/g dry & $8.34 \mathrm{E}-2$ & $9 / 16 / 08$ & $8 \mathrm{I} 15003$ & PNNL-AGG-ICP-AES \\
\hline $7439-96-5$ & Manganese & $<1.71 \mathrm{E}-2$ & ug/g dry & $1.71 \mathrm{E}-2$ & $9 / 16 / 08$ & $8 \mathrm{I} 15003$ & PNNL-AGG-ICP-AES \\
\hline $7439-98-7$ & Molybdenum & $<1.39 \mathrm{E}-1$ & ug/g dry & $1.39 \mathrm{E}-1$ & $9 / 16 / 08$ & $8 \mathrm{I} 15003$ & PNNL-AGG-ICP-AES \\
\hline $7440-02-0$ & Nickel & $<9.32 \mathrm{E}-2$ & ug/g dry & $9.32 \mathrm{E}-2$ & $9 / 16 / 08$ & $8 \mathrm{I} 15003$ & PNNL-AGG-ICP-AES \\
\hline $7723-14-0$ & Phosphorus & $<1.03 \mathrm{E} 0$ & ug/g dry & $1.03 \mathrm{E} 0$ & $9 / 16 / 08$ & $8 \mathrm{II} 15003$ & PNNL-AGG-ICP-AES \\
\hline $7439-92-1$ & Lead & $<4.32 \mathrm{E}-1$ & ug/g dry & $4.32 \mathrm{E}-1$ & $9 / 16 / 08$ & $8 \mathrm{I} 15003$ & PNNL-AGG-ICP-AES \\
\hline $7782-49-2$ & Selenium & $<1.95 \mathrm{E} 0$ & ug/g dry & $1.95 \mathrm{E} 0$ & $9 / 16 / 08$ & $8 \mathrm{I} 15003$ & PNNL-AGG-ICP-AES \\
\hline $7440-24-6$ & Strontium & $<5.22 \mathrm{E}-2$ & $\mathrm{ug} / \mathrm{g}$ dry & $5.22 \mathrm{E}-2$ & $9 / 16 / 08$ & $8 \mathrm{II} 15003$ & PNNL-AGG-ICP-AES \\
\hline $7440-28-0$ & Thallium & $<1.03 \mathrm{E} 0$ & ug/g dry & $1.03 \mathrm{E} 0$ & $9 / 16 / 08$ & $8 \mathrm{I} 15003$ & PNNL-AGG-ICP-AES \\
\hline $7440-62-2$ & Vanadium & $<4.53 \mathrm{E}-2$ & ug/g dry & 4.53E-2 & $9 / 16 / 08$ & $8 \mathrm{I} 15003$ & PNNL-AGG-ICP-AES \\
\hline $7440-66-6$ & Zinc & $<9.26 \mathrm{E}-2$ & ug/g dry & $9.26 \mathrm{E}-2$ & $9 / 16 / 08$ & $8 \mathrm{I} 15003$ & PNNL-AGG-ICP-AES \\
\hline $7440-23-5$ & Sodium & $9.29 \mathrm{E} 0$ & ug/g dry & $6.69 \mathrm{E}-1$ & $9 / 16 / 08$ & $8 \mathrm{I} 15003$ & PNNL-AGG-ICP-AES \\
\hline 7704-34-9 & Sulfur & $6.52 \mathrm{E} 0$ & ug/g dry & 3.07E0 & $9 / 16 / 08$ & $8 \mathrm{I} 15003$ & PNNL-AGG-ICP-AES \\
\hline $7440-32-6$ & Titanium & $<8.76 \mathrm{E}-3$ & ug/g dry & $8.76 \mathrm{E}-3$ & $9 / 16 / 08$ & $8 \mathrm{I} 15003$ & PNNL-AGG-ICP-AES \\
\hline $7440-67-7$ & Zirconium & $<1.50 \mathrm{E}-1$ & ug/g dry & $1.50 \mathrm{E}-1$ & $9 / 16 / 08$ & $8 \mathrm{I} 15003$ & PNNL-AGG-ICP-AES \\
\hline $7440-22-4$ & Silver & $<7.71 \mathrm{E}-2$ & $\mathrm{ug} / \mathrm{g}$ dry & 7.71E-2 & $9 / 16 / 08$ & $8 \mathrm{I} 15003$ & PNNL-AGG-ICP-AES \\
\hline $7440-15-5$ & Rhenium & $<1.52 \mathrm{E}-1$ & ug/g dry & $1.52 \mathrm{E}-1$ & $9 / 16 / 08$ & $8 \mathrm{I} 15003$ & PNNL-AGG-ICP-AES \\
\hline $7440-36-0$ & Antimony & $<6.33 \mathrm{E}-1$ & ug/g dry & $6.33 \mathrm{E}-1$ & $9 / 16 / 08$ & $8 \mathrm{II} 15003$ & PNNL-AGG-ICP-AES \\
\hline HEIS No. & B1V9B8 & \multicolumn{3}{|c|}{ Lab ID: $\quad$ 0805003-18 } & & & \\
\hline $7429-90-5$ & Aluminum & $1.89 \mathrm{E}-1$ & ug/g dry & $8.63 \mathrm{E}-2$ & $9 / 16 / 08$ & $8 \mathrm{I} 15003$ & PNNL-AGG-ICP-AES \\
\hline $7440-38-2$ & Arsenic & $<3.62 \mathrm{E}-1$ & ug/g dry & $3.62 \mathrm{E}-1$ & $9 / 16 / 08$ & $8 \mathrm{I} 15003$ & PNNL-AGG-ICP-AES \\
\hline $7440-42-8$ & Boron & $<1.95 \mathrm{E} 0$ & ug/g dry & $1.95 \mathrm{E} 0$ & $9 / 16 / 08$ & $8 \mathrm{I} 15003$ & PNNL-AGG-ICP-AES \\
\hline $7440-41-7$ & Beryllium & $<2.86 \mathrm{E}-2$ & ug/g dry & $2.86 \mathrm{E}-2$ & $9 / 16 / 08$ & $8 \mathrm{I} 15003$ & PNNL-AGG-ICP-AES \\
\hline $7440-69-9$ & Bismuth & $<1.89 \mathrm{E}-1$ & $\mathrm{ug} / \mathrm{g}$ dry & $1.89 \mathrm{E}-1$ & $9 / 16 / 08$ & $8 \mathrm{I} 15003$ & PNNL-AGG-ICP-AES \\
\hline $7440-70-2$ & Calcium & 7.14E0 & ug/g dry & $3.89 \mathrm{E}-1$ & $9 / 16 / 08$ & $8 \mathrm{I} 15003$ & PNNL-AGG-ICP-AES \\
\hline $7440-43-9$ & Cadmium & $<2.70 \mathrm{E}-2$ & ug/g dry & $2.70 \mathrm{E}-2$ & $9 / 16 / 08$ & $8 \mathrm{I} 15003$ & PNNL-AGG-ICP-AES \\
\hline $7440-48-4$ & Cobalt & $<9.65 \mathrm{E}-2$ & $\mathrm{ug} / \mathrm{g}$ dry & $9.65 \mathrm{E}-2$ & $9 / 16 / 08$ & $8 \mathrm{I} 15003$ & PNNL-AGG-ICP-AES \\
\hline $7440-47-3$ & Chromium & $<3.35 \mathrm{E}-2$ & ug/g dry & $3.35 \mathrm{E}-2$ & $9 / 16 / 08$ & $8 \mathrm{I} 15003$ & PNNL-AGG-ICP-AES \\
\hline $7440-50-8$ & Copper & $<8.08 \mathrm{E}-2$ & $\mathrm{ug} / \mathrm{g}$ dry & $8.08 \mathrm{E}-2$ & $9 / 16 / 08$ & $8 \mathrm{I} 15003$ & PNNL-AGG-ICP-AES \\
\hline $7439-89-6$ & Iron & $<1.43 \mathrm{E}-1$ & ug/g dry & $1.43 \mathrm{E}-1$ & $9 / 16 / 08$ & $8 \mathrm{I} 15003$ & PNNL-AGG-ICP-AES \\
\hline $7440-09-7$ & Potassium & $4.24 \mathrm{E} 0$ & $\mathrm{ug} / \mathrm{g}$ dry & $2.34 \mathrm{E} 0$ & $9 / 16 / 08$ & $8 \mathrm{I} 15003$ & PNNL-AGG-ICP-AES \\
\hline $7439-93-2$ & Lithium & $<5.43 \mathrm{E}-1$ & ug/g dry & $5.43 \mathrm{E}-1$ & $9 / 16 / 08$ & $8 \mathrm{I} 15003$ & PNNL-AGG-ICP-AES \\
\hline $7439-95-4$ & Magnesium & $2.90 \mathrm{E} 0$ & ug/g dry & $8.39 \mathrm{E}-2$ & $9 / 16 / 08$ & $8 \mathrm{I} 15003$ & PNNL-AGG-ICP-AES \\
\hline $7439-96-5$ & Manganese & $<1.72 \mathrm{E}-2$ & ug/g dry & $1.72 \mathrm{E}-2$ & $9 / 16 / 08$ & $8 \mathrm{I} 15003$ & PNNL-AGG-ICP-AES \\
\hline $7439-98-7$ & Molybdenum & $<1.40 \mathrm{E}-1$ & ug/g dry & $1.40 \mathrm{E}-1$ & $9 / 16 / 08$ & $8 \mathrm{I} 15003$ & PNNL-AGG-ICP-AES \\
\hline $7440-02-0$ & Nickel & $<9.38 \mathrm{E}-2$ & $\mathrm{ug} / \mathrm{g}$ dry & $9.38 \mathrm{E}-2$ & $9 / 16 / 08$ & $8 \mathrm{I} 15003$ & PNNL-AGG-ICP-AES \\
\hline $7723-14-0$ & Phosphorus & $<1.04 \mathrm{E} 0$ & $\mathrm{ug} / \mathrm{g}$ dry & $1.04 \mathrm{E} 0$ & $9 / 16 / 08$ & $8 \mathrm{I} 15003$ & PNNL-AGG-ICP-AES \\
\hline $7439-92-1$ & Lead & $<4.34 \mathrm{E}-1$ & ug/g dry & $4.34 \mathrm{E}-1$ & $9 / 16 / 08$ & $8 \mathrm{I} 15003$ & PNNL-AGG-ICP-AES \\
\hline $7782-49-2$ & Selenium & $<1.96 \mathrm{E} 0$ & $\mathrm{ug} / \mathrm{g}$ dry & $1.96 \mathrm{E} 0$ & $9 / 16 / 08$ & $8 \mathrm{I} 15003$ & PNNL-AGG-ICP-AES \\
\hline $7440-24-6$ & Strontium & $<5.25 \mathrm{E}-2$ & ug/g dry & $5.25 \mathrm{E}-2$ & $9 / 16 / 08$ & $8 \mathrm{I} 15003$ & PNNL-AGG-ICP-AES \\
\hline $7440-28-0$ & Thallium & $<1.03 \mathrm{E} 0$ & ug/g dry & $1.03 \mathrm{E} 0$ & $9 / 16 / 08$ & $8 \mathrm{I} 15003$ & PNNL-AGG-ICP-AES \\
\hline $7440-62-2$ & Vanadium & $<4.56 \mathrm{E}-2$ & ug/g dry & $4.56 \mathrm{E}-2$ & $9 / 16 / 08$ & $8 \mathrm{I} 15003$ & PNNL-AGG-ICP-AES \\
\hline $7440-66-6$ & Zinc & $<9.32 \mathrm{E}-2$ & ug/g dry & $9.32 \mathrm{E}-2$ & $9 / 16 / 08$ & $8 \mathrm{I} 15003$ & PNNL-AGG-ICP-AES \\
\hline $7440-23-5$ & Sodium & $9.63 \mathrm{E} 0$ & ug/g dry & $6.73 \mathrm{E}-1$ & $9 / 16 / 08$ & $8 \mathrm{I} 15003$ & PNNL-AGG-ICP-AES \\
\hline $7704-34-9$ & Sulfur & $5.16 \mathrm{E} 0$ & ug/g dry & $3.09 \mathrm{E} 0$ & $9 / 16 / 08$ & $8 \mathrm{I} 15003$ & PNNL-AGG-ICP-AES \\
\hline $7440-32-6$ & Titanium & $<8.81 \mathrm{E}-3$ & ug/g dry & $8.81 \mathrm{E}-3$ & $9 / 16 / 08$ & $8 \mathrm{I} 15003$ & PNNL-AGG-ICP-AES \\
\hline
\end{tabular}


Total Metals by PNNL-AGG-ICP-AES/Water Extract

\begin{tabular}{|c|c|c|c|c|c|c|c|}
\hline CAS \# & Analyte & Results & Units & EQL & Analyzed & Batch & Method \\
\hline HEIS No. & B1V9B8 & \multicolumn{3}{|c|}{ Lab ID: $\quad$ 0805003-18 } & & & \\
\hline $7440-67-7$ & Zirconium & $<1.51 \mathrm{E}-1$ & ug/g dry & $1.51 \mathrm{E}-1$ & $9 / 16 / 08$ & $8 \mathrm{I} 15003$ & PNNL-AGG-ICP-AES \\
\hline $7440-22-4$ & Silver & $<7.75 \mathrm{E}-2$ & ug/g dry & $7.75 \mathrm{E}-2$ & $9 / 16 / 08$ & $8 \mathrm{I} 15003$ & PNNL-AGG-ICP-AES \\
\hline $7440-15-5$ & Rhenium & $<1.53 \mathrm{E}-1$ & ug/g dry & $1.53 \mathrm{E}-1$ & $9 / 16 / 08$ & $8 \mathrm{I} 15003$ & PNNL-AGG-ICP-AES \\
\hline $7440-36-0$ & Antimony & $<6.37 \mathrm{E}-1$ & ug/g dry & $6.37 \mathrm{E}-1$ & $9 / 16 / 08$ & $8 \mathrm{I} 15003$ & PNNL-AGG-ICP-AES \\
\hline HEIS No. & B1V9B9 & \multicolumn{3}{|c|}{ Lab ID: $\quad$ 0805003-19 } & & & \\
\hline $7429-90-5$ & Aluminum & $2.29 \mathrm{E}-1$ & ug/g dry & $8.54 \mathrm{E}-2$ & $9 / 16 / 08$ & $8 \mathrm{I} 15003$ & PNNL-AGG-ICP-AES \\
\hline $7440-38-2$ & Arsenic & $<3.58 \mathrm{E}-1$ & ug/g dry & $3.58 \mathrm{E}-1$ & $9 / 16 / 08$ & $8 \mathrm{I} 15003$ & PNNL-AGG-ICP-AES \\
\hline $7440-42-8$ & Boron & $<1.93 \mathrm{E} 0$ & ug/g dry & $1.93 \mathrm{E} 0$ & $9 / 16 / 08$ & $8 \mathrm{I} 15003$ & PNNL-AGG-ICP-AES \\
\hline $7440-41-7$ & Beryllium & $<2.83 \mathrm{E}-2$ & ug/g dry & $2.83 \mathrm{E}-2$ & $9 / 16 / 08$ & $8 \mathrm{I} 15003$ & PNNL-AGG-ICP-AES \\
\hline $7440-69-9$ & Bismuth & $<1.87 \mathrm{E}-1$ & ug/g dry & $1.87 \mathrm{E}-1$ & $9 / 16 / 08$ & $8 \mathrm{I} 15003$ & PNNL-AGG-ICP-AES \\
\hline $7440-70-2$ & Calcium & $8.22 \mathrm{E} 0$ & ug/g dry & $3.85 \mathrm{E}-1$ & $9 / 16 / 08$ & $8 \mathrm{I} 15003$ & PNNL-AGG-ICP-AES \\
\hline $7440-43-9$ & Cadmium & $<2.67 \mathrm{E}-2$ & ug/g dry & $2.67 \mathrm{E}-2$ & $9 / 16 / 08$ & $8 \mathrm{I} 15003$ & PNNL-AGG-ICP-AES \\
\hline $7440-48-4$ & Cobalt & $<9.55 \mathrm{E}-2$ & ug/g dry & $9.55 \mathrm{E}-2$ & $9 / 16 / 08$ & $8 \mathrm{I} 15003$ & PNNL-AGG-ICP-AES \\
\hline $7440-47-3$ & Chromium & $<3.31 \mathrm{E}-2$ & ug/g dry & $3.31 \mathrm{E}-2$ & $9 / 16 / 08$ & $8 \mathrm{I} 15003$ & PNNL-AGG-ICP-AES \\
\hline $7440-50-8$ & Copper & $<8.00 \mathrm{E}-2$ & ug/g dry & $8.00 \mathrm{E}-2$ & $9 / 16 / 08$ & $8 \mathrm{I} 15003$ & PNNL-AGG-ICP-AES \\
\hline $7439-89-6$ & Iron & $<1.42 \mathrm{E}-1$ & ug/g dry & $1.42 \mathrm{E}-1$ & $9 / 16 / 08$ & $8 \mathrm{I} 15003$ & PNNL-AGG-ICP-AES \\
\hline $7440-09-7$ & Potassium & $5.07 \mathrm{E} 0$ & ug/g dry & $2.32 \mathrm{E} 0$ & $9 / 16 / 08$ & $8 \mathrm{I} 15003$ & PNNL-AGG-ICP-AES \\
\hline $7439-93-2$ & Lithium & $<5.37 \mathrm{E}-1$ & ug/g dry & $5.37 \mathrm{E}-1$ & $9 / 16 / 08$ & $8 \mathrm{I} 15003$ & PNNL-AGG-ICP-AES \\
\hline $7439-95-4$ & Magnesium & $3.35 \mathrm{E} 0$ & ug/g dry & $8.30 \mathrm{E}-2$ & $9 / 16 / 08$ & $8 \mathrm{I} 15003$ & PNNL-AGG-ICP-AES \\
\hline $7439-96-5$ & Manganese & $<1.70 \mathrm{E}-2$ & ug/g dry & $1.70 \mathrm{E}-2$ & $9 / 16 / 08$ & $8 \mathrm{I} 15003$ & PNNL-AGG-ICP-AES \\
\hline 7439-98-7 & Molybdenum & $<1.39 \mathrm{E}-1$ & ug/g dry & $1.39 \mathrm{E}-1$ & $9 / 16 / 08$ & $8 \mathrm{I} 15003$ & PNNL-AGG-ICP-AES \\
\hline $7440-02-0$ & Nickel & $<9.28 \mathrm{E}-2$ & ug/g dry & $9.28 \mathrm{E}-2$ & $9 / 16 / 08$ & $8 \mathrm{I} 15003$ & PNNL-AGG-ICP-AES \\
\hline $7723-14-0$ & Phosphorus & $<1.03 \mathrm{E} 0$ & ug/g dry & $1.03 \mathrm{E} 0$ & $9 / 16 / 08$ & $8 \mathrm{I} 15003$ & PNNL-AGG-ICP-AES \\
\hline $7439-92-1$ & Lead & $<4.30 \mathrm{E}-1$ & ug/g dry & $4.30 \mathrm{E}-1$ & $9 / 16 / 08$ & $8 \mathrm{I} 15003$ & PNNL-AGG-ICP-AES \\
\hline $7782-49-2$ & Selenium & $<1.94 \mathrm{E} 0$ & ug/g dry & $1.94 \mathrm{E} 0$ & $9 / 16 / 08$ & $8 \mathrm{I} 15003$ & PNNL-AGG-ICP-AES \\
\hline $7440-24-6$ & Strontium & $<5.19 \mathrm{E}-2$ & ug/g dry & $5.19 \mathrm{E}-2$ & $9 / 16 / 08$ & $8 \mathrm{I} 15003$ & PNNL-AGG-ICP-AES \\
\hline $7440-28-0$ & Thallium & $<1.02 \mathrm{E} 0$ & ug/g dry & $1.02 \mathrm{E} 0$ & $9 / 16 / 08$ & $8 \mathrm{I} 15003$ & PNNL-AGG-ICP-AES \\
\hline $7440-62-2$ & Vanadium & $<4.51 \mathrm{E}-2$ & ug/g dry & $4.51 \mathrm{E}-2$ & $9 / 16 / 08$ & $8 \mathrm{I} 15003$ & PNNL-AGG-ICP-AES \\
\hline $7440-66-6$ & Zinc & $<9.22 \mathrm{E}-2$ & ug/g dry & $9.22 \mathrm{E}-2$ & $9 / 16 / 08$ & $8 \mathrm{I} 15003$ & PNNL-AGG-ICP-AES \\
\hline $7440-23-5$ & Sodium & $1.14 \mathrm{E} 1$ & ug/g dry & $6.66 \mathrm{E}-1$ & $9 / 16 / 08$ & $8 \mathrm{I} 15003$ & PNNL-AGG-ICP-AES \\
\hline $7704-34-9$ & Sulfur & 7.62E0 & ug/g dry & $3.06 \mathrm{E} 0$ & $9 / 16 / 08$ & $8 \mathrm{I} 15003$ & PNNL-AGG-ICP-AES \\
\hline $7440-32-6$ & Titanium & $<8.72 \mathrm{E}-3$ & ug/g dry & $8.72 \mathrm{E}-3$ & $9 / 16 / 08$ & $8 \mathrm{I} 15003$ & PNNL-AGG-ICP-AES \\
\hline $7440-67-7$ & Zirconium & $<1.49 \mathrm{E}-1$ & ug/g dry & $1.49 \mathrm{E}-1$ & $9 / 16 / 08$ & $8 \mathrm{I} 15003$ & PNNL-AGG-ICP-AES \\
\hline $7440-22-4$ & Silver & $<7.67 \mathrm{E}-2$ & ug/g dry & 7.67E-2 & $9 / 16 / 08$ & $8 \mathrm{I} 15003$ & PNNL-AGG-ICP-AES \\
\hline $7440-15-5$ & Rhenium & $<1.51 \mathrm{E}-1$ & ug/g dry & $1.51 \mathrm{E}-1$ & $9 / 16 / 08$ & $8 \mathrm{I} 15003$ & PNNL-AGG-ICP-AES \\
\hline $7440-36-0$ & Antimony & $<6.30 \mathrm{E}-1$ & ug/g dry & $6.30 \mathrm{E}-1$ & 9/16/08 & $8 \mathrm{I} 15003$ & PNNL-AGG-ICP-AES \\
\hline HEIS No. & B1V9C0 & & b ID: & $5003-20$ & & & \\
\hline $7429-90-5$ & Aluminum & $2.37 \mathrm{E}-1$ & ug/g dry & $8.60 \mathrm{E}-2$ & $9 / 16 / 08$ & $8 \mathrm{I} 15003$ & PNNL-AGG-ICP-AES \\
\hline $7440-38-2$ & Arsenic & $<3.61 \mathrm{E}-1$ & ug/g dry & $3.61 \mathrm{E}-1$ & $9 / 16 / 08$ & $8 \mathrm{I} 15003$ & PNNL-AGG-ICP-AES \\
\hline $7440-42-8$ & Boron & $<1.95 \mathrm{E} 0$ & ug/g dry & $1.95 \mathrm{E} 0$ & $9 / 16 / 08$ & $8 \mathrm{I} 15003$ & PNNL-AGG-ICP-AES \\
\hline $7440-41-7$ & Beryllium & $<2.85 \mathrm{E}-2$ & ug/g dry & $2.85 \mathrm{E}-2$ & $9 / 16 / 08$ & $8 \mathrm{I} 15003$ & PNNL-AGG-ICP-AES \\
\hline $7440-69-9$ & Bismuth & $<1.89 \mathrm{E}-1$ & ug/g dry & $1.89 \mathrm{E}-1$ & $9 / 16 / 08$ & $8 \mathrm{I} 15003$ & PNNL-AGG-ICP-AES \\
\hline $7440-70-2$ & Calcium & $9.06 \mathrm{E} 0$ & ug/g dry & $3.88 \mathrm{E}-1$ & $9 / 16 / 08$ & $8 \mathrm{I} 15003$ & PNNL-AGG-ICP-AES \\
\hline $7440-43-9$ & Cadmium & $<2.70 \mathrm{E}-2$ & ug/g dry & $2.70 \mathrm{E}-2$ & $9 / 16 / 08$ & $8 \mathrm{I} 15003$ & PNNL-AGG-ICP-AES \\
\hline $7440-48-4$ & Cobalt & $<9.63 \mathrm{E}-2$ & ug/g dry & $9.63 \mathrm{E}-2$ & $9 / 16 / 08$ & $8 \mathrm{I} 15003$ & PNNL-AGG-ICP-AES \\
\hline $7440-47-3$ & Chromium & $<3.34 \mathrm{E}-2$ & ug/g dry & $3.34 \mathrm{E}-2$ & $9 / 16 / 08$ & $8 \mathrm{I} 15003$ & PNNL-AGG-ICP-AES \\
\hline $7440-50-8$ & Copper & $<8.06 \mathrm{E}-2$ & ug/g dry & $8.06 \mathrm{E}-2$ & $9 / 16 / 08$ & $8 \mathrm{I} 15003$ & PNNL-AGG-ICP-AES \\
\hline $7439-89-6$ & Iron & $<1.43 \mathrm{E}-1$ & ug/g dry & $1.43 \mathrm{E}-1$ & $9 / 16 / 08$ & $8 \mathrm{I} 15003$ & PNNL-AGG-ICP-AES \\
\hline
\end{tabular}


Total Metals by PNNL-AGG-ICP-AES/Water Extract

\begin{tabular}{|c|c|c|c|c|c|c|c|}
\hline CAS \# & Analyte & Results & Units & EQL & Analyzed & Batch & Method \\
\hline HEIS No. & B1V9C0 & \multicolumn{3}{|c|}{ Lab ID: $\quad$ 0805003-20 } & & & \\
\hline $7440-09-7$ & Potassium & $4.83 \mathrm{E} 0$ & $\mathrm{ug} / \mathrm{g}$ dry & $2.33 \mathrm{E} 0$ & $9 / 16 / 08$ & $8 I 15003$ & PNNL-AGG-ICP-AES \\
\hline $7439-93-2$ & Lithium & $<5.42 \mathrm{E}-1$ & $\mathrm{ug} / \mathrm{g}$ dry & $5.42 \mathrm{E}-1$ & $9 / 16 / 08$ & $8 \mathrm{II} 15003$ & PNNL-AGG-ICP-AES \\
\hline $7439-95-4$ & Magnesium & $3.61 \mathrm{E} 0$ & ug/g dry & $8.36 \mathrm{E}-2$ & $9 / 16 / 08$ & $8 \mathrm{I} 15003$ & PNNL-AGG-ICP-AES \\
\hline $7439-96-5$ & Manganese & $<1.72 \mathrm{E}-2$ & $\mathrm{ug} / \mathrm{g}$ dry & $1.72 \mathrm{E}-2$ & $9 / 16 / 08$ & $8 \mathrm{I} 15003$ & PNNL-AGG-ICP-AES \\
\hline $7439-98-7$ & Molybdenum & $<1.40 \mathrm{E}-1$ & ug/g dry & $1.40 \mathrm{E}-1$ & $9 / 16 / 08$ & $8 \mathrm{I} 15003$ & PNNL-AGG-ICP-AES \\
\hline $7440-02-0$ & Nickel & $<9.36 \mathrm{E}-2$ & ug/g dry & $9.36 \mathrm{E}-2$ & $9 / 16 / 08$ & $8 \mathrm{I} 15003$ & PNNL-AGG-ICP-AES \\
\hline $7723-14-0$ & Phosphorus & $<1.03 \mathrm{E} 0$ & ug/g dry & $1.03 \mathrm{E} 0$ & $9 / 16 / 08$ & $8 \mathrm{I} 15003$ & PNNL-AGG-ICP-AES \\
\hline $7439-92-1$ & Lead & $<4.33 \mathrm{E}-1$ & $\mathrm{ug} / \mathrm{g}$ dry & $4.33 \mathrm{E}-1$ & $9 / 16 / 08$ & $8 \mathrm{II} 15003$ & PNNL-AGG-ICP-AES \\
\hline $7782-49-2$ & Selenium & $<1.96 \mathrm{E} 0$ & $\mathrm{ug} / \mathrm{g}$ dry & $1.96 \mathrm{E} 0$ & $9 / 16 / 08$ & $8 \mathrm{II} 15003$ & PNNL-AGG-ICP-AES \\
\hline $7440-24-6$ & Strontium & $<5.23 \mathrm{E}-2$ & $\mathrm{ug} / \mathrm{g}$ dry & $5.23 \mathrm{E}-2$ & $9 / 16 / 08$ & $8 \mathrm{II} 15003$ & PNNL-AGG-ICP-AES \\
\hline $7440-28-0$ & Thallium & $<1.03 \mathrm{E} 0$ & ug/g dry & $1.03 \mathrm{E} 0$ & $9 / 16 / 08$ & $8 \mathrm{I} 15003$ & PNNL-AGG-ICP-AES \\
\hline $7440-62-2$ & Vanadium & $<4.54 \mathrm{E}-2$ & $\mathrm{ug} / \mathrm{g}$ dry & $4.54 \mathrm{E}-2$ & $9 / 16 / 08$ & $8 \mathrm{II} 15003$ & PNNL-AGG-ICP-AES \\
\hline $7440-66-6$ & Zinc & $<9.30 \mathrm{E}-2$ & $\mathrm{ug} / \mathrm{g}$ dry & $9.30 \mathrm{E}-2$ & $9 / 16 / 08$ & $8 \mathrm{I} 15003$ & PNNL-AGG-ICP-AES \\
\hline $7440-23-5$ & Sodium & $1.15 \mathrm{E} 1$ & ug/g dry & $6.71 \mathrm{E}-1$ & $9 / 16 / 08$ & $8 \mathrm{I} 15003$ & PNNL-AGG-ICP-AES \\
\hline $7704-34-9$ & Sulfur & $9.61 \mathrm{E} 0$ & $\mathrm{ug} / \mathrm{g}$ dry & $3.08 \mathrm{E} 0$ & $9 / 16 / 08$ & $8 \mathrm{II} 15003$ & PNNL-AGG-ICP-AES \\
\hline $7440-32-6$ & Titanium & $<8.78 \mathrm{E}-3$ & $\mathrm{ug} / \mathrm{g}$ dry & $8.78 \mathrm{E}-3$ & $9 / 16 / 08$ & $8 I 15003$ & PNNL-AGG-ICP-AES \\
\hline $7440-67-7$ & Zirconium & $<1.50 \mathrm{E}-1$ & $\mathrm{ug} / \mathrm{g}$ dry & $1.50 \mathrm{E}-1$ & $9 / 16 / 08$ & $8 \mathrm{II} 15003$ & PNNL-AGG-ICP-AES \\
\hline $7440-22-4$ & Silver & $<7.73 \mathrm{E}-2$ & $\mathrm{ug} / \mathrm{g}$ dry & $7.73 \mathrm{E}-2$ & $9 / 16 / 08$ & $8 I 15003$ & PNNL-AGG-ICP-AES \\
\hline $7440-15-5$ & Rhenium & $<1.52 \mathrm{E}-1$ & $\mathrm{ug} / \mathrm{g}$ dry & $1.52 \mathrm{E}-1$ & $9 / 16 / 08$ & $8 \mathrm{II} 15003$ & PNNL-AGG-ICP-AES \\
\hline $7440-36-0$ & Antimony & $<6.35 \mathrm{E}-1$ & $\mathrm{ug} / \mathrm{g}$ dry & $6.35 \mathrm{E}-1$ & $9 / 16 / 08$ & $8 \mathrm{II} 15003$ & PNNL-AGG-ICP-AES \\
\hline HEIS No. & B1V9C1 & \multicolumn{3}{|c|}{ Lab ID: $\quad$ 0805003-21 } & & & \\
\hline $7429-90-5$ & Aluminum & $2.43 \mathrm{E}-1$ & $\mathrm{ug} / \mathrm{g}$ dry & $8.57 \mathrm{E}-2$ & $9 / 16 / 08$ & $8 \mathrm{II} 15003$ & PNNL-AGG-ICP-AES \\
\hline $7440-38-2$ & Arsenic & $<3.60 \mathrm{E}-1$ & ug/g dry & $3.60 \mathrm{E}-1$ & $9 / 16 / 08$ & $8 \mathrm{I} 15003$ & PNNL-AGG-ICP-AES \\
\hline $7440-42-8$ & Boron & $<1.94 \mathrm{E} 0$ & $\mathrm{ug} / \mathrm{g}$ dry & $1.94 \mathrm{E} 0$ & $9 / 16 / 08$ & $8 I 15003$ & PNNL-AGG-ICP-AES \\
\hline $7440-41-7$ & Beryllium & $<2.84 \mathrm{E}-2$ & $\mathrm{ug} / \mathrm{g}$ dry & $2.84 \mathrm{E}-2$ & $9 / 16 / 08$ & $8 \mathrm{I} 15003$ & PNNL-AGG-ICP-AES \\
\hline $7440-69-9$ & Bismuth & $<1.88 \mathrm{E}-1$ & $\mathrm{ug} / \mathrm{g}$ dry & $1.88 \mathrm{E}-1$ & $9 / 16 / 08$ & $8 \mathrm{II} 15003$ & PNNL-AGG-ICP-AES \\
\hline $7440-70-2$ & Calcium & $1.01 \mathrm{E} 1$ & $\mathrm{ug} / \mathrm{g}$ dry & $3.86 \mathrm{E}-1$ & $9 / 16 / 08$ & $8 \mathrm{II} 15003$ & PNNL-AGG-ICP-AES \\
\hline $7440-43-9$ & Cadmium & $<2.68 \mathrm{E}-2$ & $\mathrm{ug} / \mathrm{g}$ dry & $2.68 \mathrm{E}-2$ & $9 / 16 / 08$ & $8 \mathrm{II} 15003$ & PNNL-AGG-ICP-AES \\
\hline $7440-48-4$ & Cobalt & $<9.59 \mathrm{E}-2$ & ug/g dry & $9.59 \mathrm{E}-2$ & $9 / 16 / 08$ & $8 \mathrm{II} 15003$ & PNNL-AGG-ICP-AES \\
\hline $7440-47-3$ & Chromium & $<3.33 \mathrm{E}-2$ & $\mathrm{ug} / \mathrm{g}$ dry & $3.33 \mathrm{E}-2$ & $9 / 16 / 08$ & $8 I 15003$ & PNNL-AGG-ICP-AES \\
\hline $7440-50-8$ & Copper & $<8.03 \mathrm{E}-2$ & ug/g dry & 8.03E-2 & $9 / 16 / 08$ & $8 \mathrm{II} 15003$ & PNNL-AGG-ICP-AES \\
\hline $7439-89-6$ & Iron & $<1.42 \mathrm{E}-1$ & $\mathrm{ug} / \mathrm{g}$ dry & $1.42 \mathrm{E}-1$ & $9 / 16 / 08$ & $8 \mathrm{II} 15003$ & PNNL-AGG-ICP-AES \\
\hline $7440-09-7$ & Potassium & $5.05 \mathrm{E} 0$ & $\mathrm{ug} / \mathrm{g}$ dry & $2.32 \mathrm{E} 0$ & $9 / 16 / 08$ & $8 \mathrm{I} 15003$ & PNNL-AGG-ICP-AES \\
\hline $7439-93-2$ & Lithium & $<5.39 \mathrm{E}-1$ & ug/g dry & $5.39 \mathrm{E}-1$ & $9 / 16 / 08$ & $8 \mathrm{I} 15003$ & PNNL-AGG-ICP-AES \\
\hline $7439-95-4$ & Magnesium & $3.95 \mathrm{E} 0$ & $\mathrm{ug} / \mathrm{g}$ dry & $8.33 \mathrm{E}-2$ & $9 / 16 / 08$ & $8 \mathrm{II} 15003$ & PNNL-AGG-ICP-AES \\
\hline $7439-96-5$ & Manganese & $<1.71 \mathrm{E}-2$ & ug/g dry & $1.71 \mathrm{E}-2$ & $9 / 16 / 08$ & $8 \mathrm{II} 15003$ & PNNL-AGG-ICP-AES \\
\hline $7439-98-7$ & Molybdenum & $<1.39 \mathrm{E}-1$ & $\mathrm{ug} / \mathrm{g}$ dry & $1.39 \mathrm{E}-1$ & $9 / 16 / 08$ & $8 \mathrm{II} 15003$ & PNNL-AGG-ICP-AES \\
\hline $7440-02-0$ & Nickel & $<9.32 \mathrm{E}-2$ & $\mathrm{ug} / \mathrm{g}$ dry & $9.32 \mathrm{E}-2$ & $9 / 16 / 08$ & $8 \mathrm{I} 15003$ & PNNL-AGG-ICP-AES \\
\hline $7723-14-0$ & Phosphorus & $<1.03 \mathrm{E} 0$ & $\mathrm{ug} / \mathrm{g}$ dry & $1.03 \mathrm{E} 0$ & $9 / 16 / 08$ & $8 \mathrm{II} 15003$ & PNNL-AGG-ICP-AES \\
\hline $7439-92-1$ & Lead & $<4.31 \mathrm{E}-1$ & $\mathrm{ug} / \mathrm{g}$ dry & $4.31 \mathrm{E}-1$ & $9 / 16 / 08$ & $8 \mathrm{II} 15003$ & PNNL-AGG-ICP-AES \\
\hline $7782-49-2$ & Selenium & $<1.95 \mathrm{E} 0$ & ug/g dry & $1.95 \mathrm{E} 0$ & $9 / 16 / 08$ & $8 \mathrm{I} 15003$ & PNNL-AGG-ICP-AES \\
\hline $7440-24-6$ & Strontium & $<5.21 \mathrm{E}-2$ & ug/g dry & $5.21 \mathrm{E}-2$ & $9 / 16 / 08$ & $8 \mathrm{I} 15003$ & PNNL-AGG-ICP-AES \\
\hline $7440-28-0$ & Thallium & $<1.02 \mathrm{E} 0$ & $\mathrm{ug} / \mathrm{g}$ dry & $1.02 \mathrm{E} 0$ & $9 / 16 / 08$ & $8 I 15003$ & PNNL-AGG-ICP-AES \\
\hline $7440-62-2$ & Vanadium & $<4.52 \mathrm{E}-2$ & $\mathrm{ug} / \mathrm{g}$ dry & $4.52 \mathrm{E}-2$ & $9 / 16 / 08$ & $8 I 15003$ & PNNL-AGG-ICP-AES \\
\hline $7440-66-6$ & Zinc & $<9.26 \mathrm{E}-2$ & $\mathrm{ug} / \mathrm{g}$ dry & $9.26 \mathrm{E}-2$ & $9 / 16 / 08$ & $8 I 15003$ & PNNL-AGG-ICP-AES \\
\hline $7440-23-5$ & Sodium & $1.14 \mathrm{E} 1$ & ug/g dry & $6.68 \mathrm{E}-1$ & $9 / 16 / 08$ & $8 \mathrm{I} 15003$ & PNNL-AGG-ICP-AES \\
\hline 7704-34-9 & Sulfur & $1.20 \mathrm{E} 1$ & $\mathrm{ug} / \mathrm{g}$ dry & $3.07 \mathrm{E} 0$ & $9 / 16 / 08$ & $8 I 15003$ & PNNL-AGG-ICP-AES \\
\hline $7440-32-6$ & Titanium & $<8.75 \mathrm{E}-3$ & $\mathrm{ug} / \mathrm{g}$ dry & $8.75 \mathrm{E}-3$ & $9 / 16 / 08$ & $8 \mathrm{II} 15003$ & PNNL-AGG-ICP-AES \\
\hline
\end{tabular}


Total Metals by PNNL-AGG-ICP-AES/Water Extract

\begin{tabular}{|c|c|c|c|c|c|c|c|}
\hline CAS \# & Analyte & Results & Units & EQL & Analyzed & Batch & Method \\
\hline HEIS No. & B1V9C1 & \multicolumn{2}{|c|}{ Lab ID: } & $5003-21$ & & & \\
\hline $7440-67-7$ & Zirconium & $<1.50 \mathrm{E}-1$ & ug/g dry & $1.50 \mathrm{E}-1$ & $9 / 16 / 08$ & $8 \mathrm{I} 15003$ & PNNL-AGG-ICP-AES \\
\hline $7440-22-4$ & Silver & $<7.70 \mathrm{E}-2$ & ug/g dry & $7.70 \mathrm{E}-2$ & $9 / 16 / 08$ & $8 \mathrm{I} 15003$ & PNNL-AGG-ICP-AES \\
\hline $7440-15-5$ & Rhenium & $<1.52 \mathrm{E}-1$ & ug/g dry & $1.52 \mathrm{E}-1$ & $9 / 16 / 08$ & $8 \mathrm{I} 15003$ & PNNL-AGG-ICP-AES \\
\hline $7440-36-0$ & Antimony & $<6.32 \mathrm{E}-1$ & ug/g dry & $6.32 \mathrm{E}-1$ & $9 / 16 / 08$ & $8 \mathrm{I} 15003$ & PNNL-AGG-ICP-AES \\
\hline HEIS No. & B1V9C2 & \multicolumn{3}{|c|}{ Lab ID: $\quad$ 0805003-22 } & & & \\
\hline $7429-90-5$ & Aluminum & $2.48 \mathrm{E}-1$ & ug/g dry & $8.57 \mathrm{E}-2$ & $9 / 16 / 08$ & $8 \mathrm{I} 15003$ & PNNL-AGG-ICP-AES \\
\hline $7440-38-2$ & Arsenic & $<3.60 \mathrm{E}-1$ & ug/g dry & $3.60 \mathrm{E}-1$ & $9 / 16 / 08$ & $8 \mathrm{I} 15003$ & PNNL-AGG-ICP-AES \\
\hline $7440-42-8$ & Boron & $<1.94 \mathrm{E} 0$ & ug/g dry & $1.94 \mathrm{E} 0$ & $9 / 16 / 08$ & $8 \mathrm{I} 15003$ & PNNL-AGG-ICP-AES \\
\hline $7440-41-7$ & Beryllium & $<2.84 \mathrm{E}-2$ & ug/g dry & $2.84 \mathrm{E}-2$ & $9 / 16 / 08$ & $8 \mathrm{I} 15003$ & PNNL-AGG-ICP-AES \\
\hline $7440-69-9$ & Bismuth & $<1.88 \mathrm{E}-1$ & ug/g dry & $1.88 \mathrm{E}-1$ & $9 / 16 / 08$ & $8 \mathrm{I} 15003$ & PNNL-AGG-ICP-AES \\
\hline $7440-70-2$ & Calcium & $1.13 \mathrm{E} 1$ & ug/g dry & $3.87 \mathrm{E}-1$ & $9 / 16 / 08$ & $8 \mathrm{I} 15003$ & PNNL-AGG-ICP-AES \\
\hline $7440-43-9$ & Cadmium & $<2.68 \mathrm{E}-2$ & ug/g dry & $2.68 \mathrm{E}-2$ & $9 / 16 / 08$ & $8 \mathrm{I} 15003$ & PNNL-AGG-ICP-AES \\
\hline $7440-48-4$ & Cobalt & $<9.59 \mathrm{E}-2$ & ug/g dry & $9.59 \mathrm{E}-2$ & $9 / 16 / 08$ & $8 \mathrm{I} 15003$ & PNNL-AGG-ICP-AES \\
\hline $7440-47-3$ & Chromium & $<3.33 \mathrm{E}-2$ & ug/g dry & $3.33 \mathrm{E}-2$ & $9 / 16 / 08$ & $8 \mathrm{I} 15003$ & PNNL-AGG-ICP-AES \\
\hline $7440-50-8$ & Copper & $<8.03 \mathrm{E}-2$ & ug/g dry & $8.03 \mathrm{E}-2$ & $9 / 16 / 08$ & $8 \mathrm{I} 15003$ & PNNL-AGG-ICP-AES \\
\hline $7439-89-6$ & Iron & $<1.42 \mathrm{E}-1$ & ug/g dry & $1.42 \mathrm{E}-1$ & $9 / 16 / 08$ & $8 \mathrm{I} 15003$ & PNNL-AGG-ICP-AES \\
\hline $7440-09-7$ & Potassium & $4.88 \mathrm{E} 0$ & ug/g dry & $2.33 \mathrm{E} 0$ & $9 / 16 / 08$ & $8 \mathrm{I} 15003$ & PNNL-AGG-ICP-AES \\
\hline $7439-93-2$ & Lithium & $<5.39 \mathrm{E}-1$ & ug/g dry & $5.39 \mathrm{E}-1$ & $9 / 16 / 08$ & $8 \mathrm{I} 15003$ & PNNL-AGG-ICP-AES \\
\hline $7439-95-4$ & Magnesium & 4.04E0 & ug/g dry & $8.33 \mathrm{E}-2$ & $9 / 16 / 08$ & $8 \mathrm{I} 15003$ & PNNL-AGG-ICP-AES \\
\hline $7439-96-5$ & Manganese & $<1.71 \mathrm{E}-2$ & ug/g dry & $1.71 \mathrm{E}-2$ & $9 / 16 / 08$ & $8 \mathrm{I} 15003$ & PNNL-AGG-ICP-AES \\
\hline 7439-98-7 & Molybdenum & $<1.39 \mathrm{E}-1$ & ug/g dry & $1.39 \mathrm{E}-1$ & $9 / 16 / 08$ & $8 \mathrm{I} 15003$ & PNNL-AGG-ICP-AES \\
\hline $7440-02-0$ & Nickel & $<9.32 \mathrm{E}-2$ & ug/g dry & $9.32 \mathrm{E}-2$ & $9 / 16 / 08$ & $8 \mathrm{I} 15003$ & PNNL-AGG-ICP-AES \\
\hline $7723-14-0$ & Phosphorus & $<1.03 \mathrm{E} 0$ & ug/g dry & $1.03 \mathrm{E} 0$ & $9 / 16 / 08$ & $8 \mathrm{I} 15003$ & PNNL-AGG-ICP-AES \\
\hline $7439-92-1$ & Lead & $<4.31 \mathrm{E}-1$ & ug/g dry & $4.31 \mathrm{E}-1$ & $9 / 16 / 08$ & $8 \mathrm{I} 15003$ & PNNL-AGG-ICP-AES \\
\hline $7782-49-2$ & Selenium & $<1.95 \mathrm{E} 0$ & ug/g dry & $1.95 \mathrm{E} 0$ & $9 / 16 / 08$ & $8 \mathrm{I} 15003$ & PNNL-AGG-ICP-AES \\
\hline $7440-24-6$ & Strontium & $5.28 \mathrm{E}-2$ & ug/g dry & $5.21 \mathrm{E}-2$ & $9 / 16 / 08$ & $8 \mathrm{I} 15003$ & PNNL-AGG-ICP-AES \\
\hline $7440-28-0$ & Thallium & $<1.02 \mathrm{E} 0$ & ug/g dry & $1.02 \mathrm{E} 0$ & $9 / 16 / 08$ & $8 \mathrm{I} 15003$ & PNNL-AGG-ICP-AES \\
\hline $7440-62-2$ & Vanadium & $<4.52 \mathrm{E}-2$ & ug/g dry & $4.52 \mathrm{E}-2$ & $9 / 16 / 08$ & $8 \mathrm{I} 15003$ & PNNL-AGG-ICP-AES \\
\hline $7440-66-6$ & Zinc & $<9.26 \mathrm{E}-2$ & ug/g dry & $9.26 \mathrm{E}-2$ & $9 / 16 / 08$ & $8 \mathrm{I} 15003$ & PNNL-AGG-ICP-AES \\
\hline $7440-23-5$ & Sodium & $1.16 \mathrm{E} 1$ & ug/g dry & $6.68 \mathrm{E}-1$ & $9 / 16 / 08$ & $8 \mathrm{I} 15003$ & PNNL-AGG-ICP-AES \\
\hline $7704-34-9$ & Sulfur & 1.19E1 & ug/g dry & 3.07E0 & $9 / 16 / 08$ & $8 \mathrm{I} 15003$ & PNNL-AGG-ICP-AES \\
\hline $7440-32-6$ & Titanium & $<8.75 \mathrm{E}-3$ & ug/g dry & $8.75 \mathrm{E}-3$ & $9 / 16 / 08$ & $8 \mathrm{I} 15003$ & PNNL-AGG-ICP-AES \\
\hline $7440-67-7$ & Zirconium & $<1.50 \mathrm{E}-1$ & ug/g dry & $1.50 \mathrm{E}-1$ & $9 / 16 / 08$ & $8 \mathrm{I} 15003$ & PNNL-AGG-ICP-AES \\
\hline $7440-22-4$ & Silver & $<7.70 \mathrm{E}-2$ & ug/g dry & $7.70 \mathrm{E}-2$ & $9 / 16 / 08$ & $8 \mathrm{I} 15003$ & PNNL-AGG-ICP-AES \\
\hline $7440-15-5$ & Rhenium & $<1.52 \mathrm{E}-1$ & ug/g dry & $1.52 \mathrm{E}-1$ & $9 / 16 / 08$ & $8 \mathrm{I} 15003$ & PNNL-AGG-ICP-AES \\
\hline $7440-36-0$ & Antimony & $<6.32 \mathrm{E}-1$ & ug/g dry & $6.32 \mathrm{E}-1$ & 9/16/08 & $8 \mathrm{I} 15003$ & PNNL-AGG-ICP-AES \\
\hline HEIS No. & B1V9C3 & & b ID: & $5003-23$ & & & \\
\hline $7429-90-5$ & Aluminum & $1.64 \mathrm{E}-1$ & ug/g dry & $8.55 \mathrm{E}-2$ & $9 / 16 / 08$ & $8 \mathrm{I} 15003$ & PNNL-AGG-ICP-AES \\
\hline $7440-38-2$ & Arsenic & $<3.59 \mathrm{E}-1$ & ug/g dry & $3.59 \mathrm{E}-1$ & $9 / 16 / 08$ & $8 \mathrm{I} 15003$ & PNNL-AGG-ICP-AES \\
\hline $7440-42-8$ & Boron & $<1.93 \mathrm{E} 0$ & ug/g dry & $1.93 \mathrm{E} 0$ & $9 / 16 / 08$ & $8 \mathrm{I} 15003$ & PNNL-AGG-ICP-AES \\
\hline $7440-41-7$ & Beryllium & $<2.83 \mathrm{E}-2$ & ug/g dry & $2.83 \mathrm{E}-2$ & $9 / 16 / 08$ & $8 \mathrm{I} 15003$ & PNNL-AGG-ICP-AES \\
\hline $7440-69-9$ & Bismuth & $<1.87 \mathrm{E}-1$ & ug/g dry & $1.87 \mathrm{E}-1$ & $9 / 16 / 08$ & $8 \mathrm{I} 15003$ & PNNL-AGG-ICP-AES \\
\hline $7440-70-2$ & Calcium & 7.84E0 & ug/g dry & $3.86 \mathrm{E}-1$ & $9 / 16 / 08$ & $8 \mathrm{I} 15003$ & PNNL-AGG-ICP-AES \\
\hline $7440-43-9$ & Cadmium & $<2.68 \mathrm{E}-2$ & ug/g dry & $2.68 \mathrm{E}-2$ & $9 / 16 / 08$ & $8 \mathrm{I} 15003$ & PNNL-AGG-ICP-AES \\
\hline $7440-48-4$ & Cobalt & $<9.56 \mathrm{E}-2$ & ug/g dry & $9.56 \mathrm{E}-2$ & $9 / 16 / 08$ & $8 \mathrm{I} 15003$ & PNNL-AGG-ICP-AES \\
\hline $7440-47-3$ & Chromium & $<3.32 \mathrm{E}-2$ & ug/g dry & $3.32 \mathrm{E}-2$ & $9 / 16 / 08$ & $8 \mathrm{I} 15003$ & PNNL-AGG-ICP-AES \\
\hline $7440-50-8$ & Copper & $<8.01 \mathrm{E}-2$ & ug/g dry & $8.01 \mathrm{E}-2$ & $9 / 16 / 08$ & $8 \mathrm{I} 15003$ & PNNL-AGG-ICP-AES \\
\hline $7439-89-6$ & Iron & $<1.42 \mathrm{E}-1$ & ug/g dry & $1.42 \mathrm{E}-1$ & $9 / 16 / 08$ & $8 \mathrm{I} 15003$ & PNNL-AGG-ICP-AES \\
\hline
\end{tabular}


Total Metals by PNNL-AGG-ICP-AES/Water Extract

\begin{tabular}{|c|c|c|c|c|c|c|c|}
\hline CAS \# & Analyte & Results & Units & EQL & Analyzed & Batch & Method \\
\hline HEIS No. & B1V9C3 & & b ID: & $55003-23$ & & & \\
\hline 7440-09-7 & Potassium & $4.01 \mathrm{E} 0$ & $\mathrm{ug} / \mathrm{g}$ dry & $2.32 \mathrm{E} 0$ & $9 / 16 / 08$ & $8 \mathrm{I} 15003$ & PNNL-AGG-ICP-AES \\
\hline $7439-93-2$ & Lithium & $<5.38 \mathrm{E}-1$ & ug/g dry & $5.38 \mathrm{E}-1$ & $9 / 16 / 08$ & $8 \mathrm{I} 15003$ & PNNL-AGG-ICP-AES \\
\hline $7439-95-4$ & Magnesium & $2.59 \mathrm{E} 0$ & ug/g dry & $8.31 \mathrm{E}-2$ & $9 / 16 / 08$ & $8 \mathrm{I} 15003$ & PNNL-AGG-ICP-AES \\
\hline $7439-96-5$ & Manganese & $<1.71 \mathrm{E}-2$ & ug/g dry & $1.71 \mathrm{E}-2$ & $9 / 16 / 08$ & $8 \mathrm{I} 15003$ & PNNL-AGG-ICP-AES \\
\hline $7439-98-7$ & Molybdenum & $<1.39 \mathrm{E}-1$ & ug/g dry & $1.39 \mathrm{E}-1$ & $9 / 16 / 08$ & $8 \mathrm{I} 15003$ & PNNL-AGG-ICP-AES \\
\hline $7440-02-0$ & Nickel & $<9.29 \mathrm{E}-2$ & ug/g dry & $9.29 \mathrm{E}-2$ & $9 / 16 / 08$ & $8 \mathrm{I} 15003$ & PNNL-AGG-ICP-AES \\
\hline $7723-14-0$ & Phosphorus & $<1.03 \mathrm{E} 0$ & ug/g dry & $1.03 \mathrm{E} 0$ & $9 / 16 / 08$ & $8 \mathrm{I} 15003$ & PNNL-AGG-ICP-AES \\
\hline $7439-92-1$ & Lead & $<4.30 \mathrm{E}-1$ & ug/g dry & $4.30 \mathrm{E}-1$ & $9 / 16 / 08$ & $8 \mathrm{I} 15003$ & PNNL-AGG-ICP-AES \\
\hline $7782-49-2$ & Selenium & $<1.94 \mathrm{E} 0$ & ug/g dry & $1.94 \mathrm{E} 0$ & $9 / 16 / 08$ & $8 \mathrm{I} 15003$ & PNNL-AGG-ICP-AES \\
\hline $7440-24-6$ & Strontium & $<5.20 \mathrm{E}-2$ & $\mathrm{ug} / \mathrm{g}$ dry & $5.20 \mathrm{E}-2$ & $9 / 16 / 08$ & $8 \mathrm{II} 15003$ & PNNL-AGG-ICP-AES \\
\hline $7440-28-0$ & Thallium & $<1.02 \mathrm{E} 0$ & ug/g dry & $1.02 \mathrm{E} 0$ & $9 / 16 / 08$ & $8 \mathrm{I} 15003$ & PNNL-AGG-ICP-AES \\
\hline $7440-62-2$ & Vanadium & $<4.51 \mathrm{E}-2$ & ug/g dry & $4.51 \mathrm{E}-2$ & $9 / 16 / 08$ & $8 \mathrm{I} 15003$ & PNNL-AGG-ICP-AES \\
\hline $7440-66-6$ & Zinc & $<9.23 \mathrm{E}-2$ & ug/g dry & $9.23 \mathrm{E}-2$ & $9 / 16 / 08$ & $8 \mathrm{I} 15003$ & PNNL-AGG-ICP-AES \\
\hline $7440-23-5$ & Sodium & $1.04 \mathrm{E} 1$ & ug/g dry & $6.66 \mathrm{E}-1$ & $9 / 16 / 08$ & $8 \mathrm{I} 15003$ & PNNL-AGG-ICP-AES \\
\hline $7704-34-9$ & Sulfur & $6.48 \mathrm{E} 0$ & $\mathrm{ug} / \mathrm{g}$ dry & $3.06 \mathrm{E} 0$ & $9 / 16 / 08$ & $8 \mathrm{II} 15003$ & PNNL-AGG-ICP-AES \\
\hline $7440-32-6$ & Titanium & $<8.73 \mathrm{E}-3$ & ug/g dry & $8.73 \mathrm{E}-3$ & $9 / 16 / 08$ & $8 \mathrm{I} 15003$ & PNNL-AGG-ICP-AES \\
\hline $7440-67-7$ & Zirconium & $<1.49 \mathrm{E}-1$ & ug/g dry & $1.49 \mathrm{E}-1$ & $9 / 16 / 08$ & $8 \mathrm{I} 15003$ & PNNL-AGG-ICP-AES \\
\hline $7440-22-4$ & Silver & $<7.68 \mathrm{E}-2$ & $\mathrm{ug} / \mathrm{g}$ dry & $7.68 \mathrm{E}-2$ & $9 / 16 / 08$ & $8 \mathrm{I} 15003$ & PNNL-AGG-ICP-AES \\
\hline $7440-15-5$ & Rhenium & $<1.51 \mathrm{E}-1$ & ug/g dry & $1.51 \mathrm{E}-1$ & $9 / 16 / 08$ & $8 \mathrm{I} 15003$ & PNNL-AGG-ICP-AES \\
\hline $7440-36-0$ & Antimony & $<6.31 \mathrm{E}-1$ & ug/g dry & $6.31 \mathrm{E}-1$ & $9 / 16 / 08$ & $8 \mathrm{II} 15003$ & PNNL-AGG-ICP-AES \\
\hline HEIS No. & B1V9C4 & & b ID: & 5003-24 & & & \\
\hline $7429-90-5$ & Aluminum & $1.81 \mathrm{E}-1$ & ug/g dry & $8.59 \mathrm{E}-2$ & $9 / 16 / 08$ & $8 \mathrm{I} 15003$ & PNNL-AGG-ICP-AES \\
\hline $7440-38-2$ & Arsenic & $<3.60 \mathrm{E}-1$ & ug/g dry & $3.60 \mathrm{E}-1$ & $9 / 16 / 08$ & $8 \mathrm{I} 15003$ & PNNL-AGG-ICP-AES \\
\hline $7440-42-8$ & Boron & $<1.94 \mathrm{E} 0$ & $\mathrm{ug} / \mathrm{g}$ dry & $1.94 \mathrm{E} 0$ & $9 / 16 / 08$ & $8 \mathrm{I} 15003$ & PNNL-AGG-ICP-AES \\
\hline $7440-41-7$ & Beryllium & $<2.84 \mathrm{E}-2$ & ug/g dry & $2.84 \mathrm{E}-2$ & $9 / 16 / 08$ & $8 \mathrm{I} 15003$ & PNNL-AGG-ICP-AES \\
\hline $7440-69-9$ & Bismuth & $<1.88 \mathrm{E}-1$ & $\mathrm{ug} / \mathrm{g}$ dry & $1.88 \mathrm{E}-1$ & $9 / 16 / 08$ & $8 \mathrm{I} 15003$ & PNNL-AGG-ICP-AES \\
\hline $7440-70-2$ & Calcium & $8.28 \mathrm{E} 0$ & ug/g dry & $3.87 \mathrm{E}-1$ & $9 / 16 / 08$ & $8 \mathrm{I} 15003$ & PNNL-AGG-ICP-AES \\
\hline $7440-43-9$ & Cadmium & $<2.69 \mathrm{E}-2$ & ug/g dry & $2.69 \mathrm{E}-2$ & $9 / 16 / 08$ & $8 \mathrm{I} 15003$ & PNNL-AGG-ICP-AES \\
\hline $7440-48-4$ & Cobalt & $<9.61 \mathrm{E}-2$ & ug/g dry & $9.61 \mathrm{E}-2$ & $9 / 16 / 08$ & $8 \mathrm{I} 15003$ & PNNL-AGG-ICP-AES \\
\hline $7440-47-3$ & Chromium & $<3.33 \mathrm{E}-2$ & ug/g dry & 3.33E-2 & $9 / 16 / 08$ & $8 \mathrm{I} 15003$ & PNNL-AGG-ICP-AES \\
\hline $7440-50-8$ & Copper & $<8.05 \mathrm{E}-2$ & ug/g dry & $8.05 \mathrm{E}-2$ & $9 / 16 / 08$ & $8 \mathrm{I} 15003$ & PNNL-AGG-ICP-AES \\
\hline $7439-89-6$ & Iron & $<1.42 \mathrm{E}-1$ & ug/g dry & $1.42 \mathrm{E}-1$ & $9 / 16 / 08$ & $8 \mathrm{I} 15003$ & PNNL-AGG-ICP-AES \\
\hline $7440-09-7$ & Potassium & $4.45 \mathrm{E} 0$ & $\mathrm{ug} / \mathrm{g}$ dry & $2.33 \mathrm{E} 0$ & $9 / 16 / 08$ & $8 \mathrm{I} 15003$ & PNNL-AGG-ICP-AES \\
\hline $7439-93-2$ & Lithium & $<5.41 \mathrm{E}-1$ & ug/g dry & $5.41 \mathrm{E}-1$ & $9 / 16 / 08$ & $8 \mathrm{I} 15003$ & PNNL-AGG-ICP-AES \\
\hline $7439-95-4$ & Magnesium & $2.70 \mathrm{E} 0$ & ug/g dry & $8.35 \mathrm{E}-2$ & $9 / 16 / 08$ & $8 \mathrm{I} 15003$ & PNNL-AGG-ICP-AES \\
\hline $7439-96-5$ & Manganese & $<1.71 \mathrm{E}-2$ & ug/g dry & $1.71 \mathrm{E}-2$ & $9 / 16 / 08$ & $8 \mathrm{I} 15003$ & PNNL-AGG-ICP-AES \\
\hline $7439-98-7$ & Molybdenum & $<1.39 \mathrm{E}-1$ & ug/g dry & $1.39 \mathrm{E}-1$ & $9 / 16 / 08$ & $8 \mathrm{I} 15003$ & PNNL-AGG-ICP-AES \\
\hline $7440-02-0$ & Nickel & $<9.34 \mathrm{E}-2$ & $\mathrm{ug} / \mathrm{g}$ dry & $9.34 \mathrm{E}-2$ & $9 / 16 / 08$ & $8 \mathrm{I} 15003$ & PNNL-AGG-ICP-AES \\
\hline $7723-14-0$ & Phosphorus & $<1.03 \mathrm{E} 0$ & $\mathrm{ug} / \mathrm{g}$ dry & $1.03 \mathrm{E} 0$ & $9 / 16 / 08$ & $8 \mathrm{I} 15003$ & PNNL-AGG-ICP-AES \\
\hline $7439-92-1$ & Lead & $<4.32 \mathrm{E}-1$ & ug/g dry & $4.32 \mathrm{E}-1$ & $9 / 16 / 08$ & $8 \mathrm{I} 15003$ & PNNL-AGG-ICP-AES \\
\hline $7782-49-2$ & Selenium & $<1.95 \mathrm{E} 0$ & $\mathrm{ug} / \mathrm{g}$ dry & $1.95 \mathrm{E} 0$ & $9 / 16 / 08$ & $8 \mathrm{I} 15003$ & PNNL-AGG-ICP-AES \\
\hline $7440-24-6$ & Strontium & $<5.23 \mathrm{E}-2$ & ug/g dry & $5.23 \mathrm{E}-2$ & $9 / 16 / 08$ & $8 \mathrm{I} 15003$ & PNNL-AGG-ICP-AES \\
\hline $7440-28-0$ & Thallium & $<1.03 \mathrm{E} 0$ & ug/g dry & $1.03 \mathrm{E} 0$ & $9 / 16 / 08$ & $8 \mathrm{I} 15003$ & PNNL-AGG-ICP-AES \\
\hline $7440-62-2$ & Vanadium & $<4.53 \mathrm{E}-2$ & ug/g dry & $4.53 \mathrm{E}-2$ & $9 / 16 / 08$ & $8 \mathrm{I} 15003$ & PNNL-AGG-ICP-AES \\
\hline $7440-66-6$ & Zinc & $<9.28 \mathrm{E}-2$ & ug/g dry & $9.28 \mathrm{E}-2$ & $9 / 16 / 08$ & $8 \mathrm{I} 15003$ & PNNL-AGG-ICP-AES \\
\hline $7440-23-5$ & Sodium & $1.10 \mathrm{E} 1$ & ug/g dry & $6.70 \mathrm{E}-1$ & $9 / 16 / 08$ & $8 \mathrm{I} 15003$ & PNNL-AGG-ICP-AES \\
\hline $7704-34-9$ & Sulfur & $6.26 \mathrm{E} 0$ & ug/g dry & $3.08 \mathrm{E} 0$ & $9 / 16 / 08$ & $8 \mathrm{I} 15003$ & PNNL-AGG-ICP-AES \\
\hline $7440-32-6$ & Titanium & $<8.77 \mathrm{E}-3$ & ug/g dry & $8.77 \mathrm{E}-3$ & $9 / 16 / 08$ & $8 \mathrm{I} 15003$ & PNNL-AGG-ICP-AES \\
\hline
\end{tabular}


Total Metals by PNNL-AGG-ICP-AES/Water Extract

\begin{tabular}{|c|c|c|c|c|c|c|c|}
\hline CAS \# & Analyte & Results & Units & EQL & Analyzed & Batch & Method \\
\hline HEIS No. & B1V9C4 & \multicolumn{3}{|c|}{ Lab ID: $\quad$ 0805003-24 } & & & \\
\hline $7440-67-7$ & Zirconium & $<1.50 \mathrm{E}-1$ & ug/g dry & $1.50 \mathrm{E}-1$ & $9 / 16 / 08$ & $8 \mathrm{I} 15003$ & PNNL-AGG-ICP-AES \\
\hline $7440-22-4$ & Silver & $<7.72 \mathrm{E}-2$ & ug/g dry & $7.72 \mathrm{E}-2$ & $9 / 16 / 08$ & $8 \mathrm{I} 15003$ & PNNL-AGG-ICP-AES \\
\hline $7440-15-5$ & Rhenium & $<1.52 \mathrm{E}-1$ & ug/g dry & $1.52 \mathrm{E}-1$ & $9 / 16 / 08$ & $8 \mathrm{I} 15003$ & PNNL-AGG-ICP-AES \\
\hline $7440-36-0$ & Antimony & $<6.34 \mathrm{E}-1$ & ug/g dry & $6.34 \mathrm{E}-1$ & $9 / 16 / 08$ & $8 \mathrm{I} 15003$ & PNNL-AGG-ICP-AES \\
\hline HEIS No. & B1V2V5 & \multicolumn{3}{|c|}{ Lab ID: $\quad$ 0805003-25 } & & & \\
\hline $7429-90-5$ & Aluminum & $1.76 \mathrm{E}-1$ & ug/g dry & $8.55 \mathrm{E}-2$ & $9 / 16 / 08$ & $8 \mathrm{I} 15003$ & PNNL-AGG-ICP-AES \\
\hline $7440-38-2$ & Arsenic & $<3.59 \mathrm{E}-1$ & ug/g dry & $3.59 \mathrm{E}-1$ & $9 / 16 / 08$ & $8 \mathrm{I} 15003$ & PNNL-AGG-ICP-AES \\
\hline $7440-42-8$ & Boron & $<1.93 \mathrm{E} 0$ & ug/g dry & $1.93 \mathrm{E} 0$ & $9 / 16 / 08$ & $8 \mathrm{I} 15003$ & PNNL-AGG-ICP-AES \\
\hline $7440-41-7$ & Beryllium & $<2.83 \mathrm{E}-2$ & ug/g dry & $2.83 \mathrm{E}-2$ & $9 / 16 / 08$ & $8 \mathrm{I} 15003$ & PNNL-AGG-ICP-AES \\
\hline $7440-69-9$ & Bismuth & $<1.87 \mathrm{E}-1$ & ug/g dry & $1.87 \mathrm{E}-1$ & $9 / 16 / 08$ & $8 \mathrm{I} 15003$ & PNNL-AGG-ICP-AES \\
\hline $7440-70-2$ & Calcium & $5.90 \mathrm{E} 0$ & ug/g dry & $3.86 \mathrm{E}-1$ & $9 / 16 / 08$ & $8 \mathrm{I} 15003$ & PNNL-AGG-ICP-AES \\
\hline $7440-43-9$ & Cadmium & $<2.68 \mathrm{E}-2$ & ug/g dry & $2.68 \mathrm{E}-2$ & $9 / 16 / 08$ & $8 \mathrm{I} 15003$ & PNNL-AGG-ICP-AES \\
\hline $7440-48-4$ & Cobalt & $<9.57 \mathrm{E}-2$ & ug/g dry & $9.57 \mathrm{E}-2$ & $9 / 16 / 08$ & $8 \mathrm{I} 15003$ & PNNL-AGG-ICP-AES \\
\hline $7440-47-3$ & Chromium & $<3.32 \mathrm{E}-2$ & ug/g dry & $3.32 \mathrm{E}-2$ & $9 / 16 / 08$ & $8 \mathrm{I} 15003$ & PNNL-AGG-ICP-AES \\
\hline $7440-50-8$ & Copper & $<8.01 \mathrm{E}-2$ & ug/g dry & $8.01 \mathrm{E}-2$ & $9 / 16 / 08$ & $8 \mathrm{I} 15003$ & PNNL-AGG-ICP-AES \\
\hline $7439-89-6$ & Iron & $<1.42 \mathrm{E}-1$ & ug/g dry & $1.42 \mathrm{E}-1$ & $9 / 16 / 08$ & $8 \mathrm{I} 15003$ & PNNL-AGG-ICP-AES \\
\hline $7440-09-7$ & Potassium & $3.36 \mathrm{E} 0$ & ug/g dry & $2.32 \mathrm{E} 0$ & $9 / 16 / 08$ & $8 \mathrm{I} 15003$ & PNNL-AGG-ICP-AES \\
\hline $7439-93-2$ & Lithium & $<5.38 \mathrm{E}-1$ & ug/g dry & $5.38 \mathrm{E}-1$ & $9 / 16 / 08$ & $8 \mathrm{I} 15003$ & PNNL-AGG-ICP-AES \\
\hline $7439-95-4$ & Magnesium & $1.89 \mathrm{E} 0$ & ug/g dry & $8.31 \mathrm{E}-2$ & $9 / 16 / 08$ & $8 \mathrm{I} 15003$ & PNNL-AGG-ICP-AES \\
\hline $7439-96-5$ & Manganese & $<1.71 \mathrm{E}-2$ & ug/g dry & $1.71 \mathrm{E}-2$ & $9 / 16 / 08$ & $8 \mathrm{I} 15003$ & PNNL-AGG-ICP-AES \\
\hline 7439-98-7 & Molybdenum & $<1.39 \mathrm{E}-1$ & ug/g dry & $1.39 \mathrm{E}-1$ & $9 / 16 / 08$ & $8 \mathrm{I} 15003$ & PNNL-AGG-ICP-AES \\
\hline $7440-02-0$ & Nickel & $<9.30 \mathrm{E}-2$ & ug/g dry & $9.30 \mathrm{E}-2$ & $9 / 16 / 08$ & $8 \mathrm{I} 15003$ & PNNL-AGG-ICP-AES \\
\hline $7723-14-0$ & Phosphorus & $<1.03 \mathrm{E} 0$ & ug/g dry & $1.03 \mathrm{E} 0$ & $9 / 16 / 08$ & $8 \mathrm{I} 15003$ & PNNL-AGG-ICP-AES \\
\hline $7439-92-1$ & Lead & $<4.30 \mathrm{E}-1$ & ug/g dry & $4.30 \mathrm{E}-1$ & $9 / 16 / 08$ & $8 \mathrm{I} 15003$ & PNNL-AGG-ICP-AES \\
\hline $7782-49-2$ & Selenium & $<1.94 \mathrm{E} 0$ & ug/g dry & $1.94 \mathrm{E} 0$ & $9 / 16 / 08$ & $8 \mathrm{I} 15003$ & PNNL-AGG-ICP-AES \\
\hline $7440-24-6$ & Strontium & $<5.20 \mathrm{E}-2$ & ug/g dry & $5.20 \mathrm{E}-2$ & $9 / 16 / 08$ & $8 \mathrm{I} 15003$ & PNNL-AGG-ICP-AES \\
\hline $7440-28-0$ & Thallium & $<1.02 \mathrm{E} 0$ & ug/g dry & $1.02 \mathrm{E} 0$ & $9 / 16 / 08$ & $8 \mathrm{I} 15003$ & PNNL-AGG-ICP-AES \\
\hline $7440-62-2$ & Vanadium & $<4.51 \mathrm{E}-2$ & ug/g dry & $4.51 \mathrm{E}-2$ & $9 / 16 / 08$ & $8 \mathrm{I} 15003$ & PNNL-AGG-ICP-AES \\
\hline $7440-66-6$ & Zinc & $<9.24 \mathrm{E}-2$ & ug/g dry & $9.24 \mathrm{E}-2$ & $9 / 16 / 08$ & $8 \mathrm{I} 15003$ & PNNL-AGG-ICP-AES \\
\hline $7440-23-5$ & Sodium & 7.41E0 & ug/g dry & $6.67 \mathrm{E}-1$ & $9 / 16 / 08$ & $8 \mathrm{I} 15003$ & PNNL-AGG-ICP-AES \\
\hline $7704-34-9$ & Sulfur & $3.16 \mathrm{E} 0$ & ug/g dry & $3.06 \mathrm{E} 0$ & $9 / 16 / 08$ & $8 \mathrm{I} 15003$ & PNNL-AGG-ICP-AES \\
\hline $7440-32-6$ & Titanium & $<8.73 \mathrm{E}-3$ & ug/g dry & $8.73 \mathrm{E}-3$ & $9 / 16 / 08$ & $8 \mathrm{I} 15003$ & PNNL-AGG-ICP-AES \\
\hline $7440-67-7$ & Zirconium & $<1.49 \mathrm{E}-1$ & ug/g dry & $1.49 \mathrm{E}-1$ & $9 / 16 / 08$ & $8 \mathrm{I} 15003$ & PNNL-AGG-ICP-AES \\
\hline $7440-22-4$ & Silver & $<7.68 \mathrm{E}-2$ & ug/g dry & $7.68 \mathrm{E}-2$ & $9 / 16 / 08$ & $8 \mathrm{I} 15003$ & PNNL-AGG-ICP-AES \\
\hline $7440-15-5$ & Rhenium & $<1.51 \mathrm{E}-1$ & ug/g dry & $1.51 \mathrm{E}-1$ & $9 / 16 / 08$ & $8 \mathrm{I} 15003$ & PNNL-AGG-ICP-AES \\
\hline $7440-36-0$ & Antimony & $<6.31 \mathrm{E}-1$ & ug/g dry & $6.31 \mathrm{E}-1$ & $9 / 16 / 08$ & $8 \mathrm{I} 15003$ & PNNL-AGG-ICP-AES \\
\hline
\end{tabular}


Total Metals by PNNL-AGG-ICP-AES/Acid Extract

\begin{tabular}{|c|c|c|c|c|c|c|c|}
\hline CAS \# & Analyte & Results & Units & EQL & Analyzed & Batch & Method \\
\hline HEIS No. & B1V993 & \multicolumn{3}{|c|}{ Lab ID: $\quad$ 0805003-03 } & & & \\
\hline $7429-90-5$ & Aluminum & $4.54 \mathrm{E} 3$ & ug/g dry & $2.21 \mathrm{E} 1$ & $3 / 16 / 09$ & 8L12002 & PNNL-AGG-ICP-AES \\
\hline $7440-38-2$ & Arsenic & $<3.31 \mathrm{E} 1$ & ug/g dry & $3.31 \mathrm{E} 1$ & $3 / 16 / 09$ & 8L12002 & PNNL-AGG-ICP-AES \\
\hline $7440-42-8$ & Boron & $<9.47 \mathrm{E} 1$ & ug/g dry & $9.47 \mathrm{E} 1$ & $3 / 16 / 09$ & 8L12002 & PNNL-AGG-ICP-AES \\
\hline $7440-39-3$ & Barium & $4.80 \mathrm{E} 1$ & ug/g dry & $1.35 \mathrm{E} 0$ & $3 / 16 / 09$ & $8 \mathrm{~L} 12002$ & PNNL-AGG-ICP-AES \\
\hline $7440-41-7$ & Beryllium & $<7.55 \mathrm{E}-1$ & $\mathrm{ug} / \mathrm{g}$ dry & $7.55 \mathrm{E}-1$ & $3 / 16 / 09$ & 8L12002 & PNNL-AGG-ICP-AES \\
\hline $7440-69-9$ & Bismuth & $<9.31 \mathrm{E} 0$ & ug/g dry & $9.31 \mathrm{E} 0$ & $3 / 16 / 09$ & 8L12002 & PNNL-AGG-ICP-AES \\
\hline $7440-70-2$ & Calcium & $5.00 \mathrm{E} 3$ & $\mathrm{ug} / \mathrm{g}$ dry & $2.29 \mathrm{E} 1$ & $3 / 16 / 09$ & 8L12002 & PNNL-AGG-ICP-AES \\
\hline $7440-43-9$ & Cadmium & $<1.20 \mathrm{E} 0$ & ug/g dry & $1.20 \mathrm{E} 0$ & $3 / 16 / 09$ & 8L12002 & PNNL-AGG-ICP-AES \\
\hline $7440-48-4$ & Cobalt & $6.73 \mathrm{E} 0$ & $\mathrm{ug} / \mathrm{g}$ dry & 4.72E0 & $3 / 16 / 09$ & 8L12002 & PNNL-AGG-ICP-AES \\
\hline $7440-47-3$ & Chromium & $5.40 \mathrm{E} 0$ & $\mathrm{ug} / \mathrm{g}$ dry & $1.93 \mathrm{E} 0$ & $3 / 16 / 09$ & 8L12002 & PNNL-AGG-ICP-AES \\
\hline $7440-50-8$ & Copper & 7.18E0 & $\mathrm{ug} / \mathrm{g}$ dry & $6.32 \mathrm{E} 0$ & $3 / 16 / 09$ & 8L12002 & PNNL-AGG-ICP-AES \\
\hline $7439-89-6$ & Iron & $1.36 \mathrm{E} 4$ & ug/g dry & $5.94 \mathrm{E} 0$ & $3 / 16 / 09$ & $8 \mathrm{~L} 12002$ & PNNL-AGG-ICP-AES \\
\hline $7440-09-7$ & Potassium & 7.03E2 & ug/g dry & $8.45 \mathrm{E} 1$ & $3 / 16 / 09$ & 8L12002 & PNNL-AGG-ICP-AES \\
\hline $7439-93-2$ & Lithium & $<2.05 \mathrm{E} 1$ & ug/g dry & $2.05 \mathrm{E} 1$ & $3 / 16 / 09$ & 8L12002 & PNNL-AGG-ICP-AES \\
\hline $7439-95-4$ & Magnesium & $3.25 \mathrm{E} 3$ & ug/g dry & $6.29 \mathrm{E} 0$ & $3 / 16 / 09$ & 8L12002 & PNNL-AGG-ICP-AES \\
\hline $7439-96-5$ & Manganese & $2.28 \mathrm{E} 2$ & ug/g dry & $1.97 \mathrm{E} 0$ & $3 / 16 / 09$ & 8L12002 & PNNL-AGG-ICP-AES \\
\hline $7439-98-7$ & Molybdenum & $<7.50 \mathrm{E} 0$ & ug/g dry & $7.50 \mathrm{E} 0$ & $3 / 16 / 09$ & 8L12002 & PNNL-AGG-ICP-AES \\
\hline $7440-02-0$ & Nickel & $6.90 \mathrm{E} 0$ & $\mathrm{ug} / \mathrm{g}$ dry & $4.58 \mathrm{E} 0$ & $3 / 16 / 09$ & 8L12002 & PNNL-AGG-ICP-AES \\
\hline $7723-14-0$ & Phosphorus & $4.51 \mathrm{E} 2$ & ug/g dry & $2.92 \mathrm{E} 1$ & $3 / 16 / 09$ & 8L12002 & PNNL-AGG-ICP-AES \\
\hline $7439-92-1$ & Lead & $<9.88 \mathrm{E} 0$ & ug/g dry & $9.88 \mathrm{E} 0$ & $3 / 16 / 09$ & 8L12002 & PNNL-AGG-ICP-AES \\
\hline $7782-49-2$ & Selenium & $<5.60 \mathrm{E} 1$ & $\mathrm{ug} / \mathrm{g}$ dry & $5.60 \mathrm{E} 1$ & $3 / 16 / 09$ & 8L12002 & PNNL-AGG-ICP-AES \\
\hline $7440-24-6$ & Strontium & $1.95 \mathrm{E} 1$ & ug/g dry & $1.44 \mathrm{E} 0$ & $3 / 16 / 09$ & 8L12002 & PNNL-AGG-ICP-AES \\
\hline $7440-28-0$ & Thallium & $<3.17 \mathrm{E} 1$ & ug/g dry & $3.17 \mathrm{E} 1$ & $3 / 16 / 09$ & 8L12002 & PNNL-AGG-ICP-AES \\
\hline $7440-62-2$ & Vanadium & $2.67 \mathrm{E} 1$ & $\mathrm{ug} / \mathrm{g}$ dry & 3.95E0 & $3 / 16 / 09$ & $8 \mathrm{~L} 12002$ & PNNL-AGG-ICP-AES \\
\hline $7440-66-6$ & Zinc & $2.98 \mathrm{E} 1$ & $\mathrm{ug} / \mathrm{g}$ dry & $3.60 \mathrm{E} 0$ & $3 / 16 / 09$ & 8L12002 & PNNL-AGG-ICP-AES \\
\hline $7440-23-5$ & Sodium & $2.16 \mathrm{E} 2$ & ug/g dry & $6.22 \mathrm{E} 1$ & $3 / 16 / 09$ & 8L12002 & PNNL-AGG-ICP-AES \\
\hline $7440-21-3$ & Silicon & $<1.60 \mathrm{E} 2$ & ug/g dry & $1.60 \mathrm{E} 2$ & $3 / 16 / 09$ & 8L12002 & PNNL-AGG-ICP-AES \\
\hline $7704-34-9$ & Sulfur & $<1.05 \mathrm{E} 2$ & ug/g dry & $1.05 \mathrm{E} 2$ & $3 / 16 / 09$ & 8L12002 & PNNL-AGG-ICP-AES \\
\hline $7440-32-6$ & Titanium & $5.01 \mathrm{E} 2$ & $\mathrm{ug} / \mathrm{g}$ dry & $5.06 \mathrm{E} 0$ & $3 / 16 / 09$ & 8L12002 & PNNL-AGG-ICP-AES \\
\hline $7440-67-7$ & Zirconium & $2.88 \mathrm{E} 0$ & ug/g dry & $2.65 \mathrm{E} 0$ & $3 / 16 / 09$ & 8L12002 & PNNL-AGG-ICP-AES \\
\hline $7440-22-4$ & Silver & $<2.99 \mathrm{E} 0$ & $\mathrm{ug} / \mathrm{g}$ dry & 2.99E0 & $3 / 16 / 09$ & 8L12002 & PNNL-AGG-ICP-AES \\
\hline $7440-15-5$ & Rhenium & $<9.19 \mathrm{E} 0$ & ug/g dry & $9.19 \mathrm{E} 0$ & $3 / 16 / 09$ & $8 \mathrm{~L} 12002$ & PNNL-AGG-ICP-AES \\
\hline $7440-36-0$ & Antimony & $<2.19 \mathrm{E} 1$ & ug/g dry & $2.19 \mathrm{E} 1$ & $3 / 16 / 09$ & 8L12002 & PNNL-AGG-ICP-AES \\
\hline HEIS No. & B1V995 & & b ID: & 5003-05 & & & \\
\hline $7429-90-5$ & Aluminum & $5.45 \mathrm{E} 3$ & ug/g dry & $2.33 \mathrm{E} 1$ & $3 / 16 / 09$ & 8L12002 & PNNL-AGG-ICP-AES \\
\hline $7440-38-2$ & Arsenic & $<3.49 \mathrm{E} 1$ & ug/g dry & $3.49 \mathrm{E} 1$ & $3 / 16 / 09$ & $8 \mathrm{~L} 12002$ & PNNL-AGG-ICP-AES \\
\hline $7440-42-8$ & Boron & $<9.97 \mathrm{E} 1$ & ug/g dry & $9.97 \mathrm{E} 1$ & $3 / 16 / 09$ & 8L12002 & PNNL-AGG-ICP-AES \\
\hline $7440-39-3$ & Barium & $5.24 \mathrm{E} 1$ & ug/g dry & $1.42 \mathrm{E} 0$ & $3 / 16 / 09$ & 8L12002 & PNNL-AGG-ICP-AES \\
\hline $7440-41-7$ & Beryllium & $<7.95 \mathrm{E}-1$ & ug/g dry & $7.95 \mathrm{E}-1$ & $3 / 16 / 09$ & 8L12002 & PNNL-AGG-ICP-AES \\
\hline $7440-69-9$ & Bismuth & $<9.80 \mathrm{E} 0$ & $\mathrm{ug} / \mathrm{g}$ dry & $9.80 \mathrm{E} 0$ & $3 / 16 / 09$ & 8L12002 & PNNL-AGG-ICP-AES \\
\hline $7440-70-2$ & Calcium & $7.24 \mathrm{E} 3$ & $\mathrm{ug} / \mathrm{g}$ dry & $2.41 \mathrm{E} 1$ & $3 / 16 / 09$ & $8 \mathrm{~L} 12002$ & PNNL-AGG-ICP-AES \\
\hline $7440-43-9$ & Cadmium & $<1.27 \mathrm{E} 0$ & ug/g dry & $1.27 \mathrm{E} 0$ & $3 / 16 / 09$ & 8L12002 & PNNL-AGG-ICP-AES \\
\hline $7440-48-4$ & Cobalt & $6.41 \mathrm{E} 0$ & ug/g dry & 4.97E0 & $3 / 16 / 09$ & 8L12002 & PNNL-AGG-ICP-AES \\
\hline $7440-47-3$ & Chromium & $6.34 \mathrm{E} 0$ & ug/g dry & $2.03 \mathrm{E} 0$ & $3 / 16 / 09$ & 8L12002 & PNNL-AGG-ICP-AES \\
\hline $7440-50-8$ & Copper & 7.77E0 & $\mathrm{ug} / \mathrm{g}$ dry & $6.65 \mathrm{E} 0$ & $3 / 16 / 09$ & $8 \mathrm{~L} 12002$ & PNNL-AGG-ICP-AES \\
\hline $7439-89-6$ & Iron & $1.39 \mathrm{E} 4$ & ug/g dry & $6.25 \mathrm{E} 0$ & $3 / 16 / 09$ & 8L12002 & PNNL-AGG-ICP-AES \\
\hline $7440-09-7$ & Potassium & 8.94E2 & $\mathrm{ug} / \mathrm{g}$ dry & $8.90 \mathrm{E} 1$ & $3 / 16 / 09$ & $8 \mathrm{~L} 12002$ & PNNL-AGG-ICP-AES \\
\hline $7439-93-2$ & Lithium & $<2.16 \mathrm{E} 1$ & ug/g dry & $2.16 \mathrm{E} 1$ & $3 / 16 / 09$ & 8L12002 & PNNL-AGG-ICP-AES \\
\hline
\end{tabular}


Total Metals by PNNL-AGG-ICP-AES/Acid Extract

\begin{tabular}{|c|c|c|c|c|c|c|c|}
\hline CAS \# & Analyte & Results & Units & EQL & Analyzed & Batch & Method \\
\hline HEIS No. & B1V995 & & b ID: & $5003-05$ & & & \\
\hline $7439-95-4$ & Magnesium & $3.53 \mathrm{E} 3$ & ug/g dry & $6.62 \mathrm{E} 0$ & $3 / 16 / 09$ & 8L12002 & PNNL-AGG-ICP-AES \\
\hline $7439-96-5$ & Manganese & 2.39E2 & ug/g dry & $2.08 \mathrm{E} 0$ & $3 / 16 / 09$ & 8L12002 & PNNL-AGG-ICP-AES \\
\hline $7439-98-7$ & Molybdenum & $<7.89 \mathrm{E} 0$ & ug/g dry & 7.89E0 & $3 / 16 / 09$ & 8L12002 & PNNL-AGG-ICP-AES \\
\hline $7440-02-0$ & Nickel & 7.56E0 & $\mathrm{ug} / \mathrm{g}$ dry & 4.82E0 & $3 / 16 / 09$ & 8L12002 & PNNL-AGG-ICP-AES \\
\hline $7723-14-0$ & Phosphorus & $5.21 \mathrm{E} 2$ & $\mathrm{ug} / \mathrm{g}$ dry & 3.07E1 & $3 / 16 / 09$ & 8L12002 & PNNL-AGG-ICP-AES \\
\hline $7439-92-1$ & Lead & $<1.04 \mathrm{E} 1$ & ug/g dry & $1.04 \mathrm{E} 1$ & $3 / 16 / 09$ & 8L12002 & PNNL-AGG-ICP-AES \\
\hline $7782-49-2$ & Selenium & $<5.90 \mathrm{E} 1$ & ug/g dry & $5.90 \mathrm{E} 1$ & $3 / 16 / 09$ & 8L12002 & PNNL-AGG-ICP-AES \\
\hline $7440-24-6$ & Strontium & $2.52 \mathrm{E} 1$ & ug/g dry & $1.52 \mathrm{E} 0$ & $3 / 16 / 09$ & 8L12002 & PNNL-AGG-ICP-AES \\
\hline $7440-28-0$ & Thallium & $<3.34 \mathrm{E} 1$ & ug/g dry & 3.34E1 & $3 / 16 / 09$ & 8L12002 & PNNL-AGG-ICP-AES \\
\hline $7440-62-2$ & Vanadium & $2.44 \mathrm{E} 1$ & $\mathrm{ug} / \mathrm{g}$ dry & $4.16 \mathrm{E} 0$ & $3 / 16 / 09$ & 8L12002 & PNNL-AGG-ICP-AES \\
\hline $7440-66-6$ & Zinc & 2.92E1 & $\mathrm{ug} / \mathrm{g}$ dry & 3.79E0 & $3 / 16 / 09$ & $8 \mathrm{~L} 12002$ & PNNL-AGG-ICP-AES \\
\hline $7440-23-5$ & Sodium & $2.43 \mathrm{E} 2$ & ug/g dry & $6.55 \mathrm{E} 1$ & $3 / 16 / 09$ & 8L12002 & PNNL-AGG-ICP-AES \\
\hline $7440-21-3$ & Silicon & $<1.68 \mathrm{E} 2$ & ug/g dry & $1.68 \mathrm{E} 2$ & $3 / 16 / 09$ & $8 \mathrm{~L} 12002$ & PNNL-AGG-ICP-AES \\
\hline $7704-34-9$ & Sulfur & $<1.11 \mathrm{E} 2$ & ug/g dry & $1.11 \mathrm{E} 2$ & $3 / 16 / 09$ & 8L12002 & PNNL-AGG-ICP-AES \\
\hline $7440-32-6$ & Titanium & $5.56 \mathrm{E} 2$ & ug/g dry & 5.33E0 & $3 / 16 / 09$ & 8L12002 & PNNL-AGG-ICP-AES \\
\hline $7440-67-7$ & Zirconium & 4.14E0 & $\mathrm{ug} / \mathrm{g}$ dry & $2.78 \mathrm{E} 0$ & $3 / 16 / 09$ & 8L12002 & PNNL-AGG-ICP-AES \\
\hline $7440-22-4$ & Silver & $<3.15 \mathrm{E} 0$ & ug/g dry & $3.15 \mathrm{E} 0$ & $3 / 16 / 09$ & 8L12002 & PNNL-AGG-ICP-AES \\
\hline $7440-15-5$ & Rhenium & $<9.67 \mathrm{E} 0$ & $\mathrm{ug} / \mathrm{g}$ dry & $9.67 \mathrm{E} 0$ & $3 / 16 / 09$ & 8L12002 & PNNL-AGG-ICP-AES \\
\hline $7440-36-0$ & Antimony & $<2.31 \mathrm{E} 1$ & ug/g dry & $2.31 \mathrm{E} 1$ & $3 / 16 / 09$ & 8L12002 & PNNL-AGG-ICP-AES \\
\hline HEIS No. & B1V997 & & b ID: & 5003-07 & & & \\
\hline $7429-90-5$ & Aluminum & $5.55 \mathrm{E} 3$ & ug/g dry & $2.35 \mathrm{E} 1$ & $3 / 16 / 09$ & 8L12002 & PNNL-AGG-ICP-AES \\
\hline $7440-38-2$ & Arsenic & $<3.52 \mathrm{E} 1$ & $\mathrm{ug} / \mathrm{g}$ dry & $3.52 \mathrm{E} 1$ & $3 / 16 / 09$ & $8 \mathrm{~L} 12002$ & PNNL-AGG-ICP-AES \\
\hline $7440-42-8$ & Boron & $<1.01 \mathrm{E} 2$ & ug/g dry & $1.01 \mathrm{E} 2$ & $3 / 16 / 09$ & $8 \mathrm{~L} 12002$ & PNNL-AGG-ICP-AES \\
\hline $7440-39-3$ & Barium & $5.98 \mathrm{E} 1$ & ug/g dry & $1.43 \mathrm{E} 0$ & $3 / 16 / 09$ & 8L12002 & PNNL-AGG-ICP-AES \\
\hline $7440-41-7$ & Beryllium & $<8.02 \mathrm{E}-1$ & ug/g dry & $8.02 \mathrm{E}-1$ & $3 / 16 / 09$ & $8 \mathrm{~L} 12002$ & PNNL-AGG-ICP-AES \\
\hline $7440-69-9$ & Bismuth & $<9.89 \mathrm{E} 0$ & $\mathrm{ug} / \mathrm{g}$ dry & $9.89 \mathrm{E} 0$ & $3 / 16 / 09$ & 8L12002 & PNNL-AGG-ICP-AES \\
\hline $7440-70-2$ & Calcium & $6.67 \mathrm{E} 3$ & ug/g dry & $2.44 \mathrm{E} 1$ & $3 / 16 / 09$ & 8L12002 & PNNL-AGG-ICP-AES \\
\hline $7440-43-9$ & Cadmium & $<1.28 \mathrm{E} 0$ & ug/g dry & $1.28 \mathrm{E} 0$ & $3 / 16 / 09$ & $8 \mathrm{~L} 12002$ & PNNL-AGG-ICP-AES \\
\hline $7440-48-4$ & Cobalt & $6.25 \mathrm{E} 0$ & ug/g dry & $5.02 \mathrm{E} 0$ & $3 / 16 / 09$ & 8L12002 & PNNL-AGG-ICP-AES \\
\hline $7440-47-3$ & Chromium & $8.78 \mathrm{E} 0$ & $\mathrm{ug} / \mathrm{g}$ dry & $2.05 \mathrm{E} 0$ & $3 / 16 / 09$ & 8L12002 & PNNL-AGG-ICP-AES \\
\hline $7440-50-8$ & Copper & 7.14E0 & ug/g dry & $6.71 \mathrm{E} 0$ & $3 / 16 / 09$ & 8L12002 & PNNL-AGG-ICP-AES \\
\hline $7439-89-6$ & Iron & $1.23 \mathrm{E} 4$ & ug/g dry & $6.31 \mathrm{E} 0$ & $3 / 16 / 09$ & 8L12002 & PNNL-AGG-ICP-AES \\
\hline $7440-09-7$ & Potassium & $9.91 \mathrm{E} 2$ & $\mathrm{ug} / \mathrm{g}$ dry & $8.98 \mathrm{E} 1$ & $3 / 16 / 09$ & $8 \mathrm{~L} 12002$ & PNNL-AGG-ICP-AES \\
\hline $7439-93-2$ & Lithium & $<2.18 \mathrm{E} 1$ & $\mathrm{ug} / \mathrm{g}$ dry & $2.18 \mathrm{E} 1$ & $3 / 16 / 09$ & 8L12002 & PNNL-AGG-ICP-AES \\
\hline $7439-95-4$ & Magnesium & $3.54 \mathrm{E} 3$ & ug/g dry & $6.68 \mathrm{E} 0$ & $3 / 16 / 09$ & 8L12002 & PNNL-AGG-ICP-AES \\
\hline $7439-96-5$ & Manganese & $2.31 \mathrm{E} 2$ & ug/g dry & $2.10 \mathrm{E} 0$ & $3 / 16 / 09$ & $8 \mathrm{~L} 12002$ & PNNL-AGG-ICP-AES \\
\hline $7439-98-7$ & Molybdenum & $<7.96 \mathrm{E} 0$ & $\mathrm{ug} / \mathrm{g}$ dry & 7.96E0 & $3 / 16 / 09$ & 8L12002 & PNNL-AGG-ICP-AES \\
\hline $7440-02-0$ & Nickel & $8.75 \mathrm{E} 0$ & ug/g dry & $4.86 \mathrm{E} 0$ & $3 / 16 / 09$ & 8L12002 & PNNL-AGG-ICP-AES \\
\hline $7723-14-0$ & Phosphorus & $5.22 \mathrm{E} 2$ & ug/g dry & $3.10 \mathrm{E} 1$ & $3 / 16 / 09$ & 8L12002 & PNNL-AGG-ICP-AES \\
\hline $7439-92-1$ & Lead & $<1.05 \mathrm{E} 1$ & $\mathrm{ug} / \mathrm{g}$ dry & $1.05 \mathrm{E} 1$ & $3 / 16 / 09$ & 8L12002 & PNNL-AGG-ICP-AES \\
\hline $7782-49-2$ & Selenium & $<5.95 \mathrm{E} 1$ & $\mathrm{ug} / \mathrm{g}$ dry & $5.95 \mathrm{E} 1$ & $3 / 16 / 09$ & $8 \mathrm{~L} 12002$ & PNNL-AGG-ICP-AES \\
\hline $7440-24-6$ & Strontium & $2.50 \mathrm{E} 1$ & ug/g dry & $1.53 \mathrm{E} 0$ & $3 / 16 / 09$ & 8L12002 & PNNL-AGG-ICP-AES \\
\hline $7440-28-0$ & Thallium & $<3.37 \mathrm{E} 1$ & ug/g dry & $3.37 \mathrm{E} 1$ & $3 / 16 / 09$ & 8L12002 & PNNL-AGG-ICP-AES \\
\hline $7440-62-2$ & Vanadium & $2.02 \mathrm{E} 1$ & ug/g dry & $4.20 \mathrm{E} 0$ & $3 / 16 / 09$ & 8L12002 & PNNL-AGG-ICP-AES \\
\hline $7440-66-6$ & Zinc & $2.76 \mathrm{E} 1$ & $\mathrm{ug} / \mathrm{g}$ dry & $3.82 \mathrm{E} 0$ & $3 / 16 / 09$ & $8 \mathrm{~L} 12002$ & PNNL-AGG-ICP-AES \\
\hline $7440-23-5$ & Sodium & $2.42 \mathrm{E} 2$ & ug/g dry & $6.61 \mathrm{E} 1$ & $3 / 16 / 09$ & 8L12002 & PNNL-AGG-ICP-AES \\
\hline $7440-21-3$ & Silicon & $<1.70 \mathrm{E} 2$ & $\mathrm{ug} / \mathrm{g}$ dry & $1.70 \mathrm{E} 2$ & $3 / 16 / 09$ & $8 \mathrm{~L} 12002$ & PNNL-AGG-ICP-AES \\
\hline $7704-34-9$ & Sulfur & $<1.12 \mathrm{E} 2$ & ug/g dry & $1.12 \mathrm{E} 2$ & $3 / 16 / 09$ & 8L12002 & PNNL-AGG-ICP-AES \\
\hline
\end{tabular}


Total Metals by PNNL-AGG-ICP-AES/Acid Extract

\begin{tabular}{|c|c|c|c|c|c|c|c|}
\hline CAS \# & Analyte & Results & Units & EQL & Analyzed & Batch & Method \\
\hline HEIS No. & B1V997 & \multicolumn{3}{|c|}{ Lab ID: $\quad$ 0805003-07 } & & & \\
\hline $7440-32-6$ & Titanium & $5.63 \mathrm{E} 2$ & $\mathrm{ug} / \mathrm{g}$ dry & $5.37 \mathrm{E} 0$ & $3 / 16 / 09$ & 8L12002 & PNNL-AGG-ICP-AES \\
\hline $7440-67-7$ & Zirconium & $5.07 \mathrm{E} 0$ & ug/g dry & $2.81 \mathrm{E} 0$ & $3 / 16 / 09$ & 8L12002 & PNNL-AGG-ICP-AES \\
\hline $7440-22-4$ & Silver & $<3.18 \mathrm{E} 0$ & ug/g dry & $3.18 \mathrm{E} 0$ & $3 / 16 / 09$ & 8L12002 & PNNL-AGG-ICP-AES \\
\hline $7440-15-5$ & Rhenium & $<9.76 \mathrm{E} 0$ & $\mathrm{ug} / \mathrm{g}$ dry & $9.76 \mathrm{E} 0$ & $3 / 16 / 09$ & 8L12002 & PNNL-AGG-ICP-AES \\
\hline $7440-36-0$ & Antimony & $<2.33 \mathrm{E} 1$ & ug/g dry & $2.33 \mathrm{E} 1$ & $3 / 16 / 09$ & 8L12002 & PNNL-AGG-ICP-AES \\
\hline HEIS No. & $\begin{array}{l}\text { B1V999 } \\
\end{array}$ & \multicolumn{3}{|c|}{ Lab ID: $\quad$ 0805003-09 } & & & \\
\hline $7429-90-5$ & Aluminum & $5.06 \mathrm{E} 3$ & ug/g dry & $2.34 \mathrm{E} 1$ & $3 / 16 / 09$ & 8L12002 & PNNL-AGG-ICP-AES \\
\hline $7440-38-2$ & Arsenic & $<3.51 \mathrm{E} 1$ & ug/g dry & $3.51 \mathrm{E} 1$ & $3 / 16 / 09$ & $8 \mathrm{~L} 12002$ & PNNL-AGG-ICP-AES \\
\hline $7440-42-8$ & Boron & $<1.00 \mathrm{E} 2$ & $\mathrm{ug} / \mathrm{g}$ dry & $1.00 \mathrm{E} 2$ & $3 / 16 / 09$ & $8 \mathrm{~L} 12002$ & PNNL-AGG-ICP-AES \\
\hline $7440-39-3$ & Barium & $8.02 \mathrm{E} 1$ & ug/g dry & $1.43 \mathrm{E} 0$ & $3 / 16 / 09$ & 8L12002 & PNNL-AGG-ICP-AES \\
\hline $7440-41-7$ & Beryllium & $<8.00 \mathrm{E}-1$ & $\mathrm{ug} / \mathrm{g}$ dry & $8.00 \mathrm{E}-1$ & $3 / 16 / 09$ & $8 \mathrm{~L} 12002$ & PNNL-AGG-ICP-AES \\
\hline $7440-69-9$ & Bismuth & $<9.86 \mathrm{E} 0$ & ug/g dry & $9.86 \mathrm{E} 0$ & $3 / 16 / 09$ & $8 \mathrm{~L} 12002$ & PNNL-AGG-ICP-AES \\
\hline $7440-70-2$ & Calcium & $5.75 \mathrm{E} 3$ & ug/g dry & $2.43 \mathrm{E} 1$ & $3 / 16 / 09$ & 8L12002 & PNNL-AGG-ICP-AES \\
\hline $7440-43-9$ & Cadmium & $<1.27 \mathrm{E} 0$ & ug/g dry & $1.27 \mathrm{E} 0$ & $3 / 16 / 09$ & 8L12002 & PNNL-AGG-ICP-AES \\
\hline $7440-48-4$ & Cobalt & 7.49E0 & ug/g dry & $5.00 \mathrm{E} 0$ & $3 / 16 / 09$ & 8L12002 & PNNL-AGG-ICP-AES \\
\hline $7440-47-3$ & Chromium & $5.29 \mathrm{E} 0$ & ug/g dry & $2.04 \mathrm{E} 0$ & $3 / 16 / 09$ & 8L12002 & PNNL-AGG-ICP-AES \\
\hline $7440-50-8$ & Copper & 8.69E0 & ug/g dry & $6.69 \mathrm{E} 0$ & $3 / 16 / 09$ & 8L12002 & PNNL-AGG-ICP-AES \\
\hline $7439-89-6$ & Iron & $1.48 \mathrm{E} 4$ & ug/g dry & $6.29 \mathrm{E} 0$ & $3 / 16 / 09$ & 8L12002 & PNNL-AGG-ICP-AES \\
\hline $7440-09-7$ & Potassium & 8.07E2 & $\mathrm{ug} / \mathrm{g}$ dry & $8.96 \mathrm{E} 1$ & $3 / 16 / 09$ & $8 \mathrm{~L} 12002$ & PNNL-AGG-ICP-AES \\
\hline $7439-93-2$ & Lithium & $<2.17 \mathrm{E} 1$ & ug/g dry & $2.17 \mathrm{E} 1$ & $3 / 16 / 09$ & 8L12002 & PNNL-AGG-ICP-AES \\
\hline $7439-95-4$ & Magnesium & $3.28 \mathrm{E} 3$ & ug/g dry & $6.66 \mathrm{E} 0$ & $3 / 16 / 09$ & 8L12002 & PNNL-AGG-ICP-AES \\
\hline $7439-96-5$ & Manganese & $2.34 \mathrm{E} 2$ & ug/g dry & 2.09E0 & $3 / 16 / 09$ & 8L12002 & PNNL-AGG-ICP-AES \\
\hline $7439-98-7$ & Molybdenum & $<7.94 \mathrm{E} 0$ & ug/g dry & 7.94E0 & $3 / 16 / 09$ & $8 \mathrm{~L} 12002$ & PNNL-AGG-ICP-AES \\
\hline $7440-02-0$ & Nickel & $6.51 \mathrm{E} 0$ & $\mathrm{ug} / \mathrm{g}$ dry & $4.85 \mathrm{E} 0$ & $3 / 16 / 09$ & $8 \mathrm{~L} 12002$ & PNNL-AGG-ICP-AES \\
\hline $7723-14-0$ & Phosphorus & $5.44 \mathrm{E} 2$ & ug/g dry & $3.09 \mathrm{E} 1$ & $3 / 16 / 09$ & 8L12002 & PNNL-AGG-ICP-AES \\
\hline $7439-92-1$ & Lead & $<1.05 \mathrm{E} 1$ & ug/g dry & $1.05 \mathrm{E} 1$ & $3 / 16 / 09$ & 8L12002 & PNNL-AGG-ICP-AES \\
\hline $7782-49-2$ & Selenium & $<5.94 \mathrm{E} 1$ & ug/g dry & $5.94 \mathrm{E} 1$ & $3 / 16 / 09$ & 8L12002 & PNNL-AGG-ICP-AES \\
\hline $7440-24-6$ & Strontium & $2.49 \mathrm{E} 1$ & ug/g dry & $1.53 \mathrm{E} 0$ & $3 / 16 / 09$ & 8L12002 & PNNL-AGG-ICP-AES \\
\hline $7440-28-0$ & Thallium & $<3.36 \mathrm{E} 1$ & ug/g dry & $3.36 \mathrm{E} 1$ & $3 / 16 / 09$ & 8L12002 & PNNL-AGG-ICP-AES \\
\hline $7440-62-2$ & Vanadium & $2.88 \mathrm{E} 1$ & ug/g dry & 4.19E0 & $3 / 16 / 09$ & $8 \mathrm{~L} 12002$ & PNNL-AGG-ICP-AES \\
\hline $7440-66-6$ & Zinc & $3.09 \mathrm{E} 1$ & ug/g dry & $3.82 \mathrm{E} 0$ & $3 / 16 / 09$ & 8L12002 & PNNL-AGG-ICP-AES \\
\hline $7440-23-5$ & Sodium & $3.61 \mathrm{E} 2$ & $\mathrm{ug} / \mathrm{g}$ dry & $6.59 \mathrm{E} 1$ & $3 / 16 / 09$ & 8L12002 & PNNL-AGG-ICP-AES \\
\hline $7440-21-3$ & Silicon & $<1.69 \mathrm{E} 2$ & ug/g dry & $1.69 \mathrm{E} 2$ & $3 / 16 / 09$ & 8L12002 & PNNL-AGG-ICP-AES \\
\hline $7704-34-9$ & Sulfur & $<1.12 \mathrm{E} 2$ & ug/g dry & $1.12 \mathrm{E} 2$ & $3 / 16 / 09$ & 8L12002 & PNNL-AGG-ICP-AES \\
\hline $7440-32-6$ & Titanium & $6.71 \mathrm{E} 2$ & $\mathrm{ug} / \mathrm{g}$ dry & $5.36 \mathrm{E} 0$ & $3 / 16 / 09$ & $8 \mathrm{~L} 12002$ & PNNL-AGG-ICP-AES \\
\hline $7440-67-7$ & Zirconium & $6.21 \mathrm{E} 0$ & ug/g dry & $2.80 \mathrm{E} 0$ & $3 / 16 / 09$ & 8L12002 & PNNL-AGG-ICP-AES \\
\hline $7440-22-4$ & Silver & $<3.17 \mathrm{E} 0$ & ug/g dry & $3.17 \mathrm{E} 0$ & 3/16/09 & 8L12002 & PNNL-AGG-ICP-AES \\
\hline $7440-15-5$ & Rhenium & $<9.74 \mathrm{E} 0$ & $\mathrm{ug} / \mathrm{g}$ dry & $9.74 \mathrm{E} 0$ & $3 / 16 / 09$ & 8L12002 & PNNL-AGG-ICP-AES \\
\hline $7440-36-0$ & Antimony & $<2.32 \mathrm{E} 1$ & ug/g dry & $2.32 \mathrm{E} 1$ & $3 / 16 / 09$ & 8L12002 & PNNL-AGG-ICP-AES \\
\hline HEIS No. & B1V9B1 & & b ID: & 5003-11 & & & \\
\hline $7429-90-5$ & Aluminum & $5.24 \mathrm{E} 3$ & ug/g dry & $2.38 \mathrm{E} 1$ & $3 / 16 / 09$ & 8L12002 & PNNL-AGG-ICP-AES \\
\hline $7440-38-2$ & Arsenic & $<3.58 \mathrm{E} 1$ & ug/g dry & $3.58 \mathrm{E} 1$ & $3 / 16 / 09$ & 8L12002 & PNNL-AGG-ICP-AES \\
\hline $7440-42-8$ & Boron & $<1.02 \mathrm{E} 2$ & ug/g dry & $1.02 \mathrm{E} 2$ & $3 / 16 / 09$ & 8L12002 & PNNL-AGG-ICP-AES \\
\hline $7440-39-3$ & Barium & $5.85 \mathrm{E} 1$ & ug/g dry & $1.45 \mathrm{E} 0$ & $3 / 16 / 09$ & 8L12002 & PNNL-AGG-ICP-AES \\
\hline $7440-41-7$ & Beryllium & $<8.15 \mathrm{E}-1$ & ug/g dry & $8.15 \mathrm{E}-1$ & $3 / 16 / 09$ & 8L12002 & PNNL-AGG-ICP-AES \\
\hline $7440-69-9$ & Bismuth & $<1.00 \mathrm{E} 1$ & $\mathrm{ug} / \mathrm{g}$ dry & $1.00 \mathrm{E} 1$ & $3 / 16 / 09$ & $8 \mathrm{~L} 12002$ & PNNL-AGG-ICP-AES \\
\hline $7440-70-2$ & Calcium & $6.53 \mathrm{E} 3$ & $\mathrm{ug} / \mathrm{g}$ dry & $2.47 \mathrm{E} 1$ & $3 / 16 / 09$ & $8 \mathrm{~L} 12002$ & PNNL-AGG-ICP-AES \\
\hline $7440-43-9$ & Cadmium & $<1.30 \mathrm{E} 0$ & ug/g dry & $1.30 \mathrm{E} 0$ & $3 / 16 / 09$ & 8L12002 & PNNL-AGG-ICP-AES \\
\hline
\end{tabular}


Total Metals by PNNL-AGG-ICP-AES/Acid Extract

\begin{tabular}{|c|c|c|c|c|c|c|c|}
\hline CAS \# & Analyte & Results & Units & EQL & Analyzed & Batch & Method \\
\hline HEIS No. & B1V9B1 & \multicolumn{3}{|c|}{ Lab ID: $\quad$ 0805003-11 } & & & \\
\hline $7440-48-4$ & Cobalt & $6.38 \mathrm{E} 0$ & $\mathrm{ug} / \mathrm{g}$ dry & $5.10 \mathrm{E} 0$ & $3 / 16 / 09$ & 8L12002 & PNNL-AGG-ICP-AES \\
\hline $7440-47-3$ & Chromium & $5.09 \mathrm{E} 0$ & $\mathrm{ug} / \mathrm{g}$ dry & $2.08 \mathrm{E} 0$ & $3 / 16 / 09$ & 8L12002 & PNNL-AGG-ICP-AES \\
\hline $7440-50-8$ & Copper & $7.65 \mathrm{E} 0$ & ug/g dry & $6.82 \mathrm{E} 0$ & $3 / 16 / 09$ & 8L12002 & PNNL-AGG-ICP-AES \\
\hline $7439-89-6$ & Iron & $1.27 \mathrm{E} 4$ & ug/g dry & $6.41 \mathrm{E} 0$ & $3 / 16 / 09$ & 8L12002 & PNNL-AGG-ICP-AES \\
\hline $7440-09-7$ & Potassium & $8.58 \mathrm{E} 2$ & ug/g dry & $9.12 \mathrm{E} 1$ & $3 / 16 / 09$ & 8L12002 & PNNL-AGG-ICP-AES \\
\hline $7439-93-2$ & Lithium & $<2.22 \mathrm{E} 1$ & ug/g dry & $2.22 \mathrm{E} 1$ & $3 / 16 / 09$ & $8 \mathrm{~L} 12002$ & PNNL-AGG-ICP-AES \\
\hline $7439-95-4$ & Magnesium & $3.16 \mathrm{E} 3$ & ug/g dry & $6.79 \mathrm{E} 0$ & $3 / 16 / 09$ & $8 \mathrm{~L} 12002$ & PNNL-AGG-ICP-AES \\
\hline $7439-96-5$ & Manganese & $2.28 \mathrm{E} 2$ & $\mathrm{ug} / \mathrm{g}$ dry & $2.13 \mathrm{E} 0$ & $3 / 16 / 09$ & 8L12002 & PNNL-AGG-ICP-AES \\
\hline 7439-98-7 & Molybdenum & $<8.09 \mathrm{E} 0$ & $\mathrm{ug} / \mathrm{g}$ dry & 8.09E0 & $3 / 16 / 09$ & 8L12002 & PNNL-AGG-ICP-AES \\
\hline $7440-02-0$ & Nickel & $6.79 \mathrm{E} 0$ & $\mathrm{ug} / \mathrm{g}$ dry & 4.94E0 & $3 / 16 / 09$ & 8L12002 & PNNL-AGG-ICP-AES \\
\hline $7723-14-0$ & Phosphorus & 4.54E2 & ug/g dry & $3.15 \mathrm{E} 1$ & $3 / 16 / 09$ & 8L12002 & PNNL-AGG-ICP-AES \\
\hline $7439-92-1$ & Lead & $<1.07 \mathrm{E} 1$ & $\mathrm{ug} / \mathrm{g}$ dry & $1.07 \mathrm{E} 1$ & $3 / 16 / 09$ & 8L12002 & PNNL-AGG-ICP-AES \\
\hline $7782-49-2$ & Selenium & $<6.05 \mathrm{E} 1$ & $\mathrm{ug} / \mathrm{g}$ dry & $6.05 \mathrm{E} 1$ & $3 / 16 / 09$ & 8L12002 & PNNL-AGG-ICP-AES \\
\hline $7440-24-6$ & Strontium & $2.58 \mathrm{E} 1$ & ug/g dry & $1.55 \mathrm{E} 0$ & $3 / 16 / 09$ & $8 \mathrm{~L} 12002$ & PNNL-AGG-ICP-AES \\
\hline $7440-28-0$ & Thallium & $<3.42 \mathrm{E} 1$ & $\mathrm{ug} / \mathrm{g}$ dry & $3.42 \mathrm{E} 1$ & $3 / 16 / 09$ & 8L12002 & PNNL-AGG-ICP-AES \\
\hline $7440-62-2$ & Vanadium & $2.20 \mathrm{E} 1$ & $\mathrm{ug} / \mathrm{g}$ dry & 4.26E0 & $3 / 16 / 09$ & 8L12002 & PNNL-AGG-ICP-AES \\
\hline $7440-66-6$ & Zinc & $2.75 \mathrm{E} 1$ & $\mathrm{ug} / \mathrm{g}$ dry & $3.89 \mathrm{E} 0$ & $3 / 16 / 09$ & 8L12002 & PNNL-AGG-ICP-AES \\
\hline $7440-23-5$ & Sodium & $2.70 \mathrm{E} 2$ & $\mathrm{ug} / \mathrm{g}$ dry & $6.72 \mathrm{E} 1$ & $3 / 16 / 09$ & 8L12002 & PNNL-AGG-ICP-AES \\
\hline $7440-21-3$ & Silicon & $<1.72 \mathrm{E} 2$ & $\mathrm{ug} / \mathrm{g}$ dry & $1.72 \mathrm{E} 2$ & $3 / 16 / 09$ & 8L12002 & PNNL-AGG-ICP-AES \\
\hline 7704-34-9 & Sulfur & $<1.14 \mathrm{E} 2$ & $\mathrm{ug} / \mathrm{g}$ dry & $1.14 \mathrm{E} 2$ & $3 / 16 / 09$ & 8L12002 & PNNL-AGG-ICP-AES \\
\hline $7440-32-6$ & Titanium & $5.72 \mathrm{E} 2$ & ug/g dry & $5.46 \mathrm{E} 0$ & $3 / 16 / 09$ & $8 \mathrm{~L} 12002$ & PNNL-AGG-ICP-AES \\
\hline $7440-67-7$ & Zirconium & $5.98 \mathrm{E} 0$ & ug/g dry & $2.85 \mathrm{E} 0$ & $3 / 16 / 09$ & 8L12002 & PNNL-AGG-ICP-AES \\
\hline $7440-22-4$ & Silver & $<3.23 \mathrm{E} 0$ & $\mathrm{ug} / \mathrm{g}$ dry & $3.23 \mathrm{E} 0$ & $3 / 16 / 09$ & 8L12002 & PNNL-AGG-ICP-AES \\
\hline $7440-15-5$ & Rhenium & $<9.92 \mathrm{E} 0$ & $\mathrm{ug} / \mathrm{g}$ dry & $9.92 \mathrm{E} 0$ & $3 / 16 / 09$ & 8L12002 & PNNL-AGG-ICP-AES \\
\hline $7440-36-0$ & Antimony & $<2.37 \mathrm{E} 1$ & ug/g dry & $2.37 \mathrm{E} 1$ & $3 / 16 / 09$ & $8 \mathrm{~L} 12002$ & PNNL-AGG-ICP-AES \\
\hline HEIS No. & B1V9B3 & & b ID: & $5003-13$ & & & \\
\hline $7429-90-5$ & Aluminum & $5.15 \mathrm{E} 3$ & $\mathrm{ug} / \mathrm{g}$ dry & $2.33 \mathrm{E} 1$ & $3 / 16 / 09$ & 8L12002 & PNNL-AGG-ICP-AES \\
\hline $7440-38-2$ & Arsenic & $<3.50 \mathrm{E} 1$ & $\mathrm{ug} / \mathrm{g}$ dry & $3.50 \mathrm{E} 1$ & $3 / 16 / 09$ & 8L12002 & PNNL-AGG-ICP-AES \\
\hline $7440-42-8$ & Boron & $<1.00 \mathrm{E} 2$ & $\mathrm{ug} / \mathrm{g}$ dry & $1.00 \mathrm{E} 2$ & $3 / 16 / 09$ & 8L12002 & PNNL-AGG-ICP-AES \\
\hline $7440-39-3$ & Barium & $6.29 \mathrm{E} 1$ & ug/g dry & $1.42 \mathrm{E} 0$ & $3 / 16 / 09$ & 8L12002 & PNNL-AGG-ICP-AES \\
\hline $7440-41-7$ & Beryllium & $<7.97 \mathrm{E}-1$ & ug/g dry & 7.97E-1 & $3 / 16 / 09$ & 8L12002 & PNNL-AGG-ICP-AES \\
\hline $7440-69-9$ & Bismuth & $<9.83 \mathrm{E} 0$ & $\mathrm{ug} / \mathrm{g}$ dry & $9.83 \mathrm{E} 0$ & 3/16/09 & 8L12002 & PNNL-AGG-ICP-AES \\
\hline $7440-70-2$ & Calcium & $6.00 \mathrm{E} 3$ & ug/g dry & $2.42 \mathrm{E} 1$ & $3 / 16 / 09$ & 8L12002 & PNNL-AGG-ICP-AES \\
\hline $7440-43-9$ & Cadmium & $<1.27 \mathrm{E} 0$ & ug/g dry & $1.27 \mathrm{E} 0$ & $3 / 16 / 09$ & 8L12002 & PNNL-AGG-ICP-AES \\
\hline $7440-48-4$ & Cobalt & $6.25 \mathrm{E} 0$ & $\mathrm{ug} / \mathrm{g}$ dry & 4.99E0 & $3 / 16 / 09$ & 8L12002 & PNNL-AGG-ICP-AES \\
\hline $7440-47-3$ & Chromium & $5.81 \mathrm{E} 0$ & ug/g dry & $2.04 \mathrm{E} 0$ & $3 / 16 / 09$ & 8L12002 & PNNL-AGG-ICP-AES \\
\hline $7440-50-8$ & Copper & $6.70 \mathrm{E} 0$ & $\mathrm{ug} / \mathrm{g}$ dry & $6.67 \mathrm{E} 0$ & $3 / 16 / 09$ & 8L12002 & PNNL-AGG-ICP-AES \\
\hline $7439-89-6$ & Iron & $1.36 \mathrm{E} 4$ & $\mathrm{ug} / \mathrm{g}$ dry & $6.27 \mathrm{E} 0$ & $3 / 16 / 09$ & $8 \mathrm{~L} 12002$ & PNNL-AGG-ICP-AES \\
\hline $7440-09-7$ & Potassium & $9.14 \mathrm{E} 2$ & $\mathrm{ug} / \mathrm{g}$ dry & 8.93E1 & $3 / 16 / 09$ & 8L12002 & PNNL-AGG-ICP-AES \\
\hline $7439-93-2$ & Lithium & $<2.17 \mathrm{E} 1$ & ug/g dry & $2.17 \mathrm{E} 1$ & $3 / 16 / 09$ & $8 \mathrm{~L} 12002$ & PNNL-AGG-ICP-AES \\
\hline $7439-95-4$ & Magnesium & $3.18 \mathrm{E} 3$ & $\mathrm{ug} / \mathrm{g}$ dry & $6.65 \mathrm{E} 0$ & $3 / 16 / 09$ & $8 \mathrm{~L} 12002$ & PNNL-AGG-ICP-AES \\
\hline $7439-96-5$ & Manganese & $2.28 \mathrm{E} 2$ & $\mathrm{ug} / \mathrm{g}$ dry & $2.09 \mathrm{E} 0$ & $3 / 16 / 09$ & 8L12002 & PNNL-AGG-ICP-AES \\
\hline $7439-98-7$ & Molybdenum & $<7.92 \mathrm{E} 0$ & $\mathrm{ug} / \mathrm{g}$ dry & 7.92E0 & $3 / 16 / 09$ & 8L12002 & PNNL-AGG-ICP-AES \\
\hline $7440-02-0$ & Nickel & $6.20 \mathrm{E} 0$ & ug/g dry & 4.84E0 & $3 / 16 / 09$ & 8L12002 & PNNL-AGG-ICP-AES \\
\hline $7723-14-0$ & Phosphorus & $4.28 \mathrm{E} 2$ & ug/g dry & $3.08 \mathrm{E} 1$ & $3 / 16 / 09$ & 8L12002 & PNNL-AGG-ICP-AES \\
\hline $7439-92-1$ & Lead & $<1.04 \mathrm{E} 1$ & $\mathrm{ug} / \mathrm{g}$ dry & $1.04 \mathrm{E} 1$ & $3 / 16 / 09$ & 8L12002 & PNNL-AGG-ICP-AES \\
\hline $7782-49-2$ & Selenium & $<5.92 \mathrm{E} 1$ & $\mathrm{ug} / \mathrm{g}$ dry & $5.92 \mathrm{E} 1$ & $3 / 16 / 09$ & 8L12002 & PNNL-AGG-ICP-AES \\
\hline $7440-24-6$ & Strontium & $2.82 \mathrm{E} 1$ & $\mathrm{ug} / \mathrm{g}$ dry & $1.52 \mathrm{E} 0$ & $3 / 16 / 09$ & 8L12002 & PNNL-AGG-ICP-AES \\
\hline
\end{tabular}


Total Metals by PNNL-AGG-ICP-AES/Acid Extract

\begin{tabular}{|c|c|c|c|c|c|c|c|}
\hline CAS \# & Analyte & Results & Units & EQL & Analyzed & Batch & Method \\
\hline HEIS No. & B1V9B3 & \multicolumn{3}{|c|}{ Lab ID: $\quad$ 0805003-13 } & & & \\
\hline $7440-28-0$ & Thallium & $<3.35 \mathrm{E} 1$ & $\mathrm{ug} / \mathrm{g}$ dry & $3.35 \mathrm{E} 1$ & $3 / 16 / 09$ & 8L12002 & PNNL-AGG-ICP-AES \\
\hline $7440-62-2$ & Vanadium & $2.49 \mathrm{E} 1$ & ug/g dry & $4.17 \mathrm{E} 0$ & 3/16/09 & 8L12002 & PNNL-AGG-ICP-AES \\
\hline $7440-66-6$ & Zinc & $2.65 \mathrm{E} 1$ & ug/g dry & $3.80 \mathrm{E} 0$ & 3/16/09 & 8L12002 & PNNL-AGG-ICP-AES \\
\hline $7440-23-5$ & Sodium & $3.74 \mathrm{E} 2$ & $\mathrm{ug} / \mathrm{g}$ dry & $6.57 \mathrm{E} 1$ & 3/16/09 & 8L12002 & PNNL-AGG-ICP-AES \\
\hline $7440-21-3$ & Silicon & $<1.69 \mathrm{E} 2$ & ug/g dry & $1.69 \mathrm{E} 2$ & 3/16/09 & 8L12002 & PNNL-AGG-ICP-AES \\
\hline $7704-34-9$ & Sulfur & $<1.11 \mathrm{E} 2$ & ug/g dry & $1.11 \mathrm{E} 2$ & 3/16/09 & 8L12002 & PNNL-AGG-ICP-AES \\
\hline $7440-32-6$ & Titanium & $6.00 \mathrm{E} 2$ & $\mathrm{ug} / \mathrm{g}$ dry & $5.34 \mathrm{E} 0$ & 3/16/09 & 8L12002 & PNNL-AGG-ICP-AES \\
\hline $7440-67-7$ & Zirconium & $5.10 \mathrm{E} 0$ & $\mathrm{ug} / \mathrm{g}$ dry & $2.79 \mathrm{E} 0$ & 3/16/09 & $8 \mathrm{~L} 12002$ & PNNL-AGG-ICP-AES \\
\hline $7440-22-4$ & Silver & $<3.16 \mathrm{E} 0$ & ug/g dry & $3.16 \mathrm{E} 0$ & 3/16/09 & 8L12002 & PNNL-AGG-ICP-AES \\
\hline $7440-15-5$ & Rhenium & $<9.71 \mathrm{E} 0$ & $\mathrm{ug} / \mathrm{g}$ dry & $9.71 \mathrm{E} 0$ & 3/16/09 & 8L12002 & PNNL-AGG-ICP-AES \\
\hline $7440-36-0$ & Antimony & $<2.32 \mathrm{E} 1$ & ug/g dry & $2.32 \mathrm{E} 1$ & 3/16/09 & 8L12002 & PNNL-AGG-ICP-AES \\
\hline HEIS No. & B1V9B5 & \multicolumn{3}{|c|}{ Lab ID: $\quad$ 0805003-15 } & & & \\
\hline $7429-90-5$ & Aluminum & $4.41 \mathrm{E} 3$ & ug/g dry & $2.28 \mathrm{E} 1$ & 3/16/09 & 8L12002 & PNNL-AGG-ICP-AES \\
\hline $7440-38-2$ & Arsenic & $<3.42 \mathrm{E} 1$ & ug/g dry & $3.42 \mathrm{E} 1$ & 3/16/09 & 8L12002 & PNNL-AGG-ICP-AES \\
\hline $7440-42-8$ & Boron & $<9.78 \mathrm{E} 1$ & ug/g dry & $9.78 \mathrm{E} 1$ & 3/16/09 & 8L12002 & PNNL-AGG-ICP-AES \\
\hline $7440-39-3$ & Barium & 3.64E1 & ug/g dry & $1.39 \mathrm{E} 0$ & 3/16/09 & 8L12002 & PNNL-AGG-ICP-AES \\
\hline $7440-41-7$ & Beryllium & $<7.79 \mathrm{E}-1$ & ug/g dry & $7.79 \mathrm{E}-1$ & $3 / 16 / 09$ & 8L12002 & PNNL-AGG-ICP-AES \\
\hline $7440-69-9$ & Bismuth & $<9.61 \mathrm{E} 0$ & $\mathrm{ug} / \mathrm{g}$ dry & $9.61 \mathrm{E} 0$ & 3/16/09 & 8L12002 & PNNL-AGG-ICP-AES \\
\hline $7440-70-2$ & Calcium & $6.54 \mathrm{E} 3$ & $\mathrm{ug} / \mathrm{g}$ dry & $2.37 \mathrm{E} 1$ & 3/16/09 & $8 \mathrm{~L} 12002$ & PNNL-AGG-ICP-AES \\
\hline $7440-43-9$ & Cadmium & $<1.24 \mathrm{E} 0$ & $\mathrm{ug} / \mathrm{g}$ dry & $1.24 \mathrm{E} 0$ & 3/16/09 & 8L12002 & PNNL-AGG-ICP-AES \\
\hline $7440-48-4$ & Cobalt & $<4.87 \mathrm{E} 0$ & ug/g dry & 4.87E0 & 3/16/09 & 8L12002 & PNNL-AGG-ICP-AES \\
\hline $7440-47-3$ & Chromium & $6.63 \mathrm{E} 0$ & ug/g dry & $1.99 \mathrm{E} 0$ & 3/16/09 & 8L12002 & PNNL-AGG-ICP-AES \\
\hline $7440-50-8$ & Copper & $<6.52 \mathrm{E} 0$ & ug/g dry & $6.52 \mathrm{E} 0$ & 3/16/09 & $8 \mathrm{~L} 12002$ & PNNL-AGG-ICP-AES \\
\hline $7439-89-6$ & Iron & $1.09 \mathrm{E} 4$ & $\mathrm{ug} / \mathrm{g}$ dry & $6.13 \mathrm{E} 0$ & 3/16/09 & $8 \mathrm{~L} 12002$ & PNNL-AGG-ICP-AES \\
\hline 7440-09-7 & Potassium & $7.41 \mathrm{E} 2$ & ug/g dry & $8.73 \mathrm{E} 1$ & 3/16/09 & 8L12002 & PNNL-AGG-ICP-AES \\
\hline $7439-93-2$ & Lithium & $<2.12 \mathrm{E} 1$ & ug/g dry & $2.12 \mathrm{E} 1$ & 3/16/09 & 8L12002 & PNNL-AGG-ICP-AES \\
\hline $7439-95-4$ & Magnesium & $3.55 \mathrm{E} 3$ & $\mathrm{ug} / \mathrm{g}$ dry & $6.49 \mathrm{E} 0$ & 3/16/09 & $8 \mathrm{~L} 12002$ & PNNL-AGG-ICP-AES \\
\hline $7439-96-5$ & Manganese & 2.03E2 & ug/g dry & $2.04 \mathrm{E} 0$ & 3/16/09 & 8L12002 & PNNL-AGG-ICP-AES \\
\hline $7439-98-7$ & Molybdenum & $<7.74 \mathrm{E} 0$ & ug/g dry & $7.74 \mathrm{E} 0$ & 3/16/09 & 8L12002 & PNNL-AGG-ICP-AES \\
\hline $7440-02-0$ & Nickel & $7.80 \mathrm{E} 0$ & ug/g dry & 4.73E0 & 3/16/09 & $8 \mathrm{~L} 12002$ & PNNL-AGG-ICP-AES \\
\hline $7723-14-0$ & Phosphorus & $4.04 \mathrm{E} 2$ & ug/g dry & $3.01 \mathrm{E} 1$ & 3/16/09 & $8 \mathrm{~L} 12002$ & PNNL-AGG-ICP-AES \\
\hline $7439-92-1$ & Lead & $<1.02 \mathrm{E} 1$ & $\mathrm{ug} / \mathrm{g}$ dry & $1.02 \mathrm{E} 1$ & 3/16/09 & 8L12002 & PNNL-AGG-ICP-AES \\
\hline $7782-49-2$ & Selenium & $<5.79 \mathrm{E} 1$ & ug/g dry & $5.79 \mathrm{E} 1$ & 3/16/09 & 8L12002 & PNNL-AGG-ICP-AES \\
\hline $7440-24-6$ & Strontium & $1.99 \mathrm{E} 1$ & ug/g dry & $1.49 \mathrm{E} 0$ & 3/16/09 & 8L12002 & PNNL-AGG-ICP-AES \\
\hline $7440-28-0$ & Thallium & $<3.27 \mathrm{E} 1$ & $\mathrm{ug} / \mathrm{g}$ dry & $3.27 \mathrm{E} 1$ & 3/16/09 & $8 \mathrm{~L} 12002$ & PNNL-AGG-ICP-AES \\
\hline $7440-62-2$ & Vanadium & $1.69 \mathrm{E} 1$ & ug/g dry & $4.08 \mathrm{E} 0$ & 3/16/09 & 8L12002 & PNNL-AGG-ICP-AES \\
\hline $7440-66-6$ & Zinc & $2.40 \mathrm{E} 1$ & ug/g dry & $3.72 \mathrm{E} 0$ & 3/16/09 & 8L12002 & PNNL-AGG-ICP-AES \\
\hline $7440-23-5$ & Sodium & $1.12 \mathrm{E} 2$ & $\mathrm{ug} / \mathrm{g}$ dry & $6.42 \mathrm{E} 1$ & 3/16/09 & 8L12002 & PNNL-AGG-ICP-AES \\
\hline $7440-21-3$ & Silicon & $<1.65 \mathrm{E} 2$ & ug/g dry & $1.65 \mathrm{E} 2$ & 3/16/09 & 8L12002 & PNNL-AGG-ICP-AES \\
\hline $7704-34-9$ & Sulfur & $<1.09 \mathrm{E} 2$ & ug/g dry & $1.09 \mathrm{E} 2$ & 3/16/09 & 8L12002 & PNNL-AGG-ICP-AES \\
\hline $7440-32-6$ & Titanium & $4.74 \mathrm{E} 2$ & ug/g dry & $5.22 \mathrm{E} 0$ & 3/16/09 & 8L12002 & PNNL-AGG-ICP-AES \\
\hline $7440-67-7$ & Zirconium & 4.61E0 & ug/g dry & $2.73 \mathrm{E} 0$ & 3/16/09 & 8L12002 & PNNL-AGG-ICP-AES \\
\hline $7440-22-4$ & Silver & $<3.09 \mathrm{E} 0$ & ug/g dry & $3.09 \mathrm{E} 0$ & 3/16/09 & 8L12002 & PNNL-AGG-ICP-AES \\
\hline $7440-15-5$ & Rhenium & $<9.48 \mathrm{E} 0$ & ug/g dry & $9.48 \mathrm{E} 0$ & 3/16/09 & 8L12002 & PNNL-AGG-ICP-AES \\
\hline $7440-36-0$ & Antimony & $<2.26 \mathrm{E} 1$ & ug/g dry & $2.26 \mathrm{E} 1$ & 3/16/09 & 8L12002 & PNNL-AGG-ICP-AES \\
\hline HEIS No. & B1V9B7 & & b ID: & 5003-17 & & & \\
\hline $7429-90-5$ & Aluminum & $5.01 \mathrm{E} 3$ & ug/g dry & $2.26 \mathrm{E} 1$ & 3/16/09 & $8 \mathrm{~L} 12002$ & PNNL-AGG-ICP-AES \\
\hline $7440-38-2$ & Arsenic & $<3.39 \mathrm{E} 1$ & ug/g dry & $3.39 \mathrm{E} 1$ & 3/16/09 & 8L12002 & PNNL-AGG-ICP-AES \\
\hline
\end{tabular}


Total Metals by PNNL-AGG-ICP-AES/Acid Extract

\begin{tabular}{|c|c|c|c|c|c|c|c|}
\hline CAS \# & Analyte & Results & Units & EQL & Analyzed & Batch & Method \\
\hline HEIS No. & B1V9B7 & \multicolumn{3}{|c|}{ Lab ID: $\quad$ 0805003-17 } & & & \\
\hline $7440-42-8$ & Boron & $<9.67 \mathrm{E} 1$ & ug/g dry & $9.67 \mathrm{E} 1$ & $3 / 16 / 09$ & 8L12002 & PNNL-AGG-ICP-AES \\
\hline $7440-39-3$ & Barium & $3.65 \mathrm{E} 1$ & ug/g dry & $1.38 \mathrm{E} 0$ & $3 / 16 / 09$ & 8L12002 & PNNL-AGG-ICP-AES \\
\hline $7440-41-7$ & Beryllium & $<7.71 \mathrm{E}-1$ & ug/g dry & $7.71 \mathrm{E}-1$ & $3 / 16 / 09$ & 8L12002 & PNNL-AGG-ICP-AES \\
\hline $7440-69-9$ & Bismuth & $<9.51 \mathrm{E} 0$ & ug/g dry & $9.51 \mathrm{E} 0$ & $3 / 16 / 09$ & $8 \mathrm{~L} 12002$ & PNNL-AGG-ICP-AES \\
\hline $7440-70-2$ & Calcium & 7.15E3 & $\mathrm{ug} / \mathrm{g}$ dry & $2.34 \mathrm{E} 1$ & $3 / 16 / 09$ & 8L12002 & PNNL-AGG-ICP-AES \\
\hline $7440-43-9$ & Cadmium & $<1.23 \mathrm{E} 0$ & $\mathrm{ug} / \mathrm{g}$ dry & $1.23 \mathrm{E} 0$ & $3 / 16 / 09$ & 8L12002 & PNNL-AGG-ICP-AES \\
\hline $7440-48-4$ & Cobalt & $5.23 \mathrm{E} 0$ & $\mathrm{ug} / \mathrm{g}$ dry & 4.82E0 & $3 / 16 / 09$ & 8L12002 & PNNL-AGG-ICP-AES \\
\hline $7440-47-3$ & Chromium & 8.07E0 & ug/g dry & $1.97 \mathrm{E} 0$ & $3 / 16 / 09$ & 8L12002 & PNNL-AGG-ICP-AES \\
\hline $7440-50-8$ & Copper & 7.02E0 & $\mathrm{ug} / \mathrm{g}$ dry & $6.45 \mathrm{E} 0$ & $3 / 16 / 09$ & 8L12002 & PNNL-AGG-ICP-AES \\
\hline $7439-89-6$ & Iron & $1.25 \mathrm{E} 4$ & $\mathrm{ug} / \mathrm{g}$ dry & $6.07 \mathrm{E} 0$ & $3 / 16 / 09$ & 8L12002 & PNNL-AGG-ICP-AES \\
\hline $7440-09-7$ & Potassium & $8.00 \mathrm{E} 2$ & ug/g dry & $8.64 \mathrm{E} 1$ & $3 / 16 / 09$ & 8L12002 & PNNL-AGG-ICP-AES \\
\hline $7439-93-2$ & Lithium & $<2.10 \mathrm{E} 1$ & ug/g dry & $2.10 \mathrm{E} 1$ & $3 / 16 / 09$ & 8L12002 & PNNL-AGG-ICP-AES \\
\hline $7439-95-4$ & Magnesium & $4.18 \mathrm{E} 3$ & ug/g dry & $6.43 \mathrm{E} 0$ & $3 / 16 / 09$ & $8 \mathrm{~L} 12002$ & PNNL-AGG-ICP-AES \\
\hline $7439-96-5$ & Manganese & $2.43 \mathrm{E} 2$ & ug/g dry & $2.02 \mathrm{E} 0$ & $3 / 16 / 09$ & 8L12002 & PNNL-AGG-ICP-AES \\
\hline $7439-98-7$ & Molybdenum & $<7.66 \mathrm{E} 0$ & ug/g dry & $7.66 \mathrm{E} 0$ & $3 / 16 / 09$ & 8L12002 & PNNL-AGG-ICP-AES \\
\hline $7440-02-0$ & Nickel & $8.87 \mathrm{E} 0$ & $\mathrm{ug} / \mathrm{g}$ dry & 4.68E0 & $3 / 16 / 09$ & 8L12002 & PNNL-AGG-ICP-AES \\
\hline $7723-14-0$ & Phosphorus & $4.52 \mathrm{E} 2$ & ug/g dry & $2.98 \mathrm{E} 1$ & $3 / 16 / 09$ & 8L12002 & PNNL-AGG-ICP-AES \\
\hline $7439-92-1$ & Lead & $<1.01 \mathrm{E} 1$ & $\mathrm{ug} / \mathrm{g}$ dry & $1.01 \mathrm{E} 1$ & $3 / 16 / 09$ & 8L12002 & PNNL-AGG-ICP-AES \\
\hline $7782-49-2$ & Selenium & $<5.73 \mathrm{E} 1$ & ug/g dry & $5.73 \mathrm{E} 1$ & $3 / 16 / 09$ & 8L12002 & PNNL-AGG-ICP-AES \\
\hline $7440-24-6$ & Strontium & $2.25 \mathrm{E} 1$ & ug/g dry & $1.47 \mathrm{E} 0$ & $3 / 16 / 09$ & 8L12002 & PNNL-AGG-ICP-AES \\
\hline $7440-28-0$ & Thallium & $<3.24 \mathrm{E} 1$ & $\mathrm{ug} / \mathrm{g}$ dry & $3.24 \mathrm{E} 1$ & $3 / 16 / 09$ & 8L12002 & PNNL-AGG-ICP-AES \\
\hline $7440-62-2$ & Vanadium & $1.68 \mathrm{E} 1$ & ug/g dry & 4.04E0 & $3 / 16 / 09$ & 8L12002 & PNNL-AGG-ICP-AES \\
\hline $7440-66-6$ & Zinc & $3.22 \mathrm{E} 1$ & ug/g dry & $3.68 \mathrm{E} 0$ & $3 / 16 / 09$ & 8L12002 & PNNL-AGG-ICP-AES \\
\hline $7440-23-5$ & Sodium & $1.25 \mathrm{E} 2$ & $\mathrm{ug} / \mathrm{g}$ dry & $6.36 \mathrm{E} 1$ & $3 / 16 / 09$ & $8 \mathrm{~L} 12002$ & PNNL-AGG-ICP-AES \\
\hline $7440-21-3$ & Silicon & $<1.63 \mathrm{E} 2$ & $\mathrm{ug} / \mathrm{g}$ dry & $1.63 \mathrm{E} 2$ & $3 / 16 / 09$ & 8L12002 & PNNL-AGG-ICP-AES \\
\hline $7704-34-9$ & Sulfur & $<1.08 \mathrm{E} 2$ & ug/g dry & $1.08 \mathrm{E} 2$ & $3 / 16 / 09$ & 8L12002 & PNNL-AGG-ICP-AES \\
\hline $7440-32-6$ & Titanium & $4.40 \mathrm{E} 2$ & ug/g dry & $5.17 \mathrm{E} 0$ & $3 / 16 / 09$ & 8L12002 & PNNL-AGG-ICP-AES \\
\hline $7440-67-7$ & Zirconium & $4.51 \mathrm{E} 0$ & ug/g dry & $2.70 \mathrm{E} 0$ & $3 / 16 / 09$ & $8 \mathrm{~L} 12002$ & PNNL-AGG-ICP-AES \\
\hline $7440-22-4$ & Silver & $<3.06 \mathrm{E} 0$ & ug/g dry & $3.06 \mathrm{E} 0$ & $3 / 16 / 09$ & $8 \mathrm{~L} 12002$ & PNNL-AGG-ICP-AES \\
\hline $7440-15-5$ & Rhenium & $<9.39 \mathrm{E} 0$ & ug/g dry & 9.39E0 & $3 / 16 / 09$ & 8L12002 & PNNL-AGG-ICP-AES \\
\hline $7440-36-0$ & Antimony & $<2.24 \mathrm{E} 1$ & ug/g dry & $2.24 \mathrm{E} 1$ & $3 / 16 / 09$ & $8 \mathrm{~L} 12002$ & PNNL-AGG-ICP-AES \\
\hline HEIS No. & B1V9B9 & & b ID: & 5003-19 & & & \\
\hline $7429-90-5$ & Aluminum & 4.91E3 & $\mathrm{ug} / \mathrm{g}$ dry & $2.30 \mathrm{E} 1$ & $3 / 16 / 09$ & $8 \mathrm{~L} 12002$ & PNNL-AGG-ICP-AES \\
\hline $7440-38-2$ & Arsenic & $<3.45 \mathrm{E} 1$ & $\mathrm{ug} / \mathrm{g}$ dry & $3.45 \mathrm{E} 1$ & $3 / 16 / 09$ & $8 \mathrm{~L} 12002$ & PNNL-AGG-ICP-AES \\
\hline $7440-42-8$ & Boron & $<9.86 \mathrm{E} 1$ & ug/g dry & $9.86 \mathrm{E} 1$ & $3 / 16 / 09$ & 8L12002 & PNNL-AGG-ICP-AES \\
\hline $7440-39-3$ & Barium & $4.44 \mathrm{E} 1$ & ug/g dry & $1.40 \mathrm{E} 0$ & $3 / 16 / 09$ & $8 \mathrm{~L} 12002$ & PNNL-AGG-ICP-AES \\
\hline $7440-41-7$ & Beryllium & $<7.86 \mathrm{E}-1$ & $\mathrm{ug} / \mathrm{g}$ dry & $7.86 \mathrm{E}-1$ & $3 / 16 / 09$ & 8L12002 & PNNL-AGG-ICP-AES \\
\hline $7440-69-9$ & Bismuth & $<9.69 \mathrm{E} 0$ & ug/g dry & $9.69 \mathrm{E} 0$ & $3 / 16 / 09$ & 8L12002 & PNNL-AGG-ICP-AES \\
\hline $7440-70-2$ & Calcium & $7.27 \mathrm{E} 3$ & ug/g dry & $2.39 \mathrm{E} 1$ & $3 / 16 / 09$ & 8L12002 & PNNL-AGG-ICP-AES \\
\hline $7440-43-9$ & Cadmium & $<1.25 \mathrm{E} 0$ & $\mathrm{ug} / \mathrm{g}$ dry & $1.25 \mathrm{E} 0$ & $3 / 16 / 09$ & $8 \mathrm{~L} 12002$ & PNNL-AGG-ICP-AES \\
\hline $7440-48-4$ & Cobalt & $<4.92 \mathrm{E} 0$ & $\mathrm{ug} / \mathrm{g}$ dry & 4.92E0 & $3 / 16 / 09$ & $8 \mathrm{~L} 12002$ & PNNL-AGG-ICP-AES \\
\hline $7440-47-3$ & Chromium & $6.95 \mathrm{E} 0$ & ug/g dry & $2.01 \mathrm{E} 0$ & $3 / 16 / 09$ & 8L12002 & PNNL-AGG-ICP-AES \\
\hline $7440-50-8$ & Copper & 7.44E0 & ug/g dry & $6.58 \mathrm{E} 0$ & $3 / 16 / 09$ & 8L12002 & PNNL-AGG-ICP-AES \\
\hline $7439-89-6$ & Iron & $1.11 \mathrm{E} 4$ & ug/g dry & $6.19 \mathrm{E} 0$ & $3 / 16 / 09$ & 8L12002 & PNNL-AGG-ICP-AES \\
\hline $7440-09-7$ & Potassium & $8.47 \mathrm{E} 2$ & $\mathrm{ug} / \mathrm{g}$ dry & $8.81 \mathrm{E} 1$ & $3 / 16 / 09$ & $8 \mathrm{~L} 12002$ & PNNL-AGG-ICP-AES \\
\hline $7439-93-2$ & Lithium & $<2.14 \mathrm{E} 1$ & ug/g dry & $2.14 \mathrm{E} 1$ & $3 / 16 / 09$ & 8L12002 & PNNL-AGG-ICP-AES \\
\hline $7439-95-4$ & Magnesium & $3.85 \mathrm{E} 3$ & $\mathrm{ug} / \mathrm{g}$ dry & $6.55 \mathrm{E} 0$ & $3 / 16 / 09$ & $8 \mathrm{~L} 12002$ & PNNL-AGG-ICP-AES \\
\hline $7439-96-5$ & Manganese & $2.32 \mathrm{E} 2$ & ug/g dry & $2.06 \mathrm{E} 0$ & $3 / 16 / 09$ & 8L12002 & PNNL-AGG-ICP-AES \\
\hline
\end{tabular}


Total Metals by PNNL-AGG-ICP-AES/Acid Extract

\begin{tabular}{|c|c|c|c|c|c|c|c|}
\hline CAS \# & Analyte & Results & Units & EQL & Analyzed & Batch & Method \\
\hline HEIS No. & B1V9B9 & \multicolumn{3}{|c|}{ Lab ID: $\quad$ 0805003-19 } & & & \\
\hline 7439-98-7 & Molybdenum & $<7.81 \mathrm{E} 0$ & ug/g dry & $7.81 \mathrm{E} 0$ & $3 / 16 / 09$ & 8L12002 & PNNL-AGG-ICP-AES \\
\hline $7440-02-0$ & Nickel & 7.24E0 & ug/g dry & 4.77E0 & $3 / 16 / 09$ & 8L12002 & PNNL-AGG-ICP-AES \\
\hline $7723-14-0$ & Phosphorus & $4.09 \mathrm{E} 2$ & ug/g dry & 3.04E1 & $3 / 16 / 09$ & 8L12002 & PNNL-AGG-ICP-AES \\
\hline $7439-92-1$ & Lead & $<1.03 \mathrm{E} 1$ & $\mathrm{ug} / \mathrm{g}$ dry & $1.03 \mathrm{E} 1$ & $3 / 16 / 09$ & 8L12002 & PNNL-AGG-ICP-AES \\
\hline $7782-49-2$ & Selenium & $<5.84 \mathrm{E} 1$ & $\mathrm{ug} / \mathrm{g}$ dry & $5.84 \mathrm{E} 1$ & $3 / 16 / 09$ & 8L12002 & PNNL-AGG-ICP-AES \\
\hline $7440-24-6$ & Strontium & $2.64 \mathrm{E} 1$ & ug/g dry & $1.50 \mathrm{E} 0$ & $3 / 16 / 09$ & 8L12002 & PNNL-AGG-ICP-AES \\
\hline $7440-28-0$ & Thallium & $<3.30 \mathrm{E} 1$ & $\mathrm{ug} / \mathrm{g}$ dry & $3.30 \mathrm{E} 1$ & $3 / 16 / 09$ & 8L12002 & PNNL-AGG-ICP-AES \\
\hline $7440-62-2$ & Vanadium & $1.51 \mathrm{E} 1$ & ug/g dry & $4.11 \mathrm{E} 0$ & $3 / 16 / 09$ & 8L12002 & PNNL-AGG-ICP-AES \\
\hline $7440-66-6$ & Zinc & $2.57 \mathrm{E} 1$ & $\mathrm{ug} / \mathrm{g}$ dry & $3.75 \mathrm{E} 0$ & $3 / 16 / 09$ & 8L12002 & PNNL-AGG-ICP-AES \\
\hline $7440-23-5$ & Sodium & $1.32 \mathrm{E} 2$ & $\mathrm{ug} / \mathrm{g}$ dry & $6.48 \mathrm{E} 1$ & $3 / 16 / 09$ & 8L12002 & PNNL-AGG-ICP-AES \\
\hline $7440-21-3$ & Silicon & $<1.66 \mathrm{E} 2$ & $\mathrm{ug} / \mathrm{g}$ dry & $1.66 \mathrm{E} 2$ & $3 / 16 / 09$ & $8 \mathrm{~L} 12002$ & PNNL-AGG-ICP-AES \\
\hline 7704-34-9 & Sulfur & $<1.10 \mathrm{E} 2$ & $\mathrm{ug} / \mathrm{g}$ dry & $1.10 \mathrm{E} 2$ & $3 / 16 / 09$ & 8L12002 & PNNL-AGG-ICP-AES \\
\hline $7440-32-6$ & Titanium & $4.38 \mathrm{E} 2$ & ug/g dry & $5.27 \mathrm{E} 0$ & $3 / 16 / 09$ & $8 \mathrm{~L} 12002$ & PNNL-AGG-ICP-AES \\
\hline $7440-67-7$ & Zirconium & 4.66E0 & ug/g dry & $2.76 \mathrm{E} 0$ & $3 / 16 / 09$ & 8L12002 & PNNL-AGG-ICP-AES \\
\hline $7440-22-4$ & Silver & $<3.12 \mathrm{E} 0$ & ug/g dry & $3.12 \mathrm{E} 0$ & $3 / 16 / 09$ & 8L12002 & PNNL-AGG-ICP-AES \\
\hline $7440-15-5$ & Rhenium & $<9.57 \mathrm{E} 0$ & $\mathrm{ug} / \mathrm{g}$ dry & $9.57 \mathrm{E} 0$ & $3 / 16 / 09$ & 8L12002 & PNNL-AGG-ICP-AES \\
\hline $7440-36-0$ & Antimony & $<2.28 \mathrm{E} 1$ & ug/g dry & $2.28 \mathrm{E} 1$ & $3 / 16 / 09$ & 8L12002 & PNNL-AGG-ICP-AES \\
\hline HEIS No. & B1V9C1 & \multicolumn{3}{|c|}{ Lab ID: $\quad$ 0805003-21 } & & & \\
\hline $7429-90-5$ & Aluminum & $4.66 \mathrm{E} 3$ & ug/g dry & $2.27 \mathrm{E} 1$ & $3 / 16 / 09$ & 8L12002 & PNNL-AGG-ICP-AES \\
\hline $7440-38-2$ & Arsenic & $<3.41 \mathrm{E} 1$ & $\mathrm{ug} / \mathrm{g}$ dry & $3.41 \mathrm{E} 1$ & $3 / 16 / 09$ & 8L12002 & PNNL-AGG-ICP-AES \\
\hline $7440-42-8$ & Boron & $<9.75 \mathrm{E} 1$ & ug/g dry & $9.75 \mathrm{E} 1$ & $3 / 16 / 09$ & 8L12002 & PNNL-AGG-ICP-AES \\
\hline $7440-39-3$ & Barium & $3.94 \mathrm{E} 1$ & $\mathrm{ug} / \mathrm{g}$ dry & $1.39 \mathrm{E} 0$ & $3 / 16 / 09$ & $8 \mathrm{~L} 12002$ & PNNL-AGG-ICP-AES \\
\hline $7440-41-7$ & Beryllium & $<7.77 \mathrm{E}-1$ & ug/g dry & 7.77E-1 & $3 / 16 / 09$ & $8 \mathrm{~L} 12002$ & PNNL-AGG-ICP-AES \\
\hline $7440-69-9$ & Bismuth & $<9.58 \mathrm{E} 0$ & ug/g dry & $9.58 \mathrm{E} 0$ & $3 / 16 / 09$ & 8L12002 & PNNL-AGG-ICP-AES \\
\hline $7440-70-2$ & Calcium & 7.01E3 & ug/g dry & $2.36 \mathrm{E} 1$ & $3 / 16 / 09$ & $8 \mathrm{~L} 12002$ & PNNL-AGG-ICP-AES \\
\hline $7440-43-9$ & Cadmium & $<1.24 \mathrm{E} 0$ & $\mathrm{ug} / \mathrm{g}$ dry & $1.24 \mathrm{E} 0$ & $3 / 16 / 09$ & 8L12002 & PNNL-AGG-ICP-AES \\
\hline $7440-48-4$ & Cobalt & $<4.86 \mathrm{E} 0$ & ug/g dry & $4.86 \mathrm{E} 0$ & $3 / 16 / 09$ & 8L12002 & PNNL-AGG-ICP-AES \\
\hline $7440-47-3$ & Chromium & $8.00 \mathrm{E} 0$ & ug/g dry & $1.98 \mathrm{E} 0$ & $3 / 16 / 09$ & $8 \mathrm{~L} 12002$ & PNNL-AGG-ICP-AES \\
\hline $7440-50-8$ & Copper & $<6.50 \mathrm{E} 0$ & ug/g dry & $6.50 \mathrm{E} 0$ & $3 / 16 / 09$ & 8L12002 & PNNL-AGG-ICP-AES \\
\hline $7439-89-6$ & Iron & $1.08 \mathrm{E} 4$ & $\mathrm{ug} / \mathrm{g}$ dry & $6.11 \mathrm{E} 0$ & 3/16/09 & 8L12002 & PNNL-AGG-ICP-AES \\
\hline $7440-09-7$ & Potassium & $8.00 \mathrm{E} 2$ & ug/g dry & $8.70 \mathrm{E} 1$ & $3 / 16 / 09$ & 8L12002 & PNNL-AGG-ICP-AES \\
\hline $7439-93-2$ & Lithium & $<2.11 \mathrm{E} 1$ & ug/g dry & $2.11 \mathrm{E} 1$ & $3 / 16 / 09$ & 8L12002 & PNNL-AGG-ICP-AES \\
\hline $7439-95-4$ & Magnesium & $4.07 \mathrm{E} 3$ & $\mathrm{ug} / \mathrm{g}$ dry & $6.48 \mathrm{E} 0$ & $3 / 16 / 09$ & $8 \mathrm{~L} 12002$ & PNNL-AGG-ICP-AES \\
\hline $7439-96-5$ & Manganese & $2.03 \mathrm{E} 2$ & $\mathrm{ug} / \mathrm{g}$ dry & 2.03E0 & $3 / 16 / 09$ & 8L12002 & PNNL-AGG-ICP-AES \\
\hline $7439-98-7$ & Molybdenum & $<7.72 \mathrm{E} 0$ & ug/g dry & 7.72E0 & $3 / 16 / 09$ & 8L12002 & PNNL-AGG-ICP-AES \\
\hline $7440-02-0$ & Nickel & $9.04 \mathrm{E} 0$ & ug/g dry & 4.71E0 & $3 / 16 / 09$ & $8 \mathrm{~L} 12002$ & PNNL-AGG-ICP-AES \\
\hline $7723-14-0$ & Phosphorus & $3.82 \mathrm{E} 2$ & $\mathrm{ug} / \mathrm{g}$ dry & $3.00 \mathrm{E} 1$ & $3 / 16 / 09$ & 8L12002 & PNNL-AGG-ICP-AES \\
\hline $7439-92-1$ & Lead & $<1.02 \mathrm{E} 1$ & ug/g dry & $1.02 \mathrm{E} 1$ & $3 / 16 / 09$ & 8L12002 & PNNL-AGG-ICP-AES \\
\hline $7782-49-2$ & Selenium & $<5.77 \mathrm{E} 1$ & ug/g dry & $5.77 \mathrm{E} 1$ & $3 / 16 / 09$ & 8L12002 & PNNL-AGG-ICP-AES \\
\hline $7440-24-6$ & Strontium & $2.18 \mathrm{E} 1$ & $\mathrm{ug} / \mathrm{g}$ dry & $1.48 \mathrm{E} 0$ & $3 / 16 / 09$ & 8L12002 & PNNL-AGG-ICP-AES \\
\hline $7440-28-0$ & Thallium & $<3.26 \mathrm{E} 1$ & $\mathrm{ug} / \mathrm{g}$ dry & $3.26 \mathrm{E} 1$ & $3 / 16 / 09$ & $8 \mathrm{~L} 12002$ & PNNL-AGG-ICP-AES \\
\hline $7440-62-2$ & Vanadium & $1.47 \mathrm{E} 1$ & ug/g dry & 4.07E0 & $3 / 16 / 09$ & 8L12002 & PNNL-AGG-ICP-AES \\
\hline $7440-66-6$ & Zinc & $2.36 \mathrm{E} 1$ & ug/g dry & $3.71 \mathrm{E} 0$ & $3 / 16 / 09$ & 8L12002 & PNNL-AGG-ICP-AES \\
\hline $7440-23-5$ & Sodium & $1.30 \mathrm{E} 2$ & ug/g dry & $6.41 \mathrm{E} 1$ & $3 / 16 / 09$ & 8L12002 & PNNL-AGG-ICP-AES \\
\hline $7440-21-3$ & Silicon & $<1.65 \mathrm{E} 2$ & $\mathrm{ug} / \mathrm{g}$ dry & $1.65 \mathrm{E} 2$ & $3 / 16 / 09$ & $8 \mathrm{~L} 12002$ & PNNL-AGG-ICP-AES \\
\hline $7704-34-9$ & Sulfur & $<1.08 \mathrm{E} 2$ & ug/g dry & $1.08 \mathrm{E} 2$ & $3 / 16 / 09$ & 8L12002 & PNNL-AGG-ICP-AES \\
\hline $7440-32-6$ & Titanium & $4.40 \mathrm{E} 2$ & $\mathrm{ug} / \mathrm{g}$ dry & $5.21 \mathrm{E} 0$ & $3 / 16 / 09$ & $8 \mathrm{~L} 12002$ & PNNL-AGG-ICP-AES \\
\hline $7440-67-7$ & Zirconium & $4.51 \mathrm{E} 0$ & ug/g dry & $2.72 \mathrm{E} 0$ & $3 / 16 / 09$ & 8L12002 & PNNL-AGG-ICP-AES \\
\hline
\end{tabular}


Total Metals by PNNL-AGG-ICP-AES/Acid Extract

\begin{tabular}{|c|c|c|c|c|c|c|c|}
\hline CAS \# & Analyte & Results & Units & EQL & Analyzed & Batch & Method \\
\hline HEIS No. & B1V9C1 & \multicolumn{3}{|c|}{ Lab ID: $\quad$ 0805003-21 } & & & \\
\hline $7440-22-4$ & Silver & $<3.08 \mathrm{E} 0$ & ug/g dry & $3.08 \mathrm{E} 0$ & $3 / 16 / 09$ & 8L12002 & PNNL-AGG-ICP-AES \\
\hline $7440-15-5$ & Rhenium & $<9.46 \mathrm{E} 0$ & ug/g dry & $9.46 \mathrm{E} 0$ & $3 / 16 / 09$ & 8L12002 & PNNL-AGG-ICP-AES \\
\hline $7440-36-0$ & Antimony & $<2.26 \mathrm{E} 1$ & ug/g dry & $2.26 \mathrm{E} 1$ & $3 / 16 / 09$ & 8L12002 & PNNL-AGG-ICP-AES \\
\hline HEIS No. & B1V9C3 & \multicolumn{3}{|c|}{ Lab ID: $\quad$ 0805003-23 } & & & \\
\hline $7429-90-5$ & Aluminum & $4.60 \mathrm{E} 3$ & ug/g dry & $2.32 \mathrm{E} 1$ & $3 / 16 / 09$ & 8L12002 & PNNL-AGG-ICP-AES \\
\hline $7440-38-2$ & Arsenic & $<3.48 \mathrm{E} 1$ & ug/g dry & $3.48 \mathrm{E} 1$ & $3 / 16 / 09$ & 8L12002 & PNNL-AGG-ICP-AES \\
\hline $7440-42-8$ & Boron & $<9.95 \mathrm{E} 1$ & ug/g dry & $9.95 \mathrm{E} 1$ & $3 / 16 / 09$ & 8L12002 & PNNL-AGG-ICP-AES \\
\hline $7440-39-3$ & Barium & $3.15 \mathrm{E} 1$ & ug/g dry & $1.41 \mathrm{E} 0$ & $3 / 16 / 09$ & 8L12002 & PNNL-AGG-ICP-AES \\
\hline $7440-41-7$ & Beryllium & $<7.93 \mathrm{E}-1$ & ug/g dry & $7.93 \mathrm{E}-1$ & $3 / 16 / 09$ & 8L12002 & PNNL-AGG-ICP-AES \\
\hline $7440-69-9$ & Bismuth & $<9.78 \mathrm{E} 0$ & ug/g dry & $9.78 \mathrm{E} 0$ & $3 / 16 / 09$ & 8L12002 & PNNL-AGG-ICP-AES \\
\hline $7440-70-2$ & Calcium & $5.75 \mathrm{E} 3$ & ug/g dry & $2.41 \mathrm{E} 1$ & $3 / 16 / 09$ & 8L12002 & PNNL-AGG-ICP-AES \\
\hline $7440-43-9$ & Cadmium & $<1.26 \mathrm{E} 0$ & ug/g dry & $1.26 \mathrm{E} 0$ & $3 / 16 / 09$ & 8L12002 & PNNL-AGG-ICP-AES \\
\hline $7440-48-4$ & Cobalt & $5.00 \mathrm{E} 0$ & ug/g dry & $4.96 \mathrm{E} 0$ & $3 / 16 / 09$ & 8L12002 & PNNL-AGG-ICP-AES \\
\hline $7440-47-3$ & Chromium & 7.83E0 & ug/g dry & $2.02 \mathrm{E} 0$ & $3 / 16 / 09$ & 8L12002 & PNNL-AGG-ICP-AES \\
\hline $7440-50-8$ & Copper & $<6.64 \mathrm{E} 0$ & ug/g dry & $6.64 \mathrm{E} 0$ & $3 / 16 / 09$ & 8L12002 & PNNL-AGG-ICP-AES \\
\hline $7439-89-6$ & Iron & $1.05 \mathrm{E} 4$ & ug/g dry & $6.24 \mathrm{E} 0$ & $3 / 16 / 09$ & 8L12002 & PNNL-AGG-ICP-AES \\
\hline 7440-09-7 & Potassium & $7.88 \mathrm{E} 2$ & ug/g dry & $8.88 \mathrm{E} 1$ & $3 / 16 / 09$ & 8L12002 & PNNL-AGG-ICP-AES \\
\hline $7439-93-2$ & Lithium & $<2.16 \mathrm{E} 1$ & ug/g dry & $2.16 \mathrm{E} 1$ & $3 / 16 / 09$ & 8L12002 & PNNL-AGG-ICP-AES \\
\hline $7439-95-4$ & Magnesium & $3.50 \mathrm{E} 3$ & ug/g dry & $6.61 \mathrm{E} 0$ & $3 / 16 / 09$ & 8L12002 & PNNL-AGG-ICP-AES \\
\hline $7439-96-5$ & Manganese & $1.96 \mathrm{E} 2$ & ug/g dry & $2.07 \mathrm{E} 0$ & $3 / 16 / 09$ & 8L12002 & PNNL-AGG-ICP-AES \\
\hline $7439-98-7$ & Molybdenum & $<7.88 \mathrm{E} 0$ & ug/g dry & $7.88 \mathrm{E} 0$ & $3 / 16 / 09$ & 8L12002 & PNNL-AGG-ICP-AES \\
\hline $7440-02-0$ & Nickel & $1.30 \mathrm{E} 1$ & ug/g dry & $4.81 \mathrm{E} 0$ & $3 / 16 / 09$ & 8L12002 & PNNL-AGG-ICP-AES \\
\hline $7723-14-0$ & Phosphorus & $3.54 \mathrm{E} 2$ & ug/g dry & $3.07 \mathrm{E} 1$ & $3 / 16 / 09$ & 8L12002 & PNNL-AGG-ICP-AES \\
\hline $7439-92-1$ & Lead & $<1.04 \mathrm{E} 1$ & ug/g dry & $1.04 \mathrm{E} 1$ & $3 / 16 / 09$ & 8L12002 & PNNL-AGG-ICP-AES \\
\hline $7782-49-2$ & Selenium & $<5.89 \mathrm{E} 1$ & ug/g dry & $5.89 \mathrm{E} 1$ & $3 / 16 / 09$ & 8L12002 & PNNL-AGG-ICP-AES \\
\hline $7440-24-6$ & Strontium & $2.00 \mathrm{E} 1$ & ug/g dry & $1.51 \mathrm{E} 0$ & $3 / 16 / 09$ & 8L12002 & PNNL-AGG-ICP-AES \\
\hline $7440-28-0$ & Thallium & $<3.33 \mathrm{E} 1$ & ug/g dry & $3.33 \mathrm{E} 1$ & $3 / 16 / 09$ & 8L12002 & PNNL-AGG-ICP-AES \\
\hline $7440-62-2$ & Vanadium & $1.63 \mathrm{E} 1$ & ug/g dry & $4.15 \mathrm{E} 0$ & $3 / 16 / 09$ & 8L12002 & PNNL-AGG-ICP-AES \\
\hline $7440-66-6$ & Zinc & $2.32 \mathrm{E} 1$ & ug/g dry & $3.78 \mathrm{E} 0$ & $3 / 16 / 09$ & 8L12002 & PNNL-AGG-ICP-AES \\
\hline $7440-23-5$ & Sodium & $1.31 \mathrm{E} 2$ & ug/g dry & $6.54 \mathrm{E} 1$ & $3 / 16 / 09$ & 8L12002 & PNNL-AGG-ICP-AES \\
\hline $7440-21-3$ & Silicon & $<1.68 \mathrm{E} 2$ & ug/g dry & $1.68 \mathrm{E} 2$ & $3 / 16 / 09$ & 8L12002 & PNNL-AGG-ICP-AES \\
\hline 7704-34-9 & Sulfur & $<1.11 \mathrm{E} 2$ & ug/g dry & $1.11 \mathrm{E} 2$ & $3 / 16 / 09$ & 8L12002 & PNNL-AGG-ICP-AES \\
\hline $7440-32-6$ & Titanium & 4.64E2 & ug/g dry & $5.31 \mathrm{E} 0$ & $3 / 16 / 09$ & 8L12002 & PNNL-AGG-ICP-AES \\
\hline $7440-67-7$ & Zirconium & $4.97 \mathrm{E} 0$ & ug/g dry & $2.78 \mathrm{E} 0$ & $3 / 16 / 09$ & 8L12002 & PNNL-AGG-ICP-AES \\
\hline $7440-22-4$ & Silver & $<3.14 \mathrm{E} 0$ & ug/g dry & $3.14 \mathrm{E} 0$ & $3 / 16 / 09$ & 8L12002 & PNNL-AGG-ICP-AES \\
\hline $7440-15-5$ & Rhenium & $<9.65 \mathrm{E} 0$ & ug/g dry & $9.65 \mathrm{E} 0$ & $3 / 16 / 09$ & 8L12002 & PNNL-AGG-ICP-AES \\
\hline $7440-36-0$ & Antimony & $<2.30 \mathrm{E} 1$ & ug/g dry & $2.30 \mathrm{E} 1$ & $3 / 16 / 09$ & 8L12002 & PNNL-AGG-ICP-AES \\
\hline HEIS No. & B1V2V5 & & b ID: & $5003-25$ & & & \\
\hline $7429-90-5$ & Aluminum & 4.72E3 & ug/g dry & $2.29 \mathrm{E} 1$ & $3 / 16 / 09$ & 8L12002 & PNNL-AGG-ICP-AES \\
\hline $7440-38-2$ & Arsenic & $<3.43 \mathrm{E} 1$ & ug/g dry & $3.43 \mathrm{E} 1$ & $3 / 16 / 09$ & 8L12002 & PNNL-AGG-ICP-AES \\
\hline $7440-42-8$ & Boron & $<9.81 \mathrm{E} 1$ & ug/g dry & $9.81 \mathrm{E} 1$ & $3 / 16 / 09$ & 8L12002 & PNNL-AGG-ICP-AES \\
\hline $7440-39-3$ & Barium & $4.01 \mathrm{E} 1$ & ug/g dry & $1.39 \mathrm{E} 0$ & $3 / 16 / 09$ & 8L12002 & PNNL-AGG-ICP-AES \\
\hline $7440-41-7$ & Beryllium & $<7.82 \mathrm{E}-1$ & ug/g dry & $7.82 \mathrm{E}-1$ & $3 / 16 / 09$ & 8L12002 & PNNL-AGG-ICP-AES \\
\hline $7440-69-9$ & Bismuth & $<9.64 \mathrm{E} 0$ & ug/g dry & $9.64 \mathrm{E} 0$ & $3 / 16 / 09$ & 8L12002 & PNNL-AGG-ICP-AES \\
\hline $7440-70-2$ & Calcium & $6.67 \mathrm{E} 3$ & ug/g dry & $2.37 \mathrm{E} 1$ & $3 / 16 / 09$ & $8 \mathrm{~L} 12002$ & PNNL-AGG-ICP-AES \\
\hline $7440-43-9$ & Cadmium & $<1.25 \mathrm{E} 0$ & ug/g dry & $1.25 \mathrm{E} 0$ & $3 / 16 / 09$ & 8L12002 & PNNL-AGG-ICP-AES \\
\hline $7440-48-4$ & Cobalt & $5.11 \mathrm{E} 0$ & ug/g dry & 4.89E0 & $3 / 16 / 09$ & 8L12002 & PNNL-AGG-ICP-AES \\
\hline $7440-47-3$ & Chromium & $6.57 \mathrm{E} 0$ & ug/g dry & $1.99 \mathrm{E} 0$ & $3 / 16 / 09$ & 8L12002 & PNNL-AGG-ICP-AES \\
\hline
\end{tabular}


Total Metals by PNNL-AGG-ICP-AES/Acid Extract

\begin{tabular}{|c|c|c|c|c|c|c|c|}
\hline CAS \# & Analyte & Results & Units & EQL & Analyzed & Batch & Method \\
\hline HEIS No. & B1V2V5 & \multicolumn{3}{|c|}{ Lab ID: $\quad$ 0805003-25 } & & & \\
\hline $7440-50-8$ & Copper & $7.47 \mathrm{E} 0$ & ug/g dry & $6.54 \mathrm{E} 0$ & $3 / 16 / 09$ & 8L12002 & PNNL-AGG-ICP-AES \\
\hline $7439-89-6$ & Iron & $1.11 \mathrm{E} 4$ & ug/g dry & $6.15 \mathrm{E} 0$ & $3 / 16 / 09$ & 8L12002 & PNNL-AGG-ICP-AES \\
\hline $7440-09-7$ & Potassium & $7.66 \mathrm{E} 2$ & ug/g dry & $8.76 \mathrm{E} 1$ & $3 / 16 / 09$ & 8L12002 & PNNL-AGG-ICP-AES \\
\hline $7439-93-2$ & Lithium & $<2.13 \mathrm{E} 1$ & ug/g dry & $2.13 \mathrm{E} 1$ & $3 / 16 / 09$ & 8L12002 & PNNL-AGG-ICP-AES \\
\hline $7439-95-4$ & Magnesium & $3.70 \mathrm{E} 3$ & ug/g dry & $6.51 \mathrm{E} 0$ & $3 / 16 / 09$ & 8L12002 & PNNL-AGG-ICP-AES \\
\hline $7439-96-5$ & Manganese & $2.32 \mathrm{E} 2$ & ug/g dry & 2.04E0 & $3 / 16 / 09$ & 8L12002 & PNNL-AGG-ICP-AES \\
\hline 7439-98-7 & Molybdenum & $<7.76 \mathrm{E} 0$ & ug/g dry & $7.76 \mathrm{E} 0$ & $3 / 16 / 09$ & 8L12002 & PNNL-AGG-ICP-AES \\
\hline $7440-02-0$ & Nickel & $8.95 \mathrm{E} 0$ & ug/g dry & 4.74E0 & $3 / 16 / 09$ & 8L12002 & PNNL-AGG-ICP-AES \\
\hline $7723-14-0$ & Phosphorus & $4.00 \mathrm{E} 2$ & ug/g dry & $3.02 \mathrm{E} 1$ & $3 / 16 / 09$ & 8L12002 & PNNL-AGG-ICP-AES \\
\hline $7439-92-1$ & Lead & $<1.02 \mathrm{E} 1$ & ug/g dry & $1.02 \mathrm{E} 1$ & $3 / 16 / 09$ & 8L12002 & PNNL-AGG-ICP-AES \\
\hline $7782-49-2$ & Selenium & $<5.80 \mathrm{E} 1$ & ug/g dry & $5.80 \mathrm{E} 1$ & $3 / 16 / 09$ & 8L12002 & PNNL-AGG-ICP-AES \\
\hline $7440-24-6$ & Strontium & $2.13 \mathrm{E} 1$ & ug/g dry & $1.49 \mathrm{E} 0$ & $3 / 16 / 09$ & 8L12002 & PNNL-AGG-ICP-AES \\
\hline $7440-28-0$ & Thallium & $<3.28 \mathrm{E} 1$ & ug/g dry & $3.28 \mathrm{E} 1$ & $3 / 16 / 09$ & 8L12002 & PNNL-AGG-ICP-AES \\
\hline $7440-62-2$ & Vanadium & $1.61 \mathrm{E} 1$ & ug/g dry & 4.09E0 & $3 / 16 / 09$ & 8L12002 & PNNL-AGG-ICP-AES \\
\hline $7440-66-6$ & Zinc & $2.51 \mathrm{E} 1$ & ug/g dry & $3.73 \mathrm{E} 0$ & $3 / 16 / 09$ & 8L12002 & PNNL-AGG-ICP-AES \\
\hline $7440-23-5$ & Sodium & $1.41 \mathrm{E} 2$ & ug/g dry & $6.44 \mathrm{E} 1$ & $3 / 16 / 09$ & 8L12002 & PNNL-AGG-ICP-AES \\
\hline $7440-21-3$ & Silicon & $<1.66 \mathrm{E} 2$ & ug/g dry & $1.66 \mathrm{E} 2$ & $3 / 16 / 09$ & 8L12002 & PNNL-AGG-ICP-AES \\
\hline $7704-34-9$ & Sulfur & $<1.09 \mathrm{E} 2$ & ug/g dry & $1.09 \mathrm{E} 2$ & $3 / 16 / 09$ & 8L12002 & PNNL-AGG-ICP-AES \\
\hline $7440-32-6$ & Titanium & 4.47E2 & ug/g dry & $5.24 \mathrm{E} 0$ & $3 / 16 / 09$ & 8L12002 & PNNL-AGG-ICP-AES \\
\hline $7440-67-7$ & Zirconium & $4.87 \mathrm{E} 0$ & ug/g dry & $2.74 \mathrm{E} 0$ & $3 / 16 / 09$ & 8L12002 & PNNL-AGG-ICP-AES \\
\hline $7440-22-4$ & Silver & $<3.10 \mathrm{E} 0$ & ug/g dry & $3.10 \mathrm{E} 0$ & $3 / 16 / 09$ & 8L12002 & PNNL-AGG-ICP-AES \\
\hline $7440-15-5$ & Rhenium & $<9.52 \mathrm{E} 0$ & ug/g dry & $9.52 \mathrm{E} 0$ & $3 / 16 / 09$ & 8L12002 & PNNL-AGG-ICP-AES \\
\hline $7440-36-0$ & Antimony & $<2.27 \mathrm{E} 1$ & ug/g dry & $2.27 \mathrm{E} 1$ & $3 / 16 / 09$ & 8L12002 & PNNL-AGG-ICP-AES \\
\hline
\end{tabular}




\section{Radionuclides by ICP-MS/Acid Extract}

\begin{tabular}{|c|c|c|c|c|c|c|c|}
\hline CAS \# & Analyte & Results & Units & EQL & Analyzed & Batch & Method \\
\hline \multirow{3}{*}{$\begin{array}{l}\text { HEIS No. } \\
14133-76-7\end{array}$} & B1V993 & \multicolumn{3}{|c|}{ Lab ID: $\quad$ 0805003-03 } & & & \\
\hline & Technetium-99 & $<3.89 \mathrm{E}-3$ & ug/g dry & $3.89 \mathrm{E}-3$ & $12 / 12 / 08$ & 8L12001 & PNNL-AGG-415 \\
\hline & Uranium 238 & $2.84 \mathrm{E}-1$ & ug/g dry & $2.82 \mathrm{E}-2$ & $12 / 12 / 08$ & 8L12001 & PNNL-AGG-415 \\
\hline \multirow{3}{*}{$\begin{array}{l}\text { HEIS No. } \\
14133-76-7\end{array}$} & B1V995 & \multicolumn{3}{|c|}{ Lab ID: $\quad$ 0805003-05 } & & & \\
\hline & Technetium-99 & $<4.09 \mathrm{E}-3$ & ug/g dry & $4.09 \mathrm{E}-3$ & $12 / 12 / 08$ & 8L12001 & PNNL-AGG-415 \\
\hline & Uranium 238 & $3.36 \mathrm{E}-1$ & ug/g dry & $2.97 \mathrm{E}-2$ & $12 / 12 / 08$ & 8L12001 & PNNL-AGG-415 \\
\hline \multirow{3}{*}{$\begin{array}{l}\text { HEIS No. } \\
14133-76-7\end{array}$} & B1V997 & \multicolumn{3}{|c|}{ Lab ID: $\quad$ 0805003-07 } & & & \\
\hline & Technetium-99 & $<4.13 \mathrm{E}-3$ & ug/g dry & $4.13 \mathrm{E}-3$ & $12 / 12 / 08$ & 8L12001 & PNNL-AGG-415 \\
\hline & Uranium 238 & $4.25 \mathrm{E}-1$ & ug/g dry & $3.00 \mathrm{E}-2$ & $12 / 12 / 08$ & 8L12001 & PNNL-AGG-415 \\
\hline \multirow{3}{*}{$\begin{array}{l}\text { HEIS No. } \\
14133-76-7\end{array}$} & B1V999 & \multicolumn{3}{|c|}{ Lab ID: $\quad$ 0805003-09 } & & & \\
\hline & Technetium-99 & $<4.12 \mathrm{E}-3$ & ug/g dry & $4.12 \mathrm{E}-3$ & $12 / 12 / 08$ & 8L12001 & PNNL-AGG-415 \\
\hline & Uranium 238 & $3.67 \mathrm{E}-1$ & ug/g dry & $2.99 \mathrm{E}-2$ & $12 / 12 / 08$ & 8L12001 & PNNL-AGG-415 \\
\hline \multirow{3}{*}{$\begin{array}{l}\text { HEIS No. } \\
14133-76-7\end{array}$} & B1V9B1 & \multicolumn{3}{|c|}{ Lab ID: $\quad$ 0805003-11 } & & & \\
\hline & Technetium-99 & $<4.20 \mathrm{E}-3$ & ug/g dry & $4.20 \mathrm{E}-3$ & $12 / 12 / 08$ & 8L12001 & PNNL-AGG-415 \\
\hline & Uranium 238 & $3.36 \mathrm{E}-1$ & ug/g dry & $3.05 \mathrm{E}-2$ & $12 / 12 / 08$ & 8L12001 & PNNL-AGG-415 \\
\hline \multirow{3}{*}{$\begin{array}{l}\text { HEIS No. } \\
14133-76-7\end{array}$} & B1V9B3 & \multicolumn{3}{|c|}{ Lab ID: $\quad$ 0805003-13 } & & & \\
\hline & Technetium-99 & $<4.11 \mathrm{E}-3$ & ug/g dry & $4.11 \mathrm{E}-3$ & $12 / 12 / 08$ & 8L12001 & PNNL-AGG-415 \\
\hline & Uranium 238 & $3.44 \mathrm{E}-1$ & ug/g dry & $2.98 \mathrm{E}-2$ & $12 / 12 / 08$ & 8L12001 & PNNL-AGG-415 \\
\hline \multirow{3}{*}{$\begin{array}{l}\text { HEIS No. } \\
14133-76-7\end{array}$} & B1V9B5 & \multicolumn{3}{|c|}{ Lab ID: $\quad$ 0805003-15 } & & & \\
\hline & Technetium-99 & $<4.01 \mathrm{E}-3$ & ug/g dry & $4.01 \mathrm{E}-3$ & $12 / 12 / 08$ & 8L12001 & PNNL-AGG-415 \\
\hline & Uranium 238 & $3.35 \mathrm{E}-1$ & ug/g dry & $2.91 \mathrm{E}-2$ & $12 / 12 / 08$ & 8L12001 & PNNL-AGG-415 \\
\hline \multirow{3}{*}{$\begin{array}{l}\text { HEIS No. } \\
14133-76-7\end{array}$} & B1V9B7 & \multicolumn{3}{|c|}{ Lab ID: $\quad$ 0805003-17 } & & & \\
\hline & Technetium-99 & $<3.97 \mathrm{E}-3$ & ug/g dry & $3.97 \mathrm{E}-3$ & $12 / 12 / 08$ & 8L12001 & PNNL-AGG-415 \\
\hline & Uranium 238 & $3.87 \mathrm{E}-1$ & ug/g dry & $2.88 \mathrm{E}-2$ & $12 / 12 / 08$ & 8L12001 & PNNL-AGG-415 \\
\hline \multirow{3}{*}{$\begin{array}{l}\text { HEIS No. } \\
14133-76-7\end{array}$} & B1V9B9 & \multicolumn{3}{|c|}{ Lab ID: $\quad$ 0805003-19 } & & & \\
\hline & Technetium-99 & $<4.05 \mathrm{E}-3$ & ug/g dry & $4.05 \mathrm{E}-3$ & $12 / 12 / 08$ & 8L12001 & PNNL-AGG-415 \\
\hline & Uranium 238 & $4.10 \mathrm{E}-1$ & ug/g dry & $2.94 \mathrm{E}-2$ & $12 / 12 / 08$ & 8L12001 & PNNL-AGG-415 \\
\hline \multirow{3}{*}{$\begin{array}{l}\text { HEIS No. } \\
14133-76-7\end{array}$} & B1V9C1 & \multicolumn{3}{|c|}{ Lab ID: $\quad$ 0805003-21 } & & & \\
\hline & Technetium-99 & $<4.00 \mathrm{E}-3$ & ug/g dry & $4.00 \mathrm{E}-3$ & $12 / 12 / 08$ & 8L12001 & PNNL-AGG-415 \\
\hline & Uranium 238 & $3.45 \mathrm{E}-1$ & ug/g dry & $2.91 \mathrm{E}-2$ & $12 / 12 / 08$ & $8 \mathrm{~L} 12001$ & PNNL-AGG-415 \\
\hline \multirow{3}{*}{$\begin{array}{l}\text { HEIS No. } \\
14133-76-7\end{array}$} & B1V9C3 & \multicolumn{3}{|c|}{ Lab ID: $\quad$ 0805003-23 } & & & \\
\hline & Technetium-99 & $<4.09 \mathrm{E}-3$ & ug/g dry & $4.09 \mathrm{E}-3$ & $12 / 12 / 08$ & 8L12001 & PNNL-AGG-415 \\
\hline & Uranium 238 & $2.54 \mathrm{E}-1$ & ug/g dry & $2.97 \mathrm{E}-2$ & $12 / 12 / 08$ & 8L12001 & PNNL-AGG-415 \\
\hline \multirow{3}{*}{$\begin{array}{l}\text { HEIS No. } \\
14133-76-7\end{array}$} & B1V2V5 & \multicolumn{3}{|c|}{ Lab ID: $\quad$ 0805003-25 } & & & \\
\hline & Technetium-99 & $<4.03 \mathrm{E}-3$ & ug/g dry & $4.03 \mathrm{E}-3$ & $12 / 12 / 08$ & 8L12001 & PNNL-AGG-415 \\
\hline & Uranium 238 & $3.27 \mathrm{E}-1$ & ug/g dry & $2.92 \mathrm{E}-2$ & $12 / 12 / 08$ & 8L12001 & PNNL-AGG-415 \\
\hline
\end{tabular}




\section{Radionuclides by ICP-MS/Water Extract}

\begin{tabular}{|c|c|c|c|c|c|c|}
\hline CAS \# & Analyte & Results & EQL & Analyzed & Batch & Method \\
\hline \multirow{3}{*}{$\begin{array}{l}\text { HEIS No. } \\
14133-76-7\end{array}$} & B1VDW5 & \multicolumn{2}{|c|}{ Lab ID: $\quad$ 0805001-01 } & & & \\
\hline & Technetium-99 & $<2.29 \mathrm{E}-5 \quad \mathrm{ug} / \mathrm{g}$ dry & $2.29 \mathrm{E}-5$ & $8 / 27 / 08$ & $8 \mathrm{H} 27003$ & PNNL-AGG-415 \\
\hline & Uranium 238 & $<5.61 \mathrm{E}-4 \quad$ ug/g dry & $5.61 \mathrm{E}-4$ & $8 / 27 / 08$ & $8 \mathrm{H} 27003$ & PNNL-AGG-415 \\
\hline \multirow{3}{*}{$\begin{array}{l}\text { HEIS No. } \\
14133-76-7\end{array}$} & B1VDW6 & \multicolumn{2}{|c|}{ Lab ID: $\quad$ 0805001-02 } & & & \\
\hline & Technetium-99 & $<2.25 \mathrm{E}-5 \mathrm{ug} / \mathrm{g}$ dry & $2.25 \mathrm{E}-5$ & $8 / 27 / 08$ & $8 \mathrm{H} 27003$ & PNNL-AGG-415 \\
\hline & Uranium 238 & $<5.50 \mathrm{E}-4 \quad$ ug/g dry & $5.50 \mathrm{E}-4$ & $8 / 27 / 08$ & $8 \mathrm{H} 27003$ & PNNL-AGG-415 \\
\hline \multirow{3}{*}{$\begin{array}{l}\text { HEIS No. } \\
14133-76-7\end{array}$} & B1VDW7 & \multicolumn{2}{|c|}{ Lab ID: $\quad$ 0805001-03 } & & & \\
\hline & Technetium-99 & $<2.28 \mathrm{E}-5 \quad \mathrm{ug} / \mathrm{g}$ dry & $2.28 \mathrm{E}-5$ & $8 / 27 / 08$ & $8 \mathrm{H} 27003$ & PNNL-AGG-415 \\
\hline & Uranium 238 & $<5.60 \mathrm{E}-4 \quad \mathrm{ug} / \mathrm{g}$ dry & $5.60 \mathrm{E}-4$ & $8 / 27 / 08$ & $8 \mathrm{H} 27003$ & PNNL-AGG-415 \\
\hline \multirow{3}{*}{$\begin{array}{l}\text { HEIS No. } \\
14133-76-7\end{array}$} & B1VDW8 & \multicolumn{2}{|c|}{ Lab ID: $\quad$ 0805001-04 } & & & \\
\hline & Technetium-99 & $<2.26 \mathrm{E}-5 \quad$ ug/g dry & $2.26 \mathrm{E}-5$ & $8 / 27 / 08$ & $8 \mathrm{H} 27003$ & PNNL-AGG-415 \\
\hline & Uranium 238 & $<5.55 \mathrm{E}-4$ ug/g dry & $5.55 \mathrm{E}-4$ & $8 / 27 / 08$ & $8 \mathrm{H} 27003$ & PNNL-AGG-415 \\
\hline \multirow{3}{*}{$\begin{array}{l}\text { HEIS No. } \\
14133-76-7\end{array}$} & B1VDW9 & \multicolumn{2}{|c|}{ Lab ID: $\quad$ 0805001-05 } & & & \\
\hline & Technetium-99 & $<2.27 \mathrm{E}-5 \quad \mathrm{ug} / \mathrm{g}$ dry & $2.27 \mathrm{E}-5$ & $8 / 27 / 08$ & $8 \mathrm{H} 27003$ & PNNL-AGG-415 \\
\hline & Uranium 238 & $<5.56 \mathrm{E}-4 \quad$ ug/g dry & $5.56 \mathrm{E}-4$ & $8 / 27 / 08$ & $8 \mathrm{H} 27003$ & PNNL-AGG-415 \\
\hline \multirow{3}{*}{$\begin{array}{l}\text { HEIS No. } \\
14133-76-7\end{array}$} & B1VDX0 & \multicolumn{2}{|c|}{ Lab ID: $\quad$ 0805001-06 } & & & \\
\hline & Technetium-99 & $<2.48 \mathrm{E}-5 \quad$ ug/g dry & $2.48 \mathrm{E}-5$ & $8 / 27 / 08$ & $8 \mathrm{H} 27003$ & PNNL-AGG-415 \\
\hline & Uranium 238 & $<6.08 \mathrm{E}-4$ ug/g dry & $6.08 \mathrm{E}-4$ & $8 / 27 / 08$ & $8 \mathrm{H} 27003$ & PNNL-AGG-415 \\
\hline \multirow{3}{*}{$\begin{array}{l}\text { HEIS No. } \\
14133-76-7\end{array}$} & B1V991 & \multicolumn{2}{|c|}{ Lab ID: $\quad$ 0805003-01 } & & & \\
\hline & Technetium-99 & $<2.30 \mathrm{E}-5 \mathrm{ug} / \mathrm{g}$ dry & $2.30 \mathrm{E}-5$ & $8 / 27 / 08$ & $8 \mathrm{H} 27003$ & PNNL-AGG-415 \\
\hline & Uranium 238 & $<5.64 \mathrm{E}-4$ ug/g dry & $5.64 \mathrm{E}-4$ & $8 / 27 / 08$ & $8 \mathrm{H} 27003$ & PNNL-AGG-415 \\
\hline \multirow{3}{*}{$\begin{array}{l}\text { HEIS No. } \\
14133-76-7\end{array}$} & B1V992 & \multicolumn{2}{|c|}{ Lab ID: $\quad$ 0805003-02 } & & & \\
\hline & Technetium-99 & $<2.29 \mathrm{E}-5 \quad$ ug/g dry & $2.29 \mathrm{E}-5$ & $8 / 27 / 08$ & $8 \mathrm{H} 27003$ & PNNL-AGG-415 \\
\hline & Uranium 238 & $<5.62 \mathrm{E}-4$ ug/g dry & $5.62 \mathrm{E}-4$ & $8 / 27 / 08$ & $8 \mathrm{H} 27003$ & PNNL-AGG-415 \\
\hline \multirow{3}{*}{$\begin{array}{l}\text { HEIS No. } \\
14133-76-7\end{array}$} & B1V993 & \multicolumn{2}{|c|}{ Lab ID: $\quad$ 0805003-03 } & & & \\
\hline & Technetium-99 & $<2.28 \mathrm{E}-5 \mathrm{ug} / \mathrm{g}$ dry & $2.28 \mathrm{E}-5$ & $8 / 27 / 08$ & $8 \mathrm{H} 27003$ & PNNL-AGG-415 \\
\hline & Uranium 238 & $<5.59 \mathrm{E}-4 \quad$ ug/g dry & $5.59 \mathrm{E}-4$ & $8 / 27 / 08$ & $8 \mathrm{H} 27003$ & PNNL-AGG-415 \\
\hline \multirow{3}{*}{$\begin{array}{l}\text { HEIS No. } \\
14133-76-7\end{array}$} & B1V994 & \multicolumn{2}{|c|}{ Lab ID: $\quad$ 0805003-04 } & & & \\
\hline & Technetium-99 & $<2.29 \mathrm{E}-5 \quad \mathrm{ug} / \mathrm{g}$ dry & $2.29 \mathrm{E}-5$ & $8 / 27 / 08$ & $8 \mathrm{H} 27003$ & PNNL-AGG-415 \\
\hline & Uranium 238 & $7.53 \mathrm{E}-4 \mathrm{ug} / \mathrm{g}$ dry & $5.61 \mathrm{E}-4$ & $8 / 27 / 08$ & $8 \mathrm{H} 27003$ & PNNL-AGG-415 \\
\hline \multirow{3}{*}{$\begin{array}{l}\text { HEIS No. } \\
14133-76-7\end{array}$} & B1V995 & \multicolumn{2}{|c|}{ Lab ID: $\quad$ 0805003-05 } & & & \\
\hline & Technetium-99 & $<2.30 \mathrm{E}-5 \quad$ ug/g dry & $2.30 \mathrm{E}-5$ & $8 / 27 / 08$ & $8 \mathrm{H} 27003$ & PNNL-AGG-415 \\
\hline & Uranium 238 & 7.72E-4 ug/g dry & $5.63 \mathrm{E}-4$ & $8 / 27 / 08$ & $8 \mathrm{H} 27003$ & PNNL-AGG-415 \\
\hline HEIS No. & B1V996 & Lab ID: & 5003-06 & & & \\
\hline $14133-76-7$ & Technetium-99 & $<2.30 \mathrm{E}-5 \quad$ ug/g dry & $2.30 \mathrm{E}-5$ & $8 / 27 / 08$ & $8 \mathrm{H} 27005$ & PNNL-AGG-415 \\
\hline & Uranium 238 & $2.82 \mathrm{E}-3 \mathrm{ug} / \mathrm{g}$ dry & $5.64 \mathrm{E}-4$ & $8 / 27 / 08$ & $8 \mathrm{H} 27005$ & PNNL-AGG-415 \\
\hline HEIS No. & B1V997 & Lab ID: & 5003-07 & & & \\
\hline $14133-76-7$ & Technetium-99 & $<2.30 \mathrm{E}-5 \quad \mathrm{ug} / \mathrm{g}$ dry & $2.30 \mathrm{E}-5$ & $8 / 27 / 08$ & $8 \mathrm{H} 27005$ & PNNL-AGG-415 \\
\hline & Uranium 238 & $2.88 \mathrm{E}-3 \mathrm{ug} / \mathrm{g}$ dry & $5.63 \mathrm{E}-4$ & $8 / 27 / 08$ & $8 \mathrm{H} 27005$ & PNNL-AGG-415 \\
\hline HEIS No. & B1V998 & Lab ID: & $5003-08$ & & & \\
\hline $14133-76-7$ & Technetium-99 & $<2.30 \mathrm{E}-5 \mathrm{ug} / \mathrm{g}$ dry & $2.30 \mathrm{E}-5$ & $8 / 27 / 08$ & $8 \mathrm{H} 27005$ & PNNL-AGG-415 \\
\hline & Uranium 238 & $1.16 \mathrm{E}-3 \mathrm{ug} / \mathrm{g}$ dry & $5.64 \mathrm{E}-4$ & $8 / 27 / 08$ & $8 \mathrm{H} 27005$ & PNNL-AGG-415 \\
\hline HEIS No. & B1V999 & Lab ID: & 5003-09 & & & \\
\hline $14133-76-7$ & Technetium-99 & $<2.29 \mathrm{E}-5 \quad$ ug/g dry & $2.29 \mathrm{E}-5$ & $8 / 27 / 08$ & $8 \mathrm{H} 27005$ & PNNL-AGG-415 \\
\hline & Uranium 238 & 7.74E-4 ug/g dry & $5.62 \mathrm{E}-4$ & $8 / 27 / 08$ & $8 \mathrm{H} 27005$ & PNNL-AGG-415 \\
\hline HEIS No. & B1V9B0 & Lab ID: & 5003-10 & & & \\
\hline $14133-76-7$ & Technetium-99 & $<2.29 \mathrm{E}-5 \mathrm{ug} / \mathrm{g}$ dry & $2.29 \mathrm{E}-5$ & $8 / 27 / 08$ & $8 \mathrm{H} 27005$ & PNNL-AGG-415 \\
\hline
\end{tabular}




\section{Radionuclides by ICP-MS/Water Extract}

\begin{tabular}{|c|c|c|c|c|c|c|c|}
\hline CAS \# & Analyte & Results & Units & EQL & Analyzed & Batch & Method \\
\hline \multirow[t]{2}{*}{ HEIS No. } & B1V9B0 & \multicolumn{3}{|c|}{ Lab ID: $\quad$ 0805003-10 } & & & \\
\hline & Uranium 238 & 7.38E-4 & ug/g dry & $5.62 \mathrm{E}-4$ & $8 / 27 / 08$ & $8 \mathrm{H} 27005$ & PNNL-AGG-415 \\
\hline \multirow{3}{*}{$\begin{array}{l}\text { HEIS No. } \\
14133-76-7\end{array}$} & B1V9B1 & \multicolumn{3}{|c|}{ Lab ID: $\quad$ 0805003-11 } & & & \\
\hline & Technetium-99 & $<2.29 \mathrm{E}-5$ & ug/g dry & $2.29 \mathrm{E}-5$ & $8 / 27 / 08$ & $8 \mathrm{H} 27005$ & PNNL-AGG-415 \\
\hline & Uranium 238 & $8.77 \mathrm{E}-4$ & ug/g dry & $5.62 \mathrm{E}-4$ & $8 / 27 / 08$ & $8 \mathrm{H} 27005$ & PNNL-AGG-415 \\
\hline \multirow{3}{*}{$\begin{array}{l}\text { HEIS No. } \\
14133-76-7\end{array}$} & B1V9B2 & \multicolumn{3}{|c|}{ Lab ID: $\quad$ 0805003-12 } & & & \\
\hline & Technetium-99 & $<2.30 \mathrm{E}-5$ & ug/g dry & $2.30 \mathrm{E}-5$ & $8 / 27 / 08$ & $8 \mathrm{H} 27005$ & PNNL-AGG-415 \\
\hline & Uranium 238 & $<5.64 \mathrm{E}-4$ & ug/g dry & $5.64 \mathrm{E}-4$ & $8 / 27 / 08$ & $8 \mathrm{H} 27005$ & PNNL-AGG-415 \\
\hline \multirow{3}{*}{$\begin{array}{l}\text { HEIS No. } \\
14133-76-7\end{array}$} & B1V9B3 & \multicolumn{3}{|c|}{ Lab ID: $\quad$ 0805003-13 } & & & \\
\hline & Technetium-99 & $<2.30 \mathrm{E}-5$ & ug/g dry & $2.30 \mathrm{E}-5$ & $8 / 27 / 08$ & $8 \mathrm{H} 27005$ & PNNL-AGG-415 \\
\hline & Uranium 238 & $<5.64 \mathrm{E}-4$ & ug/g dry & $5.64 \mathrm{E}-4$ & $8 / 27 / 08$ & $8 \mathrm{H} 27005$ & PNNL-AGG-415 \\
\hline \multirow{3}{*}{$\begin{array}{l}\text { HEIS No. } \\
14133-76-7\end{array}$} & B1V9B4 & \multicolumn{3}{|c|}{ Lab ID: $\quad$ 0805003-14 } & & & \\
\hline & Technetium-99 & $<2.30 \mathrm{E}-5$ & ug/g dry & $2.30 \mathrm{E}-5$ & $8 / 27 / 08$ & $8 \mathrm{H} 27005$ & PNNL-AGG-415 \\
\hline & Uranium 238 & $1.20 \mathrm{E}-3$ & ug/g dry & $5.62 \mathrm{E}-4$ & $8 / 27 / 08$ & $8 \mathrm{H} 27005$ & PNNL-AGG-415 \\
\hline \multirow{3}{*}{$\begin{array}{l}\text { HEIS No. } \\
14133-76-7\end{array}$} & B1V9B5 & \multicolumn{3}{|c|}{ Lab ID: $\quad$ 0805003-15 } & & & \\
\hline & Technetium-99 & $<2.30 \mathrm{E}-5$ & ug/g dry & $2.30 \mathrm{E}-5$ & $8 / 27 / 08$ & $8 \mathrm{H} 27005$ & PNNL-AGG-415 \\
\hline & Uranium 238 & $<5.63 \mathrm{E}-4$ & ug/g dry & $5.63 \mathrm{E}-4$ & $8 / 27 / 08$ & $8 \mathrm{H} 27005$ & PNNL-AGG-415 \\
\hline \multirow{3}{*}{$\begin{array}{l}\text { HEIS No. } \\
14133-76-7\end{array}$} & B1V9B6 & \multicolumn{3}{|c|}{ Lab ID: $\quad$ 0805003-16 } & & & \\
\hline & Technetium-99 & $<2.29 \mathrm{E}-5$ & ug/g dry & $2.29 \mathrm{E}-5$ & $8 / 27 / 08$ & $8 \mathrm{H} 27005$ & PNNL-AGG-415 \\
\hline & Uranium 238 & $<5.62 \mathrm{E}-4$ & ug/g dry & $5.62 \mathrm{E}-4$ & $8 / 27 / 08$ & $8 \mathrm{H} 27005$ & PNNL-AGG-415 \\
\hline \multirow{3}{*}{$\begin{array}{l}\text { HEIS No. } \\
14133-76-7\end{array}$} & B1V9B7 & \multicolumn{3}{|c|}{ Lab ID: $\quad$ 0805003-17 } & & & \\
\hline & Technetium-99 & $<2.30 \mathrm{E}-5$ & ug/g dry & $2.30 \mathrm{E}-5$ & $8 / 27 / 08$ & $8 \mathrm{H} 27005$ & PNNL-AGG-415 \\
\hline & Uranium 238 & $<5.63 \mathrm{E}-4$ & ug/g dry & $5.63 \mathrm{E}-4$ & $8 / 27 / 08$ & $8 \mathrm{H} 27005$ & PNNL-AGG-415 \\
\hline \multirow{3}{*}{$\begin{array}{l}\text { HEIS No. } \\
14133-76-7\end{array}$} & B1V9B8 & \multicolumn{3}{|c|}{ Lab ID: $\quad$ 0805003-18 } & & & \\
\hline & Technetium-99 & $<2.31 \mathrm{E}-5$ & ug/g dry & $2.31 \mathrm{E}-5$ & $8 / 27 / 08$ & $8 \mathrm{H} 27005$ & PNNL-AGG-415 \\
\hline & Uranium 238 & $<5.67 \mathrm{E}-4$ & ug/g dry & $5.67 \mathrm{E}-4$ & $8 / 27 / 08$ & $8 \mathrm{H} 27005$ & PNNL-AGG-415 \\
\hline \multirow{3}{*}{$\begin{array}{l}\text { HEIS No. } \\
14133-76-7\end{array}$} & B1V9B9 & \multicolumn{3}{|c|}{ Lab ID: $\quad$ 0805003-19 } & & & \\
\hline & Technetium-99 & $<2.29 \mathrm{E}-5$ & ug/g dry & $2.29 \mathrm{E}-5$ & $8 / 27 / 08$ & $8 \mathrm{H} 27005$ & PNNL-AGG-415 \\
\hline & Uranium 238 & $<5.61 \mathrm{E}-4$ & ug/g dry & $5.61 \mathrm{E}-4$ & $8 / 27 / 08$ & $8 \mathrm{H} 27005$ & PNNL-AGG-415 \\
\hline \multirow{3}{*}{$\begin{array}{l}\text { HEIS No. } \\
14133-76-7\end{array}$} & B1V9C0 & & b ID: & $5003-20$ & & & \\
\hline & Technetium-99 & $<2.31 \mathrm{E}-5$ & ug/g dry & $2.31 \mathrm{E}-5$ & $8 / 27 / 08$ & $8 \mathrm{H} 27005$ & PNNL-AGG-415 \\
\hline & Uranium 238 & $<5.65 \mathrm{E}-4$ & ug/g dry & $5.65 \mathrm{E}-4$ & $8 / 27 / 08$ & $8 \mathrm{H} 27005$ & PNNL-AGG-415 \\
\hline HEIS No. & B1V9C1 & & b ID: & $5003-21$ & & & \\
\hline $14133-76-7$ & Technetium-99 & $<2.30 \mathrm{E}-5$ & ug/g dry & $2.30 \mathrm{E}-5$ & $8 / 27 / 08$ & $8 \mathrm{H} 27005$ & PNNL-AGG-415 \\
\hline & Uranium 238 & $<5.63 \mathrm{E}-4$ & ug/g dry & $5.63 \mathrm{E}-4$ & $8 / 27 / 08$ & $8 \mathrm{H} 27005$ & PNNL-AGG-415 \\
\hline HEIS No. & B1V9C2 & & b ID: & $5003-22$ & & & \\
\hline $14133-76-7$ & Technetium-99 & $<2.30 \mathrm{E}-5$ & ug/g dry & $2.30 \mathrm{E}-5$ & $8 / 27 / 08$ & $8 \mathrm{H} 27005$ & PNNL-AGG-415 \\
\hline & Uranium 238 & $<5.63 \mathrm{E}-4$ & ug/g dry & $5.63 \mathrm{E}-4$ & $8 / 27 / 08$ & $8 \mathrm{H} 27005$ & PNNL-AGG-415 \\
\hline HEIS No. & B1V9C3 & & b ID: & $5003-23$ & & & \\
\hline $14133-76-7$ & Technetium-99 & $<2.29 \mathrm{E}-5$ & ug/g dry & $2.29 \mathrm{E}-5$ & $8 / 27 / 08$ & $8 \mathrm{H} 27005$ & PNNL-AGG-415 \\
\hline & Uranium 238 & $<5.61 \mathrm{E}-4$ & ug/g dry & $5.61 \mathrm{E}-4$ & $8 / 27 / 08$ & $8 \mathrm{H} 27005$ & PNNL-AGG-415 \\
\hline HEIS No. & B1V9C4 & & b ID: & $5003-24$ & & & \\
\hline $14133-76-7$ & Technetium-99 & $<2.30 \mathrm{E}-5$ & ug/g dry & $2.30 \mathrm{E}-5$ & $8 / 27 / 08$ & $8 \mathrm{H} 27005$ & PNNL-AGG-415 \\
\hline & Uranium 238 & $<5.64 \mathrm{E}-4$ & ug/g dry & $5.64 \mathrm{E}-4$ & $8 / 27 / 08$ & $8 \mathrm{H} 27005$ & PNNL-AGG-415 \\
\hline HEIS No. & B1V2V5 & & b ID: & $5003-25$ & & & \\
\hline $14133-76-7$ & Technetium-99 & $<2.29 \mathrm{E}-5$ & ug/g dry & $2.29 \mathrm{E}-5$ & $8 / 27 / 08$ & $8 \mathrm{H} 27005$ & PNNL-AGG-415 \\
\hline & Uranium 238 & $<5.62 \mathrm{E}-4$ & ug/g dry & $5.62 \mathrm{E}-4$ & $8 / 27 / 08$ & $8 \mathrm{H} 27005$ & PNNL-AGG-415 \\
\hline
\end{tabular}


RCRA Metals By PNNL-AGG-415/Water Extract

\begin{tabular}{|c|c|c|c|c|c|c|c|}
\hline CAS \# & Analyte & Results & Units & EQL & Analyzed & Batch & Method \\
\hline HEIS No. & B1VDW5 & \multicolumn{3}{|c|}{ Lab ID: $\quad$ 0805001-01 } & & & \\
\hline $14092-98-9$ & Chromium 52 & $<2.05 \mathrm{E}-3$ & ug/g dry & $2.05 \mathrm{E}-3$ & $8 / 26 / 08$ & $8 \mathrm{H} 26016$ & PNNL-AGG-415 \\
\hline $13981-78-7$ & Chromium 53 & $<6.38 \mathrm{E}-3$ & ug/g dry & $6.38 \mathrm{E}-3$ & $8 / 26 / 08$ & $8 \mathrm{H} 26016$ & PNNL-AGG-415 \\
\hline $14191-84-5$ & Copper 63 & $<3.47 \mathrm{E}-3$ & ug/g dry & $3.47 \mathrm{E}-3$ & $8 / 26 / 08$ & $8 \mathrm{H} 26016$ & PNNL-AGG-415 \\
\hline $14119-06-3$ & Copper 65 & $<4.82 \mathrm{E}-3$ & ug/g dry & $4.82 \mathrm{E}-3$ & $8 / 26 / 08$ & $8 \mathrm{H} 26016$ & PNNL-AGG-415 \\
\hline $7440-38-2$ & Arsenic 75 & $<6.23 \mathrm{E}-3$ & ug/g dry & $6.23 \mathrm{E}-3$ & $8 / 26 / 08$ & $8 \mathrm{H} 26016$ & PNNL-AGG-415 \\
\hline $14687-58-2$ & Selenium 82 & $<1.10 \mathrm{E}-2$ & ug/g dry & $1.10 \mathrm{E}-2$ & $8 / 26 / 08$ & $8 \mathrm{H} 26016$ & PNNL-AGG-415 \\
\hline $14392-17-7$ & Molybdenum 95 & $1.64 \mathrm{E}-3$ & ug/g dry & $1.60 \mathrm{E}-3$ & $8 / 26 / 08$ & $8 \mathrm{H} 26016$ & PNNL-AGG-415 \\
\hline 14392-19-9 & Molybdenum 97 & $<2.04 \mathrm{E}-3$ & ug/g dry & $2.04 \mathrm{E}-3$ & $8 / 26 / 08$ & $8 \mathrm{H} 26016$ & PNNL-AGG-415 \\
\hline $14392-20-2$ & Molybdenum 98 & $1.74 \mathrm{E}-3$ & ug/g dry & $1.60 \mathrm{E}-3$ & $8 / 26 / 08$ & $8 \mathrm{H} 26016$ & PNNL-AGG-415 \\
\hline $14914-61-5$ & Ruthenium 101 & $<8.17 \mathrm{E}-4$ & ug/g dry & $8.17 \mathrm{E}-4$ & $8 / 26 / 08$ & $8 \mathrm{H} 26016$ & PNNL-AGG-415 \\
\hline $14914-62-6$ & Ruthenium 102 & $<5.63 \mathrm{E}-4$ & ug/g dry & $5.63 \mathrm{E}-4$ & $8 / 26 / 08$ & $8 \mathrm{H} 26016$ & PNNL-AGG-415 \\
\hline $15766-01-5$ & Ruthenium 104 & $<3.64 \mathrm{E}-4$ & ug/g dry & $3.64 \mathrm{E}-4$ & $8 / 26 / 08$ & $8 \mathrm{H} 26016$ & PNNL-AGG-415 \\
\hline $14378-37-1$ & Silver 107 & $<9.22 \mathrm{E}-4$ & ug/g dry & $9.22 \mathrm{E}-4$ & $8 / 26 / 08$ & $8 \mathrm{H} 26016$ & PNNL-AGG-415 \\
\hline $14378-38-2$ & Silver 109 & $<1.07 \mathrm{E}-3$ & ug/g dry & $1.07 \mathrm{E}-3$ & $8 / 26 / 08$ & $8 \mathrm{H} 26016$ & PNNL-AGG-415 \\
\hline $14336-64-2$ & Cadmium 111 & $<2.94 \mathrm{E}-4$ & ug/g dry & $2.94 \mathrm{E}-4$ & $8 / 26 / 08$ & $8 \mathrm{H} 26016$ & PNNL-AGG-415 \\
\hline $14041-58-8$ & Cadmium 114 & $<6.23 \mathrm{E}-4$ & ug/g dry & $6.23 \mathrm{E}-4$ & $8 / 26 / 08$ & $8 \mathrm{H} 26016$ & PNNL-AGG-415 \\
\hline $14265-72-6$ & Antimony 121 & $<5.38 \mathrm{E}-4$ & ug/g dry & $5.38 \mathrm{E}-4$ & $8 / 26 / 08$ & $8 \mathrm{H} 26016$ & PNNL-AGG-415 \\
\hline $14119-16-5$ & Antimony 123 & $<4.28 \mathrm{E}-4$ & ug/g dry & $4.28 \mathrm{E}-4$ & $8 / 26 / 08$ & $8 \mathrm{H} 26016$ & PNNL-AGG-415 \\
\hline $13966-27-3$ & Lead 206 & $<7.17 \mathrm{E}-4$ & ug/g dry & 7.17E-4 & $8 / 26 / 08$ & $8 \mathrm{H} 26016$ & PNNL-AGG-415 \\
\hline $13966-28-4$ & Lead 208 & $<5.58 \mathrm{E}-4$ & ug/g dry & $5.58 \mathrm{E}-4$ & $8 / 26 / 08$ & $8 \mathrm{H} 26016$ & PNNL-AGG-415 \\
\hline HEIS No. & B1VDW6 & \multicolumn{3}{|c|}{ Lab ID: $\quad$ 0805001-02 } & & & \\
\hline $14092-98-9$ & Chromium 52 & $<2.01 \mathrm{E}-3$ & ug/g dry & $2.01 \mathrm{E}-3$ & $8 / 26 / 08$ & $8 \mathrm{H} 26016$ & PNNL-AGG-415 \\
\hline $13981-78-7$ & Chromium 53 & $<6.25 \mathrm{E}-3$ & ug/g dry & $6.25 \mathrm{E}-3$ & $8 / 26 / 08$ & $8 \mathrm{H} 26016$ & PNNL-AGG-415 \\
\hline $14191-84-5$ & Copper 63 & $<3.40 \mathrm{E}-3$ & ug/g dry & $3.40 \mathrm{E}-3$ & $8 / 26 / 08$ & $8 \mathrm{H} 26016$ & PNNL-AGG-415 \\
\hline $14119-06-3$ & Copper 65 & $<4.73 \mathrm{E}-3$ & ug/g dry & $4.73 \mathrm{E}-3$ & $8 / 26 / 08$ & $8 \mathrm{H} 26016$ & PNNL-AGG-415 \\
\hline $7440-38-2$ & Arsenic 75 & $<6.10 \mathrm{E}-3$ & ug/g dry & $6.10 \mathrm{E}-3$ & $8 / 26 / 08$ & $8 \mathrm{H} 26016$ & PNNL-AGG-415 \\
\hline $14687-58-2$ & Selenium 82 & $<1.08 \mathrm{E}-2$ & ug/g dry & $1.08 \mathrm{E}-2$ & $8 / 26 / 08$ & $8 \mathrm{H} 26016$ & PNNL-AGG-415 \\
\hline $14392-17-7$ & Molybdenum 95 & $<1.57 \mathrm{E}-3$ & ug/g dry & $1.57 \mathrm{E}-3$ & $8 / 26 / 08$ & $8 \mathrm{H} 26016$ & PNNL-AGG-415 \\
\hline 14392-19-9 & Molybdenum 97 & $<2.00 \mathrm{E}-3$ & ug/g dry & $2.00 \mathrm{E}-3$ & $8 / 26 / 08$ & $8 \mathrm{H} 26016$ & PNNL-AGG-415 \\
\hline $14392-20-2$ & Molybdenum 98 & $<1.57 \mathrm{E}-3$ & ug/g dry & $1.57 \mathrm{E}-3$ & $8 / 26 / 08$ & $8 \mathrm{H} 26016$ & PNNL-AGG-415 \\
\hline 14914-61-5 & Ruthenium 101 & $<8.01 \mathrm{E}-4$ & ug/g dry & $8.01 \mathrm{E}-4$ & $8 / 26 / 08$ & $8 \mathrm{H} 26016$ & PNNL-AGG-415 \\
\hline $14914-62-6$ & Ruthenium 102 & $<5.52 \mathrm{E}-4$ & ug/g dry & $5.52 \mathrm{E}-4$ & $8 / 26 / 08$ & $8 \mathrm{H} 26016$ & PNNL-AGG-415 \\
\hline $15766-01-5$ & Ruthenium 104 & $<3.56 \mathrm{E}-4$ & ug/g dry & $3.56 \mathrm{E}-4$ & $8 / 26 / 08$ & $8 \mathrm{H} 26016$ & PNNL-AGG-415 \\
\hline $14378-37-1$ & Silver 107 & $<9.03 \mathrm{E}-4$ & ug/g dry & $9.03 \mathrm{E}-4$ & $8 / 26 / 08$ & $8 \mathrm{H} 26016$ & PNNL-AGG-415 \\
\hline $14378-38-2$ & Silver 109 & $<1.04 \mathrm{E}-3$ & ug/g dry & $1.04 \mathrm{E}-3$ & $8 / 26 / 08$ & $8 \mathrm{H} 26016$ & PNNL-AGG-415 \\
\hline $14336-64-2$ & Cadmium 111 & $<2.88 \mathrm{E}-4$ & ug/g dry & $2.88 \mathrm{E}-4$ & $8 / 26 / 08$ & $8 \mathrm{H} 26016$ & PNNL-AGG-415 \\
\hline $14041-58-8$ & Cadmium 114 & $<6.10 \mathrm{E}-4$ & ug/g dry & $6.10 \mathrm{E}-4$ & $8 / 26 / 08$ & $8 \mathrm{H} 26016$ & PNNL-AGG-415 \\
\hline $14265-72-6$ & Antimony 121 & $<5.27 \mathrm{E}-4$ & $\mathrm{ug} / \mathrm{g}$ dry & $5.27 \mathrm{E}-4$ & $8 / 26 / 08$ & $8 \mathrm{H} 26016$ & PNNL-AGG-415 \\
\hline $14119-16-5$ & Antimony 123 & $<4.20 \mathrm{E}-4$ & ug/g dry & $4.20 \mathrm{E}-4$ & $8 / 26 / 08$ & $8 \mathrm{H} 26016$ & PNNL-AGG-415 \\
\hline $13966-27-3$ & Lead 206 & $<7.03 \mathrm{E}-4$ & ug/g dry & 7.03E-4 & $8 / 26 / 08$ & $8 \mathrm{H} 26016$ & PNNL-AGG-415 \\
\hline $13966-28-4$ & Lead 208 & $<5.47 \mathrm{E}-4$ & ug/g dry & $5.47 \mathrm{E}-4$ & $8 / 26 / 08$ & $8 \mathrm{H} 26016$ & PNNL-AGG-415 \\
\hline HEIS No. & B1VDW7 & \multicolumn{3}{|c|}{ Lab ID: $\quad$ 0805001-03 } & & & \\
\hline $14092-98-9$ & Chromium 52 & $<2.04 \mathrm{E}-3$ & ug/g dry & $2.04 \mathrm{E}-3$ & $8 / 26 / 08$ & $8 \mathrm{H} 26016$ & PNNL-AGG-415 \\
\hline $13981-78-7$ & Chromium 53 & $<6.36 \mathrm{E}-3$ & ug/g dry & $6.36 \mathrm{E}-3$ & $8 / 26 / 08$ & $8 \mathrm{H} 26016$ & PNNL-AGG-415 \\
\hline $14191-84-5$ & Copper 63 & $<3.46 \mathrm{E}-3$ & ug/g dry & $3.46 \mathrm{E}-3$ & $8 / 26 / 08$ & $8 \mathrm{H} 26016$ & PNNL-AGG-415 \\
\hline $14119-06-3$ & Copper 65 & $<4.81 \mathrm{E}-3$ & ug/g dry & $4.81 \mathrm{E}-3$ & $8 / 26 / 08$ & $8 \mathrm{H} 26016$ & PNNL-AGG-415 \\
\hline $7440-38-2$ & Arsenic 75 & $6.99 \mathrm{E}-3$ & ug/g dry & $6.21 \mathrm{E}-3$ & $8 / 26 / 08$ & $8 \mathrm{H} 26016$ & PNNL-AGG-415 \\
\hline $14687-58-2$ & Selenium 82 & $<1.10 \mathrm{E}-2$ & ug/g dry & $1.10 \mathrm{E}-2$ & $8 / 26 / 08$ & $8 \mathrm{H} 26016$ & PNNL-AGG-415 \\
\hline
\end{tabular}


RCRA Metals By PNNL-AGG-415/Water Extract

\begin{tabular}{|c|c|c|c|c|c|c|c|}
\hline CAS \# & Analyte & Results & Units & EQL & Analyzed & Batch & Method \\
\hline HEIS No. & B1VDW7 & \multicolumn{3}{|c|}{ Lab ID: $\quad$ 0805001-03 } & & & \\
\hline $14392-17-7$ & Molybdenum 95 & $1.73 \mathrm{E}-3$ & ug/g dry & $1.59 \mathrm{E}-3$ & $8 / 26 / 08$ & $8 \mathrm{H} 26016$ & PNNL-AGG-415 \\
\hline 14392-19-9 & Molybdenum 97 & $<2.03 \mathrm{E}-3$ & ug/g dry & $2.03 \mathrm{E}-3$ & $8 / 26 / 08$ & $8 \mathrm{H} 26016$ & PNNL-AGG-415 \\
\hline $14392-20-2$ & Molybdenum 98 & $1.80 \mathrm{E}-3$ & ug/g dry & $1.59 \mathrm{E}-3$ & $8 / 26 / 08$ & $8 \mathrm{H} 26016$ & PNNL-AGG-415 \\
\hline $14914-61-5$ & Ruthenium 101 & $<8.15 \mathrm{E}-4$ & ug/g dry & $8.15 \mathrm{E}-4$ & $8 / 26 / 08$ & $8 \mathrm{H} 26016$ & PNNL-AGG-415 \\
\hline $14914-62-6$ & Ruthenium 102 & $<5.61 \mathrm{E}-4$ & ug/g dry & $5.61 \mathrm{E}-4$ & $8 / 26 / 08$ & $8 \mathrm{H} 26016$ & PNNL-AGG-415 \\
\hline $15766-01-5$ & Ruthenium 104 & $<3.63 \mathrm{E}-4$ & ug/g dry & $3.63 \mathrm{E}-4$ & $8 / 26 / 08$ & $8 \mathrm{H} 26016$ & PNNL-AGG-415 \\
\hline $14378-37-1$ & Silver 107 & $<9.19 \mathrm{E}-4$ & ug/g dry & $9.19 \mathrm{E}-4$ & $8 / 26 / 08$ & $8 \mathrm{H} 26016$ & PNNL-AGG-415 \\
\hline $14378-38-2$ & Silver 109 & $<1.06 \mathrm{E}-3$ & ug/g dry & $1.06 \mathrm{E}-3$ & $8 / 26 / 08$ & $8 \mathrm{H} 26016$ & PNNL-AGG-415 \\
\hline $14336-64-2$ & Cadmium 111 & $<2.93 \mathrm{E}-4$ & ug/g dry & $2.93 \mathrm{E}-4$ & $8 / 26 / 08$ & $8 \mathrm{H} 26016$ & PNNL-AGG-415 \\
\hline $14041-58-8$ & Cadmium 114 & $<6.21 \mathrm{E}-4$ & ug/g dry & $6.21 \mathrm{E}-4$ & $8 / 26 / 08$ & $8 \mathrm{H} 26016$ & PNNL-AGG-415 \\
\hline $14265-72-6$ & Antimony 121 & $<5.36 \mathrm{E}-4$ & ug/g dry & $5.36 \mathrm{E}-4$ & $8 / 26 / 08$ & $8 \mathrm{H} 26016$ & PNNL-AGG-415 \\
\hline $14119-16-5$ & Antimony 123 & $<4.27 \mathrm{E}-4$ & ug/g dry & $4.27 \mathrm{E}-4$ & $8 / 26 / 08$ & $8 \mathrm{H} 26016$ & PNNL-AGG-415 \\
\hline $13966-27-3$ & Lead 206 & $<7.15 \mathrm{E}-4$ & ug/g dry & 7.15E-4 & $8 / 26 / 08$ & $8 \mathrm{H} 26016$ & PNNL-AGG-415 \\
\hline $13966-28-4$ & Lead 208 & $<5.56 \mathrm{E}-4$ & ug/g dry & $5.56 \mathrm{E}-4$ & $8 / 26 / 08$ & $8 \mathrm{H} 26016$ & PNNL-AGG-415 \\
\hline HEIS No. & B1VDW8 & \multicolumn{3}{|c|}{ Lab ID: $\quad$ 0805001-04 } & & & \\
\hline 14092-98-9 & Chromium 52 & $<2.02 \mathrm{E}-3$ & ug/g dry & $2.02 \mathrm{E}-3$ & $8 / 26 / 08$ & $8 \mathrm{H} 26016$ & PNNL-AGG-415 \\
\hline $13981-78-7$ & Chromium 53 & $<6.30 \mathrm{E}-3$ & ug/g dry & $6.30 \mathrm{E}-3$ & $8 / 26 / 08$ & $8 \mathrm{H} 26016$ & PNNL-AGG-415 \\
\hline $14191-84-5$ & Copper 63 & $<3.43 \mathrm{E}-3$ & ug/g dry & $3.43 \mathrm{E}-3$ & $8 / 26 / 08$ & $8 \mathrm{H} 26016$ & PNNL-AGG-415 \\
\hline 14119-06-3 & Copper 65 & $<4.77 \mathrm{E}-3$ & ug/g dry & $4.77 \mathrm{E}-3$ & $8 / 26 / 08$ & $8 \mathrm{H} 26016$ & PNNL-AGG-415 \\
\hline $7440-38-2$ & Arsenic 75 & $8.74 \mathrm{E}-3$ & ug/g dry & $6.15 \mathrm{E}-3$ & $8 / 26 / 08$ & $8 \mathrm{H} 26016$ & PNNL-AGG-415 \\
\hline $14687-58-2$ & Selenium 82 & $<1.09 \mathrm{E}-2$ & ug/g dry & $1.09 \mathrm{E}-2$ & $8 / 26 / 08$ & $8 \mathrm{H} 26016$ & PNNL-AGG-415 \\
\hline $14392-17-7$ & Molybdenum 95 & $<1.58 \mathrm{E}-3$ & ug/g dry & $1.58 \mathrm{E}-3$ & $8 / 26 / 08$ & $8 \mathrm{H} 26016$ & PNNL-AGG-415 \\
\hline 14392-19-9 & Molybdenum 97 & $<2.01 \mathrm{E}-3$ & ug/g dry & $2.01 \mathrm{E}-3$ & $8 / 26 / 08$ & $8 \mathrm{H} 26016$ & PNNL-AGG-415 \\
\hline $14392-20-2$ & Molybdenum 98 & $<1.58 \mathrm{E}-3$ & ug/g dry & $1.58 \mathrm{E}-3$ & $8 / 26 / 08$ & $8 \mathrm{H} 26016$ & PNNL-AGG-415 \\
\hline $14914-61-5$ & Ruthenium 101 & $<8.07 \mathrm{E}-4$ & ug/g dry & $8.07 \mathrm{E}-4$ & $8 / 26 / 08$ & $8 \mathrm{H} 26016$ & PNNL-AGG-415 \\
\hline $14914-62-6$ & Ruthenium 102 & $<5.56 \mathrm{E}-4$ & ug/g dry & $5.56 \mathrm{E}-4$ & $8 / 26 / 08$ & $8 \mathrm{H} 26016$ & PNNL-AGG-415 \\
\hline $15766-01-5$ & Ruthenium 104 & $<3.59 \mathrm{E}-4$ & ug/g dry & $3.59 \mathrm{E}-4$ & $8 / 26 / 08$ & $8 \mathrm{H} 26016$ & PNNL-AGG-415 \\
\hline $14378-37-1$ & Silver 107 & $<9.11 \mathrm{E}-4$ & ug/g dry & $9.11 \mathrm{E}-4$ & $8 / 26 / 08$ & $8 \mathrm{H} 26016$ & PNNL-AGG-415 \\
\hline $14378-38-2$ & Silver 109 & $<1.05 \mathrm{E}-3$ & ug/g dry & $1.05 \mathrm{E}-3$ & $8 / 26 / 08$ & $8 \mathrm{H} 26016$ & PNNL-AGG-415 \\
\hline $14336-64-2$ & Cadmium 111 & $<2.90 \mathrm{E}-4$ & ug/g dry & $2.90 \mathrm{E}-4$ & $8 / 26 / 08$ & $8 \mathrm{H} 26016$ & PNNL-AGG-415 \\
\hline $14041-58-8$ & Cadmium 114 & $<6.15 \mathrm{E}-4$ & ug/g dry & $6.15 \mathrm{E}-4$ & $8 / 26 / 08$ & $8 \mathrm{H} 26016$ & PNNL-AGG-415 \\
\hline $14265-72-6$ & Antimony 121 & $<5.32 \mathrm{E}-4$ & ug/g dry & $5.32 \mathrm{E}-4$ & $8 / 26 / 08$ & $8 \mathrm{H} 26016$ & PNNL-AGG-415 \\
\hline $14119-16-5$ & Antimony 123 & $<4.23 \mathrm{E}-4$ & ug/g dry & $4.23 \mathrm{E}-4$ & $8 / 26 / 08$ & $8 \mathrm{H} 26016$ & PNNL-AGG-415 \\
\hline $13966-27-3$ & Lead 206 & $<7.09 \mathrm{E}-4$ & ug/g dry & $7.09 \mathrm{E}-4$ & $8 / 26 / 08$ & $8 \mathrm{H} 26016$ & PNNL-AGG-415 \\
\hline $13966-28-4$ & Lead 208 & $<5.51 \mathrm{E}-4$ & ug/g dry & $5.51 \mathrm{E}-4$ & $8 / 26 / 08$ & $8 \mathrm{H} 26016$ & PNNL-AGG-415 \\
\hline HEIS No. & B1VDW9 & \multicolumn{3}{|c|}{ Lab ID: $\quad$ 0805001-05 } & & & \\
\hline $14092-98-9$ & Chromium 52 & $<2.03 \mathrm{E}-3$ & ug/g dry & $2.03 \mathrm{E}-3$ & $8 / 26 / 08$ & $8 \mathrm{H} 26016$ & PNNL-AGG-415 \\
\hline 13981-78-7 & Chromium 53 & $<6.31 \mathrm{E}-3$ & ug/g dry & $6.31 \mathrm{E}-3$ & $8 / 26 / 08$ & $8 \mathrm{H} 26016$ & PNNL-AGG-415 \\
\hline $14191-84-5$ & Copper 63 & $<3.43 \mathrm{E}-3$ & ug/g dry & $3.43 \mathrm{E}-3$ & $8 / 26 / 08$ & $8 \mathrm{H} 26016$ & PNNL-AGG-415 \\
\hline 14119-06-3 & Copper 65 & $<4.77 \mathrm{E}-3$ & ug/g dry & $4.77 \mathrm{E}-3$ & $8 / 26 / 08$ & $8 \mathrm{H} 26016$ & PNNL-AGG-415 \\
\hline $7440-38-2$ & Arsenic 75 & $1.37 \mathrm{E}-2$ & ug/g dry & $6.17 \mathrm{E}-3$ & $8 / 26 / 08$ & $8 \mathrm{H} 26016$ & PNNL-AGG-415 \\
\hline $14687-58-2$ & Selenium 82 & $<1.09 \mathrm{E}-2$ & ug/g dry & $1.09 \mathrm{E}-2$ & $8 / 26 / 08$ & $8 \mathrm{H} 26016$ & PNNL-AGG-415 \\
\hline $14392-17-7$ & Molybdenum 95 & $<1.58 \mathrm{E}-3$ & ug/g dry & $1.58 \mathrm{E}-3$ & $8 / 26 / 08$ & $8 \mathrm{H} 26016$ & PNNL-AGG-415 \\
\hline $14392-19-9$ & Molybdenum 97 & $<2.02 \mathrm{E}-3$ & ug/g dry & $2.02 \mathrm{E}-3$ & $8 / 26 / 08$ & $8 \mathrm{H} 26016$ & PNNL-AGG-415 \\
\hline $14392-20-2$ & Molybdenum 98 & $<1.58 \mathrm{E}-3$ & ug/g dry & $1.58 \mathrm{E}-3$ & $8 / 26 / 08$ & $8 \mathrm{H} 26016$ & PNNL-AGG-415 \\
\hline $14914-61-5$ & Ruthenium 101 & $<8.09 \mathrm{E}-4$ & ug/g dry & $8.09 \mathrm{E}-4$ & $8 / 26 / 08$ & $8 \mathrm{H} 26016$ & PNNL-AGG-415 \\
\hline $14914-62-6$ & Ruthenium 102 & $<5.57 \mathrm{E}-4$ & ug/g dry & $5.57 \mathrm{E}-4$ & $8 / 26 / 08$ & $8 \mathrm{H} 26016$ & PNNL-AGG-415 \\
\hline $15766-01-5$ & Ruthenium 104 & $<3.60 \mathrm{E}-4$ & ug/g dry & $3.60 \mathrm{E}-4$ & $8 / 26 / 08$ & $8 \mathrm{H} 26016$ & PNNL-AGG-415 \\
\hline
\end{tabular}


RCRA Metals By PNNL-AGG-415/Water Extract

\begin{tabular}{|c|c|c|c|c|c|c|c|}
\hline CAS \# & Analyte & Results & Units & EQL & Analyzed & Batch & Method \\
\hline HEIS No. & B1VDW9 & \multicolumn{3}{|c|}{ Lab ID: $\quad$ 0805001-05 } & & & \\
\hline $14378-37-1$ & Silver 107 & $<9.13 \mathrm{E}-4$ & ug/g dry & $9.13 \mathrm{E}-4$ & $8 / 26 / 08$ & $8 \mathrm{H} 26016$ & PNNL-AGG-415 \\
\hline $14378-38-2$ & Silver 109 & $<1.06 \mathrm{E}-3$ & ug/g dry & $1.06 \mathrm{E}-3$ & $8 / 26 / 08$ & $8 \mathrm{H} 26016$ & PNNL-AGG-415 \\
\hline $14336-64-2$ & Cadmium 111 & $<2.91 \mathrm{E}-4$ & ug/g dry & $2.91 \mathrm{E}-4$ & $8 / 26 / 08$ & $8 \mathrm{H} 26016$ & PNNL-AGG-415 \\
\hline $14041-58-8$ & Cadmium 114 & $<6.17 \mathrm{E}-4$ & ug/g dry & $6.17 \mathrm{E}-4$ & $8 / 26 / 08$ & $8 \mathrm{H} 26016$ & PNNL-AGG-415 \\
\hline $14265-72-6$ & Antimony 121 & $<5.33 \mathrm{E}-4$ & ug/g dry & $5.33 \mathrm{E}-4$ & $8 / 26 / 08$ & $8 \mathrm{H} 26016$ & PNNL-AGG-415 \\
\hline $14119-16-5$ & Antimony 123 & $<4.24 \mathrm{E}-4$ & ug/g dry & $4.24 \mathrm{E}-4$ & $8 / 26 / 08$ & $8 \mathrm{H} 26016$ & PNNL-AGG-415 \\
\hline $13966-27-3$ & Lead 206 & $<7.10 \mathrm{E}-4$ & ug/g dry & 7.10E-4 & $8 / 26 / 08$ & $8 \mathrm{H} 26016$ & PNNL-AGG-415 \\
\hline $13966-28-4$ & Lead 208 & $<5.52 \mathrm{E}-4$ & ug/g dry & $5.52 \mathrm{E}-4$ & $8 / 26 / 08$ & $8 \mathrm{H} 26016$ & PNNL-AGG-415 \\
\hline HEIS No. & B1VDX0 & \multicolumn{3}{|c|}{ Lab ID: $\quad$ 0805001-06 } & & & \\
\hline $14092-98-9$ & Chromium 52 & $<2.22 \mathrm{E}-3$ & ug/g dry & $2.22 \mathrm{E}-3$ & $8 / 26 / 08$ & $8 \mathrm{H} 26016$ & PNNL-AGG-415 \\
\hline $13981-78-7$ & Chromium 53 & $<6.90 \mathrm{E}-3$ & ug/g dry & $6.90 \mathrm{E}-3$ & $8 / 26 / 08$ & $8 \mathrm{H} 26016$ & PNNL-AGG-415 \\
\hline $14191-84-5$ & Copper 63 & $<3.75 \mathrm{E}-3$ & ug/g dry & $3.75 \mathrm{E}-3$ & $8 / 26 / 08$ & $8 \mathrm{H} 26016$ & PNNL-AGG-415 \\
\hline $14119-06-3$ & Copper 65 & $<5.22 \mathrm{E}-3$ & ug/g dry & $5.22 \mathrm{E}-3$ & $8 / 26 / 08$ & $8 \mathrm{H} 26016$ & PNNL-AGG-415 \\
\hline $7440-38-2$ & Arsenic 75 & $1.31 \mathrm{E}-2$ & ug/g dry & $6.74 \mathrm{E}-3$ & $8 / 26 / 08$ & $8 \mathrm{H} 26016$ & PNNL-AGG-415 \\
\hline $14687-58-2$ & Selenium 82 & $<1.19 \mathrm{E}-2$ & ug/g dry & $1.19 \mathrm{E}-2$ & $8 / 26 / 08$ & $8 \mathrm{H} 26016$ & PNNL-AGG-415 \\
\hline $14392-17-7$ & Molybdenum 95 & $<1.73 \mathrm{E}-3$ & ug/g dry & $1.73 \mathrm{E}-3$ & $8 / 26 / 08$ & $8 \mathrm{H} 26016$ & PNNL-AGG-415 \\
\hline $14392-19-9$ & Molybdenum 97 & $<2.21 \mathrm{E}-3$ & ug/g dry & $2.21 \mathrm{E}-3$ & $8 / 26 / 08$ & $8 \mathrm{H} 26016$ & PNNL-AGG-415 \\
\hline $14392-20-2$ & Molybdenum 98 & $<1.73 \mathrm{E}-3$ & ug/g dry & $1.73 \mathrm{E}-3$ & $8 / 26 / 08$ & $8 \mathrm{H} 26016$ & PNNL-AGG-415 \\
\hline $14914-61-5$ & Ruthenium 101 & $<8.84 \mathrm{E}-4$ & ug/g dry & $8.84 \mathrm{E}-4$ & $8 / 26 / 08$ & $8 \mathrm{H} 26016$ & PNNL-AGG-415 \\
\hline $14914-62-6$ & Ruthenium 102 & $<6.09 \mathrm{E}-4$ & ug/g dry & $6.09 \mathrm{E}-4$ & $8 / 26 / 08$ & $8 \mathrm{H} 26016$ & PNNL-AGG-415 \\
\hline $15766-01-5$ & Ruthenium 104 & $<3.94 \mathrm{E}-4$ & ug/g dry & $3.94 \mathrm{E}-4$ & $8 / 26 / 08$ & $8 \mathrm{H} 26016$ & PNNL-AGG-415 \\
\hline $14378-37-1$ & Silver 107 & $<9.97 \mathrm{E}-4$ & ug/g dry & $9.97 \mathrm{E}-4$ & $8 / 26 / 08$ & $8 \mathrm{H} 26016$ & PNNL-AGG-415 \\
\hline $14378-38-2$ & Silver 109 & $<1.15 \mathrm{E}-3$ & ug/g dry & $1.15 \mathrm{E}-3$ & $8 / 26 / 08$ & $8 \mathrm{H} 26016$ & PNNL-AGG-415 \\
\hline $14336-64-2$ & Cadmium 111 & $<3.18 \mathrm{E}-4$ & ug/g dry & $3.18 \mathrm{E}-4$ & $8 / 26 / 08$ & $8 \mathrm{H} 26016$ & PNNL-AGG-415 \\
\hline $14041-58-8$ & Cadmium 114 & $<6.74 \mathrm{E}-4$ & ug/g dry & $6.74 \mathrm{E}-4$ & $8 / 26 / 08$ & $8 \mathrm{H} 26016$ & PNNL-AGG-415 \\
\hline $14265-72-6$ & Antimony 121 & $<5.82 \mathrm{E}-4$ & ug/g dry & $5.82 \mathrm{E}-4$ & $8 / 26 / 08$ & $8 \mathrm{H} 26016$ & PNNL-AGG-415 \\
\hline $14119-16-5$ & Antimony 123 & $<4.64 \mathrm{E}-4$ & ug/g dry & 4.64E-4 & $8 / 26 / 08$ & $8 \mathrm{H} 26016$ & PNNL-AGG-415 \\
\hline $13966-27-3$ & Lead 206 & $<7.76 \mathrm{E}-4$ & ug/g dry & $7.76 \mathrm{E}-4$ & $8 / 26 / 08$ & $8 \mathrm{H} 26016$ & PNNL-AGG-415 \\
\hline $13966-28-4$ & Lead 208 & $<6.04 \mathrm{E}-4$ & ug/g dry & $6.04 \mathrm{E}-4$ & $8 / 26 / 08$ & $8 \mathrm{H} 26016$ & PNNL-AGG-415 \\
\hline HEIS No. & B1V991 & \multicolumn{3}{|c|}{ Lab ID: $\quad$ 0805003-01 } & & & \\
\hline $14092-98-9$ & Chromium 52 & $<2.06 \mathrm{E}-3$ & ug/g dry & $2.06 \mathrm{E}-3$ & $8 / 26 / 08$ & $8 \mathrm{H} 26016$ & PNNL-AGG-415 \\
\hline $13981-78-7$ & Chromium 53 & $<6.40 \mathrm{E}-3$ & ug/g dry & $6.40 \mathrm{E}-3$ & $8 / 26 / 08$ & $8 \mathrm{H} 26016$ & PNNL-AGG-415 \\
\hline $14191-84-5$ & Copper 63 & $4.61 \mathrm{E}-3$ & ug/g dry & $3.48 \mathrm{E}-3$ & $8 / 26 / 08$ & $8 \mathrm{H} 26016$ & PNNL-AGG-415 \\
\hline $14119-06-3$ & Copper 65 & $<4.84 \mathrm{E}-3$ & ug/g dry & $4.84 \mathrm{E}-3$ & $8 / 26 / 08$ & $8 \mathrm{H} 26016$ & PNNL-AGG-415 \\
\hline $7440-38-2$ & Arsenic 75 & $<6.25 \mathrm{E}-3$ & ug/g dry & $6.25 \mathrm{E}-3$ & $8 / 26 / 08$ & $8 \mathrm{H} 26016$ & PNNL-AGG-415 \\
\hline $14687-58-2$ & Selenium 82 & $<1.11 \mathrm{E}-2$ & ug/g dry & $1.11 \mathrm{E}-2$ & $8 / 26 / 08$ & $8 \mathrm{H} 26016$ & PNNL-AGG-415 \\
\hline $14392-17-7$ & Molybdenum 95 & $<1.61 \mathrm{E}-3$ & ug/g dry & $1.61 \mathrm{E}-3$ & $8 / 26 / 08$ & $8 \mathrm{H} 26016$ & PNNL-AGG-415 \\
\hline $14392-19-9$ & Molybdenum 97 & $<2.05 \mathrm{E}-3$ & ug/g dry & $2.05 \mathrm{E}-3$ & $8 / 26 / 08$ & $8 \mathrm{H} 26016$ & PNNL-AGG-415 \\
\hline $14392-20-2$ & Molybdenum 98 & $<1.61 \mathrm{E}-3$ & ug/g dry & $1.61 \mathrm{E}-3$ & $8 / 26 / 08$ & $8 \mathrm{H} 26016$ & PNNL-AGG-415 \\
\hline $14914-61-5$ & Ruthenium 101 & $<8.20 \mathrm{E}-4$ & ug/g dry & $8.20 \mathrm{E}-4$ & $8 / 26 / 08$ & $8 \mathrm{H} 26016$ & PNNL-AGG-415 \\
\hline $14914-62-6$ & Ruthenium 102 & $<5.65 \mathrm{E}-4$ & ug/g dry & $5.65 \mathrm{E}-4$ & $8 / 26 / 08$ & $8 \mathrm{H} 26016$ & PNNL-AGG-415 \\
\hline $15766-01-5$ & Ruthenium 104 & $<3.65 \mathrm{E}-4$ & ug/g dry & $3.65 \mathrm{E}-4$ & $8 / 26 / 08$ & $8 \mathrm{H} 26016$ & PNNL-AGG-415 \\
\hline $14378-37-1$ & Silver 107 & $<9.25 \mathrm{E}-4$ & ug/g dry & $9.25 \mathrm{E}-4$ & $8 / 26 / 08$ & $8 \mathrm{H} 26016$ & PNNL-AGG-415 \\
\hline $14378-38-2$ & Silver 109 & $<1.07 \mathrm{E}-3$ & ug/g dry & $1.07 \mathrm{E}-3$ & $8 / 26 / 08$ & $8 \mathrm{H} 26016$ & PNNL-AGG-415 \\
\hline $14336-64-2$ & Cadmium 111 & $<2.95 \mathrm{E}-4$ & ug/g dry & $2.95 \mathrm{E}-4$ & $8 / 26 / 08$ & $8 \mathrm{H} 26016$ & PNNL-AGG-415 \\
\hline $14041-58-8$ & Cadmium 114 & $<6.25 \mathrm{E}-4$ & ug/g dry & $6.25 \mathrm{E}-4$ & $8 / 26 / 08$ & $8 \mathrm{H} 26016$ & PNNL-AGG-415 \\
\hline $14265-72-6$ & Antimony 121 & $<5.40 \mathrm{E}-4$ & ug/g dry & $5.40 \mathrm{E}-4$ & $8 / 26 / 08$ & $8 \mathrm{H} 26016$ & PNNL-AGG-415 \\
\hline $13966-27-3$ & Lead 206 & $<7.20 \mathrm{E}-4$ & ug/g dry & $7.20 \mathrm{E}-4$ & $8 / 26 / 08$ & $8 \mathrm{H} 26016$ & PNNL-AGG-415 \\
\hline
\end{tabular}


RCRA Metals By PNNL-AGG-415/Water Extract

\begin{tabular}{|c|c|c|c|c|c|c|c|}
\hline CAS \# & Analyte & Results & Units & EQL & Analyzed & Batch & Method \\
\hline HEIS No. & B1V991 & \multicolumn{3}{|c|}{ Lab ID: $\quad$ 0805003-01 } & & & \\
\hline $13966-28-4$ & Lead 208 & $<5.60 \mathrm{E}-4$ & ug/g dry & $5.60 \mathrm{E}-4$ & $8 / 26 / 08$ & $8 \mathrm{H} 26016$ & PNNL-AGG-415 \\
\hline HEIS No. & B1V992 & \multicolumn{3}{|c|}{ Lab ID: $\quad$ 0805003-02 } & & & \\
\hline $14092-98-9$ & Chromium 52 & $<2.05 \mathrm{E}-3$ & ug/g dry & $2.05 \mathrm{E}-3$ & $8 / 26 / 08$ & $8 \mathrm{H} 26016$ & PNNL-AGG-415 \\
\hline $13981-78-7$ & Chromium 53 & $<6.38 \mathrm{E}-3$ & ug/g dry & $6.38 \mathrm{E}-3$ & $8 / 26 / 08$ & $8 \mathrm{H} 26016$ & PNNL-AGG-415 \\
\hline $14191-84-5$ & Copper 63 & $<3.47 \mathrm{E}-3$ & ug/g dry & $3.47 \mathrm{E}-3$ & $8 / 26 / 08$ & $8 \mathrm{H} 26016$ & PNNL-AGG-415 \\
\hline $14119-06-3$ & Copper 65 & $<4.83 \mathrm{E}-3$ & ug/g dry & $4.83 \mathrm{E}-3$ & $8 / 26 / 08$ & $8 \mathrm{H} 26016$ & PNNL-AGG-415 \\
\hline $7440-38-2$ & Arsenic 75 & $1.13 \mathrm{E}-2$ & ug/g dry & $6.23 \mathrm{E}-3$ & $8 / 26 / 08$ & $8 \mathrm{H} 26016$ & PNNL-AGG-415 \\
\hline $14687-58-2$ & Selenium 82 & $<1.10 \mathrm{E}-2$ & ug/g dry & $1.10 \mathrm{E}-2$ & $8 / 26 / 08$ & $8 \mathrm{H} 26016$ & PNNL-AGG-415 \\
\hline $14392-17-7$ & Molybdenum 95 & $<1.60 \mathrm{E}-3$ & $\mathrm{ug} / \mathrm{g}$ dry & $1.60 \mathrm{E}-3$ & $8 / 26 / 08$ & $8 \mathrm{H} 26016$ & PNNL-AGG-415 \\
\hline $14392-19-9$ & Molybdenum 97 & $<2.04 \mathrm{E}-3$ & ug/g dry & $2.04 \mathrm{E}-3$ & $8 / 26 / 08$ & $8 \mathrm{H} 26016$ & PNNL-AGG-415 \\
\hline $14392-20-2$ & Molybdenum 98 & $<1.60 \mathrm{E}-3$ & ug/g dry & $1.60 \mathrm{E}-3$ & $8 / 26 / 08$ & $8 \mathrm{H} 26016$ & PNNL-AGG-415 \\
\hline $14914-61-5$ & Ruthenium 101 & $<8.18 \mathrm{E}-4$ & ug/g dry & $8.18 \mathrm{E}-4$ & $8 / 26 / 08$ & $8 \mathrm{H} 26016$ & PNNL-AGG-415 \\
\hline $14914-62-6$ & Ruthenium 102 & $<5.63 \mathrm{E}-4$ & ug/g dry & $5.63 \mathrm{E}-4$ & $8 / 26 / 08$ & $8 \mathrm{H} 26016$ & PNNL-AGG-415 \\
\hline $15766-01-5$ & Ruthenium 104 & $<3.64 \mathrm{E}-4$ & ug/g dry & $3.64 \mathrm{E}-4$ & $8 / 26 / 08$ & $8 \mathrm{H} 26016$ & PNNL-AGG-415 \\
\hline $14378-37-1$ & Silver 107 & $<9.22 \mathrm{E}-4$ & ug/g dry & $9.22 \mathrm{E}-4$ & $8 / 26 / 08$ & $8 \mathrm{H} 26016$ & PNNL-AGG-415 \\
\hline $14378-38-2$ & Silver 109 & $<1.07 \mathrm{E}-3$ & ug/g dry & $1.07 \mathrm{E}-3$ & $8 / 26 / 08$ & $8 \mathrm{H} 26016$ & PNNL-AGG-415 \\
\hline $14336-64-2$ & Cadmium 111 & $<2.94 \mathrm{E}-4$ & ug/g dry & $2.94 \mathrm{E}-4$ & $8 / 26 / 08$ & $8 \mathrm{H} 26016$ & PNNL-AGG-415 \\
\hline $14041-58-8$ & Cadmium 114 & $<6.23 \mathrm{E}-4$ & ug/g dry & $6.23 \mathrm{E}-4$ & $8 / 26 / 08$ & $8 \mathrm{H} 26016$ & PNNL-AGG-415 \\
\hline $14265-72-6$ & Antimony 121 & $<5.38 \mathrm{E}-4$ & ug/g dry & $5.38 \mathrm{E}-4$ & $8 / 26 / 08$ & $8 \mathrm{H} 26016$ & PNNL-AGG-415 \\
\hline $13966-27-3$ & Lead 206 & $<7.18 \mathrm{E}-4$ & $\mathrm{ug} / \mathrm{g}$ dry & $7.18 \mathrm{E}-4$ & $8 / 26 / 08$ & $8 \mathrm{H} 26016$ & PNNL-AGG-415 \\
\hline $13966-28-4$ & Lead 208 & $<5.58 \mathrm{E}-4$ & ug/g dry & $5.58 \mathrm{E}-4$ & $8 / 26 / 08$ & $8 \mathrm{H} 26016$ & PNNL-AGG-415 \\
\hline HEIS No. & B1V993 & \multicolumn{3}{|c|}{ Lab ID: $\quad$ 0805003-03 } & & & \\
\hline $14092-98-9$ & Chromium 52 & $<2.04 \mathrm{E}-3$ & ug/g dry & $2.04 \mathrm{E}-3$ & $8 / 26 / 08$ & $8 \mathrm{H} 26016$ & PNNL-AGG-415 \\
\hline $13981-78-7$ & Chromium 53 & $<6.35 \mathrm{E}-3$ & ug/g dry & $6.35 \mathrm{E}-3$ & $8 / 26 / 08$ & $8 \mathrm{H} 26016$ & PNNL-AGG-415 \\
\hline $14191-84-5$ & Copper 63 & $<3.45 \mathrm{E}-3$ & ug/g dry & $3.45 \mathrm{E}-3$ & $8 / 26 / 08$ & $8 \mathrm{H} 26016$ & PNNL-AGG-415 \\
\hline $14119-06-3$ & Copper 65 & $<4.80 \mathrm{E}-3$ & ug/g dry & $4.80 \mathrm{E}-3$ & $8 / 26 / 08$ & $8 \mathrm{H} 26016$ & PNNL-AGG-415 \\
\hline $7440-38-2$ & Arsenic 75 & $7.55 \mathrm{E}-3$ & ug/g dry & $6.20 \mathrm{E}-3$ & $8 / 26 / 08$ & $8 \mathrm{H} 26016$ & PNNL-AGG-415 \\
\hline $14687-58-2$ & Selenium 82 & $<1.10 \mathrm{E}-2$ & $\mathrm{ug} / \mathrm{g}$ dry & $1.10 \mathrm{E}-2$ & $8 / 26 / 08$ & $8 \mathrm{H} 26016$ & PNNL-AGG-415 \\
\hline $14392-17-7$ & Molybdenum 95 & $<1.59 \mathrm{E}-3$ & ug/g dry & $1.59 \mathrm{E}-3$ & $8 / 26 / 08$ & $8 \mathrm{H} 26016$ & PNNL-AGG-415 \\
\hline $14392-19-9$ & Molybdenum 97 & $<2.03 \mathrm{E}-3$ & ug/g dry & $2.03 \mathrm{E}-3$ & $8 / 26 / 08$ & $8 \mathrm{H} 26016$ & PNNL-AGG-415 \\
\hline $14392-20-2$ & Molybdenum 98 & $<1.59 \mathrm{E}-3$ & $\mathrm{ug} / \mathrm{g}$ dry & $1.59 \mathrm{E}-3$ & $8 / 26 / 08$ & $8 \mathrm{H} 26016$ & PNNL-AGG-415 \\
\hline $14914-61-5$ & Ruthenium 101 & $<8.13 \mathrm{E}-4$ & ug/g dry & $8.13 \mathrm{E}-4$ & $8 / 26 / 08$ & $8 \mathrm{H} 26016$ & PNNL-AGG-415 \\
\hline $14914-62-6$ & Ruthenium 102 & $<5.60 \mathrm{E}-4$ & ug/g dry & $5.60 \mathrm{E}-4$ & $8 / 26 / 08$ & $8 \mathrm{H} 26016$ & PNNL-AGG-415 \\
\hline $15766-01-5$ & Ruthenium 104 & $<3.62 \mathrm{E}-4$ & ug/g dry & $3.62 \mathrm{E}-4$ & $8 / 26 / 08$ & $8 \mathrm{H} 26016$ & PNNL-AGG-415 \\
\hline $14378-37-1$ & Silver 107 & $<9.18 \mathrm{E}-4$ & ug/g dry & $9.18 \mathrm{E}-4$ & $8 / 26 / 08$ & $8 \mathrm{H} 26016$ & PNNL-AGG-415 \\
\hline $14378-38-2$ & Silver 109 & $<1.06 \mathrm{E}-3$ & ug/g dry & $1.06 \mathrm{E}-3$ & $8 / 26 / 08$ & $8 \mathrm{H} 26016$ & PNNL-AGG-415 \\
\hline $14336-64-2$ & Cadmium 111 & $<2.93 \mathrm{E}-4$ & ug/g dry & $2.93 \mathrm{E}-4$ & $8 / 26 / 08$ & $8 \mathrm{H} 26016$ & PNNL-AGG-415 \\
\hline $14041-58-8$ & Cadmium 114 & $<6.20 \mathrm{E}-4$ & ug/g dry & $6.20 \mathrm{E}-4$ & $8 / 26 / 08$ & $8 \mathrm{H} 26016$ & PNNL-AGG-415 \\
\hline $14265-72-6$ & Antimony 121 & $<5.36 \mathrm{E}-4$ & ug/g dry & $5.36 \mathrm{E}-4$ & $8 / 26 / 08$ & $8 \mathrm{H} 26016$ & PNNL-AGG-415 \\
\hline $13966-27-3$ & Lead 206 & $<7.14 \mathrm{E}-4$ & ug/g dry & $7.14 \mathrm{E}-4$ & $8 / 26 / 08$ & $8 \mathrm{H} 26016$ & PNNL-AGG-415 \\
\hline $13966-28-4$ & Lead 208 & $<5.56 \mathrm{E}-4$ & ug/g dry & $5.56 \mathrm{E}-4$ & $8 / 26 / 08$ & $8 \mathrm{H} 26016$ & PNNL-AGG-415 \\
\hline HEIS No. & B1V994 & & b ID: & 5003-04 & & & \\
\hline $14092-98-9$ & Chromium 52 & $<2.05 \mathrm{E}-3$ & ug/g dry & $2.05 \mathrm{E}-3$ & $8 / 26 / 08$ & $8 \mathrm{H} 26016$ & PNNL-AGG-415 \\
\hline $13981-78-7$ & Chromium 53 & $<6.38 \mathrm{E}-3$ & ug/g dry & $6.38 \mathrm{E}-3$ & $8 / 26 / 08$ & $8 \mathrm{H} 26016$ & PNNL-AGG-415 \\
\hline $14191-84-5$ & Copper 63 & $<3.47 \mathrm{E}-3$ & ug/g dry & $3.47 \mathrm{E}-3$ & $8 / 26 / 08$ & $8 \mathrm{H} 26016$ & PNNL-AGG-415 \\
\hline $14119-06-3$ & Copper 65 & $<4.82 \mathrm{E}-3$ & ug/g dry & $4.82 \mathrm{E}-3$ & $8 / 26 / 08$ & $8 \mathrm{H} 26016$ & PNNL-AGG-415 \\
\hline $7440-38-2$ & Arsenic 75 & $9.99 \mathrm{E}-3$ & ug/g dry & $6.23 \mathrm{E}-3$ & $8 / 26 / 08$ & $8 \mathrm{H} 26016$ & PNNL-AGG-415 \\
\hline $14687-58-2$ & Selenium 82 & $<1.10 \mathrm{E}-2$ & ug/g dry & $1.10 \mathrm{E}-2$ & $8 / 26 / 08$ & $8 \mathrm{H} 26016$ & PNNL-AGG-415 \\
\hline
\end{tabular}


RCRA Metals By PNNL-AGG-415/Water Extract

\begin{tabular}{|c|c|c|c|c|c|c|c|}
\hline CAS \# & Analyte & Results & Units & EQL & Analyzed & Batch & Method \\
\hline HEIS No. & B1V994 & \multicolumn{3}{|c|}{ Lab ID: $\quad$ 0805003-04 } & & & \\
\hline $14392-17-7$ & Molybdenum 95 & $1.81 \mathrm{E}-3$ & $\mathrm{ug} / \mathrm{g}$ dry & $1.60 \mathrm{E}-3$ & $8 / 26 / 08$ & $8 \mathrm{H} 26016$ & PNNL-AGG-415 \\
\hline 14392-19-9 & Molybdenum 97 & $<2.04 \mathrm{E}-3$ & ug/g dry & $2.04 \mathrm{E}-3$ & $8 / 26 / 08$ & $8 \mathrm{H} 26016$ & PNNL-AGG-415 \\
\hline $14392-20-2$ & Molybdenum 98 & $1.89 \mathrm{E}-3$ & ug/g dry & $1.60 \mathrm{E}-3$ & $8 / 26 / 08$ & $8 \mathrm{H} 26016$ & PNNL-AGG-415 \\
\hline $14914-61-5$ & Ruthenium 101 & $<8.17 \mathrm{E}-4$ & $\mathrm{ug} / \mathrm{g}$ dry & $8.17 \mathrm{E}-4$ & $8 / 26 / 08$ & $8 \mathrm{H} 26016$ & PNNL-AGG-415 \\
\hline $14914-62-6$ & Ruthenium 102 & $<5.63 \mathrm{E}-4$ & ug/g dry & $5.63 \mathrm{E}-4$ & $8 / 26 / 08$ & $8 \mathrm{H} 26016$ & PNNL-AGG-415 \\
\hline $15766-01-5$ & Ruthenium 104 & $<3.64 \mathrm{E}-4$ & $\mathrm{ug} / \mathrm{g}$ dry & $3.64 \mathrm{E}-4$ & $8 / 26 / 08$ & $8 \mathrm{H} 26016$ & PNNL-AGG-415 \\
\hline $14378-37-1$ & Silver 107 & $<9.21 \mathrm{E}-4$ & $\mathrm{ug} / \mathrm{g}$ dry & $9.21 \mathrm{E}-4$ & $8 / 26 / 08$ & $8 \mathrm{H} 26016$ & PNNL-AGG-415 \\
\hline $14378-38-2$ & Silver 109 & $<1.07 \mathrm{E}-3$ & $\mathrm{ug} / \mathrm{g}$ dry & $1.07 \mathrm{E}-3$ & $8 / 26 / 08$ & $8 \mathrm{H} 26016$ & PNNL-AGG-415 \\
\hline $14336-64-2$ & Cadmium 111 & $<2.94 \mathrm{E}-4$ & $\mathrm{ug} / \mathrm{g}$ dry & $2.94 \mathrm{E}-4$ & $8 / 26 / 08$ & $8 \mathrm{H} 26016$ & PNNL-AGG-415 \\
\hline $14041-58-8$ & Cadmium 114 & $<6.23 \mathrm{E}-4$ & $\mathrm{ug} / \mathrm{g}$ dry & $6.23 \mathrm{E}-4$ & $8 / 26 / 08$ & $8 \mathrm{H} 26016$ & PNNL-AGG-415 \\
\hline $14265-72-6$ & Antimony 121 & $<5.38 \mathrm{E}-4$ & $\mathrm{ug} / \mathrm{g}$ dry & $5.38 \mathrm{E}-4$ & $8 / 26 / 08$ & $8 \mathrm{H} 26016$ & PNNL-AGG-415 \\
\hline $13966-27-3$ & Lead 206 & $<7.17 \mathrm{E}-4$ & $\mathrm{ug} / \mathrm{g}$ dry & 7.17E-4 & $8 / 26 / 08$ & $8 \mathrm{H} 26016$ & PNNL-AGG-415 \\
\hline $13966-28-4$ & Lead 208 & $<5.58 \mathrm{E}-4$ & ug/g dry & $5.58 \mathrm{E}-4$ & $8 / 26 / 08$ & $8 \mathrm{H} 26016$ & PNNL-AGG-415 \\
\hline HEIS No. & B1V995 & \multicolumn{3}{|c|}{ Lab ID: $\quad$ 0805003-05 } & & & \\
\hline $14092-98-9$ & Chromium 52 & $<2.05 \mathrm{E}-3$ & ug/g dry & $2.05 \mathrm{E}-3$ & $8 / 26 / 08$ & $8 \mathrm{H} 26016$ & PNNL-AGG-415 \\
\hline $13981-78-7$ & Chromium 53 & $<6.40 \mathrm{E}-3$ & ug/g dry & $6.40 \mathrm{E}-3$ & $8 / 26 / 08$ & $8 \mathrm{H} 26016$ & PNNL-AGG-415 \\
\hline $14191-84-5$ & Copper 63 & $<3.48 \mathrm{E}-3$ & ug/g dry & $3.48 \mathrm{E}-3$ & $8 / 26 / 08$ & $8 \mathrm{H} 26016$ & PNNL-AGG-415 \\
\hline $14119-06-3$ & Copper 65 & $<4.84 \mathrm{E}-3$ & $\mathrm{ug} / \mathrm{g}$ dry & $4.84 \mathrm{E}-3$ & $8 / 26 / 08$ & $8 \mathrm{H} 26016$ & PNNL-AGG-415 \\
\hline $7440-38-2$ & Arsenic 75 & $1.05 \mathrm{E}-2$ & $\mathrm{ug} / \mathrm{g}$ dry & $6.25 \mathrm{E}-3$ & $8 / 26 / 08$ & $8 \mathrm{H} 26016$ & PNNL-AGG-415 \\
\hline $14687-58-2$ & Selenium 82 & $<1.10 \mathrm{E}-2$ & ug/g dry & $1.10 \mathrm{E}-2$ & $8 / 26 / 08$ & $8 \mathrm{H} 26016$ & PNNL-AGG-415 \\
\hline $14392-17-7$ & Molybdenum 95 & $2.30 \mathrm{E}-3$ & $\mathrm{ug} / \mathrm{g}$ dry & $1.60 \mathrm{E}-3$ & $8 / 26 / 08$ & $8 \mathrm{H} 26016$ & PNNL-AGG-415 \\
\hline 14392-19-9 & Molybdenum 97 & $2.40 \mathrm{E}-3$ & $\mathrm{ug} / \mathrm{g}$ dry & $2.04 \mathrm{E}-3$ & $8 / 26 / 08$ & $8 \mathrm{H} 26016$ & PNNL-AGG-415 \\
\hline $14392-20-2$ & Molybdenum 98 & $2.38 \mathrm{E}-3$ & ug/g dry & $1.60 \mathrm{E}-3$ & $8 / 26 / 08$ & $8 \mathrm{H} 26016$ & PNNL-AGG-415 \\
\hline $14914-61-5$ & Ruthenium 101 & $<8.20 \mathrm{E}-4$ & ug/g dry & $8.20 \mathrm{E}-4$ & $8 / 26 / 08$ & $8 \mathrm{H} 26016$ & PNNL-AGG-415 \\
\hline $14914-62-6$ & Ruthenium 102 & $<5.65 \mathrm{E}-4$ & ug/g dry & $5.65 \mathrm{E}-4$ & $8 / 26 / 08$ & $8 \mathrm{H} 26016$ & PNNL-AGG-415 \\
\hline $15766-01-5$ & Ruthenium 104 & $<3.65 \mathrm{E}-4$ & ug/g dry & $3.65 \mathrm{E}-4$ & $8 / 26 / 08$ & $8 \mathrm{H} 26016$ & PNNL-AGG-415 \\
\hline $14378-37-1$ & Silver 107 & $<9.25 \mathrm{E}-4$ & ug/g dry & $9.25 \mathrm{E}-4$ & $8 / 26 / 08$ & $8 \mathrm{H} 26016$ & PNNL-AGG-415 \\
\hline $14378-38-2$ & Silver 109 & $<1.07 \mathrm{E}-3$ & $\mathrm{ug} / \mathrm{g}$ dry & $1.07 \mathrm{E}-3$ & $8 / 26 / 08$ & $8 \mathrm{H} 26016$ & PNNL-AGG-415 \\
\hline $14336-64-2$ & Cadmium 111 & $<2.95 \mathrm{E}-4$ & ug/g dry & $2.95 \mathrm{E}-4$ & $8 / 26 / 08$ & $8 \mathrm{H} 26016$ & PNNL-AGG-415 \\
\hline $14041-58-8$ & Cadmium 114 & $<6.25 \mathrm{E}-4$ & ug/g dry & $6.25 \mathrm{E}-4$ & $8 / 26 / 08$ & $8 \mathrm{H} 26016$ & PNNL-AGG-415 \\
\hline $14265-72-6$ & Antimony 121 & $<5.40 \mathrm{E}-4$ & ug/g dry & $5.40 \mathrm{E}-4$ & $8 / 26 / 08$ & $8 \mathrm{H} 26016$ & PNNL-AGG-415 \\
\hline $13966-27-3$ & Lead 206 & $<7.20 \mathrm{E}-4$ & $\mathrm{ug} / \mathrm{g}$ dry & $7.20 \mathrm{E}-4$ & $8 / 26 / 08$ & $8 \mathrm{H} 26016$ & PNNL-AGG-415 \\
\hline $13966-28-4$ & Lead 208 & $<5.60 \mathrm{E}-4$ & ug/g dry & $5.60 \mathrm{E}-4$ & $8 / 26 / 08$ & $8 \mathrm{H} 26016$ & PNNL-AGG-415 \\
\hline HEIS No. & B1V996 & \multicolumn{3}{|c|}{ Lab ID: $\quad$ 0805003-06 } & & & \\
\hline $14092-98-9$ & Chromium 52 & $<2.06 \mathrm{E}-3$ & ug/g dry & $2.06 \mathrm{E}-3$ & $8 / 26 / 08$ & $8 \mathrm{H} 26017$ & PNNL-AGG-415 \\
\hline $13981-78-7$ & Chromium 53 & $<6.40 \mathrm{E}-3$ & $\mathrm{ug} / \mathrm{g}$ dry & $6.40 \mathrm{E}-3$ & $8 / 26 / 08$ & $8 \mathrm{H} 26017$ & PNNL-AGG-415 \\
\hline $14191-84-5$ & Copper 63 & $5.79 \mathrm{E}-3$ & ug/g dry & $3.48 \mathrm{E}-3$ & $8 / 26 / 08$ & $8 \mathrm{H} 26017$ & PNNL-AGG-415 \\
\hline $14119-06-3$ & Copper 65 & $5.49 \mathrm{E}-3$ & $\mathrm{ug} / \mathrm{g}$ dry & $4.84 \mathrm{E}-3$ & $8 / 26 / 08$ & $8 \mathrm{H} 26017$ & PNNL-AGG-415 \\
\hline $7440-38-2$ & Arsenic 75 & $1.45 \mathrm{E}-2$ & $\mathrm{ug} / \mathrm{g}$ dry & $6.25 \mathrm{E}-3$ & $8 / 26 / 08$ & $8 \mathrm{H} 26017$ & PNNL-AGG-415 \\
\hline $14687-58-2$ & Selenium 82 & $<1.11 \mathrm{E}-2$ & $\mathrm{ug} / \mathrm{g}$ dry & $1.11 \mathrm{E}-2$ & $8 / 26 / 08$ & $8 \mathrm{H} 26017$ & PNNL-AGG-415 \\
\hline $14392-17-7$ & Molybdenum 95 & $2.77 \mathrm{E}-3$ & $\mathrm{ug} / \mathrm{g}$ dry & $1.61 \mathrm{E}-3$ & $8 / 26 / 08$ & $8 \mathrm{H} 26017$ & PNNL-AGG-415 \\
\hline $14392-19-9$ & Molybdenum 97 & $2.87 \mathrm{E}-3$ & $\mathrm{ug} / \mathrm{g}$ dry & $2.05 \mathrm{E}-3$ & $8 / 26 / 08$ & $8 \mathrm{H} 26017$ & PNNL-AGG-415 \\
\hline $14392-20-2$ & Molybdenum 98 & $2.89 \mathrm{E}-3$ & ug/g dry & $1.61 \mathrm{E}-3$ & $8 / 26 / 08$ & $8 \mathrm{H} 26017$ & PNNL-AGG-415 \\
\hline $14914-61-5$ & Ruthenium 101 & $<8.20 \mathrm{E}-4$ & $\mathrm{ug} / \mathrm{g}$ dry & $8.20 \mathrm{E}-4$ & $8 / 26 / 08$ & $8 \mathrm{H} 26017$ & PNNL-AGG-415 \\
\hline $14914-62-6$ & Ruthenium 102 & $<5.65 \mathrm{E}-4$ & ug/g dry & $5.65 \mathrm{E}-4$ & $8 / 26 / 08$ & $8 \mathrm{H} 26017$ & PNNL-AGG-415 \\
\hline $15766-01-5$ & Ruthenium 104 & $<3.65 \mathrm{E}-4$ & ug/g dry & $3.65 \mathrm{E}-4$ & $8 / 26 / 08$ & $8 \mathrm{H} 26017$ & PNNL-AGG-415 \\
\hline $14378-37-1$ & Silver 107 & $<9.25 \mathrm{E}-4$ & ug/g dry & $9.25 \mathrm{E}-4$ & $8 / 26 / 08$ & $8 \mathrm{H} 26017$ & PNNL-AGG-415 \\
\hline $14378-38-2$ & Silver 109 & $<1.07 \mathrm{E}-3$ & ug/g dry & $1.07 \mathrm{E}-3$ & $8 / 26 / 08$ & $8 \mathrm{H} 26017$ & PNNL-AGG-415 \\
\hline
\end{tabular}


RCRA Metals By PNNL-AGG-415/Water Extract

\begin{tabular}{|c|c|c|c|c|c|c|c|}
\hline CAS \# & Analyte & Results & Units & EQL & Analyzed & Batch & Method \\
\hline HEIS No. & B1V996 & \multicolumn{3}{|c|}{ Lab ID: $\quad$ 0805003-06 } & & & \\
\hline $14336-64-2$ & Cadmium 111 & $<2.95 \mathrm{E}-4$ & ug/g dry & $2.95 \mathrm{E}-4$ & $8 / 26 / 08$ & $8 \mathrm{H} 26017$ & PNNL-AGG-415 \\
\hline $14041-58-8$ & Cadmium 114 & $<6.25 \mathrm{E}-4$ & ug/g dry & $6.25 \mathrm{E}-4$ & $8 / 26 / 08$ & $8 \mathrm{H} 26017$ & PNNL-AGG-415 \\
\hline $14265-72-6$ & Antimony 121 & $<5.40 \mathrm{E}-4$ & ug/g dry & $5.40 \mathrm{E}-4$ & $8 / 26 / 08$ & $8 \mathrm{H} 26017$ & PNNL-AGG-415 \\
\hline $13966-27-3$ & Lead 206 & $<7.20 \mathrm{E}-4$ & ug/g dry & $7.20 \mathrm{E}-4$ & $8 / 26 / 08$ & $8 \mathrm{H} 26017$ & PNNL-AGG-415 \\
\hline $13966-28-4$ & Lead 208 & $<5.60 \mathrm{E}-4$ & ug/g dry & $5.60 \mathrm{E}-4$ & $8 / 26 / 08$ & $8 \mathrm{H} 26017$ & PNNL-AGG-415 \\
\hline HEIS No. & $\begin{array}{l}\text { B1V997 } \\
\end{array}$ & \multicolumn{3}{|c|}{ Lab ID: $\quad$ 0805003-07 } & & & \\
\hline $14092-98-9$ & Chromium 52 & $<2.05 \mathrm{E}-3$ & ug/g dry & $2.05 \mathrm{E}-3$ & $8 / 26 / 08$ & $8 \mathrm{H} 26017$ & PNNL-AGG-415 \\
\hline $13981-78-7$ & Chromium 53 & $<6.39 \mathrm{E}-3$ & ug/g dry & $6.39 \mathrm{E}-3$ & $8 / 26 / 08$ & $8 \mathrm{H} 26017$ & PNNL-AGG-415 \\
\hline $14191-84-5$ & Copper 63 & $4.98 \mathrm{E}-3$ & ug/g dry & $3.48 \mathrm{E}-3$ & $8 / 26 / 08$ & $8 \mathrm{H} 26017$ & PNNL-AGG-415 \\
\hline $14119-06-3$ & Copper 65 & $<4.84 \mathrm{E}-3$ & ug/g dry & $4.84 \mathrm{E}-3$ & $8 / 26 / 08$ & $8 \mathrm{H} 26017$ & PNNL-AGG-415 \\
\hline $7440-38-2$ & Arsenic 75 & $1.38 \mathrm{E}-2$ & ug/g dry & $6.24 \mathrm{E}-3$ & $8 / 26 / 08$ & $8 \mathrm{H} 26017$ & PNNL-AGG-415 \\
\hline $14687-58-2$ & Selenium 82 & $<1.10 \mathrm{E}-2$ & ug/g dry & $1.10 \mathrm{E}-2$ & $8 / 26 / 08$ & $8 \mathrm{H} 26017$ & PNNL-AGG-415 \\
\hline $14392-17-7$ & Molybdenum 95 & $3.13 \mathrm{E}-3$ & ug/g dry & $1.60 \mathrm{E}-3$ & $8 / 26 / 08$ & $8 \mathrm{H} 26017$ & PNNL-AGG-415 \\
\hline $14392-19-9$ & Molybdenum 97 & $3.24 \mathrm{E}-3$ & ug/g dry & $2.04 \mathrm{E}-3$ & $8 / 26 / 08$ & $8 \mathrm{H} 26017$ & PNNL-AGG-415 \\
\hline $14392-20-2$ & Molybdenum 98 & $3.22 \mathrm{E}-3$ & ug/g dry & $1.60 \mathrm{E}-3$ & $8 / 26 / 08$ & $8 \mathrm{H} 26017$ & PNNL-AGG-415 \\
\hline $14914-61-5$ & Ruthenium 101 & $<8.19 \mathrm{E}-4$ & ug/g dry & $8.19 \mathrm{E}-4$ & $8 / 26 / 08$ & $8 \mathrm{H} 26017$ & PNNL-AGG-415 \\
\hline $14914-62-6$ & Ruthenium 102 & $<5.65 \mathrm{E}-4$ & ug/g dry & $5.65 \mathrm{E}-4$ & $8 / 26 / 08$ & $8 \mathrm{H} 26017$ & PNNL-AGG-415 \\
\hline $15766-01-5$ & Ruthenium 104 & $<3.65 \mathrm{E}-4$ & ug/g dry & $3.65 \mathrm{E}-4$ & $8 / 26 / 08$ & $8 \mathrm{H} 26017$ & PNNL-AGG-415 \\
\hline $14378-37-1$ & Silver 107 & $<9.24 \mathrm{E}-4$ & ug/g dry & $9.24 \mathrm{E}-4$ & $8 / 26 / 08$ & $8 \mathrm{H} 26017$ & PNNL-AGG-415 \\
\hline $14378-38-2$ & Silver 109 & $<1.07 \mathrm{E}-3$ & ug/g dry & $1.07 \mathrm{E}-3$ & $8 / 26 / 08$ & $8 \mathrm{H} 26017$ & PNNL-AGG-415 \\
\hline $14336-64-2$ & Cadmium 111 & $<2.95 \mathrm{E}-4$ & ug/g dry & $2.95 \mathrm{E}-4$ & $8 / 26 / 08$ & $8 \mathrm{H} 26017$ & PNNL-AGG-415 \\
\hline $14041-58-8$ & Cadmium 114 & $<6.24 \mathrm{E}-4$ & ug/g dry & $6.24 \mathrm{E}-4$ & $8 / 26 / 08$ & $8 \mathrm{H} 26017$ & PNNL-AGG-415 \\
\hline $14265-72-6$ & Antimony 121 & $<5.40 \mathrm{E}-4$ & ug/g dry & $5.40 \mathrm{E}-4$ & $8 / 26 / 08$ & $8 \mathrm{H} 26017$ & PNNL-AGG-415 \\
\hline $13966-27-3$ & Lead 206 & $<7.19 \mathrm{E}-4$ & ug/g dry & $7.19 \mathrm{E}-4$ & $8 / 26 / 08$ & $8 \mathrm{H} 26017$ & PNNL-AGG-415 \\
\hline $13966-28-4$ & Lead 208 & $<5.60 \mathrm{E}-4$ & ug/g dry & $5.60 \mathrm{E}-4$ & $8 / 26 / 08$ & $8 \mathrm{H} 26017$ & PNNL-AGG-415 \\
\hline HEIS No. & $\begin{array}{l}\text { B1V998 } \\
\end{array}$ & \multicolumn{3}{|c|}{ Lab ID: $\quad$ 0805003-08 } & & & \\
\hline $14092-98-9$ & Chromium 52 & $<2.06 \mathrm{E}-3$ & ug/g dry & $2.06 \mathrm{E}-3$ & $8 / 26 / 08$ & $8 \mathrm{H} 26017$ & PNNL-AGG-415 \\
\hline $13981-78-7$ & Chromium 53 & $<6.41 \mathrm{E}-3$ & ug/g dry & $6.41 \mathrm{E}-3$ & $8 / 26 / 08$ & $8 \mathrm{H} 26017$ & PNNL-AGG-415 \\
\hline $14191-84-5$ & Copper 63 & $1.47 \mathrm{E}-2$ & ug/g dry & $3.48 \mathrm{E}-3$ & $8 / 26 / 08$ & $8 \mathrm{H} 26017$ & PNNL-AGG-415 \\
\hline $14119-06-3$ & Copper 65 & $1.44 \mathrm{E}-2$ & ug/g dry & $4.84 \mathrm{E}-3$ & $8 / 26 / 08$ & $8 \mathrm{H} 26017$ & PNNL-AGG-415 \\
\hline $7440-38-2$ & Arsenic 75 & $1.34 \mathrm{E}-2$ & ug/g dry & $6.26 \mathrm{E}-3$ & $8 / 26 / 08$ & $8 \mathrm{H} 26017$ & PNNL-AGG-415 \\
\hline $14687-58-2$ & Selenium 82 & $<1.11 \mathrm{E}-2$ & ug/g dry & $1.11 \mathrm{E}-2$ & $8 / 26 / 08$ & $8 \mathrm{H} 26017$ & PNNL-AGG-415 \\
\hline $14392-17-7$ & Molybdenum 95 & $5.45 \mathrm{E}-3$ & ug/g dry & $1.61 \mathrm{E}-3$ & $8 / 26 / 08$ & $8 \mathrm{H} 26017$ & PNNL-AGG-415 \\
\hline $14392-19-9$ & Molybdenum 97 & $5.58 \mathrm{E}-3$ & ug/g dry & $2.05 \mathrm{E}-3$ & $8 / 26 / 08$ & $8 \mathrm{H} 26017$ & PNNL-AGG-415 \\
\hline $14392-20-2$ & Molybdenum 98 & $5.57 \mathrm{E}-3$ & ug/g dry & $1.61 \mathrm{E}-3$ & $8 / 26 / 08$ & $8 \mathrm{H} 26017$ & PNNL-AGG-415 \\
\hline $14914-61-5$ & Ruthenium 101 & $<8.21 \mathrm{E}-4$ & ug/g dry & $8.21 \mathrm{E}-4$ & $8 / 26 / 08$ & $8 \mathrm{H} 26017$ & PNNL-AGG-415 \\
\hline $14914-62-6$ & Ruthenium 102 & $<5.66 \mathrm{E}-4$ & ug/g dry & $5.66 \mathrm{E}-4$ & $8 / 26 / 08$ & $8 \mathrm{H} 26017$ & PNNL-AGG-415 \\
\hline $15766-01-5$ & Ruthenium 104 & $<3.65 \mathrm{E}-4$ & ug/g dry & $3.65 \mathrm{E}-4$ & $8 / 26 / 08$ & $8 \mathrm{H} 26017$ & PNNL-AGG-415 \\
\hline $14378-37-1$ & Silver 107 & $<9.26 \mathrm{E}-4$ & ug/g dry & $9.26 \mathrm{E}-4$ & $8 / 26 / 08$ & $8 \mathrm{H} 26017$ & PNNL-AGG-415 \\
\hline $14378-38-2$ & Silver 109 & $<1.07 \mathrm{E}-3$ & ug/g dry & $1.07 \mathrm{E}-3$ & $8 / 26 / 08$ & $8 \mathrm{H} 26017$ & PNNL-AGG-415 \\
\hline $14336-64-2$ & Cadmium 111 & $<2.95 \mathrm{E}-4$ & ug/g dry & $2.95 \mathrm{E}-4$ & $8 / 26 / 08$ & $8 \mathrm{H} 26017$ & PNNL-AGG-415 \\
\hline $14041-58-8$ & Cadmium 114 & $<6.26 \mathrm{E}-4$ & $\mathrm{ug} / \mathrm{g}$ dry & $6.26 \mathrm{E}-4$ & $8 / 26 / 08$ & $8 \mathrm{H} 26017$ & PNNL-AGG-415 \\
\hline $14265-72-6$ & Antimony 121 & $<5.40 \mathrm{E}-4$ & ug/g dry & $5.40 \mathrm{E}-4$ & $8 / 26 / 08$ & $8 \mathrm{H} 26017$ & PNNL-AGG-415 \\
\hline $13966-27-3$ & Lead 206 & $<7.21 \mathrm{E}-4$ & ug/g dry & $7.21 \mathrm{E}-4$ & $8 / 26 / 08$ & $8 \mathrm{H} 26017$ & PNNL-AGG-415 \\
\hline $13966-28-4$ & Lead 208 & $<5.61 \mathrm{E}-4$ & ug/g dry & $5.61 \mathrm{E}-4$ & $8 / 26 / 08$ & $8 \mathrm{H} 26017$ & PNNL-AGG-415 \\
\hline HEIS No. & B1V999 & & b ID: & 5003-09 & & & \\
\hline $14092-98-9$ & Chromium 52 & $<2.05 \mathrm{E}-3$ & ug/g dry & $2.05 \mathrm{E}-3$ & $8 / 26 / 08$ & $8 \mathrm{H} 26017$ & PNNL-AGG-415 \\
\hline $13981-78-7$ & Chromium 53 & $<6.38 \mathrm{E}-3$ & ug/g dry & $6.38 \mathrm{E}-3$ & $8 / 26 / 08$ & $8 \mathrm{H} 26017$ & PNNL-AGG-415 \\
\hline
\end{tabular}


RCRA Metals By PNNL-AGG-415/Water Extract

\begin{tabular}{|c|c|c|c|c|c|c|c|}
\hline CAS \# & Analyte & Results & Units & EQL & Analyzed & Batch & Method \\
\hline HEIS No. & B1V999 & \multicolumn{3}{|c|}{ Lab ID: $\quad$ 0805003-09 } & & & \\
\hline $14191-84-5$ & Copper 63 & $<3.47 \mathrm{E}-3$ & ug/g dry & $3.47 \mathrm{E}-3$ & $8 / 26 / 08$ & $8 \mathrm{H} 26017$ & PNNL-AGG-415 \\
\hline 14119-06-3 & Copper 65 & $<4.83 \mathrm{E}-3$ & ug/g dry & $4.83 \mathrm{E}-3$ & $8 / 26 / 08$ & $8 \mathrm{H} 26017$ & PNNL-AGG-415 \\
\hline $7440-38-2$ & Arsenic 75 & $8.86 \mathrm{E}-3$ & ug/g dry & $6.23 \mathrm{E}-3$ & $8 / 26 / 08$ & $8 \mathrm{H} 26017$ & PNNL-AGG-415 \\
\hline $14687-58-2$ & Selenium 82 & $<1.10 \mathrm{E}-2$ & ug/g dry & $1.10 \mathrm{E}-2$ & $8 / 26 / 08$ & $8 \mathrm{H} 26017$ & PNNL-AGG-415 \\
\hline $14392-17-7$ & Molybdenum 95 & $5.62 \mathrm{E}-3$ & ug/g dry & $1.60 \mathrm{E}-3$ & $8 / 26 / 08$ & $8 \mathrm{H} 26017$ & PNNL-AGG-415 \\
\hline 14392-19-9 & Molybdenum 97 & $5.63 \mathrm{E}-3$ & ug/g dry & $2.04 \mathrm{E}-3$ & $8 / 26 / 08$ & $8 \mathrm{H} 26017$ & PNNL-AGG-415 \\
\hline $14392-20-2$ & Molybdenum 98 & $5.65 \mathrm{E}-3$ & ug/g dry & $1.60 \mathrm{E}-3$ & $8 / 26 / 08$ & $8 \mathrm{H} 26017$ & PNNL-AGG-415 \\
\hline $14914-61-5$ & Ruthenium 101 & $<8.18 \mathrm{E}-4$ & ug/g dry & $8.18 \mathrm{E}-4$ & $8 / 26 / 08$ & $8 \mathrm{H} 26017$ & PNNL-AGG-415 \\
\hline $14914-62-6$ & Ruthenium 102 & $<5.63 \mathrm{E}-4$ & ug/g dry & $5.63 \mathrm{E}-4$ & $8 / 26 / 08$ & $8 \mathrm{H} 26017$ & PNNL-AGG-415 \\
\hline $15766-01-5$ & Ruthenium 104 & $<3.64 \mathrm{E}-4$ & ug/g dry & $3.64 \mathrm{E}-4$ & $8 / 26 / 08$ & $8 \mathrm{H} 26017$ & PNNL-AGG-415 \\
\hline $14378-37-1$ & Silver 107 & $<9.22 \mathrm{E}-4$ & ug/g dry & $9.22 \mathrm{E}-4$ & $8 / 26 / 08$ & $8 \mathrm{H} 26017$ & PNNL-AGG-415 \\
\hline $14378-38-2$ & Silver 109 & $<1.07 \mathrm{E}-3$ & ug/g dry & $1.07 \mathrm{E}-3$ & $8 / 26 / 08$ & $8 \mathrm{H} 26017$ & PNNL-AGG-415 \\
\hline $14336-64-2$ & Cadmium 111 & $<2.94 \mathrm{E}-4$ & ug/g dry & $2.94 \mathrm{E}-4$ & $8 / 26 / 08$ & $8 \mathrm{H} 26017$ & PNNL-AGG-415 \\
\hline $14041-58-8$ & Cadmium 114 & $<6.23 \mathrm{E}-4$ & ug/g dry & $6.23 \mathrm{E}-4$ & $8 / 26 / 08$ & $8 \mathrm{H} 26017$ & PNNL-AGG-415 \\
\hline $14265-72-6$ & Antimony 121 & $<5.38 \mathrm{E}-4$ & ug/g dry & $5.38 \mathrm{E}-4$ & $8 / 26 / 08$ & $8 \mathrm{H} 26017$ & PNNL-AGG-415 \\
\hline $13966-27-3$ & Lead 206 & $<7.18 \mathrm{E}-4$ & ug/g dry & $7.18 \mathrm{E}-4$ & $8 / 26 / 08$ & $8 \mathrm{H} 26017$ & PNNL-AGG-415 \\
\hline $13966-28-4$ & Lead 208 & $<5.58 \mathrm{E}-4$ & ug/g dry & $5.58 \mathrm{E}-4$ & $8 / 26 / 08$ & $8 \mathrm{H} 26017$ & PNNL-AGG-415 \\
\hline HEIS No. & B1V9B0 & \multicolumn{3}{|c|}{ Lab ID: $\quad$ 0805003-10 } & & & \\
\hline $14092-98-9$ & Chromium 52 & $<2.05 \mathrm{E}-3$ & ug/g dry & $2.05 \mathrm{E}-3$ & $8 / 26 / 08$ & $8 \mathrm{H} 26017$ & PNNL-AGG-415 \\
\hline $13981-78-7$ & Chromium 53 & $<6.38 \mathrm{E}-3$ & ug/g dry & $6.38 \mathrm{E}-3$ & $8 / 26 / 08$ & $8 \mathrm{H} 26017$ & PNNL-AGG-415 \\
\hline $14191-84-5$ & Copper 63 & $<3.47 \mathrm{E}-3$ & ug/g dry & $3.47 \mathrm{E}-3$ & $8 / 26 / 08$ & $8 \mathrm{H} 26017$ & PNNL-AGG-415 \\
\hline $14119-06-3$ & Copper 65 & $<4.82 \mathrm{E}-3$ & ug/g dry & $4.82 \mathrm{E}-3$ & $8 / 26 / 08$ & $8 \mathrm{H} 26017$ & PNNL-AGG-415 \\
\hline $7440-38-2$ & Arsenic 75 & $7.67 \mathrm{E}-3$ & ug/g dry & $6.23 \mathrm{E}-3$ & $8 / 26 / 08$ & $8 \mathrm{H} 26017$ & PNNL-AGG-415 \\
\hline $14687-58-2$ & Selenium 82 & $<1.10 \mathrm{E}-2$ & ug/g dry & $1.10 \mathrm{E}-2$ & $8 / 26 / 08$ & $8 \mathrm{H} 26017$ & PNNL-AGG-415 \\
\hline $14392-17-7$ & Molybdenum 95 & $5.72 \mathrm{E}-3$ & ug/g dry & $1.60 \mathrm{E}-3$ & $8 / 26 / 08$ & $8 \mathrm{H} 26017$ & PNNL-AGG-415 \\
\hline $14392-19-9$ & Molybdenum 97 & $5.72 \mathrm{E}-3$ & ug/g dry & $2.04 \mathrm{E}-3$ & $8 / 26 / 08$ & $8 \mathrm{H} 26017$ & PNNL-AGG-415 \\
\hline $14392-20-2$ & Molybdenum 98 & $5.65 \mathrm{E}-3$ & ug/g dry & $1.60 \mathrm{E}-3$ & $8 / 26 / 08$ & $8 \mathrm{H} 26017$ & PNNL-AGG-415 \\
\hline $14914-61-5$ & Ruthenium 101 & $<8.17 \mathrm{E}-4$ & ug/g dry & $8.17 \mathrm{E}-4$ & $8 / 26 / 08$ & $8 \mathrm{H} 26017$ & PNNL-AGG-415 \\
\hline $14914-62-6$ & Ruthenium 102 & $<5.63 \mathrm{E}-4$ & ug/g dry & $5.63 \mathrm{E}-4$ & $8 / 26 / 08$ & $8 \mathrm{H} 26017$ & PNNL-AGG-415 \\
\hline $15766-01-5$ & Ruthenium 104 & $<3.64 \mathrm{E}-4$ & ug/g dry & $3.64 \mathrm{E}-4$ & $8 / 26 / 08$ & $8 \mathrm{H} 26017$ & PNNL-AGG-415 \\
\hline $14378-37-1$ & Silver 107 & $<9.22 \mathrm{E}-4$ & ug/g dry & $9.22 \mathrm{E}-4$ & $8 / 26 / 08$ & $8 \mathrm{H} 26017$ & PNNL-AGG-415 \\
\hline $14378-38-2$ & Silver 109 & $<1.07 \mathrm{E}-3$ & ug/g dry & $1.07 \mathrm{E}-3$ & $8 / 26 / 08$ & $8 \mathrm{H} 26017$ & PNNL-AGG-415 \\
\hline $14336-64-2$ & Cadmium 111 & $<2.94 \mathrm{E}-4$ & ug/g dry & $2.94 \mathrm{E}-4$ & $8 / 26 / 08$ & $8 \mathrm{H} 26017$ & PNNL-AGG-415 \\
\hline $14041-58-8$ & Cadmium 114 & $<6.23 \mathrm{E}-4$ & ug/g dry & $6.23 \mathrm{E}-4$ & $8 / 26 / 08$ & $8 \mathrm{H} 26017$ & PNNL-AGG-415 \\
\hline $14265-72-6$ & Antimony 121 & $<5.38 \mathrm{E}-4$ & ug/g dry & $5.38 \mathrm{E}-4$ & $8 / 26 / 08$ & $8 \mathrm{H} 26017$ & PNNL-AGG-415 \\
\hline $13966-27-3$ & Lead 206 & $<7.18 \mathrm{E}-4$ & ug/g dry & $7.18 \mathrm{E}-4$ & $8 / 26 / 08$ & $8 \mathrm{H} 26017$ & PNNL-AGG-415 \\
\hline $13966-28-4$ & Lead 208 & $<5.58 \mathrm{E}-4$ & ug/g dry & $5.58 \mathrm{E}-4$ & $8 / 26 / 08$ & $8 \mathrm{H} 26017$ & PNNL-AGG-415 \\
\hline HEIS No. & B1V9B1 & \multicolumn{3}{|c|}{ Lab ID: $\quad$ 0805003-11 } & & & \\
\hline 14092-98-9 & Chromium 52 & $<2.05 \mathrm{E}-3$ & ug/g dry & $2.05 \mathrm{E}-3$ & $8 / 26 / 08$ & $8 \mathrm{H} 26017$ & PNNL-AGG-415 \\
\hline $13981-78-7$ & Chromium 53 & $<6.38 \mathrm{E}-3$ & ug/g dry & $6.38 \mathrm{E}-3$ & $8 / 26 / 08$ & $8 \mathrm{H} 26017$ & PNNL-AGG-415 \\
\hline $14191-84-5$ & Copper 63 & $<3.47 \mathrm{E}-3$ & ug/g dry & $3.47 \mathrm{E}-3$ & $8 / 26 / 08$ & $8 \mathrm{H} 26017$ & PNNL-AGG-415 \\
\hline $14119-06-3$ & Copper 65 & $<4.83 \mathrm{E}-3$ & $\mathrm{ug} / \mathrm{g}$ dry & $4.83 \mathrm{E}-3$ & $8 / 26 / 08$ & $8 \mathrm{H} 26017$ & PNNL-AGG-415 \\
\hline $7440-38-2$ & Arsenic 75 & $9.30 \mathrm{E}-3$ & ug/g dry & $6.23 \mathrm{E}-3$ & $8 / 26 / 08$ & $8 \mathrm{H} 26017$ & PNNL-AGG-415 \\
\hline $14687-58-2$ & Selenium 82 & $<1.10 \mathrm{E}-2$ & ug/g dry & $1.10 \mathrm{E}-2$ & $8 / 26 / 08$ & $8 \mathrm{H} 26017$ & PNNL-AGG-415 \\
\hline $14392-17-7$ & Molybdenum 95 & $7.18 \mathrm{E}-3$ & ug/g dry & $1.60 \mathrm{E}-3$ & $8 / 26 / 08$ & $8 \mathrm{H} 26017$ & PNNL-AGG-415 \\
\hline 14392-19-9 & Molybdenum 97 & $7.20 \mathrm{E}-3$ & ug/g dry & $2.04 \mathrm{E}-3$ & $8 / 26 / 08$ & $8 \mathrm{H} 26017$ & PNNL-AGG-415 \\
\hline $14392-20-2$ & Molybdenum 98 & $7.24 \mathrm{E}-3$ & ug/g dry & $1.60 \mathrm{E}-3$ & $8 / 26 / 08$ & $8 \mathrm{H} 26017$ & PNNL-AGG-415 \\
\hline $14914-61-5$ & Ruthenium 101 & $<8.18 \mathrm{E}-4$ & ug/g dry & $8.18 \mathrm{E}-4$ & $8 / 26 / 08$ & $8 \mathrm{H} 26017$ & PNNL-AGG-415 \\
\hline
\end{tabular}


RCRA Metals By PNNL-AGG-415/Water Extract

\begin{tabular}{|c|c|c|c|c|c|c|c|}
\hline CAS \# & Analyte & Results & Units & EQL & Analyzed & Batch & Method \\
\hline HEIS No. & B1V9B1 & \multicolumn{3}{|c|}{ Lab ID: $\quad$ 0805003-11 } & & & \\
\hline $14914-62-6$ & Ruthenium 102 & $<5.64 \mathrm{E}-4$ & ug/g dry & $5.64 \mathrm{E}-4$ & $8 / 26 / 08$ & $8 \mathrm{H} 26017$ & PNNL-AGG-415 \\
\hline $15766-01-5$ & Ruthenium 104 & $<3.64 \mathrm{E}-4$ & ug/g dry & $3.64 \mathrm{E}-4$ & $8 / 26 / 08$ & $8 \mathrm{H} 26017$ & PNNL-AGG-415 \\
\hline $14378-37-1$ & Silver 107 & $<9.23 \mathrm{E}-4$ & ug/g dry & $9.23 \mathrm{E}-4$ & $8 / 26 / 08$ & $8 \mathrm{H} 26017$ & PNNL-AGG-415 \\
\hline $14378-38-2$ & Silver 109 & $<1.07 \mathrm{E}-3$ & ug/g dry & $1.07 \mathrm{E}-3$ & $8 / 26 / 08$ & $8 \mathrm{H} 26017$ & PNNL-AGG-415 \\
\hline $14336-64-2$ & Cadmium 111 & $<2.94 \mathrm{E}-4$ & ug/g dry & $2.94 \mathrm{E}-4$ & $8 / 26 / 08$ & $8 \mathrm{H} 26017$ & PNNL-AGG-415 \\
\hline $14041-58-8$ & Cadmium 114 & $<6.23 \mathrm{E}-4$ & ug/g dry & $6.23 \mathrm{E}-4$ & $8 / 26 / 08$ & $8 \mathrm{H} 26017$ & PNNL-AGG-415 \\
\hline $14265-72-6$ & Antimony 121 & $<5.39 \mathrm{E}-4$ & ug/g dry & $5.39 \mathrm{E}-4$ & $8 / 26 / 08$ & $8 \mathrm{H} 26017$ & PNNL-AGG-415 \\
\hline $13966-27-3$ & Lead 206 & $<7.18 \mathrm{E}-4$ & ug/g dry & $7.18 \mathrm{E}-4$ & $8 / 26 / 08$ & $8 \mathrm{H} 26017$ & PNNL-AGG-415 \\
\hline $13966-28-4$ & Lead 208 & $<5.59 \mathrm{E}-4$ & ug/g dry & $5.59 \mathrm{E}-4$ & $8 / 26 / 08$ & $8 \mathrm{H} 26017$ & PNNL-AGG-415 \\
\hline HEIS No. & B1V9B2 & \multicolumn{3}{|c|}{ Lab ID: $\quad$ 0805003-12 } & & & \\
\hline $14092-98-9$ & Chromium 52 & $<2.06 \mathrm{E}-3$ & ug/g dry & $2.06 \mathrm{E}-3$ & $8 / 26 / 08$ & $8 \mathrm{H} 26017$ & PNNL-AGG-415 \\
\hline $13981-78-7$ & Chromium 53 & $<6.40 \mathrm{E}-3$ & ug/g dry & $6.40 \mathrm{E}-3$ & $8 / 26 / 08$ & $8 \mathrm{H} 26017$ & PNNL-AGG-415 \\
\hline $14191-84-5$ & Copper 63 & $<3.48 \mathrm{E}-3$ & ug/g dry & $3.48 \mathrm{E}-3$ & $8 / 26 / 08$ & $8 \mathrm{H} 26017$ & PNNL-AGG-415 \\
\hline $14119-06-3$ & Copper 65 & $<4.84 \mathrm{E}-3$ & ug/g dry & $4.84 \mathrm{E}-3$ & $8 / 26 / 08$ & $8 \mathrm{H} 26017$ & PNNL-AGG-415 \\
\hline $7440-38-2$ & Arsenic 75 & $6.97 \mathrm{E}-3$ & ug/g dry & $6.25 \mathrm{E}-3$ & $8 / 26 / 08$ & $8 \mathrm{H} 26017$ & PNNL-AGG-415 \\
\hline $14687-58-2$ & Selenium 82 & $<1.11 \mathrm{E}-2$ & ug/g dry & $1.11 \mathrm{E}-2$ & $8 / 26 / 08$ & $8 \mathrm{H} 26017$ & PNNL-AGG-415 \\
\hline $14392-17-7$ & Molybdenum 95 & $4.72 \mathrm{E}-3$ & ug/g dry & $1.61 \mathrm{E}-3$ & $8 / 26 / 08$ & $8 \mathrm{H} 26017$ & PNNL-AGG-415 \\
\hline $14392-19-9$ & Molybdenum 97 & $4.94 \mathrm{E}-3$ & ug/g dry & $2.05 \mathrm{E}-3$ & $8 / 26 / 08$ & $8 \mathrm{H} 26017$ & PNNL-AGG-415 \\
\hline $14392-20-2$ & Molybdenum 98 & $4.82 \mathrm{E}-3$ & ug/g dry & $1.61 \mathrm{E}-3$ & $8 / 26 / 08$ & $8 \mathrm{H} 26017$ & PNNL-AGG-415 \\
\hline $14914-61-5$ & Ruthenium 101 & $<8.20 \mathrm{E}-4$ & ug/g dry & $8.20 \mathrm{E}-4$ & $8 / 26 / 08$ & $8 \mathrm{H} 26017$ & PNNL-AGG-415 \\
\hline $14914-62-6$ & Ruthenium 102 & $<5.65 \mathrm{E}-4$ & ug/g dry & $5.65 \mathrm{E}-4$ & $8 / 26 / 08$ & $8 \mathrm{H} 26017$ & PNNL-AGG-415 \\
\hline $15766-01-5$ & Ruthenium 104 & $<3.65 \mathrm{E}-4$ & ug/g dry & $3.65 \mathrm{E}-4$ & $8 / 26 / 08$ & $8 \mathrm{H} 26017$ & PNNL-AGG-415 \\
\hline $14378-37-1$ & Silver 107 & $<9.25 \mathrm{E}-4$ & ug/g dry & $9.25 \mathrm{E}-4$ & $8 / 26 / 08$ & $8 \mathrm{H} 26017$ & PNNL-AGG-415 \\
\hline $14378-38-2$ & Silver 109 & $<1.07 \mathrm{E}-3$ & ug/g dry & $1.07 \mathrm{E}-3$ & $8 / 26 / 08$ & $8 \mathrm{H} 26017$ & PNNL-AGG-415 \\
\hline $14336-64-2$ & Cadmium 111 & $<2.95 \mathrm{E}-4$ & ug/g dry & $2.95 \mathrm{E}-4$ & $8 / 26 / 08$ & $8 \mathrm{H} 26017$ & PNNL-AGG-415 \\
\hline $14041-58-8$ & Cadmium 114 & $<6.25 \mathrm{E}-4$ & ug/g dry & $6.25 \mathrm{E}-4$ & $8 / 26 / 08$ & $8 \mathrm{H} 26017$ & PNNL-AGG-415 \\
\hline $14265-72-6$ & Antimony 121 & $<5.40 \mathrm{E}-4$ & ug/g dry & $5.40 \mathrm{E}-4$ & $8 / 26 / 08$ & $8 \mathrm{H} 26017$ & PNNL-AGG-415 \\
\hline $13966-27-3$ & Lead 206 & $<7.20 \mathrm{E}-4$ & ug/g dry & $7.20 \mathrm{E}-4$ & $8 / 26 / 08$ & $8 \mathrm{H} 26017$ & PNNL-AGG-415 \\
\hline $13966-28-4$ & Lead 208 & $<5.60 \mathrm{E}-4$ & ug/g dry & $5.60 \mathrm{E}-4$ & $8 / 26 / 08$ & $8 \mathrm{H} 26017$ & PNNL-AGG-415 \\
\hline HEIS No. & B1V9B3 & & b ID: & $5003-13$ & & & \\
\hline $14092-98-9$ & Chromium 52 & $<2.06 \mathrm{E}-3$ & ug/g dry & $2.06 \mathrm{E}-3$ & $8 / 26 / 08$ & $8 \mathrm{H} 26017$ & PNNL-AGG-415 \\
\hline $13981-78-7$ & Chromium 53 & $<6.40 \mathrm{E}-3$ & ug/g dry & $6.40 \mathrm{E}-3$ & $8 / 26 / 08$ & $8 \mathrm{H} 26017$ & PNNL-AGG-415 \\
\hline $14191-84-5$ & Copper 63 & $<3.48 \mathrm{E}-3$ & ug/g dry & $3.48 \mathrm{E}-3$ & $8 / 26 / 08$ & $8 \mathrm{H} 26017$ & PNNL-AGG-415 \\
\hline $14119-06-3$ & Copper 65 & $<4.84 \mathrm{E}-3$ & ug/g dry & $4.84 \mathrm{E}-3$ & $8 / 26 / 08$ & $8 \mathrm{H} 26017$ & PNNL-AGG-415 \\
\hline $7440-38-2$ & Arsenic 75 & $6.79 \mathrm{E}-3$ & ug/g dry & $6.25 \mathrm{E}-3$ & $8 / 26 / 08$ & $8 \mathrm{H} 26017$ & PNNL-AGG-415 \\
\hline $14687-58-2$ & Selenium 82 & $<1.11 \mathrm{E}-2$ & ug/g dry & $1.11 \mathrm{E}-2$ & $8 / 26 / 08$ & $8 \mathrm{H} 26017$ & PNNL-AGG-415 \\
\hline $14392-17-7$ & Molybdenum 95 & $2.04 \mathrm{E}-2$ & ug/g dry & $1.61 \mathrm{E}-3$ & $8 / 26 / 08$ & $8 \mathrm{H} 26017$ & PNNL-AGG-415 \\
\hline $14392-19-9$ & Molybdenum 97 & $2.04 \mathrm{E}-2$ & ug/g dry & $2.05 \mathrm{E}-3$ & $8 / 26 / 08$ & $8 \mathrm{H} 26017$ & PNNL-AGG-415 \\
\hline $14392-20-2$ & Molybdenum 98 & $2.06 \mathrm{E}-2$ & ug/g dry & $1.61 \mathrm{E}-3$ & $8 / 26 / 08$ & $8 \mathrm{H} 26017$ & PNNL-AGG-415 \\
\hline $14914-61-5$ & Ruthenium 101 & $<8.20 \mathrm{E}-4$ & ug/g dry & $8.20 \mathrm{E}-4$ & $8 / 26 / 08$ & $8 \mathrm{H} 26017$ & PNNL-AGG-415 \\
\hline $14914-62-6$ & Ruthenium 102 & $<5.65 \mathrm{E}-4$ & ug/g dry & $5.65 \mathrm{E}-4$ & $8 / 26 / 08$ & $8 \mathrm{H} 26017$ & PNNL-AGG-415 \\
\hline $15766-01-5$ & Ruthenium 104 & $<3.65 \mathrm{E}-4$ & ug/g dry & $3.65 \mathrm{E}-4$ & $8 / 26 / 08$ & $8 \mathrm{H} 26017$ & PNNL-AGG-415 \\
\hline $14378-37-1$ & Silver 107 & $<9.26 \mathrm{E}-4$ & ug/g dry & $9.26 \mathrm{E}-4$ & $8 / 26 / 08$ & $8 \mathrm{H} 26017$ & PNNL-AGG-415 \\
\hline $14378-38-2$ & Silver 109 & $<1.07 \mathrm{E}-3$ & ug/g dry & $1.07 \mathrm{E}-3$ & $8 / 26 / 08$ & $8 \mathrm{H} 26017$ & PNNL-AGG-415 \\
\hline $14336-64-2$ & Cadmium 111 & $<2.95 \mathrm{E}-4$ & ug/g dry & $2.95 \mathrm{E}-4$ & $8 / 26 / 08$ & $8 \mathrm{H} 26017$ & PNNL-AGG-415 \\
\hline $14041-58-8$ & Cadmium 114 & $<6.25 \mathrm{E}-4$ & ug/g dry & $6.25 \mathrm{E}-4$ & $8 / 26 / 08$ & $8 \mathrm{H} 26017$ & PNNL-AGG-415 \\
\hline $14265-72-6$ & Antimony 121 & $<5.40 \mathrm{E}-4$ & ug/g dry & $5.40 \mathrm{E}-4$ & $8 / 26 / 08$ & $8 \mathrm{H} 26017$ & PNNL-AGG-415 \\
\hline $13966-27-3$ & Lead 206 & $<7.20 \mathrm{E}-4$ & ug/g dry & $7.20 \mathrm{E}-4$ & $8 / 26 / 08$ & $8 \mathrm{H} 26017$ & PNNL-AGG-415 \\
\hline
\end{tabular}


RCRA Metals By PNNL-AGG-415/Water Extract

\begin{tabular}{|c|c|c|c|c|c|c|c|}
\hline CAS \# & Analyte & Results & Units & EQL & Analyzed & Batch & Method \\
\hline HEIS No. & B1V9B3 & \multicolumn{3}{|c|}{ Lab ID: $\quad$ 0805003-13 } & & & \\
\hline $13966-28-4$ & Lead 208 & $<5.60 \mathrm{E}-4$ & ug/g dry & $5.60 \mathrm{E}-4$ & $8 / 26 / 08$ & $8 \mathrm{H} 26017$ & PNNL-AGG-415 \\
\hline HEIS No. & B1V9B4 & \multicolumn{3}{|c|}{ Lab ID: $\quad$ 0805003-14 } & & & \\
\hline $14092-98-9$ & Chromium 52 & $<2.05 \mathrm{E}-3$ & ug/g dry & $2.05 \mathrm{E}-3$ & $8 / 26 / 08$ & $8 \mathrm{H} 26017$ & PNNL-AGG-415 \\
\hline $13981-78-7$ & Chromium 53 & $<6.39 \mathrm{E}-3$ & ug/g dry & $6.39 \mathrm{E}-3$ & $8 / 26 / 08$ & $8 \mathrm{H} 26017$ & PNNL-AGG-415 \\
\hline $14191-84-5$ & Copper 63 & $<3.47 \mathrm{E}-3$ & ug/g dry & $3.47 \mathrm{E}-3$ & $8 / 26 / 08$ & $8 \mathrm{H} 26017$ & PNNL-AGG-415 \\
\hline $14119-06-3$ & Copper 65 & $<4.83 \mathrm{E}-3$ & ug/g dry & $4.83 \mathrm{E}-3$ & $8 / 26 / 08$ & $8 \mathrm{H} 26017$ & PNNL-AGG-415 \\
\hline $7440-38-2$ & Arsenic 75 & $1.42 \mathrm{E}-2$ & ug/g dry & $6.24 \mathrm{E}-3$ & $8 / 26 / 08$ & $8 \mathrm{H} 26017$ & PNNL-AGG-415 \\
\hline $14687-58-2$ & Selenium 82 & $<1.10 \mathrm{E}-2$ & ug/g dry & $1.10 \mathrm{E}-2$ & $8 / 26 / 08$ & $8 \mathrm{H} 26017$ & PNNL-AGG-415 \\
\hline $14392-17-7$ & Molybdenum 95 & $1.67 \mathrm{E}-2$ & $\mathrm{ug} / \mathrm{g}$ dry & $1.60 \mathrm{E}-3$ & $8 / 26 / 08$ & $8 \mathrm{H} 26017$ & PNNL-AGG-415 \\
\hline $14392-19-9$ & Molybdenum 97 & $1.67 \mathrm{E}-2$ & ug/g dry & $2.04 \mathrm{E}-3$ & $8 / 26 / 08$ & $8 \mathrm{H} 26017$ & PNNL-AGG-415 \\
\hline $14392-20-2$ & Molybdenum 98 & $1.68 \mathrm{E}-2$ & ug/g dry & $1.60 \mathrm{E}-3$ & $8 / 26 / 08$ & $8 \mathrm{H} 26017$ & PNNL-AGG-415 \\
\hline $14914-61-5$ & Ruthenium 101 & $<8.19 \mathrm{E}-4$ & ug/g dry & $8.19 \mathrm{E}-4$ & $8 / 26 / 08$ & $8 \mathrm{H} 26017$ & PNNL-AGG-415 \\
\hline $14914-62-6$ & Ruthenium 102 & $<5.64 \mathrm{E}-4$ & ug/g dry & $5.64 \mathrm{E}-4$ & $8 / 26 / 08$ & $8 \mathrm{H} 26017$ & PNNL-AGG-415 \\
\hline $15766-01-5$ & Ruthenium 104 & $<3.64 \mathrm{E}-4$ & ug/g dry & $3.64 \mathrm{E}-4$ & $8 / 26 / 08$ & $8 \mathrm{H} 26017$ & PNNL-AGG-415 \\
\hline $14378-37-1$ & Silver 107 & $<9.23 \mathrm{E}-4$ & ug/g dry & $9.23 \mathrm{E}-4$ & $8 / 26 / 08$ & $8 \mathrm{H} 26017$ & PNNL-AGG-415 \\
\hline $14378-38-2$ & Silver 109 & $<1.07 \mathrm{E}-3$ & ug/g dry & $1.07 \mathrm{E}-3$ & $8 / 26 / 08$ & $8 \mathrm{H} 26017$ & PNNL-AGG-415 \\
\hline $14336-64-2$ & Cadmium 111 & $<2.94 \mathrm{E}-4$ & ug/g dry & $2.94 \mathrm{E}-4$ & $8 / 26 / 08$ & $8 \mathrm{H} 26017$ & PNNL-AGG-415 \\
\hline $14041-58-8$ & Cadmium 114 & $<6.24 \mathrm{E}-4$ & ug/g dry & $6.24 \mathrm{E}-4$ & $8 / 26 / 08$ & $8 \mathrm{H} 26017$ & PNNL-AGG-415 \\
\hline $14265-72-6$ & Antimony 121 & $<5.39 \mathrm{E}-4$ & ug/g dry & $5.39 \mathrm{E}-4$ & $8 / 26 / 08$ & $8 \mathrm{H} 26017$ & PNNL-AGG-415 \\
\hline $13966-27-3$ & Lead 206 & $<7.19 \mathrm{E}-4$ & $\mathrm{ug} / \mathrm{g}$ dry & $7.19 \mathrm{E}-4$ & $8 / 26 / 08$ & $8 \mathrm{H} 26017$ & PNNL-AGG-415 \\
\hline $13966-28-4$ & Lead 208 & $<5.59 \mathrm{E}-4$ & ug/g dry & $5.59 \mathrm{E}-4$ & $8 / 26 / 08$ & $8 \mathrm{H} 26017$ & PNNL-AGG-415 \\
\hline HEIS No. & B1V9B5 & \multicolumn{3}{|c|}{ Lab ID: $\quad$ 0805003-15 } & & & \\
\hline $14092-98-9$ & Chromium 52 & $<2.05 \mathrm{E}-3$ & ug/g dry & $2.05 \mathrm{E}-3$ & $8 / 27 / 08$ & $8 \mathrm{H} 26017$ & PNNL-AGG-415 \\
\hline $13981-78-7$ & Chromium 53 & $<6.40 \mathrm{E}-3$ & ug/g dry & $6.40 \mathrm{E}-3$ & $8 / 27 / 08$ & $8 \mathrm{H} 26017$ & PNNL-AGG-415 \\
\hline $14191-84-5$ & Copper 63 & $<3.48 \mathrm{E}-3$ & ug/g dry & $3.48 \mathrm{E}-3$ & $8 / 27 / 08$ & $8 \mathrm{H} 26017$ & PNNL-AGG-415 \\
\hline $14119-06-3$ & Copper 65 & $<4.84 \mathrm{E}-3$ & ug/g dry & $4.84 \mathrm{E}-3$ & $8 / 27 / 08$ & $8 \mathrm{H} 26017$ & PNNL-AGG-415 \\
\hline $7440-38-2$ & Arsenic 75 & $8.37 \mathrm{E}-3$ & ug/g dry & $6.25 \mathrm{E}-3$ & $8 / 27 / 08$ & $8 \mathrm{H} 26017$ & PNNL-AGG-415 \\
\hline $14687-58-2$ & Selenium 82 & $<1.10 \mathrm{E}-2$ & $\mathrm{ug} / \mathrm{g}$ dry & $1.10 \mathrm{E}-2$ & $8 / 27 / 08$ & $8 \mathrm{H} 26017$ & PNNL-AGG-415 \\
\hline $14392-17-7$ & Molybdenum 95 & $2.60 \mathrm{E}-3$ & ug/g dry & $1.60 \mathrm{E}-3$ & $8 / 27 / 08$ & $8 \mathrm{H} 26017$ & PNNL-AGG-415 \\
\hline $14392-19-9$ & Molybdenum 97 & $2.73 \mathrm{E}-3$ & ug/g dry & $2.04 \mathrm{E}-3$ & $8 / 27 / 08$ & $8 \mathrm{H} 26017$ & PNNL-AGG-415 \\
\hline $14392-20-2$ & Molybdenum 98 & $2.70 \mathrm{E}-3$ & $\mathrm{ug} / \mathrm{g}$ dry & $1.60 \mathrm{E}-3$ & $8 / 27 / 08$ & $8 \mathrm{H} 26017$ & PNNL-AGG-415 \\
\hline $14914-61-5$ & Ruthenium 101 & $<8.19 \mathrm{E}-4$ & ug/g dry & $8.19 \mathrm{E}-4$ & $8 / 27 / 08$ & $8 \mathrm{H} 26017$ & PNNL-AGG-415 \\
\hline $14914-62-6$ & Ruthenium 102 & $<5.65 \mathrm{E}-4$ & ug/g dry & $5.65 \mathrm{E}-4$ & $8 / 27 / 08$ & $8 \mathrm{H} 26017$ & PNNL-AGG-415 \\
\hline $15766-01-5$ & Ruthenium 104 & $<3.65 \mathrm{E}-4$ & ug/g dry & $3.65 \mathrm{E}-4$ & $8 / 27 / 08$ & $8 \mathrm{H} 26017$ & PNNL-AGG-415 \\
\hline $14378-37-1$ & Silver 107 & $<9.24 \mathrm{E}-4$ & ug/g dry & $9.24 \mathrm{E}-4$ & $8 / 27 / 08$ & $8 \mathrm{H} 26017$ & PNNL-AGG-415 \\
\hline $14378-38-2$ & Silver 109 & $<1.07 \mathrm{E}-3$ & ug/g dry & $1.07 \mathrm{E}-3$ & $8 / 27 / 08$ & $8 \mathrm{H} 26017$ & PNNL-AGG-415 \\
\hline $14336-64-2$ & Cadmium 111 & $<2.95 \mathrm{E}-4$ & ug/g dry & $2.95 \mathrm{E}-4$ & $8 / 27 / 08$ & $8 \mathrm{H} 26017$ & PNNL-AGG-415 \\
\hline $14041-58-8$ & Cadmium 114 & $<6.25 \mathrm{E}-4$ & ug/g dry & $6.25 \mathrm{E}-4$ & $8 / 27 / 08$ & $8 \mathrm{H} 26017$ & PNNL-AGG-415 \\
\hline $14265-72-6$ & Antimony 121 & $<5.40 \mathrm{E}-4$ & ug/g dry & $5.40 \mathrm{E}-4$ & $8 / 27 / 08$ & $8 \mathrm{H} 26017$ & PNNL-AGG-415 \\
\hline $13966-27-3$ & Lead 206 & $<7.20 \mathrm{E}-4$ & ug/g dry & $7.20 \mathrm{E}-4$ & $8 / 27 / 08$ & $8 \mathrm{H} 26017$ & PNNL-AGG-415 \\
\hline $13966-28-4$ & Lead 208 & $<5.60 \mathrm{E}-4$ & ug/g dry & $5.60 \mathrm{E}-4$ & $8 / 27 / 08$ & $8 \mathrm{H} 26017$ & PNNL-AGG-415 \\
\hline HEIS No. & B1V9B6 & & b ID: & $5003-16$ & & & \\
\hline $14092-98-9$ & Chromium 52 & $<2.05 \mathrm{E}-3$ & ug/g dry & $2.05 \mathrm{E}-3$ & $8 / 27 / 08$ & $8 \mathrm{H} 26017$ & PNNL-AGG-415 \\
\hline $13981-78-7$ & Chromium 53 & $<6.38 \mathrm{E}-3$ & ug/g dry & $6.38 \mathrm{E}-3$ & $8 / 27 / 08$ & $8 \mathrm{H} 26017$ & PNNL-AGG-415 \\
\hline $14191-84-5$ & Copper 63 & $<3.47 \mathrm{E}-3$ & ug/g dry & $3.47 \mathrm{E}-3$ & $8 / 27 / 08$ & $8 \mathrm{H} 26017$ & PNNL-AGG-415 \\
\hline $14119-06-3$ & Copper 65 & $<4.82 \mathrm{E}-3$ & ug/g dry & $4.82 \mathrm{E}-3$ & $8 / 27 / 08$ & $8 \mathrm{H} 26017$ & PNNL-AGG-415 \\
\hline $7440-38-2$ & Arsenic 75 & $7.88 \mathrm{E}-3$ & ug/g dry & $6.23 \mathrm{E}-3$ & $8 / 27 / 08$ & $8 \mathrm{H} 26017$ & PNNL-AGG-415 \\
\hline $14687-58-2$ & Selenium 82 & $<1.10 \mathrm{E}-2$ & ug/g dry & $1.10 \mathrm{E}-2$ & $8 / 27 / 08$ & $8 \mathrm{H} 26017$ & PNNL-AGG-415 \\
\hline
\end{tabular}


RCRA Metals By PNNL-AGG-415/Water Extract

\begin{tabular}{|c|c|c|c|c|c|c|c|}
\hline CAS \# & Analyte & Results & Units & EQL & Analyzed & Batch & Method \\
\hline HEIS No. & B1V9B6 & \multicolumn{3}{|c|}{ Lab ID: $\quad$ 0805003-16 } & & & \\
\hline $14392-17-7$ & Molybdenum 95 & $3.60 \mathrm{E}-3$ & $\mathrm{ug} / \mathrm{g}$ dry & $1.60 \mathrm{E}-3$ & $8 / 27 / 08$ & $8 \mathrm{H} 26017$ & PNNL-AGG-415 \\
\hline 14392-19-9 & Molybdenum 97 & $3.73 \mathrm{E}-3$ & ug/g dry & $2.04 \mathrm{E}-3$ & $8 / 27 / 08$ & $8 \mathrm{H} 26017$ & PNNL-AGG-415 \\
\hline $14392-20-2$ & Molybdenum 98 & $3.66 \mathrm{E}-3$ & ug/g dry & $1.60 \mathrm{E}-3$ & $8 / 27 / 08$ & $8 \mathrm{H} 26017$ & PNNL-AGG-415 \\
\hline $14914-61-5$ & Ruthenium 101 & $<8.17 \mathrm{E}-4$ & $\mathrm{ug} / \mathrm{g}$ dry & $8.17 \mathrm{E}-4$ & $8 / 27 / 08$ & $8 \mathrm{H} 26017$ & PNNL-AGG-415 \\
\hline $14914-62-6$ & Ruthenium 102 & $<5.63 \mathrm{E}-4$ & $\mathrm{ug} / \mathrm{g}$ dry & $5.63 \mathrm{E}-4$ & $8 / 27 / 08$ & $8 \mathrm{H} 26017$ & PNNL-AGG-415 \\
\hline $15766-01-5$ & Ruthenium 104 & $<3.64 \mathrm{E}-4$ & $\mathrm{ug} / \mathrm{g}$ dry & $3.64 \mathrm{E}-4$ & $8 / 27 / 08$ & $8 \mathrm{H} 26017$ & PNNL-AGG-415 \\
\hline $14378-37-1$ & Silver 107 & $<9.22 \mathrm{E}-4$ & $\mathrm{ug} / \mathrm{g}$ dry & $9.22 \mathrm{E}-4$ & $8 / 27 / 08$ & $8 \mathrm{H} 26017$ & PNNL-AGG-415 \\
\hline $14378-38-2$ & Silver 109 & $<1.07 \mathrm{E}-3$ & $\mathrm{ug} / \mathrm{g}$ dry & $1.07 \mathrm{E}-3$ & $8 / 27 / 08$ & $8 \mathrm{H} 26017$ & PNNL-AGG-415 \\
\hline $14336-64-2$ & Cadmium 111 & $<2.94 \mathrm{E}-4$ & $\mathrm{ug} / \mathrm{g}$ dry & $2.94 \mathrm{E}-4$ & $8 / 27 / 08$ & $8 \mathrm{H} 26017$ & PNNL-AGG-415 \\
\hline $14041-58-8$ & Cadmium 114 & $<6.23 \mathrm{E}-4$ & $\mathrm{ug} / \mathrm{g}$ dry & $6.23 \mathrm{E}-4$ & $8 / 27 / 08$ & $8 \mathrm{H} 26017$ & PNNL-AGG-415 \\
\hline $14265-72-6$ & Antimony 121 & $<5.38 \mathrm{E}-4$ & $\mathrm{ug} / \mathrm{g}$ dry & $5.38 \mathrm{E}-4$ & $8 / 27 / 08$ & $8 \mathrm{H} 26017$ & PNNL-AGG-415 \\
\hline $13966-27-3$ & Lead 206 & $<7.18 \mathrm{E}-4$ & $\mathrm{ug} / \mathrm{g}$ dry & $7.18 \mathrm{E}-4$ & $8 / 27 / 08$ & $8 \mathrm{H} 26017$ & PNNL-AGG-415 \\
\hline $13966-28-4$ & Lead 208 & $<5.58 \mathrm{E}-4$ & ug/g dry & $5.58 \mathrm{E}-4$ & $8 / 27 / 08$ & $8 \mathrm{H} 26017$ & PNNL-AGG-415 \\
\hline HEIS No. & B1V9B7 & \multicolumn{3}{|c|}{ Lab ID: $\quad$ 0805003-17 } & & & \\
\hline $14092-98-9$ & Chromium 52 & $<2.05 \mathrm{E}-3$ & ug/g dry & $2.05 \mathrm{E}-3$ & $8 / 27 / 08$ & $8 \mathrm{H} 26017$ & PNNL-AGG-415 \\
\hline $13981-78-7$ & Chromium 53 & $<6.40 \mathrm{E}-3$ & ug/g dry & $6.40 \mathrm{E}-3$ & $8 / 27 / 08$ & $8 \mathrm{H} 26017$ & PNNL-AGG-415 \\
\hline $14191-84-5$ & Copper 63 & $<3.48 \mathrm{E}-3$ & ug/g dry & $3.48 \mathrm{E}-3$ & $8 / 27 / 08$ & $8 \mathrm{H} 26017$ & PNNL-AGG-415 \\
\hline $14119-06-3$ & Copper 65 & $<4.84 \mathrm{E}-3$ & $\mathrm{ug} / \mathrm{g}$ dry & $4.84 \mathrm{E}-3$ & $8 / 27 / 08$ & $8 \mathrm{H} 26017$ & PNNL-AGG-415 \\
\hline $7440-38-2$ & Arsenic 75 & $6.66 \mathrm{E}-3$ & $\mathrm{ug} / \mathrm{g}$ dry & $6.25 \mathrm{E}-3$ & $8 / 27 / 08$ & $8 \mathrm{H} 26017$ & PNNL-AGG-415 \\
\hline $14687-58-2$ & Selenium 82 & $<1.10 \mathrm{E}-2$ & ug/g dry & $1.10 \mathrm{E}-2$ & $8 / 27 / 08$ & $8 \mathrm{H} 26017$ & PNNL-AGG-415 \\
\hline $14392-17-7$ & Molybdenum 95 & $2.86 \mathrm{E}-3$ & $\mathrm{ug} / \mathrm{g}$ dry & $1.60 \mathrm{E}-3$ & $8 / 27 / 08$ & $8 \mathrm{H} 26017$ & PNNL-AGG-415 \\
\hline 14392-19-9 & Molybdenum 97 & $2.92 \mathrm{E}-3$ & $\mathrm{ug} / \mathrm{g}$ dry & $2.04 \mathrm{E}-3$ & $8 / 27 / 08$ & $8 \mathrm{H} 26017$ & PNNL-AGG-415 \\
\hline $14392-20-2$ & Molybdenum 98 & $2.96 \mathrm{E}-3$ & ug/g dry & $1.60 \mathrm{E}-3$ & $8 / 27 / 08$ & $8 \mathrm{H} 26017$ & PNNL-AGG-415 \\
\hline $14914-61-5$ & Ruthenium 101 & $<8.20 \mathrm{E}-4$ & ug/g dry & $8.20 \mathrm{E}-4$ & $8 / 27 / 08$ & $8 \mathrm{H} 26017$ & PNNL-AGG-415 \\
\hline $14914-62-6$ & Ruthenium 102 & $<5.65 \mathrm{E}-4$ & ug/g dry & $5.65 \mathrm{E}-4$ & $8 / 27 / 08$ & $8 \mathrm{H} 26017$ & PNNL-AGG-415 \\
\hline $15766-01-5$ & Ruthenium 104 & $<3.65 \mathrm{E}-4$ & ug/g dry & $3.65 \mathrm{E}-4$ & $8 / 27 / 08$ & $8 \mathrm{H} 26017$ & PNNL-AGG-415 \\
\hline $14378-37-1$ & Silver 107 & $<9.24 \mathrm{E}-4$ & ug/g dry & $9.24 \mathrm{E}-4$ & $8 / 27 / 08$ & $8 \mathrm{H} 26017$ & PNNL-AGG-415 \\
\hline $14378-38-2$ & Silver 109 & $<1.07 \mathrm{E}-3$ & $\mathrm{ug} / \mathrm{g}$ dry & $1.07 \mathrm{E}-3$ & $8 / 27 / 08$ & $8 \mathrm{H} 26017$ & PNNL-AGG-415 \\
\hline $14336-64-2$ & Cadmium 111 & $<2.95 \mathrm{E}-4$ & ug/g dry & $2.95 \mathrm{E}-4$ & $8 / 27 / 08$ & $8 \mathrm{H} 26017$ & PNNL-AGG-415 \\
\hline $14041-58-8$ & Cadmium 114 & $<6.25 \mathrm{E}-4$ & ug/g dry & $6.25 \mathrm{E}-4$ & $8 / 27 / 08$ & $8 \mathrm{H} 26017$ & PNNL-AGG-415 \\
\hline $14265-72-6$ & Antimony 121 & $<5.40 \mathrm{E}-4$ & ug/g dry & $5.40 \mathrm{E}-4$ & $8 / 27 / 08$ & $8 \mathrm{H} 26017$ & PNNL-AGG-415 \\
\hline $13966-27-3$ & Lead 206 & $<7.20 \mathrm{E}-4$ & $\mathrm{ug} / \mathrm{g}$ dry & $7.20 \mathrm{E}-4$ & $8 / 27 / 08$ & $8 \mathrm{H} 26017$ & PNNL-AGG-415 \\
\hline $13966-28-4$ & Lead 208 & $<5.60 \mathrm{E}-4$ & ug/g dry & $5.60 \mathrm{E}-4$ & $8 / 27 / 08$ & $8 \mathrm{H} 26017$ & PNNL-AGG-415 \\
\hline HEIS No. & B1V9B8 & \multicolumn{3}{|c|}{ Lab ID: $\quad$ 0805003-18 } & & & \\
\hline $14092-98-9$ & Chromium 52 & $<2.07 \mathrm{E}-3$ & ug/g dry & $2.07 \mathrm{E}-3$ & $8 / 27 / 08$ & $8 \mathrm{H} 26017$ & PNNL-AGG-415 \\
\hline $13981-78-7$ & Chromium 53 & $<6.44 \mathrm{E}-3$ & $\mathrm{ug} / \mathrm{g}$ dry & $6.44 \mathrm{E}-3$ & $8 / 27 / 08$ & $8 \mathrm{H} 26017$ & PNNL-AGG-415 \\
\hline $14191-84-5$ & Copper 63 & $<3.50 \mathrm{E}-3$ & ug/g dry & $3.50 \mathrm{E}-3$ & $8 / 27 / 08$ & $8 \mathrm{H} 26017$ & PNNL-AGG-415 \\
\hline $14119-06-3$ & Copper 65 & $<4.87 \mathrm{E}-3$ & $\mathrm{ug} / \mathrm{g}$ dry & $4.87 \mathrm{E}-3$ & $8 / 27 / 08$ & $8 \mathrm{H} 26017$ & PNNL-AGG-415 \\
\hline $7440-38-2$ & Arsenic 75 & $7.09 \mathrm{E}-3$ & $\mathrm{ug} / \mathrm{g}$ dry & $6.28 \mathrm{E}-3$ & $8 / 27 / 08$ & $8 \mathrm{H} 26017$ & PNNL-AGG-415 \\
\hline $14687-58-2$ & Selenium 82 & $<1.11 \mathrm{E}-2$ & $\mathrm{ug} / \mathrm{g}$ dry & $1.11 \mathrm{E}-2$ & $8 / 27 / 08$ & $8 \mathrm{H} 26017$ & PNNL-AGG-415 \\
\hline $14392-17-7$ & Molybdenum 95 & $2.39 \mathrm{E}-3$ & $\mathrm{ug} / \mathrm{g}$ dry & $1.61 \mathrm{E}-3$ & $8 / 27 / 08$ & $8 \mathrm{H} 26017$ & PNNL-AGG-415 \\
\hline $14392-19-9$ & Molybdenum 97 & $2.49 \mathrm{E}-3$ & $\mathrm{ug} / \mathrm{g}$ dry & $2.06 \mathrm{E}-3$ & $8 / 27 / 08$ & $8 \mathrm{H} 26017$ & PNNL-AGG-415 \\
\hline $14392-20-2$ & Molybdenum 98 & $2.46 \mathrm{E}-3$ & ug/g dry & $1.61 \mathrm{E}-3$ & $8 / 27 / 08$ & $8 \mathrm{H} 26017$ & PNNL-AGG-415 \\
\hline $14914-61-5$ & Ruthenium 101 & $<8.25 \mathrm{E}-4$ & $\mathrm{ug} / \mathrm{g}$ dry & $8.25 \mathrm{E}-4$ & $8 / 27 / 08$ & $8 \mathrm{H} 26017$ & PNNL-AGG-415 \\
\hline $14914-62-6$ & Ruthenium 102 & $<5.68 \mathrm{E}-4$ & ug/g dry & $5.68 \mathrm{E}-4$ & $8 / 27 / 08$ & $8 \mathrm{H} 26017$ & PNNL-AGG-415 \\
\hline $15766-01-5$ & Ruthenium 104 & $<3.67 \mathrm{E}-4$ & ug/g dry & $3.67 \mathrm{E}-4$ & $8 / 27 / 08$ & $8 \mathrm{H} 26017$ & PNNL-AGG-415 \\
\hline $14378-37-1$ & Silver 107 & $<9.30 \mathrm{E}-4$ & ug/g dry & $9.30 \mathrm{E}-4$ & $8 / 27 / 08$ & $8 \mathrm{H} 26017$ & PNNL-AGG-415 \\
\hline $14378-38-2$ & Silver 109 & $<1.08 \mathrm{E}-3$ & ug/g dry & $1.08 \mathrm{E}-3$ & $8 / 27 / 08$ & $8 \mathrm{H} 26017$ & PNNL-AGG-415 \\
\hline
\end{tabular}


RCRA Metals By PNNL-AGG-415/Water Extract

\begin{tabular}{|c|c|c|c|c|c|c|c|}
\hline CAS \# & Analyte & Results & Units & EQL & Analyzed & Batch & Method \\
\hline HEIS No. & B1V9B8 & \multicolumn{3}{|c|}{ Lab ID: $\quad$ 0805003-18 } & & & \\
\hline $14336-64-2$ & Cadmium 111 & $<2.97 \mathrm{E}-4$ & ug/g dry & $2.97 \mathrm{E}-4$ & $8 / 27 / 08$ & $8 \mathrm{H} 26017$ & PNNL-AGG-415 \\
\hline $14041-58-8$ & Cadmium 114 & $<6.28 \mathrm{E}-4$ & ug/g dry & $6.28 \mathrm{E}-4$ & $8 / 27 / 08$ & $8 \mathrm{H} 26017$ & PNNL-AGG-415 \\
\hline $14265-72-6$ & Antimony 121 & $<5.43 \mathrm{E}-4$ & ug/g dry & $5.43 \mathrm{E}-4$ & $8 / 27 / 08$ & $8 \mathrm{H} 26017$ & PNNL-AGG-415 \\
\hline $13966-27-3$ & Lead 206 & $<7.24 \mathrm{E}-4$ & ug/g dry & 7.24E-4 & $8 / 27 / 08$ & $8 \mathrm{H} 26017$ & PNNL-AGG-415 \\
\hline $13966-28-4$ & Lead 208 & $<5.63 \mathrm{E}-4$ & ug/g dry & $5.63 \mathrm{E}-4$ & $8 / 27 / 08$ & $8 \mathrm{H} 26017$ & PNNL-AGG-415 \\
\hline HEIS No. & B1V9B9 & \multicolumn{3}{|c|}{ Lab ID: $\quad$ 0805003-19 } & & & \\
\hline $14092-98-9$ & Chromium 52 & $<2.04 \mathrm{E}-3$ & ug/g dry & $2.04 \mathrm{E}-3$ & $8 / 27 / 08$ & $8 \mathrm{H} 26017$ & PNNL-AGG-415 \\
\hline $13981-78-7$ & Chromium 53 & $<6.37 \mathrm{E}-3$ & ug/g dry & $6.37 \mathrm{E}-3$ & $8 / 27 / 08$ & $8 \mathrm{H} 26017$ & PNNL-AGG-415 \\
\hline $14191-84-5$ & Copper 63 & $<3.46 \mathrm{E}-3$ & ug/g dry & $3.46 \mathrm{E}-3$ & $8 / 27 / 08$ & $8 \mathrm{H} 26017$ & PNNL-AGG-415 \\
\hline $14119-06-3$ & Copper 65 & $<4.82 \mathrm{E}-3$ & ug/g dry & $4.82 \mathrm{E}-3$ & $8 / 27 / 08$ & $8 \mathrm{H} 26017$ & PNNL-AGG-415 \\
\hline $7440-38-2$ & Arsenic 75 & $<6.22 \mathrm{E}-3$ & ug/g dry & $6.22 \mathrm{E}-3$ & $8 / 27 / 08$ & $8 \mathrm{H} 26017$ & PNNL-AGG-415 \\
\hline $14687-58-2$ & Selenium 82 & $<1.10 \mathrm{E}-2$ & ug/g dry & $1.10 \mathrm{E}-2$ & $8 / 27 / 08$ & $8 \mathrm{H} 26017$ & PNNL-AGG-415 \\
\hline $14392-17-7$ & Molybdenum 95 & $3.68 \mathrm{E}-3$ & ug/g dry & $1.60 \mathrm{E}-3$ & $8 / 27 / 08$ & $8 \mathrm{H} 26017$ & PNNL-AGG-415 \\
\hline $14392-19-9$ & Molybdenum 97 & $3.77 \mathrm{E}-3$ & ug/g dry & $2.04 \mathrm{E}-3$ & $8 / 27 / 08$ & $8 \mathrm{H} 26017$ & PNNL-AGG-415 \\
\hline $14392-20-2$ & Molybdenum 98 & $3.70 \mathrm{E}-3$ & ug/g dry & $1.60 \mathrm{E}-3$ & $8 / 27 / 08$ & $8 \mathrm{H} 26017$ & PNNL-AGG-415 \\
\hline $14914-61-5$ & Ruthenium 101 & $<8.16 \mathrm{E}-4$ & ug/g dry & $8.16 \mathrm{E}-4$ & $8 / 27 / 08$ & $8 \mathrm{H} 26017$ & PNNL-AGG-415 \\
\hline $14914-62-6$ & Ruthenium 102 & $<5.62 \mathrm{E}-4$ & ug/g dry & $5.62 \mathrm{E}-4$ & $8 / 27 / 08$ & $8 \mathrm{H} 26017$ & PNNL-AGG-415 \\
\hline $15766-01-5$ & Ruthenium 104 & $<3.63 \mathrm{E}-4$ & ug/g dry & $3.63 \mathrm{E}-4$ & $8 / 27 / 08$ & $8 \mathrm{H} 26017$ & PNNL-AGG-415 \\
\hline $14378-37-1$ & Silver 107 & $<9.20 \mathrm{E}-4$ & ug/g dry & $9.20 \mathrm{E}-4$ & $8 / 27 / 08$ & $8 \mathrm{H} 26017$ & PNNL-AGG-415 \\
\hline $14378-38-2$ & Silver 109 & $<1.06 \mathrm{E}-3$ & ug/g dry & $1.06 \mathrm{E}-3$ & $8 / 27 / 08$ & $8 \mathrm{H} 26017$ & PNNL-AGG-415 \\
\hline $14336-64-2$ & Cadmium 111 & $<2.94 \mathrm{E}-4$ & ug/g dry & $2.94 \mathrm{E}-4$ & $8 / 27 / 08$ & $8 \mathrm{H} 26017$ & PNNL-AGG-415 \\
\hline $14041-58-8$ & Cadmium 114 & $<6.22 \mathrm{E}-4$ & ug/g dry & $6.22 \mathrm{E}-4$ & $8 / 27 / 08$ & $8 \mathrm{H} 26017$ & PNNL-AGG-415 \\
\hline $14265-72-6$ & Antimony 121 & $<5.37 \mathrm{E}-4$ & ug/g dry & $5.37 \mathrm{E}-4$ & $8 / 27 / 08$ & $8 \mathrm{H} 26017$ & PNNL-AGG-415 \\
\hline $13966-27-3$ & Lead 206 & $<7.16 \mathrm{E}-4$ & ug/g dry & $7.16 \mathrm{E}-4$ & $8 / 27 / 08$ & $8 \mathrm{H} 26017$ & PNNL-AGG-415 \\
\hline $13966-28-4$ & Lead 208 & $<5.57 \mathrm{E}-4$ & ug/g dry & $5.57 \mathrm{E}-4$ & $8 / 27 / 08$ & $8 \mathrm{H} 26017$ & PNNL-AGG-415 \\
\hline HEIS No. & B1V9C0 & \multicolumn{3}{|c|}{ Lab ID: $\quad$ 0805003-20 } & & & \\
\hline $14092-98-9$ & Chromium 52 & $<2.06 \mathrm{E}-3$ & ug/g dry & $2.06 \mathrm{E}-3$ & $8 / 27 / 08$ & $8 \mathrm{H} 26017$ & PNNL-AGG-415 \\
\hline $13981-78-7$ & Chromium 53 & $<6.42 \mathrm{E}-3$ & ug/g dry & $6.42 \mathrm{E}-3$ & $8 / 27 / 08$ & $8 \mathrm{H} 26017$ & PNNL-AGG-415 \\
\hline $14191-84-5$ & Copper 63 & $<3.49 \mathrm{E}-3$ & ug/g dry & $3.49 \mathrm{E}-3$ & $8 / 27 / 08$ & $8 \mathrm{H} 26017$ & PNNL-AGG-415 \\
\hline $14119-06-3$ & Copper 65 & $<4.85 \mathrm{E}-3$ & ug/g dry & $4.85 \mathrm{E}-3$ & $8 / 27 / 08$ & $8 \mathrm{H} 26017$ & PNNL-AGG-415 \\
\hline $7440-38-2$ & Arsenic 75 & $6.69 \mathrm{E}-3$ & ug/g dry & $6.27 \mathrm{E}-3$ & $8 / 27 / 08$ & $8 \mathrm{H} 26017$ & PNNL-AGG-415 \\
\hline $14687-58-2$ & Selenium 82 & $<1.11 \mathrm{E}-2$ & ug/g dry & $1.11 \mathrm{E}-2$ & $8 / 27 / 08$ & $8 \mathrm{H} 26017$ & PNNL-AGG-415 \\
\hline $14392-17-7$ & Molybdenum 95 & $2.56 \mathrm{E}-3$ & ug/g dry & $1.61 \mathrm{E}-3$ & $8 / 27 / 08$ & $8 \mathrm{H} 26017$ & PNNL-AGG-415 \\
\hline $14392-19-9$ & Molybdenum 97 & $2.58 \mathrm{E}-3$ & ug/g dry & $2.05 \mathrm{E}-3$ & $8 / 27 / 08$ & $8 \mathrm{H} 26017$ & PNNL-AGG-415 \\
\hline $14392-20-2$ & Molybdenum 98 & $2.63 \mathrm{E}-3$ & ug/g dry & $1.61 \mathrm{E}-3$ & $8 / 27 / 08$ & $8 \mathrm{H} 26017$ & PNNL-AGG-415 \\
\hline $14914-61-5$ & Ruthenium 101 & $<8.22 \mathrm{E}-4$ & ug/g dry & $8.22 \mathrm{E}-4$ & $8 / 27 / 08$ & $8 \mathrm{H} 26017$ & PNNL-AGG-415 \\
\hline $14914-62-6$ & Ruthenium 102 & $<5.67 \mathrm{E}-4$ & ug/g dry & $5.67 \mathrm{E}-4$ & $8 / 27 / 08$ & $8 \mathrm{H} 26017$ & PNNL-AGG-415 \\
\hline $15766-01-5$ & Ruthenium 104 & $<3.66 \mathrm{E}-4$ & ug/g dry & $3.66 \mathrm{E}-4$ & $8 / 27 / 08$ & $8 \mathrm{H} 26017$ & PNNL-AGG-415 \\
\hline $14378-37-1$ & Silver 107 & $<9.28 \mathrm{E}-4$ & ug/g dry & $9.28 \mathrm{E}-4$ & $8 / 27 / 08$ & $8 \mathrm{H} 26017$ & PNNL-AGG-415 \\
\hline $14378-38-2$ & Silver 109 & $<1.07 \mathrm{E}-3$ & ug/g dry & $1.07 \mathrm{E}-3$ & $8 / 27 / 08$ & $8 \mathrm{H} 26017$ & PNNL-AGG-415 \\
\hline $14336-64-2$ & Cadmium 111 & $<2.96 \mathrm{E}-4$ & ug/g dry & $2.96 \mathrm{E}-4$ & $8 / 27 / 08$ & $8 \mathrm{H} 26017$ & PNNL-AGG-415 \\
\hline $14041-58-8$ & Cadmium 114 & $<6.27 \mathrm{E}-4$ & $\mathrm{ug} / \mathrm{g}$ dry & $6.27 \mathrm{E}-4$ & $8 / 27 / 08$ & $8 \mathrm{H} 26017$ & PNNL-AGG-415 \\
\hline $14265-72-6$ & Antimony 121 & $<5.42 \mathrm{E}-4$ & ug/g dry & $5.42 \mathrm{E}-4$ & $8 / 27 / 08$ & $8 \mathrm{H} 26017$ & PNNL-AGG-415 \\
\hline $13966-27-3$ & Lead 206 & $1.20 \mathrm{E}-3$ & ug/g dry & $7.22 \mathrm{E}-4$ & $8 / 27 / 08$ & $8 \mathrm{H} 26017$ & PNNL-AGG-415 \\
\hline $13966-28-4$ & Lead 208 & $1.20 \mathrm{E}-3$ & ug/g dry & $5.62 \mathrm{E}-4$ & $8 / 27 / 08$ & $8 \mathrm{H} 26017$ & PNNL-AGG-415 \\
\hline HEIS No. & B1V9C1 & & b ID: & $5003-21$ & & & \\
\hline $14092-98-9$ & Chromium 52 & $<2.05 \mathrm{E}-3$ & ug/g dry & $2.05 \mathrm{E}-3$ & $8 / 27 / 08$ & $8 \mathrm{H} 26017$ & PNNL-AGG-415 \\
\hline $13981-78-7$ & Chromium 53 & $<6.39 \mathrm{E}-3$ & ug/g dry & $6.39 \mathrm{E}-3$ & $8 / 27 / 08$ & $8 \mathrm{H} 26017$ & PNNL-AGG-415 \\
\hline
\end{tabular}


RCRA Metals By PNNL-AGG-415/Water Extract

\begin{tabular}{|c|c|c|c|c|c|c|c|}
\hline CAS \# & Analyte & Results & Units & EQL & Analyzed & Batch & Method \\
\hline HEIS No. & B1V9C1 & \multicolumn{3}{|c|}{ Lab ID: $\quad$ 0805003-21 } & & & \\
\hline $14191-84-5$ & Copper 63 & $<3.48 \mathrm{E}-3$ & $\mathrm{ug} / \mathrm{g}$ dry & $3.48 \mathrm{E}-3$ & $8 / 27 / 08$ & $8 \mathrm{H} 26017$ & PNNL-AGG-415 \\
\hline $14119-06-3$ & Copper 65 & $<4.83 \mathrm{E}-3$ & ug/g dry & $4.83 \mathrm{E}-3$ & $8 / 27 / 08$ & $8 \mathrm{H} 26017$ & PNNL-AGG-415 \\
\hline $7440-38-2$ & Arsenic 75 & $6.34 \mathrm{E}-3$ & ug/g dry & $6.24 \mathrm{E}-3$ & $8 / 27 / 08$ & $8 \mathrm{H} 26017$ & PNNL-AGG-415 \\
\hline $14687-58-2$ & Selenium 82 & $<1.10 \mathrm{E}-2$ & $\mathrm{ug} / \mathrm{g}$ dry & $1.10 \mathrm{E}-2$ & $8 / 27 / 08$ & $8 \mathrm{H} 26017$ & PNNL-AGG-415 \\
\hline $14392-17-7$ & Molybdenum 95 & $2.57 \mathrm{E}-3$ & ug/g dry & $1.60 \mathrm{E}-3$ & $8 / 27 / 08$ & $8 \mathrm{H} 26017$ & PNNL-AGG-415 \\
\hline 14392-19-9 & Molybdenum 97 & $2.64 \mathrm{E}-3$ & ug/g dry & $2.04 \mathrm{E}-3$ & $8 / 27 / 08$ & $8 \mathrm{H} 26017$ & PNNL-AGG-415 \\
\hline $14392-20-2$ & Molybdenum 98 & $2.63 \mathrm{E}-3$ & $\mathrm{ug} / \mathrm{g}$ dry & $1.60 \mathrm{E}-3$ & $8 / 27 / 08$ & $8 \mathrm{H} 26017$ & PNNL-AGG-415 \\
\hline $14914-61-5$ & Ruthenium 101 & $<8.19 \mathrm{E}-4$ & $\mathrm{ug} / \mathrm{g}$ dry & $8.19 \mathrm{E}-4$ & $8 / 27 / 08$ & $8 \mathrm{H} 26017$ & PNNL-AGG-415 \\
\hline $14914-62-6$ & Ruthenium 102 & $<5.64 \mathrm{E}-4$ & ug/g dry & $5.64 \mathrm{E}-4$ & $8 / 27 / 08$ & $8 \mathrm{H} 26017$ & PNNL-AGG-415 \\
\hline $15766-01-5$ & Ruthenium 104 & $<3.65 \mathrm{E}-4$ & $\mathrm{ug} / \mathrm{g}$ dry & $3.65 \mathrm{E}-4$ & $8 / 27 / 08$ & $8 \mathrm{H} 26017$ & PNNL-AGG-415 \\
\hline $14378-37-1$ & Silver 107 & $<9.24 \mathrm{E}-4$ & ug/g dry & $9.24 \mathrm{E}-4$ & $8 / 27 / 08$ & $8 \mathrm{H} 26017$ & PNNL-AGG-415 \\
\hline $14378-38-2$ & Silver 109 & $<1.07 \mathrm{E}-3$ & ug/g dry & $1.07 \mathrm{E}-3$ & $8 / 27 / 08$ & $8 \mathrm{H} 26017$ & PNNL-AGG-415 \\
\hline $14336-64-2$ & Cadmium 111 & $<2.95 \mathrm{E}-4$ & ug/g dry & $2.95 \mathrm{E}-4$ & $8 / 27 / 08$ & $8 \mathrm{H} 26017$ & PNNL-AGG-415 \\
\hline $14041-58-8$ & Cadmium 114 & $<6.24 \mathrm{E}-4$ & ug/g dry & $6.24 \mathrm{E}-4$ & $8 / 27 / 08$ & $8 \mathrm{H} 26017$ & PNNL-AGG-415 \\
\hline $14265-72-6$ & Antimony 121 & $<5.39 \mathrm{E}-4$ & ug/g dry & $5.39 \mathrm{E}-4$ & $8 / 27 / 08$ & $8 \mathrm{H} 26017$ & PNNL-AGG-415 \\
\hline $13966-27-3$ & Lead 206 & $<7.19 \mathrm{E}-4$ & ug/g dry & $7.19 \mathrm{E}-4$ & $8 / 27 / 08$ & $8 \mathrm{H} 26017$ & PNNL-AGG-415 \\
\hline $13966-28-4$ & Lead 208 & $<5.59 \mathrm{E}-4$ & ug/g dry & $5.59 \mathrm{E}-4$ & $8 / 27 / 08$ & $8 \mathrm{H} 26017$ & PNNL-AGG-415 \\
\hline HEIS No. & B1V9C2 & \multicolumn{3}{|c|}{ Lab ID: $\quad$ 0805003-22 } & & & \\
\hline $14092-98-9$ & Chromium 52 & $<2.05 \mathrm{E}-3$ & ug/g dry & $2.05 \mathrm{E}-3$ & $8 / 27 / 08$ & $8 \mathrm{H} 26017$ & PNNL-AGG-415 \\
\hline $13981-78-7$ & Chromium 53 & $<6.39 \mathrm{E}-3$ & $\mathrm{ug} / \mathrm{g}$ dry & $6.39 \mathrm{E}-3$ & $8 / 27 / 08$ & $8 \mathrm{H} 26017$ & PNNL-AGG-415 \\
\hline $14191-84-5$ & Copper 63 & $<3.48 \mathrm{E}-3$ & ug/g dry & $3.48 \mathrm{E}-3$ & $8 / 27 / 08$ & $8 \mathrm{H} 26017$ & PNNL-AGG-415 \\
\hline $14119-06-3$ & Copper 65 & $<4.83 \mathrm{E}-3$ & ug/g dry & $4.83 \mathrm{E}-3$ & $8 / 27 / 08$ & $8 \mathrm{H} 26017$ & PNNL-AGG-415 \\
\hline $7440-38-2$ & Arsenic 75 & $7.26 \mathrm{E}-3$ & ug/g dry & $6.24 \mathrm{E}-3$ & $8 / 27 / 08$ & $8 \mathrm{H} 26017$ & PNNL-AGG-415 \\
\hline $14687-58-2$ & Selenium 82 & $<1.10 \mathrm{E}-2$ & $\mathrm{ug} / \mathrm{g}$ dry & $1.10 \mathrm{E}-2$ & $8 / 27 / 08$ & $8 \mathrm{H} 26017$ & PNNL-AGG-415 \\
\hline $14392-17-7$ & Molybdenum 95 & $2.09 \mathrm{E}-3$ & ug/g dry & $1.60 \mathrm{E}-3$ & $8 / 27 / 08$ & $8 \mathrm{H} 26017$ & PNNL-AGG-415 \\
\hline 14392-19-9 & Molybdenum 97 & $2.21 \mathrm{E}-3$ & ug/g dry & $2.04 \mathrm{E}-3$ & $8 / 27 / 08$ & $8 \mathrm{H} 26017$ & PNNL-AGG-415 \\
\hline $14392-20-2$ & Molybdenum 98 & $2.19 \mathrm{E}-3$ & ug/g dry & $1.60 \mathrm{E}-3$ & $8 / 27 / 08$ & $8 \mathrm{H} 26017$ & PNNL-AGG-415 \\
\hline $14914-61-5$ & Ruthenium 101 & $<8.19 \mathrm{E}-4$ & ug/g dry & $8.19 \mathrm{E}-4$ & $8 / 27 / 08$ & $8 \mathrm{H} 26017$ & PNNL-AGG-415 \\
\hline $14914-62-6$ & Ruthenium 102 & $<5.64 \mathrm{E}-4$ & ug/g dry & $5.64 \mathrm{E}-4$ & $8 / 27 / 08$ & $8 \mathrm{H} 26017$ & PNNL-AGG-415 \\
\hline $15766-01-5$ & Ruthenium 104 & $<3.65 \mathrm{E}-4$ & ug/g dry & $3.65 \mathrm{E}-4$ & $8 / 27 / 08$ & $8 \mathrm{H} 26017$ & PNNL-AGG-415 \\
\hline $14378-37-1$ & Silver 107 & $<9.24 \mathrm{E}-4$ & ug/g dry & $9.24 \mathrm{E}-4$ & $8 / 27 / 08$ & $8 \mathrm{H} 26017$ & PNNL-AGG-415 \\
\hline $14378-38-2$ & Silver 109 & $<1.07 \mathrm{E}-3$ & ug/g dry & $1.07 \mathrm{E}-3$ & $8 / 27 / 08$ & $8 \mathrm{H} 26017$ & PNNL-AGG-415 \\
\hline $14336-64-2$ & Cadmium 111 & $<2.95 \mathrm{E}-4$ & ug/g dry & $2.95 \mathrm{E}-4$ & $8 / 27 / 08$ & $8 \mathrm{H} 26017$ & PNNL-AGG-415 \\
\hline $14041-58-8$ & Cadmium 114 & $<6.24 \mathrm{E}-4$ & ug/g dry & $6.24 \mathrm{E}-4$ & $8 / 27 / 08$ & $8 \mathrm{H} 26017$ & PNNL-AGG-415 \\
\hline $14265-72-6$ & Antimony 121 & $<5.39 \mathrm{E}-4$ & $\mathrm{ug} / \mathrm{g}$ dry & $5.39 \mathrm{E}-4$ & $8 / 27 / 08$ & $8 \mathrm{H} 26017$ & PNNL-AGG-415 \\
\hline $13966-27-3$ & Lead 206 & $<7.19 \mathrm{E}-4$ & ug/g dry & $7.19 \mathrm{E}-4$ & $8 / 27 / 08$ & $8 \mathrm{H} 26017$ & PNNL-AGG-415 \\
\hline $13966-28-4$ & Lead 208 & $<5.59 \mathrm{E}-4$ & ug/g dry & $5.59 \mathrm{E}-4$ & $8 / 27 / 08$ & $8 \mathrm{H} 26017$ & PNNL-AGG-415 \\
\hline HEIS No. & B1V9C3 & \multicolumn{3}{|c|}{ Lab ID: $\quad$ 0805003-23 } & & & \\
\hline $14092-98-9$ & Chromium 52 & $<2.05 \mathrm{E}-3$ & ug/g dry & $2.05 \mathrm{E}-3$ & $8 / 27 / 08$ & $8 \mathrm{H} 26017$ & PNNL-AGG-415 \\
\hline $13981-78-7$ & Chromium 53 & $<6.38 \mathrm{E}-3$ & ug/g dry & $6.38 \mathrm{E}-3$ & $8 / 27 / 08$ & $8 \mathrm{H} 26017$ & PNNL-AGG-415 \\
\hline $14191-84-5$ & Copper 63 & $<3.47 \mathrm{E}-3$ & ug/g dry & $3.47 \mathrm{E}-3$ & $8 / 27 / 08$ & $8 \mathrm{H} 26017$ & PNNL-AGG-415 \\
\hline $14119-06-3$ & Copper 65 & $<4.82 \mathrm{E}-3$ & $\mathrm{ug} / \mathrm{g}$ dry & $4.82 \mathrm{E}-3$ & $8 / 27 / 08$ & $8 \mathrm{H} 26017$ & PNNL-AGG-415 \\
\hline $7440-38-2$ & Arsenic 75 & $7.64 \mathrm{E}-3$ & ug/g dry & $6.23 \mathrm{E}-3$ & $8 / 27 / 08$ & $8 \mathrm{H} 26017$ & PNNL-AGG-415 \\
\hline $14687-58-2$ & Selenium 82 & $<1.10 \mathrm{E}-2$ & ug/g dry & $1.10 \mathrm{E}-2$ & $8 / 27 / 08$ & $8 \mathrm{H} 26017$ & PNNL-AGG-415 \\
\hline $14392-17-7$ & Molybdenum 95 & $1.83 \mathrm{E}-3$ & $\mathrm{ug} / \mathrm{g}$ dry & $1.60 \mathrm{E}-3$ & $8 / 27 / 08$ & $8 \mathrm{H} 26017$ & PNNL-AGG-415 \\
\hline 14392-19-9 & Molybdenum 97 & $<2.04 \mathrm{E}-3$ & $\mathrm{ug} / \mathrm{g}$ dry & $2.04 \mathrm{E}-3$ & $8 / 27 / 08$ & $8 \mathrm{H} 26017$ & PNNL-AGG-415 \\
\hline $14392-20-2$ & Molybdenum 98 & $1.97 \mathrm{E}-3$ & $\mathrm{ug} / \mathrm{g}$ dry & $1.60 \mathrm{E}-3$ & $8 / 27 / 08$ & $8 \mathrm{H} 26017$ & PNNL-AGG-415 \\
\hline $14914-61-5$ & Ruthenium 101 & $<8.17 \mathrm{E}-4$ & ug/g dry & $8.17 \mathrm{E}-4$ & $8 / 27 / 08$ & $8 \mathrm{H} 26017$ & PNNL-AGG-415 \\
\hline
\end{tabular}


RCRA Metals By PNNL-AGG-415/Water Extract

\begin{tabular}{|c|c|c|c|c|c|c|c|}
\hline CAS \# & Analyte & Results & Units & EQL & Analyzed & Batch & Method \\
\hline HEIS No. & B1V9C3 & \multicolumn{3}{|c|}{ Lab ID: $\quad$ 0805003-23 } & & & \\
\hline $14914-62-6$ & Ruthenium 102 & $<5.63 \mathrm{E}-4$ & ug/g dry & $5.63 \mathrm{E}-4$ & $8 / 27 / 08$ & $8 \mathrm{H} 26017$ & PNNL-AGG-415 \\
\hline $15766-01-5$ & Ruthenium 104 & $<3.64 \mathrm{E}-4$ & ug/g dry & $3.64 \mathrm{E}-4$ & $8 / 27 / 08$ & $8 \mathrm{H} 26017$ & PNNL-AGG-415 \\
\hline $14378-37-1$ & Silver 107 & $<9.21 \mathrm{E}-4$ & ug/g dry & $9.21 \mathrm{E}-4$ & $8 / 27 / 08$ & $8 \mathrm{H} 26017$ & PNNL-AGG-415 \\
\hline $14378-38-2$ & Silver 109 & $<1.07 \mathrm{E}-3$ & ug/g dry & $1.07 \mathrm{E}-3$ & $8 / 27 / 08$ & $8 \mathrm{H} 26017$ & PNNL-AGG-415 \\
\hline $14336-64-2$ & Cadmium 111 & $<2.94 \mathrm{E}-4$ & ug/g dry & $2.94 \mathrm{E}-4$ & $8 / 27 / 08$ & $8 \mathrm{H} 26017$ & PNNL-AGG-415 \\
\hline $14041-58-8$ & Cadmium 114 & $<6.23 \mathrm{E}-4$ & ug/g dry & $6.23 \mathrm{E}-4$ & $8 / 27 / 08$ & $8 \mathrm{H} 26017$ & PNNL-AGG-415 \\
\hline $14265-72-6$ & Antimony 121 & $<5.38 \mathrm{E}-4$ & ug/g dry & $5.38 \mathrm{E}-4$ & $8 / 27 / 08$ & $8 \mathrm{H} 26017$ & PNNL-AGG-415 \\
\hline $13966-27-3$ & Lead 206 & $<7.17 \mathrm{E}-4$ & ug/g dry & $7.17 \mathrm{E}-4$ & $8 / 27 / 08$ & $8 \mathrm{H} 26017$ & PNNL-AGG-415 \\
\hline $13966-28-4$ & Lead 208 & $<5.58 \mathrm{E}-4$ & ug/g dry & $5.58 \mathrm{E}-4$ & $8 / 27 / 08$ & $8 \mathrm{H} 26017$ & PNNL-AGG-415 \\
\hline HEIS No. & B1V9C4 & \multicolumn{3}{|c|}{ Lab ID: $\quad$ 0805003-24 } & & & \\
\hline $14092-98-9$ & Chromium 52 & $<2.06 \mathrm{E}-3$ & ug/g dry & $2.06 \mathrm{E}-3$ & $8 / 27 / 08$ & $8 \mathrm{H} 26017$ & PNNL-AGG-415 \\
\hline $13981-78-7$ & Chromium 53 & $<6.41 \mathrm{E}-3$ & ug/g dry & $6.41 \mathrm{E}-3$ & $8 / 27 / 08$ & $8 \mathrm{H} 26017$ & PNNL-AGG-415 \\
\hline $14191-84-5$ & Copper 63 & $<3.48 \mathrm{E}-3$ & ug/g dry & $3.48 \mathrm{E}-3$ & $8 / 27 / 08$ & $8 \mathrm{H} 26017$ & PNNL-AGG-415 \\
\hline $14119-06-3$ & Copper 65 & $<4.85 \mathrm{E}-3$ & ug/g dry & $4.85 \mathrm{E}-3$ & $8 / 27 / 08$ & $8 \mathrm{H} 26017$ & PNNL-AGG-415 \\
\hline $7440-38-2$ & Arsenic 75 & $<6.26 \mathrm{E}-3$ & ug/g dry & $6.26 \mathrm{E}-3$ & $8 / 27 / 08$ & $8 \mathrm{H} 26017$ & PNNL-AGG-415 \\
\hline $14687-58-2$ & Selenium 82 & $<1.11 \mathrm{E}-2$ & ug/g dry & $1.11 \mathrm{E}-2$ & $8 / 27 / 08$ & $8 \mathrm{H} 26017$ & PNNL-AGG-415 \\
\hline $14392-17-7$ & Molybdenum 95 & $5.79 \mathrm{E}-3$ & ug/g dry & $1.61 \mathrm{E}-3$ & $8 / 27 / 08$ & $8 \mathrm{H} 26017$ & PNNL-AGG-415 \\
\hline $14392-19-9$ & Molybdenum 97 & $5.99 \mathrm{E}-3$ & ug/g dry & $2.05 \mathrm{E}-3$ & $8 / 27 / 08$ & $8 \mathrm{H} 26017$ & PNNL-AGG-415 \\
\hline $14392-20-2$ & Molybdenum 98 & $5.86 \mathrm{E}-3$ & ug/g dry & $1.61 \mathrm{E}-3$ & $8 / 27 / 08$ & $8 \mathrm{H} 26017$ & PNNL-AGG-415 \\
\hline $14914-61-5$ & Ruthenium 101 & $<8.21 \mathrm{E}-4$ & ug/g dry & $8.21 \mathrm{E}-4$ & $8 / 27 / 08$ & $8 \mathrm{H} 26017$ & PNNL-AGG-415 \\
\hline $14914-62-6$ & Ruthenium 102 & $<5.66 \mathrm{E}-4$ & ug/g dry & $5.66 \mathrm{E}-4$ & $8 / 27 / 08$ & $8 \mathrm{H} 26017$ & PNNL-AGG-415 \\
\hline $15766-01-5$ & Ruthenium 104 & $<3.65 \mathrm{E}-4$ & ug/g dry & $3.65 \mathrm{E}-4$ & $8 / 27 / 08$ & $8 \mathrm{H} 26017$ & PNNL-AGG-415 \\
\hline $14378-37-1$ & Silver 107 & $<9.26 \mathrm{E}-4$ & ug/g dry & $9.26 \mathrm{E}-4$ & $8 / 27 / 08$ & $8 \mathrm{H} 26017$ & PNNL-AGG-415 \\
\hline $14378-38-2$ & Silver 109 & $<1.07 \mathrm{E}-3$ & ug/g dry & $1.07 \mathrm{E}-3$ & $8 / 27 / 08$ & $8 \mathrm{H} 26017$ & PNNL-AGG-415 \\
\hline $14336-64-2$ & Cadmium 111 & $<2.95 \mathrm{E}-4$ & ug/g dry & $2.95 \mathrm{E}-4$ & $8 / 27 / 08$ & $8 \mathrm{H} 26017$ & PNNL-AGG-415 \\
\hline $14041-58-8$ & Cadmium 114 & $<6.26 \mathrm{E}-4$ & ug/g dry & $6.26 \mathrm{E}-4$ & $8 / 27 / 08$ & $8 \mathrm{H} 26017$ & PNNL-AGG-415 \\
\hline $14265-72-6$ & Antimony 121 & $<5.41 \mathrm{E}-4$ & ug/g dry & $5.41 \mathrm{E}-4$ & $8 / 27 / 08$ & $8 \mathrm{H} 26017$ & PNNL-AGG-415 \\
\hline $13966-27-3$ & Lead 206 & $<7.21 \mathrm{E}-4$ & ug/g dry & $7.21 \mathrm{E}-4$ & $8 / 27 / 08$ & $8 \mathrm{H} 26017$ & PNNL-AGG-415 \\
\hline $13966-28-4$ & Lead 208 & $<5.61 \mathrm{E}-4$ & ug/g dry & $5.61 \mathrm{E}-4$ & $8 / 27 / 08$ & $8 \mathrm{H} 26017$ & PNNL-AGG-415 \\
\hline HEIS No. & B1V2V5 & & b ID: & $5003-25$ & & & \\
\hline $14092-98-9$ & Chromium 52 & $<2.05 \mathrm{E}-3$ & ug/g dry & $2.05 \mathrm{E}-3$ & $8 / 27 / 08$ & $8 \mathrm{H} 26017$ & PNNL-AGG-415 \\
\hline $13981-78-7$ & Chromium 53 & $<6.38 \mathrm{E}-3$ & ug/g dry & $6.38 \mathrm{E}-3$ & $8 / 27 / 08$ & $8 \mathrm{H} 26017$ & PNNL-AGG-415 \\
\hline $14191-84-5$ & Copper 63 & $<3.47 \mathrm{E}-3$ & ug/g dry & $3.47 \mathrm{E}-3$ & $8 / 27 / 08$ & $8 \mathrm{H} 26017$ & PNNL-AGG-415 \\
\hline $14119-06-3$ & Copper 65 & $<4.82 \mathrm{E}-3$ & ug/g dry & $4.82 \mathrm{E}-3$ & $8 / 27 / 08$ & $8 \mathrm{H} 26017$ & PNNL-AGG-415 \\
\hline $7440-38-2$ & Arsenic 75 & $8.48 \mathrm{E}-3$ & ug/g dry & $6.23 \mathrm{E}-3$ & $8 / 27 / 08$ & $8 \mathrm{H} 26017$ & PNNL-AGG-415 \\
\hline $14687-58-2$ & Selenium 82 & $<1.10 \mathrm{E}-2$ & ug/g dry & $1.10 \mathrm{E}-2$ & $8 / 27 / 08$ & $8 \mathrm{H} 26017$ & PNNL-AGG-415 \\
\hline $14392-17-7$ & Molybdenum 95 & $1.79 \mathrm{E}-3$ & ug/g dry & $1.60 \mathrm{E}-3$ & $8 / 27 / 08$ & $8 \mathrm{H} 26017$ & PNNL-AGG-415 \\
\hline $14392-19-9$ & Molybdenum 97 & $<2.04 \mathrm{E}-3$ & ug/g dry & $2.04 \mathrm{E}-3$ & $8 / 27 / 08$ & $8 \mathrm{H} 26017$ & PNNL-AGG-415 \\
\hline $14392-20-2$ & Molybdenum 98 & $1.93 \mathrm{E}-3$ & ug/g dry & $1.60 \mathrm{E}-3$ & $8 / 27 / 08$ & $8 \mathrm{H} 26017$ & PNNL-AGG-415 \\
\hline $14914-61-5$ & Ruthenium 101 & $<8.17 \mathrm{E}-4$ & ug/g dry & $8.17 \mathrm{E}-4$ & $8 / 27 / 08$ & $8 \mathrm{H} 26017$ & PNNL-AGG-415 \\
\hline $14914-62-6$ & Ruthenium 102 & $<5.63 \mathrm{E}-4$ & ug/g dry & $5.63 \mathrm{E}-4$ & $8 / 27 / 08$ & $8 \mathrm{H} 26017$ & PNNL-AGG-415 \\
\hline $15766-01-5$ & Ruthenium 104 & $<3.64 \mathrm{E}-4$ & ug/g dry & $3.64 \mathrm{E}-4$ & $8 / 27 / 08$ & $8 \mathrm{H} 26017$ & PNNL-AGG-415 \\
\hline $14378-37-1$ & Silver 107 & $<9.22 \mathrm{E}-4$ & ug/g dry & $9.22 \mathrm{E}-4$ & $8 / 27 / 08$ & $8 \mathrm{H} 26017$ & PNNL-AGG-415 \\
\hline $14378-38-2$ & Silver 109 & $<1.07 \mathrm{E}-3$ & ug/g dry & $1.07 \mathrm{E}-3$ & $8 / 27 / 08$ & $8 \mathrm{H} 26017$ & PNNL-AGG-415 \\
\hline $14336-64-2$ & Cadmium 111 & $<2.94 \mathrm{E}-4$ & ug/g dry & $2.94 \mathrm{E}-4$ & $8 / 27 / 08$ & $8 \mathrm{H} 26017$ & PNNL-AGG-415 \\
\hline $14041-58-8$ & Cadmium 114 & $<6.23 \mathrm{E}-4$ & ug/g dry & $6.23 \mathrm{E}-4$ & $8 / 27 / 08$ & $8 \mathrm{H} 26017$ & PNNL-AGG-415 \\
\hline $14265-72-6$ & Antimony 121 & $<5.38 \mathrm{E}-4$ & ug/g dry & $5.38 \mathrm{E}-4$ & $8 / 27 / 08$ & $8 \mathrm{H} 26017$ & PNNL-AGG-415 \\
\hline $13966-27-3$ & Lead 206 & $<7.17 \mathrm{E}-4$ & ug/g dry & $7.17 \mathrm{E}-4$ & $8 / 27 / 08$ & $8 \mathrm{H} 26017$ & PNNL-AGG-415 \\
\hline
\end{tabular}


RCRA Metals By PNNL-AGG-415/Water Extract

\begin{tabular}{|c|c|c|c|c|c|c|c|}
\hline CAS \# & Analyte & Results & Units & EQL & Analyzed & Batch & Method \\
\hline HEIS No. & B1V2V5 & & & $5003-25$ & & & \\
\hline $13966-28-4$ & Lead 208 & $<5.58 \mathrm{E}-4$ & ag/g dry & $5.58 \mathrm{E}-4$ & $8 / 27 / 08$ & $8 \mathrm{H} 26017$ & PNNL-AGG-415 \\
\hline
\end{tabular}


RCRA Metals By PNNL-AGG-415/Acid Extract

\begin{tabular}{|c|c|c|c|c|c|c|c|}
\hline CAS \# & Analyte & Results & Units & EQL & Analyzed & Batch & Method \\
\hline HEIS No. & B1V993 & \multicolumn{3}{|c|}{ Lab ID: $\quad$ 0805003-03 } & & & \\
\hline $14092-98-9$ & Chromium 52 & $5.03 \mathrm{E} 0$ & ug/g dry & $1.78 \mathrm{E}-1$ & $12 / 15 / 08$ & 8L15001 & PNNL-AGG-415 \\
\hline $14191-84-5$ & Copper 63 & $7.85 \mathrm{E} 0$ & ug/g dry & $6.31 \mathrm{E}-1$ & $12 / 15 / 08$ & 8L15001 & PNNL-AGG-415 \\
\hline $14119-06-3$ & Copper 65 & $8.18 \mathrm{E} 0$ & ug/g dry & $5.62 \mathrm{E}-1$ & $12 / 15 / 08$ & 8L15001 & PNNL-AGG-415 \\
\hline $7440-38-2$ & Arsenic 75 & 7.45E-1 & ug/g dry & $3.92 \mathrm{E}-1$ & $12 / 15 / 08$ & 8L15001 & PNNL-AGG-415 \\
\hline $14687-58-2$ & Selenium 82 & $<1.07 \mathrm{E} 0$ & ug/g dry & $1.07 \mathrm{E} 0$ & $12 / 15 / 08$ & 8L15001 & PNNL-AGG-415 \\
\hline $14392-17-7$ & Molybdenum 95 & $<2.20 \mathrm{E}-1$ & ug/g dry & $2.20 \mathrm{E}-1$ & $12 / 15 / 08$ & 8L15001 & PNNL-AGG-415 \\
\hline 14392-19-9 & Molybdenum 97 & $<2.01 \mathrm{E}-1$ & ug/g dry & $2.01 \mathrm{E}-1$ & $12 / 15 / 08$ & 8L15001 & PNNL-AGG-415 \\
\hline $14392-20-2$ & Molybdenum 98 & $1.80 \mathrm{E}-1$ & ug/g dry & $8.33 \mathrm{E}-2$ & $12 / 15 / 08$ & 8L15001 & PNNL-AGG-415 \\
\hline $14914-61-5$ & Ruthenium 101 & $<4.22 \mathrm{E}-2$ & ug/g dry & $4.22 \mathrm{E}-2$ & $12 / 15 / 08$ & $8 \mathrm{~L} 15001$ & PNNL-AGG-415 \\
\hline $14914-62-6$ & Ruthenium 102 & $<2.55 \mathrm{E}-2$ & ug/g dry & $2.55 \mathrm{E}-2$ & $12 / 15 / 08$ & $8 \mathrm{~L} 15001$ & PNNL-AGG-415 \\
\hline $15766-01-5$ & Ruthenium 104 & $<7.12 \mathrm{E}-2$ & ug/g dry & 7.12E-2 & $12 / 15 / 08$ & 8L15001 & PNNL-AGG-415 \\
\hline $14378-37-1$ & Silver 107 & $<6.50 \mathrm{E}-2$ & ug/g dry & $6.50 \mathrm{E}-2$ & $12 / 15 / 08$ & $8 \mathrm{~L} 15001$ & PNNL-AGG-415 \\
\hline $14378-38-2$ & Silver 109 & $<4.25 \mathrm{E}-2$ & ug/g dry & $4.25 \mathrm{E}-2$ & $12 / 15 / 08$ & 8L15001 & PNNL-AGG-415 \\
\hline $14336-64-2$ & Cadmium 111 & $<4.61 \mathrm{E}-2$ & ug/g dry & $4.61 \mathrm{E}-2$ & $12 / 15 / 08$ & 8L15001 & PNNL-AGG-415 \\
\hline $14041-58-8$ & Cadmium 114 & 7.73E-2 & ug/g dry & $1.05 \mathrm{E}-2$ & $12 / 15 / 08$ & $8 \mathrm{~L} 15001$ & PNNL-AGG-415 \\
\hline $14265-72-6$ & Antimony 121 & $<7.45 \mathrm{E}-2$ & ug/g dry & $7.45 \mathrm{E}-2$ & $12 / 15 / 08$ & 8L15001 & PNNL-AGG-415 \\
\hline $13966-27-3$ & Lead 206 & $2.20 \mathrm{E} 0$ & ug/g dry & $7.32 \mathrm{E}-2$ & $12 / 15 / 08$ & 8L15001 & PNNL-AGG-415 \\
\hline $13966-28-4$ & Lead 208 & $2.18 \mathrm{E} 0$ & ug/g dry & $3.46 \mathrm{E}-2$ & $12 / 15 / 08$ & $8 \mathrm{~L} 15001$ & PNNL-AGG-415 \\
\hline HEIS No. & B1V995 & \multicolumn{3}{|c|}{ Lab ID: $\quad$ 0805003-05 } & & & \\
\hline $14092-98-9$ & Chromium 52 & $5.75 \mathrm{E} 0$ & ug/g dry & $1.88 \mathrm{E}-1$ & $12 / 15 / 08$ & $8 \mathrm{~L} 15001$ & PNNL-AGG-415 \\
\hline $14191-84-5$ & Copper 63 & 8.37E0 & ug/g dry & $6.64 \mathrm{E}-1$ & $12 / 15 / 08$ & 8L15001 & PNNL-AGG-415 \\
\hline $14119-06-3$ & Copper 65 & $8.86 \mathrm{E} 0$ & ug/g dry & $5.92 \mathrm{E}-1$ & $12 / 15 / 08$ & 8L15001 & PNNL-AGG-415 \\
\hline $7440-38-2$ & Arsenic 75 & $1.23 \mathrm{E} 0$ & ug/g dry & 4.13E-1 & $12 / 15 / 08$ & 8L15001 & PNNL-AGG-415 \\
\hline $14687-58-2$ & Selenium 82 & $<1.13 \mathrm{E} 0$ & ug/g dry & $1.13 \mathrm{E} 0$ & $12 / 15 / 08$ & $8 \mathrm{~L} 15001$ & PNNL-AGG-415 \\
\hline $14392-17-7$ & Molybdenum 95 & $2.73 \mathrm{E}-1$ & ug/g dry & $2.31 \mathrm{E}-1$ & $12 / 15 / 08$ & 8L15001 & PNNL-AGG-415 \\
\hline $14392-19-9$ & Molybdenum 97 & $2.75 \mathrm{E}-1$ & ug/g dry & $2.12 \mathrm{E}-1$ & $12 / 15 / 08$ & 8L15001 & PNNL-AGG-415 \\
\hline $14392-20-2$ & Molybdenum 98 & $2.62 \mathrm{E}-1$ & ug/g dry & $8.77 \mathrm{E}-2$ & $12 / 15 / 08$ & $8 \mathrm{~L} 15001$ & PNNL-AGG-415 \\
\hline $14914-61-5$ & Ruthenium 101 & $<4.44 \mathrm{E}-2$ & ug/g dry & $4.44 \mathrm{E}-2$ & $12 / 15 / 08$ & $8 \mathrm{~L} 15001$ & PNNL-AGG-415 \\
\hline $14914-62-6$ & Ruthenium 102 & $<2.68 \mathrm{E}-2$ & ug/g dry & $2.68 \mathrm{E}-2$ & $12 / 15 / 08$ & 8L15001 & PNNL-AGG-415 \\
\hline $15766-01-5$ & Ruthenium 104 & $<7.50 \mathrm{E}-2$ & ug/g dry & $7.50 \mathrm{E}-2$ & $12 / 15 / 08$ & $8 \mathrm{~L} 15001$ & PNNL-AGG-415 \\
\hline $14378-37-1$ & Silver 107 & $<6.85 \mathrm{E}-2$ & ug/g dry & $6.85 \mathrm{E}-2$ & $12 / 15 / 08$ & 8L15001 & PNNL-AGG-415 \\
\hline $14378-38-2$ & Silver 109 & $<4.47 \mathrm{E}-2$ & ug/g dry & $4.47 \mathrm{E}-2$ & $12 / 15 / 08$ & 8L15001 & PNNL-AGG-415 \\
\hline $14336-64-2$ & Cadmium 111 & $<4.85 \mathrm{E}-2$ & ug/g dry & $4.85 \mathrm{E}-2$ & $12 / 15 / 08$ & $8 \mathrm{~L} 15001$ & PNNL-AGG-415 \\
\hline $14041-58-8$ & Cadmium 114 & $1.73 \mathrm{E}-1$ & ug/g dry & $1.10 \mathrm{E}-2$ & $12 / 15 / 08$ & 8L15001 & PNNL-AGG-415 \\
\hline $14265-72-6$ & Antimony 121 & $<7.84 \mathrm{E}-2$ & ug/g dry & $7.84 \mathrm{E}-2$ & $12 / 15 / 08$ & 8L15001 & PNNL-AGG-415 \\
\hline $13966-27-3$ & Lead 206 & $3.18 \mathrm{E} 0$ & ug/g dry & $7.71 \mathrm{E}-2$ & $12 / 15 / 08$ & 8L15001 & PNNL-AGG-415 \\
\hline $13966-28-4$ & Lead 208 & $3.15 \mathrm{E} 0$ & ug/g dry & $3.65 \mathrm{E}-2$ & $12 / 15 / 08$ & 8L15001 & PNNL-AGG-415 \\
\hline HEIS No. & B1V997 & \multicolumn{3}{|c|}{ Lab ID: $\quad$ 0805003-07 } & & & \\
\hline 14092-98-9 & Chromium 52 & $8.20 \mathrm{E} 0$ & ug/g dry & $1.90 \mathrm{E}-1$ & $12 / 15 / 08$ & $8 \mathrm{~L} 15001$ & PNNL-AGG-415 \\
\hline $14191-84-5$ & Copper 63 & 7.52E0 & ug/g dry & $6.70 \mathrm{E}-1$ & $12 / 15 / 08$ & 8L15001 & PNNL-AGG-415 \\
\hline $14119-06-3$ & Copper 65 & 7.82E0 & ug/g dry & $5.97 \mathrm{E}-1$ & $12 / 15 / 08$ & 8L15001 & PNNL-AGG-415 \\
\hline $7440-38-2$ & Arsenic 75 & $1.64 \mathrm{E} 0$ & $\mathrm{ug} / \mathrm{g}$ dry & $4.16 \mathrm{E}-1$ & $12 / 15 / 08$ & $8 \mathrm{~L} 15001$ & PNNL-AGG-415 \\
\hline $14687-58-2$ & Selenium 82 & $<1.14 \mathrm{E} 0$ & ug/g dry & $1.14 \mathrm{E} 0$ & $12 / 15 / 08$ & 8L15001 & PNNL-AGG-415 \\
\hline $14392-17-7$ & Molybdenum 95 & $<2.33 \mathrm{E}-1$ & ug/g dry & $2.33 \mathrm{E}-1$ & $12 / 15 / 08$ & $8 \mathrm{~L} 15001$ & PNNL-AGG-415 \\
\hline $14392-19-9$ & Molybdenum 97 & $<2.14 \mathrm{E}-1$ & ug/g dry & $2.14 \mathrm{E}-1$ & $12 / 15 / 08$ & $8 \mathrm{~L} 15001$ & PNNL-AGG-415 \\
\hline $14392-20-2$ & Molybdenum 98 & $1.58 \mathrm{E}-1$ & ug/g dry & $8.85 \mathrm{E}-2$ & $12 / 15 / 08$ & 8L15001 & PNNL-AGG-415 \\
\hline $14914-61-5$ & Ruthenium 101 & $<4.48 \mathrm{E}-2$ & ug/g dry & $4.48 \mathrm{E}-2$ & $12 / 15 / 08$ & $8 \mathrm{~L} 15001$ & PNNL-AGG-415 \\
\hline $14914-62-6$ & Ruthenium 102 & $<2.71 \mathrm{E}-2$ & $\mathrm{ug} / \mathrm{g}$ dry & $2.71 \mathrm{E}-2$ & $12 / 15 / 08$ & 8L15001 & PNNL-AGG-415 \\
\hline
\end{tabular}


RCRA Metals By PNNL-AGG-415/Acid Extract

\begin{tabular}{|c|c|c|c|c|c|c|c|}
\hline CAS \# & Analyte & Results & Units & EQL & Analyzed & Batch & Method \\
\hline HEIS No. & B1V997 & \multicolumn{3}{|c|}{ Lab ID: $\quad$ 0805003-07 } & & & \\
\hline $15766-01-5$ & Ruthenium 104 & $<7.57 \mathrm{E}-2$ & ug/g dry & $7.57 \mathrm{E}-2$ & $12 / 15 / 08$ & 8L15001 & PNNL-AGG-415 \\
\hline $14378-37-1$ & Silver 107 & $<6.91 \mathrm{E}-2$ & ug/g dry & $6.91 \mathrm{E}-2$ & $12 / 15 / 08$ & 8L15001 & PNNL-AGG-415 \\
\hline $14378-38-2$ & Silver 109 & $<4.51 \mathrm{E}-2$ & ug/g dry & $4.51 \mathrm{E}-2$ & $12 / 15 / 08$ & 8L15001 & PNNL-AGG-415 \\
\hline $14336-64-2$ & Cadmium 111 & $<4.89 \mathrm{E}-2$ & ug/g dry & $4.89 \mathrm{E}-2$ & $12 / 15 / 08$ & 8L15001 & PNNL-AGG-415 \\
\hline $14041-58-8$ & Cadmium 114 & $9.79 \mathrm{E}-2$ & ug/g dry & $1.11 \mathrm{E}-2$ & $12 / 15 / 08$ & 8L15001 & PNNL-AGG-415 \\
\hline $14265-72-6$ & Antimony 121 & $<7.91 \mathrm{E}-2$ & ug/g dry & $7.91 \mathrm{E}-2$ & $12 / 15 / 08$ & 8L15001 & PNNL-AGG-415 \\
\hline $13966-27-3$ & Lead 206 & $3.15 \mathrm{E} 0$ & ug/g dry & 7.77E-2 & $12 / 15 / 08$ & $8 \mathrm{~L} 15001$ & PNNL-AGG-415 \\
\hline $13966-28-4$ & Lead 208 & 3.07E0 & ug/g dry & $3.68 \mathrm{E}-2$ & $12 / 15 / 08$ & $8 \mathrm{~L} 15001$ & PNNL-AGG-415 \\
\hline HEIS No. & B1V999 & \multicolumn{3}{|c|}{ Lab ID: $\quad$ 0805003-09 } & & & \\
\hline $14092-98-9$ & Chromium 52 & $5.29 \mathrm{E} 0$ & ug/g dry & $1.89 \mathrm{E}-1$ & $12 / 15 / 08$ & 8L15001 & PNNL-AGG-415 \\
\hline $14191-84-5$ & Copper 63 & $9.80 \mathrm{E} 0$ & $\mathrm{ug} / \mathrm{g}$ dry & $6.68 \mathrm{E}-1$ & $12 / 15 / 08$ & $8 \mathrm{~L} 15001$ & PNNL-AGG-415 \\
\hline $14119-06-3$ & Copper 65 & $1.02 \mathrm{E} 1$ & ug/g dry & $5.96 \mathrm{E}-1$ & $12 / 15 / 08$ & 8L15001 & PNNL-AGG-415 \\
\hline $7440-38-2$ & Arsenic 75 & $7.48 \mathrm{E}-1$ & ug/g dry & $4.15 \mathrm{E}-1$ & $12 / 15 / 08$ & 8L15001 & PNNL-AGG-415 \\
\hline $14687-58-2$ & Selenium 82 & $<1.14 \mathrm{E} 0$ & ug/g dry & $1.14 \mathrm{E} 0$ & $12 / 15 / 08$ & 8L15001 & PNNL-AGG-415 \\
\hline $14392-17-7$ & Molybdenum 95 & $<2.33 \mathrm{E}-1$ & ug/g dry & $2.33 \mathrm{E}-1$ & $12 / 15 / 08$ & 8L15001 & PNNL-AGG-415 \\
\hline $14392-19-9$ & Molybdenum 97 & $<2.13 \mathrm{E}-1$ & ug/g dry & $2.13 \mathrm{E}-1$ & $12 / 15 / 08$ & 8L15001 & PNNL-AGG-415 \\
\hline $14392-20-2$ & Molybdenum 98 & $1.99 \mathrm{E}-1$ & ug/g dry & $8.83 \mathrm{E}-2$ & $12 / 15 / 08$ & 8L15001 & PNNL-AGG-415 \\
\hline $14914-61-5$ & Ruthenium 101 & $<4.47 \mathrm{E}-2$ & ug/g dry & $4.47 \mathrm{E}-2$ & $12 / 15 / 08$ & 8L15001 & PNNL-AGG-415 \\
\hline $14914-62-6$ & Ruthenium 102 & $<2.70 \mathrm{E}-2$ & $\mathrm{ug} / \mathrm{g}$ dry & $2.70 \mathrm{E}-2$ & $12 / 15 / 08$ & $8 \mathrm{~L} 15001$ & PNNL-AGG-415 \\
\hline $15766-01-5$ & Ruthenium 104 & $<7.55 \mathrm{E}-2$ & ug/g dry & $7.55 \mathrm{E}-2$ & $12 / 15 / 08$ & 8L15001 & PNNL-AGG-415 \\
\hline $14378-37-1$ & Silver 107 & $<6.89 \mathrm{E}-2$ & ug/g dry & $6.89 \mathrm{E}-2$ & $12 / 15 / 08$ & 8L15001 & PNNL-AGG-415 \\
\hline $14378-38-2$ & Silver 109 & $<4.50 \mathrm{E}-2$ & ug/g dry & $4.50 \mathrm{E}-2$ & $12 / 15 / 08$ & $8 \mathrm{~L} 15001$ & PNNL-AGG-415 \\
\hline $14336-64-2$ & Cadmium 111 & $<4.88 \mathrm{E}-2$ & ug/g dry & $4.88 \mathrm{E}-2$ & $12 / 15 / 08$ & 8L15001 & PNNL-AGG-415 \\
\hline $14041-58-8$ & Cadmium 114 & $1.31 \mathrm{E}-1$ & ug/g dry & $1.11 \mathrm{E}-2$ & $12 / 15 / 08$ & 8L15001 & PNNL-AGG-415 \\
\hline $14265-72-6$ & Antimony 121 & $<7.89 \mathrm{E}-2$ & ug/g dry & $7.89 \mathrm{E}-2$ & $12 / 15 / 08$ & 8L15001 & PNNL-AGG-415 \\
\hline $13966-27-3$ & Lead 206 & $2.24 \mathrm{E} 0$ & ug/g dry & $7.76 \mathrm{E}-2$ & $12 / 15 / 08$ & $8 \mathrm{~L} 15001$ & PNNL-AGG-415 \\
\hline $13966-28-4$ & Lead 208 & $2.25 \mathrm{E} 0$ & $\mathrm{ug} / \mathrm{g}$ dry & $3.67 \mathrm{E}-2$ & $12 / 15 / 08$ & $8 \mathrm{~L} 15001$ & PNNL-AGG-415 \\
\hline HEIS No. & B1V9B1 & \multicolumn{3}{|c|}{ Lab ID: $\quad$ 0805003-11 } & & & \\
\hline $14092-98-9$ & Chromium 52 & \multicolumn{3}{|c|}{$4.98 \mathrm{E} 0 \quad u g / g$ dry $1.93 \mathrm{E}-1$} & $12 / 15 / 08$ & 8L15001 & PNNL-AGG-415 \\
\hline $14191-84-5$ & Copper 63 & 8.62E0 & ug/g dry & $6.81 \mathrm{E}-1$ & $12 / 15 / 08$ & 8L15001 & PNNL-AGG-415 \\
\hline 14119-06-3 & Copper 65 & 8.98E0 & $\mathrm{ug} / \mathrm{g}$ dry & $6.07 \mathrm{E}-1$ & $12 / 15 / 08$ & $8 \mathrm{~L} 15001$ & PNNL-AGG-415 \\
\hline $7440-38-2$ & Arsenic 75 & $9.85 \mathrm{E}-1$ & $\mathrm{ug} / \mathrm{g}$ dry & $4.23 \mathrm{E}-1$ & $12 / 15 / 08$ & $8 \mathrm{~L} 15001$ & PNNL-AGG-415 \\
\hline $14687-58-2$ & Selenium 82 & $<1.16 \mathrm{E} 0$ & ug/g dry & $1.16 \mathrm{E} 0$ & $12 / 15 / 08$ & 8L15001 & PNNL-AGG-415 \\
\hline $14392-17-7$ & Molybdenum 95 & $<2.37 \mathrm{E}-1$ & $\mathrm{ug} / \mathrm{g}$ dry & $2.37 \mathrm{E}-1$ & $12 / 15 / 08$ & $8 \mathrm{~L} 15001$ & PNNL-AGG-415 \\
\hline 14392-19-9 & Molybdenum 97 & $<2.17 \mathrm{E}-1$ & ug/g dry & $2.17 \mathrm{E}-1$ & $12 / 15 / 08$ & 8L15001 & PNNL-AGG-415 \\
\hline $14392-20-2$ & Molybdenum 98 & $1.62 \mathrm{E}-1$ & ug/g dry & $8.99 \mathrm{E}-2$ & $12 / 15 / 08$ & 8L15001 & PNNL-AGG-415 \\
\hline $14914-61-5$ & Ruthenium 101 & $<4.55 \mathrm{E}-2$ & ug/g dry & $4.55 \mathrm{E}-2$ & $12 / 15 / 08$ & 8L15001 & PNNL-AGG-415 \\
\hline $14914-62-6$ & Ruthenium 102 & $<2.75 \mathrm{E}-2$ & ug/g dry & $2.75 \mathrm{E}-2$ & $12 / 15 / 08$ & $8 \mathrm{~L} 15001$ & PNNL-AGG-415 \\
\hline $15766-01-5$ & Ruthenium 104 & $<7.69 \mathrm{E}-2$ & ug/g dry & $7.69 \mathrm{E}-2$ & $12 / 15 / 08$ & 8L15001 & PNNL-AGG-415 \\
\hline $14378-37-1$ & Silver 107 & $<7.02 \mathrm{E}-2$ & ug/g dry & $7.02 \mathrm{E}-2$ & $12 / 15 / 08$ & 8L15001 & PNNL-AGG-415 \\
\hline $14378-38-2$ & Silver 109 & $<4.58 \mathrm{E}-2$ & ug/g dry & $4.58 \mathrm{E}-2$ & $12 / 15 / 08$ & 8L15001 & PNNL-AGG-415 \\
\hline $14336-64-2$ & Cadmium 111 & $<4.97 \mathrm{E}-2$ & ug/g dry & $4.97 \mathrm{E}-2$ & $12 / 15 / 08$ & $8 \mathrm{~L} 15001$ & PNNL-AGG-415 \\
\hline $14041-58-8$ & Cadmium 114 & $9.98 \mathrm{E}-2$ & ug/g dry & $1.13 \mathrm{E}-2$ & $12 / 15 / 08$ & 8L15001 & PNNL-AGG-415 \\
\hline $14265-72-6$ & Antimony 121 & $<8.04 \mathrm{E}-2$ & ug/g dry & $8.04 \mathrm{E}-2$ & $12 / 15 / 08$ & 8L15001 & PNNL-AGG-415 \\
\hline $13966-27-3$ & Lead 206 & $2.66 \mathrm{E} 0$ & ug/g dry & $7.90 \mathrm{E}-2$ & $12 / 15 / 08$ & $8 \mathrm{~L} 15001$ & PNNL-AGG-415 \\
\hline $13966-28-4$ & Lead 208 & $2.55 \mathrm{E} 0$ & ug/g dry & $3.74 \mathrm{E}-2$ & $12 / 15 / 08$ & 8L15001 & PNNL-AGG-415 \\
\hline HEIS No. & B1V9B3 & \multicolumn{3}{|c|}{ Lab ID: $\quad 0805003-13$} & & & \\
\hline $14092-98-9$ & Chromium 52 & $5.98 \mathrm{E} 0$ & ug/g dry & $1.88 \mathrm{E}-1$ & $12 / 15 / 08$ & 8L15001 & PNNL-AGG-415 \\
\hline
\end{tabular}


RCRA Metals By PNNL-AGG-415/Acid Extract

\begin{tabular}{|c|c|c|c|c|c|c|c|}
\hline CAS \# & Analyte & Results & Units & EQL & Analyzed & Batch & Method \\
\hline HEIS No. & B1V9B3 & \multicolumn{3}{|c|}{ Lab ID: $\quad$ 0805003-13 } & & & \\
\hline $14191-84-5$ & Copper 63 & $8.36 \mathrm{E} 0$ & ug/g dry & $6.66 \mathrm{E}-1$ & $12 / 15 / 08$ & 8L15001 & PNNL-AGG-415 \\
\hline 14119-06-3 & Copper 65 & $8.50 \mathrm{E} 0$ & ug/g dry & $5.94 \mathrm{E}-1$ & $12 / 15 / 08$ & 8L15001 & PNNL-AGG-415 \\
\hline $7440-38-2$ & Arsenic 75 & $1.08 \mathrm{E} 0$ & ug/g dry & $4.14 \mathrm{E}-1$ & $12 / 15 / 08$ & 8L15001 & PNNL-AGG-415 \\
\hline $14687-58-2$ & Selenium 82 & $<1.13 \mathrm{E} 0$ & ug/g dry & $1.13 \mathrm{E} 0$ & $12 / 15 / 08$ & 8L15001 & PNNL-AGG-415 \\
\hline 14392-17-7 & Molybdenum 95 & $7.69 \mathrm{E}-1$ & ug/g dry & $2.32 \mathrm{E}-1$ & $12 / 15 / 08$ & 8L15001 & PNNL-AGG-415 \\
\hline 14392-19-9 & Molybdenum 97 & $7.55 \mathrm{E}-1$ & ug/g dry & $2.13 \mathrm{E}-1$ & $12 / 15 / 08$ & 8L15001 & PNNL-AGG-415 \\
\hline $14392-20-2$ & Molybdenum 98 & $7.16 \mathrm{E}-1$ & ug/g dry & $8.80 \mathrm{E}-2$ & $12 / 15 / 08$ & $8 \mathrm{~L} 15001$ & PNNL-AGG-415 \\
\hline $14914-61-5$ & Ruthenium 101 & $<4.45 \mathrm{E}-2$ & ug/g dry & $4.45 \mathrm{E}-2$ & $12 / 15 / 08$ & 8L15001 & PNNL-AGG-415 \\
\hline $14914-62-6$ & Ruthenium 102 & $<2.69 \mathrm{E}-2$ & ug/g dry & $2.69 \mathrm{E}-2$ & $12 / 15 / 08$ & $8 \mathrm{~L} 15001$ & PNNL-AGG-415 \\
\hline $15766-01-5$ & Ruthenium 104 & $<7.53 \mathrm{E}-2$ & ug/g dry & $7.53 \mathrm{E}-2$ & $12 / 15 / 08$ & $8 \mathrm{~L} 15001$ & PNNL-AGG-415 \\
\hline $14378-37-1$ & Silver 107 & $<6.87 \mathrm{E}-2$ & ug/g dry & $6.87 \mathrm{E}-2$ & $12 / 15 / 08$ & 8L15001 & PNNL-AGG-415 \\
\hline $14378-38-2$ & Silver 109 & $<4.49 \mathrm{E}-2$ & ug/g dry & $4.49 \mathrm{E}-2$ & $12 / 15 / 08$ & $8 \mathrm{~L} 15001$ & PNNL-AGG-415 \\
\hline $14336-64-2$ & Cadmium 111 & $<4.87 \mathrm{E}-2$ & ug/g dry & 4.87E-2 & $12 / 15 / 08$ & 8L15001 & PNNL-AGG-415 \\
\hline $14041-58-8$ & Cadmium 114 & $5.57 \mathrm{E}-2$ & ug/g dry & $1.10 \mathrm{E}-2$ & $12 / 15 / 08$ & 8L15001 & PNNL-AGG-415 \\
\hline $14265-72-6$ & Antimony 121 & $<7.87 \mathrm{E}-2$ & ug/g dry & $7.87 \mathrm{E}-2$ & $12 / 15 / 08$ & 8L15001 & PNNL-AGG-415 \\
\hline $13966-27-3$ & Lead 206 & $2.22 \mathrm{E} 0$ & ug/g dry & $7.73 \mathrm{E}-2$ & $12 / 15 / 08$ & 8L15001 & PNNL-AGG-415 \\
\hline $13966-28-4$ & Lead 208 & $2.21 \mathrm{E} 0$ & ug/g dry & $3.66 \mathrm{E}-2$ & $12 / 15 / 08$ & 8L15001 & PNNL-AGG-415 \\
\hline HEIS No. & B1V9B5 & \multicolumn{3}{|c|}{ Lab ID: $\quad$ 0805003-15 } & & & \\
\hline $14092-98-9$ & Chromium 52 & $6.64 \mathrm{E} 0$ & ug/g dry & $1.84 \mathrm{E}-1$ & $12 / 15 / 08$ & 8L15001 & PNNL-AGG-415 \\
\hline $14191-84-5$ & Copper 63 & $6.87 \mathrm{E} 0$ & ug/g dry & $6.51 \mathrm{E}-1$ & $12 / 15 / 08$ & 8L15001 & PNNL-AGG-415 \\
\hline $14119-06-3$ & Copper 65 & 7.09E0 & ug/g dry & $5.80 \mathrm{E}-1$ & $12 / 15 / 08$ & 8L15001 & PNNL-AGG-415 \\
\hline $7440-38-2$ & Arsenic 75 & $1.58 \mathrm{E} 0$ & ug/g dry & $4.05 \mathrm{E}-1$ & $12 / 15 / 08$ & 8L15001 & PNNL-AGG-415 \\
\hline $14687-58-2$ & Selenium 82 & $<1.11 \mathrm{E} 0$ & ug/g dry & $1.11 \mathrm{E} 0$ & $12 / 15 / 08$ & 8L15001 & PNNL-AGG-415 \\
\hline $14392-17-7$ & Molybdenum 95 & $<2.27 \mathrm{E}-1$ & ug/g dry & $2.27 \mathrm{E}-1$ & $12 / 15 / 08$ & $8 \mathrm{~L} 15001$ & PNNL-AGG-415 \\
\hline 14392-19-9 & Molybdenum 97 & $<2.08 \mathrm{E}-1$ & ug/g dry & $2.08 \mathrm{E}-1$ & $12 / 15 / 08$ & 8L15001 & PNNL-AGG-415 \\
\hline $14392-20-2$ & Molybdenum 98 & $1.52 \mathrm{E}-1$ & ug/g dry & $8.60 \mathrm{E}-2$ & $12 / 15 / 08$ & 8L15001 & PNNL-AGG-415 \\
\hline $14914-61-5$ & Ruthenium 101 & $<4.35 \mathrm{E}-2$ & ug/g dry & $4.35 \mathrm{E}-2$ & $12 / 15 / 08$ & $8 \mathrm{~L} 15001$ & PNNL-AGG-415 \\
\hline $14914-62-6$ & Ruthenium 102 & $<2.63 \mathrm{E}-2$ & ug/g dry & $2.63 \mathrm{E}-2$ & $12 / 15 / 08$ & $8 \mathrm{~L} 15001$ & PNNL-AGG-415 \\
\hline $15766-01-5$ & Ruthenium 104 & $<7.35 \mathrm{E}-2$ & ug/g dry & $7.35 \mathrm{E}-2$ & $12 / 15 / 08$ & 8L15001 & PNNL-AGG-415 \\
\hline $14378-37-1$ & Silver 107 & $<6.71 \mathrm{E}-2$ & ug/g dry & $6.71 \mathrm{E}-2$ & $12 / 15 / 08$ & $8 \mathrm{~L} 15001$ & PNNL-AGG-415 \\
\hline $14378-38-2$ & Silver 109 & $<4.38 \mathrm{E}-2$ & ug/g dry & $4.38 \mathrm{E}-2$ & $12 / 15 / 08$ & 8L15001 & PNNL-AGG-415 \\
\hline $14336-64-2$ & Cadmium 111 & $<4.76 \mathrm{E}-2$ & ug/g dry & $4.76 \mathrm{E}-2$ & $12 / 15 / 08$ & 8L15001 & PNNL-AGG-415 \\
\hline $14041-58-8$ & Cadmium 114 & $5.78 \mathrm{E}-2$ & ug/g dry & $1.08 \mathrm{E}-2$ & $12 / 15 / 08$ & $8 \mathrm{~L} 15001$ & PNNL-AGG-415 \\
\hline $14265-72-6$ & Antimony 121 & $<7.69 \mathrm{E}-2$ & ug/g dry & $7.69 \mathrm{E}-2$ & $12 / 15 / 08$ & 8L15001 & PNNL-AGG-415 \\
\hline $13966-27-3$ & Lead 206 & $2.43 \mathrm{E} 0$ & ug/g dry & $7.56 \mathrm{E}-2$ & $12 / 15 / 08$ & 8L15001 & PNNL-AGG-415 \\
\hline $13966-28-4$ & Lead 208 & $2.38 \mathrm{E} 0$ & ug/g dry & $3.58 \mathrm{E}-2$ & $12 / 15 / 08$ & $8 \mathrm{~L} 15001$ & PNNL-AGG-415 \\
\hline HEIS No. & B1V9B7 & \multicolumn{3}{|c|}{ Lab ID: $\quad$ 0805003-17 } & & & \\
\hline $14092-98-9$ & Chromium 52 & 7.87E0 & ug/g dry & $1.82 \mathrm{E}-1$ & $12 / 15 / 08$ & $8 \mathrm{~L} 15001$ & PNNL-AGG-415 \\
\hline $14191-84-5$ & Copper 63 & $8.18 \mathrm{E} 0$ & ug/g dry & $6.44 \mathrm{E}-1$ & $12 / 15 / 08$ & $8 \mathrm{~L} 15001$ & PNNL-AGG-415 \\
\hline 14119-06-3 & Copper 65 & $8.25 \mathrm{E} 0$ & ug/g dry & $5.74 \mathrm{E}-1$ & $12 / 15 / 08$ & $8 \mathrm{~L} 15001$ & PNNL-AGG-415 \\
\hline $7440-38-2$ & Arsenic 75 & $2.14 \mathrm{E} 0$ & ug/g dry & $4.01 \mathrm{E}-1$ & $12 / 15 / 08$ & 8L15001 & PNNL-AGG-415 \\
\hline $14687-58-2$ & Selenium 82 & $<1.09 \mathrm{E} 0$ & $\mathrm{ug} / \mathrm{g}$ dry & $1.09 \mathrm{E} 0$ & $12 / 15 / 08$ & $8 \mathrm{~L} 15001$ & PNNL-AGG-415 \\
\hline $14392-17-7$ & Molybdenum 95 & $<2.24 \mathrm{E}-1$ & ug/g dry & $2.24 \mathrm{E}-1$ & $12 / 15 / 08$ & 8L15001 & PNNL-AGG-415 \\
\hline $14392-19-9$ & Molybdenum 97 & $2.13 \mathrm{E}-1$ & ug/g dry & $2.06 \mathrm{E}-1$ & $12 / 15 / 08$ & $8 \mathrm{~L} 15001$ & PNNL-AGG-415 \\
\hline $14392-20-2$ & Molybdenum 98 & $2.15 \mathrm{E}-1$ & ug/g dry & $8.51 \mathrm{E}-2$ & $12 / 15 / 08$ & $8 \mathrm{~L} 15001$ & PNNL-AGG-415 \\
\hline $14914-61-5$ & Ruthenium 101 & $<4.31 \mathrm{E}-2$ & ug/g dry & $4.31 \mathrm{E}-2$ & $12 / 15 / 08$ & 8L15001 & PNNL-AGG-415 \\
\hline $14914-62-6$ & Ruthenium 102 & $<2.60 \mathrm{E}-2$ & ug/g dry & $2.60 \mathrm{E}-2$ & $12 / 15 / 08$ & $8 \mathrm{~L} 15001$ & PNNL-AGG-415 \\
\hline $15766-01-5$ & Ruthenium 104 & $<7.28 \mathrm{E}-2$ & ug/g dry & $7.28 \mathrm{E}-2$ & $12 / 15 / 08$ & 8L15001 & PNNL-AGG-415 \\
\hline
\end{tabular}


RCRA Metals By PNNL-AGG-415/Acid Extract

\begin{tabular}{|c|c|c|c|c|c|c|c|}
\hline CAS \# & Analyte & Results & Units & EQL & Analyzed & Batch & Method \\
\hline HEIS No. & B1V9B7 & \multicolumn{3}{|c|}{ Lab ID: $\quad$ 0805003-17 } & & & \\
\hline $14378-37-1$ & Silver 107 & $<6.64 \mathrm{E}-2$ & ug/g dry & $6.64 \mathrm{E}-2$ & $12 / 15 / 08$ & 8L15001 & PNNL-AGG-415 \\
\hline $14378-38-2$ & Silver 109 & $<4.34 \mathrm{E}-2$ & ug/g dry & $4.34 \mathrm{E}-2$ & $12 / 15 / 08$ & 8L15001 & PNNL-AGG-415 \\
\hline $14336-64-2$ & Cadmium 111 & $<4.71 \mathrm{E}-2$ & ug/g dry & $4.71 \mathrm{E}-2$ & $12 / 15 / 08$ & 8L15001 & PNNL-AGG-415 \\
\hline $14041-58-8$ & Cadmium 114 & $5.84 \mathrm{E}-2$ & ug/g dry & $1.07 \mathrm{E}-2$ & $12 / 15 / 08$ & 8L15001 & PNNL-AGG-415 \\
\hline $14265-72-6$ & Antimony 121 & $<7.61 \mathrm{E}-2$ & ug/g dry & $7.61 \mathrm{E}-2$ & $12 / 15 / 08$ & 8L15001 & PNNL-AGG-415 \\
\hline $13966-27-3$ & Lead 206 & 3.27E0 & ug/g dry & $7.48 \mathrm{E}-2$ & $12 / 15 / 08$ & 8L15001 & PNNL-AGG-415 \\
\hline $13966-28-4$ & Lead 208 & $3.16 \mathrm{E} 0$ & ug/g dry & $3.54 \mathrm{E}-2$ & $12 / 15 / 08$ & $8 \mathrm{~L} 15001$ & PNNL-AGG-415 \\
\hline HEIS No. & B1V9B9 & \multicolumn{3}{|c|}{ Lab ID: $\quad$ 0805003-19 } & & & \\
\hline $14092-98-9$ & Chromium 52 & $7.41 \mathrm{E} 0$ & ug/g dry & $1.86 \mathrm{E}-1$ & $12 / 15 / 08$ & 8L15001 & PNNL-AGG-415 \\
\hline $14191-84-5$ & Copper 63 & 8.31E0 & ug/g dry & $6.57 \mathrm{E}-1$ & $12 / 15 / 08$ & 8L15001 & PNNL-AGG-415 \\
\hline 14119-06-3 & Copper 65 & $8.40 \mathrm{E} 0$ & $\mathrm{ug} / \mathrm{g}$ dry & $5.85 \mathrm{E}-1$ & $12 / 15 / 08$ & $8 \mathrm{~L} 15001$ & PNNL-AGG-415 \\
\hline $7440-38-2$ & Arsenic 75 & $1.89 \mathrm{E} 0$ & ug/g dry & $4.08 \mathrm{E}-1$ & $12 / 15 / 08$ & 8L15001 & PNNL-AGG-415 \\
\hline $14687-58-2$ & Selenium 82 & $<1.12 \mathrm{E} 0$ & ug/g dry & $1.12 \mathrm{E} 0$ & $12 / 15 / 08$ & 8L15001 & PNNL-AGG-415 \\
\hline $14392-17-7$ & Molybdenum 95 & $<2.29 \mathrm{E}-1$ & ug/g dry & $2.29 \mathrm{E}-1$ & $12 / 15 / 08$ & 8L15001 & PNNL-AGG-415 \\
\hline $14392-19-9$ & Molybdenum 97 & $<2.10 \mathrm{E}-1$ & ug/g dry & $2.10 \mathrm{E}-1$ & $12 / 15 / 08$ & $8 \mathrm{~L} 15001$ & PNNL-AGG-415 \\
\hline $14392-20-2$ & Molybdenum 98 & $1.68 \mathrm{E}-1$ & ug/g dry & $8.68 \mathrm{E}-2$ & $12 / 15 / 08$ & 8L15001 & PNNL-AGG-415 \\
\hline $14914-61-5$ & Ruthenium 101 & $<4.39 \mathrm{E}-2$ & ug/g dry & $4.39 \mathrm{E}-2$ & $12 / 15 / 08$ & 8L15001 & PNNL-AGG-415 \\
\hline $14914-62-6$ & Ruthenium 102 & $<2.65 \mathrm{E}-2$ & ug/g dry & $2.65 \mathrm{E}-2$ & $12 / 15 / 08$ & 8L15001 & PNNL-AGG-415 \\
\hline $15766-01-5$ & Ruthenium 104 & $<7.42 \mathrm{E}-2$ & $\mathrm{ug} / \mathrm{g}$ dry & 7.42E-2 & $12 / 15 / 08$ & $8 \mathrm{~L} 15001$ & PNNL-AGG-415 \\
\hline $14378-37-1$ & Silver 107 & $<6.77 \mathrm{E}-2$ & ug/g dry & $6.77 \mathrm{E}-2$ & $12 / 15 / 08$ & $8 \mathrm{~L} 15001$ & PNNL-AGG-415 \\
\hline $14378-38-2$ & Silver 109 & $<4.42 \mathrm{E}-2$ & ug/g dry & $4.42 \mathrm{E}-2$ & $12 / 15 / 08$ & 8L15001 & PNNL-AGG-415 \\
\hline $14336-64-2$ & Cadmium 111 & $<4.80 \mathrm{E}-2$ & ug/g dry & $4.80 \mathrm{E}-2$ & $12 / 15 / 08$ & $8 \mathrm{~L} 15001$ & PNNL-AGG-415 \\
\hline $14041-58-8$ & Cadmium 114 & $4.97 \mathrm{E}-2$ & ug/g dry & $1.09 \mathrm{E}-2$ & $12 / 15 / 08$ & 8L15001 & PNNL-AGG-415 \\
\hline $14265-72-6$ & Antimony 121 & $<7.76 \mathrm{E}-2$ & ug/g dry & $7.76 \mathrm{E}-2$ & $12 / 15 / 08$ & 8L15001 & PNNL-AGG-415 \\
\hline $13966-27-3$ & Lead 206 & $2.68 \mathrm{E} 0$ & ug/g dry & $7.62 \mathrm{E}-2$ & $12 / 15 / 08$ & 8L15001 & PNNL-AGG-415 \\
\hline $13966-28-4$ & Lead 208 & $2.61 \mathrm{E} 0$ & $\mathrm{ug} / \mathrm{g}$ dry & $3.61 \mathrm{E}-2$ & $12 / 15 / 08$ & $8 \mathrm{~L} 15001$ & PNNL-AGG-415 \\
\hline HEIS No. & B1V9C1 & \multicolumn{3}{|c|}{ Lab ID: $\quad$ 0805003-21 } & & & \\
\hline $14092-98-9$ & Chromium 52 & $7.58 \mathrm{E} 0$ & ug/g dry & $1.84 \mathrm{E}-1$ & $12 / 15 / 08$ & 8L15001 & PNNL-AGG-415 \\
\hline $14191-84-5$ & Copper 63 & $6.68 \mathrm{E} 0$ & ug/g dry & $6.49 \mathrm{E}-1$ & $12 / 15 / 08$ & 8L15001 & PNNL-AGG-415 \\
\hline $14119-06-3$ & Copper 65 & $6.80 \mathrm{E} 0$ & ug/g dry & $5.79 \mathrm{E}-1$ & $12 / 15 / 08$ & 8L15001 & PNNL-AGG-415 \\
\hline $7440-38-2$ & Arsenic 75 & $1.47 \mathrm{E} 0$ & $\mathrm{ug} / \mathrm{g}$ dry & $4.04 \mathrm{E}-1$ & $12 / 15 / 08$ & $8 \mathrm{~L} 15001$ & PNNL-AGG-415 \\
\hline $14687-58-2$ & Selenium 82 & $<1.10 \mathrm{E} 0$ & $\mathrm{ug} / \mathrm{g}$ dry & $1.10 \mathrm{E} 0$ & $12 / 15 / 08$ & $8 \mathrm{~L} 15001$ & PNNL-AGG-415 \\
\hline $14392-17-7$ & Molybdenum 95 & $<2.26 \mathrm{E}-1$ & ug/g dry & $2.26 \mathrm{E}-1$ & $12 / 15 / 08$ & 8L15001 & PNNL-AGG-415 \\
\hline $14392-19-9$ & Molybdenum 97 & $<2.07 \mathrm{E}-1$ & $\mathrm{ug} / \mathrm{g}$ dry & $2.07 \mathrm{E}-1$ & $12 / 15 / 08$ & $8 \mathrm{~L} 15001$ & PNNL-AGG-415 \\
\hline $14392-20-2$ & Molybdenum 98 & $1.36 \mathrm{E}-1$ & ug/g dry & $8.58 \mathrm{E}-2$ & $12 / 15 / 08$ & 8L15001 & PNNL-AGG-415 \\
\hline $14914-61-5$ & Ruthenium 101 & $<4.34 \mathrm{E}-2$ & ug/g dry & $4.34 \mathrm{E}-2$ & $12 / 15 / 08$ & 8L15001 & PNNL-AGG-415 \\
\hline $14914-62-6$ & Ruthenium 102 & $<2.62 \mathrm{E}-2$ & ug/g dry & $2.62 \mathrm{E}-2$ & $12 / 15 / 08$ & 8L15001 & PNNL-AGG-415 \\
\hline $15766-01-5$ & Ruthenium 104 & $<7.33 \mathrm{E}-2$ & $\mathrm{ug} / \mathrm{g}$ dry & 7.33E-2 & $12 / 15 / 08$ & $8 \mathrm{~L} 15001$ & PNNL-AGG-415 \\
\hline $14378-37-1$ & Silver 107 & $<6.69 \mathrm{E}-2$ & $\mathrm{ug} / \mathrm{g}$ dry & $6.69 \mathrm{E}-2$ & $12 / 15 / 08$ & 8L15001 & PNNL-AGG-415 \\
\hline $14378-38-2$ & Silver 109 & $<4.37 \mathrm{E}-2$ & ug/g dry & $4.37 \mathrm{E}-2$ & $12 / 15 / 08$ & 8L15001 & PNNL-AGG-415 \\
\hline $14336-64-2$ & Cadmium 111 & $<4.74 \mathrm{E}-2$ & ug/g dry & $4.74 \mathrm{E}-2$ & $12 / 15 / 08$ & 8L15001 & PNNL-AGG-415 \\
\hline $14041-58-8$ & Cadmium 114 & 4.93E-2 & $\mathrm{ug} / \mathrm{g}$ dry & $1.08 \mathrm{E}-2$ & $12 / 15 / 08$ & $8 \mathrm{~L} 15001$ & PNNL-AGG-415 \\
\hline $14265-72-6$ & Antimony 121 & $<7.67 \mathrm{E}-2$ & ug/g dry & $7.67 \mathrm{E}-2$ & $12 / 15 / 08$ & 8L15001 & PNNL-AGG-415 \\
\hline $13966-27-3$ & Lead 206 & $2.25 \mathrm{E} 0$ & ug/g dry & $7.54 \mathrm{E}-2$ & $12 / 15 / 08$ & 8L15001 & PNNL-AGG-415 \\
\hline $13966-28-4$ & Lead 208 & $2.18 \mathrm{E} 0$ & $\mathrm{ug} / \mathrm{g}$ dry & $3.57 \mathrm{E}-2$ & $12 / 15 / 08$ & $8 \mathrm{~L} 15001$ & PNNL-AGG-415 \\
\hline HEIS No. & B1V9C3 & \multicolumn{3}{|c|}{ Lab ID: $\quad$ 0805003-23 } & & & \\
\hline 14092-98-9 & Chromium 52 & 7.19E0 & ug/g dry & $1.87 \mathrm{E}-1$ & $12 / 15 / 08$ & $8 \mathrm{~L} 15001$ & PNNL-AGG-415 \\
\hline $14191-84-5$ & Copper 63 & $6.73 \mathrm{E} 0$ & ug/g dry & $6.63 \mathrm{E}-1$ & $12 / 15 / 08$ & 8L15001 & PNNL-AGG-415 \\
\hline
\end{tabular}


RCRA Metals By PNNL-AGG-415/Acid Extract

\begin{tabular}{|c|c|c|c|c|c|c|c|}
\hline CAS \# & Analyte & Results & Units & EQL & Analyzed & Batch & Method \\
\hline HEIS No. & B1V9C3 & \multicolumn{3}{|c|}{ Lab ID: $\quad$ 0805003-23 } & & & \\
\hline 14119-06-3 & Copper 65 & $6.89 \mathrm{E} 0$ & $u g / g$ dry & $5.90 \mathrm{E}-1$ & $12 / 15 / 08$ & 8L15001 & PNNL-AGG-415 \\
\hline $7440-38-2$ & Arsenic 75 & $1.31 \mathrm{E} 0$ & ug/g dry & $4.12 \mathrm{E}-1$ & $12 / 15 / 08$ & 8L15001 & PNNL-AGG-415 \\
\hline $14687-58-2$ & Selenium 82 & $<1.13 \mathrm{E} 0$ & ug/g dry & $1.13 \mathrm{E} 0$ & $12 / 15 / 08$ & 8L15001 & PNNL-AGG-415 \\
\hline $14392-17-7$ & Molybdenum 95 & $<2.31 \mathrm{E}-1$ & $\mathrm{ug} / \mathrm{g}$ dry & $2.31 \mathrm{E}-1$ & $12 / 15 / 08$ & 8L15001 & PNNL-AGG-415 \\
\hline $14392-19-9$ & Molybdenum 97 & $<2.11 \mathrm{E}-1$ & ug/g dry & $2.11 \mathrm{E}-1$ & $12 / 15 / 08$ & $8 \mathrm{~L} 15001$ & PNNL-AGG-415 \\
\hline $14392-20-2$ & Molybdenum 98 & $1.22 \mathrm{E}-1$ & ug/g dry & $8.75 \mathrm{E}-2$ & $12 / 15 / 08$ & 8L15001 & PNNL-AGG-415 \\
\hline $14914-61-5$ & Ruthenium 101 & $<4.43 \mathrm{E}-2$ & ug/g dry & $4.43 \mathrm{E}-2$ & $12 / 15 / 08$ & 8L15001 & PNNL-AGG-415 \\
\hline $14914-62-6$ & Ruthenium 102 & $<2.68 \mathrm{E}-2$ & ug/g dry & $2.68 \mathrm{E}-2$ & $12 / 15 / 08$ & $8 \mathrm{~L} 15001$ & PNNL-AGG-415 \\
\hline $15766-01-5$ & Ruthenium 104 & $<7.48 \mathrm{E}-2$ & ug/g dry & $7.48 \mathrm{E}-2$ & $12 / 15 / 08$ & 8L15001 & PNNL-AGG-415 \\
\hline $14378-37-1$ & Silver 107 & $<6.83 \mathrm{E}-2$ & ug/g dry & $6.83 \mathrm{E}-2$ & $12 / 15 / 08$ & $8 \mathrm{~L} 15001$ & PNNL-AGG-415 \\
\hline $14378-38-2$ & Silver 109 & $<4.46 \mathrm{E}-2$ & ug/g dry & $4.46 \mathrm{E}-2$ & $12 / 15 / 08$ & $8 \mathrm{~L} 15001$ & PNNL-AGG-415 \\
\hline $14336-64-2$ & Cadmium 111 & $<4.84 \mathrm{E}-2$ & $\mathrm{ug} / \mathrm{g}$ dry & $4.84 \mathrm{E}-2$ & $12 / 15 / 08$ & $8 \mathrm{~L} 15001$ & PNNL-AGG-415 \\
\hline $14041-58-8$ & Cadmium 114 & $4.30 \mathrm{E}-2$ & ug/g dry & $1.10 \mathrm{E}-2$ & $12 / 15 / 08$ & 8L15001 & PNNL-AGG-415 \\
\hline $14265-72-6$ & Antimony 121 & $<7.83 \mathrm{E}-2$ & ug/g dry & $7.83 \mathrm{E}-2$ & $12 / 15 / 08$ & 8L15001 & PNNL-AGG-415 \\
\hline $13966-27-3$ & Lead 206 & $2.45 \mathrm{E} 0$ & ug/g dry & $7.69 \mathrm{E}-2$ & $12 / 15 / 08$ & 8L15001 & PNNL-AGG-415 \\
\hline $13966-28-4$ & Lead 208 & 2.29E0 & ug/g dry & $3.64 \mathrm{E}-2$ & $12 / 15 / 08$ & $8 \mathrm{~L} 15001$ & PNNL-AGG-415 \\
\hline HEIS No. & B1V2V5 & \multicolumn{3}{|c|}{ Lab ID: $\quad$ 0805003-25 } & & & \\
\hline 14092-98-9 & Chromium 52 & 7.12E0 & ug/g dry & $1.85 \mathrm{E}-1$ & $12 / 15 / 08$ & 8L15001 & PNNL-AGG-415 \\
\hline $14191-84-5$ & Copper 63 & $8.51 \mathrm{E} 0$ & ug/g dry & $6.53 \mathrm{E}-1$ & $12 / 15 / 08$ & $8 \mathrm{~L} 15001$ & PNNL-AGG-415 \\
\hline $14119-06-3$ & Copper 65 & $8.60 \mathrm{E} 0$ & ug/g dry & $5.82 \mathrm{E}-1$ & $12 / 15 / 08$ & $8 \mathrm{~L} 15001$ & PNNL-AGG-415 \\
\hline $7440-38-2$ & Arsenic 75 & $1.49 \mathrm{E} 0$ & ug/g dry & 4.06E-1 & $12 / 15 / 08$ & 8L15001 & PNNL-AGG-415 \\
\hline $14687-58-2$ & Selenium 82 & $<1.11 \mathrm{E} 0$ & ug/g dry & $1.11 \mathrm{E} 0$ & $12 / 15 / 08$ & 8L15001 & PNNL-AGG-415 \\
\hline $14392-17-7$ & Molybdenum 95 & $<2.27 \mathrm{E}-1$ & ug/g dry & $2.27 \mathrm{E}-1$ & $12 / 15 / 08$ & 8L15001 & PNNL-AGG-415 \\
\hline 14392-19-9 & Molybdenum 97 & $<2.08 \mathrm{E}-1$ & ug/g dry & $2.08 \mathrm{E}-1$ & $12 / 15 / 08$ & 8L15001 & PNNL-AGG-415 \\
\hline $14392-20-2$ & Molybdenum 98 & $1.89 \mathrm{E}-1$ & ug/g dry & $8.63 \mathrm{E}-2$ & $12 / 15 / 08$ & 8L15001 & PNNL-AGG-415 \\
\hline $14914-61-5$ & Ruthenium 101 & $<4.37 \mathrm{E}-2$ & ug/g dry & $4.37 \mathrm{E}-2$ & $12 / 15 / 08$ & 8L15001 & PNNL-AGG-415 \\
\hline $14914-62-6$ & Ruthenium 102 & $<2.64 \mathrm{E}-2$ & ug/g dry & $2.64 \mathrm{E}-2$ & $12 / 15 / 08$ & $8 \mathrm{~L} 15001$ & PNNL-AGG-415 \\
\hline $15766-01-5$ & Ruthenium 104 & $<7.38 \mathrm{E}-2$ & ug/g dry & $7.38 \mathrm{E}-2$ & $12 / 15 / 08$ & $8 \mathrm{~L} 15001$ & PNNL-AGG-415 \\
\hline $14378-37-1$ & Silver 107 & $<6.73 \mathrm{E}-2$ & ug/g dry & $6.73 \mathrm{E}-2$ & $12 / 15 / 08$ & $8 \mathrm{~L} 15001$ & PNNL-AGG-415 \\
\hline $14378-38-2$ & Silver 109 & $<4.40 \mathrm{E}-2$ & ug/g dry & $4.40 \mathrm{E}-2$ & $12 / 15 / 08$ & $8 \mathrm{~L} 15001$ & PNNL-AGG-415 \\
\hline $14336-64-2$ & Cadmium 111 & $<4.77 \mathrm{E}-2$ & ug/g dry & 4.77E-2 & $12 / 15 / 08$ & 8L15001 & PNNL-AGG-415 \\
\hline $14041-58-8$ & Cadmium 114 & $4.79 \mathrm{E}-2$ & ug/g dry & $1.08 \mathrm{E}-2$ & $12 / 15 / 08$ & 8L15001 & PNNL-AGG-415 \\
\hline $14265-72-6$ & Antimony 121 & $<7.72 \mathrm{E}-2$ & ug/g dry & $7.72 \mathrm{E}-2$ & $12 / 15 / 08$ & $8 \mathrm{~L} 15001$ & PNNL-AGG-415 \\
\hline $13966-27-3$ & Lead 206 & $2.53 \mathrm{E} 0$ & ug/g dry & $7.58 \mathrm{E}-2$ & $12 / 15 / 08$ & 8L15001 & PNNL-AGG-415 \\
\hline $13966-28-4$ & Lead 208 & $2.46 \mathrm{E} 0$ & ug/g dry & $3.59 \mathrm{E}-2$ & $12 / 15 / 08$ & 8L15001 & PNNL-AGG-415 \\
\hline
\end{tabular}




\section{Carbon Analysis/Soil}

\begin{tabular}{|c|c|c|c|c|c|}
\hline $\begin{array}{l}\text { Total Org } \\
\text { Lab ID }\end{array}$ & $\begin{array}{c}\text { c Carbon } \\
\text { HEIS No. }\end{array}$ & $\begin{array}{l}001 \\
\text { Results }\end{array}$ & EQL & Analyzed & Batch \\
\hline 0805003-03 & B1V993 & $5.18 \mathrm{E} 2$ & $2.00 \mathrm{E} 2$ & $1 / 13 / 09$ & {$[\mathrm{CALC}]$} \\
\hline 0805003-05 & B1V995 & $2.89 \mathrm{E} 2$ & $2.00 \mathrm{E} 2$ & $1 / 13 / 09$ & {$[\mathrm{CALC}]$} \\
\hline 0805003-07 & B1V997 & $4.49 \mathrm{E} 2$ & $2.00 \mathrm{E} 2$ & $1 / 13 / 09$ & {$[\mathrm{CALC}]$} \\
\hline 0805003-09 & B1V999 & $<2.00 \mathrm{E} 2$ & $2.00 \mathrm{E} 2$ & $1 / 13 / 09$ & {$[\mathrm{CALC}]$} \\
\hline 0805003-11 & B1V9B1 & $<2.00 \mathrm{E} 2$ & $2.00 \mathrm{E} 2$ & $1 / 13 / 09$ & {$[\mathrm{CALC}]$} \\
\hline 0805003-13 & B1V9B3 & $<2.00 \mathrm{E} 2$ & $2.00 \mathrm{E} 2$ & $1 / 13 / 09$ & {$[\mathrm{CALC}]$} \\
\hline 0805003-15 & B1V9B5 & $<2.00 \mathrm{E} 2$ & $2.00 \mathrm{E} 2$ & $1 / 13 / 09$ & {$[\mathrm{CALC}]$} \\
\hline 0805003-17 & B1V9B7 & $<2.00 \mathrm{E} 2$ & $2.00 \mathrm{E} 2$ & $1 / 13 / 09$ & {$[\mathrm{CALC}]$} \\
\hline 0805003-19 & B1V9B9 & $<2.00 \mathrm{E} 2$ & $2.00 \mathrm{E} 2$ & $1 / 13 / 09$ & {$[\mathrm{CALC}]$} \\
\hline 0805003-21 & $\mathrm{B} 1 \mathrm{~V} 9 \mathrm{C} 1$ & $<2.00 \mathrm{E} 2$ & $2.00 \mathrm{E} 2$ & $1 / 13 / 09$ & {$[\mathrm{CALC}]$} \\
\hline 0805003-23 & B1V9C3 & $<2.00 \mathrm{E} 2$ & $2.00 \mathrm{E} 2$ & $1 / 13 / 09$ & {$[\mathrm{CALC}]$} \\
\hline 0805003-25 & B1V2V5 & $<2.00 \mathrm{E} 2$ & $2.00 \mathrm{E} 2$ & $1 / 13 / 09$ & [CALC] \\
\hline
\end{tabular}




\section{Carbon Analysis/Soil}

\begin{tabular}{|c|c|c|c|c|c|}
\hline $\begin{array}{l}\text { Total Carl } \\
\text { Lab ID }\end{array}$ & $\begin{array}{l}(\mathrm{ug} / \mathrm{g}) \text { by AGG-TOC-001 } \\
\text { HEIS No. }\end{array}$ & Results & EQL & Analyzed & Batch \\
\hline 0805003-03 & B1V993 & $1.39 \mathrm{E} 3$ & $2.00 \mathrm{E} 2$ & $1 / 12 / 09$ & 9A12001 \\
\hline 0805003-05 & B1V995 & $2.00 \mathrm{E} 3$ & $2.00 \mathrm{E} 2$ & $1 / 12 / 09$ & 9A12001 \\
\hline 0805003-07 & B1V997 & $2.17 \mathrm{E} 3$ & $2.00 \mathrm{E} 2$ & $1 / 12 / 09$ & 9A12001 \\
\hline 0805003-09 & B1V999 & $1.20 \mathrm{E} 3$ & $2.00 \mathrm{E} 2$ & $1 / 12 / 09$ & 9A12001 \\
\hline 0805003-11 & B1V9B1 & $1.42 \mathrm{E} 3$ & $2.00 \mathrm{E} 2$ & $1 / 12 / 09$ & 9A12001 \\
\hline 0805003-13 & B1V9B3 & $1.36 \mathrm{E} 3$ & $2.00 \mathrm{E} 2$ & $1 / 12 / 09$ & 9A12001 \\
\hline 0805003-15 & B1V9B5 & $2.43 \mathrm{E} 3$ & $2.00 \mathrm{E} 2$ & $1 / 12 / 09$ & 9A12001 \\
\hline 0805003-17 & B1V9B7 & $2.47 \mathrm{E} 3$ & $2.00 \mathrm{E} 2$ & $1 / 12 / 09$ & 9A12001 \\
\hline 0805003-19 & B1V9B9 & $2.30 \mathrm{E} 3$ & $2.00 \mathrm{E} 2$ & $1 / 12 / 09$ & 9A12001 \\
\hline 0805003-21 & $\mathrm{B} 1 \mathrm{~V} 9 \mathrm{C} 1$ & $2.76 \mathrm{E} 3$ & $2.00 \mathrm{E} 2$ & $1 / 12 / 09$ & 9A12001 \\
\hline 0805003-23 & B1V9C3 & $1.74 \mathrm{E} 3$ & $2.00 \mathrm{E} 2$ & $1 / 12 / 09$ & 9A12001 \\
\hline 0805003-25 & B1V2V5 & $2.03 \mathrm{E} 3$ & $2.00 \mathrm{E} 2$ & $1 / 12 / 09$ & 9A12001 \\
\hline
\end{tabular}




\begin{tabular}{|c|c|c|c|c|c|}
\hline \multicolumn{6}{|c|}{ Carbon Analysis/Soil } \\
\hline \multicolumn{6}{|c|}{ Total Inorganic Carbon (ug/g) by AGG-TOC-001 } \\
\hline Lab ID & HEIS No. & Results & EQL & Analyzed & Batch \\
\hline 0805003-03 & B1V993 & $8.71 \mathrm{E} 2$ & $2.00 \mathrm{E} 2$ & $1 / 13 / 09$ & 9A13003 \\
\hline 0805003-05 & B1V995 & $1.71 \mathrm{E} 3$ & $2.00 \mathrm{E} 2$ & $1 / 13 / 09$ & 9A13003 \\
\hline 0805003-07 & B1V997 & $1.72 \mathrm{E} 3$ & $2.00 \mathrm{E} 2$ & $1 / 13 / 09$ & 9A13003 \\
\hline 0805003-09 & B1V999 & $1.02 \mathrm{E} 3$ & $2.00 \mathrm{E} 2$ & $1 / 13 / 09$ & 9A13003 \\
\hline 0805003-11 & B1V9B1 & $1.68 \mathrm{E} 3$ & $2.00 \mathrm{E} 2$ & $1 / 13 / 09$ & 9A13003 \\
\hline 0805003-13 & B1V9B3 & $1.31 \mathrm{E} 3$ & $2.00 \mathrm{E} 2$ & $1 / 13 / 09$ & $9 A 13003$ \\
\hline 0805003-15 & B1V9B5 & $2.66 \mathrm{E} 3$ & $2.00 \mathrm{E} 2$ & $1 / 13 / 09$ & 9A13003 \\
\hline 0805003-17 & B1V9B7 & $2.71 \mathrm{E} 3$ & $2.00 \mathrm{E} 2$ & $1 / 13 / 09$ & 9A13003 \\
\hline 0805003-19 & B1V9B9 & $2.55 \mathrm{E} 3$ & $2.00 \mathrm{E} 2$ & $1 / 13 / 09$ & 9A13003 \\
\hline 0805003-21 & B1V9C1 & $3.02 \mathrm{E} 3$ & $2.00 \mathrm{E} 2$ & $1 / 13 / 09$ & 9A13003 \\
\hline 0805003-23 & B1V9C3 & $1.80 \mathrm{E} 3$ & $2.00 \mathrm{E} 2$ & $1 / 13 / 09$ & 9A13003 \\
\hline 0805003-25 & B1V2V5 & $2.16 \mathrm{E} 3$ & $2.00 \mathrm{E} 2$ & $1 / 13 / 09$ & 9A13003 \\
\hline
\end{tabular}


GEA/Soil

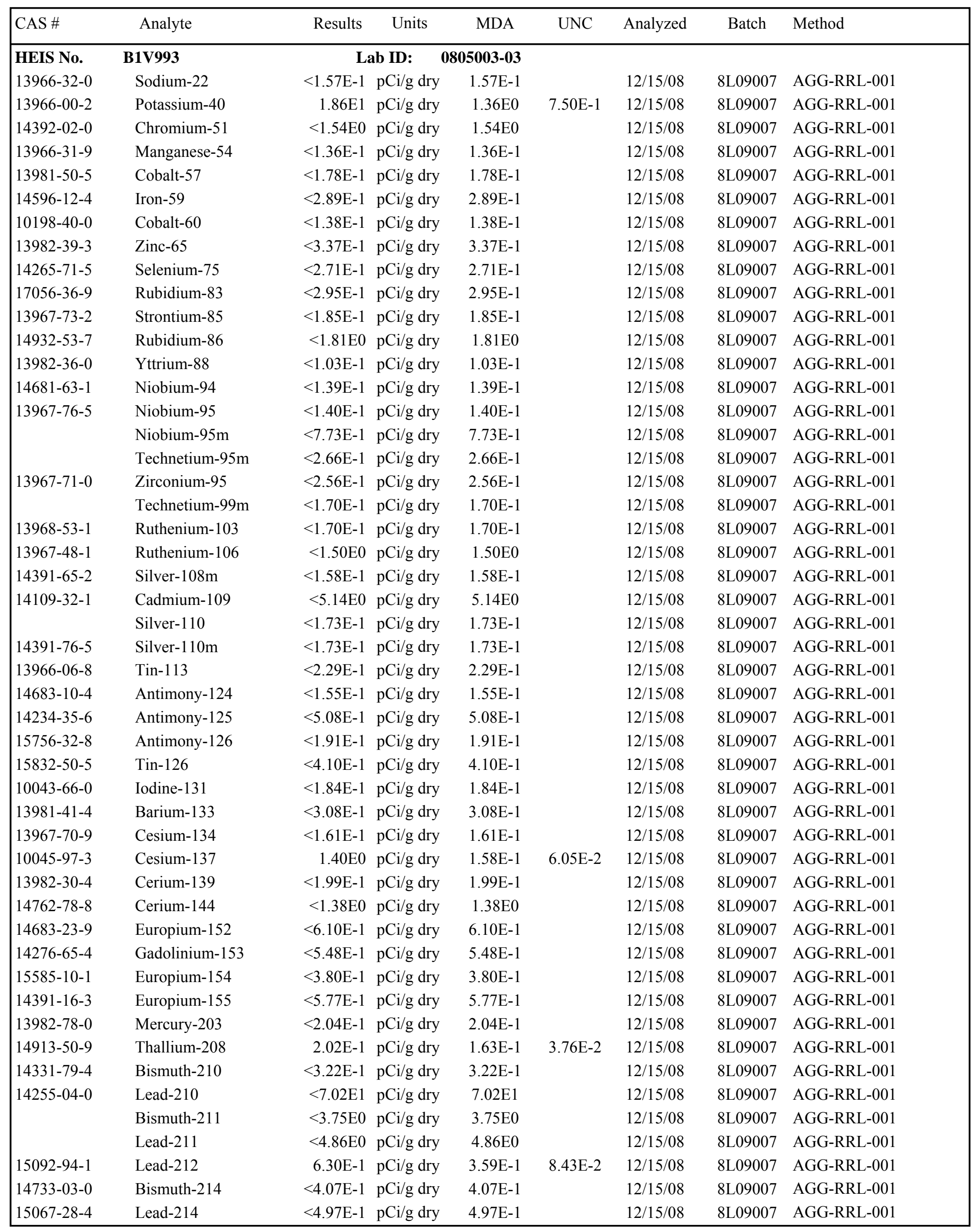


GEA/Soil

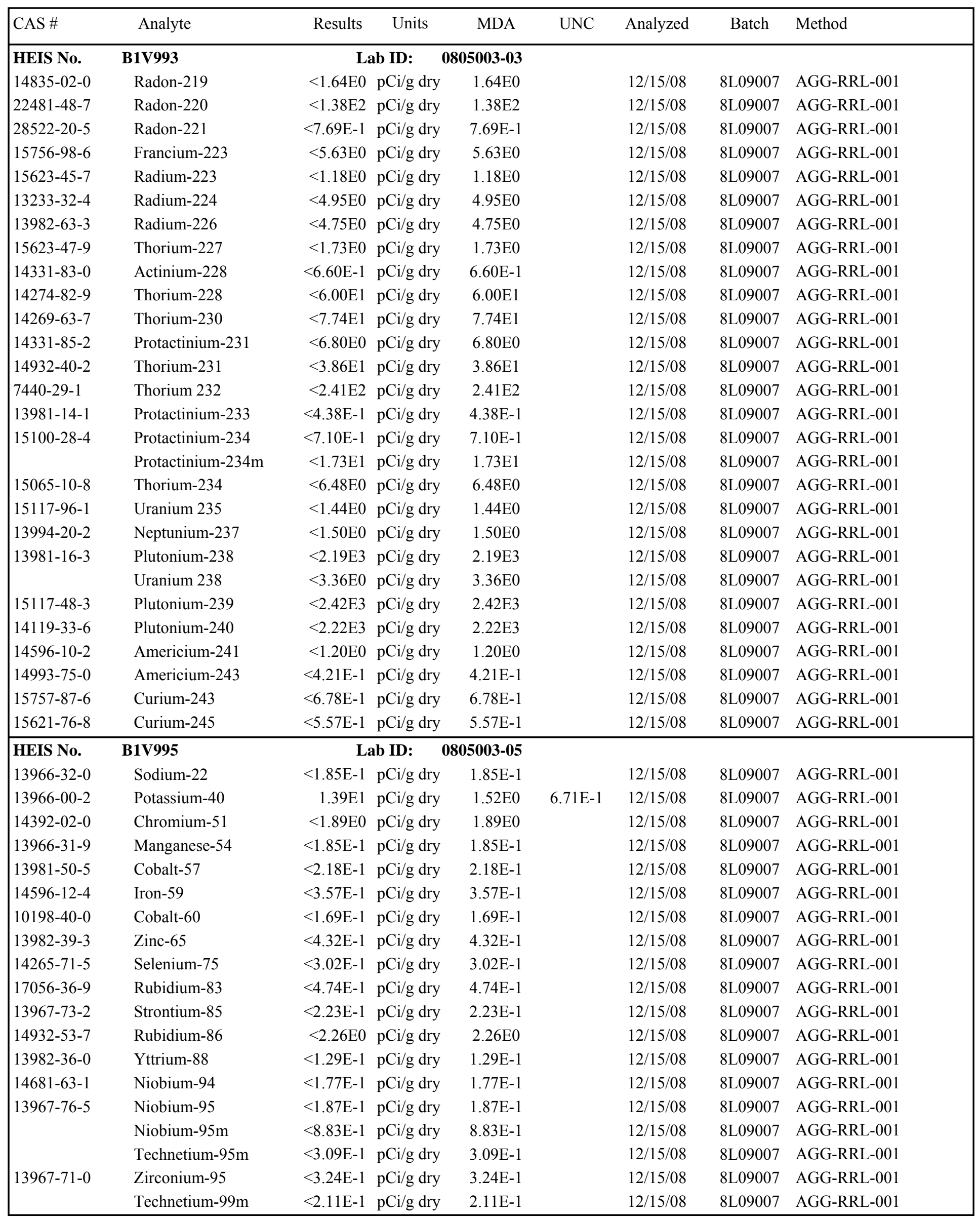


GEA/Soil

\begin{tabular}{|c|c|c|c|c|c|c|c|c|}
\hline CAS \# & Analyte & Results & Units & MDA & UNC & Analyzed & Batch & Method \\
\hline HEIS No. & B1V995 & & ab ID: & $5003-05$ & & & & \\
\hline 13968-53-1 & Ruthenium-103 & $<2.16 \mathrm{E}-1$ & pCi/g dry & $2.16 \mathrm{E}-1$ & & $12 / 15 / 08$ & 8L09007 & AGG-RRL-001 \\
\hline $13967-48-1$ & Ruthenium-106 & $<2.14 \mathrm{E} 0$ & pCi/g dry & $2.14 \mathrm{E} 0$ & & $12 / 15 / 08$ & 8L09007 & AGG-RRL-001 \\
\hline $14391-65-2$ & Silver-108m & $<1.91 \mathrm{E}-1$ & pCi $/ g$ dry & $1.91 \mathrm{E}-1$ & & $12 / 15 / 08$ & 8L09007 & AGG-RRL-001 \\
\hline \multirow[t]{2}{*}{ 14109-32-1 } & Cadmium-109 & $<6.63 \mathrm{E} 0$ & pCi/g dry & $6.63 \mathrm{E} 0$ & & $12 / 15 / 08$ & 8L09007 & AGG-RRL-001 \\
\hline & Silver-110 & $<2.26 \mathrm{E}-1$ & pCi/g dry & $2.26 \mathrm{E}-1$ & & $12 / 15 / 08$ & 8L09007 & AGG-RRL-001 \\
\hline $14391-76-5$ & Silver-110m & $<2.27 \mathrm{E}-1$ & pCi/g dry & $2.27 \mathrm{E}-1$ & & $12 / 15 / 08$ & 8L09007 & AGG-RRL-001 \\
\hline 13966-06-8 & Tin-113 & $<2.81 \mathrm{E}-1$ & pCi/g dry & $2.81 \mathrm{E}-1$ & & $12 / 15 / 08$ & 8L09007 & AGG-RRL-001 \\
\hline 14683-10-4 & Antimony-124 & $<2.30 \mathrm{E}-1$ & pCi/g dry & $2.30 \mathrm{E}-1$ & & $12 / 15 / 08$ & 8L09007 & AGG-RRL-001 \\
\hline $14234-35-6$ & Antimony-125 & $<6.16 \mathrm{E}-1$ & pCi/g dry & $6.16 \mathrm{E}-1$ & & $12 / 15 / 08$ & 8L09007 & AGG-RRL-001 \\
\hline $15756-32-8$ & Antimony-126 & $<2.10 \mathrm{E}-1$ & pCi/g dry & $2.10 \mathrm{E}-1$ & & $12 / 15 / 08$ & 8L09007 & AGG-RRL-001 \\
\hline $15832-50-5$ & Tin-126 & $<5.42 \mathrm{E}-1$ & pCi/g dry & $5.42 \mathrm{E}-1$ & & $12 / 15 / 08$ & 8L09007 & AGG-RRL-001 \\
\hline 10043-66-0 & Iodine-131 & $<2.46 \mathrm{E}-1$ & pCi/g dry & $2.46 \mathrm{E}-1$ & & $12 / 15 / 08$ & 8L09007 & AGG-RRL-001 \\
\hline 13981-41-4 & Barium-133 & $<3.50 \mathrm{E}-1$ & pCi/g dry & $3.50 \mathrm{E}-1$ & & $12 / 15 / 08$ & 8L09007 & AGG-RRL-001 \\
\hline $13967-70-9$ & Cesium-134 & $<2.12 \mathrm{E}-1$ & pCi/g dry & $2.12 \mathrm{E}-1$ & & $12 / 15 / 08$ & 8L09007 & AGG-RRL-001 \\
\hline $10045-97-3$ & Cesium-137 & $<2.49 \mathrm{E}-1$ & pCi/g dry & $2.49 \mathrm{E}-1$ & & $12 / 15 / 08$ & 8L09007 & AGG-RRL-001 \\
\hline 13982-30-4 & Cerium-139 & $<2.40 \mathrm{E}-1$ & pCi/g dry & $2.40 \mathrm{E}-1$ & & $12 / 15 / 08$ & 8L09007 & AGG-RRL-001 \\
\hline $14762-78-8$ & Cerium-144 & $<1.74 \mathrm{E} 0$ & pCi/g dry & $1.74 \mathrm{E} 0$ & & $12 / 15 / 08$ & 8L09007 & AGG-RRL-001 \\
\hline $14683-23-9$ & Europium-152 & $<8.09 \mathrm{E}-1$ & pCi/g dry & $8.09 \mathrm{E}-1$ & & $12 / 15 / 08$ & 8L09007 & AGG-RRL-001 \\
\hline $14276-65-4$ & Gadolinium-153 & $<7.09 \mathrm{E}-1$ & pCi/g dry & $7.09 \mathrm{E}-1$ & & $12 / 15 / 08$ & 8L09007 & AGG-RRL-001 \\
\hline 15585-10-1 & Europium-154 & $<4.64 \mathrm{E}-1$ & pCi/g dry & 4.64E-1 & & $12 / 15 / 08$ & 8L09007 & AGG-RRL-001 \\
\hline $14391-16-3$ & Europium-155 & $<7.69 \mathrm{E}-1$ & pCi/g dry & $7.69 \mathrm{E}-1$ & & $12 / 15 / 08$ & 8L09007 & AGG-RRL-001 \\
\hline 13982-78-0 & Mercury-203 & $<2.42 \mathrm{E}-1$ & pCi/g dry & $2.42 \mathrm{E}-1$ & & $12 / 15 / 08$ & 8L09007 & AGG-RRL-001 \\
\hline $14913-50-9$ & Thallium-208 & $<2.46 \mathrm{E}-1$ & pCi/g dry & $2.46 \mathrm{E}-1$ & & $12 / 15 / 08$ & 8L09007 & AGG-RRL-001 \\
\hline $14331-79-4$ & Bismuth-210 & $<3.60 \mathrm{E}-1$ & pCi/g dry & $3.60 \mathrm{E}-1$ & & $12 / 15 / 08$ & 8L09007 & AGG-RRL-001 \\
\hline \multirow[t]{3}{*}{$14255-04-0$} & Lead-210 & $<4.00 \mathrm{E} 1$ & pCi/g dry & $4.00 \mathrm{E} 1$ & & $12 / 15 / 08$ & 8L09007 & AGG-RRL-001 \\
\hline & Bismuth-211 & $<4.36 \mathrm{E} 0$ & pCi/g dry & $4.36 \mathrm{E} 0$ & & $12 / 15 / 08$ & 8L09007 & AGG-RRL-001 \\
\hline & Lead-211 & $<5.97 \mathrm{E} 0$ & pCi/g dry & $5.97 \mathrm{E} 0$ & & $12 / 15 / 08$ & 8L09007 & AGG-RRL-001 \\
\hline 15092-94-1 & Lead-212 & $<5.13 \mathrm{E}-1$ & pCi/g dry & $5.13 \mathrm{E}-1$ & & $12 / 15 / 08$ & 8L09007 & AGG-RRL-001 \\
\hline $14733-03-0$ & Bismuth-214 & $<4.84 \mathrm{E}-1$ & pCi/g dry & $4.84 \mathrm{E}-1$ & & $12 / 15 / 08$ & 8L09007 & AGG-RRL-001 \\
\hline $15067-28-4$ & Lead-214 & $<5.60 \mathrm{E}-1$ & pCi/g dry & $5.60 \mathrm{E}-1$ & & $12 / 15 / 08$ & 8L09007 & AGG-RRL-001 \\
\hline $14835-02-0$ & Radon-219 & $<1.88 \mathrm{E} 0$ & pCi/g dry & $1.88 \mathrm{E} 0$ & & $12 / 15 / 08$ & 8L09007 & AGG-RRL-001 \\
\hline $22481-48-7$ & Radon-220 & $<1.81 \mathrm{E} 2$ & pCi/g dry & $1.81 \mathrm{E} 2$ & & $12 / 15 / 08$ & 8L09007 & AGG-RRL-001 \\
\hline $28522-20-5$ & Radon-221 & $<9.33 \mathrm{E}-1$ & pCi/g dry & $9.33 \mathrm{E}-1$ & & $12 / 15 / 08$ & 8L09007 & AGG-RRL-001 \\
\hline $15756-98-6$ & Francium-223 & $<6.49 \mathrm{E} 0$ & pCi/g dry & $6.49 \mathrm{E} 0$ & & $12 / 15 / 08$ & 8L09007 & AGG-RRL-001 \\
\hline $15623-45-7$ & Radium-223 & $<1.34 \mathrm{E} 0$ & pCi/g dry & $1.34 \mathrm{E} 0$ & & $12 / 15 / 08$ & 8L09007 & AGG-RRL-001 \\
\hline $13233-32-4$ & Radium-224 & $<5.58 \mathrm{E} 0$ & pCi/g dry & $5.58 \mathrm{E} 0$ & & $12 / 15 / 08$ & 8L09007 & AGG-RRL-001 \\
\hline 13982-63-3 & Radium-226 & $<5.75 \mathrm{E} 0$ & pCi/g dry & $5.75 \mathrm{E} 0$ & & $12 / 15 / 08$ & 8L09007 & AGG-RRL-001 \\
\hline $15623-47-9$ & Thorium-227 & $<1.97 \mathrm{E} 0$ & pCi/g dry & $1.97 \mathrm{E} 0$ & & $12 / 15 / 08$ & 8L09007 & AGG-RRL-001 \\
\hline $14331-83-0$ & Actinium-228 & $<7.97 \mathrm{E}-1$ & pCi/g dry & 7.97E-1 & & $12 / 15 / 08$ & 8L09007 & AGG-RRL-001 \\
\hline $14274-82-9$ & Thorium-228 & $<7.10 \mathrm{E} 1$ & pCi/g dry & $7.10 \mathrm{E} 1$ & & $12 / 15 / 08$ & 8L09007 & AGG-RRL-001 \\
\hline $14269-63-7$ & Thorium-230 & $<9.47 \mathrm{E} 1$ & pCi/g dry & $9.47 \mathrm{E} 1$ & & $12 / 15 / 08$ & 8L09007 & AGG-RRL-001 \\
\hline $14331-85-2$ & Protactinium-231 & $<8.63 \mathrm{E} 0$ & pCi/g dry & $8.63 \mathrm{E} 0$ & & $12 / 15 / 08$ & 8L09007 & AGG-RRL-001 \\
\hline $14932-40-2$ & Thorium-231 & $<4.95 \mathrm{E} 1$ & pCi/g dry & $4.95 \mathrm{E} 1$ & & $12 / 15 / 08$ & 8L09007 & AGG-RRL-001 \\
\hline $7440-29-1$ & Thorium 232 & $<2.50 \mathrm{E} 2$ & pCi/g dry & $2.50 \mathrm{E} 2$ & & $12 / 15 / 08$ & 8L09007 & AGG-RRL-001 \\
\hline 13981-14-1 & Protactinium-233 & $<5.25 \mathrm{E}-1$ & pCi/g dry & $5.25 \mathrm{E}-1$ & & $12 / 15 / 08$ & 8L09007 & AGG-RRL-001 \\
\hline \multirow[t]{2}{*}{$15100-28-4$} & Protactinium-234 & $<9.18 \mathrm{E}-1$ & pCi/g dry & $9.18 \mathrm{E}-1$ & & $12 / 15 / 08$ & 8L09007 & AGG-RRL-001 \\
\hline & Protactinium-234m & $<2.21 \mathrm{E} 1$ & pCi/g dry & $2.21 \mathrm{E} 1$ & & $12 / 15 / 08$ & 8L09007 & AGG-RRL-001 \\
\hline $15065-10-8$ & Thorium-234 & $<8.08 \mathrm{E} 0$ & pCi/g dry & $8.08 \mathrm{E} 0$ & & $12 / 15 / 08$ & 8L09007 & AGG-RRL-001 \\
\hline $15117-96-1$ & Uranium 235 & $<1.77 \mathrm{E} 0$ & pCi/g dry & $1.77 \mathrm{E} 0$ & & $12 / 15 / 08$ & 8L09007 & AGG-RRL-001 \\
\hline
\end{tabular}


GEA/Soil

\begin{tabular}{|c|c|c|c|c|c|c|c|c|}
\hline CAS \# & Analyte & Results & Units & MDA & UNC & Analyzed & Batch & Method \\
\hline HEIS No. & B1V995 & & ab ID: & 5003-05 & & & & \\
\hline 13994-20-2 & Neptunium-237 & $<2.00 \mathrm{E} 0$ & pCi/g dry & $2.00 \mathrm{E} 0$ & & $12 / 15 / 08$ & 8L09007 & AGG-RRL-001 \\
\hline \multirow[t]{2}{*}{ 13981-16-3 } & Plutonium-238 & $<2.88 \mathrm{E} 3$ & pCi/g dry & $2.88 \mathrm{E} 3$ & & $12 / 15 / 08$ & 8L09007 & AGG-RRL-001 \\
\hline & Uranium 238 & $<4.17 \mathrm{E} 0$ & pCi/g dry & $4.17 \mathrm{E} 0$ & & $12 / 15 / 08$ & 8L09007 & AGG-RRL-001 \\
\hline $15117-48-3$ & Plutonium-239 & $<3.02 \mathrm{E} 3$ & pCi $/ g$ dry & $3.02 \mathrm{E} 3$ & & $12 / 15 / 08$ & 8L09007 & AGG-RRL-001 \\
\hline $14119-33-6$ & Plutonium-240 & $<2.84 \mathrm{E} 3$ & pCi/g dry & $2.84 \mathrm{E} 3$ & & $12 / 15 / 08$ & 8L09007 & AGG-RRL-001 \\
\hline $14596-10-2$ & Americium-241 & $<1.28 \mathrm{E} 0$ & pCi/g dry & $1.28 \mathrm{E} 0$ & & $12 / 15 / 08$ & 8L09007 & AGG-RRL-001 \\
\hline 14993-75-0 & Americium-243 & $<5.03 \mathrm{E}-1$ & pCi/g dry & $5.03 \mathrm{E}-1$ & & $12 / 15 / 08$ & 8L09007 & AGG-RRL-001 \\
\hline $15757-87-6$ & Curium-243 & $<8.66 \mathrm{E}-1$ & pCi $/ g$ dry & $8.66 \mathrm{E}-1$ & & $12 / 15 / 08$ & 8L09007 & AGG-RRL-001 \\
\hline $15621-76-8$ & Curium-245 & $<7.11 \mathrm{E}-1$ & pCi/g dry & 7.11E-1 & & $12 / 15 / 08$ & 8L09007 & AGG-RRL-001 \\
\hline HEIS No. & B1V997 & & ab ID: & 5003-07 & & & & \\
\hline 13966-32-0 & Sodium-22 & $<1.98 \mathrm{E}-1$ & pCi/g dry & $1.98 \mathrm{E}-1$ & & $12 / 15 / 08$ & 8L09007 & AGG-RRL-001 \\
\hline $13966-00-2$ & Potassium-40 & $1.64 \mathrm{E} 1$ & pCi $/ g$ dry & $1.39 \mathrm{E} 0$ & 7.73E-1 & $12 / 15 / 08$ & 8L09007 & AGG-RRL-001 \\
\hline 14392-02-0 & Chromium-51 & $<1.86 \mathrm{E} 0$ & pCi/g dry & $1.86 \mathrm{E} 0$ & & $12 / 15 / 08$ & 8L09007 & AGG-RRL-001 \\
\hline 13966-31-9 & Manganese-54 & $<1.93 \mathrm{E}-1$ & pCi/g dry & $1.93 \mathrm{E}-1$ & & $12 / 15 / 08$ & 8L09007 & AGG-RRL-001 \\
\hline $13981-50-5$ & Cobalt-57 & $<2.27 \mathrm{E}-1$ & pCi/g dry & $2.27 \mathrm{E}-1$ & & $12 / 15 / 08$ & 8L09007 & AGG-RRL-001 \\
\hline $14596-12-4$ & Iron-59 & $<3.49 \mathrm{E}-1$ & pCi/g dry & $3.49 \mathrm{E}-1$ & & $12 / 15 / 08$ & 8L09007 & AGG-RRL-001 \\
\hline 10198-40-0 & Cobalt- 60 & $<1.81 \mathrm{E}-1$ & pCi/g dry & $1.81 \mathrm{E}-1$ & & $12 / 15 / 08$ & 8L09007 & AGG-RRL-001 \\
\hline $13982-39-3$ & Zinc-65 & $<4.67 \mathrm{E}-1$ & $\mathrm{pCi} / \mathrm{g}$ dry & $4.67 \mathrm{E}-1$ & & $12 / 15 / 08$ & 8L09007 & AGG-RRL-001 \\
\hline $14265-71-5$ & Selenium-75 & $<3.31 \mathrm{E}-1$ & pCi/g dry & $3.31 \mathrm{E}-1$ & & $12 / 15 / 08$ & 8L09007 & AGG-RRL-001 \\
\hline $17056-36-9$ & Rubidium-83 & $<3.73 \mathrm{E}-1$ & pCi $/ g$ dry & $3.73 \mathrm{E}-1$ & & $12 / 15 / 08$ & 8L09007 & AGG-RRL-001 \\
\hline 13967-73-2 & Strontium-85 & $<2.58 \mathrm{E}-1$ & pCi/g dry & $2.58 \mathrm{E}-1$ & & $12 / 15 / 08$ & 8L09007 & AGG-RRL-001 \\
\hline $14932-53-7$ & Rubidium-86 & $<2.24 \mathrm{E} 0$ & pCi/g dry & $2.24 \mathrm{E} 0$ & & $12 / 15 / 08$ & 8L09007 & AGG-RRL-001 \\
\hline 13982-36-0 & Yttrium-88 & $<1.33 \mathrm{E}-1$ & pCi/g dry & $1.33 \mathrm{E}-1$ & & $12 / 15 / 08$ & 8L09007 & AGG-RRL-001 \\
\hline 14681-63-1 & Niobium-94 & $<1.86 \mathrm{E}-1$ & pCi/g dry & $1.86 \mathrm{E}-1$ & & $12 / 15 / 08$ & 8L09007 & AGG-RRL-001 \\
\hline \multirow[t]{3}{*}{$13967-76-5$} & Niobium-95 & $<1.81 \mathrm{E}-1$ & pCi/g dry & $1.81 \mathrm{E}-1$ & & $12 / 15 / 08$ & 8L09007 & AGG-RRL-001 \\
\hline & Niobium-95m & $<9.98 \mathrm{E}-1$ & $\mathrm{pCi} / \mathrm{g}$ dry & $9.98 \mathrm{E}-1$ & & $12 / 15 / 08$ & 8L09007 & AGG-RRL-001 \\
\hline & Technetium-95m & $<3.36 \mathrm{E}-1$ & pCi/g dry & $3.36 \mathrm{E}-1$ & & $12 / 15 / 08$ & 8L09007 & AGG-RRL-001 \\
\hline \multirow[t]{2}{*}{$13967-71-0$} & Zirconium-95 & $<3.10 \mathrm{E}-1$ & pCi/g dry & $3.10 \mathrm{E}-1$ & & $12 / 15 / 08$ & 8L09007 & AGG-RRL-001 \\
\hline & Technetium-99m & $<2.21 \mathrm{E}-1$ & pCi/g dry & $2.21 \mathrm{E}-1$ & & $12 / 15 / 08$ & 8L09007 & AGG-RRL-001 \\
\hline $13968-53-1$ & Ruthenium-103 & $<1.98 \mathrm{E}-1$ & $\mathrm{pCi} / \mathrm{g}$ dry & $1.98 \mathrm{E}-1$ & & $12 / 15 / 08$ & 8L09007 & AGG-RRL-001 \\
\hline $13967-48-1$ & Ruthenium-106 & $<1.88 \mathrm{E} 0$ & pCi/g dry & $1.88 \mathrm{E} 0$ & & $12 / 15 / 08$ & 8L09007 & AGG-RRL-001 \\
\hline $14391-65-2$ & Silver-108m & $<2.01 \mathrm{E}-1$ & pCi/g dry & $2.01 \mathrm{E}-1$ & & $12 / 15 / 08$ & 8L09007 & AGG-RRL-001 \\
\hline \multirow[t]{2}{*}{$14109-32-1$} & Cadmium-109 & $<6.73 \mathrm{E} 0$ & pCi/g dry & $6.73 \mathrm{E} 0$ & & $12 / 15 / 08$ & 8L09007 & AGG-RRL-001 \\
\hline & Silver-110 & $<1.91 \mathrm{E}-1$ & pCi/g dry & $1.91 \mathrm{E}-1$ & & $12 / 15 / 08$ & 8L09007 & AGG-RRL-001 \\
\hline $14391-76-5$ & Silver-110m & $<1.93 \mathrm{E}-1$ & pCi/g dry & $1.93 \mathrm{E}-1$ & & $12 / 15 / 08$ & 8L09007 & AGG-RRL-001 \\
\hline 13966-06-8 & Tin-113 & $<2.76 \mathrm{E}-1$ & pCi/g dry & $2.76 \mathrm{E}-1$ & & $12 / 15 / 08$ & 8L09007 & AGG-RRL-001 \\
\hline $14683-10-4$ & Antimony-124 & $<1.83 \mathrm{E}-1$ & pCi/g dry & $1.83 \mathrm{E}-1$ & & $12 / 15 / 08$ & 8L09007 & AGG-RRL-001 \\
\hline $14234-35-6$ & Antimony-125 & $<6.34 \mathrm{E}-1$ & pCi/g dry & $6.34 \mathrm{E}-1$ & & $12 / 15 / 08$ & 8L09007 & AGG-RRL-001 \\
\hline $15756-32-8$ & Antimony-126 & $<1.74 \mathrm{E}-1$ & pCi/g dry & $1.74 \mathrm{E}-1$ & & $12 / 15 / 08$ & 8L09007 & AGG-RRL-001 \\
\hline $15832-50-5$ & Tin-126 & $<5.38 \mathrm{E}-1$ & pCi/g dry & $5.38 \mathrm{E}-1$ & & $12 / 15 / 08$ & 8L09007 & AGG-RRL-001 \\
\hline $10043-66-0$ & Iodine-131 & $<2.28 \mathrm{E}-1$ & $\mathrm{pCi} / \mathrm{g}$ dry & $2.28 \mathrm{E}-1$ & & $12 / 15 / 08$ & 8L09007 & AGG-RRL-001 \\
\hline 13981-41-4 & Barium-133 & $<3.88 \mathrm{E}-1$ & pCi/g dry & $3.88 \mathrm{E}-1$ & & $12 / 15 / 08$ & 8L09007 & AGG-RRL-001 \\
\hline $13967-70-9$ & Cesium-134 & $<2.15 \mathrm{E}-1$ & pCi/g dry & $2.15 \mathrm{E}-1$ & & $12 / 15 / 08$ & 8L09007 & AGG-RRL-001 \\
\hline $10045-97-3$ & Cesium-137 & $<2.16 \mathrm{E}-1$ & pCi/g dry & $2.16 \mathrm{E}-1$ & & $12 / 15 / 08$ & 8L09007 & AGG-RRL-001 \\
\hline 13982-30-4 & Cerium-139 & $<2.50 \mathrm{E}-1$ & pCi/g dry & $2.50 \mathrm{E}-1$ & & $12 / 15 / 08$ & 8L09007 & AGG-RRL-001 \\
\hline $14762-78-8$ & Cerium-144 & $<1.80 \mathrm{E} 0$ & pCi/g dry & $1.80 \mathrm{E} 0$ & & $12 / 15 / 08$ & 8L09007 & AGG-RRL-001 \\
\hline $14683-23-9$ & Europium-152 & $<7.64 \mathrm{E}-1$ & pCi/g dry & 7.64E-1 & & $12 / 15 / 08$ & 8L09007 & AGG-RRL-001 \\
\hline $14276-65-4$ & Gadolinium-153 & $<6.91 \mathrm{E}-1$ & pCi/g dry & $6.91 \mathrm{E}-1$ & & $12 / 15 / 08$ & 8L09007 & AGG-RRL-001 \\
\hline
\end{tabular}


GEA/Soil

\begin{tabular}{|c|c|c|c|c|c|c|c|c|}
\hline CAS \# & Analyte & Results & Units & MDA & UNC & Analyzed & Batch & Method \\
\hline HEIS No. & B1V997 & & b ID: & $5003-07$ & & & & \\
\hline $15585-10-1$ & Europium-154 & $<4.79 \mathrm{E}-1$ & pCi/g dry & $4.79 \mathrm{E}-1$ & & $12 / 15 / 08$ & 8L09007 & AGG-RRL-001 \\
\hline 14391-16-3 & Europium-155 & $<7.54 \mathrm{E}-1$ & pCi/g dry & $7.54 \mathrm{E}-1$ & & $12 / 15 / 08$ & 8L09007 & AGG-RRL-001 \\
\hline $13982-78-0$ & Mercury-203 & $<2.52 \mathrm{E}-1$ & pCi/g dry & $2.52 \mathrm{E}-1$ & & $12 / 15 / 08$ & 8L09007 & AGG-RRL-001 \\
\hline $14913-50-9$ & Thallium-208 & $2.03 \mathrm{E}-1$ & pCi $/ g$ dry & $1.93 \mathrm{E}-1$ & $4.26 \mathrm{E}-2$ & $12 / 15 / 08$ & 8L09007 & AGG-RRL-001 \\
\hline 14331-79-4 & Bismuth-210 & $<3.95 \mathrm{E}-1$ & pCi $/ g$ dry & $3.95 \mathrm{E}-1$ & & $12 / 15 / 08$ & 8L09007 & AGG-RRL-001 \\
\hline \multirow[t]{3}{*}{$14255-04-0$} & Lead-210 & $<9.06 \mathrm{E} 1$ & pCi/g dry & $9.06 \mathrm{E} 1$ & & $12 / 15 / 08$ & 8L09007 & AGG-RRL-001 \\
\hline & Bismuth-211 & $<4.65 \mathrm{E} 0$ & pCi/g dry & $4.65 \mathrm{E} 0$ & & $12 / 15 / 08$ & 8L09007 & AGG-RRL-001 \\
\hline & Lead-211 & $<6.35 \mathrm{E} 0$ & pCi $/ g$ dry & $6.35 \mathrm{E} 0$ & & $12 / 15 / 08$ & 8L09007 & AGG-RRL-001 \\
\hline 15092-94-1 & Lead-212 & $8.55 \mathrm{E}-1$ & pCi/g dry & $3.56 \mathrm{E}-1$ & $1.63 \mathrm{E}-1$ & $12 / 15 / 08$ & 8L09007 & AGG-RRL-001 \\
\hline 14733-03-0 & Bismuth-214 & $<4.93 \mathrm{E}-1$ & pCi/g dry & $4.93 \mathrm{E}-1$ & & $12 / 15 / 08$ & 8L09007 & AGG-RRL-001 \\
\hline $15067-28-4$ & Lead-214 & $1.19 \mathrm{E} 0$ & pCi/g dry & $5.40 \mathrm{E}-1$ & $4.07 \mathrm{E}-1$ & $12 / 15 / 08$ & 8L09007 & AGG-RRL-001 \\
\hline $14835-02-0$ & Radon-219 & $<1.99 \mathrm{E} 0$ & pCi/g dry & $1.99 \mathrm{E} 0$ & & $12 / 15 / 08$ & 8L09007 & AGG-RRL-001 \\
\hline $22481-48-7$ & Radon-220 & $<1.66 \mathrm{E} 2$ & pCi $/ g$ dry & $1.66 \mathrm{E} 2$ & & $12 / 15 / 08$ & 8L09007 & AGG-RRL-001 \\
\hline $28522-20-5$ & Radon-221 & $<9.68 \mathrm{E}-1$ & pCi/g dry & $9.68 \mathrm{E}-1$ & & $12 / 15 / 08$ & 8L09007 & AGG-RRL-001 \\
\hline $15756-98-6$ & Francium-223 & $<7.22 \mathrm{E} 0$ & pCi/g dry & $7.22 \mathrm{E} 0$ & & $12 / 15 / 08$ & 8L09007 & AGG-RRL-001 \\
\hline $15623-45-7$ & Radium-223 & $<1.45 \mathrm{E} 0$ & pCi/g dry & $1.45 \mathrm{E} 0$ & & $12 / 15 / 08$ & 8L09007 & AGG-RRL-001 \\
\hline $13233-32-4$ & Radium-224 & $<3.94 \mathrm{E} 0$ & pCi/g dry & $3.94 \mathrm{E} 0$ & & $12 / 15 / 08$ & 8L09007 & AGG-RRL-001 \\
\hline $13982-63-3$ & Radium-226 & $<5.97 \mathrm{E} 0$ & pCi/g dry & $5.97 \mathrm{E} 0$ & & $12 / 15 / 08$ & 8L09007 & AGG-RRL-001 \\
\hline $15623-47-9$ & Thorium-227 & $<2.23 \mathrm{E} 0$ & pCi $/ g$ dry & $2.23 \mathrm{E} 0$ & & $12 / 15 / 08$ & 8L09007 & AGG-RRL-001 \\
\hline $14331-83-0$ & Actinium-228 & $<8.83 \mathrm{E}-1$ & pCi/g dry & $8.83 \mathrm{E}-1$ & & $12 / 15 / 08$ & 8L09007 & AGG-RRL-001 \\
\hline $14274-82-9$ & Thorium-228 & $<7.70 \mathrm{E} 1$ & $\mathrm{pCi} / \mathrm{g}$ dry & $7.70 \mathrm{E} 1$ & & $12 / 15 / 08$ & 8L09007 & AGG-RRL-001 \\
\hline $14269-63-7$ & Thorium-230 & $<1.01 \mathrm{E} 2$ & $\mathrm{pCi} / \mathrm{g}$ dry & $1.01 \mathrm{E} 2$ & & $12 / 15 / 08$ & 8L09007 & AGG-RRL-001 \\
\hline $14331-85-2$ & Protactinium-231 & $<8.31 \mathrm{E} 0$ & pCi/g dry & $8.31 \mathrm{E} 0$ & & $12 / 15 / 08$ & 8L09007 & AGG-RRL-001 \\
\hline $14932-40-2$ & Thorium-231 & $<4.88 \mathrm{E} 1$ & $\mathrm{pCi} / \mathrm{g}$ dry & $4.88 \mathrm{E} 1$ & & $12 / 15 / 08$ & 8L09007 & AGG-RRL-001 \\
\hline $7440-29-1$ & Thorium 232 & $<3.19 \mathrm{E} 2$ & $\mathrm{pCi} / \mathrm{g}$ dry & $3.19 \mathrm{E} 2$ & & $12 / 15 / 08$ & 8L09007 & AGG-RRL-001 \\
\hline 13981-14-1 & Protactinium-233 & $<5.11 \mathrm{E}-1$ & $\mathrm{pCi} / \mathrm{g}$ dry & $5.11 \mathrm{E}-1$ & & $12 / 15 / 08$ & 8L09007 & AGG-RRL-001 \\
\hline \multirow{2}{*}{$15100-28-4$} & Protactinium-234 & $<8.87 \mathrm{E}-1$ & pCi $/ g$ dry & $8.87 \mathrm{E}-1$ & & $12 / 15 / 08$ & 8L09007 & AGG-RRL-001 \\
\hline & Protactinium-234m & $<2.18 \mathrm{E} 1$ & $\mathrm{pCi} / \mathrm{g}$ dry & $2.18 \mathrm{E} 1$ & & $12 / 15 / 08$ & 8L09007 & AGG-RRL-001 \\
\hline $15065-10-8$ & Thorium-234 & $<8.13 \mathrm{E} 0$ & pCi/g dry & $8.13 \mathrm{E} 0$ & & $12 / 15 / 08$ & 8L09007 & AGG-RRL-001 \\
\hline 15117-96-1 & Uranium 235 & $<1.85 \mathrm{E} 0$ & pCi/g dry & $1.85 \mathrm{E} 0$ & & $12 / 15 / 08$ & 8L09007 & AGG-RRL-001 \\
\hline $13994-20-2$ & Neptunium-237 & $<1.97 \mathrm{E} 0$ & pCi/g dry & $1.97 \mathrm{E} 0$ & & $12 / 15 / 08$ & 8L09007 & AGG-RRL-001 \\
\hline \multirow[t]{2}{*}{ 13981-16-3 } & Plutonium-238 & $<2.79 \mathrm{E} 3$ & $\mathrm{pCi} / \mathrm{g}$ dry & $2.79 \mathrm{E} 3$ & & $12 / 15 / 08$ & 8L09007 & AGG-RRL-001 \\
\hline & Uranium 238 & $<4.21 \mathrm{E} 0$ & pCi/g dry & $4.21 \mathrm{E} 0$ & & $12 / 15 / 08$ & 8L09007 & AGG-RRL-001 \\
\hline $15117-48-3$ & Plutonium-239 & $<3.19 \mathrm{E} 3$ & pCi/g dry & $3.19 \mathrm{E} 3$ & & $12 / 15 / 08$ & 8L09007 & AGG-RRL-001 \\
\hline $14119-33-6$ & Plutonium-240 & $<2.78 \mathrm{E} 3$ & pCi/g dry & $2.78 \mathrm{E} 3$ & & $12 / 15 / 08$ & 8L09007 & AGG-RRL-001 \\
\hline $14596-10-2$ & Americium-241 & $<1.60 \mathrm{E} 0$ & pCi/g dry & $1.60 \mathrm{E} 0$ & & $12 / 15 / 08$ & 8L09007 & AGG-RRL-001 \\
\hline $14993-75-0$ & Americium-243 & $<5.39 \mathrm{E}-1$ & pCi/g dry & $5.39 \mathrm{E}-1$ & & $12 / 15 / 08$ & 8L09007 & AGG-RRL-001 \\
\hline $15757-87-6$ & Curium-243 & $<8.48 \mathrm{E}-1$ & $\mathrm{pCi} / \mathrm{g}$ dry & $8.48 \mathrm{E}-1$ & & $12 / 15 / 08$ & 8L09007 & AGG-RRL-001 \\
\hline $15621-76-8$ & Curium-245 & $<6.96 \mathrm{E}-1$ & pCi/g dry & $6.96 \mathrm{E}-1$ & & $12 / 15 / 08$ & 8L09007 & AGG-RRL-001 \\
\hline HEIS No. & B1V999 & & b ID: & 5003-09 & & & & \\
\hline $13966-32-0$ & Sodium-22 & $<1.82 \mathrm{E}-1$ & $\mathrm{pCi} / \mathrm{g}$ dry & $1.82 \mathrm{E}-1$ & & $12 / 15 / 08$ & 8L09007 & AGG-RRL-001 \\
\hline 13966-00-2 & Potassium-40 & $1.26 \mathrm{E} 1$ & pCi/g dry & $1.42 \mathrm{E} 0$ & $6.18 \mathrm{E}-1$ & $12 / 15 / 08$ & 8L09007 & AGG-RRL-001 \\
\hline $14392-02-0$ & Chromium-51 & $<1.71 \mathrm{E} 0$ & pCi/g dry & $1.71 \mathrm{E} 0$ & & $12 / 15 / 08$ & 8L09007 & AGG-RRL-001 \\
\hline 13966-31-9 & Manganese-54 & $<1.72 \mathrm{E}-1$ & pCi/g dry & $1.72 \mathrm{E}-1$ & & $12 / 15 / 08$ & 8L09007 & AGG-RRL-001 \\
\hline $13981-50-5$ & Cobalt-57 & $<1.99 \mathrm{E}-1$ & pCi/g dry & $1.99 \mathrm{E}-1$ & & $12 / 15 / 08$ & 8L09007 & AGG-RRL-001 \\
\hline $14596-12-4$ & Iron-59 & $<3.13 \mathrm{E}-1$ & pCi/g dry & $3.13 \mathrm{E}-1$ & & $12 / 15 / 08$ & 8L09007 & AGG-RRL-001 \\
\hline 10198-40-0 & Cobalt-60 & $<1.60 \mathrm{E}-1$ & pCi $/ g$ dry & $1.60 \mathrm{E}-1$ & & $12 / 15 / 08$ & 8L09007 & AGG-RRL-001 \\
\hline $13982-39-3$ & Zinc-65 & $<4.07 \mathrm{E}-1$ & pCi/g dry & $4.07 \mathrm{E}-1$ & & $12 / 15 / 08$ & 8L09007 & AGG-RRL-001 \\
\hline
\end{tabular}


GEA/Soil

\begin{tabular}{|c|c|c|c|c|c|c|c|c|}
\hline CAS \# & Analyte & Results & Units & MDA & UNC & Analyzed & Batch & Method \\
\hline HEIS No. & B1V999 & & ab ID: & $5003-09$ & & & & \\
\hline $14265-71-5$ & Selenium-75 & $<2.86 \mathrm{E}-1$ & pCi/g dry & $2.86 \mathrm{E}-1$ & & $12 / 15 / 08$ & 8L09007 & AGG-RRL-001 \\
\hline 17056-36-9 & Rubidium-83 & $<4.26 \mathrm{E}-1$ & pCi/g dry & $4.26 \mathrm{E}-1$ & & $12 / 15 / 08$ & 8L09007 & AGG-RRL-001 \\
\hline $13967-73-2$ & Strontium-85 & $<1.96 \mathrm{E}-1$ & pCi $/ g$ dry & $1.96 \mathrm{E}-1$ & & $12 / 15 / 08$ & 8L09007 & AGG-RRL-001 \\
\hline $14932-53-7$ & Rubidium-86 & $<2.01 \mathrm{E} 0$ & pCi/g dry & $2.01 \mathrm{E} 0$ & & $12 / 15 / 08$ & 8L09007 & AGG-RRL-001 \\
\hline 13982-36-0 & Yttrium-88 & $<1.36 \mathrm{E}-1$ & pCi/g dry & $1.36 \mathrm{E}-1$ & & $12 / 15 / 08$ & 8L09007 & AGG-RRL-001 \\
\hline 14681-63-1 & Niobium-94 & $<1.58 \mathrm{E}-1$ & pCi/g dry & $1.58 \mathrm{E}-1$ & & $12 / 15 / 08$ & 8L09007 & AGG-RRL-001 \\
\hline \multirow[t]{3}{*}{$13967-76-5$} & Niobium-95 & $<1.74 \mathrm{E}-1$ & pCi/g dry & $1.74 \mathrm{E}-1$ & & $12 / 15 / 08$ & 8L09007 & AGG-RRL-001 \\
\hline & Niobium-95m & $<8.04 \mathrm{E}-1$ & pCi/g dry & $8.04 \mathrm{E}-1$ & & $12 / 15 / 08$ & 8L09007 & AGG-RRL-001 \\
\hline & Technetium-95m & $<2.82 \mathrm{E}-1$ & pCi/g dry & $2.82 \mathrm{E}-1$ & & $12 / 15 / 08$ & 8L09007 & AGG-RRL-001 \\
\hline \multirow[t]{2}{*}{$13967-71-0$} & Zirconium-95 & $<3.07 \mathrm{E}-1$ & pCi/g dry & $3.07 \mathrm{E}-1$ & & $12 / 15 / 08$ & 8L09007 & AGG-RRL-001 \\
\hline & Technetium-99m & $<1.93 \mathrm{E}-1$ & pCi/g dry & $1.93 \mathrm{E}-1$ & & $12 / 15 / 08$ & 8L09007 & AGG-RRL-001 \\
\hline 13968-53-1 & Ruthenium-103 & $<1.93 \mathrm{E}-1$ & pCi/g dry & $1.93 \mathrm{E}-1$ & & $12 / 15 / 08$ & 8L09007 & AGG-RRL-001 \\
\hline $13967-48-1$ & Ruthenium-106 & $<2.04 \mathrm{E} 0$ & pCi/g dry & $2.04 \mathrm{E} 0$ & & $12 / 15 / 08$ & 8L09007 & AGG-RRL-001 \\
\hline $14391-65-2$ & Silver-108m & $<1.76 \mathrm{E}-1$ & pCi/g dry & $1.76 \mathrm{E}-1$ & & $12 / 15 / 08$ & 8L09007 & AGG-RRL-001 \\
\hline \multirow[t]{2}{*}{$14109-32-1$} & Cadmium-109 & $<6.14 \mathrm{E} 0$ & pCi/g dry & $6.14 \mathrm{E} 0$ & & $12 / 15 / 08$ & 8L09007 & AGG-RRL-001 \\
\hline & Silver-110 & $<1.78 \mathrm{E}-1$ & pCi/g dry & $1.78 \mathrm{E}-1$ & & $12 / 15 / 08$ & 8L09007 & AGG-RRL-001 \\
\hline $14391-76-5$ & Silver-110m & $<1.78 \mathrm{E}-1$ & pCi/g dry & $1.78 \mathrm{E}-1$ & & $12 / 15 / 08$ & 8L09007 & AGG-RRL-001 \\
\hline 13966-06-8 & Tin-113 & $<2.57 \mathrm{E}-1$ & pCi/g dry & $2.57 \mathrm{E}-1$ & & $12 / 15 / 08$ & 8L09007 & AGG-RRL-001 \\
\hline 14683-10-4 & Antimony-124 & $<2.17 \mathrm{E}-1$ & pCi/g dry & $2.17 \mathrm{E}-1$ & & $12 / 15 / 08$ & 8L09007 & AGG-RRL-001 \\
\hline $14234-35-6$ & Antimony-125 & $<5.49 \mathrm{E}-1$ & pCi/g dry & $5.49 \mathrm{E}-1$ & & $12 / 15 / 08$ & 8L09007 & AGG-RRL-001 \\
\hline $15756-32-8$ & Antimony-126 & $<1.62 \mathrm{E}-1$ & pCi/g dry & $1.62 \mathrm{E}-1$ & & $12 / 15 / 08$ & 8L09007 & AGG-RRL-001 \\
\hline $15832-50-5$ & Tin-126 & $<5.03 \mathrm{E}-1$ & pCi/g dry & $5.03 \mathrm{E}-1$ & & $12 / 15 / 08$ & 8L09007 & AGG-RRL-001 \\
\hline 10043-66-0 & Iodine-131 & $<2.24 \mathrm{E}-1$ & pCi/g dry & $2.24 \mathrm{E}-1$ & & $12 / 15 / 08$ & 8L09007 & AGG-RRL-001 \\
\hline 13981-41-4 & Barium-133 & $<3.11 \mathrm{E}-1$ & pCi/g dry & $3.11 \mathrm{E}-1$ & & $12 / 15 / 08$ & 8L09007 & AGG-RRL-001 \\
\hline $13967-70-9$ & Cesium-134 & $<1.93 \mathrm{E}-1$ & pCi/g dry & $1.93 \mathrm{E}-1$ & & $12 / 15 / 08$ & 8L09007 & AGG-RRL-001 \\
\hline $10045-97-3$ & Cesium-137 & $<1.99 \mathrm{E}-1$ & pCi/g dry & $1.99 \mathrm{E}-1$ & & $12 / 15 / 08$ & 8L09007 & AGG-RRL-001 \\
\hline 13982-30-4 & Cerium-139 & $<2.20 \mathrm{E}-1$ & pCi/g dry & $2.20 \mathrm{E}-1$ & & $12 / 15 / 08$ & 8L09007 & AGG-RRL-001 \\
\hline $14762-78-8$ & Cerium-144 & $<1.58 \mathrm{E} 0$ & pCi/g dry & $1.58 \mathrm{E} 0$ & & $12 / 15 / 08$ & 8L09007 & AGG-RRL-001 \\
\hline $14683-23-9$ & Europium-152 & $<7.10 \mathrm{E}-1$ & pCi/g dry & $7.10 \mathrm{E}-1$ & & $12 / 15 / 08$ & 8L09007 & AGG-RRL-001 \\
\hline $14276-65-4$ & Gadolinium-153 & $<6.52 \mathrm{E}-1$ & pCi/g dry & $6.52 \mathrm{E}-1$ & & $12 / 15 / 08$ & 8L09007 & AGG-RRL-001 \\
\hline 15585-10-1 & Europium-154 & $<4.20 \mathrm{E}-1$ & pCi/g dry & $4.20 \mathrm{E}-1$ & & $12 / 15 / 08$ & 8L09007 & AGG-RRL-001 \\
\hline 14391-16-3 & Europium-155 & $<7.13 \mathrm{E}-1$ & pCi/g dry & 7.13E-1 & & $12 / 15 / 08$ & 8L09007 & AGG-RRL-001 \\
\hline 13982-78-0 & Mercury-203 & $<2.19 \mathrm{E}-1$ & pCi/g dry & $2.19 \mathrm{E}-1$ & & $12 / 15 / 08$ & 8L09007 & AGG-RRL-001 \\
\hline $14913-50-9$ & Thallium-208 & $<2.28 \mathrm{E}-1$ & pCi/g dry & $2.28 \mathrm{E}-1$ & & $12 / 15 / 08$ & 8L09007 & AGG-RRL-001 \\
\hline $14331-79-4$ & Bismuth-210 & $<3.44 \mathrm{E}-1$ & pCi/g dry & $3.44 \mathrm{E}-1$ & & $12 / 15 / 08$ & 8L09007 & AGG-RRL-001 \\
\hline \multirow[t]{3}{*}{$14255-04-0$} & Lead-210 & $<3.67 \mathrm{E} 1$ & pCi/g dry & $3.67 \mathrm{E} 1$ & & $12 / 15 / 08$ & 8L09007 & AGG-RRL-001 \\
\hline & Bismuth-211 & $<3.96 \mathrm{E} 0$ & pCi/g dry & $3.96 \mathrm{E} 0$ & & $12 / 15 / 08$ & 8L09007 & AGG-RRL-001 \\
\hline & Lead-211 & $<5.41 \mathrm{E} 0$ & pCi/g dry & $5.41 \mathrm{E} 0$ & & $12 / 15 / 08$ & 8L09007 & AGG-RRL-001 \\
\hline 15092-94-1 & Lead-212 & $<4.64 \mathrm{E}-1$ & pCi/g dry & 4.64E-1 & & $12 / 15 / 08$ & 8L09007 & AGG-RRL-001 \\
\hline 14733-03-0 & Bismuth-214 & $<4.69 \mathrm{E}-1$ & pCi/g dry & $4.69 \mathrm{E}-1$ & & $12 / 15 / 08$ & 8L09007 & AGG-RRL-001 \\
\hline $15067-28-4$ & Lead-214 & $<5.03 \mathrm{E}-1$ & pCi/g dry & $5.03 \mathrm{E}-1$ & & $12 / 15 / 08$ & 8L09007 & AGG-RRL-001 \\
\hline $14835-02-0$ & Radon-219 & $<1.75 \mathrm{E} 0$ & $\mathrm{pCi} / \mathrm{g}$ dry & $1.75 \mathrm{E} 0$ & & $12 / 15 / 08$ & 8L09007 & AGG-RRL-001 \\
\hline $22481-48-7$ & Radon-220 & $<1.75 \mathrm{E} 2$ & pCi/g dry & $1.75 \mathrm{E} 2$ & & $12 / 15 / 08$ & 8L09007 & AGG-RRL-001 \\
\hline $28522-20-5$ & Radon-221 & $<8.53 \mathrm{E}-1$ & pCi/g dry & $8.53 \mathrm{E}-1$ & & $12 / 15 / 08$ & 8L09007 & AGG-RRL-001 \\
\hline $15756-98-6$ & Francium-223 & $<5.91 \mathrm{E} 0$ & pCi/g dry & $5.91 \mathrm{E} 0$ & & $12 / 15 / 08$ & 8L09007 & AGG-RRL-001 \\
\hline $15623-45-7$ & Radium-223 & $<1.27 \mathrm{E} 0$ & pCi/g dry & $1.27 \mathrm{E} 0$ & & $12 / 15 / 08$ & 8L09007 & AGG-RRL-001 \\
\hline $13233-32-4$ & Radium-224 & $<5.00 \mathrm{E} 0$ & pCi/g dry & $5.00 \mathrm{E} 0$ & & $12 / 15 / 08$ & 8L09007 & AGG-RRL-001 \\
\hline $13982-63-3$ & Radium-226 & $<5.25 \mathrm{E} 0$ & pCi/g dry & $5.25 \mathrm{E} 0$ & & $12 / 15 / 08$ & 8L09007 & AGG-RRL-001 \\
\hline $15623-47-9$ & Thorium-227 & $<1.80 \mathrm{E} 0$ & pCi/g dry & $1.80 \mathrm{E} 0$ & & $12 / 15 / 08$ & 8L09007 & AGG-RRL-001 \\
\hline
\end{tabular}


GEA/Soil

\begin{tabular}{|c|c|c|c|c|c|c|c|c|}
\hline CAS \# & Analyte & Results & Units & MDA & UNC & Analyzed & Batch & Method \\
\hline HEIS No. & B1V999 & & ab ID: & $5003-09$ & & & & \\
\hline $14331-83-0$ & Actinium-228 & $<7.13 \mathrm{E}-1$ & pCi/g dry & 7.13E-1 & & $12 / 15 / 08$ & 8L09007 & AGG-RRL-001 \\
\hline $14274-82-9$ & Thorium-228 & $<6.63 \mathrm{E} 1$ & pCi/g dry & $6.63 \mathrm{E} 1$ & & $12 / 15 / 08$ & 8L09007 & AGG-RRL-001 \\
\hline $14269-63-7$ & Thorium-230 & $<8.74 \mathrm{E} 1$ & pCi/g dry & $8.74 \mathrm{E} 1$ & & $12 / 15 / 08$ & 8L09007 & AGG-RRL-001 \\
\hline $14331-85-2$ & Protactinium-231 & $<7.58 \mathrm{E} 0$ & pCi $/ g$ dry & $7.58 \mathrm{E} 0$ & & $12 / 15 / 08$ & 8L09007 & AGG-RRL-001 \\
\hline $14932-40-2$ & Thorium-231 & $<4.59 \mathrm{E} 1$ & pCi $/ g$ dry & $4.59 \mathrm{E} 1$ & & $12 / 15 / 08$ & 8L09007 & AGG-RRL-001 \\
\hline $7440-29-1$ & Thorium 232 & $<2.21 \mathrm{E} 2$ & pCi/g dry & $2.21 \mathrm{E} 2$ & & $12 / 15 / 08$ & 8L09007 & AGG-RRL-001 \\
\hline 13981-14-1 & Protactinium-233 & $<4.65 \mathrm{E}-1$ & $\mathrm{pCi} / \mathrm{g}$ dry & $4.65 \mathrm{E}-1$ & & $12 / 15 / 08$ & 8L09007 & AGG-RRL-001 \\
\hline \multirow[t]{2}{*}{$15100-28-4$} & Protactinium-234 & $<8.51 \mathrm{E}-1$ & pCi $/ g$ dry & $8.51 \mathrm{E}-1$ & & $12 / 15 / 08$ & 8L09007 & AGG-RRL-001 \\
\hline & Protactinium-234m & $<2.01 \mathrm{E} 1$ & $\mathrm{pCi} / \mathrm{g}$ dry & $2.01 \mathrm{E} 1$ & & $12 / 15 / 08$ & 8L09007 & AGG-RRL-001 \\
\hline $15065-10-8$ & Thorium-234 & $<7.42 \mathrm{E} 0$ & $\mathrm{pCi} / \mathrm{g}$ dry & $7.42 \mathrm{E} 0$ & & $12 / 15 / 08$ & 8L09007 & AGG-RRL-001 \\
\hline $15117-96-1$ & Uranium 235 & $<1.64 \mathrm{E} 0$ & pCi/g dry & $1.64 \mathrm{E} 0$ & & $12 / 15 / 08$ & 8L09007 & AGG-RRL-001 \\
\hline 13994-20-2 & Neptunium-237 & $<1.85 \mathrm{E} 0$ & pCi/g dry & $1.85 \mathrm{E} 0$ & & $12 / 15 / 08$ & 8L09007 & AGG-RRL-001 \\
\hline \multirow[t]{2}{*}{ 13981-16-3 } & Plutonium-238 & $<2.65 \mathrm{E} 3$ & pCi/g dry & $2.65 \mathrm{E} 3$ & & $12 / 15 / 08$ & 8L09007 & AGG-RRL-001 \\
\hline & Uranium 238 & $<3.82 \mathrm{E} 0$ & pCi/g dry & $3.82 \mathrm{E} 0$ & & $12 / 15 / 08$ & 8L09007 & AGG-RRL-001 \\
\hline $15117-48-3$ & Plutonium-239 & $<2.74 \mathrm{E} 3$ & pCi/g dry & $2.74 \mathrm{E} 3$ & & $12 / 15 / 08$ & 8L09007 & AGG-RRL-001 \\
\hline $14119-33-6$ & Plutonium-240 & $<2.63 \mathrm{E} 3$ & pCi/g dry & $2.63 \mathrm{E} 3$ & & $12 / 15 / 08$ & 8L09007 & AGG-RRL-001 \\
\hline $14596-10-2$ & Americium-241 & $<1.13 \mathrm{E} 0$ & pCi/g dry & $1.13 \mathrm{E} 0$ & & $12 / 15 / 08$ & 8L09007 & AGG-RRL-001 \\
\hline $14993-75-0$ & Americium-243 & $<4.66 \mathrm{E}-1$ & pCi/g dry & 4.66E-1 & & $12 / 15 / 08$ & 8L09007 & AGG-RRL-001 \\
\hline $15757-87-6$ & Curium-243 & $<8.10 \mathrm{E}-1$ & pCi $/ g$ dry & $8.10 \mathrm{E}-1$ & & $12 / 15 / 08$ & 8L09007 & AGG-RRL-001 \\
\hline $15621-76-8$ & Curium-245 & $<6.65 \mathrm{E}-1$ & pCi/g dry & $6.65 \mathrm{E}-1$ & & $12 / 15 / 08$ & 8L09007 & AGG-RRL-001 \\
\hline HEIS No. & B1V9B1 & & ab ID: & $5003-11$ & & & & \\
\hline $13966-32-0$ & Sodium-22 & $<1.85 \mathrm{E}-1$ & pCi/g dry & $1.85 \mathrm{E}-1$ & & $12 / 15 / 08$ & 8L09007 & AGG-RRL-001 \\
\hline $13966-00-2$ & Potassium-40 & $1.54 \mathrm{E} 1$ & pCi/g dry & $1.99 \mathrm{E} 0$ & 7.79E-1 & $12 / 15 / 08$ & 8L09007 & AGG-RRL-001 \\
\hline $14392-02-0$ & Chromium-51 & $<1.68 \mathrm{E} 0$ & pCi/g dry & $1.68 \mathrm{E} 0$ & & $12 / 15 / 08$ & 8L09007 & AGG-RRL-001 \\
\hline 13966-31-9 & Manganese-54 & $<1.65 \mathrm{E}-1$ & pCi/g dry & $1.65 \mathrm{E}-1$ & & $12 / 15 / 08$ & 8L09007 & AGG-RRL-001 \\
\hline $13981-50-5$ & Cobalt-57 & $<2.02 \mathrm{E}-1$ & pCi/g dry & $2.02 \mathrm{E}-1$ & & $12 / 15 / 08$ & 8L09007 & AGG-RRL-001 \\
\hline $14596-12-4$ & Iron-59 & $<3.20 \mathrm{E}-1$ & pCi/g dry & $3.20 \mathrm{E}-1$ & & $12 / 15 / 08$ & 8L09007 & AGG-RRL-001 \\
\hline 10198-40-0 & Cobalt- 60 & $<1.56 \mathrm{E}-1$ & pCi/g dry & $1.56 \mathrm{E}-1$ & & $12 / 15 / 08$ & 8L09007 & AGG-RRL-001 \\
\hline $13982-39-3$ & Zinc-65 & $<4.46 \mathrm{E}-1$ & pCi/g dry & $4.46 \mathrm{E}-1$ & & $12 / 15 / 08$ & 8L09007 & AGG-RRL-001 \\
\hline $14265-71-5$ & Selenium-75 & $<3.04 \mathrm{E}-1$ & $\mathrm{pCi} / \mathrm{g}$ dry & $3.04 \mathrm{E}-1$ & & $12 / 15 / 08$ & 8L09007 & AGG-RRL-001 \\
\hline 17056-36-9 & Rubidium-83 & $<3.46 \mathrm{E}-1$ & pCi/g dry & $3.46 \mathrm{E}-1$ & & $12 / 15 / 08$ & 8L09007 & AGG-RRL-001 \\
\hline $13967-73-2$ & Strontium-85 & $<2.19 \mathrm{E}-1$ & pCi/g dry & $2.19 \mathrm{E}-1$ & & $12 / 15 / 08$ & 8L09007 & AGG-RRL-001 \\
\hline $14932-53-7$ & Rubidium-86 & $<1.97 \mathrm{E} 0$ & pCi/g dry & $1.97 \mathrm{E} 0$ & & $12 / 15 / 08$ & 8L09007 & AGG-RRL-001 \\
\hline $13982-36-0$ & Yttrium-88 & $<1.24 \mathrm{E}-1$ & pCi/g dry & $1.24 \mathrm{E}-1$ & & $12 / 15 / 08$ & 8L09007 & AGG-RRL-001 \\
\hline $14681-63-1$ & Niobium-94 & $<1.57 \mathrm{E}-1$ & pCi/g dry & $1.57 \mathrm{E}-1$ & & $12 / 15 / 08$ & 8L09007 & AGG-RRL-001 \\
\hline \multirow[t]{3}{*}{$13967-76-5$} & Niobium-95 & $<1.61 \mathrm{E}-1$ & pCi/g dry & $1.61 \mathrm{E}-1$ & & $12 / 15 / 08$ & 8L09007 & AGG-RRL-001 \\
\hline & Niobium-95m & $<8.99 \mathrm{E}-1$ & pCi/g dry & $8.99 \mathrm{E}-1$ & & $12 / 15 / 08$ & 8L09007 & AGG-RRL-001 \\
\hline & Technetium-95m & $<2.93 \mathrm{E}-1$ & pCi/g dry & $2.93 \mathrm{E}-1$ & & $12 / 15 / 08$ & 8L09007 & AGG-RRL-001 \\
\hline \multirow[t]{2}{*}{$13967-71-0$} & Zirconium-95 & $<2.91 \mathrm{E}-1$ & pCi/g dry & $2.91 \mathrm{E}-1$ & & $12 / 15 / 08$ & 8L09007 & AGG-RRL-001 \\
\hline & Technetium-99m & $<1.99 \mathrm{E}-1$ & pCi/g dry & $1.99 \mathrm{E}-1$ & & $12 / 15 / 08$ & 8L09007 & AGG-RRL-001 \\
\hline $13968-53-1$ & Ruthenium-103 & $<1.78 \mathrm{E}-1$ & pCi/g dry & $1.78 \mathrm{E}-1$ & & $12 / 15 / 08$ & 8L09007 & AGG-RRL-001 \\
\hline $13967-48-1$ & Ruthenium-106 & $<1.70 \mathrm{E} 0$ & pCi/g dry & $1.70 \mathrm{E} 0$ & & $12 / 15 / 08$ & 8L09007 & AGG-RRL-001 \\
\hline $14391-65-2$ & Silver-108m & $<1.76 \mathrm{E}-1$ & pCi/g dry & $1.76 \mathrm{E}-1$ & & $12 / 15 / 08$ & 8L09007 & AGG-RRL-001 \\
\hline \multirow[t]{2}{*}{$14109-32-1$} & Cadmium-109 & $<5.91 \mathrm{E} 0$ & pCi/g dry & $5.91 \mathrm{E} 0$ & & $12 / 15 / 08$ & 8L09007 & AGG-RRL-001 \\
\hline & Silver-110 & $<1.70 \mathrm{E}-1$ & pCi/g dry & $1.70 \mathrm{E}-1$ & & $12 / 15 / 08$ & 8L09007 & AGG-RRL-001 \\
\hline $14391-76-5$ & Silver-110m & $<1.71 \mathrm{E}-1$ & pCi/g dry & $1.71 \mathrm{E}-1$ & & $12 / 15 / 08$ & 8L09007 & AGG-RRL-001 \\
\hline 13966-06-8 & Tin-113 & $<2.61 \mathrm{E}-1$ & pCi/g dry & $2.61 \mathrm{E}-1$ & & $12 / 15 / 08$ & 8L09007 & AGG-RRL-001 \\
\hline $14683-10-4$ & Antimony-124 & $<1.83 \mathrm{E}-1$ & pCi/g dry & $1.83 \mathrm{E}-1$ & & $12 / 15 / 08$ & 8L09007 & AGG-RRL-001 \\
\hline
\end{tabular}


GEA/Soil

\begin{tabular}{|c|c|c|c|c|c|c|c|c|}
\hline CAS \# & Analyte & Results & Units & MDA & UNC & Analyzed & Batch & Method \\
\hline HEIS No. & B1V9B1 & \multicolumn{3}{|c|}{ Lab ID: $\quad$ 0805003-11 } & & & & \\
\hline $14234-35-6$ & Antimony-125 & $<5.44 \mathrm{E}-1$ & pCi/g dry & $5.44 \mathrm{E}-1$ & & $12 / 15 / 08$ & 8L09007 & AGG-RRL-001 \\
\hline $15756-32-8$ & Antimony-126 & $<1.73 \mathrm{E}-1$ & pCi/g dry & $1.73 \mathrm{E}-1$ & & $12 / 15 / 08$ & 8L09007 & AGG-RRL-001 \\
\hline $15832-50-5$ & Tin-126 & $<4.68 \mathrm{E}-1$ & pCi $/ g$ dry & $4.68 \mathrm{E}-1$ & & $12 / 15 / 08$ & 8L09007 & AGG-RRL-001 \\
\hline $10043-66-0$ & Iodine-131 & $<2.03 \mathrm{E}-1$ & pCi/g dry & $2.03 \mathrm{E}-1$ & & $12 / 15 / 08$ & 8L09007 & AGG-RRL-001 \\
\hline $13981-41-4$ & Barium-133 & $<3.43 \mathrm{E}-1$ & pCi/g dry & $3.43 \mathrm{E}-1$ & & $12 / 15 / 08$ & 8L09007 & AGG-RRL-001 \\
\hline 13967-70-9 & Cesium-134 & $<2.01 \mathrm{E}-1$ & pCi/g dry & $2.01 \mathrm{E}-1$ & & $12 / 15 / 08$ & 8L09007 & AGG-RRL-001 \\
\hline $10045-97-3$ & Cesium-137 & $<1.99 \mathrm{E}-1$ & pCi/g dry & $1.99 \mathrm{E}-1$ & & $12 / 15 / 08$ & 8L09007 & AGG-RRL-001 \\
\hline 13982-30-4 & Cerium-139 & $<2.29 \mathrm{E}-1$ & pCi/g dry & $2.29 \mathrm{E}-1$ & & $12 / 15 / 08$ & 8L09007 & AGG-RRL-001 \\
\hline $14762-78-8$ & Cerium-144 & $<1.60 \mathrm{E} 0$ & pCi/g dry & $1.60 \mathrm{E} 0$ & & $12 / 15 / 08$ & 8L09007 & AGG-RRL-001 \\
\hline $14683-23-9$ & Europium-152 & $<7.03 \mathrm{E}-1$ & pCi/g dry & 7.03E-1 & & $12 / 15 / 08$ & 8L09007 & AGG-RRL-001 \\
\hline $14276-65-4$ & Gadolinium-153 & $<6.28 \mathrm{E}-1$ & pCi/g dry & $6.28 \mathrm{E}-1$ & & $12 / 15 / 08$ & 8L09007 & AGG-RRL-001 \\
\hline $15585-10-1$ & Europium-154 & $<4.31 \mathrm{E}-1$ & pCi/g dry & $4.31 \mathrm{E}-1$ & & $12 / 15 / 08$ & 8L09007 & AGG-RRL-001 \\
\hline 14391-16-3 & Europium-155 & $<6.55 \mathrm{E}-1$ & pCi/g dry & $6.55 \mathrm{E}-1$ & & $12 / 15 / 08$ & 8L09007 & AGG-RRL-001 \\
\hline 13982-78-0 & Mercury-203 & $<2.27 \mathrm{E}-1$ & pCi/g dry & $2.27 \mathrm{E}-1$ & & $12 / 15 / 08$ & 8L09007 & AGG-RRL-001 \\
\hline $14913-50-9$ & Thallium-208 & $<1.70 \mathrm{E}-1$ & pCi/g dry & $1.70 \mathrm{E}-1$ & & $12 / 15 / 08$ & 8L09007 & AGG-RRL-001 \\
\hline $14331-79-4$ & Bismuth-210 & $<3.61 \mathrm{E}-1$ & pCi/g dry & $3.61 \mathrm{E}-1$ & & $12 / 15 / 08$ & 8L09007 & AGG-RRL-001 \\
\hline \multirow[t]{3}{*}{$14255-04-0$} & Lead-210 & $<8.65 \mathrm{E} 1$ & pCi/g dry & $8.65 \mathrm{E} 1$ & & $12 / 15 / 08$ & 8L09007 & AGG-RRL-001 \\
\hline & Bismuth-211 & $<4.09 \mathrm{E} 0$ & pCi/g dry & $4.09 \mathrm{E} 0$ & & $12 / 15 / 08$ & 8L09007 & AGG-RRL-001 \\
\hline & Lead-211 & $<5.58 \mathrm{E} 0$ & pCi/g dry & $5.58 \mathrm{E} 0$ & & $12 / 15 / 08$ & 8L09007 & AGG-RRL-001 \\
\hline 15092-94-1 & Lead-212 & $6.185 \mathrm{E}-1$ & pCi/g dry & $4.45 \mathrm{E}-1$ & $9.85 \mathrm{E}-2$ & $12 / 15 / 08$ & 8L09007 & AGG-RRL-001 \\
\hline 14733-03-0 & Bismuth-214 & $<4.83 \mathrm{E}-1$ & pCi/g dry & $4.83 \mathrm{E}-1$ & & $12 / 15 / 08$ & 8L09007 & AGG-RRL-001 \\
\hline $15067-28-4$ & Lead-214 & $1.34 \mathrm{E} 0$ & pCi/g dry & $3.73 \mathrm{E}-1$ & $1.33 \mathrm{E} 0$ & $12 / 15 / 08$ & 8L09007 & AGG-RRL-001 \\
\hline $14835-02-0$ & Radon-219 & $<1.79 \mathrm{E} 0$ & pCi/g dry & $1.79 \mathrm{E} 0$ & & $12 / 15 / 08$ & 8L09007 & AGG-RRL-001 \\
\hline $22481-48-7$ & Radon-220 & $<1.55 \mathrm{E} 2$ & pCi/g dry & $1.55 \mathrm{E} 2$ & & $12 / 15 / 08$ & 8L09007 & AGG-RRL-001 \\
\hline $28522-20-5$ & Radon-221 & $<8.84 \mathrm{E}-1$ & pCi/g dry & $8.84 \mathrm{E}-1$ & & $12 / 15 / 08$ & 8L09007 & AGG-RRL-001 \\
\hline $15756-98-6$ & Francium-223 & $<6.51 \mathrm{E} 0$ & pCi/g dry & $6.51 \mathrm{E} 0$ & & $12 / 15 / 08$ & 8L09007 & AGG-RRL-001 \\
\hline $15623-45-7$ & Radium-223 & $<1.30 \mathrm{E} 0$ & pCi/g dry & $1.30 \mathrm{E} 0$ & & $12 / 15 / 08$ & 8L09007 & AGG-RRL-001 \\
\hline $13233-32-4$ & Radium-224 & $<5.65 \mathrm{E} 0$ & pCi/g dry & $5.65 \mathrm{E} 0$ & & $12 / 15 / 08$ & 8L09007 & AGG-RRL-001 \\
\hline $13982-63-3$ & Radium-226 & $<5.47 \mathrm{E} 0$ & pCi/g dry & $5.47 \mathrm{E} 0$ & & $12 / 15 / 08$ & 8L09007 & AGG-RRL-001 \\
\hline $15623-47-9$ & Thorium-227 & $<2.01 \mathrm{E} 0$ & pCi/g dry & $2.01 \mathrm{E} 0$ & & $12 / 15 / 08$ & 8L09007 & AGG-RRL-001 \\
\hline 14331-83-0 & Actinium-228 & $<8.12 \mathrm{E}-1$ & pCi/g dry & $8.12 \mathrm{E}-1$ & & $12 / 15 / 08$ & 8L09007 & AGG-RRL-001 \\
\hline $14274-82-9$ & Thorium-228 & $<6.83 \mathrm{E} 1$ & pCi/g dry & $6.83 \mathrm{E} 1$ & & $12 / 15 / 08$ & 8L09007 & AGG-RRL-001 \\
\hline $14269-63-7$ & Thorium-230 & $<8.97 \mathrm{E} 1$ & pCi/g dry & $8.97 \mathrm{E} 1$ & & $12 / 15 / 08$ & 8L09007 & AGG-RRL-001 \\
\hline $14331-85-2$ & Protactinium-231 & $<7.52 \mathrm{E} 0$ & pCi/g dry & $7.52 \mathrm{E} 0$ & & $12 / 15 / 08$ & 8L09007 & AGG-RRL-001 \\
\hline $14932-40-2$ & Thorium-231 & $<4.39 \mathrm{E} 1$ & pCi/g dry & $4.39 \mathrm{E} 1$ & & $12 / 15 / 08$ & 8L09007 & AGG-RRL-001 \\
\hline $7440-29-1$ & Thorium 232 & $<2.74 \mathrm{E} 2$ & pCi/g dry & $2.74 \mathrm{E} 2$ & & $12 / 15 / 08$ & 8L09007 & AGG-RRL-001 \\
\hline 13981-14-1 & Protactinium-233 & $<4.65 \mathrm{E}-1$ & pCi/g dry & $4.65 \mathrm{E}-1$ & & $12 / 15 / 08$ & 8L09007 & AGG-RRL-001 \\
\hline \multirow[t]{2}{*}{$15100-28-4$} & Protactinium-234 & $<7.97 \mathrm{E}-1$ & pCi/g dry & 7.97E-1 & & $12 / 15 / 08$ & 8L09007 & AGG-RRL-001 \\
\hline & Protactinium-234m & $<2.11 \mathrm{E} 1$ & pCi/g dry & $2.11 \mathrm{E} 1$ & & $12 / 15 / 08$ & 8L09007 & AGG-RRL-001 \\
\hline $15065-10-8$ & Thorium-234 & $<7.46 \mathrm{E} 0$ & pCi/g dry & $7.46 \mathrm{E} 0$ & & $12 / 15 / 08$ & 8L09007 & AGG-RRL-001 \\
\hline 15117-96-1 & Uranium 235 & $<1.65 \mathrm{E} 0$ & pCi/g dry & $1.65 \mathrm{E} 0$ & & $12 / 15 / 08$ & 8L09007 & AGG-RRL-001 \\
\hline 13994-20-2 & Neptunium-237 & $<1.71 \mathrm{E} 0$ & pCi/g dry & $1.71 \mathrm{E} 0$ & & $12 / 15 / 08$ & 8L09007 & AGG-RRL-001 \\
\hline \multirow[t]{2}{*}{ 13981-16-3 } & Plutonium-238 & $<2.48 \mathrm{E} 3$ & pCi/g dry & $2.48 \mathrm{E} 3$ & & $12 / 15 / 08$ & 8L09007 & AGG-RRL-001 \\
\hline & Uranium 238 & $<3.86 \mathrm{E} 0$ & pCi/g dry & $3.86 \mathrm{E} 0$ & & $12 / 15 / 08$ & 8L09007 & AGG-RRL-001 \\
\hline $15117-48-3$ & Plutonium-239 & $<2.79 \mathrm{E} 3$ & pCi/g dry & $2.79 \mathrm{E} 3$ & & $12 / 15 / 08$ & 8L09007 & AGG-RRL-001 \\
\hline $14119-33-6$ & Plutonium-240 & $<2.51 \mathrm{E} 3$ & pCi/g dry & $2.51 \mathrm{E} 3$ & & $12 / 15 / 08$ & 8L09007 & AGG-RRL-001 \\
\hline $14596-10-2$ & Americium-241 & $<1.38 \mathrm{E} 0$ & pCi/g dry & $1.38 \mathrm{E} 0$ & & $12 / 15 / 08$ & 8L09007 & AGG-RRL-001 \\
\hline 14993-75-0 & Americium-243 & $<2.83 \mathrm{E}-1$ & pCi/g dry & $2.83 \mathrm{E}-1$ & & $12 / 15 / 08$ & 8L09007 & AGG-RRL-001 \\
\hline $15757-87-6$ & Curium-243 & $<7.66 \mathrm{E}-1$ & pCi/g dry & $7.66 \mathrm{E}-1$ & & $12 / 15 / 08$ & 8L09007 & AGG-RRL-001 \\
\hline
\end{tabular}




\section{GEA/Soil}

\begin{tabular}{|c|c|c|c|c|c|c|c|c|}
\hline CAS \# & Analyte & Results & Units & MDA & UNC & Analyzed & Batch & Method \\
\hline HEIS No. & B1V9B1 & \multicolumn{4}{|c|}{ Lab ID: $\quad$ 0805003-11 } & & & \\
\hline $15621-76-8$ & Curium-245 & $<6.29 \mathrm{E}-1$ & pCi $/ g$ dry & $6.29 \mathrm{E}-1$ & & $12 / 15 / 08$ & 8L09007 & AGG-RRL-001 \\
\hline HEIS No. & B1V9B3 & \multicolumn{4}{|c|}{ Lab ID: $\quad 0805003-13$} & & & \\
\hline $13966-32-0$ & Sodium-22 & $<2.35 \mathrm{E}-1$ & pCi/g dry & $2.35 \mathrm{E}-1$ & & $12 / 15 / 08$ & 8L09007 & AGG-RRL-001 \\
\hline $13966-00-2$ & Potassium-40 & $1.74 \mathrm{E} 1$ & $\mathrm{pCi} / \mathrm{g}$ dry & $1.42 \mathrm{E} 0$ & $7.59 \mathrm{E}-1$ & $12 / 15 / 08$ & 8L09007 & AGG-RRL-001 \\
\hline $14392-02-0$ & Chromium-51 & $<1.96 \mathrm{E} 0$ & pCi/g dry & $1.96 \mathrm{E} 0$ & & $12 / 15 / 08$ & 8L09007 & AGG-RRL-001 \\
\hline 13966-31-9 & Manganese-54 & $<2.13 \mathrm{E}-1$ & pCi/g dry & $2.13 \mathrm{E}-1$ & & $12 / 15 / 08$ & 8L09007 & AGG-RRL-001 \\
\hline $13981-50-5$ & Cobalt-57 & $<2.34 \mathrm{E}-1$ & pCi $/ g$ dry & $2.34 \mathrm{E}-1$ & & $12 / 15 / 08$ & 8L09007 & AGG-RRL-001 \\
\hline $14596-12-4$ & Iron-59 & $<3.60 \mathrm{E}-1$ & $\mathrm{pCi} / \mathrm{g}$ dry & $3.60 \mathrm{E}-1$ & & $12 / 15 / 08$ & 8L09007 & AGG-RRL-001 \\
\hline $10198-40-0$ & Cobalt-60 & $<1.86 \mathrm{E}-1$ & pCi/g dry & $1.86 \mathrm{E}-1$ & & $12 / 15 / 08$ & 8L09007 & AGG-RRL-001 \\
\hline $13982-39-3$ & Zinc-65 & $<4.45 \mathrm{E}-1$ & pCi/g dry & $4.45 \mathrm{E}-1$ & & $12 / 15 / 08$ & 8L09007 & AGG-RRL-001 \\
\hline $14265-71-5$ & Selenium-75 & $<3.28 \mathrm{E}-1$ & $\mathrm{pCi} / \mathrm{g}$ dry & $3.28 \mathrm{E}-1$ & & $12 / 15 / 08$ & 8L09007 & AGG-RRL-001 \\
\hline $17056-36-9$ & Rubidium-83 & $<5.07 \mathrm{E}-1$ & pCi/g dry & $5.07 \mathrm{E}-1$ & & $12 / 15 / 08$ & 8L09007 & AGG-RRL-001 \\
\hline $13967-73-2$ & Strontium-85 & $<2.34 \mathrm{E}-1$ & $\mathrm{pCi} / \mathrm{g}$ dry & $2.34 \mathrm{E}-1$ & & $12 / 15 / 08$ & 8L09007 & AGG-RRL-001 \\
\hline $14932-53-7$ & Rubidium-86 & $<2.52 \mathrm{E} 0$ & pCi/g dry & $2.52 \mathrm{E} 0$ & & $12 / 15 / 08$ & 8L09007 & AGG-RRL-001 \\
\hline $13982-36-0$ & Yttrium-88 & $<1.36 \mathrm{E}-1$ & pCi $/ g$ dry & $1.36 \mathrm{E}-1$ & & $12 / 15 / 08$ & 8L09007 & AGG-RRL-001 \\
\hline $14681-63-1$ & Niobium-94 & $<1.96 \mathrm{E}-1$ & pCi/g dry & $1.96 \mathrm{E}-1$ & & $12 / 15 / 08$ & 8L09007 & AGG-RRL-001 \\
\hline \multirow[t]{3}{*}{$13967-76-5$} & Niobium-95 & $<2.07 \mathrm{E}-1$ & $\mathrm{pCi} / \mathrm{g}$ dry & $2.07 \mathrm{E}-1$ & & $12 / 15 / 08$ & 8L09007 & AGG-RRL-001 \\
\hline & Niobium-95m & $<9.39 \mathrm{E}-1$ & $\mathrm{pCi} / \mathrm{g}$ dry & $9.39 \mathrm{E}-1$ & & $12 / 15 / 08$ & 8L09007 & AGG-RRL-001 \\
\hline & Technetium-95m & $<3.32 \mathrm{E}-1$ & $\mathrm{pCi} / \mathrm{g}$ dry & $3.32 \mathrm{E}-1$ & & $12 / 15 / 08$ & 8L09007 & AGG-RRL-001 \\
\hline \multirow[t]{2}{*}{$13967-71-0$} & Zirconium-95 & $<3.79 \mathrm{E}-1$ & $\mathrm{pCi} / \mathrm{g}$ dry & $3.79 \mathrm{E}-1$ & & $12 / 15 / 08$ & 8L09007 & AGG-RRL-001 \\
\hline & Technetium-99m & $<2.28 \mathrm{E}-1$ & pCi/g dry & $2.28 \mathrm{E}-1$ & & $12 / 15 / 08$ & 8L09007 & AGG-RRL-001 \\
\hline $13968-53-1$ & Ruthenium-103 & $<2.29 \mathrm{E}-1$ & pCi/g dry & $2.29 \mathrm{E}-1$ & & $12 / 15 / 08$ & 8L09007 & AGG-RRL-001 \\
\hline $13967-48-1$ & Ruthenium-106 & $<2.38 \mathrm{E} 0$ & $\mathrm{pCi} / \mathrm{g}$ dry & $2.38 \mathrm{E} 0$ & & $12 / 15 / 08$ & 8L09007 & AGG-RRL-001 \\
\hline $14391-65-2$ & Silver-108m & $<2.12 \mathrm{E}-1$ & pCi/g dry & $2.12 \mathrm{E}-1$ & & $12 / 15 / 08$ & 8L09007 & AGG-RRL-001 \\
\hline \multirow[t]{2}{*}{$14109-32-1$} & Cadmium-109 & $<7.02 \mathrm{E} 0$ & $\mathrm{pCi} / \mathrm{g}$ dry & 7.02E0 & & $12 / 15 / 08$ & 8L09007 & AGG-RRL-001 \\
\hline & Silver-110 & $<2.04 \mathrm{E}-1$ & $\mathrm{pCi} / \mathrm{g}$ dry & $2.04 \mathrm{E}-1$ & & $12 / 15 / 08$ & 8L09007 & AGG-RRL-001 \\
\hline $14391-76-5$ & Silver-110m & $<2.04 \mathrm{E}-1$ & pCi/g dry & $2.04 \mathrm{E}-1$ & & $12 / 15 / 08$ & 8L09007 & AGG-RRL-001 \\
\hline $13966-06-8$ & Tin-113 & $<3.00 \mathrm{E}-1$ & pCi/g dry & $3.00 \mathrm{E}-1$ & & $12 / 15 / 08$ & 8L09007 & AGG-RRL-001 \\
\hline $14683-10-4$ & Antimony-124 & $<2.46 \mathrm{E}-1$ & pCi/g dry & $2.46 \mathrm{E}-1$ & & $12 / 15 / 08$ & 8L09007 & AGG-RRL-001 \\
\hline $14234-35-6$ & Antimony-125 & $<6.34 \mathrm{E}-1$ & $\mathrm{pCi} / \mathrm{g}$ dry & $6.34 \mathrm{E}-1$ & & $12 / 15 / 08$ & 8L09007 & AGG-RRL-001 \\
\hline $15756-32-8$ & Antimony-126 & $<1.88 \mathrm{E}-1$ & pCi/g dry & $1.88 \mathrm{E}-1$ & & $12 / 15 / 08$ & 8L09007 & AGG-RRL-001 \\
\hline $15832-50-5$ & Tin-126 & $<5.76 \mathrm{E}-1$ & pCi/g dry & $5.76 \mathrm{E}-1$ & & $12 / 15 / 08$ & 8L09007 & AGG-RRL-001 \\
\hline $10043-66-0$ & Iodine-131 & $<2.59 \mathrm{E}-1$ & pCi/g dry & $2.59 \mathrm{E}-1$ & & $12 / 15 / 08$ & 8L09007 & AGG-RRL-001 \\
\hline $13981-41-4$ & Barium-133 & $<3.67 \mathrm{E}-1$ & $\mathrm{pCi} / \mathrm{g}$ dry & $3.67 \mathrm{E}-1$ & & $12 / 15 / 08$ & 8L09007 & AGG-RRL-001 \\
\hline $13967-70-9$ & Cesium-134 & $<2.43 \mathrm{E}-1$ & pCi/g dry & $2.43 \mathrm{E}-1$ & & $12 / 15 / 08$ & 8L09007 & AGG-RRL-001 \\
\hline $10045-97-3$ & Cesium-137 & $<2.28 \mathrm{E}-1$ & pCi/g dry & $2.28 \mathrm{E}-1$ & & $12 / 15 / 08$ & 8L09007 & AGG-RRL-001 \\
\hline $13982-30-4$ & Cerium-139 & $<2.52 \mathrm{E}-1$ & pCi/g dry & $2.52 \mathrm{E}-1$ & & $12 / 15 / 08$ & 8L09007 & AGG-RRL-001 \\
\hline $14762-78-8$ & Cerium-144 & $<1.87 \mathrm{E} 0$ & pCi/g dry & $1.87 \mathrm{E} 0$ & & $12 / 15 / 08$ & 8L09007 & AGG-RRL-001 \\
\hline $14683-23-9$ & Europium-152 & $<8.73 \mathrm{E}-1$ & pCi/g dry & $8.73 \mathrm{E}-1$ & & $12 / 15 / 08$ & 8L09007 & AGG-RRL-001 \\
\hline $14276-65-4$ & Gadolinium-153 & $<7.52 \mathrm{E}-1$ & $\mathrm{pCi} / \mathrm{g}$ dry & $7.52 \mathrm{E}-1$ & & $12 / 15 / 08$ & 8L09007 & AGG-RRL-001 \\
\hline $15585-10-1$ & Europium-154 & $<4.94 \mathrm{E}-1$ & pCi/g dry & $4.94 \mathrm{E}-1$ & & $12 / 15 / 08$ & 8L09007 & AGG-RRL-001 \\
\hline $14391-16-3$ & Europium-155 & $<8.20 \mathrm{E}-1$ & pCi $/ g$ dry & $8.20 \mathrm{E}-1$ & & $12 / 15 / 08$ & 8L09007 & AGG-RRL-001 \\
\hline $13982-78-0$ & Mercury-203 & $<2.56 \mathrm{E}-1$ & pCi/g dry & $2.56 \mathrm{E}-1$ & & $12 / 15 / 08$ & 8L09007 & AGG-RRL-001 \\
\hline $14913-50-9$ & Thallium-208 & $<2.63 \mathrm{E}-1$ & pCi/g dry & $2.63 \mathrm{E}-1$ & & $12 / 15 / 08$ & 8L09007 & AGG-RRL-001 \\
\hline $14331-79-4$ & Bismuth-210 & $<3.92 \mathrm{E}-1$ & pCi/g dry & $3.92 \mathrm{E}-1$ & & $12 / 15 / 08$ & 8L09007 & AGG-RRL-001 \\
\hline \multirow[t]{3}{*}{$14255-04-0$} & Lead-210 & $<4.16 \mathrm{E} 1$ & pCi/g dry & $4.16 \mathrm{E} 1$ & & $12 / 15 / 08$ & 8L09007 & AGG-RRL-001 \\
\hline & Bismuth-211 & $<4.64 \mathrm{E} 0$ & $\mathrm{pCi} / \mathrm{g}$ dry & 4.64E0 & & $12 / 15 / 08$ & 8L09007 & AGG-RRL-001 \\
\hline & Lead-211 & $<6.35 \mathrm{E} 0$ & pCi $/ g$ dry & $6.35 \mathrm{E} 0$ & & $12 / 15 / 08$ & 8L09007 & AGG-RRL-001 \\
\hline
\end{tabular}


GEA/Soil

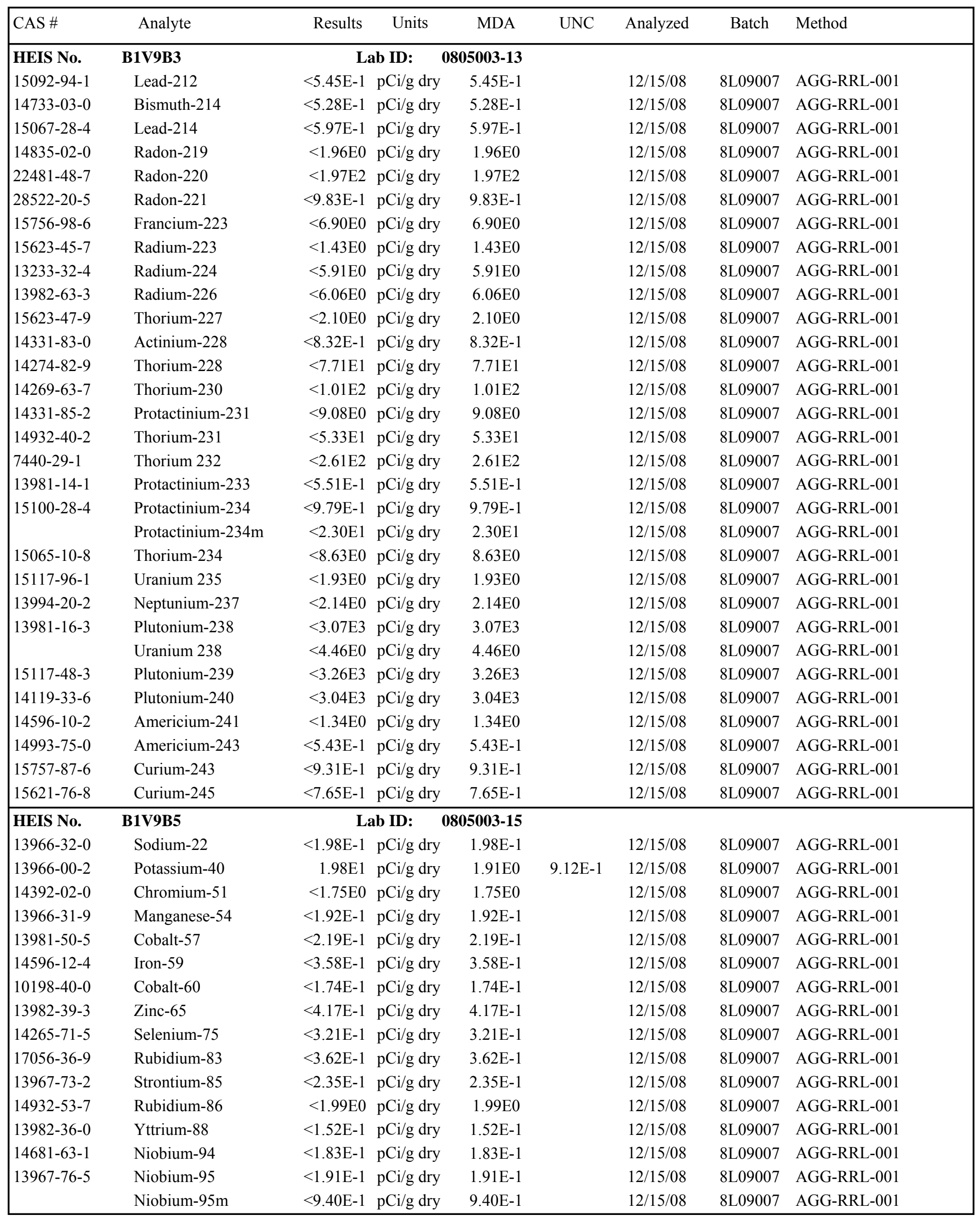


GEA/Soil

\begin{tabular}{|c|c|c|c|c|c|c|c|c|}
\hline CAS \# & Analyte & Results & Units & MDA & UNC & Analyzed & Batch & Method \\
\hline \multirow[t]{2}{*}{ HEIS No. } & B1V9B5 & \multicolumn{4}{|c|}{ Lab ID: $\quad$ 0805003-15 } & & & \\
\hline & Technetium-95m & $<3.12 \mathrm{E}-1$ & pCi/g dry & $3.12 \mathrm{E}-1$ & & $12 / 15 / 08$ & 8L09007 & AGG-RRL-001 \\
\hline \multirow[t]{2}{*}{$13967-71-0$} & Zirconium-95 & $<3.29 \mathrm{E}-1$ & pCi/g dry & $3.29 \mathrm{E}-1$ & & $12 / 15 / 08$ & 8L09007 & AGG-RRL-001 \\
\hline & Technetium-99m & $<2.15 \mathrm{E}-1$ & pCi/g dry & $2.15 \mathrm{E}-1$ & & $12 / 15 / 08$ & 8L09007 & AGG-RRL-001 \\
\hline 13968-53-1 & Ruthenium-103 & $<1.95 \mathrm{E}-1$ & pCi/g dry & $1.95 \mathrm{E}-1$ & & $12 / 15 / 08$ & 8L09007 & AGG-RRL-001 \\
\hline $13967-48-1$ & Ruthenium-106 & $<1.84 \mathrm{E} 0$ & pCi/g dry & $1.84 \mathrm{E} 0$ & & $12 / 15 / 08$ & 8L09007 & AGG-RRL-001 \\
\hline 14391-65-2 & Silver-108m & $<1.95 \mathrm{E}-1$ & pCi/g dry & $1.95 \mathrm{E}-1$ & & $12 / 15 / 08$ & 8L09007 & AGG-RRL-001 \\
\hline \multirow[t]{2}{*}{$14109-32-1$} & Cadmium-109 & $<6.36 \mathrm{E} 0$ & pCi/g dry & $6.36 \mathrm{E} 0$ & & $12 / 15 / 08$ & 8L09007 & AGG-RRL-001 \\
\hline & Silver-110 & $<1.88 \mathrm{E}-1$ & pCi/g dry & $1.88 \mathrm{E}-1$ & & $12 / 15 / 08$ & 8L09007 & AGG-RRL-001 \\
\hline $14391-76-5$ & Silver-110m & $<1.89 \mathrm{E}-1$ & pCi/g dry & $1.89 \mathrm{E}-1$ & & $12 / 15 / 08$ & 8L09007 & AGG-RRL-001 \\
\hline 13966-06-8 & Tin-113 & $<2.80 \mathrm{E}-1$ & pCi/g dry & $2.80 \mathrm{E}-1$ & & $12 / 15 / 08$ & 8L09007 & AGG-RRL-001 \\
\hline $14683-10-4$ & Antimony-124 & $<1.97 \mathrm{E}-1$ & pCi/g dry & $1.97 \mathrm{E}-1$ & & $12 / 15 / 08$ & 8L09007 & AGG-RRL-001 \\
\hline $14234-35-6$ & Antimony-125 & $<6.08 \mathrm{E}-1$ & pCi/g dry & $6.08 \mathrm{E}-1$ & & $12 / 15 / 08$ & 8L09007 & AGG-RRL-001 \\
\hline $15756-32-8$ & Antimony-126 & $<1.83 \mathrm{E}-1$ & pCi/g dry & $1.83 \mathrm{E}-1$ & & $12 / 15 / 08$ & 8L09007 & AGG-RRL-001 \\
\hline $15832-50-5$ & Tin-126 & $<5.08 \mathrm{E}-1$ & pCi/g dry & $5.08 \mathrm{E}-1$ & & $12 / 15 / 08$ & 8L09007 & AGG-RRL-001 \\
\hline 10043-66-0 & Iodine-131 & $<2.18 \mathrm{E}-1$ & pCi/g dry & $2.18 \mathrm{E}-1$ & & $12 / 15 / 08$ & 8L09007 & AGG-RRL-001 \\
\hline 13981-41-4 & Barium-133 & $<3.73 \mathrm{E}-1$ & pCi/g dry & $3.73 \mathrm{E}-1$ & & $12 / 15 / 08$ & 8L09007 & AGG-RRL-001 \\
\hline $13967-70-9$ & Cesium-134 & $<2.15 \mathrm{E}-1$ & pCi/g dry & $2.15 \mathrm{E}-1$ & & $12 / 15 / 08$ & 8L09007 & AGG-RRL-001 \\
\hline $10045-97-3$ & Cesium-137 & $<2.12 \mathrm{E}-1$ & pCi/g dry & $2.12 \mathrm{E}-1$ & & $12 / 15 / 08$ & 8L09007 & AGG-RRL-001 \\
\hline 13982-30-4 & Cerium-139 & $<2.43 \mathrm{E}-1$ & pCi/g dry & $2.43 \mathrm{E}-1$ & & $12 / 15 / 08$ & 8L09007 & AGG-RRL-001 \\
\hline $14762-78-8$ & Cerium-144 & $<1.75 \mathrm{E} 0$ & pCi/g dry & $1.75 \mathrm{E} 0$ & & $12 / 15 / 08$ & 8L09007 & AGG-RRL-001 \\
\hline $14683-23-9$ & Europium-152 & $<7.27 \mathrm{E}-1$ & pCi/g dry & $7.27 \mathrm{E}-1$ & & $12 / 15 / 08$ & 8L09007 & AGG-RRL-001 \\
\hline $14276-65-4$ & Gadolinium-153 & $<6.65 \mathrm{E}-1$ & pCi/g dry & $6.65 \mathrm{E}-1$ & & $12 / 15 / 08$ & 8L09007 & AGG-RRL-001 \\
\hline $15585-10-1$ & Europium-154 & $<4.66 \mathrm{E}-1$ & pCi/g dry & 4.66E-1 & & $12 / 15 / 08$ & 8L09007 & AGG-RRL-001 \\
\hline 14391-16-3 & Europium-155 & $<7.15 \mathrm{E}-1$ & pCi/g dry & 7.15E-1 & & $12 / 15 / 08$ & 8L09007 & AGG-RRL-001 \\
\hline 13982-78-0 & Mercury-203 & $<2.44 \mathrm{E}-1$ & pCi/g dry & $2.44 \mathrm{E}-1$ & & $12 / 15 / 08$ & 8L09007 & AGG-RRL-001 \\
\hline $14913-50-9$ & Thallium-208 & $<2.01 \mathrm{E}-1$ & pCi/g dry & $2.01 \mathrm{E}-1$ & & $12 / 15 / 08$ & 8L09007 & AGG-RRL-001 \\
\hline $14331-79-4$ & Bismuth-210 & $<3.83 \mathrm{E}-1$ & pCi/g dry & $3.83 \mathrm{E}-1$ & & $12 / 15 / 08$ & 8L09007 & AGG-RRL-001 \\
\hline \multirow[t]{3}{*}{$14255-04-0$} & Lead-210 & $<8.49 \mathrm{E} 1$ & pCi/g dry & $8.49 \mathrm{E} 1$ & & $12 / 15 / 08$ & 8L09007 & AGG-RRL-001 \\
\hline & Bismuth-211 & $<4.36 \mathrm{E} 0$ & pCi/g dry & $4.36 \mathrm{E} 0$ & & $12 / 15 / 08$ & 8L09007 & AGG-RRL-001 \\
\hline & Lead-211 & $<5.96 \mathrm{E} 0$ & pCi/g dry & $5.96 \mathrm{E} 0$ & & $12 / 15 / 08$ & 8L09007 & AGG-RRL-001 \\
\hline 15092-94-1 & Lead-212 & $<5.01 \mathrm{E}-1$ & pCi/g dry & $5.01 \mathrm{E}-1$ & & $12 / 15 / 08$ & 8L09007 & AGG-RRL-001 \\
\hline 14733-03-0 & Bismuth-214 & $<5.01 \mathrm{E}-1$ & pCi/g dry & $5.01 \mathrm{E}-1$ & & $12 / 15 / 08$ & 8L09007 & AGG-RRL-001 \\
\hline $15067-28-4$ & Lead-214 & $1.85 \mathrm{E} 0$ & pCi/g dry & $3.66 \mathrm{E}-1$ & $9.39 \mathrm{E}-1$ & $12 / 15 / 08$ & 8L09007 & AGG-RRL-001 \\
\hline $14835-02-0$ & Radon-219 & $<1.96 \mathrm{E} 0$ & pCi/g dry & $1.96 \mathrm{E} 0$ & & $12 / 15 / 08$ & 8L09007 & AGG-RRL-001 \\
\hline $22481-48-7$ & Radon-220 & $<1.74 \mathrm{E} 2$ & pCi/g dry & $1.74 \mathrm{E} 2$ & & $12 / 15 / 08$ & 8L09007 & AGG-RRL-001 \\
\hline $28522-20-5$ & Radon-221 & $<9.57 \mathrm{E}-1$ & pCi/g dry & $9.57 \mathrm{E}-1$ & & $12 / 15 / 08$ & 8L09007 & AGG-RRL-001 \\
\hline $15756-98-6$ & Francium-223 & $<6.84 \mathrm{E} 0$ & pCi/g dry & $6.84 \mathrm{E} 0$ & & $12 / 15 / 08$ & 8L09007 & AGG-RRL-001 \\
\hline $15623-45-7$ & Radium-223 & $<1.43 \mathrm{E} 0$ & pCi/g dry & $1.43 \mathrm{E} 0$ & & $12 / 15 / 08$ & 8L09007 & AGG-RRL-001 \\
\hline $13233-32-4$ & Radium-224 & $<5.99 \mathrm{E} 0$ & pCi/g dry & $5.99 \mathrm{E} 0$ & & $12 / 15 / 08$ & 8L09007 & AGG-RRL-001 \\
\hline $13982-63-3$ & Radium-226 & $<5.88 \mathrm{E} 0$ & pCi/g dry & $5.88 \mathrm{E} 0$ & & $12 / 15 / 08$ & 8L09007 & AGG-RRL-001 \\
\hline $15623-47-9$ & Thorium-227 & $<2.10 \mathrm{E} 0$ & pCi/g dry & $2.10 \mathrm{E} 0$ & & $12 / 15 / 08$ & 8L09007 & AGG-RRL-001 \\
\hline $14331-83-0$ & Actinium-228 & $<8.76 \mathrm{E}-1$ & $\mathrm{pCi} / \mathrm{g}$ dry & $8.76 \mathrm{E}-1$ & & $12 / 15 / 08$ & 8L09007 & AGG-RRL-001 \\
\hline $14274-82-9$ & Thorium-228 & $<7.44 \mathrm{E} 1$ & pCi/g dry & $7.44 \mathrm{E} 1$ & & $12 / 15 / 08$ & 8L09007 & AGG-RRL-001 \\
\hline $14269-63-7$ & Thorium-230 & $<9.82 \mathrm{E} 1$ & pCi/g dry & $9.82 \mathrm{E} 1$ & & $12 / 15 / 08$ & 8L09007 & AGG-RRL-001 \\
\hline $14331-85-2$ & Protactinium-231 & $<7.68 \mathrm{E} 0$ & pCi/g dry & $7.68 \mathrm{E} 0$ & & $12 / 15 / 08$ & 8L09007 & AGG-RRL-001 \\
\hline $14932-40-2$ & Thorium-231 & $<4.76 \mathrm{E} 1$ & pCi/g dry & $4.76 \mathrm{E} 1$ & & $12 / 15 / 08$ & 8L09007 & AGG-RRL-001 \\
\hline $7440-29-1$ & Thorium 232 & $<3.05 \mathrm{E} 2$ & pCi/g dry & $3.05 \mathrm{E} 2$ & & $12 / 15 / 08$ & 8L09007 & AGG-RRL-001 \\
\hline 13981-14-1 & Protactinium-233 & $<4.78 \mathrm{E}-1$ & pCi/g dry & $4.78 \mathrm{E}-1$ & & $12 / 15 / 08$ & 8L09007 & AGG-RRL-001 \\
\hline $15100-28-4$ & Protactinium-234 & $<8.55 \mathrm{E}-1$ & pCi/g dry & $8.55 \mathrm{E}-1$ & & $12 / 15 / 08$ & 8L09007 & AGG-RRL-001 \\
\hline
\end{tabular}


GEA/Soil

\begin{tabular}{|c|c|c|c|c|c|c|c|c|}
\hline CAS \# & Analyte & Results & Units & MDA & UNC & Analyzed & Batch & Method \\
\hline \multirow[t]{2}{*}{ HEIS No. } & B1V9B5 & \multicolumn{3}{|c|}{ Lab ID: $\quad$ 0805003-15 } & & & & \\
\hline & Protactinium-234m & $<2.20 \mathrm{E} 1$ & pCi/g dry & $2.20 \mathrm{E} 1$ & & $12 / 15 / 08$ & 8L09007 & AGG-RRL-001 \\
\hline $15065-10-8$ & Thorium-234 & $<7.98 \mathrm{E} 0$ & pCi/g dry & $7.98 \mathrm{E} 0$ & & $12 / 15 / 08$ & 8L09007 & AGG-RRL-001 \\
\hline 15117-96-1 & Uranium 235 & $<1.77 \mathrm{E} 0$ & pCi/g dry & $1.77 \mathrm{E} 0$ & & $12 / 15 / 08$ & 8L09007 & AGG-RRL-001 \\
\hline 13994-20-2 & Neptunium-237 & $<1.86 \mathrm{E} 0$ & pCi/g dry & $1.86 \mathrm{E} 0$ & & $12 / 15 / 08$ & 8L09007 & AGG-RRL-001 \\
\hline \multirow[t]{2}{*}{ 13981-16-3 } & Plutonium-238 & $<2.67 \mathrm{E} 3$ & $\mathrm{pCi} / \mathrm{g}$ dry & $2.67 \mathrm{E} 3$ & & $12 / 15 / 08$ & 8L09007 & AGG-RRL-001 \\
\hline & Uranium 238 & $<4.14 \mathrm{E} 0$ & pCi/g dry & $4.14 \mathrm{E} 0$ & & $12 / 15 / 08$ & 8L09007 & AGG-RRL-001 \\
\hline $15117-48-3$ & Plutonium-239 & $<3.07 \mathrm{E} 3$ & $\mathrm{pCi} / \mathrm{g}$ dry & $3.07 \mathrm{E} 3$ & & $12 / 15 / 08$ & 8L09007 & AGG-RRL-001 \\
\hline $14119-33-6$ & Plutonium-240 & $<2.75 \mathrm{E} 3$ & pCi $/ g$ dry & $2.75 \mathrm{E} 3$ & & $12 / 15 / 08$ & 8L09007 & AGG-RRL-001 \\
\hline $14596-10-2$ & Americium-241 & $<1.54 \mathrm{E} 0$ & $\mathrm{pCi} / \mathrm{g}$ dry & $1.54 \mathrm{E} 0$ & & $12 / 15 / 08$ & 8L09007 & AGG-RRL-001 \\
\hline $14993-75-0$ & Americium-243 & $<5.38 \mathrm{E}-1$ & $\mathrm{pCi} / \mathrm{g}$ dry & $5.38 \mathrm{E}-1$ & & $12 / 15 / 08$ & 8L09007 & AGG-RRL-001 \\
\hline $15757-87-6$ & Curium-243 & $<8.39 \mathrm{E}-1$ & pCi/g dry & $8.39 \mathrm{E}-1$ & & $12 / 15 / 08$ & 8L09007 & AGG-RRL-001 \\
\hline $15621-76-8$ & Curium-245 & $<6.90 \mathrm{E}-1$ & $\mathrm{pCi} / \mathrm{g}$ dry & $6.90 \mathrm{E}-1$ & & $12 / 15 / 08$ & 8L09007 & AGG-RRL-001 \\
\hline HEIS No. & B1V9B7 & \multicolumn{3}{|c|}{ Lab ID: $\quad$ 0805003-17 } & & & & \\
\hline $13966-32-0$ & Sodium-22 & $<2.52 \mathrm{E}-1$ & $\mathrm{pCi} / \mathrm{g}$ dry & $2.52 \mathrm{E}-1$ & & $12 / 15 / 08$ & 8L09007 & AGG-RRL-001 \\
\hline 13966-00-2 & Potassium-40 & $1.84 \mathrm{E} 1$ & pCi/g dry & $1.92 \mathrm{E} 0$ & $8.75 \mathrm{E}-1$ & $12 / 15 / 08$ & 8L09007 & AGG-RRL-001 \\
\hline $14392-02-0$ & Chromium-51 & $<2.39 \mathrm{E} 0$ & pCi/g dry & $2.39 \mathrm{E} 0$ & & $12 / 15 / 08$ & 8L09007 & AGG-RRL-001 \\
\hline 13966-31-9 & Manganese-54 & $<2.38 \mathrm{E}-1$ & pCi/g dry & $2.38 \mathrm{E}-1$ & & $12 / 15 / 08$ & 8L09007 & AGG-RRL-001 \\
\hline $13981-50-5$ & Cobalt-57 & $<2.78 \mathrm{E}-1$ & $\mathrm{pCi} / \mathrm{g}$ dry & $2.78 \mathrm{E}-1$ & & $12 / 15 / 08$ & 8L09007 & AGG-RRL-001 \\
\hline $14596-12-4$ & Iron-59 & $<4.21 \mathrm{E}-1$ & pCi/g dry & $4.21 \mathrm{E}-1$ & & $12 / 15 / 08$ & 8L09007 & AGG-RRL-001 \\
\hline $10198-40-0$ & Cobalt-60 & $<2.17 \mathrm{E}-1$ & pCi/g dry & $2.17 \mathrm{E}-1$ & & $12 / 15 / 08$ & 8L09007 & AGG-RRL-001 \\
\hline 13982-39-3 & Zinc-65 & $<5.50 \mathrm{E}-1$ & pCi/g dry & $5.50 \mathrm{E}-1$ & & $12 / 15 / 08$ & 8L09007 & AGG-RRL-001 \\
\hline $14265-71-5$ & Selenium-75 & $<4.00 \mathrm{E}-1$ & pCi/g dry & $4.00 \mathrm{E}-1$ & & $12 / 15 / 08$ & 8L09007 & AGG-RRL-001 \\
\hline 17056-36-9 & Rubidium-83 & $<5.90 \mathrm{E}-1$ & pCi/g dry & $5.90 \mathrm{E}-1$ & & $12 / 15 / 08$ & 8L09007 & AGG-RRL-001 \\
\hline $13967-73-2$ & Strontium-85 & $<2.68 \mathrm{E}-1$ & pCi/g dry & $2.68 \mathrm{E}-1$ & & $12 / 15 / 08$ & 8L09007 & AGG-RRL-001 \\
\hline $14932-53-7$ & Rubidium-86 & $<2.79 \mathrm{E} 0$ & pCi/g dry & $2.79 \mathrm{E} 0$ & & $12 / 15 / 08$ & 8L09007 & AGG-RRL-001 \\
\hline 13982-36-0 & Yttrium-88 & $<1.91 \mathrm{E}-1$ & $\mathrm{pCi} / \mathrm{g}$ dry & $1.91 \mathrm{E}-1$ & & $12 / 15 / 08$ & 8L09007 & AGG-RRL-001 \\
\hline 14681-63-1 & Niobium-94 & $<2.34 \mathrm{E}-1$ & pCi/g dry & $2.34 \mathrm{E}-1$ & & $12 / 15 / 08$ & 8L09007 & AGG-RRL-001 \\
\hline \multirow[t]{3}{*}{$13967-76-5$} & Niobium-95 & $<2.34 \mathrm{E}-1$ & pCi/g dry & $2.34 \mathrm{E}-1$ & & $12 / 15 / 08$ & 8L09007 & AGG-RRL-001 \\
\hline & Niobium-95m & $<1.16 \mathrm{E} 0$ & pCi/g dry & $1.16 \mathrm{E} 0$ & & $12 / 15 / 08$ & 8L09007 & AGG-RRL-001 \\
\hline & Technetium-95m & $<3.89 \mathrm{E}-1$ & $\mathrm{pCi} / \mathrm{g}$ dry & $3.89 \mathrm{E}-1$ & & $12 / 15 / 08$ & 8L09007 & AGG-RRL-001 \\
\hline \multirow[t]{2}{*}{ 13967-71-0 } & Zirconium-95 & $<4.21 \mathrm{E}-1$ & pCi/g dry & $4.21 \mathrm{E}-1$ & & $12 / 15 / 08$ & 8L09007 & AGG-RRL-001 \\
\hline & Technetium-99m & $<2.65 \mathrm{E}-1$ & pCi/g dry & $2.65 \mathrm{E}-1$ & & $12 / 15 / 08$ & 8L09007 & AGG-RRL-001 \\
\hline 13968-53-1 & Ruthenium-103 & $<2.65 \mathrm{E}-1$ & pCi/g dry & $2.65 \mathrm{E}-1$ & & $12 / 15 / 08$ & 8L09007 & AGG-RRL-001 \\
\hline $13967-48-1$ & Ruthenium-106 & $<2.82 \mathrm{E} 0$ & pCi/g dry & $2.82 \mathrm{E} 0$ & & $12 / 15 / 08$ & 8L09007 & AGG-RRL-001 \\
\hline $14391-65-2$ & Silver-108m & $<2.50 \mathrm{E}-1$ & pCi/g dry & $2.50 \mathrm{E}-1$ & & $12 / 15 / 08$ & 8L09007 & AGG-RRL-001 \\
\hline \multirow[t]{2}{*}{$14109-32-1$} & Cadmium-109 & $<8.58 \mathrm{E} 0$ & pCi/g dry & $8.58 \mathrm{E} 0$ & & $12 / 15 / 08$ & 8L09007 & AGG-RRL-001 \\
\hline & Silver-110 & $<2.46 \mathrm{E}-1$ & pCi/g dry & $2.46 \mathrm{E}-1$ & & $12 / 15 / 08$ & 8L09007 & AGG-RRL-001 \\
\hline 14391-76-5 & Silver-110m & $<2.47 \mathrm{E}-1$ & pCi/g dry & $2.47 \mathrm{E}-1$ & & $12 / 15 / 08$ & 8L09007 & AGG-RRL-001 \\
\hline $13966-06-8$ & Tin-113 & $<3.47 \mathrm{E}-1$ & pCi/g dry & $3.47 \mathrm{E}-1$ & & $12 / 15 / 08$ & 8L09007 & AGG-RRL-001 \\
\hline $14683-10-4$ & Antimony-124 & $<2.88 \mathrm{E}-1$ & pCi/g dry & $2.88 \mathrm{E}-1$ & & $12 / 15 / 08$ & 8L09007 & AGG-RRL-001 \\
\hline $14234-35-6$ & Antimony-125 & $<7.64 \mathrm{E}-1$ & $\mathrm{pCi} / \mathrm{g}$ dry & $7.64 \mathrm{E}-1$ & & $12 / 15 / 08$ & 8L09007 & AGG-RRL-001 \\
\hline $15756-32-8$ & Antimony-126 & $<2.42 \mathrm{E}-1$ & pCi/g dry & $2.42 \mathrm{E}-1$ & & $12 / 15 / 08$ & 8L09007 & AGG-RRL-001 \\
\hline $15832-50-5$ & Tin-126 & $<7.04 \mathrm{E}-1$ & pCi/g dry & 7.04E-1 & & $12 / 15 / 08$ & 8L09007 & AGG-RRL-001 \\
\hline $10043-66-0$ & Iodine-131 & $<3.09 \mathrm{E}-1$ & pCi/g dry & $3.09 \mathrm{E}-1$ & & $12 / 15 / 08$ & 8L09007 & AGG-RRL-001 \\
\hline 13981-41-4 & Barium-133 & $<4.40 \mathrm{E}-1$ & pCi/g dry & $4.40 \mathrm{E}-1$ & & $12 / 15 / 08$ & 8L09007 & AGG-RRL-001 \\
\hline 13967-70-9 & Cesium-134 & $<2.69 \mathrm{E}-1$ & pCi/g dry & $2.69 \mathrm{E}-1$ & & $12 / 15 / 08$ & 8L09007 & AGG-RRL-001 \\
\hline $10045-97-3$ & Cesium-137 & $<2.82 \mathrm{E}-1$ & pCi/g dry & $2.82 \mathrm{E}-1$ & & $12 / 15 / 08$ & 8L09007 & AGG-RRL-001 \\
\hline $13982-30-4$ & Cerium-139 & $<2.99 \mathrm{E}-1$ & pCi/g dry & $2.99 \mathrm{E}-1$ & & $12 / 15 / 08$ & 8L09007 & AGG-RRL-001 \\
\hline
\end{tabular}


GEA/Soil

\begin{tabular}{|c|c|c|c|c|c|c|c|c|}
\hline CAS \# & Analyte & Results & Units & MDA & UNC & Analyzed & Batch & Method \\
\hline HEIS No. & B1V9B7 & & b ID: & $5003-17$ & & & & \\
\hline $14762-78-8$ & Cerium-144 & $<2.18 \mathrm{E} 0$ & pCi/g dry & $2.18 \mathrm{E} 0$ & & $12 / 15 / 08$ & 8L09007 & AGG-RRL-001 \\
\hline $14683-23-9$ & Europium-152 & $<1.05 \mathrm{E} 0$ & pCi/g dry & $1.05 \mathrm{E} 0$ & & $12 / 15 / 08$ & 8L09007 & AGG-RRL-001 \\
\hline $14276-65-4$ & Gadolinium-153 & $<9.15 \mathrm{E}-1$ & pCi/g dry & $9.15 \mathrm{E}-1$ & & $12 / 15 / 08$ & 8L09007 & AGG-RRL-001 \\
\hline $15585-10-1$ & Europium-154 & $<5.83 \mathrm{E}-1$ & pCi $/ g$ dry & $5.83 \mathrm{E}-1$ & & $12 / 15 / 08$ & 8L09007 & AGG-RRL-001 \\
\hline 14391-16-3 & Europium-155 & $<9.99 \mathrm{E}-1$ & pCi $/ g$ dry & $9.99 \mathrm{E}-1$ & & $12 / 15 / 08$ & 8L09007 & AGG-RRL-001 \\
\hline 13982-78-0 & Mercury-203 & $<3.01 \mathrm{E}-1$ & pCi/g dry & 3.01E-1 & & $12 / 15 / 08$ & 8L09007 & AGG-RRL-001 \\
\hline $14913-50-9$ & Thallium-208 & $<3.24 \mathrm{E}-1$ & pCi/g dry & $3.24 \mathrm{E}-1$ & & $12 / 15 / 08$ & 8L09007 & AGG-RRL-001 \\
\hline $14331-79-4$ & Bismuth-210 & $<4.77 \mathrm{E}-1$ & pCi $/ g$ dry & $4.77 \mathrm{E}-1$ & & $12 / 15 / 08$ & 8L09007 & AGG-RRL-001 \\
\hline \multirow[t]{3}{*}{$14255-04-0$} & Lead-210 & $<5.04 \mathrm{E} 1$ & pCi/g dry & $5.04 \mathrm{E} 1$ & & $12 / 15 / 08$ & 8L09007 & AGG-RRL-001 \\
\hline & Bismuth-211 & $<5.66 \mathrm{E} 0$ & $\mathrm{pCi} / \mathrm{g}$ dry & $5.66 \mathrm{E} 0$ & & $12 / 15 / 08$ & 8L09007 & AGG-RRL-001 \\
\hline & Lead-211 & $<7.74 \mathrm{E} 0$ & pCi/g dry & 7.74E0 & & $12 / 15 / 08$ & 8L09007 & AGG-RRL-001 \\
\hline 15092-94-1 & Lead-212 & $<6.74 \mathrm{E}-1$ & pCi/g dry & $6.74 \mathrm{E}-1$ & & $12 / 15 / 08$ & 8L09007 & AGG-RRL-001 \\
\hline 14733-03-0 & Bismuth-214 & $<6.08 \mathrm{E}-1$ & pCi $/ g$ dry & $6.08 \mathrm{E}-1$ & & $12 / 15 / 08$ & 8L09007 & AGG-RRL-001 \\
\hline $15067-28-4$ & Lead-214 & $<7.17 \mathrm{E}-1$ & pCi/g dry & 7.17E-1 & & $12 / 15 / 08$ & 8L09007 & AGG-RRL-001 \\
\hline $14835-02-0$ & Radon-219 & $<2.38 \mathrm{E} 0$ & pCi/g dry & $2.38 \mathrm{E} 0$ & & $12 / 15 / 08$ & 8L09007 & AGG-RRL-001 \\
\hline $22481-48-7$ & Radon-220 & $<2.23 \mathrm{E} 2$ & pCi/g dry & $2.23 \mathrm{E} 2$ & & $12 / 15 / 08$ & 8L09007 & AGG-RRL-001 \\
\hline $28522-20-5$ & Radon-221 & $<1.14 \mathrm{E} 0$ & pCi/g dry & $1.14 \mathrm{E} 0$ & & $12 / 15 / 08$ & 8L09007 & AGG-RRL-001 \\
\hline $15756-98-6$ & Francium-223 & $<8.49 \mathrm{E} 0$ & pCi/g dry & $8.49 \mathrm{E} 0$ & & $12 / 15 / 08$ & 8L09007 & AGG-RRL-001 \\
\hline $15623-45-7$ & Radium-223 & $<1.74 \mathrm{E} 0$ & pCi $/ g$ dry & $1.74 \mathrm{E} 0$ & & $12 / 15 / 08$ & 8L09007 & AGG-RRL-001 \\
\hline $13233-32-4$ & Radium-224 & $<7.31 \mathrm{E} 0$ & pCi/g dry & 7.31E0 & & $12 / 15 / 08$ & 8L09007 & AGG-RRL-001 \\
\hline $13982-63-3$ & Radium-226 & $<7.03 \mathrm{E} 0$ & $\mathrm{pCi} / \mathrm{g}$ dry & 7.03E0 & & $12 / 15 / 08$ & 8L09007 & AGG-RRL-001 \\
\hline $15623-47-9$ & Thorium-227 & $<2.59 \mathrm{E} 0$ & $\mathrm{pCi} / \mathrm{g}$ dry & $2.59 \mathrm{E} 0$ & & $12 / 15 / 08$ & 8L09007 & AGG-RRL-001 \\
\hline $14331-83-0$ & Actinium-228 & $<1.05 \mathrm{E} 0$ & pCi/g dry & $1.05 \mathrm{E} 0$ & & $12 / 15 / 08$ & 8L09007 & AGG-RRL-001 \\
\hline $14274-82-9$ & Thorium-228 & $<9.19 \mathrm{E} 1$ & $\mathrm{pCi} / \mathrm{g}$ dry & $9.19 \mathrm{E} 1$ & & $12 / 15 / 08$ & 8L09007 & AGG-RRL-001 \\
\hline $14269-63-7$ & Thorium-230 & $<1.23 \mathrm{E} 2$ & $\mathrm{pCi} / \mathrm{g}$ dry & $1.23 \mathrm{E} 2$ & & $12 / 15 / 08$ & 8L09007 & AGG-RRL-001 \\
\hline $14331-85-2$ & Protactinium-231 & $<1.09 \mathrm{E} 1$ & $\mathrm{pCi} / \mathrm{g}$ dry & $1.09 \mathrm{E} 1$ & & $12 / 15 / 08$ & 8L09007 & AGG-RRL-001 \\
\hline $14932-40-2$ & Thorium-231 & $<6.38 \mathrm{E} 1$ & pCi $/ g$ dry & $6.38 \mathrm{E} 1$ & & $12 / 15 / 08$ & 8L09007 & AGG-RRL-001 \\
\hline $7440-29-1$ & Thorium 232 & $<3.21 \mathrm{E} 2$ & $\mathrm{pCi} / \mathrm{g}$ dry & $3.21 \mathrm{E} 2$ & & $12 / 15 / 08$ & 8L09007 & AGG-RRL-001 \\
\hline 13981-14-1 & Protactinium-233 & $<6.51 \mathrm{E}-1$ & $\mathrm{pCi} / \mathrm{g}$ dry & $6.51 \mathrm{E}-1$ & & $12 / 15 / 08$ & 8L09007 & AGG-RRL-001 \\
\hline \multirow[t]{2}{*}{$15100-28-4$} & Protactinium-234 & $<1.19 \mathrm{E} 0$ & pCi/g dry & $1.19 \mathrm{E} 0$ & & $12 / 15 / 08$ & 8L09007 & AGG-RRL-001 \\
\hline & Protactinium-234m & $<2.83 \mathrm{E} 1$ & pCi/g dry & $2.83 \mathrm{E} 1$ & & $12 / 15 / 08$ & 8L09007 & AGG-RRL-001 \\
\hline $15065-10-8$ & Thorium-234 & $<1.05 \mathrm{E} 1$ & $\mathrm{pCi} / \mathrm{g}$ dry & $1.05 \mathrm{E} 1$ & & $12 / 15 / 08$ & 8L09007 & AGG-RRL-001 \\
\hline 15117-96-1 & Uranium 235 & $<2.26 \mathrm{E} 0$ & pCi/g dry & $2.26 \mathrm{E} 0$ & & $12 / 15 / 08$ & 8L09007 & AGG-RRL-001 \\
\hline $13994-20-2$ & Neptunium-237 & $<2.60 \mathrm{E} 0$ & pCi/g dry & $2.60 \mathrm{E} 0$ & & $12 / 15 / 08$ & 8L09007 & AGG-RRL-001 \\
\hline \multirow[t]{2}{*}{ 13981-16-3 } & Plutonium-238 & $<3.71 \mathrm{E} 3$ & pCi/g dry & $3.71 \mathrm{E} 3$ & & $12 / 15 / 08$ & 8L09007 & AGG-RRL-001 \\
\hline & Uranium 238 & $<5.41 \mathrm{E} 0$ & pCi/g dry & $5.41 \mathrm{E} 0$ & & $12 / 15 / 08$ & 8L09007 & AGG-RRL-001 \\
\hline $15117-48-3$ & Plutonium-239 & $<3.81 \mathrm{E} 3$ & $\mathrm{pCi} / \mathrm{g}$ dry & $3.81 \mathrm{E} 3$ & & $12 / 15 / 08$ & 8L09007 & AGG-RRL-001 \\
\hline $14119-33-6$ & Plutonium-240 & $<3.68 \mathrm{E} 3$ & $\mathrm{pCi} / \mathrm{g}$ dry & $3.68 \mathrm{E} 3$ & & $12 / 15 / 08$ & 8L09007 & AGG-RRL-001 \\
\hline $14596-10-2$ & Americium-241 & $<1.64 \mathrm{E} 0$ & pCi/g dry & $1.64 \mathrm{E} 0$ & & $12 / 15 / 08$ & 8L09007 & AGG-RRL-001 \\
\hline $14993-75-0$ & Americium-243 & $<6.55 \mathrm{E}-1$ & $\mathrm{pCi} / \mathrm{g}$ dry & $6.55 \mathrm{E}-1$ & & $12 / 15 / 08$ & 8L09007 & AGG-RRL-001 \\
\hline $15757-87-6$ & Curium-243 & $<1.12 \mathrm{E} 0$ & $\mathrm{pCi} / \mathrm{g}$ dry & $1.12 \mathrm{E} 0$ & & $12 / 15 / 08$ & 8L09007 & AGG-RRL-001 \\
\hline $15621-76-8$ & Curium-245 & $<9.21 \mathrm{E}-1$ & pCi/g dry & $9.21 \mathrm{E}-1$ & & $12 / 15 / 08$ & 8L09007 & AGG-RRL-001 \\
\hline HEIS No. & B1V9B9 & & b ID: & 5003-19 & & & & \\
\hline $13966-32-0$ & Sodium-22 & $<1.93 \mathrm{E}-1$ & pCi/g dry & $1.93 \mathrm{E}-1$ & & $12 / 16 / 08$ & 8L09007 & AGG-RRL-001 \\
\hline $13966-00-2$ & Potassium-40 & $1.98 \mathrm{E} 1$ & $\mathrm{pCi} / \mathrm{g}$ dry & $1.79 \mathrm{E} 0$ & $9.10 \mathrm{E}-1$ & $12 / 16 / 08$ & 8L09007 & AGG-RRL-001 \\
\hline $14392-02-0$ & Chromium-51 & $<1.95 \mathrm{E} 0$ & pCi/g dry & $1.95 \mathrm{E} 0$ & & $12 / 16 / 08$ & 8L09007 & AGG-RRL-001 \\
\hline 13966-31-9 & Manganese-54 & $<1.76 \mathrm{E}-1$ & pCi $/ g$ dry & $1.76 \mathrm{E}-1$ & & $12 / 16 / 08$ & 8L09007 & AGG-RRL-001 \\
\hline $13981-50-5$ & Cobalt-57 & $<2.28 \mathrm{E}-1$ & pCi/g dry & $2.28 \mathrm{E}-1$ & & $12 / 16 / 08$ & 8L09007 & AGG-RRL-001 \\
\hline
\end{tabular}


GEA/Soil

\begin{tabular}{|c|c|c|c|c|c|c|c|c|}
\hline CAS \# & Analyte & Results & Units & MDA & UNC & Analyzed & Batch & Method \\
\hline HEIS No. & B1V9B9 & & ab ID: & $5003-19$ & & & & \\
\hline $14596-12-4$ & Iron-59 & $<3.60 \mathrm{E}-1$ & pCi/g dry & $3.60 \mathrm{E}-1$ & & $12 / 16 / 08$ & 8L09007 & AGG-RRL-001 \\
\hline 10198-40-0 & Cobalt-60 & $<1.75 \mathrm{E}-1$ & pCi/g dry & $1.75 \mathrm{E}-1$ & & $12 / 16 / 08$ & 8L09007 & AGG-RRL-001 \\
\hline $13982-39-3$ & Zinc-65 & $<4.75 \mathrm{E}-1$ & pCi $/ g$ dry & $4.75 \mathrm{E}-1$ & & $12 / 16 / 08$ & 8L09007 & AGG-RRL-001 \\
\hline $14265-71-5$ & Selenium-75 & $<3.41 \mathrm{E}-1$ & pCi/g dry & $3.41 \mathrm{E}-1$ & & $12 / 16 / 08$ & 8L09007 & AGG-RRL-001 \\
\hline $17056-36-9$ & Rubidium-83 & $<3.81 \mathrm{E}-1$ & pCi/g dry & $3.81 \mathrm{E}-1$ & & $12 / 16 / 08$ & 8L09007 & AGG-RRL-001 \\
\hline 13967-73-2 & Strontium-85 & $<2.47 \mathrm{E}-1$ & pCi/g dry & $2.47 \mathrm{E}-1$ & & $12 / 16 / 08$ & 8L09007 & AGG-RRL-001 \\
\hline $14932-53-7$ & Rubidium-86 & $<2.29 \mathrm{E} 0$ & pCi/g dry & $2.29 \mathrm{E} 0$ & & $12 / 16 / 08$ & 8L09007 & AGG-RRL-001 \\
\hline 13982-36-0 & Yttrium-88 & $<1.58 \mathrm{E}-1$ & pCi/g dry & $1.58 \mathrm{E}-1$ & & $12 / 16 / 08$ & 8L09007 & AGG-RRL-001 \\
\hline 14681-63-1 & Niobium-94 & $<1.83 \mathrm{E}-1$ & pCi/g dry & $1.83 \mathrm{E}-1$ & & $12 / 16 / 08$ & 8L09007 & AGG-RRL-001 \\
\hline \multirow[t]{3}{*}{$13967-76-5$} & Niobium-95 & $<2.03 \mathrm{E}-1$ & pCi/g dry & $2.03 \mathrm{E}-1$ & & $12 / 16 / 08$ & 8L09007 & AGG-RRL-001 \\
\hline & Niobium-95m & $<1.00 \mathrm{E} 0$ & pCi/g dry & $1.00 \mathrm{E} 0$ & & $12 / 16 / 08$ & 8L09007 & AGG-RRL-001 \\
\hline & Technetium-95m & $<3.28 \mathrm{E}-1$ & pCi/g dry & $3.28 \mathrm{E}-1$ & & $12 / 16 / 08$ & 8L09007 & AGG-RRL-001 \\
\hline \multirow[t]{2}{*}{ 13967-71-0 } & Zirconium-95 & $<3.38 \mathrm{E}-1$ & pCi/g dry & $3.38 \mathrm{E}-1$ & & $12 / 16 / 08$ & 8L09007 & AGG-RRL-001 \\
\hline & Technetium-99m & $<2.19 \mathrm{E}-1$ & pCi/g dry & $2.19 \mathrm{E}-1$ & & $12 / 16 / 08$ & 8L09007 & AGG-RRL-001 \\
\hline $13968-53-1$ & Ruthenium-103 & $<2.08 \mathrm{E}-1$ & $\mathrm{pCi} / \mathrm{g}$ dry & $2.08 \mathrm{E}-1$ & & $12 / 16 / 08$ & 8L09007 & AGG-RRL-001 \\
\hline 13967-48-1 & Ruthenium-106 & $<1.96 \mathrm{E} 0$ & pCi/g dry & $1.96 \mathrm{E} 0$ & & $12 / 16 / 08$ & 8L09007 & AGG-RRL-001 \\
\hline $14391-65-2$ & Silver-108m & $<1.99 \mathrm{E}-1$ & pCi/g dry & $1.99 \mathrm{E}-1$ & & $12 / 16 / 08$ & 8L09007 & AGG-RRL-001 \\
\hline \multirow[t]{2}{*}{$14109-32-1$} & Cadmium-109 & $<6.68 \mathrm{E} 0$ & pCi/g dry & $6.68 \mathrm{E} 0$ & & $12 / 16 / 08$ & 8L09007 & AGG-RRL-001 \\
\hline & Silver-110 & $<1.97 \mathrm{E}-1$ & pCi/g dry & $1.97 \mathrm{E}-1$ & & $12 / 16 / 08$ & 8L09007 & AGG-RRL-001 \\
\hline $14391-76-5$ & Silver-110m & $<1.97 \mathrm{E}-1$ & pCi/g dry & $1.97 \mathrm{E}-1$ & & $12 / 16 / 08$ & 8L09007 & AGG-RRL-001 \\
\hline 13966-06-8 & Tin-113 & $<2.94 \mathrm{E}-1$ & pCi/g dry & $2.94 \mathrm{E}-1$ & & $12 / 16 / 08$ & 8L09007 & AGG-RRL-001 \\
\hline 14683-10-4 & Antimony-124 & $<1.87 \mathrm{E}-1$ & pCi/g dry & $1.87 \mathrm{E}-1$ & & $12 / 16 / 08$ & 8L09007 & AGG-RRL-001 \\
\hline $14234-35-6$ & Antimony-125 & $<6.28 \mathrm{E}-1$ & pCi/g dry & $6.28 \mathrm{E}-1$ & & $12 / 16 / 08$ & 8L09007 & AGG-RRL-001 \\
\hline $15756-32-8$ & Antimony-126 & $<1.93 \mathrm{E}-1$ & pCi/g dry & $1.93 \mathrm{E}-1$ & & $12 / 16 / 08$ & 8L09007 & AGG-RRL-001 \\
\hline $15832-50-5$ & Tin-126 & $<5.30 \mathrm{E}-1$ & pCi/g dry & $5.30 \mathrm{E}-1$ & & $12 / 16 / 08$ & 8L09007 & AGG-RRL-001 \\
\hline 10043-66-0 & Iodine-131 & $<2.41 \mathrm{E}-1$ & pCi/g dry & $2.41 \mathrm{E}-1$ & & $12 / 16 / 08$ & 8L09007 & AGG-RRL-001 \\
\hline 13981-41-4 & Barium-133 & $<3.85 \mathrm{E}-1$ & pCi/g dry & $3.85 \mathrm{E}-1$ & & $12 / 16 / 08$ & 8L09007 & AGG-RRL-001 \\
\hline 13967-70-9 & Cesium-134 & $<2.23 \mathrm{E}-1$ & pCi/g dry & $2.23 \mathrm{E}-1$ & & $12 / 16 / 08$ & 8L09007 & AGG-RRL-001 \\
\hline $10045-97-3$ & Cesium-137 & $<2.17 \mathrm{E}-1$ & pCi/g dry & $2.17 \mathrm{E}-1$ & & $12 / 16 / 08$ & 8L09007 & AGG-RRL-001 \\
\hline 13982-30-4 & Cerium-139 & $<2.50 \mathrm{E}-1$ & pCi/g dry & $2.50 \mathrm{E}-1$ & & $12 / 16 / 08$ & 8L09007 & AGG-RRL-001 \\
\hline $14762-78-8$ & Cerium-144 & $<1.80 \mathrm{E} 0$ & pCi/g dry & $1.80 \mathrm{E} 0$ & & $12 / 16 / 08$ & 8L09007 & AGG-RRL-001 \\
\hline $14683-23-9$ & Europium-152 & $<7.56 \mathrm{E}-1$ & pCi/g dry & $7.56 \mathrm{E}-1$ & & $12 / 16 / 08$ & 8L09007 & AGG-RRL-001 \\
\hline $14276-65-4$ & Gadolinium-153 & $<7.03 \mathrm{E}-1$ & pCi/g dry & 7.03E-1 & & $12 / 16 / 08$ & 8L09007 & AGG-RRL-001 \\
\hline $15585-10-1$ & Europium-154 & $<4.78 \mathrm{E}-1$ & pCi/g dry & $4.78 \mathrm{E}-1$ & & $12 / 16 / 08$ & 8L09007 & AGG-RRL-001 \\
\hline 14391-16-3 & Europium-155 & $<7.50 \mathrm{E}-1$ & pCi/g dry & $7.50 \mathrm{E}-1$ & & $12 / 16 / 08$ & 8L09007 & AGG-RRL-001 \\
\hline $13982-78-0$ & Mercury-203 & $<2.56 \mathrm{E}-1$ & pCi/g dry & $2.56 \mathrm{E}-1$ & & $12 / 16 / 08$ & 8L09007 & AGG-RRL-001 \\
\hline 14913-50-9 & Thallium-208 & $<2.66 \mathrm{E}-1$ & pCi/g dry & $2.66 \mathrm{E}-1$ & & $12 / 16 / 08$ & 8L09007 & AGG-RRL-001 \\
\hline 14331-79-4 & Bismuth-210 & $<4.06 \mathrm{E}-1$ & pCi/g dry & $4.06 \mathrm{E}-1$ & & $12 / 16 / 08$ & 8L09007 & AGG-RRL-001 \\
\hline \multirow[t]{3}{*}{$14255-04-0$} & Lead-210 & $<9.46 \mathrm{E} 1$ & pCi/g dry & $9.46 \mathrm{E} 1$ & & $12 / 16 / 08$ & 8L09007 & AGG-RRL-001 \\
\hline & Bismuth-211 & $<4.74 \mathrm{E} 0$ & pCi/g dry & $4.74 \mathrm{E} 0$ & & $12 / 16 / 08$ & 8L09007 & AGG-RRL-001 \\
\hline & Lead-211 & $<6.47 \mathrm{E} 0$ & pCi/g dry & $6.47 \mathrm{E} 0$ & & $12 / 16 / 08$ & 8L09007 & AGG-RRL-001 \\
\hline 15092-94-1 & Lead-212 & $<5.00 \mathrm{E}-1$ & pCi/g dry & $5.00 \mathrm{E}-1$ & & $12 / 16 / 08$ & 8L09007 & AGG-RRL-001 \\
\hline 14733-03-0 & Bismuth-214 & $<5.52 \mathrm{E}-1$ & pCi/g dry & $5.52 \mathrm{E}-1$ & & $12 / 16 / 08$ & 8L09007 & AGG-RRL-001 \\
\hline $15067-28-4$ & Lead-214 & $<6.12 \mathrm{E}-1$ & pCi/g dry & $6.12 \mathrm{E}-1$ & & $12 / 16 / 08$ & 8L09007 & AGG-RRL-001 \\
\hline $14835-02-0$ & Radon-219 & $<2.07 \mathrm{E} 0$ & pCi/g dry & $2.07 \mathrm{E} 0$ & & $12 / 16 / 08$ & 8L09007 & AGG-RRL-001 \\
\hline $22481-48-7$ & Radon-220 & $<1.74 \mathrm{E} 2$ & pCi/g dry & $1.74 \mathrm{E} 2$ & & $12 / 16 / 08$ & 8L09007 & AGG-RRL-001 \\
\hline $28522-20-5$ & Radon-221 & $<9.93 \mathrm{E}-1$ & pCi/g dry & $9.93 \mathrm{E}-1$ & & $12 / 16 / 08$ & 8L09007 & AGG-RRL-001 \\
\hline 15756-98-6 & Francium-223 & $<7.23 \mathrm{E} 0$ & pCi/g dry & $7.23 \mathrm{E} 0$ & & $12 / 16 / 08$ & 8L09007 & AGG-RRL-001 \\
\hline $15623-45-7$ & Radium-223 & $<1.50 \mathrm{E} 0$ & pCi/g dry & $1.50 \mathrm{E} 0$ & & $12 / 16 / 08$ & 8L09007 & AGG-RRL-001 \\
\hline
\end{tabular}


GEA/Soil

\begin{tabular}{|c|c|c|c|c|c|c|c|c|}
\hline CAS \# & Analyte & Results & Units & MDA & $\mathrm{UNC}$ & Analyzed & Batch & Method \\
\hline HEIS No. & B1V9B9 & \multicolumn{3}{|c|}{ Lab ID: $\quad$ 0805003-19 } & & & & \\
\hline $13233-32-4$ & Radium-224 & $<6.29 \mathrm{E} 0$ & pCi/g dry & $6.29 \mathrm{E} 0$ & & $12 / 16 / 08$ & 8L09007 & AGG-RRL-001 \\
\hline $13982-63-3$ & Radium-226 & $<6.12 \mathrm{E} 0$ & pCi/g dry & $6.12 \mathrm{E} 0$ & & $12 / 16 / 08$ & 8L09007 & AGG-RRL-001 \\
\hline $15623-47-9$ & Thorium-227 & $<2.25 \mathrm{E} 0$ & pCi/g dry & $2.25 \mathrm{E} 0$ & & $12 / 16 / 08$ & 8L09007 & AGG-RRL-001 \\
\hline $14331-83-0$ & Actinium-228 & $<8.82 \mathrm{E}-1$ & pCi/g dry & $8.82 \mathrm{E}-1$ & & $12 / 16 / 08$ & 8L09007 & AGG-RRL-001 \\
\hline $14274-82-9$ & Thorium-228 & $<7.70 \mathrm{E} 1$ & pCi/g dry & $7.70 \mathrm{E} 1$ & & $12 / 16 / 08$ & 8L09007 & AGG-RRL-001 \\
\hline $14269-63-7$ & Thorium-230 & $<1.04 \mathrm{E} 2$ & pCi/g dry & $1.04 \mathrm{E} 2$ & & $12 / 16 / 08$ & 8L09007 & AGG-RRL-001 \\
\hline $14331-85-2$ & Protactinium-231 & $<8.14 \mathrm{E} 0$ & pCi/g dry & $8.14 \mathrm{E} 0$ & & $12 / 16 / 08$ & 8L09007 & AGG-RRL-001 \\
\hline $14932-40-2$ & Thorium-231 & $<4.98 \mathrm{E} 1$ & pCi/g dry & $4.98 \mathrm{E} 1$ & & $12 / 16 / 08$ & 8L09007 & AGG-RRL-001 \\
\hline $7440-29-1$ & Thorium 232 & $<3.08 \mathrm{E} 2$ & pCi/g dry & $3.08 \mathrm{E} 2$ & & $12 / 16 / 08$ & 8L09007 & AGG-RRL-001 \\
\hline 13981-14-1 & Protactinium-233 & $<5.14 \mathrm{E}-1$ & pCi/g dry & $5.14 \mathrm{E}-1$ & & $12 / 16 / 08$ & 8L09007 & AGG-RRL-001 \\
\hline \multirow[t]{2}{*}{$15100-28-4$} & Protactinium-234 & $<9.02 \mathrm{E}-1$ & pCi/g dry & $9.02 \mathrm{E}-1$ & & $12 / 16 / 08$ & 8L09007 & AGG-RRL-001 \\
\hline & Protactinium-234m & $<2.48 \mathrm{E} 1$ & pCi/g dry & $2.48 \mathrm{E} 1$ & & $12 / 16 / 08$ & 8L09007 & AGG-RRL-001 \\
\hline $15065-10-8$ & Thorium-234 & $<8.30 \mathrm{E} 0$ & pCi/g dry & $8.30 \mathrm{E} 0$ & & $12 / 16 / 08$ & 8L09007 & AGG-RRL-001 \\
\hline 15117-96-1 & Uranium 235 & $<1.87 \mathrm{E} 0$ & pCi/g dry & $1.87 \mathrm{E} 0$ & & $12 / 16 / 08$ & 8L09007 & AGG-RRL-001 \\
\hline 13994-20-2 & Neptunium-237 & $<1.95 \mathrm{E} 0$ & pCi/g dry & $1.95 \mathrm{E} 0$ & & $12 / 16 / 08$ & 8L09007 & AGG-RRL-001 \\
\hline \multirow[t]{2}{*}{ 13981-16-3 } & Plutonium-238 & $<2.83 \mathrm{E} 3$ & $\mathrm{pCi} / \mathrm{g}$ dry & $2.83 \mathrm{E} 3$ & & $12 / 16 / 08$ & 8L09007 & AGG-RRL-001 \\
\hline & Uranium 238 & $<4.30 \mathrm{E} 0$ & pCi/g dry & $4.30 \mathrm{E} 0$ & & $12 / 16 / 08$ & 8L09007 & AGG-RRL-001 \\
\hline $15117-48-3$ & Plutonium-239 & $<3.15 \mathrm{E} 3$ & pCi/g dry & $3.15 \mathrm{E} 3$ & & $12 / 16 / 08$ & 8L09007 & AGG-RRL-001 \\
\hline $14119-33-6$ & Plutonium-240 & $<2.89 \mathrm{E} 3$ & pCi/g dry & $2.89 \mathrm{E} 3$ & & $12 / 16 / 08$ & 8L09007 & AGG-RRL-001 \\
\hline $14596-10-2$ & Americium-241 & $<1.55 \mathrm{E} 0$ & pCi/g dry & $1.55 \mathrm{E} 0$ & & $12 / 16 / 08$ & 8L09007 & AGG-RRL-001 \\
\hline $14993-75-0$ & Americium-243 & $<5.71 \mathrm{E}-1$ & pCi/g dry & $5.71 \mathrm{E}-1$ & & $12 / 16 / 08$ & 8L09007 & AGG-RRL-001 \\
\hline $15757-87-6$ & Curium-243 & $<8.83 \mathrm{E}-1$ & pCi/g dry & $8.83 \mathrm{E}-1$ & & $12 / 16 / 08$ & 8L09007 & AGG-RRL-001 \\
\hline $15621-76-8$ & Curium-245 & $<7.26 \mathrm{E}-1$ & pCi/g dry & $7.26 \mathrm{E}-1$ & & $12 / 16 / 08$ & 8L09007 & AGG-RRL-001 \\
\hline HEIS No. & B1V9C1 & \multicolumn{3}{|c|}{$\begin{array}{ll}\text { Lab ID: } & 0805003-21\end{array}$} & & & & \\
\hline $13966-32-0$ & Sodium-22 & $<2.36 \mathrm{E}-1$ & pCi/g dry & $2.36 \mathrm{E}-1$ & & $12 / 16 / 08$ & 8L09007 & AGG-RRL-001 \\
\hline $13966-00-2$ & Potassium- 40 & $2.12 \mathrm{E} 1$ & pCi/g dry & $2.05 \mathrm{E} 0$ & $9.38 \mathrm{E}-1$ & $12 / 16 / 08$ & 8L09007 & AGG-RRL-001 \\
\hline 14392-02-0 & Chromium-51 & $<2.32 \mathrm{E} 0$ & pCi/g dry & $2.32 \mathrm{E} 0$ & & $12 / 16 / 08$ & 8L09007 & AGG-RRL-001 \\
\hline 13966-31-9 & Manganese-54 & $<2.33 \mathrm{E}-1$ & pCi/g dry & $2.33 \mathrm{E}-1$ & & $12 / 16 / 08$ & 8L09007 & AGG-RRL-001 \\
\hline $13981-50-5$ & Cobalt-57 & $<2.62 \mathrm{E}-1$ & pCi/g dry & $2.62 \mathrm{E}-1$ & & $12 / 16 / 08$ & 8L09007 & AGG-RRL-001 \\
\hline $14596-12-4$ & Iron-59 & $<4.33 \mathrm{E}-1$ & pCi/g dry & 4.33E-1 & & $12 / 16 / 08$ & 8L09007 & AGG-RRL-001 \\
\hline 10198-40-0 & Cobalt-60 & $<2.15 \mathrm{E}-1$ & pCi/g dry & $2.15 \mathrm{E}-1$ & & $12 / 16 / 08$ & 8L09007 & AGG-RRL-001 \\
\hline 13982-39-3 & Zinc-65 & $<5.26 \mathrm{E}-1$ & pCi/g dry & $5.26 \mathrm{E}-1$ & & $12 / 16 / 08$ & 8L09007 & AGG-RRL-001 \\
\hline $14265-71-5$ & Selenium-75 & $<3.90 \mathrm{E}-1$ & pCi/g dry & $3.90 \mathrm{E}-1$ & & $12 / 16 / 08$ & 8L09007 & AGG-RRL-001 \\
\hline 17056-36-9 & Rubidium-83 & $<5.41 \mathrm{E}-1$ & pCi/g dry & $5.41 \mathrm{E}-1$ & & $12 / 16 / 08$ & 8L09007 & AGG-RRL-001 \\
\hline $13967-73-2$ & Strontium- 85 & $<2.50 \mathrm{E}-1$ & pCi/g dry & $2.50 \mathrm{E}-1$ & & $12 / 16 / 08$ & 8L09007 & AGG-RRL-001 \\
\hline $14932-53-7$ & Rubidium-86 & $<2.80 \mathrm{E} 0$ & pCi/g dry & $2.80 \mathrm{E} 0$ & & $12 / 16 / 08$ & 8L09007 & AGG-RRL-001 \\
\hline 13982-36-0 & Yttrium-88 & $<1.78 \mathrm{E}-1$ & pCi/g dry & $1.78 \mathrm{E}-1$ & & $12 / 16 / 08$ & 8L09007 & AGG-RRL-001 \\
\hline 14681-63-1 & Niobium-94 & $<2.17 \mathrm{E}-1$ & pCi/g dry & $2.17 \mathrm{E}-1$ & & $12 / 16 / 08$ & 8L09007 & AGG-RRL-001 \\
\hline \multirow[t]{3}{*}{$13967-76-5$} & Niobium-95 & $<2.34 \mathrm{E}-1$ & pCi/g dry & $2.34 \mathrm{E}-1$ & & $12 / 16 / 08$ & 8L09007 & AGG-RRL-001 \\
\hline & Niobium-95m & $<1.09 \mathrm{E} 0$ & pCi/g dry & $1.09 \mathrm{E} 0$ & & $12 / 16 / 08$ & 8L09007 & AGG-RRL-001 \\
\hline & Technetium-95m & $<3.72 \mathrm{E}-1$ & pCi/g dry & $3.72 \mathrm{E}-1$ & & $12 / 16 / 08$ & 8L09007 & AGG-RRL-001 \\
\hline \multirow[t]{2}{*}{$13967-71-0$} & Zirconium-95 & $<3.97 \mathrm{E}-1$ & pCi/g dry & $3.97 \mathrm{E}-1$ & & $12 / 16 / 08$ & 8L09007 & AGG-RRL-001 \\
\hline & Technetium-99m & $<2.56 \mathrm{E}-1$ & pCi/g dry & $2.56 \mathrm{E}-1$ & & $12 / 16 / 08$ & 8L09007 & AGG-RRL-001 \\
\hline 13968-53-1 & Ruthenium-103 & $<2.51 \mathrm{E}-1$ & pCi/g dry & $2.51 \mathrm{E}-1$ & & $12 / 16 / 08$ & 8L09007 & AGG-RRL-001 \\
\hline $13967-48-1$ & Ruthenium-106 & $<2.65 \mathrm{E} 0$ & pCi/g dry & $2.65 \mathrm{E} 0$ & & $12 / 16 / 08$ & 8L09007 & AGG-RRL-001 \\
\hline $14391-65-2$ & Silver-108m & $<2.38 \mathrm{E}-1$ & pCi/g dry & $2.38 \mathrm{E}-1$ & & $12 / 16 / 08$ & 8L09007 & AGG-RRL-001 \\
\hline \multirow[t]{2}{*}{$14109-32-1$} & Cadmium-109 & $<8.12 \mathrm{E} 0$ & pCi/g dry & $8.12 \mathrm{E} 0$ & & $12 / 16 / 08$ & 8L09007 & AGG-RRL-001 \\
\hline & Silver-110 & $<2.24 \mathrm{E}-1$ & pCi $/ g$ dry & $2.24 \mathrm{E}-1$ & & $12 / 16 / 08$ & 8L09007 & AGG-RRL-001 \\
\hline
\end{tabular}


GEA/Soil

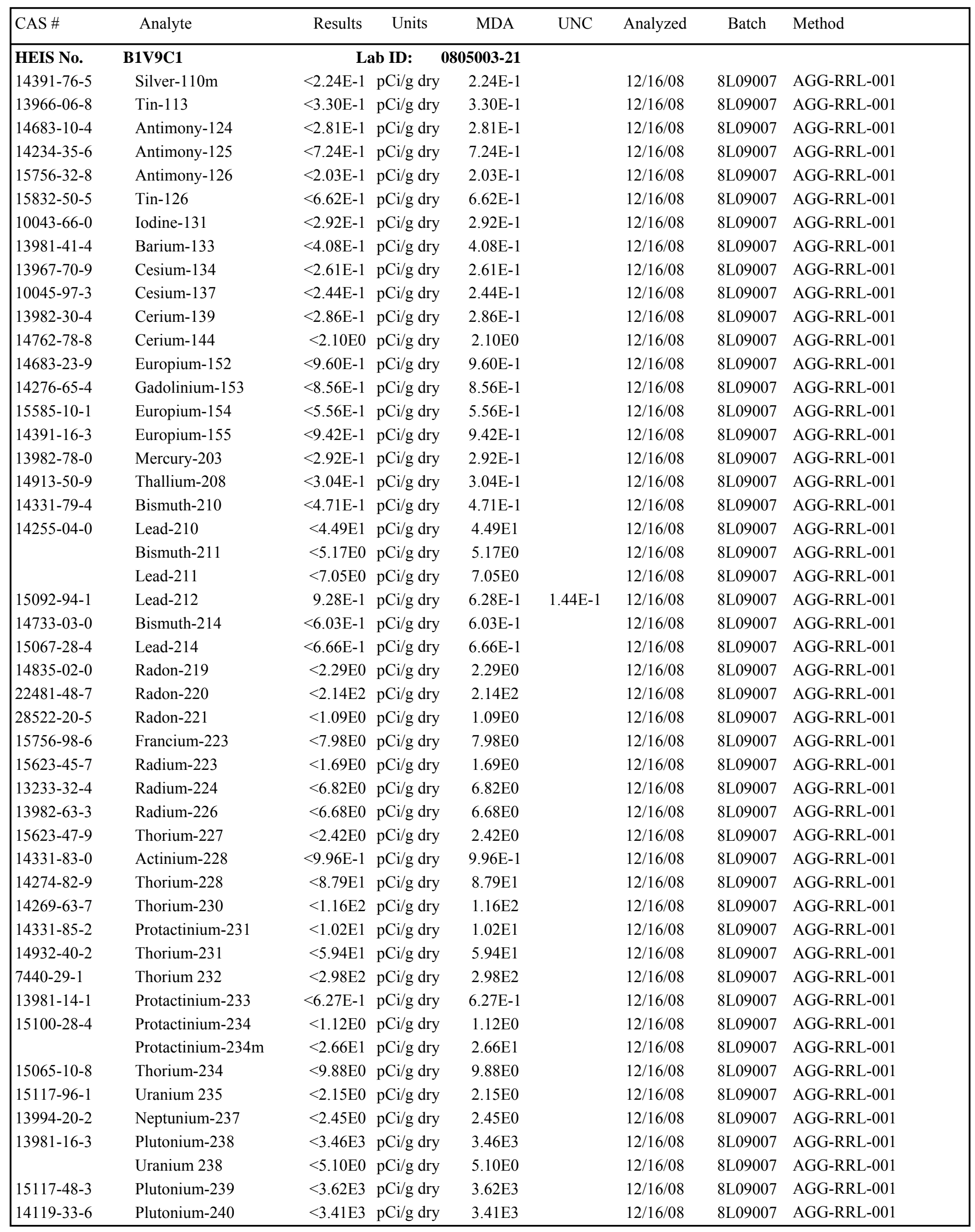


GEA/Soil

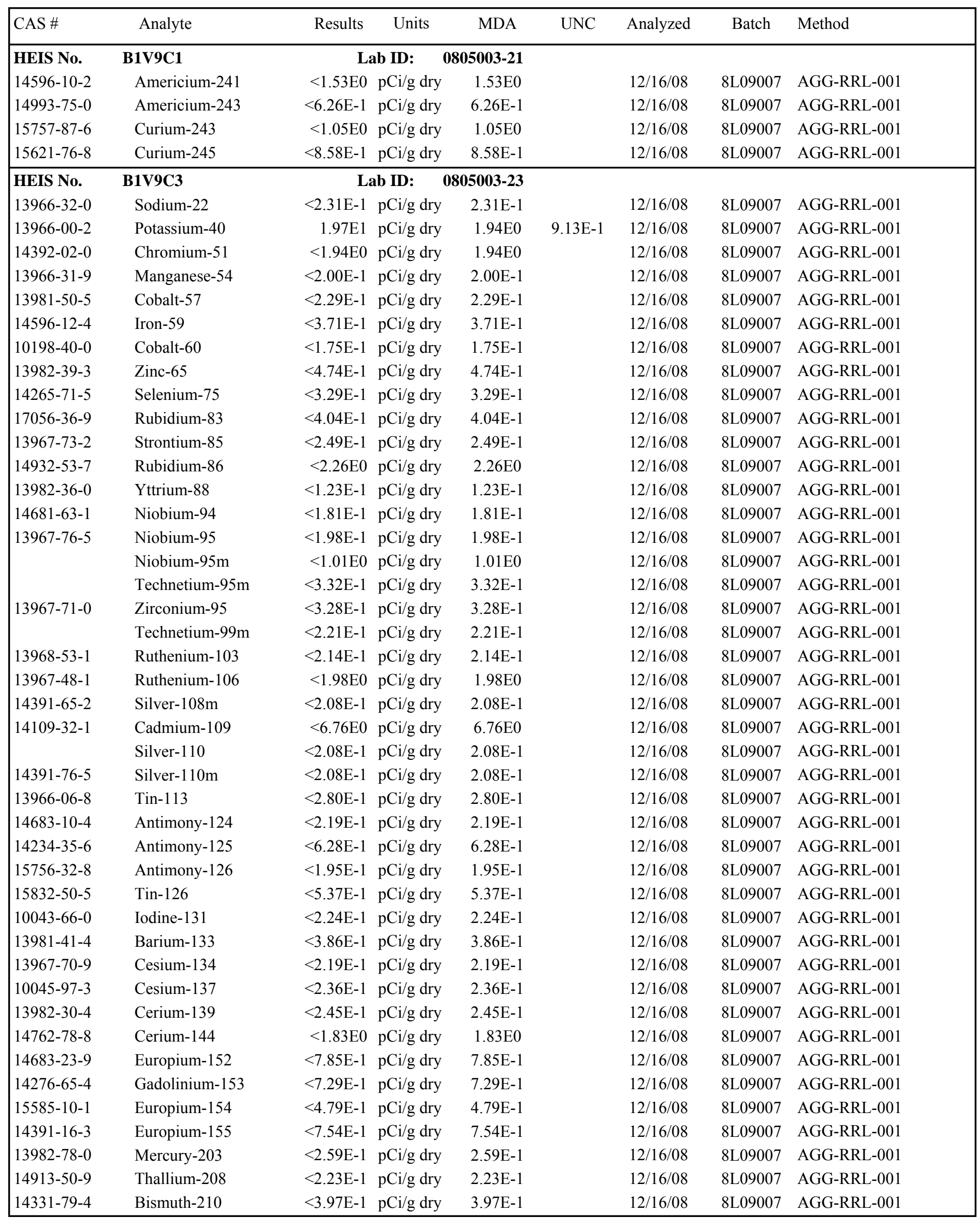


GEA/Soil

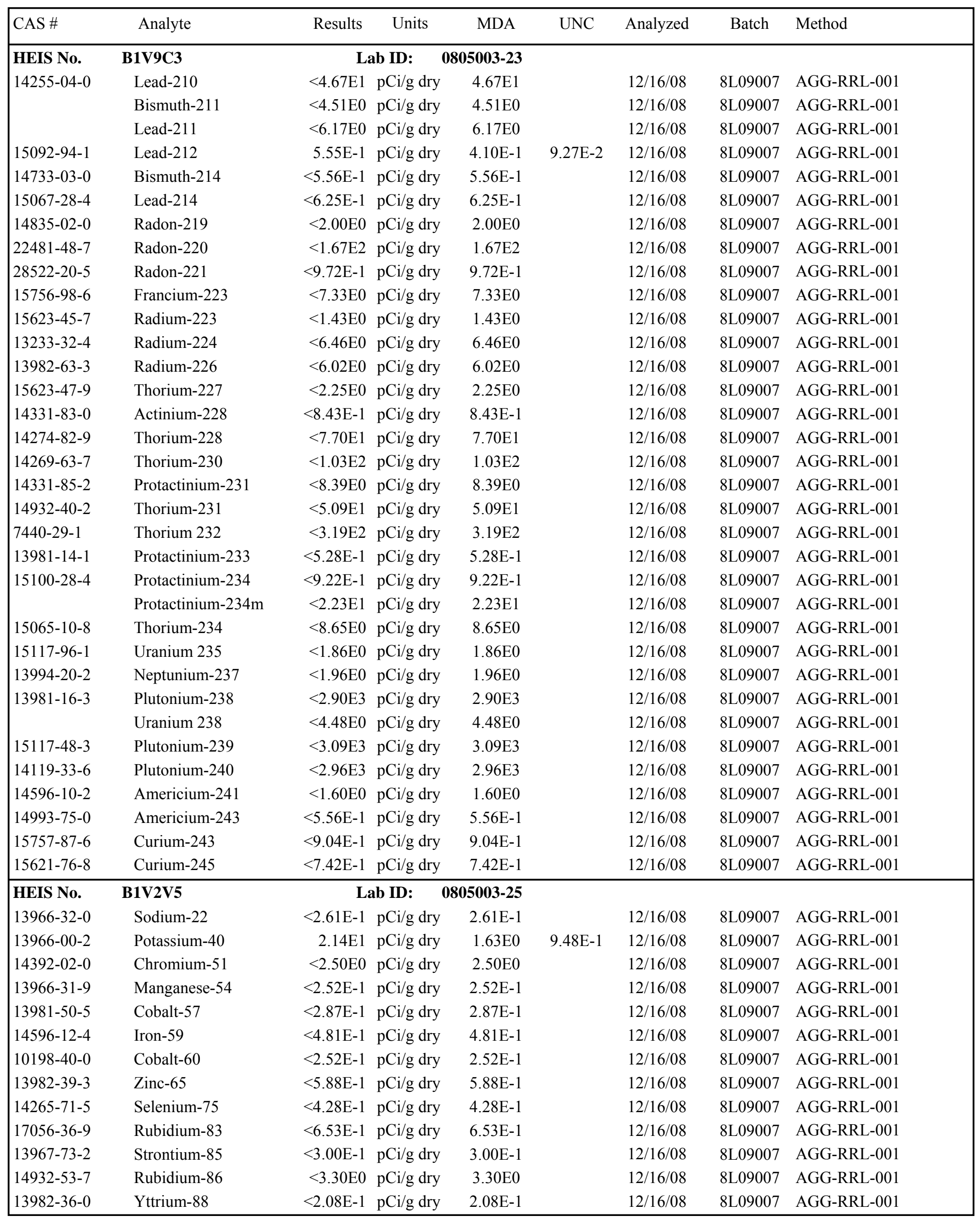


GEA/Soil

\begin{tabular}{|c|c|c|c|c|c|c|c|c|}
\hline CAS \# & Analyte & Results & Units & MDA & UNC & Analyzed & Batch & Method \\
\hline HEIS No. & B1V2V5 & & ab ID: & $5003-25$ & & & & \\
\hline 14681-63-1 & Niobium-94 & $<2.36 \mathrm{E}-1$ & pCi/g dry & $2.36 \mathrm{E}-1$ & & $12 / 16 / 08$ & 8L09007 & AGG-RRL-001 \\
\hline \multirow[t]{3}{*}{$13967-76-5$} & Niobium-95 & $<2.72 \mathrm{E}-1$ & pCi/g dry & $2.72 \mathrm{E}-1$ & & $12 / 16 / 08$ & 8L09007 & AGG-RRL-001 \\
\hline & Niobium-95m & $<1.23 \mathrm{E} 0$ & pCi/g dry & $1.23 \mathrm{E} 0$ & & $12 / 16 / 08$ & 8L09007 & AGG-RRL-001 \\
\hline & Technetium-95m & $<4.11 \mathrm{E}-1$ & pCi $/ g$ dry & $4.11 \mathrm{E}-1$ & & $12 / 16 / 08$ & 8L09007 & AGG-RRL-001 \\
\hline \multirow[t]{2}{*}{ 13967-71-0 } & Zirconium-95 & $<4.78 \mathrm{E}-1$ & $\mathrm{pCi} / \mathrm{g}$ dry & $4.78 \mathrm{E}-1$ & & $12 / 16 / 08$ & 8L09007 & AGG-RRL-001 \\
\hline & Technetium-99m & $<2.75 \mathrm{E}-1$ & pCi/g dry & $2.75 \mathrm{E}-1$ & & $12 / 16 / 08$ & 8L09007 & AGG-RRL-001 \\
\hline 13968-53-1 & Ruthenium-103 & $<2.95 \mathrm{E}-1$ & pCi/g dry & $2.95 \mathrm{E}-1$ & & $12 / 16 / 08$ & 8L09007 & AGG-RRL-001 \\
\hline 13967-48-1 & Ruthenium-106 & $<3.05 \mathrm{E} 0$ & pCi $/ g$ dry & $3.05 \mathrm{E} 0$ & & $12 / 16 / 08$ & 8L09007 & AGG-RRL-001 \\
\hline 14391-65-2 & Silver-108m & $<2.45 \mathrm{E}-1$ & pCi/g dry & $2.45 \mathrm{E}-1$ & & $12 / 16 / 08$ & 8L09007 & AGG-RRL-001 \\
\hline \multirow[t]{2}{*}{$14109-32-1$} & Cadmium-109 & $<9.03 \mathrm{E} 0$ & pCi/g dry & $9.03 \mathrm{E} 0$ & & $12 / 16 / 08$ & 8L09007 & AGG-RRL-001 \\
\hline & Silver-110 & $<2.72 \mathrm{E}-1$ & pCi/g dry & $2.72 \mathrm{E}-1$ & & $12 / 16 / 08$ & 8L09007 & AGG-RRL-001 \\
\hline $14391-76-5$ & Silver-110m & $<2.73 \mathrm{E}-1$ & pCi/g dry & $2.73 \mathrm{E}-1$ & & $12 / 16 / 08$ & 8L09007 & AGG-RRL-001 \\
\hline 13966-06-8 & Tin-113 & $<3.73 \mathrm{E}-1$ & pCi/g dry & $3.73 \mathrm{E}-1$ & & $12 / 16 / 08$ & 8L09007 & AGG-RRL-001 \\
\hline $14683-10-4$ & Antimony-124 & $<3.06 \mathrm{E}-1$ & pCi/g dry & $3.06 \mathrm{E}-1$ & & $12 / 16 / 08$ & 8L09007 & AGG-RRL-001 \\
\hline $14234-35-6$ & Antimony-125 & $<7.76 \mathrm{E}-1$ & pCi/g dry & $7.76 \mathrm{E}-1$ & & $12 / 16 / 08$ & 8L09007 & AGG-RRL-001 \\
\hline 15756-32-8 & Antimony-126 & $<2.53 \mathrm{E}-1$ & pCi/g dry & $2.53 \mathrm{E}-1$ & & $12 / 16 / 08$ & 8L09007 & AGG-RRL-001 \\
\hline $15832-50-5$ & Tin-126 & $<7.39 \mathrm{E}-1$ & pCi/g dry & $7.39 \mathrm{E}-1$ & & $12 / 16 / 08$ & 8L09007 & AGG-RRL-001 \\
\hline 10043-66-0 & Iodine-131 & $<3.40 \mathrm{E}-1$ & pCi/g dry & $3.40 \mathrm{E}-1$ & & $12 / 16 / 08$ & 8L09007 & AGG-RRL-001 \\
\hline 13981-41-4 & Barium-133 & $<4.83 \mathrm{E}-1$ & pCi/g dry & $4.83 \mathrm{E}-1$ & & $12 / 16 / 08$ & 8L09007 & AGG-RRL-001 \\
\hline 13967-70-9 & Cesium-134 & $<2.93 \mathrm{E}-1$ & pCi/g dry & $2.93 \mathrm{E}-1$ & & $12 / 16 / 08$ & 8L09007 & AGG-RRL-001 \\
\hline $10045-97-3$ & Cesium-137 & $<3.01 \mathrm{E}-1$ & pCi/g dry & $3.01 \mathrm{E}-1$ & & $12 / 16 / 08$ & 8L09007 & AGG-RRL-001 \\
\hline 13982-30-4 & Cerium-139 & $<3.20 \mathrm{E}-1$ & pCi/g dry & $3.20 \mathrm{E}-1$ & & $12 / 16 / 08$ & 8L09007 & AGG-RRL-001 \\
\hline 14762-78-8 & Cerium-144 & $<2.24 \mathrm{E} 0$ & pCi/g dry & $2.24 \mathrm{E} 0$ & & $12 / 16 / 08$ & 8L09007 & AGG-RRL-001 \\
\hline 14683-23-9 & Europium-152 & $<1.06 \mathrm{E} 0$ & pCi/g dry & $1.06 \mathrm{E} 0$ & & $12 / 16 / 08$ & 8L09007 & AGG-RRL-001 \\
\hline $14276-65-4$ & Gadolinium-153 & $<9.56 \mathrm{E}-1$ & pCi/g dry & $9.56 \mathrm{E}-1$ & & $12 / 16 / 08$ & 8L09007 & AGG-RRL-001 \\
\hline $15585-10-1$ & Europium-154 & $<6.07 \mathrm{E}-1$ & pCi/g dry & $6.07 \mathrm{E}-1$ & & $12 / 16 / 08$ & 8L09007 & AGG-RRL-001 \\
\hline 14391-16-3 & Europium-155 & $<1.05 \mathrm{E} 0$ & pCi/g dry & $1.05 \mathrm{E} 0$ & & $12 / 16 / 08$ & 8L09007 & AGG-RRL-001 \\
\hline 13982-78-0 & Mercury-203 & $<3.25 \mathrm{E}-1$ & pCi/g dry & $3.25 \mathrm{E}-1$ & & $12 / 16 / 08$ & 8L09007 & AGG-RRL-001 \\
\hline $14913-50-9$ & Thallium-208 & $<3.30 \mathrm{E}-1$ & pCi/g dry & $3.30 \mathrm{E}-1$ & & $12 / 16 / 08$ & 8L09007 & AGG-RRL-001 \\
\hline 14331-79-4 & Bismuth-210 & $<5.11 \mathrm{E}-1$ & pCi/g dry & $5.11 \mathrm{E}-1$ & & $12 / 16 / 08$ & 8L09007 & AGG-RRL-001 \\
\hline \multirow[t]{3}{*}{$14255-04-0$} & Lead-210 & $<5.28 \mathrm{E} 1$ & pCi/g dry & $5.28 \mathrm{E} 1$ & & $12 / 16 / 08$ & 8L09007 & AGG-RRL-001 \\
\hline & Bismuth-211 & $<6.09 \mathrm{E} 0$ & $\mathrm{pCi} / \mathrm{g}$ dry & $6.09 \mathrm{E} 0$ & & $12 / 16 / 08$ & 8L09007 & AGG-RRL-001 \\
\hline & Lead-211 & $<8.32 \mathrm{E} 0$ & pCi/g dry & $8.32 \mathrm{E} 0$ & & $12 / 16 / 08$ & 8L09007 & AGG-RRL-001 \\
\hline 15092-94-1 & Lead-212 & $<7.10 \mathrm{E}-1$ & pCi/g dry & $7.10 \mathrm{E}-1$ & & $12 / 16 / 08$ & 8L09007 & AGG-RRL-001 \\
\hline 14733-03-0 & Bismuth-214 & $<6.61 \mathrm{E}-1$ & pCi/g dry & $6.61 \mathrm{E}-1$ & & $12 / 16 / 08$ & 8L09007 & AGG-RRL-001 \\
\hline $15067-28-4$ & Lead-214 & $<7.77 \mathrm{E}-1$ & pCi/g dry & $7.77 \mathrm{E}-1$ & & $12 / 16 / 08$ & 8L09007 & AGG-RRL-001 \\
\hline $14835-02-0$ & Radon-219 & $<2.60 \mathrm{E} 0$ & pCi/g dry & $2.60 \mathrm{E} 0$ & & $12 / 16 / 08$ & 8L09007 & AGG-RRL-001 \\
\hline $22481-48-7$ & Radon-220 & $<2.46 \mathrm{E} 2$ & pCi/g dry & $2.46 \mathrm{E} 2$ & & $12 / 16 / 08$ & 8L09007 & AGG-RRL-001 \\
\hline $28522-20-5$ & Radon-221 & $<1.24 \mathrm{E} 0$ & pCi/g dry & $1.24 \mathrm{E} 0$ & & $12 / 16 / 08$ & 8L09007 & AGG-RRL-001 \\
\hline $15756-98-6$ & Francium-223 & $<9.07 \mathrm{E} 0$ & pCi/g dry & $9.07 \mathrm{E} 0$ & & $12 / 16 / 08$ & 8L09007 & AGG-RRL-001 \\
\hline $15623-45-7$ & Radium-223 & $<1.88 \mathrm{E} 0$ & $\mathrm{pCi} / \mathrm{g}$ dry & $1.88 \mathrm{E} 0$ & & $12 / 16 / 08$ & 8L09007 & AGG-RRL-001 \\
\hline $13233-32-4$ & Radium-224 & $<7.62 \mathrm{E} 0$ & pCi/g dry & 7.62E0 & & $12 / 16 / 08$ & 8L09007 & AGG-RRL-001 \\
\hline 13982-63-3 & Radium-226 & $<7.69 \mathrm{E} 0$ & pCi/g dry & 7.69E0 & & $12 / 16 / 08$ & 8L09007 & AGG-RRL-001 \\
\hline $15623-47-9$ & Thorium-227 & $<2.75 \mathrm{E} 0$ & pCi/g dry & $2.75 \mathrm{E} 0$ & & $12 / 16 / 08$ & 8L09007 & AGG-RRL-001 \\
\hline $14331-83-0$ & Actinium-228 & $<1.14 \mathrm{E} 0$ & pCi/g dry & $1.14 \mathrm{E} 0$ & & $12 / 16 / 08$ & 8L09007 & AGG-RRL-001 \\
\hline $14274-82-9$ & Thorium-228 & $<9.97 \mathrm{E} 1$ & pCi/g dry & $9.97 \mathrm{E} 1$ & & $12 / 16 / 08$ & 8L09007 & AGG-RRL-001 \\
\hline $14269-63-7$ & Thorium-230 & $<1.32 \mathrm{E} 2$ & pCi/g dry & $1.32 \mathrm{E} 2$ & & $12 / 16 / 08$ & 8L09007 & AGG-RRL-001 \\
\hline $14331-85-2$ & Protactinium-231 & $<1.15 \mathrm{E} 1$ & pCi/g dry & $1.15 \mathrm{E} 1$ & & $12 / 16 / 08$ & 8L09007 & AGG-RRL-001 \\
\hline 14932-40-2 & Thorium-231 & $<6.70 \mathrm{E} 1$ & pCi/g dry & $6.70 \mathrm{E} 1$ & & $12 / 16 / 08$ & 8L09007 & AGG-RRL-001 \\
\hline
\end{tabular}




\section{GEA/Soil}

\begin{tabular}{|c|c|c|c|c|c|c|c|c|}
\hline CAS \# & Analyte & Results & Units & MDA & UNC & Analyzed & Batch & Method \\
\hline HEIS No. & B1V2V5 & & ab ID: & $5003-25$ & & & & \\
\hline $7440-29-1$ & Thorium 232 & $<3.46 \mathrm{E} 2$ & pCi/g dry & $3.46 \mathrm{E} 2$ & & $12 / 16 / 08$ & 8L09007 & AGG-RRL-001 \\
\hline 13981-14-1 & Protactinium-233 & $<6.83 \mathrm{E}-1$ & $\mathrm{pCi} / \mathrm{g}$ dry & $6.83 \mathrm{E}-1$ & & $12 / 16 / 08$ & 8L09007 & AGG-RRL-001 \\
\hline \multirow[t]{2}{*}{$15100-28-4$} & Protactinium-234 & $<1.22 \mathrm{E} 0$ & pCi/g dry & $1.22 \mathrm{E} 0$ & & $12 / 16 / 08$ & 8L09007 & AGG-RRL-001 \\
\hline & Protactinium-234m & $<2.91 \mathrm{E} 1$ & pCi/g dry & $2.91 \mathrm{E} 1$ & & $12 / 16 / 08$ & 8L09007 & AGG-RRL-001 \\
\hline $15065-10-8$ & Thorium-234 & $<1.11 \mathrm{E} 1$ & pCi/g dry & $1.11 \mathrm{E} 1$ & & $12 / 16 / 08$ & 8L09007 & AGG-RRL-001 \\
\hline $15117-96-1$ & Uranium 235 & $<2.35 \mathrm{E} 0$ & pCi/g dry & $2.35 \mathrm{E} 0$ & & $12 / 16 / 08$ & 8L09007 & AGG-RRL-001 \\
\hline $13994-20-2$ & Neptunium-237 & $<2.73 \mathrm{E} 0$ & $\mathrm{pCi} / \mathrm{g}$ dry & $2.73 \mathrm{E} 0$ & & $12 / 16 / 08$ & 8L09007 & AGG-RRL-001 \\
\hline \multirow[t]{2}{*}{ 13981-16-3 } & Plutonium-238 & $<3.92 \mathrm{E} 3$ & pCi/g dry & $3.92 \mathrm{E} 3$ & & $12 / 16 / 08$ & 8L09007 & AGG-RRL-001 \\
\hline & Uranium 238 & $<5.67 \mathrm{E} 0$ & pCi/g dry & 5.67E0 & & $12 / 16 / 08$ & 8L09007 & AGG-RRL-001 \\
\hline $15117-48-3$ & Plutonium-239 & $<3.91 \mathrm{E} 3$ & pCi/g dry & $3.91 \mathrm{E} 3$ & & $12 / 16 / 08$ & 8L09007 & AGG-RRL-001 \\
\hline $14119-33-6$ & Plutonium-240 & $<3.86 \mathrm{E} 3$ & pCi/g dry & $3.86 \mathrm{E} 3$ & & $12 / 16 / 08$ & 8L09007 & AGG-RRL-001 \\
\hline $14596-10-2$ & Americium-241 & $<1.76 \mathrm{E} 0$ & $\mathrm{pCi} / \mathrm{g}$ dry & $1.76 \mathrm{E} 0$ & & $12 / 16 / 08$ & 8L09007 & AGG-RRL-001 \\
\hline $14993-75-0$ & Americium-243 & $<6.95 \mathrm{E}-1$ & pCi/g dry & $6.95 \mathrm{E}-1$ & & $12 / 16 / 08$ & 8L09007 & AGG-RRL-001 \\
\hline $15757-87-6$ & Curium-243 & $<1.18 \mathrm{E} 0$ & pCi/g dry & $1.18 \mathrm{E} 0$ & & $12 / 16 / 08$ & 8L09007 & AGG-RRL-001 \\
\hline $15621-76-8$ & Curium-245 & $<9.68 \mathrm{E}-1$ & pCi/g dry & $9.68 \mathrm{E}-1$ & & $12 / 16 / 08$ & 8L09007 & AGG-RRL-001 \\
\hline
\end{tabular}


Total Alpha Total Beta/Acid Extract

\begin{tabular}{|c|c|c|c|c|c|c|c|c|}
\hline CAS \# & Analyte & Results & Units & MDA & UNC & Analyzed & Batch & Method \\
\hline HEIS No. & B1V993 & \multicolumn{4}{|c|}{ Lab ID: $\quad$ 0805003-03 } & & & \\
\hline $12587-47-2$ & Gross Beta & $<9.78 \mathrm{E} 1$ & $\mathrm{pCi} / \mathrm{g}$ dry & $9.78 \mathrm{E} 1$ & & $3 / 17 / 09$ & $9 \mathrm{C} 16001$ & AGG-RRL-002 \\
\hline $12587-46-1$ & Gross Alpha & $<4.35 \mathrm{E} 1$ & $\mathrm{pCi} / \mathrm{g}$ dry & $4.35 \mathrm{E} 1$ & & $3 / 17 / 09$ & 9C16001 & AGG-RRL-002 \\
\hline HEIS No. & B1V995 & \multicolumn{3}{|c|}{$\begin{array}{ll}\text { Lab ID: } & \text { 0805003-05 }\end{array}$} & & & & \\
\hline $12587-47-2$ & Gross Beta & $<1.04 \mathrm{E} 2$ & pCi/g dry & $1.04 \mathrm{E} 2$ & & $3 / 17 / 09$ & $9 \mathrm{C} 16001$ & AGG-RRL-002 \\
\hline $12587-46-1$ & Gross Alpha & $<4.60 \mathrm{E} 1$ & pCi/g dry & $4.60 \mathrm{E} 1$ & & $3 / 17 / 09$ & $9 \mathrm{C} 16001$ & AGG-RRL-002 \\
\hline HEIS No. & B1V997 & \multicolumn{3}{|c|}{ Lab ID: $\quad$ 0805003-07 } & & & & \\
\hline $12587-47-2$ & Gross Beta & $<1.04 \mathrm{E} 2$ & $\mathrm{pCi} / \mathrm{g}$ dry & $1.04 \mathrm{E} 2$ & & $3 / 17 / 09$ & 9C16001 & AGG-RRL-002 \\
\hline $12587-46-1$ & Gross Alpha & $<4.64 \mathrm{E} 1$ & pCi/g dry & $4.64 \mathrm{E} 1$ & & $3 / 17 / 09$ & 9C16001 & AGG-RRL-002 \\
\hline HEIS No. & B1V999 & \multicolumn{3}{|c|}{ Lab ID: $\quad$ 0805003-09 } & & & & \\
\hline $12587-47-2$ & Gross Beta & $<1.04 \mathrm{E} 2$ & pCi/g dry & $1.04 \mathrm{E} 2$ & & $3 / 18 / 09$ & $9 \mathrm{C} 16001$ & AGG-RRL-002 \\
\hline $12587-46-1$ & Gross Alpha & $<4.63 \mathrm{E} 1$ & pCi/g dry & $4.63 \mathrm{E} 1$ & & $3 / 18 / 09$ & 9C16001 & AGG-RRL-002 \\
\hline HEIS No. & B1V9B1 & \multicolumn{3}{|c|}{ Lab ID: $\quad$ 0805003-11 } & & & & \\
\hline $12587-47-2$ & Gross Beta & $<1.06 \mathrm{E} 2$ & pCi/g dry & $1.06 \mathrm{E} 2$ & & $3 / 18 / 09$ & 9C16001 & AGG-RRL-002 \\
\hline $12587-46-1$ & Gross Alpha & $<4.71 \mathrm{E} 1$ & pCi/g dry & $4.71 \mathrm{E} 1$ & & $3 / 18 / 09$ & 9C16001 & AGG-RRL-002 \\
\hline HEIS No. & B1V9B3 & \multicolumn{3}{|c|}{ Lab ID: $\quad$ 0805003-13 } & & & & \\
\hline $12587-47-2$ & Gross Beta & $<1.03 \mathrm{E} 2$ & pCi/g dry & $1.03 \mathrm{E} 2$ & & $3 / 18 / 09$ & $9 \mathrm{C} 16001$ & AGG-RRL-002 \\
\hline $12587-46-1$ & Gross Alpha & $<4.60 \mathrm{E} 1$ & $\mathrm{pCi} / \mathrm{g}$ dry & $4.60 \mathrm{E} 1$ & & $3 / 18 / 09$ & $9 \mathrm{C} 16001$ & AGG-RRL-002 \\
\hline HEIS No. & B1V9B5 & \multicolumn{3}{|c|}{ Lab ID: $\quad$ 0805003-15 } & & & & \\
\hline $12587-47-2$ & Gross Beta & $<1.02 \mathrm{E} 2$ & pCi/g dry & $1.02 \mathrm{E} 2$ & & $3 / 18 / 09$ & $9 \mathrm{C} 16001$ & AGG-RRL-002 \\
\hline $12587-46-1$ & Gross Alpha & $<4.52 \mathrm{E} 1$ & pCi/g dry & $4.52 \mathrm{E} 1$ & & $3 / 18 / 09$ & 9C16001 & AGG-RRL-002 \\
\hline HEIS No. & B1V9B7 & \multicolumn{3}{|c|}{ Lab ID: $\quad$ 0805003-17 } & & & & \\
\hline $12587-47-2$ & Gross Beta & $<9.99 \mathrm{E} 1$ & pCi/g dry & $9.99 \mathrm{E} 1$ & & $3 / 18 / 09$ & $9 \mathrm{C} 16001$ & AGG-RRL-002 \\
\hline $12587-46-1$ & Gross Alpha & $<4.44 \mathrm{E} 1$ & pCi/g dry & $4.44 \mathrm{E} 1$ & & $3 / 18 / 09$ & 9C16001 & AGG-RRL-002 \\
\hline HEIS No. & B1V9B9 & \multicolumn{3}{|c|}{ Lab ID: $\quad$ 0805003-19 } & & & & \\
\hline $12587-47-2$ & Gross Beta & $<1.02 \mathrm{E} 2$ & pCi/g dry & $1.02 \mathrm{E} 2$ & & $3 / 18 / 09$ & 9C16001 & AGG-RRL-002 \\
\hline $12587-46-1$ & Gross Alpha & $<4.52 \mathrm{E} 1$ & pCi/g dry & $4.52 \mathrm{E} 1$ & & $3 / 18 / 09$ & 9C16001 & AGG-RRL-002 \\
\hline HEIS No. & B1V9C1 & \multicolumn{3}{|c|}{ Lab ID: $\quad$ 0805003-21 } & & & & \\
\hline $12587-47-2$ & Gross Beta & $<1.01 \mathrm{E} 2$ & pCi/g dry & $1.01 \mathrm{E} 2$ & & $3 / 18 / 09$ & $9 \mathrm{C} 16001$ & AGG-RRL-002 \\
\hline $12587-46-1$ & Gross Alpha & $<4.49 \mathrm{E} 1$ & pCi/g dry & $4.49 \mathrm{E} 1$ & & $3 / 18 / 09$ & 9C16001 & AGG-RRL-002 \\
\hline HEIS No. & B1V9C3 & \multicolumn{3}{|c|}{ Lab ID: $\quad$ 0805003-23 } & & & & \\
\hline $12587-47-2$ & Gross Beta & $<1.02 \mathrm{E} 2$ & pCi/g dry & $1.02 \mathrm{E} 2$ & & $3 / 18 / 09$ & $9 \mathrm{C} 16001$ & AGG-RRL-002 \\
\hline $12587-46-1$ & Gross Alpha & $<4.54 \mathrm{E} 1$ & pCi/g dry & $4.54 \mathrm{E} 1$ & & $3 / 18 / 09$ & 9C16001 & AGG-RRL-002 \\
\hline HEIS No. & B1V2V5 & \multicolumn{3}{|c|}{ Lab ID: $\quad$ 0805003-25 } & & & & \\
\hline $12587-47-2$ & Gross Beta & $<1.01 \mathrm{E} 2$ & pCi/g dry & $1.01 \mathrm{E} 2$ & & $3 / 18 / 09$ & 9C16001 & AGG-RRL-002 \\
\hline $12587-46-1$ & Gross Alpha & $<4.51 \mathrm{E} 1$ & pCi/g dry & $4.51 \mathrm{E} 1$ & & $3 / 18 / 09$ & $9 \mathrm{C} 16001$ & AGG-RRL-002 \\
\hline
\end{tabular}


Total Alpha Total Beta/Water Extract

\begin{tabular}{|c|c|c|c|c|c|c|c|c|}
\hline CAS \# & Analyte & Results & Units & MDA & UNC & Analyzed & Batch & Method \\
\hline HEIS No. & B1V993 & \multicolumn{4}{|c|}{ Lab ID: $\quad$ 0805003-03 } & & & \\
\hline $12587-47-2$ & Gross Beta & $<3.17 \mathrm{E} 1$ & $\mathrm{pCi} / \mathrm{g}$ dry & $3.17 \mathrm{E} 1$ & & 3/17/09 & 8L15002 & AGG-RRL-002 \\
\hline $12587-46-1$ & Gross Alpha & $<1.41 \mathrm{E} 1$ & $\mathrm{pCi} / \mathrm{g}$ dry & $1.41 \mathrm{E} 1$ & & $3 / 17 / 09$ & 8L15002 & AGG-RRL-002 \\
\hline HEIS No. & B1V995 & \multicolumn{4}{|c|}{ Lab ID: $\quad$ 0805003-05 } & & & \\
\hline $12587-47-2$ & Gross Beta & $<3.32 \mathrm{E} 1$ & $\mathrm{pCi} / \mathrm{g}$ dry & $3.32 \mathrm{E} 1$ & & 3/17/09 & 8L15002 & AGG-RRL-002 \\
\hline $12587-46-1$ & Gross Alpha & $<1.47 \mathrm{E} 1$ & $\mathrm{pCi} / \mathrm{g}$ dry & $1.47 \mathrm{E} 1$ & & $3 / 17 / 09$ & $8 \mathrm{~L} 15002$ & AGG-RRL-002 \\
\hline HEIS No. & B1V997 & \multicolumn{4}{|c|}{ Lab ID: $\quad$ 0805003-07 } & & & \\
\hline $12587-47-2$ & Gross Beta & $<3.41 \mathrm{E} 1$ & $\mathrm{pCi} / \mathrm{g}$ dry & $3.41 \mathrm{E} 1$ & & $3 / 17 / 09$ & 8L15002 & AGG-RRL-002 \\
\hline $12587-46-1$ & Gross Alpha & $<1.52 \mathrm{E} 1$ & $\mathrm{pCi} / \mathrm{g}$ dry & $1.52 \mathrm{E} 1$ & & 3/17/09 & 8L15002 & AGG-RRL-002 \\
\hline HEIS No. & $\begin{array}{l}\text { B1V999 } \\
\end{array}$ & \multicolumn{4}{|c|}{ Lab ID: $\quad$ 0805003-09 } & & & \\
\hline $12587-47-2$ & Gross Beta & $<3.31 \mathrm{E} 1$ & pCi/g dry & $3.31 \mathrm{E} 1$ & & $3 / 17 / 09$ & $8 \mathrm{~L} 15002$ & AGG-RRL-002 \\
\hline $12587-46-1$ & Gross Alpha & $<1.47 \mathrm{E} 1$ & $\mathrm{pCi} / \mathrm{g}$ dry & $1.47 \mathrm{E} 1$ & & $3 / 17 / 09$ & $8 \mathrm{~L} 15002$ & AGG-RRL-002 \\
\hline HEIS No. & B1V9B1 & \multicolumn{4}{|c|}{ Lab ID: $\quad$ 0805003-11 } & & & \\
\hline $12587-47-2$ & Gross Beta & $<3.35 \mathrm{E} 1$ & $\mathrm{pCi} / \mathrm{g}$ dry & $3.35 \mathrm{E} 1$ & & $3 / 17 / 09$ & 8L15002 & AGG-RRL-002 \\
\hline $12587-46-1$ & Gross Alpha & $<1.49 \mathrm{E} 1$ & pCi/g dry & $1.49 \mathrm{E} 1$ & & $3 / 17 / 09$ & $8 \mathrm{~L} 15002$ & AGG-RRL-002 \\
\hline HEIS No. & B1V9B3 & \multicolumn{4}{|c|}{ Lab ID: $\quad$ 0805003-13 } & & & \\
\hline $12587-47-2$ & Gross Beta & $<3.26 \mathrm{E} 1$ & $\mathrm{pCi} / \mathrm{g}$ dry & $3.26 \mathrm{E} 1$ & & 3/17/09 & 8L15002 & AGG-RRL-002 \\
\hline $12587-46-1$ & Gross Alpha & $<1.45 \mathrm{E} 1$ & pCi/g dry & $1.45 \mathrm{E} 1$ & & $3 / 17 / 09$ & $8 \mathrm{~L} 15002$ & AGG-RRL-002 \\
\hline HEIS No. & B1V9B5 & \multicolumn{4}{|c|}{ Lab ID: $\quad$ 0805003-15 } & & & \\
\hline $12587-47-2$ & Gross Beta & $<3.13 \mathrm{E} 1$ & $\mathrm{pCi} / \mathrm{g}$ dry & $3.13 \mathrm{E} 1$ & & 3/17/09 & $8 \mathrm{~L} 15002$ & AGG-RRL-002 \\
\hline $12587-46-1$ & Gross Alpha & $<1.39 \mathrm{E} 1$ & $\mathrm{pCi} / \mathrm{g}$ dry & $1.39 \mathrm{E} 1$ & & $3 / 17 / 09$ & 8L15002 & AGG-RRL-002 \\
\hline HEIS No. & B1V9B7 & \multicolumn{4}{|c|}{ Lab ID: $\quad$ 0805003-17 } & & & \\
\hline $12587-47-2$ & Gross Beta & $<3.14 \mathrm{E} 1$ & pCi/g dry & $3.14 \mathrm{E} 1$ & & $3 / 17 / 09$ & $8 \mathrm{~L} 15002$ & AGG-RRL-002 \\
\hline $12587-46-1$ & Gross Alpha & $<1.40 \mathrm{E} 1$ & $\mathrm{pCi} / \mathrm{g}$ dry & $1.40 \mathrm{E} 1$ & & $3 / 17 / 09$ & $8 \mathrm{~L} 15002$ & AGG-RRL-002 \\
\hline HEIS No. & B1V9B9 & \multicolumn{4}{|c|}{ Lab ID: $\quad$ 0805003-19 } & & & \\
\hline $12587-47-2$ & Gross Beta & $<3.15 \mathrm{E} 1$ & $\mathrm{pCi} / \mathrm{g}$ dry & $3.15 \mathrm{E} 1$ & & $3 / 17 / 09$ & 8L15002 & AGG-RRL-002 \\
\hline $12587-46-1$ & Gross Alpha & $<1.40 \mathrm{E} 1$ & $\mathrm{pCi} / \mathrm{g}$ dry & $1.40 \mathrm{E} 1$ & & $3 / 17 / 09$ & $8 \mathrm{~L} 15002$ & AGG-RRL-002 \\
\hline HEIS No. & B1V9C1 & \multicolumn{4}{|c|}{ Lab ID: $\quad$ 0805003-21 } & & & \\
\hline $12587-47-2$ & Gross Beta & $<3.14 \mathrm{E} 1$ & $\mathrm{pCi} / \mathrm{g}$ dry & $3.14 \mathrm{E} 1$ & & $3 / 17 / 09$ & 8L15002 & AGG-RRL-002 \\
\hline $12587-46-1$ & Gross Alpha & $<1.40 \mathrm{E} 1$ & pCi/g dry & $1.40 \mathrm{E} 1$ & & $3 / 17 / 09$ & $8 \mathrm{~L} 15002$ & AGG-RRL-002 \\
\hline HEIS No. & B1V9C3 & \multicolumn{4}{|c|}{ Lab ID: $\quad$ 0805003-23 } & & & \\
\hline $12587-47-2$ & Gross Beta & $<3.29 \mathrm{E} 1$ & $\mathrm{pCi} / \mathrm{g}$ dry & $3.29 \mathrm{E} 1$ & & $3 / 17 / 09$ & $8 \mathrm{~L} 15002$ & AGG-RRL-002 \\
\hline $12587-46-1$ & Gross Alpha & $<1.46 \mathrm{E} 1$ & $\mathrm{pCi} / \mathrm{g}$ dry & $1.46 \mathrm{E} 1$ & & $3 / 17 / 09$ & $8 \mathrm{~L} 15002$ & AGG-RRL-002 \\
\hline HEIS No. & B1V2V5 & \multicolumn{4}{|c|}{ Lab ID: $\quad$ 0805003-25 } & & & \\
\hline $12587-47-2$ & Gross Beta & $<3.25 \mathrm{E} 1$ & $\mathrm{pCi} / \mathrm{g}$ dry & $3.25 \mathrm{E} 1$ & & 3/17/09 & 8L15002 & AGG-RRL-002 \\
\hline $12587-46-1$ & Gross Alpha & $<1.45 \mathrm{E} 1$ & pCi/g dry & $1.45 \mathrm{E} 1$ & & $3 / 17 / 09$ & $8 \mathrm{~L} 15002$ & AGG-RRL-002 \\
\hline
\end{tabular}


Wet Chemistry - Quality Control

Environmental Science Laboratory

\begin{tabular}{|c|c|c|c|c|c|c|c|c|c|c|}
\hline Analyte & Result & $\begin{array}{r}\text { porting } \\
\text { Limit }\end{array}$ & Units & $\begin{array}{l}\text { Spike } \\
\text { Level }\end{array}$ & $\begin{array}{l}\text { Source } \\
\text { Result }\end{array}$ & $\%$ REC & $\begin{array}{l}\% \text { REC } \\
\text { Limits }\end{array}$ & RPD & $\begin{array}{l}\text { RPD } \\
\text { Limit }\end{array}$ & Notes \\
\hline
\end{tabular}

Batch 8G31008 - 1:1 Water Extract (pH_EC_Alk)

Blank (8G31008-BLK1)

Specific Conductance (EC)

$<1.00 \mathrm{E}-2 \quad 1.00 \mathrm{E}-2 \quad \mathrm{mS} / \mathrm{cm}$

Prepared \& Analyzed: 08/01/08

Duplicate (8G31008-DUP1)

Source: 0805003-15

Prepared \& Analyzed: 08/01/08

Specific Conductance (EC)

9.00E-2 $1.00 \mathrm{E}-2 \quad \mathrm{mS} / \mathrm{cm}$

9.00E-2

35

Batch 8H01005 - 1:1 Water Extract (pH_EC_Alk)

Duplicate (8H01005-DUP1)

$\mathrm{pH}$

Source: 0805003-15

Prepared \& Analyzed: 08/01/08

8.54E0 N/A pH Units

8.84E0

3.4

35

Duplicate (8H01005-DUP2)

Source: 0805003-19 Prepared \& Analyzed: 08/01/08

$\mathrm{pH}$

$8.00 \mathrm{E} 0$

N/A pH Units

8.36E0

4.40

35

Batch 8H04007 - 1:1 Water Extract (pH_EC_Alk)

Blank (8H04007-BLK1)

Prepared \& Analyzed: 08/05/08

Specific Conductance (EC)

$<1.00 \mathrm{E}-2 \quad 1.00 \mathrm{E}-2 \quad \mathrm{mS} / \mathrm{cm}$

Duplicate (8H04007-DUP1)

Specific Conductance (EC)

\begin{tabular}{cc} 
Source: & $\mathbf{0 8 0 5 0 0 1 - 0 5}$ \\
\hline $7.30 \mathrm{E}-2$ & $1.00 \mathrm{E}-2 \quad \mathrm{mS} / \mathrm{cm}$
\end{tabular}

Prepared \& Analyzed: 08/05/08

Batch 8H04008 - 1:1 Water Extract (pH_EC_Alk)

Duplicate (8H04008-DUP1)

$\mathrm{pH}$

Source: 0805001-0

Prepared \& Analyzed: 08/05/08

$\begin{array}{llll}8.63 \mathrm{E} 0 & \mathrm{~N} / \mathrm{A} & \mathrm{pH} \text { Units } & 8.57 \mathrm{E} 0\end{array}$

0.698

35 
Wet Chemistry - Quality Control

Environmental Science Laboratory

\begin{tabular}{|lcccccccccccc}
\hline & & Reporting & & Spike & Source & & \%REC & & RPD & & \\
Analyte & Result & Limit & Units & Level & Result & \%REC & Limits & RPD & Limit & Notes
\end{tabular}

Batch 8H04008 - 1:1 Water Extract (pH_EC_Alk)

Duplicate (8H04008-DUP2)

$\mathrm{pH}$

Source: 0805001-06

Prepared \& Analyzed: 08/05/08

N/A pH Units $8.66 \mathrm{E} 0$

4.61

35

Batch 8H06002 - 1:1 Water Extract (pH_EC_Alk)

Blank (8H06002-BLK1)

Alkalinity as $\mathrm{CaCO} 3$

Duplicate (8H06002-DUP1)

Alkalinity as $\mathrm{CaCO} 3$

$8.27 \mathrm{E} 0 \quad$ N/A pH Units

Prepared \& Analyzed: 08/06/08

$<2.35 \mathrm{E} 1 \quad 2.35 \mathrm{E} 1 \quad \mathrm{ug} / \mathrm{g}$ wet

Batch 8H06003 - 1:1 Water Extract (pH_EC_Alk)

Blank (8H06003-BLK1)

Alkalinity as $\mathrm{CaCO} 3$ Source: 0805003-15

Prepared \& Analyzed: 08/06/08

$3.50 \mathrm{E} 1 \quad 2.35 \mathrm{E} 1 \quad \mathrm{ug} / \mathrm{g}$ dry $3.57 \mathrm{E} 1$

Prepared \& Analyzed: 08/06/08

Duplicate (8H06003-DUP1)

Alkalinity as $\mathrm{CaCO} 3$

(2.35E1

\begin{tabular}{lll} 
& Prepared \& Analyzed: 08/06/08 \\
\hline $2.35 \mathrm{E} 1 \mathrm{ug} / \mathrm{g}$ wet &
\end{tabular}

Source: 0805001-05

Prepared \& Analyzed: 08/06/08

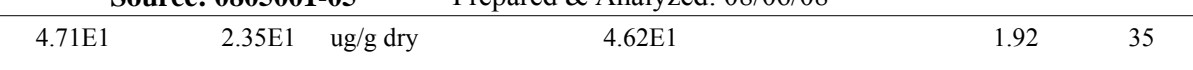

Batch 8H20001 - 1:1 Water Extract (pH_EC_Alk)

\begin{tabular}{|c|c|c|c|c|}
\hline Duplicate (8H20001-DUP2) & Source: 0805003-89 & Prepared \& Analyzed: 08/20/08 & & \\
\hline $\mathrm{pH}$ & N/A pH Units & $7.75 \mathrm{E} 0$ & 0.00 & 35 \\
\hline
\end{tabular}

Batch 8H20002 - 1:1 Water Extract (pH_EC_Alk)

Blank (8H20002-BLK1)

Prepared \& Analyzed: 08/20/08

Specific Conductance (EC)

$<1.00 \mathrm{E}-2 \quad 1.00 \mathrm{E}-2 \quad \mathrm{mS} / \mathrm{cm}$ 
Wet Chemistry - Quality Control

Environmental Science Laboratory

\begin{tabular}{|c|c|c|c|c|c|c|c|c|c|c|}
\hline & & orting & & Spike & Source & & $\%$ REC & & RPD & \\
\hline Analyte & Result & Limit & Units & Level & Result & $\%$ REC & Limits & RPD & Limit & Notes \\
\hline
\end{tabular}

Batch 8H20003 - 1:1 Water Extract (pH_EC_Alk)

Blank (8H20003-BLK1)

Alkalinity as $\mathrm{CaCO} 3$

$<2.35 \mathrm{E} 1 \quad 2.35 \mathrm{E} 1 \quad \mathrm{ug} / \mathrm{g}$ wet


Anions by Ion Chromatography - Quality Control

Environmental Science Laboratory

\begin{tabular}{|c|c|c|c|c|c|c|c|c|c|c|}
\hline Analyte & Result & $\begin{array}{r}\text { porting } \\
\text { Limit }\end{array}$ & Units & $\begin{array}{l}\text { Spike } \\
\text { Level }\end{array}$ & $\begin{array}{l}\text { Source } \\
\text { Result }\end{array}$ & $\%$ REC & $\begin{array}{l}\text { \%REC } \\
\text { Limits }\end{array}$ & RPD & $\begin{array}{l}\text { RPD } \\
\text { Limit }\end{array}$ & Notes \\
\hline
\end{tabular}

Batch 8H01004 - 1:1 Water Extract (IC)

Blank (8H01004-BLK1)

Fluoride

Chloride

Nitrite

Bromide

Nitrate

Sulfate

Phosphate

$\begin{array}{rrc}<2.00 \mathrm{E}-1 & 2.00 \mathrm{E}-1 & \text { ug/g wet } \\ <5.00 \mathrm{E}-1 & 5.00 \mathrm{E}-1 & " \\ <1.00 \mathrm{E} 0 & 1.00 \mathrm{E} 0 & " \\ <1.00 \mathrm{E} 0 & 1.00 \mathrm{E} 0 & " \\ <1.00 \mathrm{E} 0 & 1.00 \mathrm{E} 0 & " \\ <1.50 \mathrm{E} 0 & 1.50 \mathrm{E} 0 & " \\ <1.50 \mathrm{E} 0 & 1.50 \mathrm{E} 0 & "\end{array}$

Prepared: 08/01/08 Analyzed: 08/02/08

LCS (8H01004-BS1)

Prepared: 08/01/08 Analyzed: 08/02/08

\section{Fluoride \\ Chloride \\ Nitrite \\ Bromide \\ Nitrate \\ Sulfate}

Phosphate

(2)

Duplicate (8H01004-DUP1)

Fluoride

Chloride

Nitrite

Bromide

Nitrate

Sulfate

Phosphate

Post Spike (8H01004-PS1)

Fluoride

Chloride

Nitrite

Bromide

Nitrate

Sulfate

Phosphate

$2.08 \mathrm{E} 0$

$2.08 \mathrm{E} 0 \quad 2.00 \mathrm{E}-1 \quad \mathrm{ug} / \mathrm{g}$ wet

$4.93 \mathrm{E} 0$

\section{$1.10 \mathrm{E} 1$}

$9.85 \mathrm{E} 0$

$1.06 \mathrm{E} 1$

$1.51 \mathrm{E} 1$

$1.48 \mathrm{E} 1$

$5.00 \mathrm{E}-1$

$104 \quad 80-120$

$98.6 \quad 80-120$

$1.00 \mathrm{E} 0 \quad " \quad 9.99 \mathrm{E} 0$

110

$80-120$

$\begin{array}{lll}1.00 \mathrm{E} 0 & " & 9.99 \mathrm{E} 0 \\ 1.00 \mathrm{E} 0 & " & 9.99 \mathrm{E} 0\end{array}$

98.6

106

80-120

$1.00 \mathrm{E} 0 \quad " \quad 9.99 \mathrm{E} 0$

$1.50 \mathrm{E} 0$

$1.50 \mathrm{E} 1$

$100 \quad 80-120$

$1.50 \mathrm{E} 0 \quad " 1.50 \mathrm{E} 1$

$98.9 \quad 80-120$

Source: 0805003-15 Prepared: 08/01/08 Analyzed: 08/02/08

\begin{tabular}{|c|c|c|c|c|c|c|}
\hline $2.32 \mathrm{E}-1$ & $2.00 \mathrm{E}-1$ & ug/g dry & $2.34 \mathrm{E}-1$ & 0.713 & 35 & $\mathrm{D}$ \\
\hline$<5.00 \mathrm{E}-1$ & $5.00 \mathrm{E}-1$ & $"$ & ND & & 35 & \\
\hline$<1.00 \mathrm{E} 0$ & $1.00 \mathrm{E} 0$ & $"$ & ND & & 35 & \\
\hline$<1.00 \mathrm{E} 0$ & $1.00 \mathrm{E} 0$ & $"$ & ND & & 35 & \\
\hline 2.44E0 & $1.00 \mathrm{E} 0$ & $"$ & $2.46 \mathrm{E} 0$ & 0.508 & 35 & D \\
\hline 7.24E0 & $1.50 \mathrm{E} 0$ & $"$ & 7.27E0 & 0.282 & 35 & D \\
\hline$<1.50 \mathrm{E} 0$ & $1.50 \mathrm{E} 0$ & $"$ & ND & & 35 & \\
\hline
\end{tabular}

Source: 0805003-06

Prepared: 08/01/08 Analyzed: 08/02/08

\begin{tabular}{lcccccc}
\multicolumn{2}{c}{ Source: 0805003-06 } & \multicolumn{2}{c}{ Prepared: 08/01/08 } & Analyzed: 08/02/08 \\
$1.92 \mathrm{E} 0$ & N/A & ug/mL & $8.00 \mathrm{E}-1$ & $1.14 \mathrm{E} 0$ & 96.9 & $75-125$ \\
$2.13 \mathrm{E} 0$ & N/A & $"$ & $2.00 \mathrm{E} 0$ & $2.84 \mathrm{E}-1$ & 92.4 & $75-125$ \\
$3.88 \mathrm{E} 0$ & N/A & $"$ & $4.00 \mathrm{E} 0$ & $\mathrm{ND}$ & 97 & $75-125$ \\
$3.77 \mathrm{E} 0$ & $\mathrm{~N} / \mathrm{A}$ & $"$ & $4.00 \mathrm{E} 0$ & $\mathrm{ND}$ & 94.2 & $75-125$ \\
$8.35 \mathrm{E} 0$ & $\mathrm{~N} / \mathrm{A}$ & $"$ & $4.00 \mathrm{E} 0$ & $4.60 \mathrm{E} 0$ & 93.7 & $75-125$ \\
$8.33 \mathrm{E} 0$ & $\mathrm{~N}$ & $"$ & $6.00 \mathrm{E} 0$ & $2.68 \mathrm{E} 0$ & 94.2 & $75-125$ \\
$6.28 \mathrm{E} 0$ & N/A & $"$ & $6.00 \mathrm{E} 0$ & $7.90 \mathrm{E}-1$ & 91.5 & $75-125$
\end{tabular}


Anions by Ion Chromatography - Quality Control

Environmental Science Laboratory

\begin{tabular}{|c|c|c|c|c|c|c|c|c|c|c|}
\hline Analyte & Result & $\begin{array}{r}\text { porting } \\
\text { Limit }\end{array}$ & Units & $\begin{array}{l}\text { Spike } \\
\text { Level }\end{array}$ & $\begin{array}{l}\text { Source } \\
\text { Result }\end{array}$ & $\%$ REC & $\begin{array}{l}\text { \%REC } \\
\text { Limits }\end{array}$ & RPD & $\begin{array}{l}\text { RPD } \\
\text { Limit }\end{array}$ & Notes \\
\hline
\end{tabular}

Batch 8H05004 - 1:1 Water Extract (IC)

\section{Blank (8H05004-BLK1)}

Fluoride

Chloride

Nitrite

Bromide

Nitrate

Sulfate

Phosphate

$\begin{array}{lrc} & & \\ <2.00 \mathrm{E}-1 & 2.00 \mathrm{E}-1 & \text { ug/g wet } \\ <5.00 \mathrm{E}-1 & 5.00 \mathrm{E}-1 & " \\ <1.00 \mathrm{E} 0 & 1.00 \mathrm{E} 0 & " \\ <1.00 \mathrm{E} 0 & 1.00 \mathrm{E} 0 & " \\ <1.00 \mathrm{E} 0 & 1.00 \mathrm{E} 0 & " \\ <1.50 \mathrm{E} 0 & 1.50 \mathrm{E} 0 & " \\ <1.50 \mathrm{E} 0 & 1.50 \mathrm{E} 0 & \end{array}$

LCS (8H05004-BS1)

Prepared: 08/05/08 Analyzed: 08/06/08

\begin{tabular}{|c|c|c|c|c|c|c|c|}
\hline Fluoride & $2.00 \mathrm{E} 0$ & $2.00 \mathrm{E}-1$ & ug/g wet & $2.00 \mathrm{E} 0$ & & 100 & $80-120$ \\
\hline Chloride & $4.85 \mathrm{E} 0$ & $5.00 \mathrm{E}-1$ & $"$ & $5.00 \mathrm{E} 0$ & & 96.9 & $80-120$ \\
\hline Nitrite & $1.12 \mathrm{E} 1$ & $1.00 \mathrm{E} 0$ & $"$ & $1.00 \mathrm{E} 1$ & & 112 & $80-12$ \\
\hline Bromide & $9.96 \mathrm{E} 0$ & $1.00 \mathrm{E} 0$ & $"$ & $1.00 \mathrm{E} 1$ & & 99.6 & $80-12$ \\
\hline Nitrate & $1.03 \mathrm{E} 1$ & $1.00 \mathrm{E} 0$ & $"$ & $1.00 \mathrm{E} 1$ & & 103 & $80-120$ \\
\hline Sulfate & $1.49 \mathrm{E} 1$ & $1.50 \mathrm{E} 0$ & $"$ & $1.50 \mathrm{E} 1$ & & 99.5 & $80-120$ \\
\hline Phosphate & $1.51 \mathrm{E} 1$ & $1.50 \mathrm{E} 0$ & $"$ & $1.50 \mathrm{E} 1$ & & 101 & $80-120$ \\
\hline Duplicate (8H05004-DUP1) & \multicolumn{3}{|c|}{ Source: 0805001-05 } & \multicolumn{2}{|c|}{ Prepared: 08/05/08 } & Analyzec & $8 / 06 / 08$ \\
\hline Fluoride & $5.78 \mathrm{E}-1$ & $2.00 \mathrm{E}-1$ & ug/g dry & & $5.95 \mathrm{E}-1$ & & \\
\hline Chloride & $<5.00 \mathrm{E}-1$ & $5.00 \mathrm{E}-1$ & $"$ & & ND & & \\
\hline Nitrite & $<1.00 \mathrm{E} 0$ & $1.00 \mathrm{E} 0$ & $"$ & & ND & & \\
\hline Bromide & $<1.00 \mathrm{E} 0$ & $1.00 \mathrm{E} 0$ & $"$ & & ND & & \\
\hline Nitrate & $<1.00 \mathrm{E} 0$ & $1.00 \mathrm{E} 0$ & $"$ & & ND & & \\
\hline Sulfate & $<1.50 \mathrm{E} 0$ & $1.50 \mathrm{E} 0$ & $"$ & & ND & & \\
\hline Phosphate & $<1.50 \mathrm{E} 0$ & $1.50 \mathrm{E} 0$ & $"$ & & ND & & \\
\hline Post Spike (8H05004-PS1) & \multicolumn{3}{|c|}{ Source: 0805003-05 } & \multicolumn{2}{|c|}{ Prepared: 08/05/08 } & Analyzec & 8/06/08 \\
\hline Fluoride & $1.79 \mathrm{E} 0$ & $\mathrm{~N} / \mathrm{A}$ & $\mathrm{ug} / \mathrm{mL}$ & $8.00 \mathrm{E}-1$ & $1.01 \mathrm{E} 0$ & 96.9 & $75-125$ \\
\hline Chloride & $2.20 \mathrm{E} 0$ & N/A & $"$ & $2.00 \mathrm{E} 0$ & $3.92 \mathrm{E}-1$ & 90.6 & $75-125$ \\
\hline Nitrite & $3.96 \mathrm{E} 0$ & N/A & $"$ & $4.00 \mathrm{E} 0$ & ND & 99 & $75-125$ \\
\hline Bromide & $3.81 \mathrm{E} 0$ & N/A & $"$ & $4.00 \mathrm{E} 0$ & ND & 95.3 & $75-125$ \\
\hline Nitrate & $5.25 \mathrm{E} 0$ & N/A & $"$ & $4.00 \mathrm{E} 0$ & $1.44 \mathrm{E} 0$ & 95.2 & $75-125$ \\
\hline Sulfate & $7.56 \mathrm{E} 0$ & N/A & $"$ & $6.00 \mathrm{E} 0$ & $2.02 \mathrm{E} 0$ & 92.3 & $75-125$ \\
\hline Phosphate & $5.92 \mathrm{E} 0$ & N/A & $"$ & $6.00 \mathrm{E} 0$ & $4.44 \mathrm{E}-1$ & 91.3 & $75-125$ \\
\hline
\end{tabular}


Anions by Ion Chromatography - Quality Control

Environmental Science Laboratory

\begin{tabular}{|c|c|c|c|c|c|c|c|c|c|c|}
\hline Analyte & Result & $\begin{array}{r}\text { porting } \\
\text { Limit }\end{array}$ & Units & $\begin{array}{l}\text { Spike } \\
\text { Level }\end{array}$ & $\begin{array}{l}\text { Source } \\
\text { Result }\end{array}$ & $\%$ REC & $\begin{array}{l}\text { \%REC } \\
\text { Limits }\end{array}$ & RPD & $\begin{array}{l}\text { RPD } \\
\text { Limit }\end{array}$ & Notes \\
\hline
\end{tabular}

Batch 8H21001 - 1:1 Water Extract (IC)

\begin{tabular}{|c|c|c|c|c|c|c|}
\hline Blank (8H21001-BLK1) & \multicolumn{6}{|c|}{ Prepared: 08/21/08 Analyzed: 08/22/08 } \\
\hline Fluoride & $<2.00 \mathrm{E}-1$ & $2.00 \mathrm{E}-1$ & ug/g wet & & & \\
\hline Chloride & $<5.00 \mathrm{E}-1$ & $5.00 \mathrm{E}-1$ & $"$ & & & \\
\hline Nitrite & $<1.00 \mathrm{E} 0$ & $1.00 \mathrm{E} 0$ & $"$ & & & \\
\hline Bromide & $<1.00 \mathrm{E} 0$ & $1.00 \mathrm{E} 0$ & $"$ & & & \\
\hline Nitrate & $<1.00 \mathrm{E} 0$ & $1.00 \mathrm{E} 0$ & $"$ & & & \\
\hline Sulfate & $<1.50 \mathrm{E} 0$ & $1.50 \mathrm{E} 0$ & $"$ & & & \\
\hline Phosphate & $<1.50 \mathrm{E} 0$ & $1.50 \mathrm{E} 0$ & $"$ & & & \\
\hline LCS (8H21001-BS1) & \multicolumn{6}{|c|}{ Prepared: 08/21/08 Analyzed: 08/22/08 } \\
\hline Fluoride & 2.07E0 & $2.00 \mathrm{E}-1$ & ug/g wet & $2.00 \mathrm{E} 0$ & 103 & $80-120$ \\
\hline Chloride & 4.94E0 & $5.00 \mathrm{E}-1$ & $"$ & $5.00 \mathrm{E} 0$ & 98.7 & $80-120$ \\
\hline Nitrite & $1.10 \mathrm{E} 1$ & $1.00 \mathrm{E} 0$ & $"$ & $1.00 \mathrm{E} 1$ & 110 & $80-120$ \\
\hline Bromide & $1.01 \mathrm{E} 1$ & $1.00 \mathrm{E} 0$ & $"$ & $1.00 \mathrm{E} 1$ & 101 & $80-120$ \\
\hline Nitrate & $1.05 \mathrm{E} 1$ & $1.00 \mathrm{E} 0$ & $"$ & $1.00 \mathrm{E} 1$ & 105 & $80-120$ \\
\hline Sulfate & $1.54 \mathrm{E} 1$ & $1.50 \mathrm{E} 0$ & $"$ & $1.50 \mathrm{E} 1$ & 103 & $80-120$ \\
\hline Phosphate & $1.52 \mathrm{E} 1$ & $1.50 \mathrm{E} 0$ & $"$ & $1.50 \mathrm{E} 1$ & 101 & $80-120$ \\
\hline
\end{tabular}


Total Metals by PNNL-AGG-ICP-AES/Water Extract - Quality Control

Environmental Science Laboratory

\begin{tabular}{|lrrrrrrrrrrr}
\hline & & Reporting & & Spike & Source & & \%REC & & RPD \\
Analyte & Result & Limit & Units & Level & Result & $\%$ REC & Limits & RPD & Limit & Notes \\
\hline
\end{tabular}

Batch 8I15002 - 1:1 Water Extract (ICP/ICPMS)

\begin{tabular}{|c|c|c|c|}
\hline \multicolumn{4}{|c|}{ Blank (8I15002-BLK1) } \\
\hline Aluminum & $<8.58 \mathrm{E}-2$ & $8.58 \mathrm{E}-2$ & ug/g wet \\
\hline Boron & $<1.94 \mathrm{E} 0$ & $1.94 \mathrm{E} 0$ & $"$ \\
\hline Barium & $1.29 \mathrm{E}-2$ & $8.79 \mathrm{E}-3$ & $"$ \\
\hline Beryllium & $<2.84 \mathrm{E}-2$ & $2.84 \mathrm{E}-2$ & $"$ \\
\hline Bismuth & $<1.88 \mathrm{E}-1$ & $1.88 \mathrm{E}-1$ & $"$ \\
\hline Calcium & $<3.87 \mathrm{E}-1$ & $3.87 \mathrm{E}-1$ & $"$ \\
\hline Cadmium & $<2.69 \mathrm{E}-2$ & $2.69 \mathrm{E}-2$ & $"$ \\
\hline Cobalt & $<9.60 \mathrm{E}-2$ & $9.60 \mathrm{E}-2$ & $"$ \\
\hline Chromium & $<3.33 \mathrm{E}-2$ & $3.33 \mathrm{E}-2$ & $"$ \\
\hline Copper & $<8.04 \mathrm{E}-2$ & $8.04 \mathrm{E}-2$ & $"$ \\
\hline Iron & $<1.42 \mathrm{E}-1$ & $1.42 \mathrm{E}-1$ & $"$ \\
\hline Potassium & $<2.33 \mathrm{E} 0$ & 2.33E0 & $"$ \\
\hline Lithium & $<5.40 \mathrm{E}-1$ & $5.40 \mathrm{E}-1$ & $"$ \\
\hline Magnesium & $<8.34 \mathrm{E}-2$ & $8.34 \mathrm{E}-2$ & $"$ \\
\hline Manganese & $<1.71 \mathrm{E}-2$ & $1.71 \mathrm{E}-2$ & $"$ \\
\hline Molybdenum & $<1.39 \mathrm{E}-1$ & $1.39 \mathrm{E}-1$ & $"$ \\
\hline Nickel & $<9.33 \mathrm{E}-2$ & $9.33 \mathrm{E}-2$ & " \\
\hline Phosphorus & $<1.03 \mathrm{E} 0$ & $1.03 \mathrm{E} 0$ & $"$ \\
\hline Lead & $<4.32 \mathrm{E}-1$ & $4.32 \mathrm{E}-1$ & $"$ \\
\hline Selenium & $<1.95 \mathrm{E} 0$ & $1.95 \mathrm{E} 0$ & $"$ \\
\hline Strontium & $<5.22 \mathrm{E}-2$ & $5.22 \mathrm{E}-2$ & $"$ \\
\hline Thallium & $<1.03 \mathrm{E} 0$ & $1.03 \mathrm{E} 0$ & $"$ \\
\hline Vanadium & $<4.53 \mathrm{E}-2$ & $4.53 \mathrm{E}-2$ & " \\
\hline Zinc & $<9.27 \mathrm{E}-2$ & $9.27 \mathrm{E}-2$ & $"$ \\
\hline Sodium & $<6.69 \mathrm{E}-1$ & $6.69 \mathrm{E}-1$ & $"$ \\
\hline Sulfur & $<3.08 \mathrm{E} 0$ & 3.08E0 & " \\
\hline Titanium & $<8.76 \mathrm{E}-3$ & $8.76 \mathrm{E}-3$ & $"$ \\
\hline Zirconium & $<1.50 \mathrm{E}-1$ & $1.50 \mathrm{E}-1$ & $"$ \\
\hline Silver & $<7.71 \mathrm{E}-2$ & $7.71 \mathrm{E}-2$ & " \\
\hline Rhenium & $<1.52 \mathrm{E}-1$ & $1.52 \mathrm{E}-1$ & $"$ \\
\hline
\end{tabular}


Total Metals by PNNL-AGG-ICP-AES/Water Extract - Quality Control

Environmental Science Laboratory

\begin{tabular}{|lrrrrrrrrrrr}
\hline & & Reporting & & Spike & Source & & \%REC & & RPD \\
Analyte & Result & Limit & Units & Level & Result & $\%$ REC & Limits & RPD & Limit & Notes \\
\hline
\end{tabular}

Batch 8I15002 - 1:1 Water Extract (ICP/ICPMS)

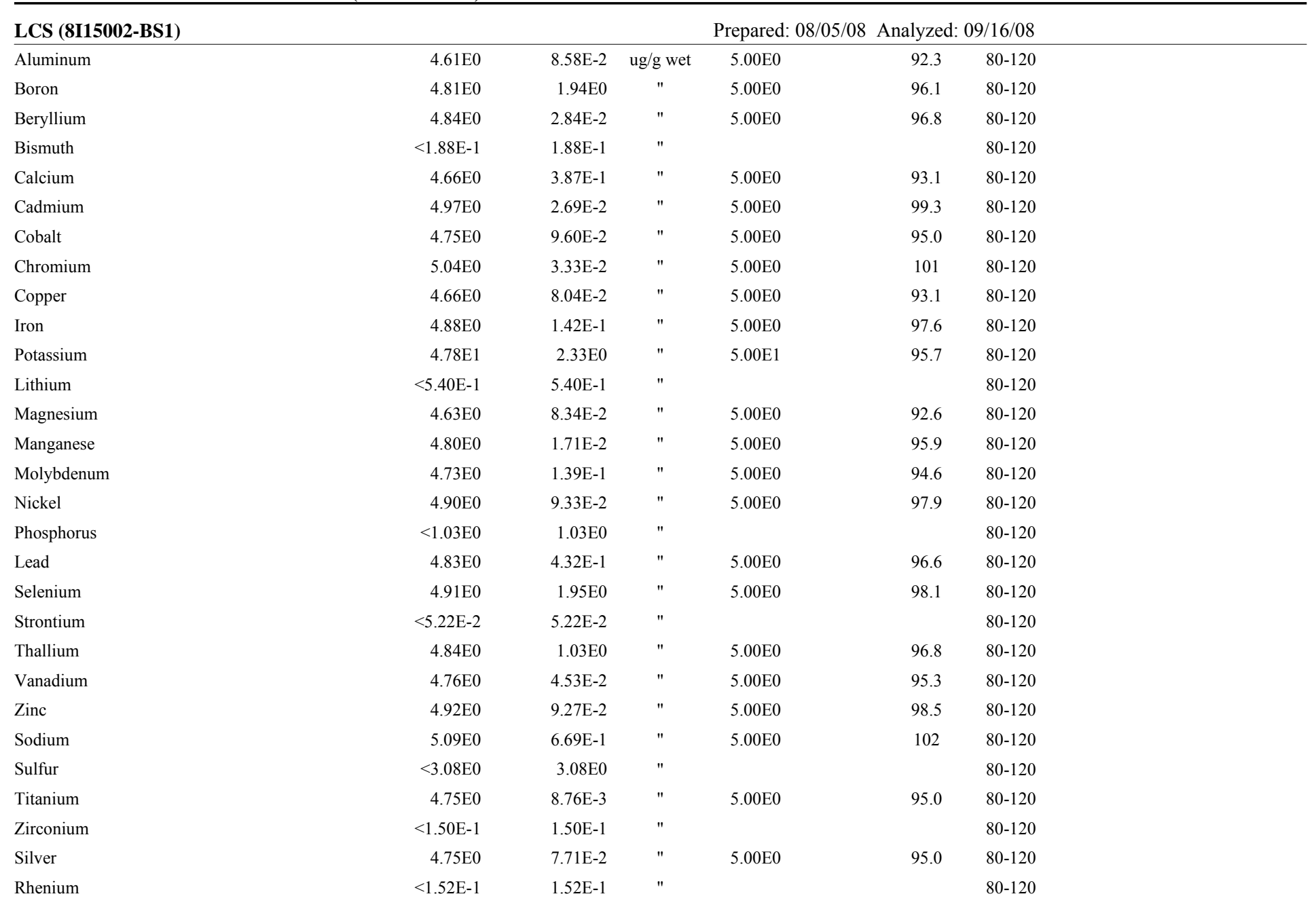


Total Metals by PNNL-AGG-ICP-AES/Water Extract - Quality Control

Environmental Science Laboratory

\begin{tabular}{|c|c|c|c|c|c|c|c|c|c|c|}
\hline Analyte & Result & $\begin{array}{r}\text { porting } \\
\text { Limit }\end{array}$ & Units & $\begin{array}{l}\text { Spike } \\
\text { Level }\end{array}$ & $\begin{array}{l}\text { Source } \\
\text { Result }\end{array}$ & $\%$ REC & $\begin{array}{l}\text { \%REC } \\
\text { Limits }\end{array}$ & RPD & $\begin{array}{l}\text { RPD } \\
\text { Limit }\end{array}$ & Notes \\
\hline
\end{tabular}

Batch 8I15002 - 1:1 Water Extract (ICP/ICPMS)

\begin{tabular}{|c|c|c|c|c|c|c|}
\hline Duplicate (8I15002-DUP1) & \multicolumn{3}{|c|}{ Source: 0805001-05 } & \multicolumn{3}{|c|}{ Prepared: 08/05/08 Analyzed: 09/16/08 } \\
\hline Aluminum & $4.78 \mathrm{E}-1$ & $8.58 \mathrm{E}-2$ & ug/g dry & $4.42 \mathrm{E}-1$ & 7.66 & 35 \\
\hline Boron & $<1.94 \mathrm{E} 0$ & $1.94 \mathrm{E} 0$ & $"$ & ND & & 35 \\
\hline Beryllium & $<2.84 \mathrm{E}-2$ & $2.84 \mathrm{E}-2$ & $"$ & ND & & 35 \\
\hline Bismuth & $<1.88 \mathrm{E}-1$ & $1.88 \mathrm{E}-1$ & $"$ & ND & & 35 \\
\hline Calcium & $7.20 \mathrm{E} 0$ & $3.87 \mathrm{E}-1$ & $"$ & 7.19E0 & 0.220 & 35 \\
\hline Cadmium & $<2.69 \mathrm{E}-2$ & $2.69 \mathrm{E}-2$ & $"$ & ND & & 35 \\
\hline Cobalt & $<9.60 \mathrm{E}-2$ & $9.60 \mathrm{E}-2$ & $"$ & ND & & 35 \\
\hline Chromium & $<3.33 \mathrm{E}-2$ & $3.33 \mathrm{E}-2$ & $"$ & ND & & 35 \\
\hline Copper & $<8.04 \mathrm{E}-2$ & $8.04 \mathrm{E}-2$ & $"$ & ND & & 35 \\
\hline Iron & $5.04 \mathrm{E}-1$ & $1.42 \mathrm{E}-1$ & $"$ & $4.86 \mathrm{E}-1$ & 3.77 & 35 \\
\hline Potassium & $2.96 \mathrm{E} 0$ & 2.33E0 & $"$ & $2.94 \mathrm{E} 0$ & 0.676 & 35 \\
\hline Lithium & $<5.40 \mathrm{E}-1$ & $5.40 \mathrm{E}-1$ & $"$ & ND & & 35 \\
\hline Magnesium & $1.12 \mathrm{E} 0$ & $8.34 \mathrm{E}-2$ & $"$ & $1.15 \mathrm{E} 0$ & 2.28 & 35 \\
\hline Manganese & $<1.71 \mathrm{E}-2$ & $1.71 \mathrm{E}-2$ & $"$ & ND & & 35 \\
\hline Molybdenum & $<1.39 \mathrm{E}-1$ & $1.39 \mathrm{E}-1$ & $"$ & ND & & 35 \\
\hline Nickel & $<9.33 \mathrm{E}-2$ & $9.33 \mathrm{E}-2$ & $"$ & ND & & 35 \\
\hline Phosphorus & $<1.03 \mathrm{E} 0$ & $1.03 \mathrm{E} 0$ & $"$ & ND & & 35 \\
\hline Lead & $<4.32 \mathrm{E}-1$ & $4.32 \mathrm{E}-1$ & $"$ & ND & & 35 \\
\hline Selenium & $<1.95 \mathrm{E} 0$ & $1.95 \mathrm{E} 0$ & $"$ & ND & & 35 \\
\hline Strontium & $<5.22 \mathrm{E}-2$ & $5.22 \mathrm{E}-2$ & $"$ & ND & & 35 \\
\hline Thallium & $<1.03 \mathrm{E} 0$ & $1.03 \mathrm{E} 0$ & $"$ & ND & & 35 \\
\hline Vanadium & $<4.53 \mathrm{E}-2$ & $4.53 \mathrm{E}-2$ & $"$ & ND & & 35 \\
\hline Zinc & $<9.27 \mathrm{E}-2$ & $9.27 \mathrm{E}-2$ & $"$ & ND & & 35 \\
\hline Sodium & $6.42 \mathrm{E} 0$ & $6.69 \mathrm{E}-1$ & $"$ & $6.60 \mathrm{E} 0$ & 2.75 & 35 \\
\hline Sulfur & $<3.07 \mathrm{E} 0$ & 3.07E0 & $"$ & ND & & 35 \\
\hline Titanium & $2.52 \mathrm{E}-2$ & $8.76 \mathrm{E}-3$ & $"$ & $2.32 \mathrm{E}-2$ & 8.30 & 35 \\
\hline Zirconium & $<1.50 \mathrm{E}-1$ & $1.50 \mathrm{E}-1$ & $"$ & ND & & 35 \\
\hline Silver & $<7.71 \mathrm{E}-2$ & $7.71 \mathrm{E}-2$ & $"$ & ND & & 35 \\
\hline Rhenium & $<1.52 \mathrm{E}-1$ & $1.52 \mathrm{E}-1$ & $"$ & ND & & 35 \\
\hline
\end{tabular}


Total Metals by PNNL-AGG-ICP-AES/Water Extract - Quality Control

Environmental Science Laboratory

\begin{tabular}{|lrrrrrrrrrrr}
\hline & & Reporting & & Spike & Source & & \%REC & & RPD \\
Analyte & Result & Limit & Units & Level & Result & $\%$ REC & Limits & RPD & Limit & Notes \\
\hline
\end{tabular}

Batch 8I15002 - 1:1 Water Extract (ICP/ICPMS)

\begin{tabular}{|c|c|c|c|c|c|c|c|}
\hline \multirow{2}{*}{$\begin{array}{l}\text { Post Spike (8I15002-PS1) } \\
\text { Aluminum }\end{array}$} & \multicolumn{3}{|c|}{ Source: 0805001-05 } & \multicolumn{2}{|c|}{ Prepared: 09/15/08 } & \multicolumn{2}{|c|}{ Analyzed: 09/16/08 } \\
\hline & $6.31 \mathrm{E} 2$ & N/A & $\mathrm{ug} / \mathrm{L}$ & $5.00 \mathrm{E} 2$ & $1.50 \mathrm{E} 2$ & 96.4 & $75-125$ \\
\hline Boron & $5.17 \mathrm{E} 2$ & N/A & $"$ & $5.00 \mathrm{E} 2$ & $1.39 \mathrm{E} 1$ & 101 & $75-125$ \\
\hline Beryllium & $2.60 \mathrm{E} 2$ & N/A & $"$ & $2.50 \mathrm{E} 2$ & $4.00 \mathrm{E}-1$ & 104 & $75-125$ \\
\hline Bismuth & $4.87 \mathrm{E} 2$ & N/A & $"$ & $5.00 \mathrm{E} 2$ & $1.62 \mathrm{E} 1$ & 94.1 & $75-125$ \\
\hline Calcium & $2.96 \mathrm{E} 3$ & N/A & $"$ & $5.00 \mathrm{E} 2$ & $2.43 \mathrm{E} 3$ & 107 & $75-125$ \\
\hline Cadmium & $2.50 \mathrm{E} 2$ & N/A & $"$ & $2.50 \mathrm{E} 2$ & ND & 100 & $75-125$ \\
\hline Cobalt & $2.48 \mathrm{E} 2$ & N/A & $"$ & $2.50 \mathrm{E} 2$ & $1.90 \mathrm{E}-1$ & 99.2 & $75-125$ \\
\hline Chromium & $1.28 \mathrm{E} 2$ & N/A & $"$ & $1.25 \mathrm{E} 2$ & ND & 103 & $75-125$ \\
\hline Copper & $4.99 \mathrm{E} 2$ & N/A & $"$ & $5.00 \mathrm{E} 2$ & $4.58 \mathrm{E} 0$ & 98.9 & $75-125$ \\
\hline Iron & $6.81 \mathrm{E} 2$ & N/A & $"$ & $5.00 \mathrm{E} 2$ & $1.64 \mathrm{E} 2$ & 103 & $75-125$ \\
\hline Potassium & $2.27 \mathrm{E} 3$ & N/A & $"$ & $1.25 \mathrm{E} 3$ & $9.92 \mathrm{E} 2$ & 102 & $75-125$ \\
\hline Lithium & $4.86 \mathrm{E} 2$ & N/A & $"$ & $5.00 \mathrm{E} 2$ & $3.12 \mathrm{E} 0$ & 96.5 & $75-125$ \\
\hline Magnesium & $8.76 \mathrm{E} 2$ & N/A & $"$ & $5.00 \mathrm{E} 2$ & $3.87 \mathrm{E} 2$ & 97.7 & $75-125$ \\
\hline Manganese & $2.59 \mathrm{E} 2$ & N/A & $"$ & $2.50 \mathrm{E} 2$ & $7.28 \mathrm{E}-1$ & 103 & $75-125$ \\
\hline Molybdenum & $4.96 \mathrm{E} 2$ & N/A & $"$ & $5.00 \mathrm{E} 2$ & $2.07 \mathrm{E} 0$ & 98.7 & $75-125$ \\
\hline Nickel & $5.06 \mathrm{E} 2$ & N/A & $"$ & $5.00 \mathrm{E} 2$ & $5.91 \mathrm{E}-2$ & 101 & $75-125$ \\
\hline Phosphorus & $1.29 \mathrm{E} 3$ & N/A & $"$ & $1.25 \mathrm{E} 3$ & $5.36 \mathrm{E} 1$ & 98.8 & $75-125$ \\
\hline Lead & $5.00 \mathrm{E} 2$ & N/A & $"$ & $5.00 \mathrm{E} 2$ & $2.21 \mathrm{E} 0$ & 99.6 & $75-125$ \\
\hline Selenium & $4.77 \mathrm{E} 2$ & N/A & $"$ & $5.00 \mathrm{E} 2$ & $2.40 \mathrm{E} 1$ & 90.6 & $75-125$ \\
\hline Strontium & $5.00 \mathrm{E} 2$ & N/A & $"$ & $5.00 \mathrm{E} 2$ & $1.15 \mathrm{E} 1$ & 97.6 & $75-125$ \\
\hline Thallium & $5.04 \mathrm{E} 2$ & N/A & $"$ & $5.00 \mathrm{E} 2$ & $2.29 \mathrm{E} 0$ & 100 & $75-125$ \\
\hline Vanadium & $2.54 \mathrm{E} 2$ & N/A & $"$ & $2.50 \mathrm{E} 2$ & $1.07 \mathrm{E} 1$ & 97.5 & $75-125$ \\
\hline Zinc & $2.89 \mathrm{E} 2$ & N/A & $"$ & $2.50 \mathrm{E} 2$ & $1.35 \mathrm{E} 1$ & 110 & $75-125$ \\
\hline Sodium & $2.80 \mathrm{E} 3$ & N/A & $"$ & $5.00 \mathrm{E} 2$ & $2.23 \mathrm{E} 3$ & 114 & $75-125$ \\
\hline Sulfur & $1.14 \mathrm{E} 3$ & N/A & $"$ & $1.00 \mathrm{E} 3$ & $1.33 \mathrm{E} 2$ & 101 & $75-125$ \\
\hline Titanium & $2.56 \mathrm{E} 2$ & N/A & $"$ & $2.50 \mathrm{E} 2$ & $7.83 \mathrm{E} 0$ & 99.4 & $75-125$ \\
\hline Zirconium & $2.60 \mathrm{E} 2$ & N/A & $"$ & $2.50 \mathrm{E} 2$ & $1.87 \mathrm{E} 1$ & 96.7 & $75-125$ \\
\hline Silver & $5.05 \mathrm{E} 2$ & N/A & $"$ & $5.00 \mathrm{E} 2$ & $1.59 \mathrm{E} 0$ & 101 & $75-125$ \\
\hline Rhenium & $5.05 \mathrm{E} 2$ & N/A & $"$ & $5.00 \mathrm{E} 2$ & $5.23 \mathrm{E} 0$ & 99.9 & $75-125$ \\
\hline
\end{tabular}


Total Metals by PNNL-AGG-ICP-AES/Water Extract - Quality Control

Environmental Science Laboratory

\begin{tabular}{|lrrrrrrrrrrr}
\hline & & Reporting & & Spike & Source & & \%REC & & RPD \\
Analyte & Result & Limit & Units & Level & Result & $\%$ REC & Limits & RPD & Limit & Notes \\
\hline
\end{tabular}

Batch 8I15003 - 1:1 Water Extract (ICP/ICPMS)

\begin{tabular}{|c|c|c|c|}
\hline \multicolumn{4}{|c|}{ Blank (8I15003-BLK1) } \\
\hline Aluminum & $<8.58 \mathrm{E}-2$ & $8.58 \mathrm{E}-2$ & ug/g wet \\
\hline Boron & $<1.94 \mathrm{E} 0$ & $1.94 \mathrm{E} 0$ & $"$ \\
\hline Beryllium & $<2.84 \mathrm{E}-2$ & $2.84 \mathrm{E}-2$ & $"$ \\
\hline Bismuth & $<1.88 \mathrm{E}-1$ & $1.88 \mathrm{E}-1$ & $"$ \\
\hline Calcium & $<3.87 \mathrm{E}-1$ & $3.87 \mathrm{E}-1$ & $"$ \\
\hline Cadmium & $<2.69 \mathrm{E}-2$ & $2.69 \mathrm{E}-2$ & $"$ \\
\hline Cobalt & $<9.60 \mathrm{E}-2$ & $9.60 \mathrm{E}-2$ & $"$ \\
\hline Chromium & $<3.33 \mathrm{E}-2$ & $3.33 \mathrm{E}-2$ & $"$ \\
\hline Iron & $<1.42 \mathrm{E}-1$ & $1.42 \mathrm{E}-1$ & $"$ \\
\hline Potassium & $<2.33 \mathrm{E} 0$ & 2.33E0 & $"$ \\
\hline Lithium & $<5.40 \mathrm{E}-1$ & $5.40 \mathrm{E}-1$ & $"$ \\
\hline Magnesium & $<8.34 \mathrm{E}-2$ & 8.34E-2 & $"$ \\
\hline Manganese & $<1.71 \mathrm{E}-2$ & $1.71 \mathrm{E}-2$ & $"$ \\
\hline Molybdenum & $<1.39 \mathrm{E}-1$ & $1.39 \mathrm{E}-1$ & $"$ \\
\hline Nickel & $<9.33 \mathrm{E}-2$ & $9.33 \mathrm{E}-2$ & $"$ \\
\hline Phosphorus & $<1.03 \mathrm{E} 0$ & $1.03 \mathrm{E} 0$ & $"$ \\
\hline Lead & $<4.32 \mathrm{E}-1$ & $4.32 \mathrm{E}-1$ & $"$ \\
\hline Selenium & $<1.95 \mathrm{E} 0$ & $1.95 \mathrm{E} 0$ & $"$ \\
\hline Strontium & $<5.22 \mathrm{E}-2$ & $5.22 \mathrm{E}-2$ & $"$ \\
\hline Thallium & $<1.03 \mathrm{E} 0$ & $1.03 \mathrm{E} 0$ & $"$ \\
\hline Vanadium & $<4.53 \mathrm{E}-2$ & $4.53 \mathrm{E}-2$ & $"$ \\
\hline Zinc & $<9.27 \mathrm{E}-2$ & $9.27 \mathrm{E}-2$ & $"$ \\
\hline Sodium & $<6.69 \mathrm{E}-1$ & $6.69 \mathrm{E}-1$ & $"$ \\
\hline Silicon & $<1.50 \mathrm{E} 0$ & $1.50 \mathrm{E} 0$ & $"$ \\
\hline Sulfur & $<3.08 \mathrm{E} 0$ & $3.08 \mathrm{E} 0$ & $"$ \\
\hline Titanium & $<8.76 \mathrm{E}-3$ & $8.76 \mathrm{E}-3$ & $"$ \\
\hline Zirconium & $<1.50 \mathrm{E}-1$ & $1.50 \mathrm{E}-1$ & $"$ \\
\hline Silver & $<7.71 \mathrm{E}-2$ & $7.71 \mathrm{E}-2$ & $"$ \\
\hline Rhenium & $<1.52 \mathrm{E}-1$ & $1.52 \mathrm{E}-1$ & $"$ \\
\hline
\end{tabular}


Total Metals by PNNL-AGG-ICP-AES/Water Extract - Quality Control

Environmental Science Laboratory

\begin{tabular}{|lrrrrrrrrrrr}
\hline & & Reporting & & Spike & Source & & \%REC & & RPD \\
Analyte & Result & Limit & Units & Level & Result & $\%$ REC & Limits & RPD & Limit & Notes \\
\hline
\end{tabular}

Batch 8I15003 - 1:1 Water Extract (ICP/ICPMS)

\begin{tabular}{|c|c|c|c|c|c|c|}
\hline \multirow{2}{*}{$\begin{array}{l}\text { LCS (8I15003-BS1) } \\
\text { Aluminum }\end{array}$} & \multicolumn{6}{|c|}{ Prepared: 08/01/08 Analyzed: 09/16/08 } \\
\hline & $4.93 \mathrm{E} 0$ & $8.58 \mathrm{E}-2$ & ug/g wet & 4.99E0 & 98.8 & $80-120$ \\
\hline Boron & $5.05 \mathrm{E} 0$ & $1.94 \mathrm{E} 0$ & $"$ & 4.99E0 & 101 & $80-120$ \\
\hline Beryllium & $5.05 \mathrm{E} 0$ & $2.84 \mathrm{E}-2$ & $"$ & 4.99E0 & 101 & $80-120$ \\
\hline Bismuth & $<1.88 \mathrm{E}-1$ & $1.88 \mathrm{E}-1$ & $"$ & & & $80-120$ \\
\hline Calcium & $5.24 \mathrm{E} 0$ & $3.87 \mathrm{E}-1$ & $"$ & 4.99E0 & 105 & $80-120$ \\
\hline Cadmium & $5.14 \mathrm{E} 0$ & $2.69 \mathrm{E}-2$ & $"$ & 4.99E0 & 103 & $80-120$ \\
\hline Cobalt & $4.95 \mathrm{E} 0$ & $9.60 \mathrm{E}-2$ & $"$ & 4.99E0 & 99.2 & $80-120$ \\
\hline Chromium & $5.19 \mathrm{E} 0$ & $3.33 \mathrm{E}-2$ & $"$ & 4.99E0 & 104 & $80-120$ \\
\hline Iron & $5.11 \mathrm{E} 0$ & $1.42 \mathrm{E}-1$ & $"$ & 4.99E0 & 102 & $80-120$ \\
\hline Potassium & $4.95 \mathrm{E} 1$ & $2.33 \mathrm{E} 0$ & $"$ & $4.99 \mathrm{E} 1$ & 99.2 & $80-120$ \\
\hline Lithium & $<5.40 \mathrm{E}-1$ & $5.40 \mathrm{E}-1$ & $"$ & & & $80-120$ \\
\hline Magnesium & $4.80 \mathrm{E} 0$ & $8.34 \mathrm{E}-2$ & $"$ & 4.99E0 & 96.3 & $80-120$ \\
\hline Manganese & $4.95 \mathrm{E} 0$ & $1.71 \mathrm{E}-2$ & $"$ & 4.99E0 & 99.2 & $80-120$ \\
\hline Molybdenum & $4.93 \mathrm{E} 0$ & $1.39 \mathrm{E}-1$ & $"$ & 4.99E0 & 98.8 & $80-120$ \\
\hline Nickel & $5.05 \mathrm{E} 0$ & $9.33 \mathrm{E}-2$ & $"$ & 4.99E0 & 101 & $80-120$ \\
\hline Phosphorus & $<1.03 \mathrm{E} 0$ & $1.03 \mathrm{E} 0$ & $"$ & & & $80-120$ \\
\hline Lead & $5.02 \mathrm{E} 0$ & $4.32 \mathrm{E}-1$ & $"$ & 4.99E0 & 101 & $80-120$ \\
\hline Selenium & $5.08 \mathrm{E} 0$ & $1.95 \mathrm{E} 0$ & $"$ & 4.99E0 & 102 & $80-120$ \\
\hline Strontium & $<5.22 \mathrm{E}-2$ & $5.22 \mathrm{E}-2$ & $"$ & & & $80-120$ \\
\hline Thallium & $4.98 \mathrm{E} 0$ & $1.03 \mathrm{E} 0$ & $"$ & 4.99E0 & 99.7 & $80-120$ \\
\hline Vanadium & $4.92 \mathrm{E} 0$ & $4.53 \mathrm{E}-2$ & $"$ & 4.99E0 & 98.6 & $80-120$ \\
\hline Zinc & $5.77 \mathrm{E} 0$ & $9.27 \mathrm{E}-2$ & $"$ & 4.99E0 & 116 & $80-120$ \\
\hline Sodium & $5.31 \mathrm{E} 0$ & $6.69 \mathrm{E}-1$ & $"$ & 4.99E0 & 106 & $80-120$ \\
\hline Silicon & $3.10 \mathrm{E} 0$ & $1.50 \mathrm{E} 0$ & $"$ & $2.50 \mathrm{E} 0$ & 124 & $80-120$ \\
\hline Sulfur & $<3.08 \mathrm{E} 0$ & $3.08 \mathrm{E} 0$ & $"$ & & & $80-120$ \\
\hline Titanium & $4.95 \mathrm{E} 0$ & $8.76 \mathrm{E}-3$ & $"$ & 4.99E0 & 99.1 & $80-120$ \\
\hline Zirconium & $<1.50 \mathrm{E}-1$ & $1.50 \mathrm{E}-1$ & $"$ & & & $80-120$ \\
\hline Silver & 4.93E0 & $7.71 \mathrm{E}-2$ & $"$ & 4.99E0 & 98.9 & $80-120$ \\
\hline Rhenium & $<1.52 \mathrm{E}-1$ & $1.52 \mathrm{E}-1$ & $"$ & & & $80-120$ \\
\hline
\end{tabular}


Total Metals by PNNL-AGG-ICP-AES/Water Extract - Quality Control

Environmental Science Laboratory

\begin{tabular}{|c|c|c|c|c|c|c|c|c|c|c|}
\hline Analyte & Result & $\begin{array}{r}\text { porting } \\
\text { Limit }\end{array}$ & Units & $\begin{array}{l}\text { Spike } \\
\text { Level }\end{array}$ & $\begin{array}{l}\text { Source } \\
\text { Result }\end{array}$ & $\%$ REC & $\begin{array}{l}\text { \%REC } \\
\text { Limits }\end{array}$ & RPD & $\begin{array}{l}\text { RPD } \\
\text { Limit }\end{array}$ & Notes \\
\hline
\end{tabular}

Batch 8I15003 - 1:1 Water Extract (ICP/ICPMS)

\begin{tabular}{|c|c|c|c|c|c|c|}
\hline Duplicate (8I15003-DUP1) & \multicolumn{3}{|c|}{ Source: 0805003-15 } & \multicolumn{3}{|c|}{ Prepared: 08/01/08 Analyzed: 09/16/08 } \\
\hline Aluminum & $1.87 \mathrm{E}-1$ & $8.59 \mathrm{E}-2$ & ug/g dry & $2.07 \mathrm{E}-1$ & 10.5 & 35 \\
\hline Boron & $<1.94 \mathrm{E} 0$ & $1.94 \mathrm{E} 0$ & $"$ & ND & & 35 \\
\hline Beryllium & $<2.84 \mathrm{E}-2$ & $2.84 \mathrm{E}-2$ & $"$ & ND & & 35 \\
\hline Bismuth & $<1.88 \mathrm{E}-1$ & $1.88 \mathrm{E}-1$ & $"$ & ND & & 35 \\
\hline Calcium & $5.22 \mathrm{E} 0$ & $3.87 \mathrm{E}-1$ & $"$ & $5.26 \mathrm{E} 0$ & 0.766 & 35 \\
\hline Cadmium & $<2.69 \mathrm{E}-2$ & $2.69 \mathrm{E}-2$ & $"$ & ND & & 35 \\
\hline Cobalt & $<9.61 \mathrm{E}-2$ & $9.61 \mathrm{E}-2$ & $"$ & ND & & 35 \\
\hline Chromium & $<3.33 \mathrm{E}-2$ & $3.33 \mathrm{E}-2$ & $"$ & ND & & 35 \\
\hline Iron & $1.55 \mathrm{E}-1$ & $1.42 \mathrm{E}-1$ & $"$ & $1.42 \mathrm{E}-1$ & 8.46 & 35 \\
\hline Potassium & $3.21 \mathrm{E} 0$ & $2.33 \mathrm{E} 0$ & $"$ & $2.88 \mathrm{E} 0$ & 10.8 & 35 \\
\hline Lithium & $<5.40 \mathrm{E}-1$ & $5.40 \mathrm{E}-1$ & $"$ & ND & & 35 \\
\hline Magnesium & $1.92 \mathrm{E} 0$ & $8.35 \mathrm{E}-2$ & $"$ & $1.96 \mathrm{E} 0$ & 2.01 & 35 \\
\hline Manganese & $<1.71 \mathrm{E}-2$ & $1.71 \mathrm{E}-2$ & $"$ & ND & & 35 \\
\hline Molybdenum & $<1.39 \mathrm{E}-1$ & $1.39 \mathrm{E}-1$ & $"$ & ND & & 35 \\
\hline Nickel & $<9.34 \mathrm{E}-2$ & $9.34 \mathrm{E}-2$ & $"$ & ND & & 35 \\
\hline Phosphorus & $<1.03 \mathrm{E} 0$ & $1.03 \mathrm{E} 0$ & $"$ & ND & & 35 \\
\hline Lead & $<4.32 \mathrm{E}-1$ & $4.32 \mathrm{E}-1$ & $"$ & ND & & 35 \\
\hline Selenium & $<1.95 \mathrm{E} 0$ & $1.95 \mathrm{E} 0$ & $"$ & ND & & 35 \\
\hline Strontium & $<5.22 \mathrm{E}-2$ & $5.22 \mathrm{E}-2$ & $"$ & ND & & 35 \\
\hline Thallium & $<1.03 \mathrm{E} 0$ & $1.03 \mathrm{E} 0$ & $"$ & ND & & 35 \\
\hline Vanadium & $<4.53 \mathrm{E}-2$ & $4.53 \mathrm{E}-2$ & $"$ & ND & & 35 \\
\hline Zinc & $<9.28 \mathrm{E}-2$ & $9.28 \mathrm{E}-2$ & $"$ & ND & & 35 \\
\hline Sodium & $5.87 \mathrm{E} 0$ & $6.70 \mathrm{E}-1$ & $"$ & $5.94 \mathrm{E} 0$ & 1.29 & 35 \\
\hline Silicon & 7.22E0 & $1.50 \mathrm{E} 0$ & $"$ & $6.88 \mathrm{E} 0$ & 4.90 & 35 \\
\hline Sulfur & $<3.08 \mathrm{E} 0$ & $3.08 \mathrm{E} 0$ & $"$ & ND & & 35 \\
\hline Titanium & $<8.77 \mathrm{E}-3$ & $8.77 \mathrm{E}-3$ & $"$ & ND & & 35 \\
\hline Zirconium & $<1.50 \mathrm{E}-1$ & $1.50 \mathrm{E}-1$ & $"$ & ND & & 35 \\
\hline Silver & $<7.72 \mathrm{E}-2$ & $7.72 \mathrm{E}-2$ & $"$ & ND & & 35 \\
\hline Rhenium & $<1.52 \mathrm{E}-1$ & $1.52 \mathrm{E}-1$ & $"$ & ND & & 35 \\
\hline
\end{tabular}


Total Metals by PNNL-AGG-ICP-AES/Water Extract - Quality Control

Environmental Science Laboratory

\begin{tabular}{|lrrrrrrrrrrr}
\hline & & Reporting & & Spike & Source & & \%REC & & RPD \\
Analyte & Result & Limit & Units & Level & Result & $\%$ REC & Limits & RPD & Limit & Notes \\
\hline
\end{tabular}

Batch 8I15003 - 1:1 Water Extract (ICP/ICPMS)

\begin{tabular}{|c|c|c|c|c|c|c|c|}
\hline \multirow{2}{*}{$\begin{array}{l}\text { Post Spike (8I15003-PS1) } \\
\text { Aluminum }\end{array}$} & \multicolumn{3}{|c|}{ Source: 0805003-15 } & \multicolumn{2}{|c|}{ Prepared: 09/15/08 } & \multicolumn{2}{|c|}{ Analyzed: 09/16/08 } \\
\hline & $5.65 \mathrm{E} 2$ & N/A & $\mathrm{ug} / \mathrm{L}$ & $5.00 \mathrm{E} 2$ & $6.91 \mathrm{E} 1$ & 99.2 & $75-125$ \\
\hline Boron & $5.16 \mathrm{E} 2$ & N/A & $"$ & $5.00 \mathrm{E} 2$ & $1.07 \mathrm{E} 0$ & 103 & $75-125$ \\
\hline Beryllium & $2.50 \mathrm{E} 2$ & N/A & $"$ & $2.50 \mathrm{E} 2$ & ND & 100 & $75-125$ \\
\hline Bismuth & $4.89 \mathrm{E} 2$ & N/A & $"$ & $5.00 \mathrm{E} 2$ & $7.65 \mathrm{E} 0$ & 96.2 & $75-125$ \\
\hline Calcium & $2.36 \mathrm{E} 3$ & N/A & $"$ & $5.00 \mathrm{E} 2$ & $1.75 \mathrm{E} 3$ & 120 & $75-125$ \\
\hline Cadmium & $2.45 \mathrm{E} 2$ & N/A & $"$ & $2.50 \mathrm{E} 2$ & $5.16 \mathrm{E}-1$ & 97.8 & $75-125$ \\
\hline Cobalt & $2.41 \mathrm{E} 2$ & N/A & $"$ & $2.50 \mathrm{E} 2$ & ND & 96.8 & $75-125$ \\
\hline Chromium & $1.23 \mathrm{E} 2$ & N/A & $"$ & $1.25 \mathrm{E} 2$ & ND & 102 & $75-125$ \\
\hline Iron & $5.55 \mathrm{E} 2$ & N/A & $"$ & $5.00 \mathrm{E} 2$ & $4.75 \mathrm{E} 1$ & 102 & $75-125$ \\
\hline Potassium & $2.34 \mathrm{E} 3$ & $\mathrm{~N} / \mathrm{A}$ & $"$ & $1.25 \mathrm{E} 3$ & $9.61 \mathrm{E} 2$ & 111 & $75-125$ \\
\hline Lithium & $5.30 \mathrm{E} 2$ & N/A & $"$ & $5.00 \mathrm{E} 2$ & $1.32 \mathrm{E} 1$ & 103 & $75-125$ \\
\hline Magnesium & $1.17 \mathrm{E} 3$ & N/A & $"$ & $5.00 \mathrm{E} 2$ & $6.53 \mathrm{E} 2$ & 103 & $75-125$ \\
\hline Manganese & $2.51 \mathrm{E} 2$ & N/A & $"$ & $2.50 \mathrm{E} 2$ & ND & 101 & $75-125$ \\
\hline Molybdenum & 4.97E2 & N/A & $"$ & $5.00 \mathrm{E} 2$ & $1.53 \mathrm{E} 0$ & 99.1 & $75-125$ \\
\hline Nickel & 4.91E2 & N/A & $"$ & $5.00 \mathrm{E} 2$ & ND & 98.4 & $75-125$ \\
\hline Phosphorus & $1.22 \mathrm{E} 3$ & N/A & $"$ & $1.25 \mathrm{E} 3$ & ND & 97.4 & $75-125$ \\
\hline Lead & $4.86 \mathrm{E} 2$ & N/A & $"$ & $5.00 \mathrm{E} 2$ & $6.31 \mathrm{E}-2$ & 97.2 & $75-125$ \\
\hline Selenium & $4.78 \mathrm{E} 2$ & N/A & $"$ & $5.00 \mathrm{E} 2$ & ND & 99.6 & $75-125$ \\
\hline Strontium & $5.27 \mathrm{E} 2$ & N/A & $"$ & $5.00 \mathrm{E} 2$ & $1.08 \mathrm{E} 1$ & 103 & $75-125$ \\
\hline Thallium & $5.00 \mathrm{E} 2$ & N/A & $"$ & $5.00 \mathrm{E} 2$ & $3.30 \mathrm{E} 0$ & 99.4 & $75-125$ \\
\hline Vanadium & $2.54 \mathrm{E} 2$ & N/A & $"$ & $2.50 \mathrm{E} 2$ & $1.07 \mathrm{E} 1$ & 97.3 & $75-125$ \\
\hline Zinc & $2.53 \mathrm{E} 2$ & N/A & $"$ & $2.50 \mathrm{E} 2$ & $9.35 \mathrm{E} 0$ & 97.6 & $75-125$ \\
\hline Sodium & $2.60 \mathrm{E} 3$ & N/A & $"$ & $5.00 \mathrm{E} 2$ & $1.98 \mathrm{E} 3$ & 123 & $75-125$ \\
\hline Sulfur & $1.81 \mathrm{E} 3$ & N/A & $"$ & $1.00 \mathrm{E} 3$ & $8.34 \mathrm{E} 2$ & 97.3 & $75-125$ \\
\hline Titanium & $2.49 \mathrm{E} 2$ & N/A & $"$ & $2.50 \mathrm{E} 2$ & $2.13 \mathrm{E} 0$ & 98.6 & $75-125$ \\
\hline Zirconium & $2.63 \mathrm{E} 2$ & N/A & $"$ & $2.50 \mathrm{E} 2$ & $1.80 \mathrm{E} 1$ & 98.1 & $75-125$ \\
\hline Silver & $5.10 \mathrm{E} 2$ & N/A & $"$ & $5.00 \mathrm{E} 2$ & $1.88 \mathrm{E} 0$ & 102 & $75-125$ \\
\hline Rhenium & $4.99 \mathrm{E} 2$ & N/A & $"$ & $5.00 \mathrm{E} 2$ & $5.28 \mathrm{E} 0$ & 98.8 & $75-125$ \\
\hline
\end{tabular}


Total Metals by PNNL-AGG-ICP-AES/Water Extract - Quality Control

Environmental Science Laboratory

\begin{tabular}{|rrrrrrrrrrrr}
\hline & & Reporting & & Spike & Source & & \%REC & & RPD \\
Analyte & Result & Limit & Units & Level & Result & $\%$ REC & Limits & RPD & Limit & Notes \\
\hline
\end{tabular}

Batch 8I16001 - 1:1 Water Extract (ICP/ICPMS)

\begin{tabular}{|c|c|c|c|}
\hline \multicolumn{4}{|c|}{ Blank (8I16001-BLK1) } \\
\hline Aluminum & $<8.58 \mathrm{E}-2$ & $8.58 \mathrm{E}-2$ & ug/g wet \\
\hline Boron & $<1.94 \mathrm{E} 0$ & $1.94 \mathrm{E} 0$ & $"$ \\
\hline Beryllium & $<2.84 \mathrm{E}-2$ & $2.84 \mathrm{E}-2$ & $"$ \\
\hline Bismuth & $<1.88 \mathrm{E}-1$ & $1.88 \mathrm{E}-1$ & $"$ \\
\hline Calcium & $<3.87 \mathrm{E}-1$ & $3.87 \mathrm{E}-1$ & $"$ \\
\hline Cadmium & $<2.69 \mathrm{E}-2$ & $2.69 \mathrm{E}-2$ & $"$ \\
\hline Cobalt & $<9.60 \mathrm{E}-2$ & $9.60 \mathrm{E}-2$ & $"$ \\
\hline Chromium & $<3.33 \mathrm{E}-2$ & $3.33 \mathrm{E}-2$ & $"$ \\
\hline Iron & $<1.42 \mathrm{E}-1$ & $1.42 \mathrm{E}-1$ & $"$ \\
\hline Potassium & $<2.33 \mathrm{E} 0$ & 2.33E0 & $"$ \\
\hline Lithium & $<5.40 \mathrm{E}-1$ & $5.40 \mathrm{E}-1$ & $"$ \\
\hline Magnesium & $<8.34 \mathrm{E}-2$ & 8.34E-2 & $"$ \\
\hline Manganese & $<1.71 \mathrm{E}-2$ & $1.71 \mathrm{E}-2$ & $"$ \\
\hline Molybdenum & $<1.39 \mathrm{E}-1$ & $1.39 \mathrm{E}-1$ & $"$ \\
\hline Nickel & $<9.33 \mathrm{E}-2$ & $9.33 \mathrm{E}-2$ & $"$ \\
\hline Phosphorus & $<1.03 \mathrm{E} 0$ & $1.03 \mathrm{E} 0$ & $"$ \\
\hline Lead & $<4.32 \mathrm{E}-1$ & $4.32 \mathrm{E}-1$ & $"$ \\
\hline Selenium & $<1.95 \mathrm{E} 0$ & $1.95 \mathrm{E} 0$ & $"$ \\
\hline Strontium & $<5.22 \mathrm{E}-2$ & $5.22 \mathrm{E}-2$ & $"$ \\
\hline Thallium & $<1.03 \mathrm{E} 0$ & $1.03 \mathrm{E} 0$ & $"$ \\
\hline Vanadium & $<4.53 \mathrm{E}-2$ & $4.53 \mathrm{E}-2$ & $"$ \\
\hline Zinc & $<9.27 \mathrm{E}-2$ & $9.27 \mathrm{E}-2$ & $"$ \\
\hline Sodium & $<6.69 \mathrm{E}-1$ & $6.69 \mathrm{E}-1$ & $"$ \\
\hline Silicon & $<1.50 \mathrm{E} 0$ & $1.50 \mathrm{E} 0$ & $"$ \\
\hline Sulfur & $<3.08 \mathrm{E} 0$ & $3.08 \mathrm{E} 0$ & $"$ \\
\hline Titanium & $<8.76 \mathrm{E}-3$ & $8.76 \mathrm{E}-3$ & $"$ \\
\hline Zirconium & $<1.50 \mathrm{E}-1$ & $1.50 \mathrm{E}-1$ & $"$ \\
\hline Silver & $<7.71 \mathrm{E}-2$ & $7.71 \mathrm{E}-2$ & $"$ \\
\hline Rhenium & $<1.52 \mathrm{E}-1$ & $1.52 \mathrm{E}-1$ & $"$ \\
\hline
\end{tabular}


Total Metals by PNNL-AGG-ICP-AES/Water Extract - Quality Control

Environmental Science Laboratory

\begin{tabular}{|lrrrrrrrrrrr}
\hline & & Reporting & & Spike & Source & & \%REC & & RPD \\
Analyte & Result & Limit & Units & Level & Result & $\%$ REC & Limits & RPD & Limit & Notes \\
\hline
\end{tabular}

Batch 8I16001 - 1:1 Water Extract (ICP/ICPMS)

\begin{tabular}{|c|c|c|c|c|c|c|}
\hline \multirow{2}{*}{$\begin{array}{l}\text { LCS (8I16001-BS1) } \\
\text { Aluminum }\end{array}$} & \multicolumn{6}{|c|}{ Prepared: 08/20/08 Analyzed: 09/16/08 } \\
\hline & $4.67 \mathrm{E} 0$ & $8.58 \mathrm{E}-2$ & ug/g wet & $4.99 \mathrm{E} 0$ & 93.5 & $80-120$ \\
\hline Boron & 4.96E0 & $1.94 \mathrm{E} 0$ & $"$ & 4.99E0 & 99.5 & $80-120$ \\
\hline Beryllium & $4.96 \mathrm{E} 0$ & $2.84 \mathrm{E}-2$ & $"$ & $4.99 \mathrm{E} 0$ & 99.4 & $80-120$ \\
\hline Bismuth & $<1.88 \mathrm{E}-1$ & $1.88 \mathrm{E}-1$ & $"$ & & & $80-120$ \\
\hline Calcium & $4.72 \mathrm{E} 0$ & 3.87E-1 & $"$ & $4.99 \mathrm{E} 0$ & 94.7 & $80-120$ \\
\hline Cadmium & $5.06 \mathrm{E} 0$ & $2.69 \mathrm{E}-2$ & $"$ & 4.99E0 & 101 & $80-120$ \\
\hline Cobalt & $4.84 \mathrm{E} 0$ & $9.60 \mathrm{E}-2$ & $"$ & $4.99 \mathrm{E} 0$ & 97.0 & $80-120$ \\
\hline Chromium & $5.09 \mathrm{E} 0$ & $3.33 \mathrm{E}-2$ & $"$ & $4.99 \mathrm{E} 0$ & 102 & $80-120$ \\
\hline Iron & $4.94 \mathrm{E} 0$ & $1.42 \mathrm{E}-1$ & $"$ & $4.99 \mathrm{E} 0$ & 99.0 & $80-120$ \\
\hline Potassium & $4.92 \mathrm{E} 1$ & $2.33 \mathrm{E} 0$ & $"$ & 4.99E1 & 98.5 & $80-120$ \\
\hline Lithium & $<5.40 \mathrm{E}-1$ & $5.40 \mathrm{E}-1$ & $"$ & & & $80-120$ \\
\hline Magnesium & $4.69 \mathrm{E} 0$ & $8.34 \mathrm{E}-2$ & $"$ & $4.99 \mathrm{E} 0$ & 93.9 & $80-120$ \\
\hline Manganese & $4.86 \mathrm{E} 0$ & $1.71 \mathrm{E}-2$ & $"$ & $4.99 \mathrm{E} 0$ & 97.3 & $80-120$ \\
\hline Molybdenum & $4.85 \mathrm{E} 0$ & $1.39 \mathrm{E}-1$ & $"$ & $4.99 \mathrm{E} 0$ & 97.1 & $80-120$ \\
\hline Nickel & $4.96 \mathrm{E} 0$ & $9.33 \mathrm{E}-2$ & $"$ & $4.99 \mathrm{E} 0$ & 99.5 & $80-120$ \\
\hline Phosphorus & $<1.03 \mathrm{E} 0$ & $1.03 \mathrm{E} 0$ & $"$ & & & $80-120$ \\
\hline Lead & $4.92 \mathrm{E} 0$ & $4.32 \mathrm{E}-1$ & $"$ & $4.99 \mathrm{E} 0$ & 98.7 & $80-120$ \\
\hline Selenium & $5.15 \mathrm{E} 0$ & $1.95 \mathrm{E} 0$ & $"$ & $4.99 \mathrm{E} 0$ & 103 & $80-120$ \\
\hline Strontium & $<5.22 \mathrm{E}-2$ & $5.22 \mathrm{E}-2$ & $"$ & & & $80-120$ \\
\hline Thallium & $4.92 \mathrm{E} 0$ & $1.03 \mathrm{E} 0$ & $"$ & $4.99 \mathrm{E} 0$ & 98.6 & $80-120$ \\
\hline Vanadium & $4.82 \mathrm{E} 0$ & $4.53 \mathrm{E}-2$ & $"$ & $4.99 \mathrm{E} 0$ & 96.6 & $80-120$ \\
\hline Zinc & $5.01 \mathrm{E} 0$ & $9.27 \mathrm{E}-2$ & $"$ & $4.99 \mathrm{E} 0$ & 100 & $80-120$ \\
\hline Sodium & $5.19 \mathrm{E} 0$ & $6.69 \mathrm{E}-1$ & $"$ & $4.99 \mathrm{E} 0$ & 104 & $80-120$ \\
\hline Silicon & $2.80 \mathrm{E} 0$ & $1.50 \mathrm{E} 0$ & $"$ & $2.50 \mathrm{E} 0$ & 112 & $80-120$ \\
\hline Sulfur & $<3.08 \mathrm{E} 0$ & $3.08 \mathrm{E} 0$ & $"$ & & & $80-120$ \\
\hline Titanium & $4.81 \mathrm{E} 0$ & $8.76 \mathrm{E}-3$ & $"$ & $4.99 \mathrm{E} 0$ & 96.5 & $80-120$ \\
\hline Zirconium & $<1.50 \mathrm{E}-1$ & $1.50 \mathrm{E}-1$ & $"$ & & & $80-120$ \\
\hline Silver & $4.83 \mathrm{E} 0$ & $7.71 \mathrm{E}-2$ & $"$ & 4.99E0 & 96.8 & $80-120$ \\
\hline Rhenium & $<1.52 \mathrm{E}-1$ & $1.52 \mathrm{E}-1$ & $"$ & & & $80-120$ \\
\hline
\end{tabular}


Total Metals by PNNL-AGG-ICP-AES/Acid Extract - Quality Control

Environmental Science Laboratory

\begin{tabular}{|lrrrrrrrrrrr}
\hline & & Reporting & & Spike & Source & & \%REC & & RPD \\
Analyte & Result & Limit & Units & Level & Result & $\%$ REC & Limits & RPD & Limit & Notes \\
\hline
\end{tabular}

Batch 8L12002 - ASTM D 5198 (ICP/ICPMS)

\begin{tabular}{|c|c|c|c|}
\hline \multicolumn{4}{|c|}{ Blank (8L12002-BLK1) } \\
\hline Aluminum & $<6.76 \mathrm{E} 0$ & $6.76 \mathrm{E} 0$ & ug/g wet \\
\hline Arsenic & $<1.01 \mathrm{E} 1$ & $1.01 \mathrm{E} 1$ & $"$ \\
\hline Boron & $<2.90 \mathrm{E} 1$ & $2.90 \mathrm{E} 1$ & $"$ \\
\hline Barium & $<4.12 \mathrm{E}-1$ & $4.12 \mathrm{E}-1$ & $"$ \\
\hline Beryllium & $<2.31 \mathrm{E}-1$ & $2.31 \mathrm{E}-1$ & $"$ \\
\hline Bismuth & $<2.85 \mathrm{E} 0$ & $2.85 \mathrm{E} 0$ & $"$ \\
\hline Calcium & $<7.02 \mathrm{E} 0$ & $7.02 \mathrm{E} 0$ & $"$ \\
\hline Cadmium & $<3.68 \mathrm{E}-1$ & $3.68 \mathrm{E}-1$ & $"$ \\
\hline Cobalt & $<1.44 \mathrm{E} 0$ & $1.44 \mathrm{E} 0$ & $"$ \\
\hline Chromium & $<5.90 \mathrm{E}-1$ & $5.90 \mathrm{E}-1$ & $"$ \\
\hline Copper & $<1.93 \mathrm{E} 0$ & $1.93 \mathrm{E} 0$ & $"$ \\
\hline Iron & $<1.82 \mathrm{E} 0$ & $1.82 \mathrm{E} 0$ & $"$ \\
\hline Potassium & $<2.59 \mathrm{E} 1$ & $2.59 \mathrm{E} 1$ & $"$ \\
\hline Lithium & $<6.28 \mathrm{E} 0$ & $6.28 \mathrm{E} 0$ & $"$ \\
\hline Magnesium & $<1.92 \mathrm{E} 0$ & $1.92 \mathrm{E} 0$ & $"$ \\
\hline Manganese & $<6.04 \mathrm{E}-1$ & $6.04 \mathrm{E}-1$ & $"$ \\
\hline Molybdenum & $<2.29 \mathrm{E} 0$ & $2.29 \mathrm{E} 0$ & $"$ \\
\hline Nickel & $<1.40 \mathrm{E} 0$ & $1.40 \mathrm{E} 0$ & $"$ \\
\hline Phosphorus & $<8.93 \mathrm{E} 0$ & 8.93E0 & $"$ \\
\hline Lead & $<3.02 \mathrm{E} 0$ & $3.02 \mathrm{E} 0$ & $"$ \\
\hline Selenium & $<1.72 \mathrm{E} 1$ & $1.72 \mathrm{E} 1$ & $"$ \\
\hline Strontium & $<4.40 \mathrm{E}-1$ & $4.40 \mathrm{E}-1$ & $"$ \\
\hline Thallium & $<9.70 \mathrm{E} 0$ & $9.70 \mathrm{E} 0$ & $"$ \\
\hline Vanadium & $<1.21 \mathrm{E} 0$ & $1.21 \mathrm{E} 0$ & $"$ \\
\hline Zinc & $<1.10 \mathrm{E} 0$ & $1.10 \mathrm{E} 0$ & $"$ \\
\hline Sodium & $<1.90 \mathrm{E} 1$ & $1.90 \mathrm{E} 1$ & $"$ \\
\hline Silicon & $<4.89 \mathrm{E} 1$ & $4.89 \mathrm{E} 1$ & $"$ \\
\hline Sulfur & $<3.22 \mathrm{E} 1$ & $3.22 \mathrm{E} 1$ & $"$ \\
\hline Titanium & $<1.55 \mathrm{E} 0$ & $1.55 \mathrm{E} 0$ & $"$ \\
\hline Zirconium & $<8.10 \mathrm{E}-1$ & $8.10 \mathrm{E}-1$ & $"$ \\
\hline Silver & $<9.16 \mathrm{E}-1$ & $9.16 \mathrm{E}-1$ & $"$ \\
\hline Rhenium & $<2.81 \mathrm{E} 0$ & $2.81 \mathrm{E} 0$ & $"$ \\
\hline Antimony & $<6.71 \mathrm{E} 0$ & $6.71 \mathrm{E} 0$ & $"$ \\
\hline
\end{tabular}


Total Metals by PNNL-AGG-ICP-AES/Acid Extract - Quality Control

Environmental Science Laboratory

\begin{tabular}{|lrrrrrrrrrrr}
\hline & & Reporting & & Spike & Source & & \%REC & & RPD \\
Analyte & Result & Limit & Units & Level & Result & \%REC & Limits & RPD & Limit & Notes \\
\hline
\end{tabular}

Batch 8L12002 - ASTM D 5198 (ICP/ICPMS)

\begin{tabular}{|c|c|c|c|c|c|c|}
\hline \multirow{2}{*}{$\frac{\text { LCS (8L12002-BS1) }}{\text { Aluminum }}$} & \multicolumn{6}{|c|}{ Prepared: 12/11/08 Analyzed: 03/16/09 } \\
\hline & $5.57 \mathrm{E} 0$ & $6.76 \mathrm{E}-1$ & ug/g wet & $6.06 \mathrm{E} 0$ & 92.0 & $80-120$ \\
\hline Arsenic & $5.95 \mathrm{E} 0$ & $1.01 \mathrm{E} 0$ & $"$ & & & $80-120$ \\
\hline Boron & $5.54 \mathrm{E} 0$ & $2.90 \mathrm{E} 0$ & $"$ & $6.06 \mathrm{E} 0$ & 91.5 & $80-120$ \\
\hline Barium & $5.76 \mathrm{E} 0$ & 4.12E-2 & $"$ & $6.06 \mathrm{E} 0$ & 95.1 & $80-120$ \\
\hline Beryllium & 5.94E0 & $2.31 \mathrm{E}-2$ & $"$ & $6.06 \mathrm{E} 0$ & 98.1 & $80-120$ \\
\hline Bismuth & $<2.85 \mathrm{E}-1$ & $2.85 \mathrm{E}-1$ & $"$ & & & $80-120$ \\
\hline Calcium & $5.65 \mathrm{E} 0$ & $7.02 \mathrm{E}-1$ & $"$ & $6.06 \mathrm{E} 0$ & 93.3 & $80-120$ \\
\hline Cadmium & $5.79 \mathrm{E} 0$ & $3.68 \mathrm{E}-2$ & $"$ & $6.06 \mathrm{E} 0$ & 95.6 & $80-120$ \\
\hline Cobalt & $6.02 \mathrm{E} 0$ & $1.44 \mathrm{E}-1$ & $"$ & $6.06 \mathrm{E} 0$ & 99.5 & $80-120$ \\
\hline Chromium & $5.98 \mathrm{E} 0$ & $5.90 \mathrm{E}-2$ & $"$ & $6.06 \mathrm{E} 0$ & 98.8 & $80-120$ \\
\hline Copper & 5.91E0 & $1.93 \mathrm{E}-1$ & $"$ & $6.06 \mathrm{E} 0$ & 97.7 & $80-120$ \\
\hline Iron & $6.09 \mathrm{E} 0$ & $1.82 \mathrm{E}-1$ & $"$ & $6.06 \mathrm{E} 0$ & 101 & $80-120$ \\
\hline Potassium & $5.65 \mathrm{E} 1$ & $2.59 \mathrm{E} 0$ & $"$ & $6.06 \mathrm{E} 1$ & 93.3 & $80-120$ \\
\hline Lithium & $<6.28 \mathrm{E}-1$ & $6.28 \mathrm{E}-1$ & $"$ & & & $80-120$ \\
\hline Magnesium & $5.46 \mathrm{E} 0$ & $1.92 \mathrm{E}-1$ & $"$ & $6.06 \mathrm{E} 0$ & 90.2 & $80-120$ \\
\hline Manganese & $6.09 \mathrm{E} 0$ & $6.04 \mathrm{E}-2$ & $"$ & $6.06 \mathrm{E} 0$ & 101 & $80-120$ \\
\hline Molybdenum & $6.04 \mathrm{E} 0$ & $2.29 \mathrm{E}-1$ & $"$ & $6.06 \mathrm{E} 0$ & 99.8 & $80-120$ \\
\hline Nickel & $5.92 \mathrm{E} 0$ & $1.40 \mathrm{E}-1$ & $"$ & $6.06 \mathrm{E} 0$ & 97.8 & $80-120$ \\
\hline Phosphorus & $<8.93 \mathrm{E}-1$ & $8.93 \mathrm{E}-1$ & $"$ & & & $80-120$ \\
\hline Lead & $5.95 \mathrm{E} 0$ & $3.02 \mathrm{E}-1$ & $"$ & $6.06 \mathrm{E} 0$ & 98.3 & $80-120$ \\
\hline Selenium & $5.14 \mathrm{E} 0$ & $1.72 \mathrm{E} 0$ & $"$ & $6.06 \mathrm{E} 0$ & 84.9 & $80-120$ \\
\hline Strontium & $<4.40 \mathrm{E}-2$ & 4.40E-2 & $"$ & & & $80-120$ \\
\hline Thallium & $5.92 \mathrm{E} 0$ & $9.70 \mathrm{E}-1$ & $"$ & $6.06 \mathrm{E} 0$ & 97.8 & $80-120$ \\
\hline Vanadium & $5.82 \mathrm{E} 0$ & $1.21 \mathrm{E}-1$ & $"$ & $6.06 \mathrm{E} 0$ & 96.1 & $80-120$ \\
\hline Zinc & $5.76 \mathrm{E} 0$ & $1.10 \mathrm{E}-1$ & $"$ & $6.06 \mathrm{E} 0$ & 95.2 & $80-120$ \\
\hline Sodium & $6.04 \mathrm{E} 0$ & $1.90 \mathrm{E} 0$ & $"$ & $6.06 \mathrm{E} 0$ & 99.7 & $80-120$ \\
\hline Silicon & $7.21 \mathrm{E} 0$ & 4.89E0 & $"$ & $6.06 \mathrm{E} 0$ & 119 & $80-120$ \\
\hline Sulfur & $<3.22 \mathrm{E} 0$ & $3.22 \mathrm{E} 0$ & $"$ & & & $80-120$ \\
\hline Titanium & $6.14 \mathrm{E} 0$ & $1.55 \mathrm{E}-1$ & $"$ & $6.06 \mathrm{E} 0$ & 101 & $80-120$ \\
\hline Zirconium & $<8.10 \mathrm{E}-2$ & $8.10 \mathrm{E}-2$ & $"$ & & & $80-120$ \\
\hline Silver & $5.65 \mathrm{E} 0$ & $9.16 \mathrm{E}-2$ & $"$ & $6.06 \mathrm{E} 0$ & 93.4 & $80-120$ \\
\hline Rhenium & $<2.81 \mathrm{E}-1$ & $2.81 \mathrm{E}-1$ & $"$ & & & $80-120$ \\
\hline Antimony & $5.83 \mathrm{E} 0$ & $6.71 \mathrm{E}-1$ & $"$ & & & $80-120$ \\
\hline
\end{tabular}


Total Metals by PNNL-AGG-ICP-AES/Acid Extract - Quality Control

Environmental Science Laboratory

\begin{tabular}{|lrrrrrrrrrrr}
\hline & & Reporting & & Spike & Source & & \%REC & & RPD \\
Analyte & Result & Limit & Units & Level & Result & $\%$ REC & Limits & RPD & Limit & Notes \\
\hline
\end{tabular}

Batch 8L12002 - ASTM D 5198 (ICP/ICPMS)

\begin{tabular}{|c|c|c|c|c|c|c|}
\hline \multirow{2}{*}{$\begin{array}{l}\text { Duplicate (8L12002-DUP1) } \\
\text { Aluminum }\end{array}$} & \multicolumn{3}{|c|}{ Source: 0805003-15 } & \multirow{2}{*}{$\begin{array}{r}\text { Prepared: } 12 / 11 / 08 \\
4.41 \mathrm{E} 3\end{array}$} & \multicolumn{2}{|l|}{ Analyzed: 03/16/09 } \\
\hline & $4.76 \mathrm{E} 3$ & $2.21 \mathrm{E} 1$ & ug/g dry & & 7.65 & 35 \\
\hline Arsenic & $<3.31 \mathrm{E} 1$ & $3.31 \mathrm{E} 1$ & $"$ & ND & & 35 \\
\hline Boron & $<9.47 \mathrm{E} 1$ & $9.47 \mathrm{E} 1$ & $"$ & ND & & 35 \\
\hline Barium & 4.09E1 & $1.35 \mathrm{E} 0$ & $"$ & $3.64 \mathrm{E} 1$ & 11.8 & 35 \\
\hline Beryllium & $<7.55 \mathrm{E}-1$ & $7.55 \mathrm{E}-1$ & $"$ & ND & & 35 \\
\hline Bismuth & $<9.31 \mathrm{E} 0$ & $9.31 \mathrm{E} 0$ & $"$ & ND & & 35 \\
\hline Calcium & $7.49 \mathrm{E} 3$ & $2.29 \mathrm{E} 1$ & $"$ & $6.54 \mathrm{E} 3$ & 13.5 & 35 \\
\hline Cadmium & $<1.20 \mathrm{E} 0$ & $1.20 \mathrm{E} 0$ & $"$ & ND & & 35 \\
\hline Cobalt & 4.93E0 & $4.72 \mathrm{E} 0$ & $"$ & ND & & 35 \\
\hline Chromium & 7.25E0 & $1.93 \mathrm{E} 0$ & $"$ & $6.63 \mathrm{E} 0$ & 8.85 & 35 \\
\hline Copper & $<6.32 \mathrm{E} 0$ & $6.32 \mathrm{E} 0$ & $"$ & ND & & 35 \\
\hline Iron & $1.12 \mathrm{E} 4$ & $5.94 \mathrm{E} 0$ & $"$ & $1.09 \mathrm{E} 4$ & 2.99 & 35 \\
\hline Potassium & 8.07E2 & $8.45 \mathrm{E} 1$ & $"$ & $7.41 \mathrm{E} 2$ & 8.57 & 35 \\
\hline Lithium & $<2.05 \mathrm{E} 1$ & $2.05 \mathrm{E} 1$ & $"$ & ND & & 35 \\
\hline Magnesium & $3.73 \mathrm{E} 3$ & $6.29 \mathrm{E} 0$ & $"$ & $3.55 \mathrm{E} 3$ & 5.02 & 35 \\
\hline Manganese & $2.12 \mathrm{E} 2$ & $1.97 \mathrm{E} 0$ & $"$ & $2.03 \mathrm{E} 2$ & 3.99 & 35 \\
\hline Molybdenum & $<7.50 \mathrm{E} 0$ & $7.50 \mathrm{E} 0$ & $"$ & ND & & 35 \\
\hline Nickel & 7.05E0 & $4.58 \mathrm{E} 0$ & $"$ & $7.80 \mathrm{E} 0$ & 10.1 & 35 \\
\hline Phosphorus & 4.43E2 & $2.92 \mathrm{E} 1$ & $"$ & $4.04 \mathrm{E} 2$ & 9.14 & 35 \\
\hline Lead & $<9.88 \mathrm{E} 0$ & $9.88 \mathrm{E} 0$ & $"$ & ND & & 35 \\
\hline Selenium & $<5.60 \mathrm{E} 1$ & $5.60 \mathrm{E} 1$ & $"$ & ND & & 35 \\
\hline Strontium & $2.41 \mathrm{E} 1$ & $1.44 \mathrm{E} 0$ & $"$ & $1.99 \mathrm{E} 1$ & 19.0 & 35 \\
\hline Thallium & $<3.17 \mathrm{E} 1$ & $3.17 \mathrm{E} 1$ & $"$ & ND & & 35 \\
\hline Vanadium & $1.68 \mathrm{E} 1$ & $3.95 \mathrm{E} 0$ & $"$ & $1.69 \mathrm{E} 1$ & 0.617 & 35 \\
\hline Zinc & $2.55 \mathrm{E} 1$ & $3.60 \mathrm{E} 0$ & $"$ & $2.40 \mathrm{E} 1$ & 6.01 & 35 \\
\hline Sodium & $1.33 \mathrm{E} 2$ & $6.22 \mathrm{E} 1$ & $"$ & $1.12 \mathrm{E} 2$ & 16.9 & 35 \\
\hline Silicon & $<1.60 \mathrm{E} 2$ & $1.60 \mathrm{E} 2$ & $"$ & ND & & 35 \\
\hline Sulfur & $<1.05 \mathrm{E} 2$ & $1.05 \mathrm{E} 2$ & $"$ & ND & & 35 \\
\hline Titanium & $4.56 \mathrm{E} 2$ & $5.06 \mathrm{E} 0$ & $"$ & 4.74E2 & 3.86 & 35 \\
\hline Zirconium & 4.40E0 & $2.65 \mathrm{E} 0$ & $"$ & 4.61E0 & 4.63 & 35 \\
\hline Silver & $<2.99 \mathrm{E} 0$ & $2.99 \mathrm{E} 0$ & $"$ & ND & & 35 \\
\hline Rhenium & $<9.19 \mathrm{E} 0$ & $9.19 \mathrm{E} 0$ & $"$ & ND & & 35 \\
\hline Antimony & $<2.19 \mathrm{E} 1$ & $2.19 \mathrm{E} 1$ & $"$ & ND & & 35 \\
\hline
\end{tabular}


Total Metals by PNNL-AGG-ICP-AES/Acid Extract - Quality Control

Environmental Science Laboratory

\begin{tabular}{|lrrrrrrrrrrr}
\hline & & Reporting & & Spike & Source & & \%REC & & RPD \\
Analyte & Result & Limit & Units & Level & Result & \%REC & Limits & RPD & Limit & Notes \\
\hline
\end{tabular}

Batch 8L12002 - ASTM D 5198 (ICP/ICPMS)

\begin{tabular}{|c|c|c|c|c|c|c|c|}
\hline \multirow{2}{*}{$\begin{array}{l}\text { Post Spike (8L12002-PS1) } \\
\text { Aluminum }\end{array}$} & \multicolumn{3}{|c|}{ Source: 0805003-15 } & \multicolumn{2}{|c|}{ Prepared: 12/12/08 } & \multicolumn{2}{|c|}{ Analyzed: 03/16/09 } \\
\hline & $2.72 \mathrm{E} 4$ & N/A & $\mathrm{ug} / \mathrm{L}$ & $5.00 \mathrm{E} 2$ & $2.62 \mathrm{E} 4$ & 207 & $75-125$ \\
\hline Arsenic & 4.07E2 & N/A & $"$ & $5.00 \mathrm{E} 2$ & ND & 95.7 & $75-125$ \\
\hline Boron & 4.99E2 & $\mathrm{N} / \mathrm{A}$ & $"$ & $5.00 \mathrm{E} 2$ & ND & 102 & $75-125$ \\
\hline Barium & 4.67E2 & $\mathrm{N} / \mathrm{A}$ & $"$ & $2.50 \mathrm{E} 2$ & $2.16 \mathrm{E} 2$ & 101 & $75-125$ \\
\hline Beryllium & 2.63E2 & N/A & $"$ & $2.50 \mathrm{E} 2$ & $3.21 \mathrm{E}-1$ & 105 & $75-125$ \\
\hline Bismuth & $5.07 \mathrm{E} 2$ & $\mathrm{~N} / \mathrm{A}$ & $"$ & $5.00 \mathrm{E} 2$ & $4.11 \mathrm{E} 1$ & 93.1 & $75-125$ \\
\hline Calcium & $3.98 \mathrm{E} 4$ & N/A & $"$ & $5.00 \mathrm{E} 2$ & $3.88 \mathrm{E} 4$ & 203 & $75-125$ \\
\hline Cadmium & $2.46 \mathrm{E} 2$ & N/A & $"$ & $2.50 \mathrm{E} 2$ & ND & 100 & $75-125$ \\
\hline Cobalt & $2.71 \mathrm{E} 2$ & $\mathrm{~N} / \mathrm{A}$ & $"$ & $2.50 \mathrm{E} 2$ & $2.81 \mathrm{E} 1$ & 97.3 & $75-125$ \\
\hline Chromium & $1.67 \mathrm{E} 2$ & N/A & $"$ & $1.25 \mathrm{E} 2$ & $3.93 \mathrm{E} 1$ & 102 & $75-125$ \\
\hline Copper & $5.42 \mathrm{E} 2$ & N/A & $"$ & $5.00 \mathrm{E} 2$ & $3.71 \mathrm{E} 1$ & 101 & $75-125$ \\
\hline Iron & $6.58 \mathrm{E} 4$ & N/A & $"$ & $5.00 \mathrm{E} 2$ & $6.46 \mathrm{E} 4$ & 251 & $75-125$ \\
\hline Potassium & $5.70 \mathrm{E} 3$ & N/A & $"$ & $1.25 \mathrm{E} 3$ & 4.39E3 & 104 & $75-125$ \\
\hline Lithium & $5.28 \mathrm{E} 2$ & N/A & $"$ & $5.00 \mathrm{E} 2$ & $3.14 \mathrm{E} 1$ & 99.3 & $75-125$ \\
\hline Magnesium & $2.18 \mathrm{E} 4$ & N/A & $"$ & $5.00 \mathrm{E} 2$ & $2.10 \mathrm{E} 4$ & 148 & $75-125$ \\
\hline Manganese & $1.48 \mathrm{E} 3$ & N/A & $"$ & $2.50 \mathrm{E} 2$ & $1.21 \mathrm{E} 3$ & 111 & $75-125$ \\
\hline Molybdenum & $5.19 \mathrm{E} 2$ & N/A & $"$ & $5.00 \mathrm{E} 2$ & $2.88 \mathrm{E} 1$ & 98 & $75-125$ \\
\hline Nickel & $5.45 \mathrm{E} 2$ & N/A & $"$ & $5.00 \mathrm{E} 2$ & 4.62E1 & 99.7 & $75-125$ \\
\hline Phosphorus & $3.64 \mathrm{E} 3$ & N/A & $"$ & $1.25 \mathrm{E} 3$ & $2.40 \mathrm{E} 3$ & 99.2 & $75-125$ \\
\hline Lead & $4.90 \mathrm{E} 2$ & N/A & $"$ & $5.00 \mathrm{E} 2$ & ND & 101 & $75-125$ \\
\hline Selenium & 4.29E2 & N/A & $"$ & $5.00 \mathrm{E} 2$ & ND & 101 & $75-125$ \\
\hline Strontium & $6.19 \mathrm{E} 2$ & N/A & $"$ & $5.00 \mathrm{E} 2$ & $1.18 \mathrm{E} 2$ & 100 & $75-125$ \\
\hline Thallium & 4.48E2 & $\mathrm{N} / \mathrm{A}$ & $"$ & $5.00 \mathrm{E} 2$ & ND & 102 & $75-125$ \\
\hline Vanadium & $3.45 \mathrm{E} 2$ & N/A & $"$ & $2.50 \mathrm{E} 2$ & $1.00 \mathrm{E} 2$ & 97.9 & $75-125$ \\
\hline Zinc & $3.85 \mathrm{E} 2$ & N/A & $"$ & $2.50 \mathrm{E} 2$ & $1.43 \mathrm{E} 2$ & 97 & $75-125$ \\
\hline Sodium & $1.16 \mathrm{E} 3$ & $\mathrm{~N} / \mathrm{A}$ & $"$ & $5.00 \mathrm{E} 2$ & $6.64 \mathrm{E} 2$ & 99.1 & $75-125$ \\
\hline Silicon & 4.71E2 & N/A & $"$ & $5.00 \mathrm{E} 2$ & ND & 109 & $75-125$ \\
\hline Sulfur & $1.16 \mathrm{E} 3$ & N/A & $"$ & $1.00 \mathrm{E} 3$ & $6.07 \mathrm{E} 1$ & 110 & $75-125$ \\
\hline Titanium & $3.09 \mathrm{E} 3$ & N/A & $"$ & $2.50 \mathrm{E} 2$ & $2.81 \mathrm{E} 3$ & 112 & $75-125$ \\
\hline Zirconium & $2.78 \mathrm{E} 2$ & N/A & $"$ & $2.50 \mathrm{E} 2$ & $2.73 \mathrm{E} 1$ & 100 & $75-125$ \\
\hline Silver & $4.24 \mathrm{E} 2$ & $\mathrm{~N} / \mathrm{A}$ & $"$ & $5.00 \mathrm{E} 2$ & ND & 88.3 & $75-125$ \\
\hline Rhenium & $4.86 \mathrm{E} 2$ & N/A & $"$ & $5.00 \mathrm{E} 2$ & ND & 98.8 & $75-125$ \\
\hline Antimony & $4.62 \mathrm{E} 2$ & $\mathrm{~N} / \mathrm{A}$ & $"$ & $5.00 \mathrm{E} 2$ & ND & 92.7 & $75-125$ \\
\hline
\end{tabular}


Radionuclides by ICP-MS/Acid Extract - Quality Control

Environmental Science Laboratory

\begin{tabular}{|c|c|c|c|c|c|c|c|c|c|c|}
\hline & & orting & & Spike & Source & & $\%$ REC & & RPD & \\
\hline Analyte & Result & Limit & Units & Level & Result & $\%$ REC & Limits & RPD & Limit & Notes \\
\hline
\end{tabular}

Batch 8L12001 - ASTM D 5198 (ICP/ICPMS)

Blank (8L12001-BLK1)

Technetium-99

Uranium 238

Duplicate (8L12001-DUP1)

Technetium-99

Uranium 238

Post Spike (8L12001-PS1)

Technetium-99

Uranium 238

$\begin{array}{llc} & & \\ & & \\ <1.19 \mathrm{E}-3 & 1.19 \mathrm{E}-3 & \text { ug } / g \text { wet } \\ <8.64 \mathrm{E}-3 & 8.64 \mathrm{E}-3 & "\end{array}$

Prepared \& Analyzed: 12/12/08

Source: 0805003-15

$<3.89 \mathrm{E}-3 \quad 3.89 \mathrm{E}-3 \quad$ ug/g dry

3.76E-1

$2.82 \mathrm{E}-2$

"

Prepared \& Analyzed: 12/12/08

$\begin{array}{ccc}\text { ND } & 35 \\ 3.35 \mathrm{E}-1 & 11.5 & 35\end{array}$

Source: 0805003-15

Prepared \& Analyzed: 12/12/08

$\begin{array}{ccccccc}4.57 \mathrm{E}-1 & \text { N/A } & \text { ug/L } & 5.00 \mathrm{E}-1 & 7.17 \mathrm{E}-4 & 91.2 & 75-125 \\ 1.47 \mathrm{E} 0 & \text { N/A } & " & 5.00 \mathrm{E}-1 & 9.94 \mathrm{E}-1 & 95.8 & 75-125\end{array}$




\section{Radionuclides by ICP-MS/Water Extract - Quality Control \\ Environmental Science Laboratory}

\begin{tabular}{|lrrrrrrrrrrr}
\hline & & Reporting & & Spike & Source & & & $\%$ REC & & RPD \\
Analyte & Result & Limit & Units & Level & Result & $\%$ REC & Limits & RPD & Limit & Notes \\
\hline
\end{tabular}

Batch 8H27003 - 1:1 Water Extract (ICP/ICPMS)

Blank (8H27003-BLK1)

Technetium-99

Uranium 238

Duplicate (8H27003-DUP1)

Technetium-99

Uranium 238

Post Spike (8H27003-PS1)

Technetium-99

Uranium 238

Batch 8H27005 - 1:1 Water Extract (ICP/ICPMS)

\section{Blank (8H27005-BLK1)}

Technetium-99

Uranium 238

Duplicate (8H27005-DUP1)

Technetium-99

Uranium 238

Post Spike (8H27005-PS1)

Technetium-99

Uranium 238

Batch 8H27006 - 1:1 Water Extract (ICP/ICPMS)

\section{Blank (8H27006-BLK1)}

Technetium-99

Uranium 238

$\begin{array}{ccc}<2.30 \mathrm{E}-5 & 2.30 \mathrm{E}-5 & \text { ug/g wet } \\ <5.64 \mathrm{E}-4 & 5.64 \mathrm{E}-4 & "\end{array}$

Prepared \& Analyzed: 08/27/08

Source: 0805001-05

$<2.30 \mathrm{E}-5 \quad 2.30 \mathrm{E}-5 \quad$ ug/g dry

$<5.63 \mathrm{E}-4$

$5.63 \mathrm{E}-4$

"

Prepared \& Analyzed: 08/27/08

\begin{tabular}{|c|c|c|}
\hline ND & & \\
\hline $\mathrm{ND}$ & & \\
\hline Inalyze & $8 / 27$ & \\
\hline ND & 102 & $75-125$ \\
\hline $.80 \mathrm{E}-2$ & 103 & $75-125$ \\
\hline
\end{tabular}

Source: 0805001-05

Prepared \& Analyzed: 08/27/08

$\begin{array}{ccccccc}5.09 \mathrm{E}-1 & \mathrm{~N} / \mathrm{A} & \mathrm{ug} / \mathrm{L} & 5.00 \mathrm{E}-1 & \mathrm{ND} & 102 & 75-125 \\ 5.75 \mathrm{E}-1 & \mathrm{~N} / \mathrm{A} & \mu & 5.00 \mathrm{E}-1 & 5.80 \mathrm{E}-2 & 103 & 75-125\end{array}$

) 
RCRA Metals By PNNL-AGG-415/Water Extract - Quality Control

Environmental Science Laboratory

\begin{tabular}{|lrrrrrrrrrrr}
\hline & & Reporting & & Spike & Source & & & $\%$ REC & & RPD \\
Analyte & Result & Limit & Units & Level & Result & $\%$ REC & Limits & RPD & Limit & Notes \\
\hline
\end{tabular}

Batch 8H26016 - 1:1 Water Extract (ICP/ICPMS)

Blank (8H26016-BLK1)

Chromium 52

Copper 63

Arsenic 75

Selenium 82

Molybdenum 95

Ruthenium 101

Silver 107

Silver 109

Cadmium 111

Antimony 121

Lead 208

LCS (8H26016-BS1)

$\begin{array}{ccc}<2.06 \mathrm{E}-3 & 2.06 \mathrm{E}-3 & \mathrm{ug} / \mathrm{g} \text { wet } \\ <3.48 \mathrm{E}-3 & 3.48 \mathrm{E}-3 & " \\ <6.25 \mathrm{E}-3 & 6.25 \mathrm{E}-3 & " \\ <1.10 \mathrm{E}-2 & 1.10 \mathrm{E}-2 & " \\ <1.60 \mathrm{E}-3 & 1.60 \mathrm{E}-3 & " \\ <8.20 \mathrm{E}-4 & 8.20 \mathrm{E}-4 & " \\ <9.25 \mathrm{E}-4 & 9.25 \mathrm{E}-4 & " \\ <1.07 \mathrm{E}-3 & 1.07 \mathrm{E}-3 & " \\ <2.95 \mathrm{E}-4 & 2.95 \mathrm{E}-4 & " \\ <5.40 \mathrm{E}-4 & 5.40 \mathrm{E}-4 & " \\ <5.60 \mathrm{E}-4 & 5.60 \mathrm{E}-4 & "\end{array}$

Chromium 52

Copper 63

Arsenic 75

Selenium 82

Molybdenum 95

Ruthenium 101

Silver 107

Silver 109

Cadmium 111

Lead 208

(

Prepared \& Analyzed: 08/26/08

Duplicate (8H26016-DUP1)

Chromium 52

Copper 63

Arsenic 75

Selenium 82

Molybdenum 95

Ruthenium 101

Silver 107

Silver 109

Cadmium 111

Antimony 121

Lead 208

95

(2)

5.16E0 2.06E-1 ug/g wet

Prepared \& Analyzed: 08/26/08

$\begin{array}{llcccc}5.16 \mathrm{E} 0 & 2.06 \mathrm{E}-1 & \text { ug } / g \text { wet } & 5.00 \mathrm{E} 0 & 103 & 80-120 \\ 5.09 \mathrm{E} 0 & 3.48 \mathrm{E}-1 & " & 5.00 \mathrm{E} 0 & 102 & 80-120\end{array}$

$\begin{array}{llllll}4.93 \mathrm{E} 0 & 6.25 \mathrm{E}-1 & " & 5.00 \mathrm{E} 0 & 98.6 & 80-120\end{array}$

$\begin{array}{llllll}5.00 \mathrm{E} 0 & 1.10 \mathrm{E} 0 & " & 5.00 \mathrm{E} 0 & 99.9 & 80-120\end{array}$

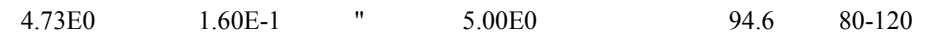

$\begin{array}{llll}<.20 \mathrm{E}-2 & 8.20 \mathrm{E}-2 \quad " \quad & 80-120\end{array}$

$\begin{array}{llllll}4.78 \mathrm{E} 0 & 9.25 \mathrm{E}-2 & \quad & 5.00 \mathrm{E} 0 & 95.6 & 80-120\end{array}$

$\begin{array}{llllll}4.78 \mathrm{E} 0 & 1.07 \mathrm{E}-1 & \quad & 5.00 \mathrm{E} 0 & 95.6 & 80-120\end{array}$

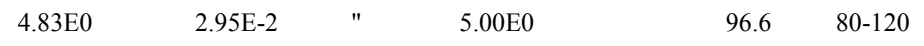

$\begin{array}{lllll}4.92 \mathrm{E} 0 & 5.60 \mathrm{E}-2 & \quad & 5.00 \mathrm{E} 0 & 98.4\end{array}$

Source: 0805001-05 Prepared \& Analyzed: 08/26/08

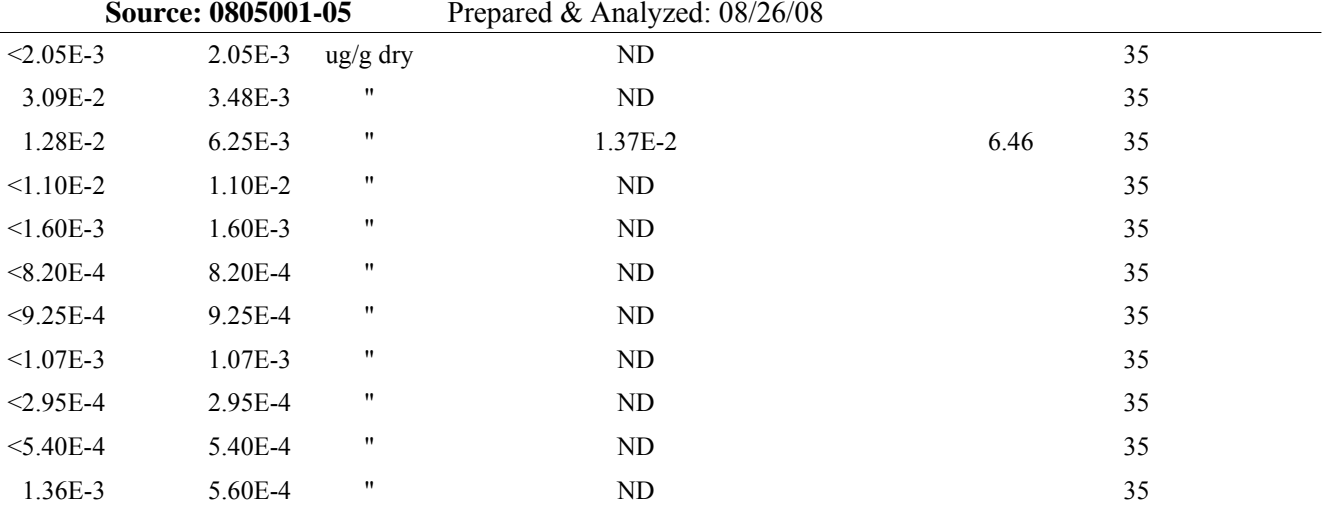


RCRA Metals By PNNL-AGG-415/Water Extract - Quality Control

Environmental Science Laboratory

\begin{tabular}{|lrrrrrrrrrrr}
\hline & & Reporting & & Spike & Source & & & $\%$ REC & & RPD \\
Analyte & Result & Limit & Units & Level & Result & $\%$ REC & Limits & RPD & Limit & Notes \\
\hline
\end{tabular}

Batch 8H26016 - 1:1 Water Extract (ICP/ICPMS)

\begin{tabular}{|c|c|c|c|c|c|c|c|}
\hline \multirow{2}{*}{$\begin{array}{l}\text { Post Spike (8H26016-PS1) } \\
\text { Chromium } 52\end{array}$} & \multicolumn{3}{|c|}{ Source: 0805001-05 } & \multicolumn{4}{|c|}{ Prepared \& Analyzed: 08/26/08 } \\
\hline & $5.27 \mathrm{E} 0$ & N/A & $\mathrm{ug} / \mathrm{L}$ & $5.00 \mathrm{E} 0$ & $1.18 \mathrm{E}-1$ & 103 & $75-125$ \\
\hline Copper 63 & $5.63 \mathrm{E} 0$ & N/A & $"$ & $5.00 \mathrm{E} 0$ & $4.03 \mathrm{E}-1$ & 105 & $75-125$ \\
\hline Arsenic 75 & $7.86 \mathrm{E} 0$ & N/A & $"$ & $5.00 \mathrm{E} 0$ & 2.77E0 & 102 & $75-125$ \\
\hline Selenium 82 & $5.39 \mathrm{E} 0$ & N/A & $"$ & $5.00 \mathrm{E} 0$ & $2.33 \mathrm{E}-2$ & 107 & $75-125$ \\
\hline Molybdenum 95 & $4.96 \mathrm{E} 0$ & N/A & $"$ & $5.00 \mathrm{E} 0$ & $1.65 \mathrm{E}-1$ & 95.8 & $75-125$ \\
\hline Ruthenium 101 & $4.80 \mathrm{E} 0$ & $\mathrm{~N} / \mathrm{A}$ & $"$ & $5.00 \mathrm{E} 0$ & $4.11 \mathrm{E}-3$ & 96 & $75-125$ \\
\hline Silver 107 & $4.80 \mathrm{E} 0$ & N/A & $"$ & $5.00 \mathrm{E} 0$ & $1.77 \mathrm{E}-2$ & 95.6 & $75-125$ \\
\hline Silver 109 & $4.81 \mathrm{E} 0$ & N/A & $"$ & $5.00 \mathrm{E} 0$ & $2.03 \mathrm{E}-2$ & 95.7 & $75-125$ \\
\hline Cadmium 111 & 4.84E0 & N/A & $"$ & $5.00 \mathrm{E} 0$ & ND & 96.8 & $75-125$ \\
\hline Antimony 121 & $4.91 \mathrm{E} 0$ & N/A & $"$ & $5.00 \mathrm{E} 0$ & $3.46 \mathrm{E}-2$ & 97.6 & $75-125$ \\
\hline Lead 208 & $4.92 \mathrm{E} 0$ & N/A & $"$ & $5.00 \mathrm{E} 0$ & $3.18 \mathrm{E}-2$ & 97.8 & $75-125$ \\
\hline
\end{tabular}

Batch 8H26017 - 1:1 Water Extract (ICP/ICPMS)

\begin{tabular}{llll} 
Blank (8H26017-BLK1) & & & \\
\hline Chromium 52 & $<2.06 \mathrm{E}-3$ & $2.06 \mathrm{E}-3$ & ug/g wet \\
Copper 63 & $<3.48 \mathrm{E}-3$ & $3.48 \mathrm{E}-3$ & $"$ \\
Arsenic 75 & $<6.25 \mathrm{E}-3$ & $6.25 \mathrm{E}-3$ & $"$ \\
Selenium 82 & $<1.10 \mathrm{E}-2$ & $1.10 \mathrm{E}-2$ & $"$ \\
Molybdenum 95 & $<1.60 \mathrm{E}-3$ & $1.60 \mathrm{E}-3$ & $"$ \\
Ruthenium 101 & $<8.20 \mathrm{E}-4$ & $8.20 \mathrm{E}-4$ & $"$ \\
Silver 107 & $<9.25 \mathrm{E}-4$ & $9.25 \mathrm{E}-4$ & $"$ \\
Silver 109 & $<1.07 \mathrm{E}-3$ & $1.07 \mathrm{E}-3$ & $"$ \\
Cadmium 111 & $<2.95 \mathrm{E}-4$ & $2.95 \mathrm{E}-4$ & $"$ \\
Antimony 121 & $<5.40 \mathrm{E}-4$ & $5.40 \mathrm{E}-4$ & $"$ \\
Lead 208 & $<5.60 \mathrm{E}-4$ & $5.60 \mathrm{E}-4$ & $"$
\end{tabular}


RCRA Metals By PNNL-AGG-415/Water Extract - Quality Control

Environmental Science Laboratory

\begin{tabular}{|lrrrrrrrrrrr}
\hline & & Reporting & & Spike & Source & & & $\%$ REC & & RPD \\
Analyte & Result & Limit & Units & Level & Result & $\%$ REC & Limits & RPD & Limit & Notes \\
\hline
\end{tabular}

Batch 8H26017 - 1:1 Water Extract (ICP/ICPMS)

\begin{tabular}{|c|c|c|c|c|c|c|c|c|c|}
\hline \multirow{2}{*}{$\begin{array}{l}\text { LCS (8H26017-BS1) } \\
\text { Chromium } 52\end{array}$} & \multicolumn{9}{|c|}{ Prepared \& Analyzed: 08/26/08 } \\
\hline & $5.22 \mathrm{E} 0$ & $2.06 \mathrm{E}-1$ & ug/g wet & 4.99E0 & & 105 & $80-120$ & & \\
\hline Copper 63 & $5.21 \mathrm{E} 0$ & $3.48 \mathrm{E}-1$ & $"$ & 4.99E0 & & 104 & $80-120$ & & \\
\hline Arsenic 75 & $5.08 \mathrm{E} 0$ & $6.25 \mathrm{E}-1$ & $"$ & 4.99E0 & & 102 & $80-120$ & & \\
\hline Selenium 82 & $5.29 \mathrm{E} 0$ & $1.10 \mathrm{E} 0$ & $"$ & 4.99E0 & & 106 & $80-120$ & & \\
\hline Molybdenum 95 & 4.87E0 & $1.60 \mathrm{E}-1$ & $"$ & 4.99E0 & & 97.6 & $80-120$ & & \\
\hline Ruthenium 101 & $<8.20 \mathrm{E}-2$ & $8.20 \mathrm{E}-2$ & $"$ & & & & $80-120$ & & \\
\hline Silver 107 & 4.87E0 & $9.25 \mathrm{E}-2$ & $"$ & 4.99E0 & & 97.7 & $80-120$ & & \\
\hline Silver 109 & $4.88 \mathrm{E} 0$ & $1.07 \mathrm{E}-1$ & $"$ & 4.99E0 & & 97.9 & $80-120$ & & \\
\hline Cadmium 111 & 4.93E0 & $2.95 \mathrm{E}-2$ & $"$ & 4.99E0 & & 98.9 & $80-120$ & & \\
\hline Lead 208 & $5.06 \mathrm{E} 0$ & $5.60 \mathrm{E}-2$ & $"$ & 4.99E0 & & 101 & $80-120$ & & \\
\hline Duplicate (8H26017-DUP1) & \multicolumn{3}{|c|}{ Source: 0805003-15 } & \multicolumn{4}{|c|}{ Prepared: 08/26/08 Analyzed: 08/27/08 } & & \\
\hline Chromium 52 & $<2.06 \mathrm{E}-3$ & $2.06 \mathrm{E}-3$ & ug/g dry & \multicolumn{3}{|c|}{ ND } & & & 35 \\
\hline Copper 63 & $<3.48 \mathrm{E}-3$ & $3.48 \mathrm{E}-3$ & $"$ & \multicolumn{3}{|c|}{ ND } & & & 35 \\
\hline Arsenic 75 & $8.60 \mathrm{E}-3$ & $6.25 \mathrm{E}-3$ & $"$ & \multicolumn{3}{|c|}{$8.37 \mathrm{E}-3$} & & 2.74 & 35 \\
\hline Selenium 82 & $<1.11 \mathrm{E}-2$ & $1.11 \mathrm{E}-2$ & $"$ & \multicolumn{3}{|c|}{ ND } & & & 35 \\
\hline Molybdenum 95 & $2.45 \mathrm{E}-3$ & $1.61 \mathrm{E}-3$ & $"$ & \multicolumn{3}{|c|}{$2.60 \mathrm{E}-3$} & & 5.94 & 35 \\
\hline Ruthenium 101 & $<8.21 \mathrm{E}-4$ & $8.21 \mathrm{E}-4$ & $"$ & \multicolumn{3}{|c|}{ ND } & & & 35 \\
\hline Silver 107 & $<9.26 \mathrm{E}-4$ & $9.26 \mathrm{E}-4$ & $"$ & \multicolumn{3}{|c|}{ ND } & & & 35 \\
\hline Silver 109 & $<1.07 \mathrm{E}-3$ & $1.07 \mathrm{E}-3$ & $"$ & \multicolumn{3}{|c|}{ ND } & & & 35 \\
\hline Cadmium 111 & $<2.95 \mathrm{E}-4$ & $2.95 \mathrm{E}-4$ & $"$ & \multicolumn{3}{|c|}{ ND } & & & 35 \\
\hline Antimony 121 & $8.86 \mathrm{E}-4$ & $5.40 \mathrm{E}-4$ & $"$ & \multicolumn{3}{|c|}{ ND } & & & 35 \\
\hline Lead 208 & $<5.60 \mathrm{E}-4$ & $5.60 \mathrm{E}-4$ & $"$ & \multicolumn{3}{|c|}{$\mathrm{ND}$} & & & 35 \\
\hline Post Spike (8H26017-PS1) & \multicolumn{3}{|c|}{ Source: 0805003-15 } & \multicolumn{4}{|c|}{ Prepared: 08/26/08 Analyzed: 08/27/08 } & & \\
\hline Chromium 52 & $5.18 \mathrm{E} 0$ & N/A & $\mathrm{ug} / \mathrm{L}$ & $5.00 \mathrm{E} 0$ & $5.51 \mathrm{E}-2$ & 102 & $75-125$ & & \\
\hline Copper 63 & $5.54 \mathrm{E} 0$ & N/A & $"$ & $5.00 \mathrm{E} 0$ & $4.81 \mathrm{E}-1$ & 101 & $75-125$ & & \\
\hline Arsenic 75 & $6.89 \mathrm{E} 0$ & N/A & $"$ & $5.00 \mathrm{E} 0$ & $1.67 \mathrm{E} 0$ & 104 & $75-125$ & & \\
\hline Selenium 82 & $5.46 \mathrm{E} 0$ & N/A & $"$ & $5.00 \mathrm{E} 0$ & $8.49 \mathrm{E}-2$ & 107 & $75-125$ & & \\
\hline Molybdenum 95 & $5.27 \mathrm{E} 0$ & N/A & $"$ & $5.00 \mathrm{E} 0$ & $5.20 \mathrm{E}-1$ & 95.1 & $75-125$ & & \\
\hline Ruthenium 101 & $4.82 \mathrm{E} 0$ & N/A & $"$ & $5.00 \mathrm{E} 0$ & $3.66 \mathrm{E}-3$ & 96.4 & $75-125$ & & \\
\hline Silver 107 & $4.80 \mathrm{E} 0$ & N/A & $"$ & $5.00 \mathrm{E} 0$ & $1.71 \mathrm{E}-2$ & 95.7 & $75-125$ & & \\
\hline Silver 109 & $4.81 \mathrm{E} 0$ & N/A & $"$ & $5.00 \mathrm{E} 0$ & $1.89 \mathrm{E}-2$ & 95.8 & $75-125$ & & \\
\hline Cadmium 111 & 4.91E0 & N/A & $"$ & $5.00 \mathrm{E} 0$ & $1.85 \mathrm{E}-3$ & 98.1 & $75-125$ & & \\
\hline Antimony 121 & $4.95 \mathrm{E} 0$ & N/A & $"$ & $5.00 \mathrm{E} 0$ & $1.85 \mathrm{E}-2$ & 98.7 & $75-125$ & & \\
\hline Lead 208 & $4.96 \mathrm{E} 0$ & N/A & $"$ & $5.00 \mathrm{E} 0$ & $4.23 \mathrm{E}-2$ & 98.3 & $75-125$ & & \\
\hline
\end{tabular}


RCRA Metals By PNNL-AGG-415/Water Extract - Quality Control

Environmental Science Laboratory

\begin{tabular}{|lrrrrrrrrrrr}
\hline & & Reporting & & Spike & Source & & & $\%$ REC & & RPD \\
Analyte & Result & Limit & Units & Level & Result & $\%$ REC & Limits & RPD & Limit & Notes \\
\hline
\end{tabular}

Batch 8H26018 - 1:1 Water Extract (ICP/ICPMS)

Blank (8H26018-BLK1)

Chromium 52

Copper 63

Arsenic 75

Selenium 82

Molybdenum 95

Ruthenium 101

Silver 107

Silver 109

Cadmium 111

Antimony 121

Lead 208

LCS (8H26018-BS1)

Chromium 52

Copper 63

Arsenic 75

Selenium 82

Molybdenum 95

Ruthenium 101

Silver 107

Silver 109

Cadmium 111

Lead 208
Prepared: 08/26/08 Analyzed: 08/27/08

$\begin{array}{llc}<2.06 \mathrm{E}-3 & 2.06 \mathrm{E}-3 & \text { ug/g wet } \\ <3.48 \mathrm{E}-3 & 3.48 \mathrm{E}-3 & " \\ <6.25 \mathrm{E}-3 & 6.25 \mathrm{E}-3 & " \\ <1.10 \mathrm{E}-2 & 1.10 \mathrm{E}-2 & " \\ <1.60 \mathrm{E}-3 & 1.60 \mathrm{E}-3 & " \\ <8.20 \mathrm{E}-4 & 8.20 \mathrm{E}-4 & " \\ <9.25 \mathrm{E}-4 & 9.25 \mathrm{E}-4 & " \\ <1.07 \mathrm{E}-3 & 1.07 \mathrm{E}-3 & " \\ <2.95 \mathrm{E}-4 & 2.95 \mathrm{E}-4 & " \\ <5.40 \mathrm{E}-4 & 5.40 \mathrm{E}-4 & " \\ <5.60 \mathrm{E}-4 & 5.60 \mathrm{E}-4 & "\end{array}$

Prepared: 08/26/08 Analyzed: 08/27/08

$\begin{array}{rrcccc}5.09 \mathrm{E} 0 & 2.06 \mathrm{E}-1 & \text { ug } / \mathrm{g} \text { wet } & 4.99 \mathrm{E} 0 & 102 & 80-120 \\ 5.15 \mathrm{E} 0 & 3.48 \mathrm{E}-1 & " & 4.99 \mathrm{E} 0 & 103 & 80-120 \\ 5.08 \mathrm{E} 0 & 6.25 \mathrm{E}-1 & " & 4.99 \mathrm{E} 0 & 102 & 80-120 \\ 5.17 \mathrm{E} 0 & 1.10 \mathrm{E} 0 & " & 4.99 \mathrm{E} 0 & 104 & 80-120 \\ 4.77 \mathrm{E} 0 & 1.60 \mathrm{E}-1 & " & 4.99 \mathrm{E} 0 & 95.6 & 80-120 \\ <8.20 \mathrm{E}-2 & 8.20 \mathrm{E}-2 & " & & & 80-120 \\ 4.74 \mathrm{E} 0 & 9.25 \mathrm{E}-2 & " & 4.99 \mathrm{E} 0 & 94.9 & 80-120 \\ 4.78 \mathrm{E} 0 & 1.07 \mathrm{E}-1 & " & 4.99 \mathrm{E} 0 & 95.7 & 80-120 \\ 4.86 \mathrm{E} 0 & 2.95 \mathrm{E}-2 & " & 4.99 \mathrm{E} 0 & 97.4 & 80-120 \\ 5.01 \mathrm{E} 0 & 5.60 \mathrm{E}-2 & " & 4.99 \mathrm{E} 0 & 100 & 80-120\end{array}$


RCRA Metals By PNNL-AGG-415/Acid Extract - Quality Control

Environmental Science Laboratory

\begin{tabular}{|rrrrrrrrrrrr}
\hline & & Reporting & & Spike & Source & & \%REC & & RPD \\
Analyte & Result & Limit & Units & Level & Result & $\%$ REC & Limits & RPD & Limit & Notes \\
\hline
\end{tabular}

Batch 8L15001 - ASTM D 5198 (ICP/ICPMS)
Blank (8L15001-BLK1)

Chromium 52

Chromium 53

Copper 63

Copper 65

Arsenic 75

Selenium 82

Molybdenum 95

Molybdenum 97

Molybdenum 98

Ruthenium 101

Ruthenium 102

Ruthenium 104

Silver 107

Silver 109

Cadmium 111

Cadmium 114

Antimony 121

Lead 206

Lead 208
LCS (8L15001-BS1)

Chromium 52

Copper 63

Copper 65

Arsenic 75

Selenium 82

Molybdenum 95

Molybdenum 97

Molybdenum 98

Ruthenium 101

Ruthenium 102

Ruthenium 104

Silver 107

Silver 109

Cadmium 111

Cadmium 114

Antimony 121

Lead 206

Lead 208

$\begin{array}{rlc}<5.46 \mathrm{E}-2 & 5.46 \mathrm{E}-2 & \text { ug/g wet } \\ 5.75 \mathrm{E}-1 & 1.61 \mathrm{E}-1 & " \\ <1.93 \mathrm{E}-1 & 1.93 \mathrm{E}-1 & " \\ <1.72 \mathrm{E}-1 & 1.72 \mathrm{E}-1 & " \\ <1.20 \mathrm{E}-1 & 1.20 \mathrm{E}-1 & " \\ <3.28 \mathrm{E}-1 & 3.28 \mathrm{E}-1 & " \\ <6.72 \mathrm{E}-2 & 6.72 \mathrm{E}-2 & " \\ <6.16 \mathrm{E}-2 & 6.16 \mathrm{E}-2 & " \\ <2.55 \mathrm{E}-2 & 2.55 \mathrm{E}-2 & " \\ <1.29 \mathrm{E}-2 & 1.29 \mathrm{E}-2 & " \\ <7.80 \mathrm{E}-3 & 7.80 \mathrm{E}-3 & " \\ <2.18 \mathrm{E}-2 & 2.18 \mathrm{E}-2 & " \\ <1.99 \mathrm{E}-2 & 1.99 \mathrm{E}-2 & " \\ <1.30 \mathrm{E}-2 & 1.30 \mathrm{E}-2 & " \\ <1.41 \mathrm{E}-2 & 1.41 \mathrm{E}-2 & " \\ <3.20 \mathrm{E}-3 & 3.20 \mathrm{E}-3 & " \\ <2.28 \mathrm{E}-2 & 2.28 \mathrm{E}-2 & " \\ <2.24 \mathrm{E}-2 & 2.24 \mathrm{E}-2 & " \\ <1.06 \mathrm{E}-2 & 1.06 \mathrm{E}-2 & "\end{array}$

Prepared \& Analyzed: 12/15/08

Prepared \& Analyzed: 12/15/08

$\begin{array}{rccccc}6.37 \mathrm{E} 0 & 2.73 \mathrm{E}-1 & \text { ug/g wet } & 6.06 \mathrm{E} 0 & 105 & 80-120 \\ 6.88 \mathrm{E} 0 & 9.65 \mathrm{E}-1 & " & 6.06 \mathrm{E} 0 & 114 & 80-120 \\ 6.84 \mathrm{E} 0 & 8.60 \mathrm{E}-1 & " & 6.06 \mathrm{E} 0 & 113 & 80-120 \\ 6.44 \mathrm{E} 0 & 6.00 \mathrm{E}-1 & " & 6.06 \mathrm{E} 0 & 106 & 80-120 \\ 6.48 \mathrm{E} 0 & 1.64 \mathrm{E} 0 & " & 6.06 \mathrm{E} 0 & 107 & 80-120 \\ 6.76 \mathrm{E} 0 & 3.36 \mathrm{E}-1 & " & 6.06 \mathrm{E} 0 & 112 & 80-120 \\ 6.59 \mathrm{E} 0 & 3.08 \mathrm{E}-1 & " & 6.06 \mathrm{E} 0 & 109 & 80-120 \\ 5.90 \mathrm{E} 0 & 1.28 \mathrm{E}-1 & " & 6.06 \mathrm{E} 0 & 97.4 & 80-120 \\ <6.45 \mathrm{E}-2 & 6.45 \mathrm{E}-2 & " & & & 80-120 \\ <3.90 \mathrm{E}-2 & 3.90 \mathrm{E}-2 & " & & & 80-120 \\ <1.09 \mathrm{E}-1 & 1.09 \mathrm{E}-1 & " & & 112 & 80-120 \\ 6.78 \mathrm{E} 0 & 9.95 \mathrm{E}-2 & " & 6.06 \mathrm{E} 0 & 111 & 80-120 \\ 6.73 \mathrm{E} 0 & 6.50 \mathrm{E}-2 & " & 6.06 \mathrm{E} 0 & 112 & 80-120 \\ 6.76 \mathrm{E} 0 & 7.05 \mathrm{E}-2 & " & 6.06 \mathrm{E} 0 & 111 & 80-120 \\ 6.69 \mathrm{E} 0 & 1.60 \mathrm{E}-2 & " & 6.06 \mathrm{E} 0 & 105 & 80-120 \\ 6.39 \mathrm{E} 0 & 1.14 \mathrm{E}-1 & " & 6.06 \mathrm{E} 0 & 112 & 80-120 \\ 6.75 \mathrm{E} 0 & 1.12 \mathrm{E}-1 & " & 6.06 \mathrm{E} 0 & 111 & 80-120 \\ 6.71 \mathrm{E} 0 & 5.30 \mathrm{E}-2 & " & 6.06 \mathrm{E} 0 & & \end{array}$


RCRA Metals By PNNL-AGG-415/Acid Extract - Quality Control

Environmental Science Laboratory

\begin{tabular}{|c|c|c|c|c|c|c|c|c|c|c|}
\hline Analyte & Result & $\begin{array}{r}\text { porting } \\
\text { Limit }\end{array}$ & Units & $\begin{array}{l}\text { Spike } \\
\text { Level }\end{array}$ & $\begin{array}{l}\text { Source } \\
\text { Result }\end{array}$ & $\%$ REC & $\begin{array}{l}\text { \%REC } \\
\text { Limits }\end{array}$ & RPD & $\begin{array}{l}\text { RPD } \\
\text { Limit }\end{array}$ & Notes \\
\hline
\end{tabular}

Batch 8L15001 - ASTM D 5198 (ICP/ICPMS)

\begin{tabular}{|c|c|c|c|c|c|c|c|c|c|}
\hline \multirow{2}{*}{$\begin{array}{l}\text { Duplicate (8L15001-DUP1) } \\
\text { Chromium } 52\end{array}$} & \multicolumn{3}{|c|}{ Source: 0805003-15 } & \multicolumn{6}{|c|}{ Prepared \& Analyzed: 12/15/08 } \\
\hline & $7.21 \mathrm{E} 0$ & $1.78 \mathrm{E}-1$ & ug/g dry & & $6.64 \mathrm{E} 0$ & & & 8.12 & 35 \\
\hline Copper 63 & $7.50 \mathrm{E} 0$ & $6.31 \mathrm{E}-1$ & $"$ & & $6.87 \mathrm{E} 0$ & & & 8.87 & 35 \\
\hline Copper 65 & $7.54 \mathrm{E} 0$ & $5.62 \mathrm{E}-1$ & $"$ & & $7.09 \mathrm{E} 0$ & & & 6.20 & 35 \\
\hline Arsenic 75 & $2.80 \mathrm{E} 0$ & $3.92 \mathrm{E}-1$ & $"$ & & $1.58 \mathrm{E} 0$ & & & 55.9 & 35 \\
\hline Selenium 82 & $<1.07 \mathrm{E} 0$ & $1.07 \mathrm{E} 0$ & $"$ & & ND & & & & 35 \\
\hline Molybdenum 95 & $<2.20 \mathrm{E}-1$ & $2.20 \mathrm{E}-1$ & $"$ & & ND & & & & 35 \\
\hline Molybdenum 97 & $<2.01 \mathrm{E}-1$ & $2.01 \mathrm{E}-1$ & $"$ & & ND & & & & 35 \\
\hline Molybdenum 98 & $1.50 \mathrm{E}-1$ & $8.33 \mathrm{E}-2$ & $"$ & & $1.52 \mathrm{E}-1$ & & & 1.49 & 35 \\
\hline Ruthenium 101 & $<4.22 \mathrm{E}-2$ & $4.22 \mathrm{E}-2$ & $"$ & & ND & & & & 35 \\
\hline Ruthenium 102 & $<2.55 \mathrm{E}-2$ & $2.55 \mathrm{E}-2$ & $"$ & & ND & & & & 35 \\
\hline Ruthenium 104 & $<7.12 \mathrm{E}-2$ & $7.12 \mathrm{E}-2$ & $"$ & & ND & & & & 35 \\
\hline Silver 107 & $<6.50 \mathrm{E}-2$ & $6.50 \mathrm{E}-2$ & $"$ & & ND & & & & 35 \\
\hline Silver 109 & $<4.25 \mathrm{E}-2$ & $4.25 \mathrm{E}-2$ & $"$ & & ND & & & & 35 \\
\hline Cadmium 111 & $5.26 \mathrm{E}-2$ & $4.61 \mathrm{E}-2$ & $"$ & & ND & & & & 35 \\
\hline Cadmium 114 & 7.77E-2 & $1.05 \mathrm{E}-2$ & $"$ & & $5.78 \mathrm{E}-2$ & & & 29.4 & 35 \\
\hline Antimony 121 & $<7.45 \mathrm{E}-2$ & $7.45 \mathrm{E}-2$ & $"$ & & ND & & & & 35 \\
\hline Lead 206 & $5.14 \mathrm{E} 0$ & 7.32E-2 & $"$ & & $2.43 \mathrm{E} 0$ & & & 71.4 & 35 \\
\hline Lead 208 & $5.16 \mathrm{E} 0$ & $3.46 \mathrm{E}-2$ & $"$ & & $2.38 \mathrm{E} 0$ & & & 73.6 & 35 \\
\hline Post Spike (8L15001-PS1) & \multicolumn{3}{|c|}{ Source: 0805003-15 } & \multicolumn{4}{|c|}{ Prepared \& Analyzed: 12/15/08 } & & \\
\hline Chromium 52 & $2.44 \mathrm{E} 1$ & N/A & $\mathrm{ug} / \mathrm{L}$ & $5.00 \mathrm{E} 0$ & $1.97 \mathrm{E} 1$ & 94.2 & $75-125$ & & \\
\hline Copper 63 & $2.44 \mathrm{E} 1$ & N/A & $"$ & $5.00 \mathrm{E} 0$ & $2.04 \mathrm{E} 1$ & 80.7 & $75-125$ & & \\
\hline Copper 65 & $2.53 \mathrm{E} 1$ & N/A & $"$ & $5.00 \mathrm{E} 0$ & $2.10 \mathrm{E} 1$ & 85.6 & $75-125$ & & \\
\hline Arsenic 75 & $8.65 \mathrm{E} 0$ & $\mathrm{~N} / \mathrm{A}$ & $"$ & $5.00 \mathrm{E} 0$ & 4.67E0 & 79.6 & $75-125$ & & \\
\hline Selenium 82 & $4.44 \mathrm{E} 0$ & $\mathrm{~N} / \mathrm{A}$ & $"$ & $5.00 \mathrm{E} 0$ & $1.99 \mathrm{E}-1$ & 84.8 & $75-125$ & & \\
\hline Molybdenum 95 & $5.70 \mathrm{E} 0$ & N/A & $"$ & $5.00 \mathrm{E} 0$ & $4.12 \mathrm{E}-1$ & 106 & $75-125$ & & \\
\hline Molybdenum 97 & $5.66 \mathrm{E} 0$ & N/A & $"$ & $5.00 \mathrm{E} 0$ & 4.37E-1 & 104 & $75-125$ & & \\
\hline Molybdenum 98 & $5.43 \mathrm{E} 0$ & N/A & $"$ & $5.00 \mathrm{E} 0$ & $4.51 \mathrm{E}-1$ & 99.7 & $75-125$ & & \\
\hline Ruthenium 101 & $5.03 \mathrm{E} 0$ & $\mathrm{~N} / \mathrm{A}$ & $"$ & $5.00 \mathrm{E} 0$ & ND & 101 & $75-125$ & & \\
\hline Ruthenium 102 & $4.95 \mathrm{E} 0$ & N/A & $"$ & $5.00 \mathrm{E} 0$ & $2.71 \mathrm{E}-2$ & 98.4 & $75-125$ & & \\
\hline Ruthenium 104 & $5.20 \mathrm{E} 0$ & $\mathrm{~N} / \mathrm{A}$ & $"$ & $5.00 \mathrm{E} 0$ & $1.01 \mathrm{E}-1$ & 102 & $75-125$ & & \\
\hline Silver 107 & $4.82 \mathrm{E} 0$ & N/A & $"$ & $5.00 \mathrm{E} 0$ & $1.24 \mathrm{E}-1$ & 94 & $75-125$ & & \\
\hline Silver 109 & 4.73E0 & N/A & $"$ & $5.00 \mathrm{E} 0$ & $8.10 \mathrm{E}-2$ & 93 & $75-125$ & & \\
\hline Cadmium 111 & 4.96Е0 & N/A & $"$ & $5.00 \mathrm{E} 0$ & $1.09 \mathrm{E}-1$ & 97 & $75-125$ & & \\
\hline Cadmium 114 & 4.87E0 & N/A & $"$ & $5.00 \mathrm{E} 0$ & $1.71 \mathrm{E}-1$ & 94 & $75-125$ & & \\
\hline Antimony 121 & $4.56 \mathrm{E} 0$ & N/A & $"$ & $5.00 \mathrm{E} 0$ & $1.15 \mathrm{E}-1$ & 88.9 & $75-125$ & & \\
\hline Lead 206 & $1.13 \mathrm{E} 1$ & $\mathrm{~N} / \mathrm{A}$ & $"$ & $5.00 \mathrm{E} 0$ & $7.22 \mathrm{E} 0$ & 82.5 & $75-125$ & & \\
\hline Lead 208 & $1.12 \mathrm{E} 1$ & N/A & $"$ & $5.00 \mathrm{E} 0$ & 7.07E0 & 83.6 & $75-125$ & & \\
\hline
\end{tabular}


Total Alpha Total Beta/Acid Extract - Quality Control

Environmental Science Laboratory

\begin{tabular}{|lrrrrrrrrrrr}
\hline & & Reporting & & Spike & Source & & & $\%$ REC & & RPD \\
Analyte & Result & Limit & Units & Level & Result & $\%$ REC & Limits & RPD & Limit & Notes \\
\hline
\end{tabular}

Batch 9C16001 - ASTM D 5198 (RadChem)

Blank (9C16001-BLK1)

Gross Beta

Gross Alpha

$\begin{array}{lll}<2.99 \mathrm{E} 1 & 2.99 \mathrm{E} 1 & \mathrm{pCi} / \mathrm{g} \text { wet } \\ <1.33 \mathrm{E} 1 & 1.33 \mathrm{E} 1 & "\end{array}$

Prepared: 03/16/09 Analyzed: 03/18/09

Duplicate (9C16001-DUP1)

Gross Beta

Source: 0805003-15

Prepared: 03/16/09 Analyzed: 03/18/09

Gross Alpha

$<9.78 \mathrm{E} 1$

9.78E1 pCi/g dry

ND

35

$<4.35 \mathrm{E} 1$

$4.35 \mathrm{E} 1$

ND

35 
Total Alpha Total Beta/Water Extract - Quality Control

Environmental Science Laboratory

\begin{tabular}{|lrrrrrrrrrrr}
\hline & & Reporting & & Spike & Source & & & $\%$ REC & & RPD \\
Analyte & Result & Limit & Units & Level & Result & $\%$ REC & Limits & RPD & Limit & Notes \\
\hline
\end{tabular}

Batch 8L15002 - 1:1 Water Extract (RadChem)

Blank (8L15002-BLK1)

Gross Beta

Gross Alpha

Duplicate (8L15002-DUP1)

Gross Beta

Gross Alpha

$\begin{array}{ccc}<2.99 \mathrm{E} 1 & 2.99 \mathrm{E} 1 & \mathrm{pCi} / \mathrm{g} \text { wet } \\ <1.33 \mathrm{E} 1 & 1.33 \mathrm{E} 1 & "\end{array}$

Prepared: 12/15/08 Analyzed: 03/17/09

Prepared: 12/15/08 Analyzed: 03/17/09

$<2.99 \mathrm{E} 1 \quad 2.99 \mathrm{E} 1 \quad \mathrm{pCi} / \mathrm{g}$ wet

$<1.33 \mathrm{E} 1$

$1.33 \mathrm{E} 1$
35

35 


\section{PARTICLE SIZE DISTRIBUTION ANALYSIS HYDROMETER}

\begin{tabular}{|r|c|c|}
\hline Sample ID: & B1VDW5 & \\
\hline Time, min & $\mathrm{X}, \mathrm{um}$ & $\mathrm{P}, \%$ \\
\hline 0.5 & 81.1 & 1.10 \\
\hline 1 & 57.2 & 1.03 \\
\hline 3 & 33.0 & 0.96 \\
\hline 10 & 17.8 & 0.55 \\
\hline 30 & 10.3 & 0.48 \\
\hline 60 & 7.21 & 0.27 \\
\hline 90 & 5.88 & 0.27 \\
\hline 120 & 5.10 & 0.27 \\
\hline 1440 & 1.47 & 0.27 \\
\hline
\end{tabular}

\begin{tabular}{|r|c|c|}
\hline Sample ID: & B1VDW7 & \\
\hline Time, min & X, um & $\mathrm{P}, \%$ \\
\hline 0.5 & 81.8 & 5.50 \\
\hline 1 & 57.6 & 4.95 \\
\hline 3 & 33.3 & 4.95 \\
\hline 10 & 18.0 & 3.57 \\
\hline 30 & 10.3 & 2.75 \\
\hline 60 & 7.27 & 2.20 \\
\hline 90 & 5.92 & 1.92 \\
\hline 120 & 5.12 & 1.65 \\
\hline 1440 & 1.48 & 1.65 \\
\hline
\end{tabular}

\begin{tabular}{|r|c|c|}
\hline Sample ID: & B1VDW6 & \\
\hline Time, min & $\mathrm{X}, \mathrm{um}$ & $\mathrm{P}, \%$ \\
\hline 0.5 & 81.3 & 2.66 \\
\hline 1 & 57.4 & 2.51 \\
\hline 3 & 32.9 & 2.04 \\
\hline 10 & 17.9 & 1.57 \\
\hline 30 & 10.3 & 1.25 \\
\hline 60 & 7.22 & 0.78 \\
\hline 90 & 5.88 & 0.63 \\
\hline 120 & 5.10 & 0.63 \\
\hline 1440 & 1.47 & 0.63 \\
\hline
\end{tabular}

\begin{tabular}{|r|c|c|}
\hline Sample ID: & B1VDW8 & \\
\hline Time, min & $\mathrm{X}, \mathrm{um}$ & $\mathrm{P}, \%$ \\
\hline 0.5 & 83.8 & 8.65 \\
\hline 1 & 59.0 & 8.10 \\
\hline 3 & 33.6 & 6.42 \\
\hline 10 & 18.2 & 5.03 \\
\hline 30 & 10.4 & 3.91 \\
\hline 60 & 7.34 & 3.35 \\
\hline 90 & 5.97 & 2.79 \\
\hline 120 & 5.15 & 2.51 \\
\hline 1440 & 1.48 & 2.23 \\
\hline
\end{tabular}




\section{PARTICLE SIZE DISTRIBUTION ANALYSIS HYDROMETER}

\begin{tabular}{|r|c|c|}
\hline Sample ID: & B1VDW9 & \\
\hline Time, min & $\mathrm{X}, \mathrm{um}$ & $\mathrm{P}, \%$ \\
\hline 0.5 & 83.1 & 5.23 \\
\hline 1 & 58.1 & 4.26 \\
\hline 3 & 33.3 & 3.48 \\
\hline 10 & 18.1 & 2.71 \\
\hline 30 & 10.4 & 2.32 \\
\hline 60 & 7.29 & 1.74 \\
\hline 90 & 5.94 & 1.55 \\
\hline 120 & 5.14 & 1.55 \\
\hline 1440 & 1.48 & 1.35 \\
\hline
\end{tabular}

\begin{tabular}{|r|c|c|}
\hline Sample ID: & B1VDX0 & \\
\hline Time, min & X, um & $\mathrm{P}, \%$ \\
\hline 0.5 & 84.4 & 10.14 \\
\hline 1 & 59.1 & 8.69 \\
\hline 3 & 33.9 & 7.82 \\
\hline 10 & 18.3 & 5.50 \\
\hline 30 & 10.4 & 4.06 \\
\hline 60 & 7.34 & 3.48 \\
\hline 90 & 5.97 & 2.90 \\
\hline 120 & 5.15 & 2.61 \\
\hline 1440 & 1.48 & 2.32 \\
\hline
\end{tabular}




\section{PARTICLE SIZE DISTRIBUTION ANALYSIS HYDROMETER}

\begin{tabular}{|r|c|c|}
\hline Sample ID: & B1V991 & \\
\hline Time, min & $\mathrm{X}, \mathrm{um}$ & $\mathrm{P}, \%$ \\
\hline 0.5 & 83.7 & 2.62 \\
\hline 1 & 58.9 & 2.27 \\
\hline 3 & 33.8 & 1.75 \\
\hline 10 & 18.4 & 1.40 \\
\hline 30 & 10.6 & 1.40 \\
\hline 60 & 7.49 & 1.05 \\
\hline 90 & 6.10 & 0.87 \\
\hline 120 & 5.28 & 0.87 \\
\hline 1440 & 1.52 & 0.70 \\
\hline
\end{tabular}

\begin{tabular}{|r|c|c|}
\hline Sample ID: & B1V993 & \\
\hline Time, min & X, um & P, \% \\
\hline 0.5 & 85.5 & 5.82 \\
\hline 1 & 60.0 & 4.89 \\
\hline 3 & 34.3 & 3.96 \\
\hline 10 & 18.7 & 3.26 \\
\hline 30 & 10.7 & 2.56 \\
\hline 60 & 7.56 & 2.33 \\
\hline 90 & 6.16 & 2.10 \\
\hline 120 & 5.32 & 1.86 \\
\hline 1440 & 1.53 & 1.40 \\
\hline
\end{tabular}

\begin{tabular}{|r|c|c|}
\hline Sample ID: & B1V992 & \\
\hline Time, min & $\mathrm{X}, \mathrm{um}$ & $\mathrm{P}, \%$ \\
\hline 0.5 & 86.3 & 6.95 \\
\hline 1 & 60.5 & 5.99 \\
\hline 3 & 34.6 & 5.03 \\
\hline 10 & 18.8 & 4.07 \\
\hline 30 & 10.8 & 3.35 \\
\hline 60 & 7.57 & 2.64 \\
\hline 90 & 6.17 & 2.40 \\
\hline 120 & 5.33 & 2.16 \\
\hline 1440 & 1.52 & 1.20 \\
\hline
\end{tabular}

\begin{tabular}{|r|c|c|}
\hline Sample ID: & B1V994 & \\
\hline Time, min & $\mathrm{X}, \mathrm{um}$ & $\mathrm{P}, \%$ \\
\hline 0.5 & 87.7 & 9.99 \\
\hline 1 & 61.3 & 8.37 \\
\hline 3 & 34.8 & 6.21 \\
\hline 10 & 18.9 & 5.40 \\
\hline 30 & 10.9 & 4.59 \\
\hline 60 & 7.62 & 3.78 \\
\hline 90 & 6.20 & 3.24 \\
\hline 120 & 5.36 & 2.97 \\
\hline 1440 & 1.53 & 1.62 \\
\hline
\end{tabular}




\section{PARTICLE SIZE DISTRIBUTION ANALYSIS HYDROMETER}

\begin{tabular}{|r|c|c|}
\hline Sample ID: & B1V995 & \\
\hline Time, min & $\mathrm{X}, \mathrm{um}$ & $\mathrm{P}, \%$ \\
\hline 0.5 & 88.2 & 7.98 \\
\hline 1 & 61.6 & 6.78 \\
\hline 3 & 35.1 & 5.39 \\
\hline 10 & 19.0 & 4.19 \\
\hline 30 & 10.9 & 3.39 \\
\hline 60 & 7.62 & 2.79 \\
\hline 90 & 6.21 & 2.59 \\
\hline 120 & 5.36 & 2.19 \\
\hline 1440 & 1.53 & 1.40 \\
\hline
\end{tabular}

\begin{tabular}{|r|c|c|}
\hline Sample ID: & B1V997 & \\
\hline Time, min & X, um & P, \% \\
\hline 0.5 & 90.5 & 15.85 \\
\hline 1 & 62.3 & 11.67 \\
\hline 3 & 34.9 & 7.48 \\
\hline 10 & 18.9 & 5.68 \\
\hline 30 & 10.8 & 4.79 \\
\hline 60 & 7.62 & 4.19 \\
\hline 90 & 6.20 & 3.59 \\
\hline 120 & 5.36 & 3.29 \\
\hline 1440 & 1.53 & 2.09 \\
\hline
\end{tabular}

\begin{tabular}{|r|c|c|}
\hline Sample ID: & B1V996 & \\
\hline Time, min & $\mathrm{X}, \mathrm{um}$ & $\mathrm{P}, \%$ \\
\hline 0.5 & 89.8 & 14.15 \\
\hline 1 & 62.0 & 10.69 \\
\hline 3 & 34.9 & 7.22 \\
\hline 10 & 18.9 & 5.49 \\
\hline 30 & 10.8 & 4.33 \\
\hline 60 & 7.61 & 3.75 \\
\hline 90 & 6.20 & 3.47 \\
\hline 120 & 5.36 & 3.18 \\
\hline 1440 & 1.53 & 2.02 \\
\hline
\end{tabular}

\begin{tabular}{|r|c|c|}
\hline Sample ID: & B1V998 & \\
\hline Time, min & X, um & P, \% \\
\hline 0.5 & 87.0 & 8.13 \\
\hline 1 & 61.0 & 7.15 \\
\hline 3 & 34.9 & 6.16 \\
\hline 10 & 19.0 & 5.42 \\
\hline 30 & 10.9 & 4.68 \\
\hline 60 & 7.66 & 3.94 \\
\hline 90 & 6.23 & 3.45 \\
\hline 120 & 5.38 & 3.20 \\
\hline 1440 & 1.53 & 1.48 \\
\hline
\end{tabular}




\section{PARTICLE SIZE DISTRIBUTION ANALYSIS HYDROMETER}

\begin{tabular}{|r|c|c|}
\hline Sample ID: & B1V999 & \\
\hline Time, min & $\mathrm{X}, \mathrm{um}$ & $\mathrm{P}, \%$ \\
\hline 0.5 & 85.4 & 5.29 \\
\hline 1 & 60.1 & 4.85 \\
\hline 3 & 34.5 & 4.19 \\
\hline 10 & 18.8 & 3.53 \\
\hline 30 & 10.8 & 2.87 \\
\hline 60 & 7.57 & 2.42 \\
\hline 90 & 6.17 & 2.20 \\
\hline 120 & 5.33 & 1.98 \\
\hline 1440 & 1.52 & 1.10 \\
\hline
\end{tabular}

\begin{tabular}{|r|c|c|}
\hline Sample ID: & B1V9B1 & \\
\hline Time, min & X, um & $\mathrm{P}, \%$ \\
\hline 0.5 & 84.1 & 3.02 \\
\hline 1 & 59.3 & 2.84 \\
\hline 3 & 34.2 & 2.66 \\
\hline 10 & 18.6 & 2.31 \\
\hline 30 & 10.7 & 2.13 \\
\hline 60 & 7.56 & 1.78 \\
\hline 90 & 6.16 & 1.60 \\
\hline 120 & 5.32 & 1.42 \\
\hline 1440 & 1.52 & 0.89 \\
\hline
\end{tabular}

\begin{tabular}{|r|c|c|}
\hline Sample ID: & B1V9B0 & \\
\hline Time, min & $\mathrm{X}, \mathrm{um}$ & $\mathrm{P}, \%$ \\
\hline 0.5 & 84.6 & 2.40 \\
\hline 1 & 59.7 & 2.28 \\
\hline 3 & 34.2 & 1.80 \\
\hline 10 & 18.7 & 1.68 \\
\hline 30 & 10.7 & 1.44 \\
\hline 60 & 7.56 & 1.20 \\
\hline 90 & 6.16 & 1.08 \\
\hline 120 & 5.32 & 0.96 \\
\hline 1440 & 1.52 & 0.60 \\
\hline
\end{tabular}

\begin{tabular}{|r|c|c|}
\hline Sample ID: & B1V9B2 & \\
\hline Time, min & X, um & $\mathrm{P}, \%$ \\
\hline 0.5 & 84.8 & 4.44 \\
\hline 1 & 59.7 & 4.02 \\
\hline 3 & 34.2 & 3.38 \\
\hline 10 & 18.6 & 2.75 \\
\hline 30 & 10.7 & 2.33 \\
\hline 60 & 7.54 & 1.90 \\
\hline 90 & 6.14 & 1.69 \\
\hline 120 & 5.31 & 1.48 \\
\hline 1440 & 1.52 & 1.06 \\
\hline
\end{tabular}




\section{PARTICLE SIZE DISTRIBUTION ANALYSIS HYDROMETER}

\begin{tabular}{|r|c|c|}
\hline Sample ID: & B1V9B3 & \\
\hline Time, min & $\mathrm{X}, \mathrm{um}$ & $\mathrm{P}, \%$ \\
\hline 0.5 & 85.5 & 9.63 \\
\hline 1 & 60.2 & 8.86 \\
\hline 3 & 34.5 & 7.32 \\
\hline 10 & 18.8 & 6.16 \\
\hline 30 & 10.8 & 5.01 \\
\hline 60 & 7.57 & 4.24 \\
\hline 90 & 6.16 & 3.47 \\
\hline 120 & 5.33 & 3.47 \\
\hline 1440 & 1.53 & 2.31 \\
\hline
\end{tabular}

\begin{tabular}{|r|c|c|}
\hline Sample ID: & B1V9B5 & \\
\hline Time, min & X, um & P, \% \\
\hline 0.5 & 81.6 & 1.61 \\
\hline 1 & 57.6 & 1.21 \\
\hline 3 & 33.2 & 1.21 \\
\hline 10 & 18.2 & 1.21 \\
\hline 30 & 10.5 & 1.21 \\
\hline 60 & 7.43 & 1.21 \\
\hline 90 & 6.07 & 1.21 \\
\hline 120 & 5.26 & 1.21 \\
\hline 1440 & 1.52 & 1.21 \\
\hline
\end{tabular}

\begin{tabular}{|r|c|c|}
\hline Sample ID: & B1V9B4 & \\
\hline Time, min & $\mathrm{X}, \mathrm{um}$ & $\mathrm{P}, \%$ \\
\hline 0.5 & 83.9 & 9.82 \\
\hline 1 & 59.2 & 9.20 \\
\hline 3 & 34.0 & 7.98 \\
\hline 10 & 18.5 & 6.14 \\
\hline 30 & 10.7 & 5.52 \\
\hline 60 & 7.54 & 5.52 \\
\hline 90 & 6.16 & 5.52 \\
\hline 120 & 5.32 & 4.91 \\
\hline 1440 & 1.52 & 3.07 \\
\hline
\end{tabular}

\begin{tabular}{|r|c|c|}
\hline Sample ID: & B1V9B6 & \\
\hline Time, min & $\mathrm{X}, \mathrm{um}$ & $\mathrm{P}, \%$ \\
\hline 0.5 & 82.0 & 3.13 \\
\hline 1 & 57.9 & 2.61 \\
\hline 3 & 33.2 & 1.56 \\
\hline 10 & 18.2 & 1.56 \\
\hline 30 & 10.5 & 1.56 \\
\hline 60 & 7.43 & 1.56 \\
\hline 90 & 6.07 & 1.56 \\
\hline 120 & 5.26 & 1.56 \\
\hline 1440 & 1.52 & 1.56 \\
\hline
\end{tabular}




\section{PARTICLE SIZE DISTRIBUTION ANALYSIS HYDROMETER}

\begin{tabular}{|r|c|c|}
\hline Sample ID: & B1V9B7 & \\
\hline Time, min & $\mathrm{X}, \mathrm{um}$ & $\mathrm{P}, \%$ \\
\hline 0.5 & 82.2 & 3.64 \\
\hline 1 & 58.1 & 3.64 \\
\hline 3 & 33.4 & 2.60 \\
\hline 10 & 18.3 & 2.08 \\
\hline 30 & 10.5 & 2.08 \\
\hline 60 & 7.45 & 2.08 \\
\hline 90 & 6.07 & 1.56 \\
\hline 120 & 5.26 & 1.56 \\
\hline 1440 & 1.52 & 1.56 \\
\hline
\end{tabular}

\begin{tabular}{|r|c|c|}
\hline Sample ID: & B1V9B9 & \\
\hline Time, min & X, um & P, \% \\
\hline 0.5 & 83.0 & 5.91 \\
\hline 1 & 58.4 & 4.84 \\
\hline 3 & 33.6 & 3.76 \\
\hline 10 & 18.4 & 3.76 \\
\hline 30 & 10.6 & 3.23 \\
\hline 60 & 7.47 & 2.69 \\
\hline 90 & 6.10 & 2.69 \\
\hline 120 & 5.27 & 2.15 \\
\hline 1440 & 1.52 & 1.61 \\
\hline
\end{tabular}

\begin{tabular}{|r|c|c|}
\hline Sample ID: & B1V9B8 & \\
\hline Time, min & $\mathrm{X}, \mathrm{um}$ & $\mathrm{P}, \%$ \\
\hline 0.5 & 81.6 & 2.55 \\
\hline 1 & 57.6 & 1.92 \\
\hline 3 & 33.2 & 1.92 \\
\hline 10 & 18.2 & 1.92 \\
\hline 30 & 10.5 & 1.92 \\
\hline 60 & 7.43 & 1.92 \\
\hline 90 & 6.07 & 1.92 \\
\hline 120 & 5.26 & 1.92 \\
\hline 1440 & 1.52 & 1.92 \\
\hline
\end{tabular}

\begin{tabular}{|r|c|c|}
\hline Sample ID: & B1V9C0 & \\
\hline Time, min & $\mathrm{X}, \mathrm{um}$ & $\mathrm{P}, \%$ \\
\hline 0.5 & 83.0 & 6.48 \\
\hline 1 & 58.3 & 4.71 \\
\hline 3 & 33.6 & 4.12 \\
\hline 10 & 18.3 & 3.53 \\
\hline 30 & 10.6 & 2.95 \\
\hline 60 & 7.47 & 2.95 \\
\hline 90 & 6.10 & 2.95 \\
\hline 120 & 5.28 & 2.95 \\
\hline 1440 & 1.52 & 1.77 \\
\hline
\end{tabular}




\section{PARTICLE SIZE DISTRIBUTION ANALYSIS HYDROMETER}

\begin{tabular}{|r|c|c|}
\hline Sample ID: & B1V9C1 & \\
\hline Time, min & $\mathrm{X}, \mathrm{um}$ & $\mathrm{P}, \%$ \\
\hline 0.5 & 82.2 & 3.61 \\
\hline 1 & 58.0 & 3.10 \\
\hline 3 & 33.5 & 3.10 \\
\hline 10 & 18.3 & 3.10 \\
\hline 30 & 10.6 & 2.58 \\
\hline 60 & 7.45 & 2.06 \\
\hline 90 & 6.08 & 2.06 \\
\hline 120 & 5.26 & 1.55 \\
\hline 1440 & 1.52 & 1.55 \\
\hline
\end{tabular}

\begin{tabular}{|r|c|c|}
\hline Sample ID: & B1V9C3 & \\
\hline Time, min & X, um & $\mathrm{P}, \%$ \\
\hline 0.5 & 83.5 & 5.16 \\
\hline 1 & 58.7 & 4.05 \\
\hline 3 & 33.7 & 3.32 \\
\hline 10 & 18.5 & 3.32 \\
\hline 30 & 10.6 & 2.58 \\
\hline 60 & 7.49 & 2.21 \\
\hline 90 & 6.11 & 2.21 \\
\hline 120 & 5.28 & 1.84 \\
\hline 1440 & 1.52 & 1.11 \\
\hline
\end{tabular}

\begin{tabular}{|r|c|c|}
\hline Sample ID: & $\mathrm{B} 1 \mathrm{~V} 9 \mathrm{C} 2$ & \\
\hline Time, min & $\mathrm{X}, \mathrm{um}$ & $\mathrm{P}, \%$ \\
\hline 0.5 & 83.0 & 5.12 \\
\hline 1 & 58.4 & 4.19 \\
\hline 3 & 33.7 & 4.19 \\
\hline 10 & 18.4 & 3.26 \\
\hline 30 & 10.6 & 3.26 \\
\hline 60 & 7.47 & 2.33 \\
\hline 90 & 6.10 & 2.33 \\
\hline 120 & 5.26 & 1.40 \\
\hline 1440 & 1.52 & 1.40 \\
\hline
\end{tabular}

\begin{tabular}{|r|c|c|}
\hline Sample ID: & B1V9C4 & \\
\hline Time, min & $\mathrm{X}, \mathrm{um}$ & $\mathrm{P}, \%$ \\
\hline 0.5 & 83.5 & 4.90 \\
\hline 1 & 58.9 & 4.55 \\
\hline 3 & 33.9 & 3.85 \\
\hline 10 & 18.6 & 3.85 \\
\hline 30 & 10.7 & 3.15 \\
\hline 60 & 7.50 & 2.45 \\
\hline 90 & 6.13 & 2.45 \\
\hline 120 & 5.29 & 2.10 \\
\hline 1440 & 1.52 & 1.40 \\
\hline
\end{tabular}




\section{PARTICLE SIZE DISTRIBUTION ANALYSIS HYDROMETER}

\begin{tabular}{|r|c|c|}
\hline Sample ID: & B1V2V5 & \\
\hline Time, min & X, um & $\mathrm{P}, \%$ \\
\hline 0.5 & 83.1 & 5.78 \\
\hline 1 & 58.7 & 5.30 \\
\hline 3 & 33.7 & 4.33 \\
\hline 10 & 18.4 & 3.37 \\
\hline 30 & 10.6 & 2.89 \\
\hline 60 & 7.47 & 2.41 \\
\hline 90 & 6.10 & 2.41 \\
\hline 120 & 5.26 & 1.44 \\
\hline 1440 & 1.51 & 0.96 \\
\hline
\end{tabular}


PARTICLE SIZE DISTRIBUTION ANALYSIS DRY SEIVE

\begin{tabular}{|c|c|r|c|}
\hline Sample ID: & \multicolumn{1}{|l|}{ B1VDW5 } & \multicolumn{1}{l|}{} \\
\hline SIEVE NUMBER & X, um & SOIL FRACTION & PERCENT PASSING \\
\hline $21 / 2 "$ & 63000 & $0.00 \%$ & 100 \\
\hline $11 / 4 "$ & 31500 & $0.00 \%$ & 100 \\
\hline $5 / 8^{\prime \prime}$ & 16000 & $52.3 \%$ & 47.7 \\
\hline $5 / 16^{\prime \prime}$ & 8000 & $21.3 \%$ & 26.3 \\
\hline 5 & 4000 & $10.0 \%$ & 16.3 \\
\hline 10 & 2000 & $4.16 \%$ & 12.1 \\
\hline 18 & 1000 & $4.06 \%$ & 8.09 \\
\hline 35 & 500 & $2.68 \%$ & 5.40 \\
\hline 60 & 250 & $2.54 \%$ & 2.86 \\
\hline 120 & 125 & $1.33 \%$ & 1.54 \\
\hline 230 & 63 & $0.86 \%$ & 0.68 \\
\hline Pan & & $0.68 \%$ & 0.00 \\
\hline
\end{tabular}

\begin{tabular}{|c|c|r|c|}
\hline Sample ID: & \multicolumn{1}{|l|}{ B1VDW6 } & \multicolumn{1}{l|}{} \\
\hline SIEVE NUMBER & X, um & \multicolumn{1}{l|}{ SOIL FRACTION } & PERCENT PASSING \\
\hline $21 / 2^{\prime \prime}$ & 63000 & $0.00 \%$ & 100 \\
\hline $11 / 4 "$ & 31500 & $0.00 \%$ & 100 \\
\hline $5 / 8^{\prime \prime}$ & 16000 & $37.4 \%$ & 62.6 \\
\hline $5 / 16^{\prime \prime}$ & 8000 & $16.7 \%$ & 46.0 \\
\hline 5 & 4000 & $8.83 \%$ & 37.1 \\
\hline 10 & 2000 & $6.48 \%$ & 30.7 \\
\hline 18 & 1000 & $8.76 \%$ & 21.9 \\
\hline 35 & 500 & $7.03 \%$ & 14.9 \\
\hline 60 & 250 & $7.31 \%$ & 7.56 \\
\hline 120 & 125 & $3.86 \%$ & 3.71 \\
\hline 230 & 63 & $2.04 \%$ & 1.66 \\
\hline Pan & & $1.66 \%$ & 0.00 \\
\hline
\end{tabular}


PARTICLE SIZE DISTRIBUTION ANALYSIS DRY SEIVE

\begin{tabular}{|c|c|r|c|}
\hline Sample ID: & \multicolumn{1}{|l|}{ B1VDW7 } & \multicolumn{1}{l|}{} \\
\hline SIEVE NUMBER & X, um & SOIL FRACTION & PERCENT PASSING \\
\hline $21 / 2 "$ & 63000 & $0.00 \%$ & 100 \\
\hline $11 / 4 "$ & 31500 & $0.00 \%$ & 100 \\
\hline $5 / 8^{\prime \prime}$ & 16000 & $12.2 \%$ & 87.8 \\
\hline $5 / 16^{\prime \prime}$ & 8000 & $10.7 \%$ & 77.1 \\
\hline 5 & 4000 & $12.4 \%$ & 64.6 \\
\hline 10 & 2000 & $10.2 \%$ & 54.5 \\
\hline 18 & 1000 & $13.7 \%$ & 40.8 \\
\hline 35 & 500 & $12.1 \%$ & 28.7 \\
\hline 60 & 250 & $12.1 \%$ & 16.6 \\
\hline 120 & 125 & $7.76 \%$ & 8.87 \\
\hline 230 & 63 & $5.51 \%$ & 3.36 \\
\hline Pan & & $3.36 \%$ & 0.00 \\
\hline
\end{tabular}

\begin{tabular}{|c|c|r|c|}
\hline Sample ID: & \multicolumn{1}{|l|}{ B1VDW8 } & \multicolumn{1}{|l|}{} \\
\hline SIEVE NUMBER & X, um & \multicolumn{1}{|l|}{ SOIL FRACTION } & PERCENT PASSING \\
\hline $21 / 2^{\prime \prime}$ & 63000 & $0.00 \%$ & 100 \\
\hline $11 / 4 "$ & 31500 & $0.00 \%$ & 100 \\
\hline $5 / 8^{\prime \prime}$ & 16000 & $5.19 \%$ & 94.8 \\
\hline $5 / 16^{\prime \prime}$ & 8000 & $17.6 \%$ & 77.2 \\
\hline 5 & 4000 & $13.5 \%$ & 63.7 \\
\hline 10 & 2000 & $9.85 \%$ & 53.9 \\
\hline 18 & 1000 & $14.6 \%$ & 39.3 \\
\hline 35 & 500 & $12.7 \%$ & 26.6 \\
\hline 60 & 250 & $9.69 \%$ & 16.9 \\
\hline 120 & 125 & $7.77 \%$ & 9.09 \\
\hline 230 & 63 & $5.86 \%$ & 3.23 \\
\hline Pan & & $3.23 \%$ & 0.00 \\
\hline
\end{tabular}


PARTICLE SIZE DISTRIBUTION ANALYSIS DRY SEIVE

\begin{tabular}{|c|c|r|c|}
\hline Sample ID: & \multicolumn{1}{|l|}{ B1VDW9 } & \multicolumn{1}{l|}{} \\
\hline SIEVE NUMBER & X, um & SOIL FRACTION & PERCENT PASSING \\
\hline $21 / 2 "$ & 63000 & $0.00 \%$ & 100 \\
\hline $11 / 4 "$ & 31500 & $0.00 \%$ & 100 \\
\hline $5 / 8^{\prime \prime}$ & 16000 & $24.3 \%$ & 75.7 \\
\hline $5 / 16^{\prime \prime}$ & 8000 & $21.1 \%$ & 54.6 \\
\hline 5 & 4000 & $7.81 \%$ & 46.8 \\
\hline 10 & 2000 & $8.97 \%$ & 37.8 \\
\hline 18 & 1000 & $13.7 \%$ & 24.1 \\
\hline 35 & 500 & $12.1 \%$ & 12.0 \\
\hline 60 & 250 & $5.28 \%$ & 6.76 \\
\hline 120 & 125 & $3.22 \%$ & 3.54 \\
\hline 230 & 63 & $2.14 \%$ & 1.40 \\
\hline Pan & & $1.40 \%$ & 0.00 \\
\hline
\end{tabular}

\begin{tabular}{|c|c|r|c|}
\hline Sample ID: & \multicolumn{1}{|l|}{ B1VDX0 } & \multicolumn{1}{|l|}{} \\
\hline SIEVE NUMBER & X, um & \multicolumn{1}{|l|}{ SOIL FRACTION } & PERCENT PASSING \\
\hline $21 / 2^{\prime \prime}$ & 63000 & $0.00 \%$ & 100 \\
\hline $11 / 4 "$ & 31500 & $0.00 \%$ & 100 \\
\hline $5 / 8^{\prime \prime}$ & 16000 & $5.87 \%$ & 94.1 \\
\hline $5 / 16^{\prime \prime}$ & 8000 & $12.5 \%$ & 81.6 \\
\hline 5 & 4000 & $14.0 \%$ & 67.6 \\
\hline 10 & 2000 & $11.2 \%$ & 56.4 \\
\hline 18 & 1000 & $17.5 \%$ & 38.9 \\
\hline 35 & 500 & $16.5 \%$ & 22.4 \\
\hline 60 & 250 & $8.48 \%$ & 13.9 \\
\hline 120 & 125 & $4.84 \%$ & 9.09 \\
\hline 230 & 63 & $4.99 \%$ & 4.10 \\
\hline Pan & & $4.10 \%$ & 0.00 \\
\hline
\end{tabular}




\section{PARTICLE SIZE DISTRIBUTION ANALYSIS DRY SEIVE}

\begin{tabular}{|c|c|c|c|}
\hline Sample ID: & B1V991 & & \\
\hline SIEVE NUMBER & X, um & SOIL FRACTION & PERCENT PASSING \\
\hline $21 / 2 "$ & 63000 & $0.0 \%$ & 100 \\
\hline $11 / 4^{\prime \prime}$ & 31500 & $0.0 \%$ & 100 \\
\hline $5 / 8^{\prime \prime}$ & 16000 & $33.6 \%$ & 66.4 \\
\hline $5 / 16 "$ & 8000 & $15.3 \%$ & 51.2 \\
\hline 5 & 4000 & $10.4 \%$ & 40.8 \\
\hline 10 & 2000 & $8.1 \%$ & 32.7 \\
\hline 18 & 1000 & $9.5 \%$ & 23.2 \\
\hline 35 & 500 & $8.1 \%$ & 15.1 \\
\hline 60 & 250 & $7.4 \%$ & 7.72 \\
\hline 120 & 125 & $3.9 \%$ & 3.80 \\
\hline $230+$ Pan & $<125$ & $3.8 \%$ & 0.00 \\
\hline
\end{tabular}

\begin{tabular}{|c|c|c|c|}
\hline Sample ID: & B1V992 & & \\
\hline SIEVE NUMBER & X, um & SOIL FRACTION & PERCENT PASSING \\
\hline $21 / 2^{\prime \prime}$ & 63000 & $0.0 \%$ & 100 \\
\hline $11 /$ " $^{\prime \prime}$ & 31500 & $0.0 \%$ & 100 \\
\hline $5 / 8^{\prime \prime}$ & 16000 & $18.0 \%$ & 82.0 \\
\hline $5 / 16^{\prime \prime}$ & 8000 & $18.3 \%$ & 63.7 \\
\hline 5 & 4000 & $11.1 \%$ & 52.6 \\
\hline 10 & 2000 & $9.2 \%$ & 43.4 \\
\hline 18 & 1000 & $14.4 \%$ & 29.1 \\
\hline 35 & 500 & $14.4 \%$ & 14.7 \\
\hline 60 & 250 & $6.3 \%$ & 8.36 \\
\hline 120 & 125 & $3.6 \%$ & 4.72 \\
\hline $230+\operatorname{Pan}$ & $<125$ & $4.7 \%$ & 0.00 \\
\hline
\end{tabular}




\section{PARTICLE SIZE DISTRIBUTION ANALYSIS DRY SEIVE}

\begin{tabular}{|c|c|c|c|}
\hline Sample ID: & B1V993 & & \\
\hline SIEVE NUMBER & X, um & SOIL FRACTION & PERCENT PASSING \\
\hline $21 / 2 "$ & 63000 & $0.0 \%$ & 100 \\
\hline $11 / 4^{\prime \prime}$ & 31500 & $0.0 \%$ & 100 \\
\hline $5 / 8^{\prime \prime}$ & 16000 & $14.4 \%$ & 85.6 \\
\hline $5 / 16 "$ & 8000 & $17.4 \%$ & 68.2 \\
\hline 5 & 4000 & $11.5 \%$ & 56.7 \\
\hline 10 & 2000 & $8.9 \%$ & 47.8 \\
\hline 18 & 1000 & $12.5 \%$ & 35.3 \\
\hline 35 & 500 & $12.3 \%$ & 23.0 \\
\hline 60 & 250 & $9.9 \%$ & 13.1 \\
\hline 120 & 125 & $6.3 \%$ & 6.79 \\
\hline $230+$ Pan & $<125$ & $6.8 \%$ & 0.00 \\
\hline
\end{tabular}

\begin{tabular}{|c|c|c|c|}
\hline Sample ID: & B1V994 & & \\
\hline SIEVE NUMBER & X, um & SOIL FRACTION & PERCENT PASSING \\
\hline $21 / 2^{\prime \prime}$ & 63000 & $0.0 \%$ & 100 \\
\hline $11 /$ " $^{\prime \prime}$ & 31500 & $0.0 \%$ & 100 \\
\hline $5 / 8^{\prime \prime}$ & 16000 & $8.8 \%$ & 91.2 \\
\hline $5 / 16^{\prime \prime}$ & 8000 & $18.5 \%$ & 72.6 \\
\hline 5 & 4000 & $13.4 \%$ & 59.2 \\
\hline 10 & 2000 & $10.4 \%$ & 48.8 \\
\hline 18 & 1000 & $15.5 \%$ & 33.3 \\
\hline 35 & 500 & $13.6 \%$ & 19.8 \\
\hline 60 & 250 & $8.0 \%$ & 11.8 \\
\hline 120 & 125 & $5.0 \%$ & 6.78 \\
\hline $230+$ Pan & $<125$ & $6.8 \%$ & 0.00 \\
\hline
\end{tabular}




\section{PARTICLE SIZE DISTRIBUTION ANALYSIS DRY SEIVE}

\begin{tabular}{|c|c|c|c|}
\hline Sample ID: & B1V995 & & \\
\hline SIEVE NUMBER & X, um & SOIL FRACTION & PERCENT PASSING \\
\hline $21 / 2 "$ & 63000 & $0.0 \%$ & 100 \\
\hline $11 / 4 "$ & 31500 & $0.0 \%$ & 100 \\
\hline $5 / 8 "$ & 16000 & $18.7 \%$ & 81.3 \\
\hline $5 / 16 "$ & 8000 & $21.0 \%$ & 60.3 \\
\hline 5 & 4000 & $13.0 \%$ & 47.3 \\
\hline 10 & 2000 & $9.9 \%$ & 37.4 \\
\hline 18 & 1000 & $12.8 \%$ & 24.6 \\
\hline 35 & 500 & $11.5 \%$ & 13.1 \\
\hline 60 & 250 & $5.6 \%$ & 7.50 \\
\hline 120 & 125 & $3.5 \%$ & 3.98 \\
\hline $230+$ Pan & $<125$ & $4.0 \%$ & 0.00 \\
\hline
\end{tabular}

\begin{tabular}{|c|c|c|c|}
\hline Sample ID: & B1V996 & & \\
\hline SIEVE NUMBER & X, um & SOIL FRACTION & PERCENT PASSING \\
\hline $21 / 2^{\prime \prime}$ & 63000 & $0.0 \%$ & 100 \\
\hline $11 /$ " $^{\prime \prime}$ & 31500 & $0.0 \%$ & 100 \\
\hline $5 / 8^{\prime \prime}$ & 16000 & $13.6 \%$ & 86.4 \\
\hline $5 / 16^{\prime \prime}$ & 8000 & $17.3 \%$ & 69.1 \\
\hline 5 & 4000 & $7.8 \%$ & 61.3 \\
\hline 10 & 2000 & $8.1 \%$ & 53.2 \\
\hline 18 & 1000 & $16.5 \%$ & 36.8 \\
\hline 35 & 500 & $14.5 \%$ & 22.3 \\
\hline 60 & 250 & $5.4 \%$ & 16.9 \\
\hline 120 & 125 & $4.2 \%$ & 12.6 \\
\hline $230+\operatorname{Pan}$ & $<125$ & $12.6 \%$ & 0.00 \\
\hline
\end{tabular}


PARTICLE SIZE DISTRIBUTION ANALYSIS DRY SEIVE

\begin{tabular}{|c|c|c|c|}
\hline Sample ID: & B1V997 & & \\
\hline SIEVE NUMBER & X, um & SOIL FRACTION & PERCENT PASSING \\
\hline $21 / 2^{\prime \prime}$ & 63000 & $0.0 \%$ & 100 \\
\hline $11 / 4 "$ & 31500 & $0.0 \%$ & 100 \\
\hline $5 / 8^{\prime \prime}$ & 16000 & $2.6 \%$ & 97.4 \\
\hline $5 / 16^{\prime \prime}$ & 8000 & $16.9 \%$ & 80.5 \\
\hline 5 & 4000 & $10.1 \%$ & 70.4 \\
\hline 10 & 2000 & $9.9 \%$ & 60.5 \\
\hline 18 & 1000 & $21.1 \%$ & 39.4 \\
\hline 35 & 500 & $17.5 \%$ & 21.9 \\
\hline 60 & 250 & $6.7 \%$ & 15.3 \\
\hline 120 & 125 & $4.6 \%$ & 10.6 \\
\hline $230+$ Pan & $<125$ & $10.6 \%$ & 0.00 \\
\hline
\end{tabular}

\begin{tabular}{|c|c|c|c|}
\hline Sample ID: & B1V998 & & \\
\hline SIEVE NUMBER & X, um & SOIL FRACTION & PERCENT PASSING \\
\hline $21 / 2 "$ & 63000 & $0.0 \%$ & 100 \\
\hline $11 / 4^{\prime \prime}$ & 31500 & $0.0 \%$ & 100 \\
\hline $5 / 8^{\prime \prime}$ & 16000 & $7.7 \%$ & 92.3 \\
\hline $5 / 16^{\prime \prime}$ & 8000 & $12.0 \%$ & 80.4 \\
\hline 5 & 4000 & $15.9 \%$ & 64.4 \\
\hline 10 & 2000 & $18.2 \%$ & 46.2 \\
\hline 18 & 1000 & $19.3 \%$ & 26.9 \\
\hline 35 & 500 & $11.3 \%$ & 15.6 \\
\hline 60 & 250 & $6.1 \%$ & 9.49 \\
\hline 120 & 125 & $4.1 \%$ & 5.37 \\
\hline $230+$ Pan & $<125$ & $5.4 \%$ & 0.00 \\
\hline
\end{tabular}




\section{PARTICLE SIZE DISTRIBUTION ANALYSIS DRY SEIVE}

\begin{tabular}{|c|c|c|c|}
\hline Sample ID: & B1V999 & & \\
\hline SIEVE NUMBER & X, um & SOIL FRACTION & PERCENT PASSING \\
\hline $21 / 2 "$ & 63000 & $0.0 \%$ & 100 \\
\hline $11 / 4^{\prime \prime}$ & 31500 & $0.0 \%$ & 100 \\
\hline $5 / 8 "$ & 16000 & $15.8 \%$ & 84.2 \\
\hline $5 / 16 "$ & 8000 & $22.6 \%$ & 61.6 \\
\hline 5 & 4000 & $18.7 \%$ & 42.9 \\
\hline 10 & 2000 & $13.9 \%$ & 28.9 \\
\hline 18 & 1000 & $11.5 \%$ & 17.5 \\
\hline 35 & 500 & $6.6 \%$ & 10.9 \\
\hline 60 & 250 & $3.9 \%$ & 7.04 \\
\hline 120 & 125 & $2.8 \%$ & 4.28 \\
\hline $230+$ Pan & $<125$ & $4.3 \%$ & 0.00 \\
\hline
\end{tabular}

\begin{tabular}{|c|c|c|c|}
\hline Sample ID: & B1V9B0 & & \\
\hline SIEVE NUMBER & X, um & SOIL FRACTION & PERCENT PASSING \\
\hline $21 / 2^{\prime \prime}$ & 63000 & $0.0 \%$ & 100 \\
\hline $11 /$ " $^{\prime \prime}$ & 31500 & $0.0 \%$ & 100 \\
\hline $5 / 8^{\prime \prime}$ & 16000 & $22.8 \%$ & 77.2 \\
\hline $5 / 16^{\prime \prime}$ & 8000 & $42.8 \%$ & 34.4 \\
\hline 5 & 4000 & $15.0 \%$ & 19.4 \\
\hline 10 & 2000 & $6.2 \%$ & 13.3 \\
\hline 18 & 1000 & $4.4 \%$ & 8.86 \\
\hline 35 & 500 & $3.5 \%$ & 5.38 \\
\hline 60 & 250 & $2.1 \%$ & 3.28 \\
\hline 120 & 125 & $1.3 \%$ & 1.97 \\
\hline $230+$ Pan & $<125$ & $2.0 \%$ & 0.00 \\
\hline
\end{tabular}




\section{PARTICLE SIZE DISTRIBUTION ANALYSIS DRY SEIVE}

\begin{tabular}{|c|c|c|c|}
\hline Sample ID: & B1V9B1 & & \\
\hline SIEVE NUMBER & X, um & SOIL FRACTION & PERCENT PASSING \\
\hline $21 / 2 "$ & 63000 & $0.0 \%$ & 100 \\
\hline $11 / 4^{\prime \prime}$ & 31500 & $0.0 \%$ & 100 \\
\hline $5 / 8^{\prime \prime}$ & 16000 & $41.5 \%$ & 58.5 \\
\hline $5 / 16 "$ & 8000 & $19.2 \%$ & 39.3 \\
\hline 5 & 4000 & $12.8 \%$ & 26.5 \\
\hline 10 & 2000 & $5.7 \%$ & 20.8 \\
\hline 18 & 1000 & $8.3 \%$ & 12.6 \\
\hline 35 & 500 & $5.6 \%$ & 7.00 \\
\hline 60 & 250 & $3.4 \%$ & 3.60 \\
\hline 120 & 125 & $1.5 \%$ & 2.11 \\
\hline $230+$ Pan & $<125$ & $2.1 \%$ & 0.00 \\
\hline
\end{tabular}

\begin{tabular}{|c|c|c|c|}
\hline Sample ID: & B1V9B2 & & \\
\hline SIEVE NUMBER & X, um & SOIL FRACTION & PERCENT PASSING \\
\hline $21 / 2^{\prime \prime}$ & 63000 & $0.0 \%$ & 100 \\
\hline $11 /$ " $^{\prime \prime}$ & 31500 & $0.0 \%$ & 100 \\
\hline $5 / 8^{\prime \prime}$ & 16000 & $25.3 \%$ & 74.7 \\
\hline $5 / 16^{\prime \prime}$ & 8000 & $25.7 \%$ & 49.0 \\
\hline 5 & 4000 & $16.2 \%$ & 32.8 \\
\hline 10 & 2000 & $8.6 \%$ & 24.2 \\
\hline 18 & 1000 & $7.3 \%$ & 16.8 \\
\hline 35 & 500 & $6.3 \%$ & 10.6 \\
\hline 60 & 250 & $4.5 \%$ & 6.12 \\
\hline 120 & 125 & $2.6 \%$ & 3.54 \\
\hline $230+$ Pan & $<125$ & $3.5 \%$ & 0.00 \\
\hline
\end{tabular}




\section{PARTICLE SIZE DISTRIBUTION ANALYSIS DRY SEIVE}

\begin{tabular}{|c|c|c|c|}
\hline Sample ID: & B1V9B3 & & \\
\hline SIEVE NUMBER & X, um & SOIL FRACTION & PERCENT PASSING \\
\hline $21 / 2 "$ & 63000 & $0.0 \%$ & 100 \\
\hline $11 / 4^{\prime \prime}$ & 31500 & $0.0 \%$ & 100 \\
\hline $5 / 8^{\prime \prime}$ & 16000 & $15.7 \%$ & 84.3 \\
\hline $5 / 16 "$ & 8000 & $18.9 \%$ & 65.4 \\
\hline 5 & 4000 & $11.3 \%$ & 54.1 \\
\hline 10 & 2000 & $9.2 \%$ & 44.9 \\
\hline 18 & 1000 & $12.2 \%$ & 32.7 \\
\hline 35 & 500 & $12.1 \%$ & 20.6 \\
\hline 60 & 250 & $8.2 \%$ & 12.4 \\
\hline 120 & 125 & $5.2 \%$ & 7.19 \\
\hline $230+$ Pan & $<125$ & $7.2 \%$ & 0.00 \\
\hline
\end{tabular}

\begin{tabular}{|c|c|c|c|}
\hline Sample ID: & B1V9B4 & & \\
\hline SIEVE NUMBER & X, um & SOIL FRACTION & PERCENT PASSING \\
\hline $21 / 2^{\prime \prime}$ & 63000 & $0.0 \%$ & 100 \\
\hline $11 /$ " $^{\prime \prime}$ & 31500 & $0.0 \%$ & 100 \\
\hline $5 / 8^{\prime \prime}$ & 16000 & $0.0 \%$ & 100 \\
\hline $5 / 16^{\prime \prime}$ & 8000 & $0.0 \%$ & 100 \\
\hline 5 & 4000 & $2.2 \%$ & 97.8 \\
\hline 10 & 2000 & $2.8 \%$ & 95.0 \\
\hline 18 & 1000 & $11.8 \%$ & 83.2 \\
\hline 35 & 500 & $47.4 \%$ & 35.8 \\
\hline 60 & 250 & $27.6 \%$ & 8.19 \\
\hline 120 & 125 & $5.3 \%$ & 2.88 \\
\hline $230+\operatorname{Pan}$ & $<125$ & $2.9 \%$ & 0.00 \\
\hline
\end{tabular}




\section{PARTICLE SIZE DISTRIBUTION ANALYSIS DRY SEIVE}

\begin{tabular}{|c|c|c|c|}
\hline Sample ID: & B1V9B5 & & \\
\hline SIEVE NUMBER & X, um & SOIL FRACTION & PERCENT PASSING \\
\hline $21 / 2 "$ & 63000 & $0.0 \%$ & 100 \\
\hline $11 / 4^{\prime \prime}$ & 31500 & $0.0 \%$ & 100 \\
\hline $5 / 8^{\prime \prime}$ & 16000 & $0.0 \%$ & 100 \\
\hline $5 / 16 "$ & 8000 & $0.0 \%$ & 100 \\
\hline 5 & 4000 & $0.2 \%$ & 99.8 \\
\hline 10 & 2000 & $2.5 \%$ & 97.3 \\
\hline 18 & 1000 & $55.7 \%$ & 41.6 \\
\hline 35 & 500 & $35.1 \%$ & 6.52 \\
\hline 60 & 250 & $4.1 \%$ & 2.46 \\
\hline 120 & 125 & $1.2 \%$ & 1.26 \\
\hline $230+$ Pan & $<125$ & $1.3 \%$ & 0.00 \\
\hline
\end{tabular}

\begin{tabular}{|c|c|c|c|}
\hline Sample ID: & B1V9B6 & & \\
\hline SIEVE NUMBER & X, um & SOIL FRACTION & PERCENT PASSING \\
\hline $21 / 2^{\prime \prime}$ & 63000 & $0.0 \%$ & 100 \\
\hline $11 /$ " $^{\prime \prime}$ & 31500 & $0.0 \%$ & 100 \\
\hline $5 / 8^{\prime \prime}$ & 16000 & $0.0 \%$ & 100 \\
\hline $5 / 16^{\prime \prime}$ & 8000 & $0.7 \%$ & 99.3 \\
\hline 5 & 4000 & $0.3 \%$ & 99.0 \\
\hline 10 & 2000 & $3.3 \%$ & 95.7 \\
\hline 18 & 1000 & $46.0 \%$ & 49.7 \\
\hline 35 & 500 & $34.2 \%$ & 15.5 \\
\hline 60 & 250 & $8.0 \%$ & 7.53 \\
\hline 120 & 125 & $3.6 \%$ & 3.92 \\
\hline $230+\operatorname{Pan}$ & $<125$ & $3.9 \%$ & 0.00 \\
\hline
\end{tabular}




\section{PARTICLE SIZE DISTRIBUTION ANALYSIS DRY SEIVE}

\begin{tabular}{|c|c|c|c|}
\hline Sample ID: & B1V9B7 & & \\
\hline SIEVE NUMBER & X, um & SOIL FRACTION & PERCENT PASSING \\
\hline $21 / 2 "$ & 63000 & $0.0 \%$ & 100 \\
\hline $11 / 4^{\prime \prime}$ & 31500 & $0.0 \%$ & 100 \\
\hline $5 / 8^{\prime \prime}$ & 16000 & $0.0 \%$ & 100 \\
\hline $5 / 16 "$ & 8000 & $0.0 \%$ & 100 \\
\hline 5 & 4000 & $0.0 \%$ & 100 \\
\hline 10 & 2000 & $0.3 \%$ & 99.7 \\
\hline 18 & 1000 & $25.0 \%$ & 74.8 \\
\hline 35 & 500 & $61.1 \%$ & 13.6 \\
\hline 60 & 250 & $8.4 \%$ & 5.28 \\
\hline 120 & 125 & $2.6 \%$ & 2.65 \\
\hline $230+$ Pan & $<125$ & $2.6 \%$ & 0.00 \\
\hline
\end{tabular}

\begin{tabular}{|c|c|c|c|}
\hline Sample ID: & B1V9B8 & & \\
\hline SIEVE NUMBER & X, um & SOIL FRACTION & PERCENT PASSING \\
\hline $21 / 2^{\prime \prime}$ & 63000 & $0.0 \%$ & 100 \\
\hline $11 /$ " $^{\prime \prime}$ & 31500 & $0.0 \%$ & 100 \\
\hline $5 / 8^{\prime \prime}$ & 16000 & $0.0 \%$ & 100 \\
\hline $5 / 16^{\prime \prime}$ & 8000 & $0.0 \%$ & 100 \\
\hline 5 & 4000 & $0.0 \%$ & 100 \\
\hline 10 & 2000 & $0.2 \%$ & 99.8 \\
\hline 18 & 1000 & $29.2 \%$ & 70.6 \\
\hline 35 & 500 & $58.6 \%$ & 12.0 \\
\hline 60 & 250 & $8.5 \%$ & 3.51 \\
\hline 120 & 125 & $2.0 \%$ & 1.52 \\
\hline $230+\operatorname{Pan}$ & $<125$ & $1.5 \%$ & 0.00 \\
\hline
\end{tabular}




\section{PARTICLE SIZE DISTRIBUTION ANALYSIS DRY SEIVE}

\begin{tabular}{|c|c|c|c|}
\hline Sample ID: & B1V9B9 & & \\
\hline SIEVE NUMBER & X, um & SOIL FRACTION & PERCENT PASSING \\
\hline $21 / 2 "$ & 63000 & $0.0 \%$ & 100 \\
\hline $11 / 4^{\prime \prime}$ & 31500 & $0.0 \%$ & 100 \\
\hline $5 / 8^{\prime \prime}$ & 16000 & $0.0 \%$ & 100 \\
\hline $5 / 16 "$ & 8000 & $0.0 \%$ & 100 \\
\hline 5 & 4000 & $0.0 \%$ & 100 \\
\hline 10 & 2000 & $0.5 \%$ & 99.5 \\
\hline 18 & 1000 & $21.6 \%$ & 77.9 \\
\hline 35 & 500 & $56.6 \%$ & 21.3 \\
\hline 60 & 250 & $10.6 \%$ & 10.7 \\
\hline 120 & 125 & $4.5 \%$ & 6.18 \\
\hline $230+$ Pan & $<125$ & $6.2 \%$ & 0.00 \\
\hline
\end{tabular}

\begin{tabular}{|c|c|c|c|}
\hline Sample ID: & B1V9C0 & & \\
\hline SIEVE NUMBER & X, um & SOIL FRACTION & PERCENT PASSING \\
\hline $21 / 2^{\prime \prime}$ & 63000 & $0.0 \%$ & 100 \\
\hline $11 /$ " $^{\prime \prime}$ & 31500 & $0.0 \%$ & 100 \\
\hline $5 / 8^{\prime \prime}$ & 16000 & $0.0 \%$ & 100 \\
\hline $5 / 16^{\prime \prime}$ & 8000 & $0.0 \%$ & 100 \\
\hline 5 & 4000 & $0.6 \%$ & 99.4 \\
\hline 10 & 2000 & $1.4 \%$ & 98.0 \\
\hline 18 & 1000 & $34.5 \%$ & 63.5 \\
\hline 35 & 500 & $48.1 \%$ & 15.4 \\
\hline 60 & 250 & $8.7 \%$ & 6.73 \\
\hline 120 & 125 & $3.3 \%$ & 3.46 \\
\hline $230+\operatorname{Pan}$ & $<125$ & $3.5 \%$ & 0.00 \\
\hline
\end{tabular}




\section{PARTICLE SIZE DISTRIBUTION ANALYSIS DRY SEIVE}

\begin{tabular}{|c|c|c|c|}
\hline Sample ID: & B1V9C1 & & \\
\hline SIEVE NUMBER & X, um & SOIL FRACTION & PERCENT PASSING \\
\hline $21 / 2 "$ & 63000 & $0.0 \%$ & 100 \\
\hline $11 / 4^{\prime \prime}$ & 31500 & $0.0 \%$ & 100 \\
\hline $5 / 8^{\prime \prime}$ & 16000 & $0.0 \%$ & 100 \\
\hline $5 / 16 "$ & 8000 & $0.0 \%$ & 100 \\
\hline 5 & 4000 & $0.0 \%$ & 100 \\
\hline 10 & 2000 & $0.7 \%$ & 99.3 \\
\hline 18 & 1000 & $30.6 \%$ & 68.7 \\
\hline 35 & 500 & $53.6 \%$ & 15.2 \\
\hline 60 & 250 & $8.1 \%$ & 7.11 \\
\hline 120 & 125 & $3.4 \%$ & 3.73 \\
\hline $230+$ Pan & $<125$ & $3.7 \%$ & 0.00 \\
\hline
\end{tabular}

\begin{tabular}{|c|c|c|c|}
\hline Sample ID: & B1V9C2 & & \\
\hline SIEVE NUMBER & $\mathrm{X}$, um & SOIL FRACTION & PERCENT PASSING \\
\hline $21 / 2^{\prime \prime}$ & 63000 & $0.0 \%$ & 100 \\
\hline $11 /$ " $^{\prime \prime}$ & 31500 & $0.0 \%$ & 100 \\
\hline $5 / 8^{\prime \prime}$ & 16000 & $0.0 \%$ & 100 \\
\hline $5 / 16^{\prime \prime}$ & 8000 & $0.3 \%$ & 99.7 \\
\hline 5 & 4000 & $5.4 \%$ & 94.2 \\
\hline 10 & 2000 & $11.3 \%$ & 82.9 \\
\hline 18 & 1000 & $18.4 \%$ & 64.5 \\
\hline 35 & 500 & $35.1 \%$ & 29.4 \\
\hline 60 & 250 & $21.6 \%$ & 7.76 \\
\hline 120 & 125 & $4.4 \%$ & 3.32 \\
\hline $230+\operatorname{Pan}$ & $<125$ & $3.3 \%$ & 0.00 \\
\hline
\end{tabular}




\section{PARTICLE SIZE DISTRIBUTION ANALYSIS DRY SEIVE}

\begin{tabular}{|c|c|c|c|}
\hline Sample ID: & B1V9C3 & & \\
\hline SIEVE NUMBER & X, um & SOIL FRACTION & PERCENT PASSING \\
\hline $21 / 2 "$ & 63000 & $0.0 \%$ & 100 \\
\hline $11 / 4^{\prime \prime}$ & 31500 & $0.0 \%$ & 100 \\
\hline $5 / 8^{\prime \prime}$ & 16000 & $0.0 \%$ & 100 \\
\hline $5 / 16 "$ & 8000 & $0.9 \%$ & 99.1 \\
\hline 5 & 4000 & $8.0 \%$ & 91.1 \\
\hline 10 & 2000 & $10.5 \%$ & 80.7 \\
\hline 18 & 1000 & $20.1 \%$ & 60.6 \\
\hline 35 & 500 & $36.7 \%$ & 23.9 \\
\hline 60 & 250 & $17.0 \%$ & 6.90 \\
\hline 120 & 125 & $4.2 \%$ & 2.71 \\
\hline $230+$ Pan & $<125$ & $2.7 \%$ & 0.00 \\
\hline
\end{tabular}

\begin{tabular}{|c|c|c|c|}
\hline Sample ID: & B1V9C4 & & \\
\hline SIEVE NUMBER & X, um & SOIL FRACTION & PERCENT PASSING \\
\hline $21 / 2^{\prime \prime}$ & 63000 & $0.0 \%$ & 100 \\
\hline $11 /$ " $^{\prime \prime}$ & 31500 & $0.0 \%$ & 100 \\
\hline $5 / 8^{\prime \prime}$ & 16000 & $8.6 \%$ & 91.4 \\
\hline $5 / 16^{\prime \prime}$ & 8000 & $6.4 \%$ & 85.0 \\
\hline 5 & 4000 & $13.2 \%$ & 71.8 \\
\hline 10 & 2000 & $9.2 \%$ & 62.6 \\
\hline 18 & 1000 & $12.5 \%$ & 50.1 \\
\hline 35 & 500 & $26.9 \%$ & 23.2 \\
\hline 60 & 250 & $15.8 \%$ & 7.34 \\
\hline 120 & 125 & $3.8 \%$ & 3.54 \\
\hline $230+$ Pan & $<125$ & $3.5 \%$ & 0.00 \\
\hline
\end{tabular}




\section{PARTICLE SIZE DISTRIBUTION ANALYSIS DRY SEIVE}

\begin{tabular}{|c|c|c|c|}
\hline Sample ID: & B1V2V5 & & \\
\hline SIEVE NUMBER & $\mathrm{X}$, um & SOIL FRACTION & PERCENT PASSING \\
\hline $21 / 2^{\prime \prime}$ & 63000 & $0.0 \%$ & 100 \\
\hline $11 / 4^{\prime \prime}$ & 31500 & $0.0 \%$ & 100 \\
\hline $5 / 8^{\prime \prime}$ & 16000 & $0.0 \%$ & 100 \\
\hline $5 / 16^{\prime \prime}$ & 8000 & $0.0 \%$ & 100 \\
\hline 5 & 4000 & $1.0 \%$ & 99.0 \\
\hline 10 & 2000 & $5.2 \%$ & 93.8 \\
\hline 18 & 1000 & $39.3 \%$ & 54.5 \\
\hline 35 & 500 & $41.3 \%$ & 13.3 \\
\hline 60 & 250 & $6.8 \%$ & 6.46 \\
\hline 120 & 125 & $3.2 \%$ & 3.27 \\
\hline $230+$ Pan & $<125$ & $3.3 \%$ & 0.00 \\
\hline
\end{tabular}



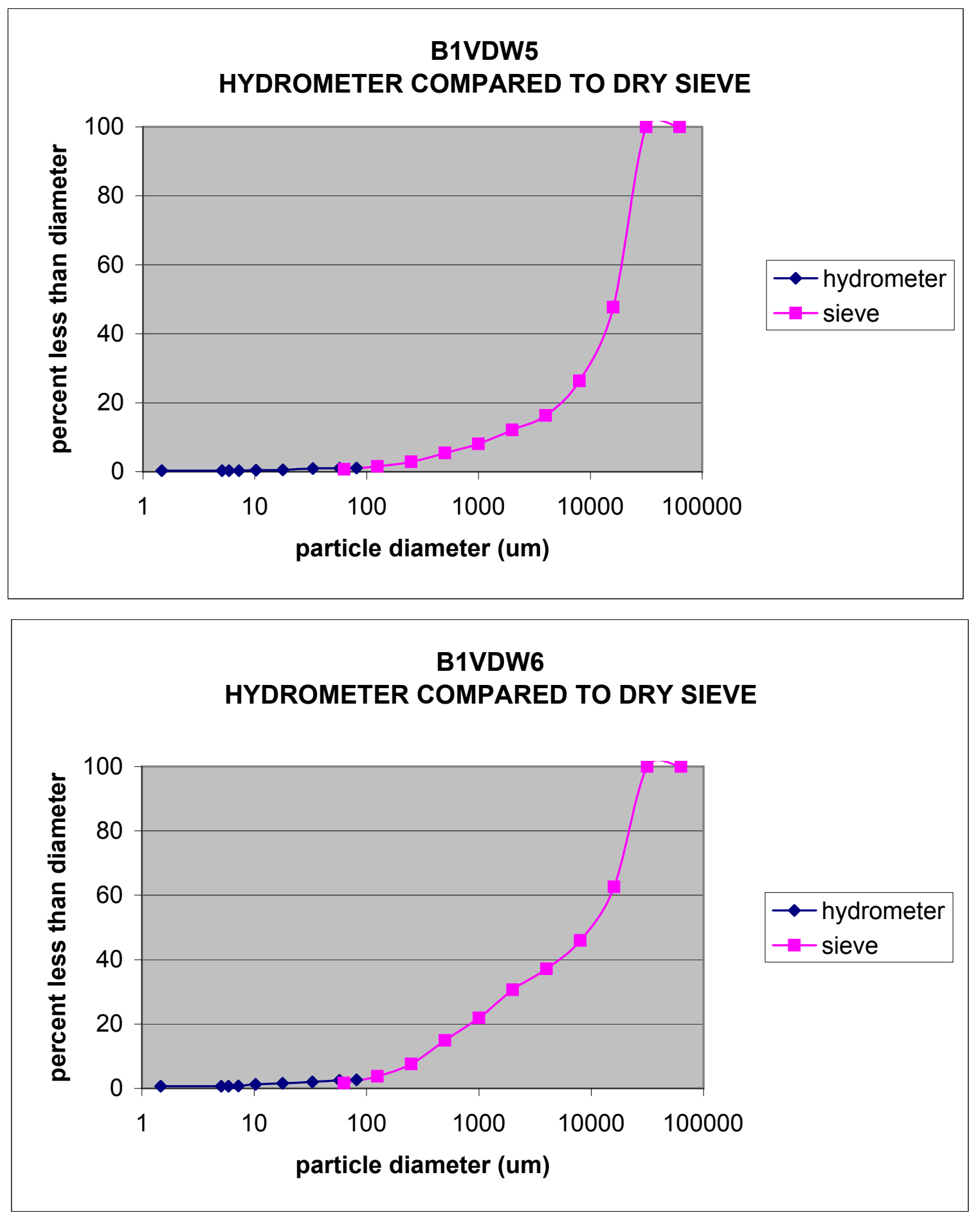

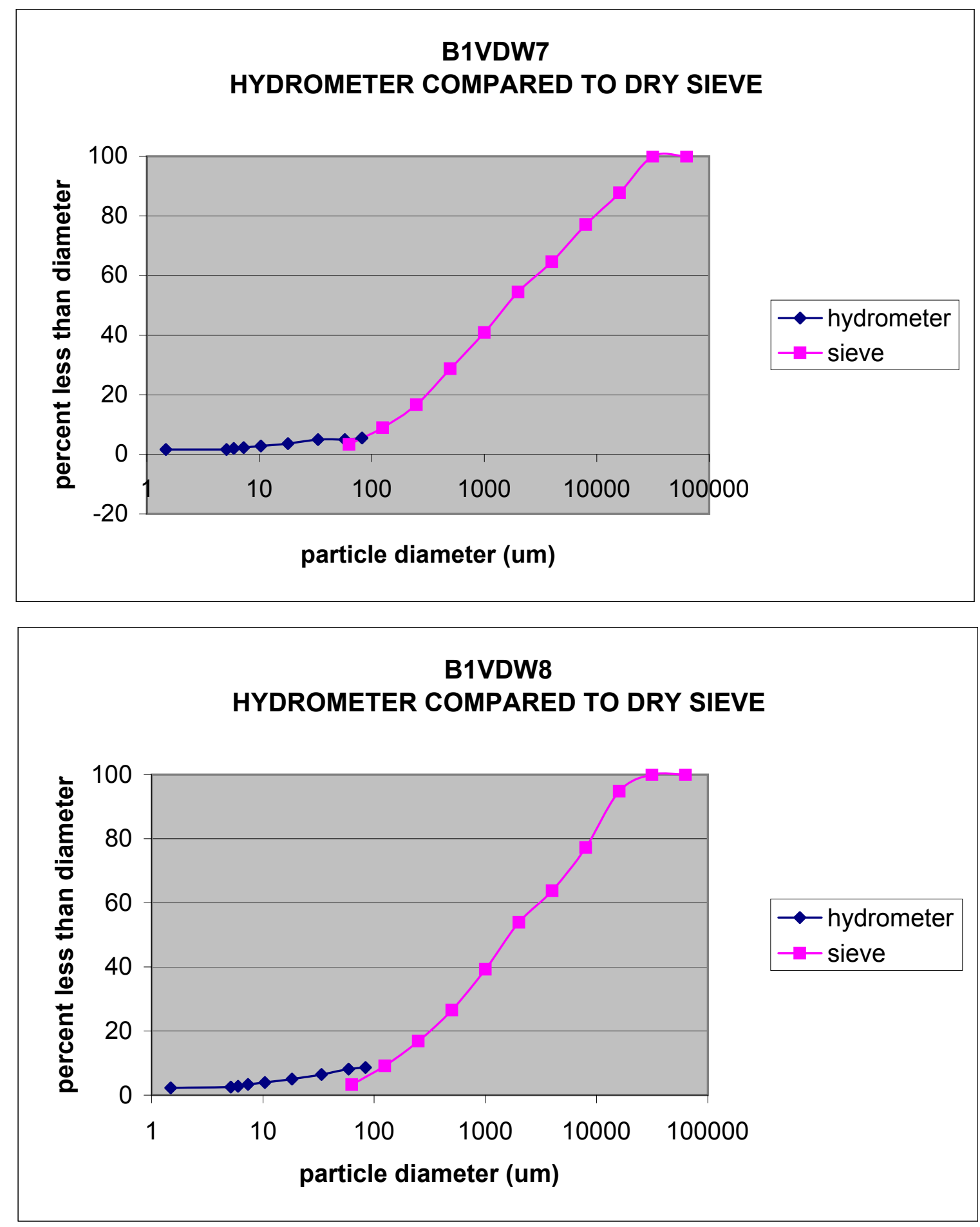

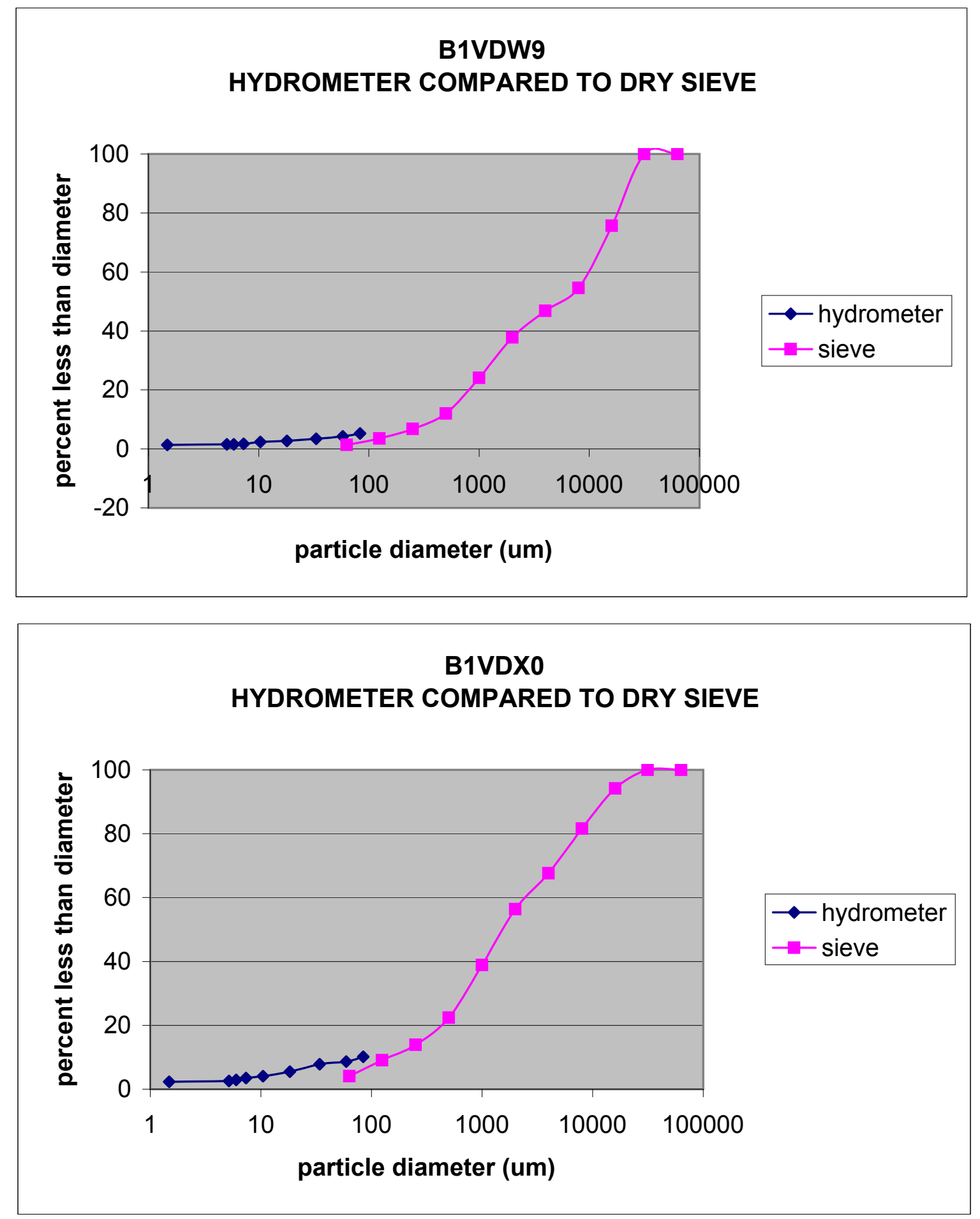

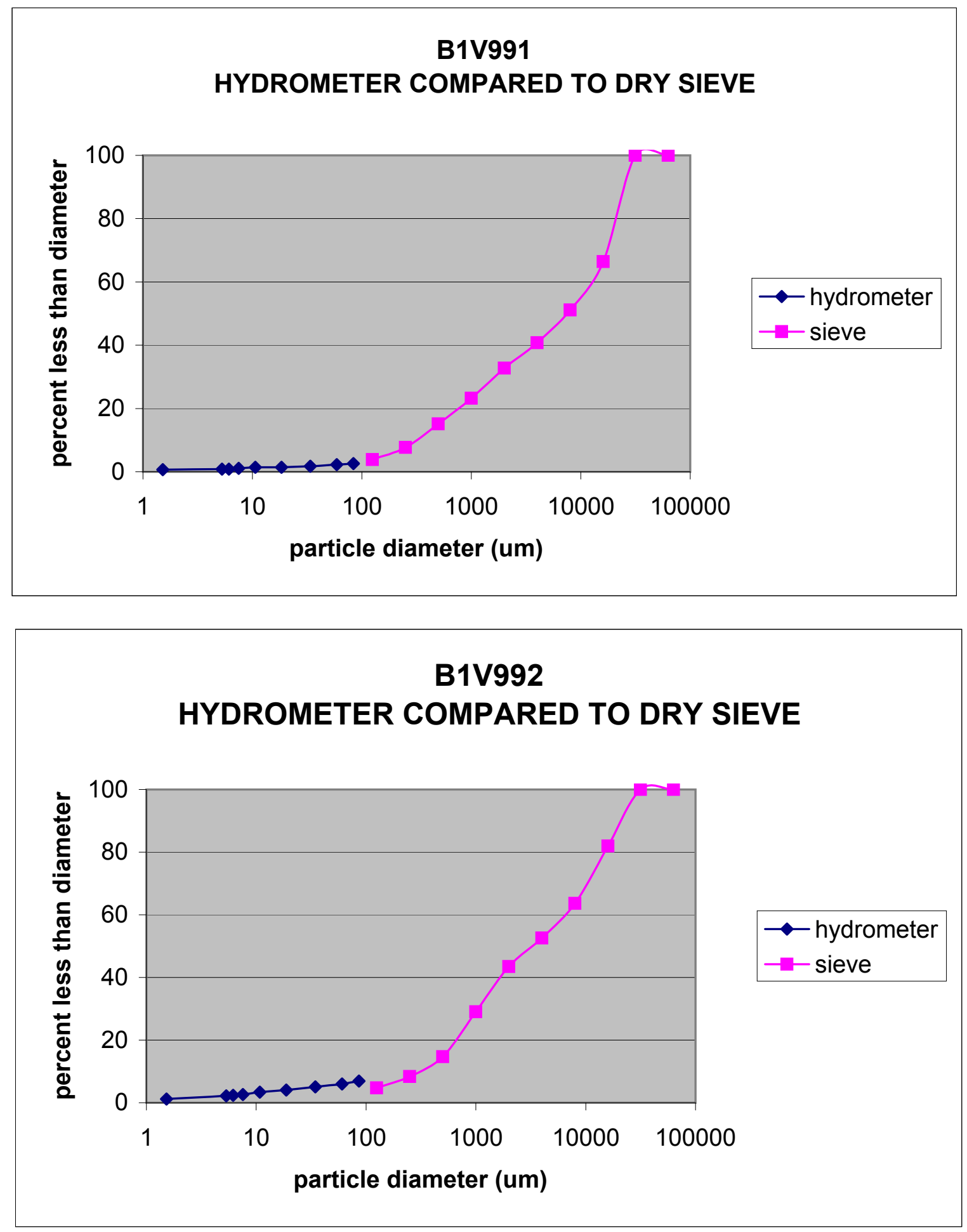

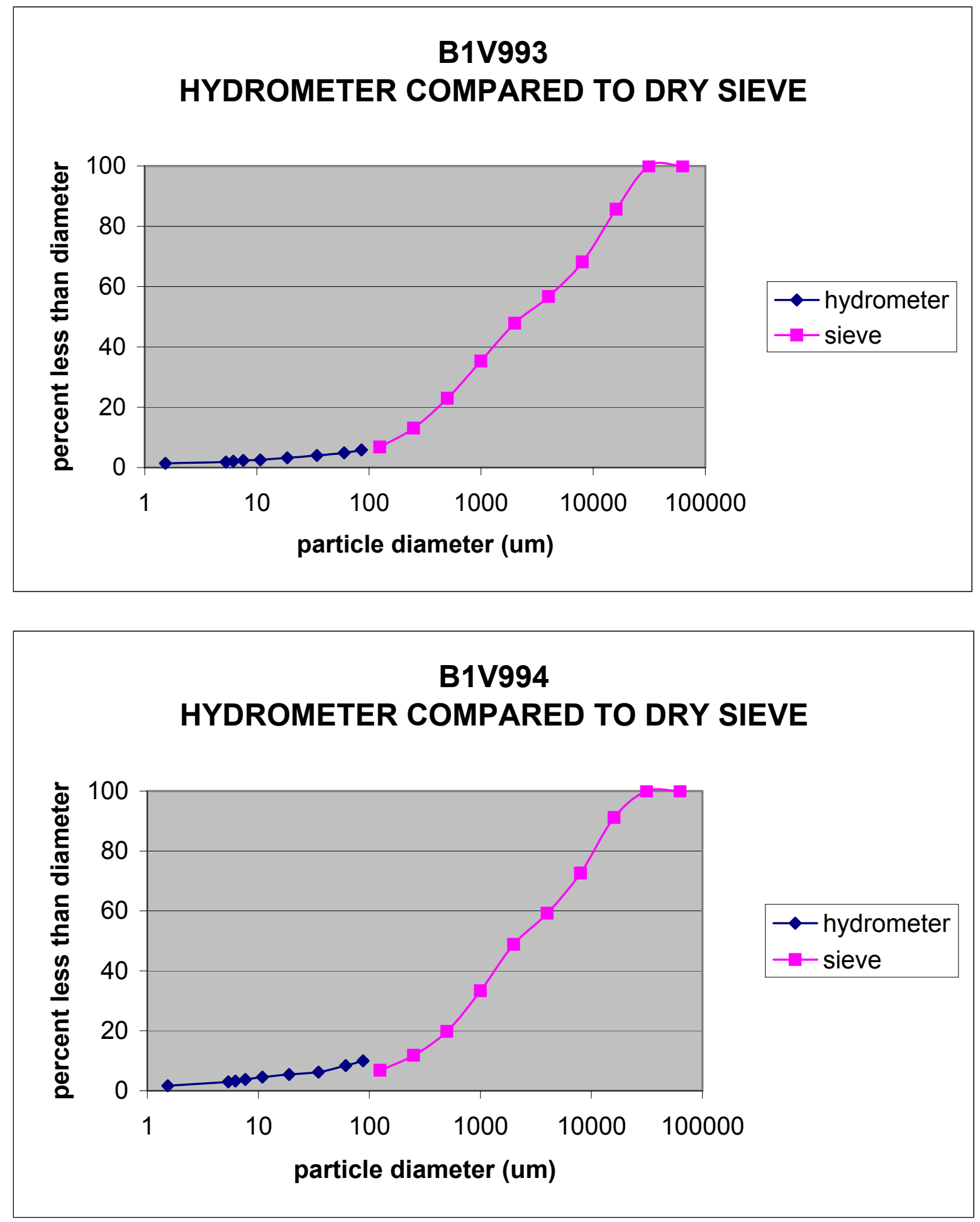

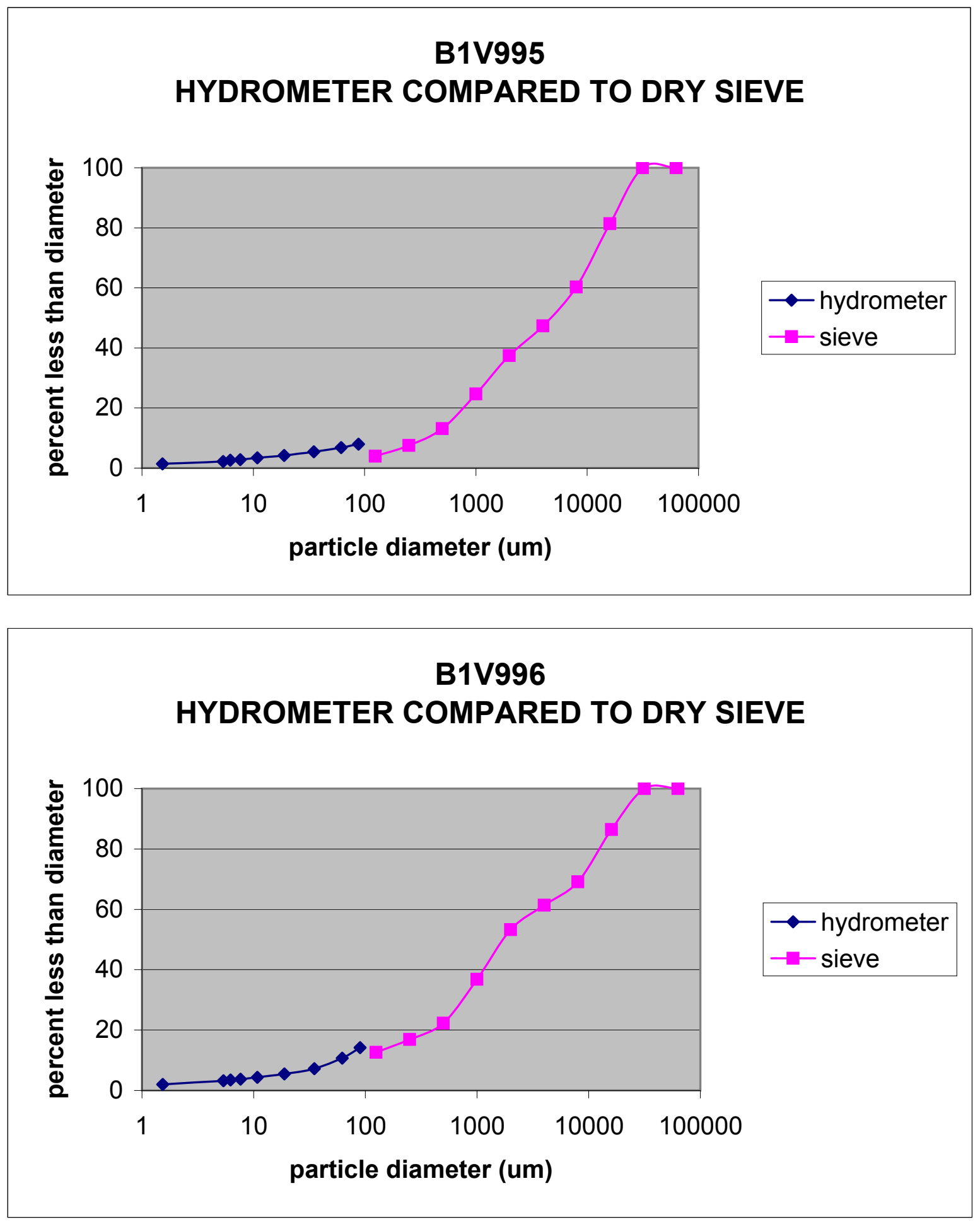

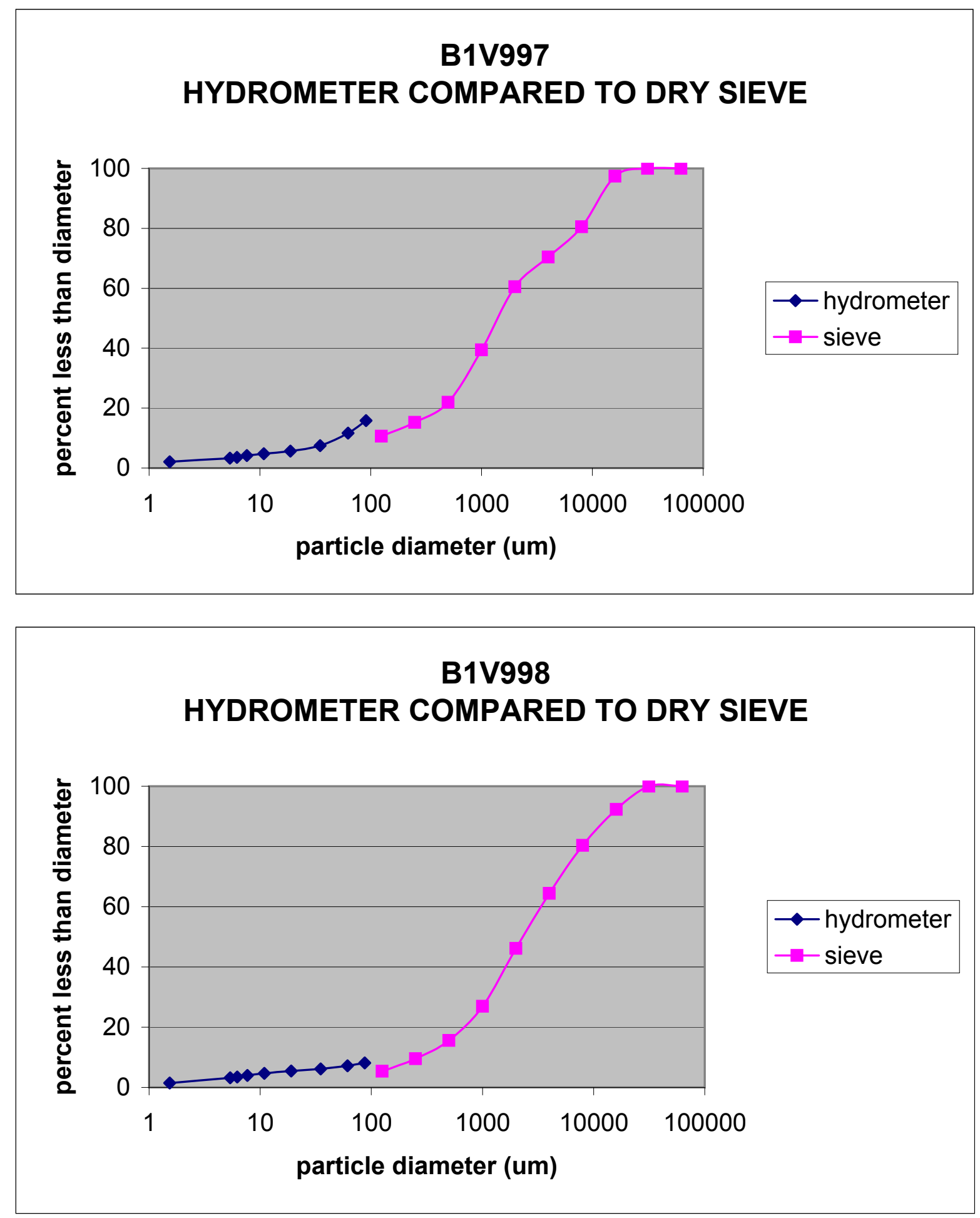

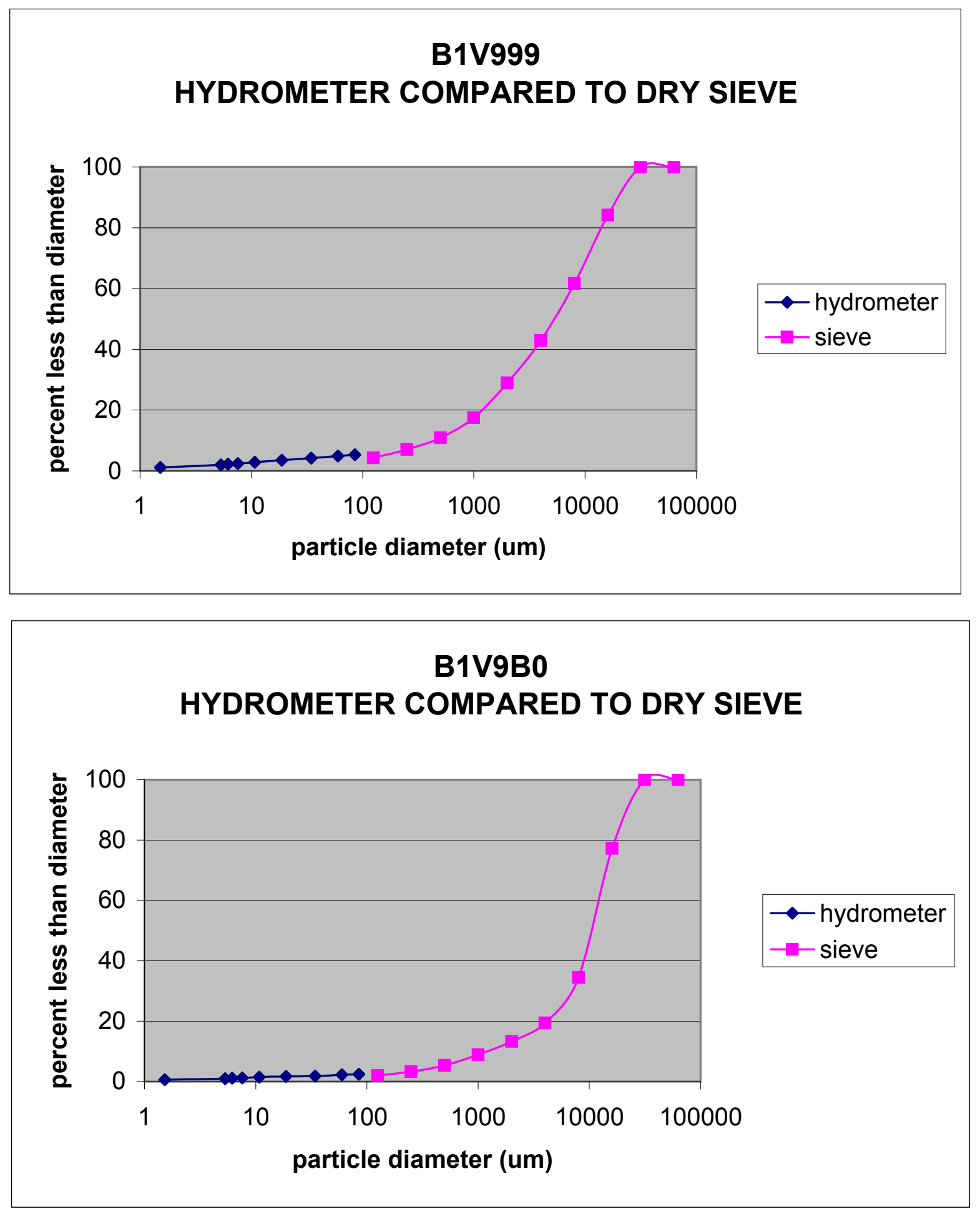


\section{B1V9B1 \\ HYDROMETER COMPARED TO DRY SIEVE}

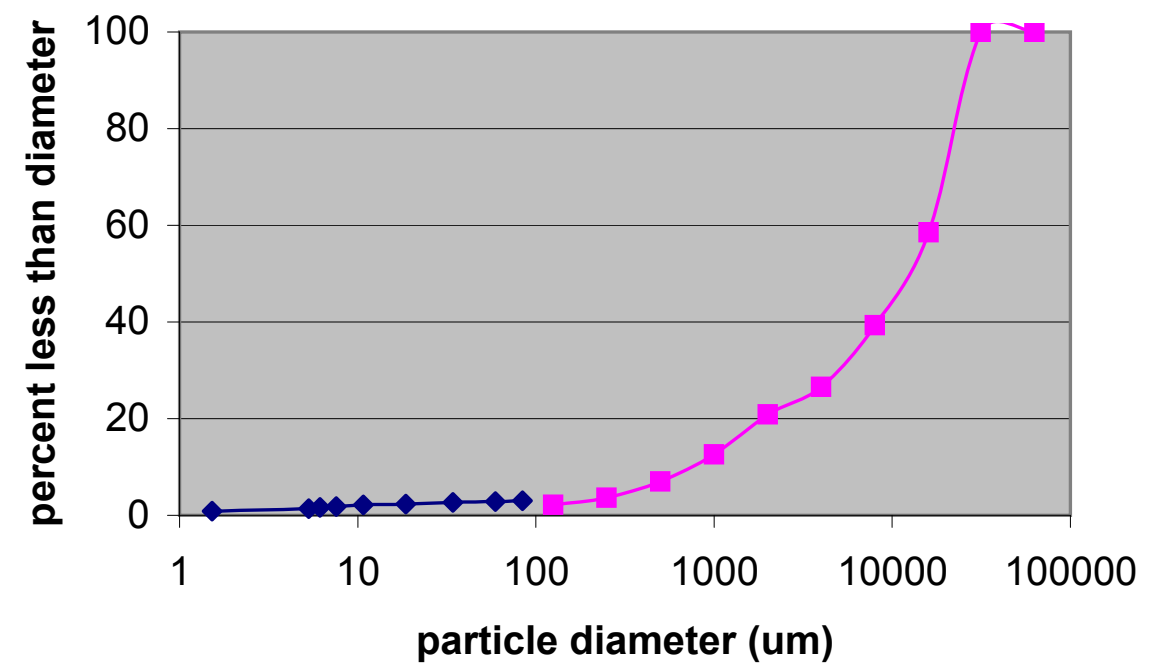

- hydrometer

- - sieve

\section{B1V9B2}

HYDROMETER COMPARED TO DRY SIEVE

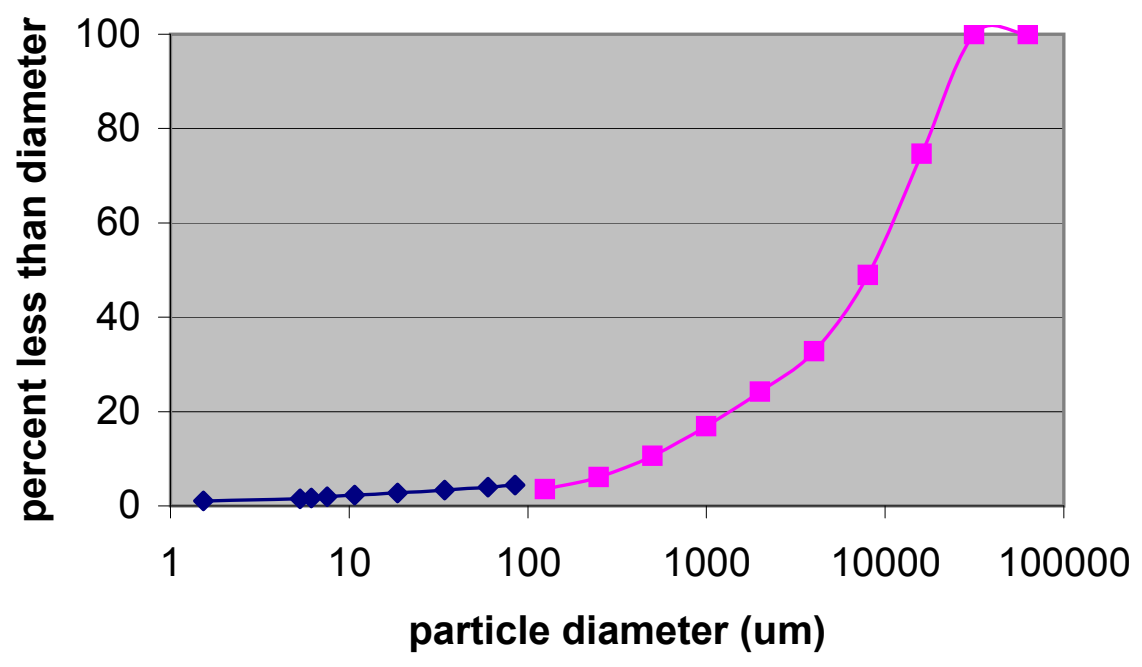

$\multimap$ hydrometer

$\rightarrow-$ sieve 

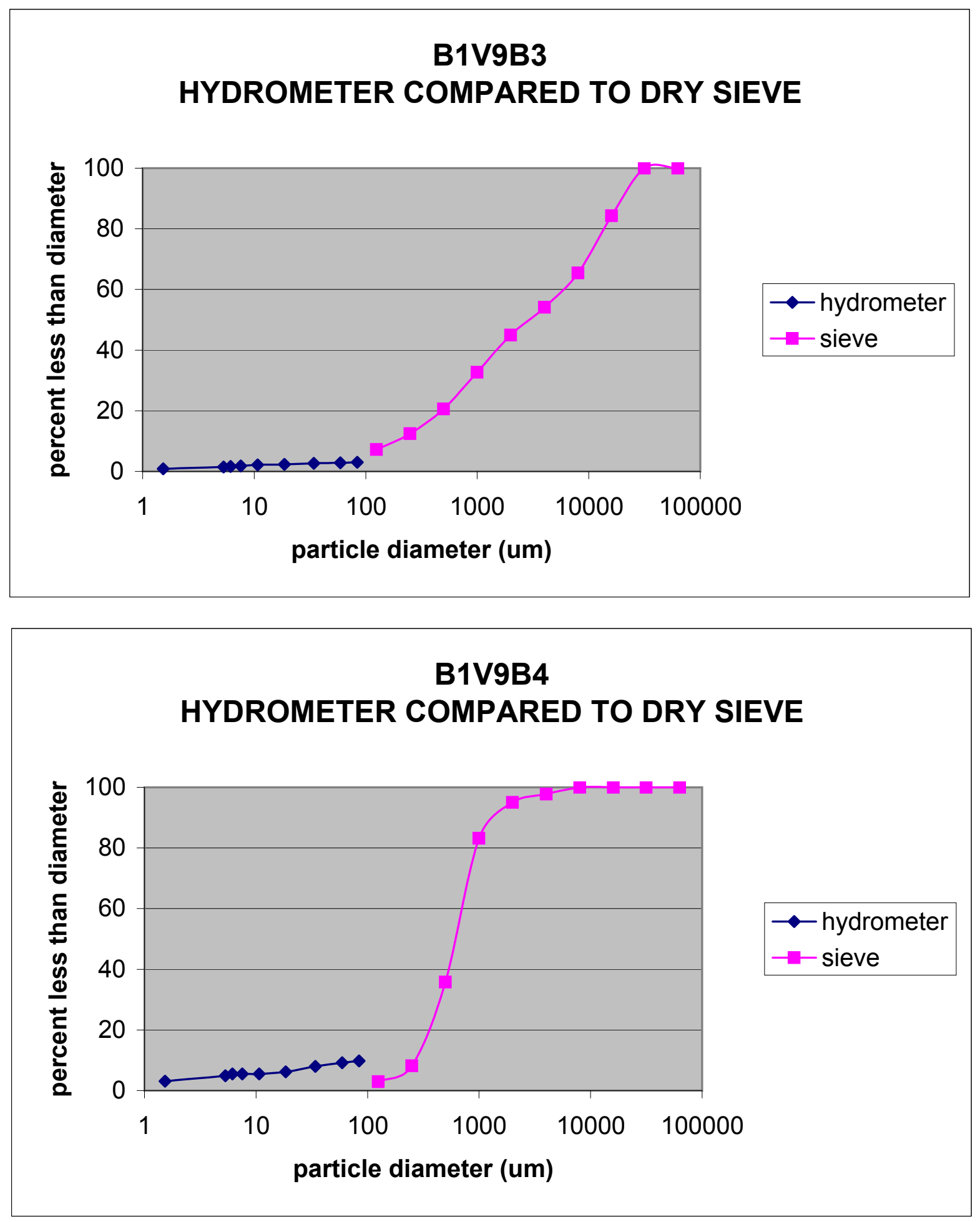

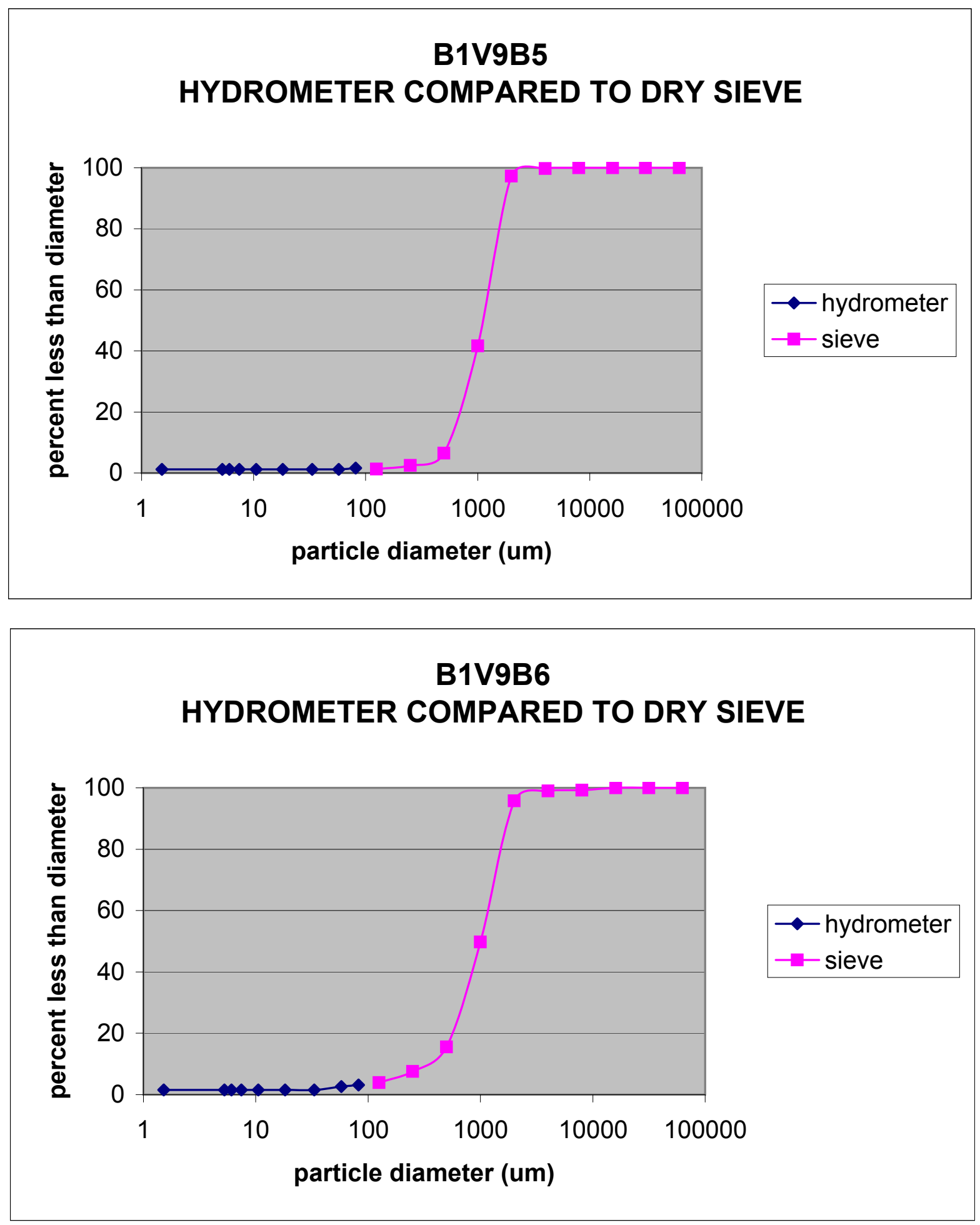

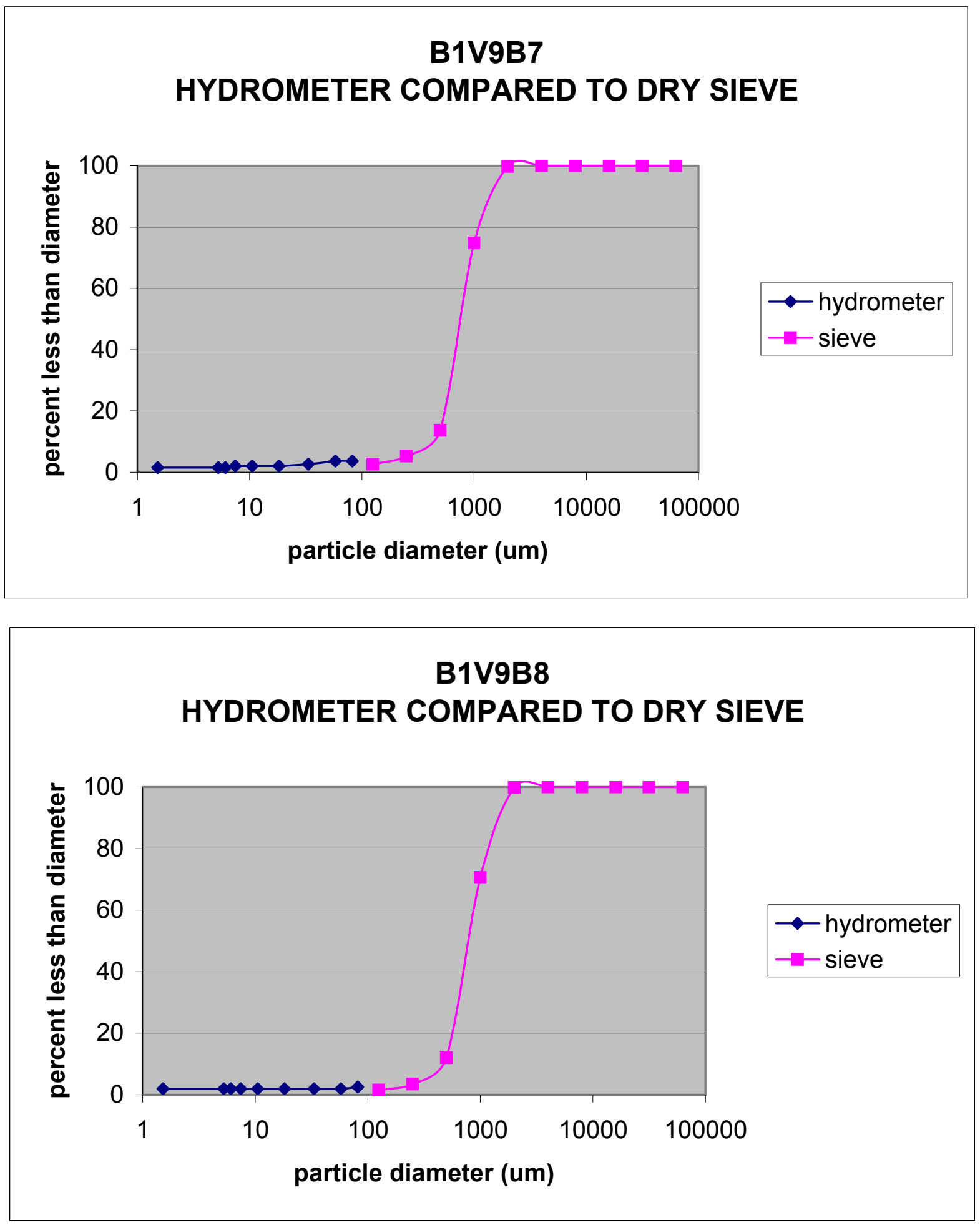

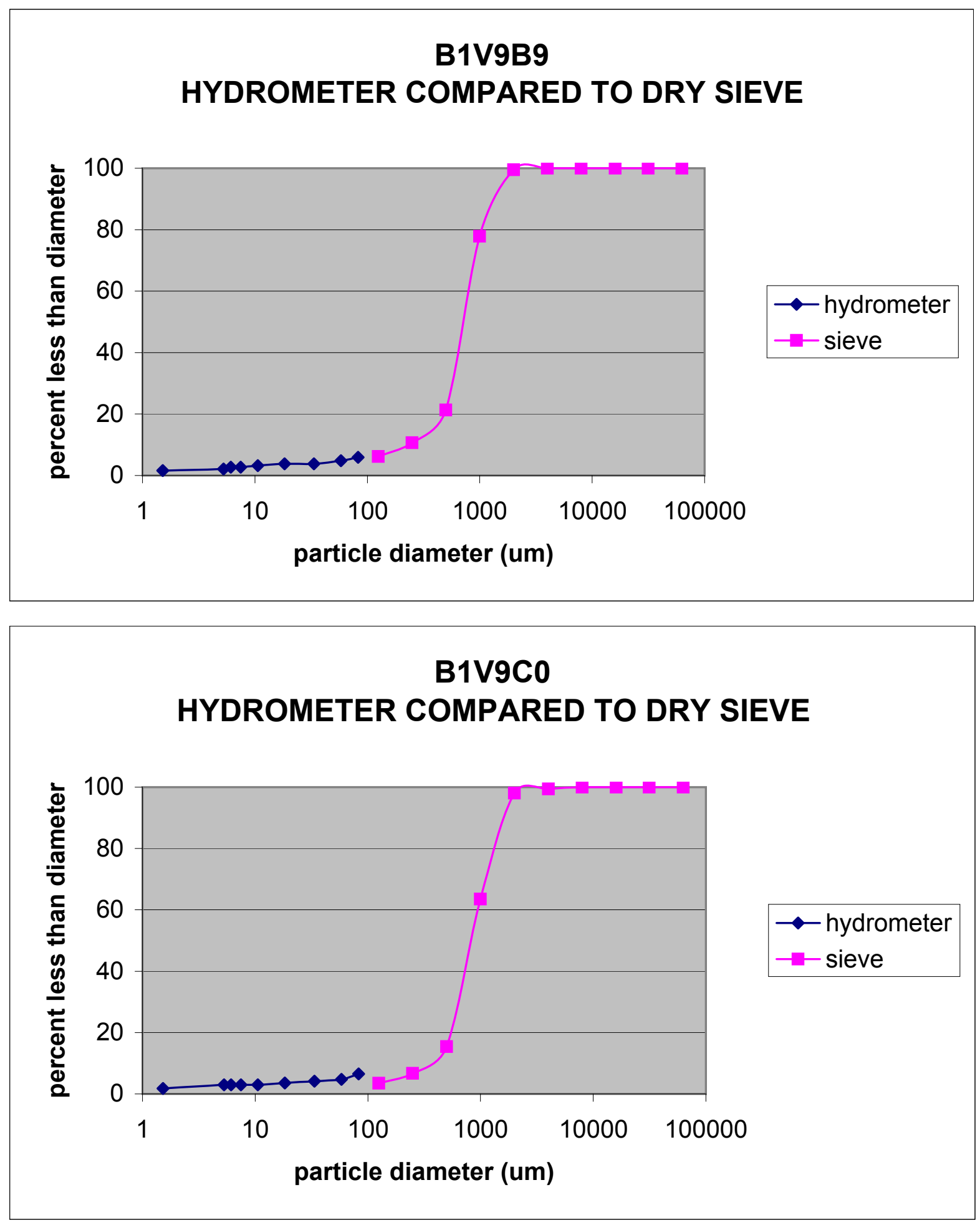

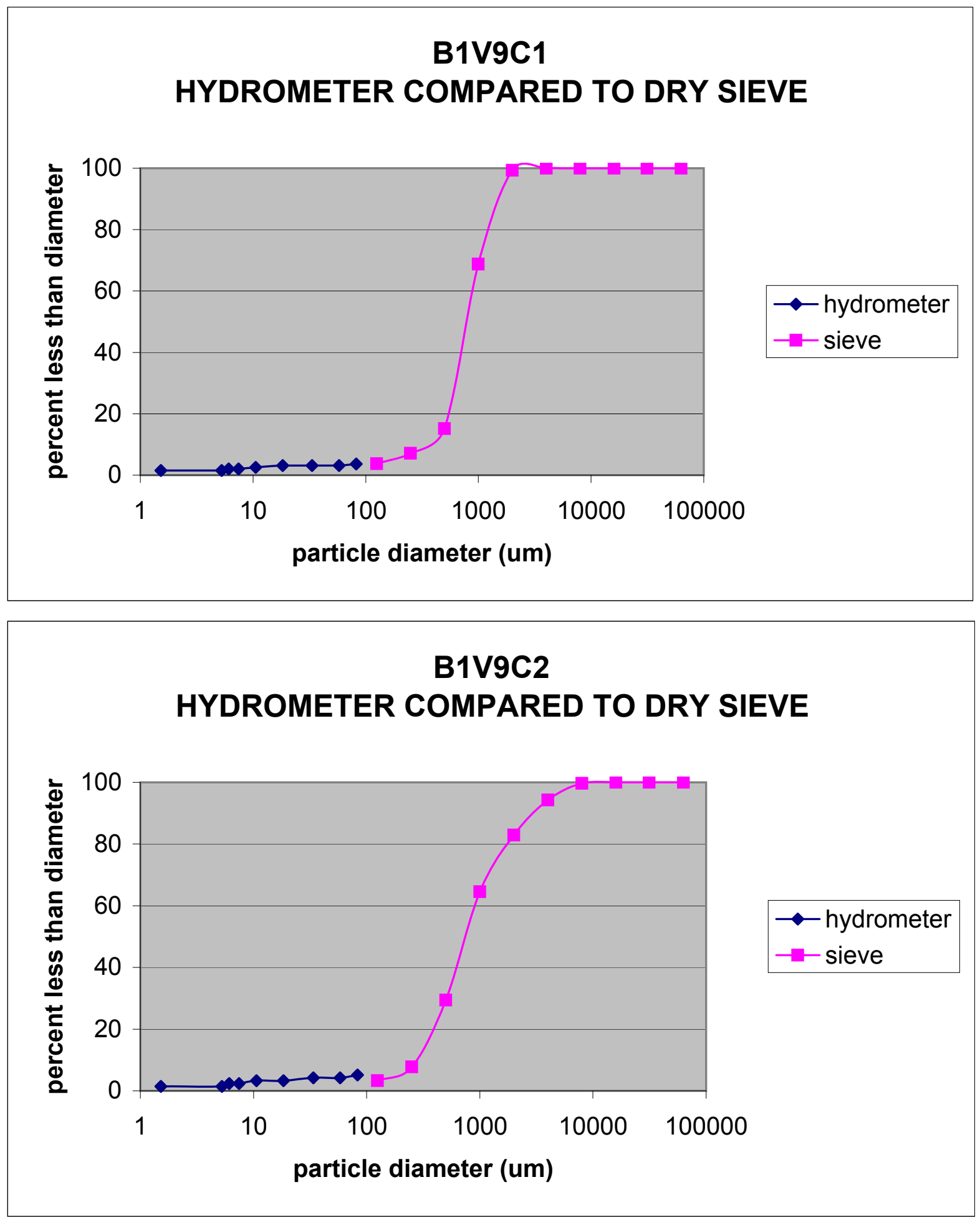

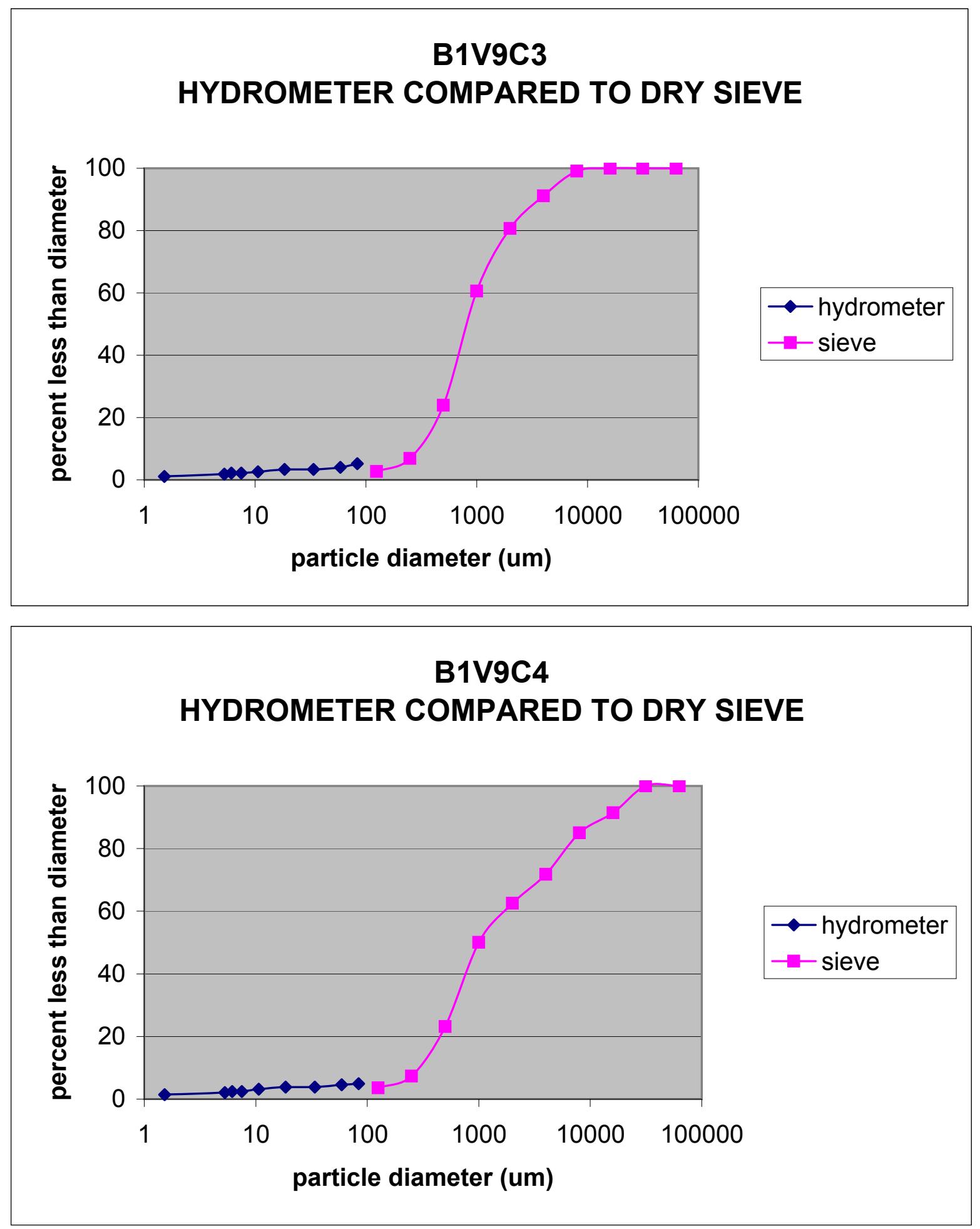


\section{B1V2V5 \\ HYDROMETER COMPARED TO DRY SIEVE}

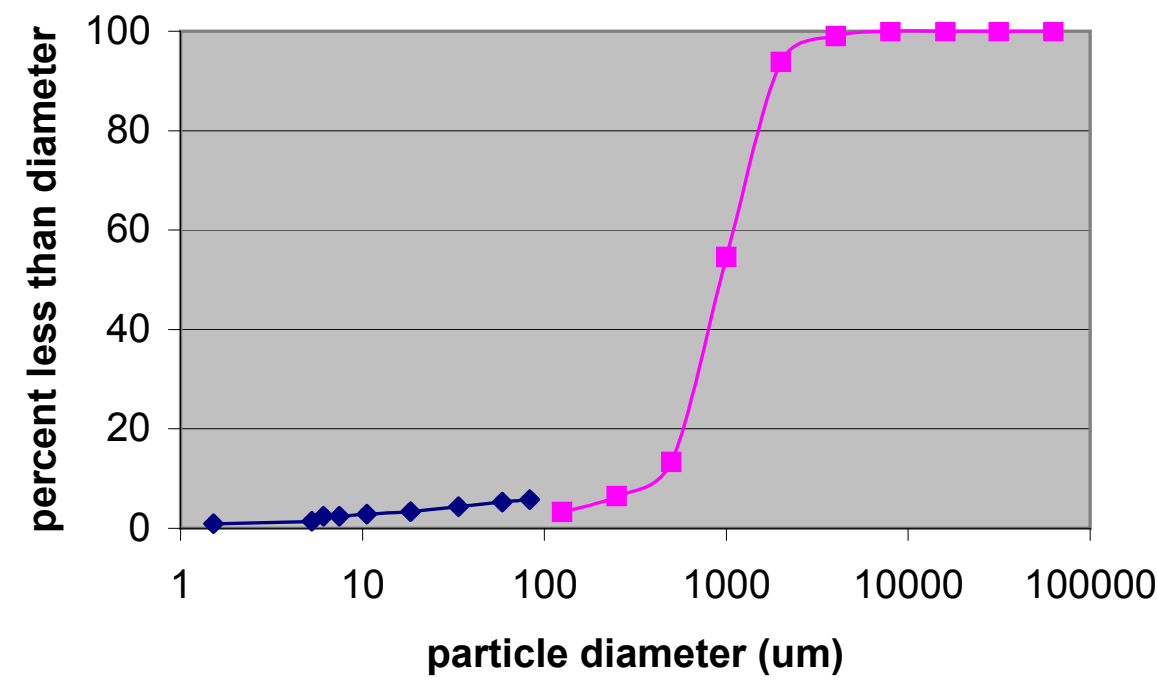

$\multimap$ hydrometer

- - sieve 


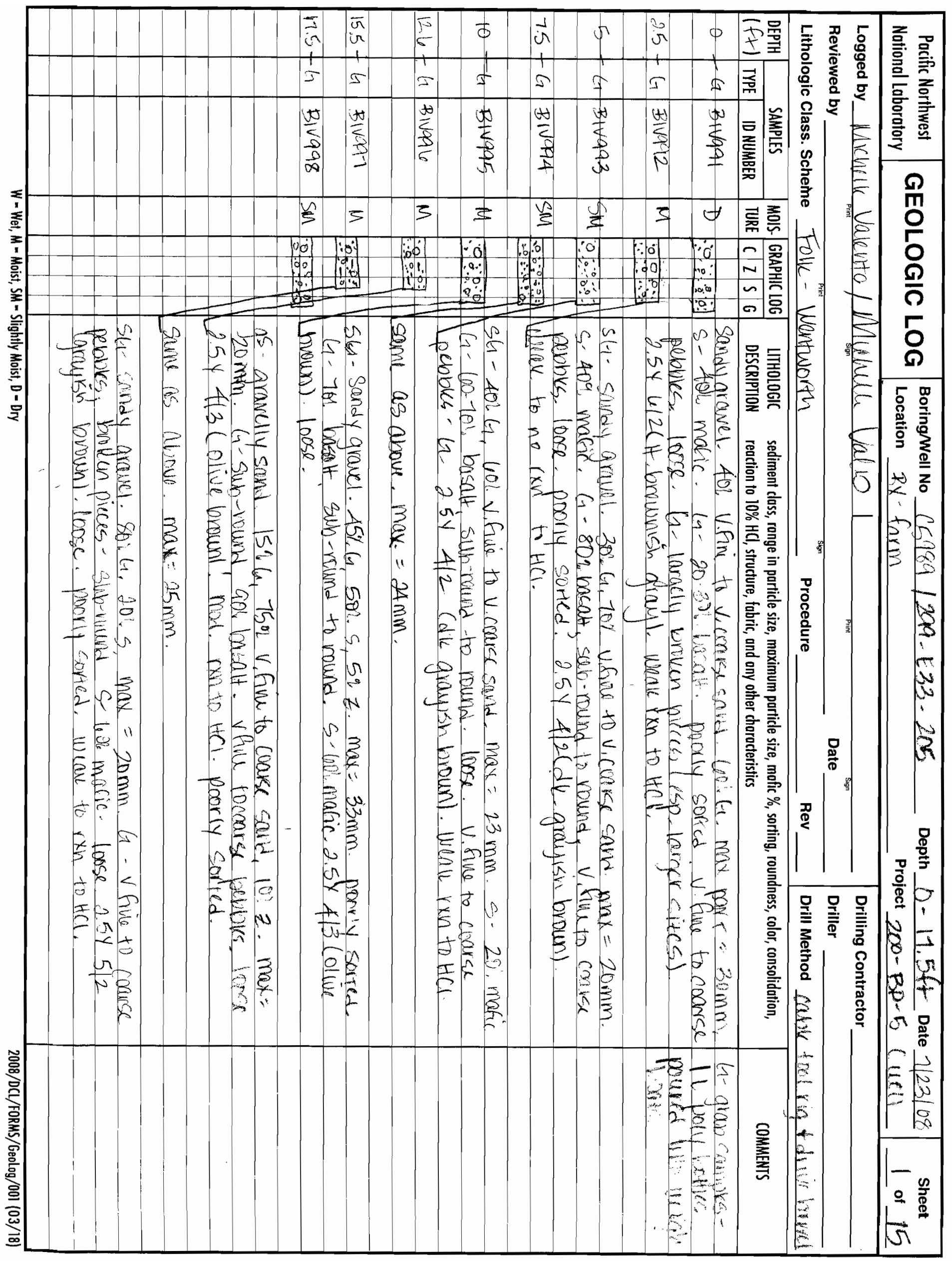




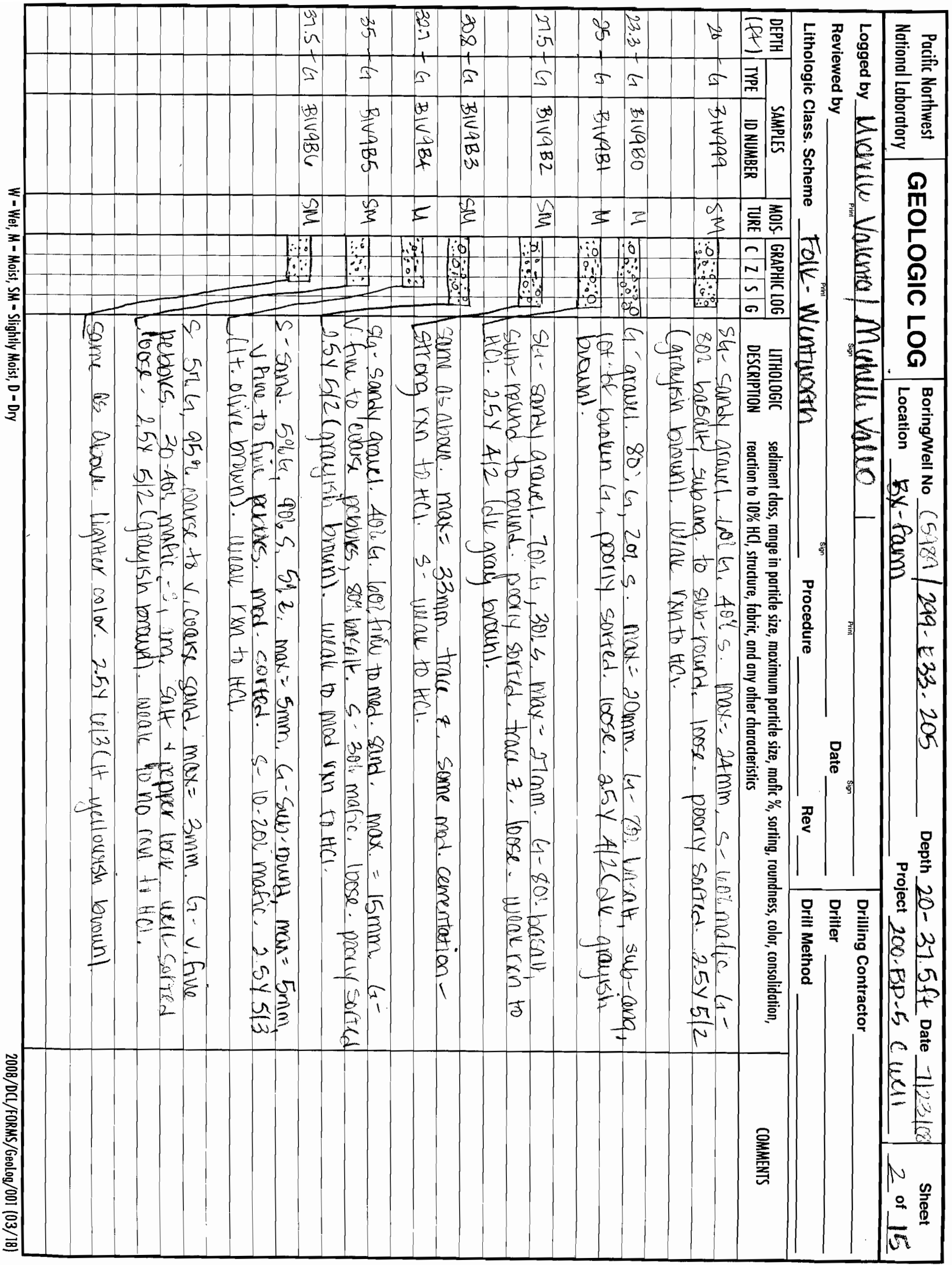




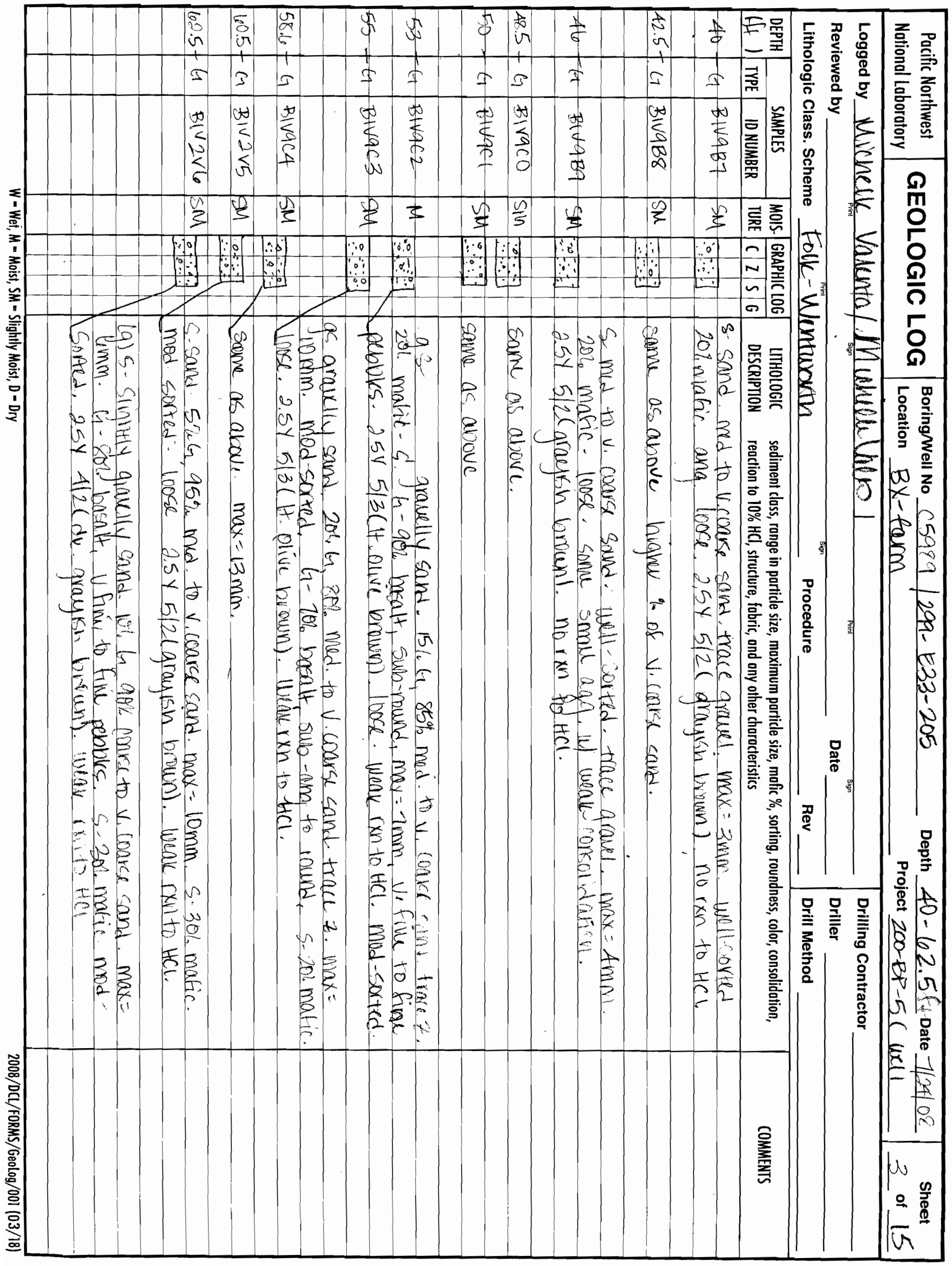




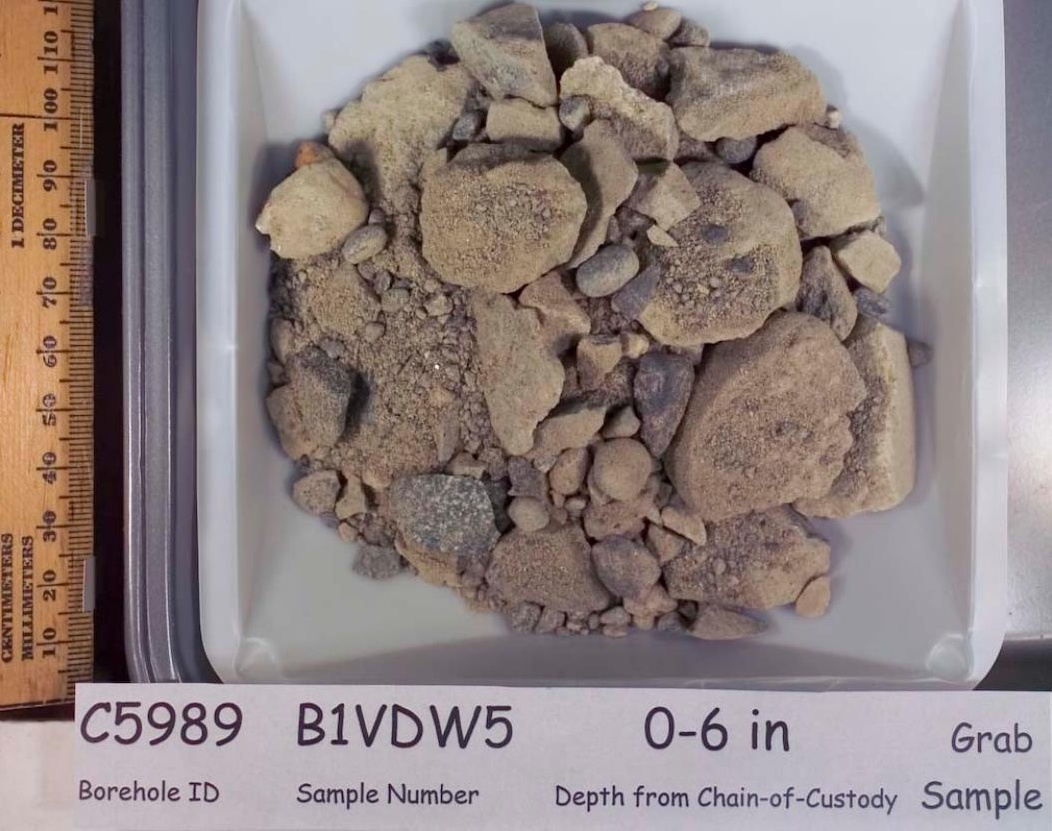




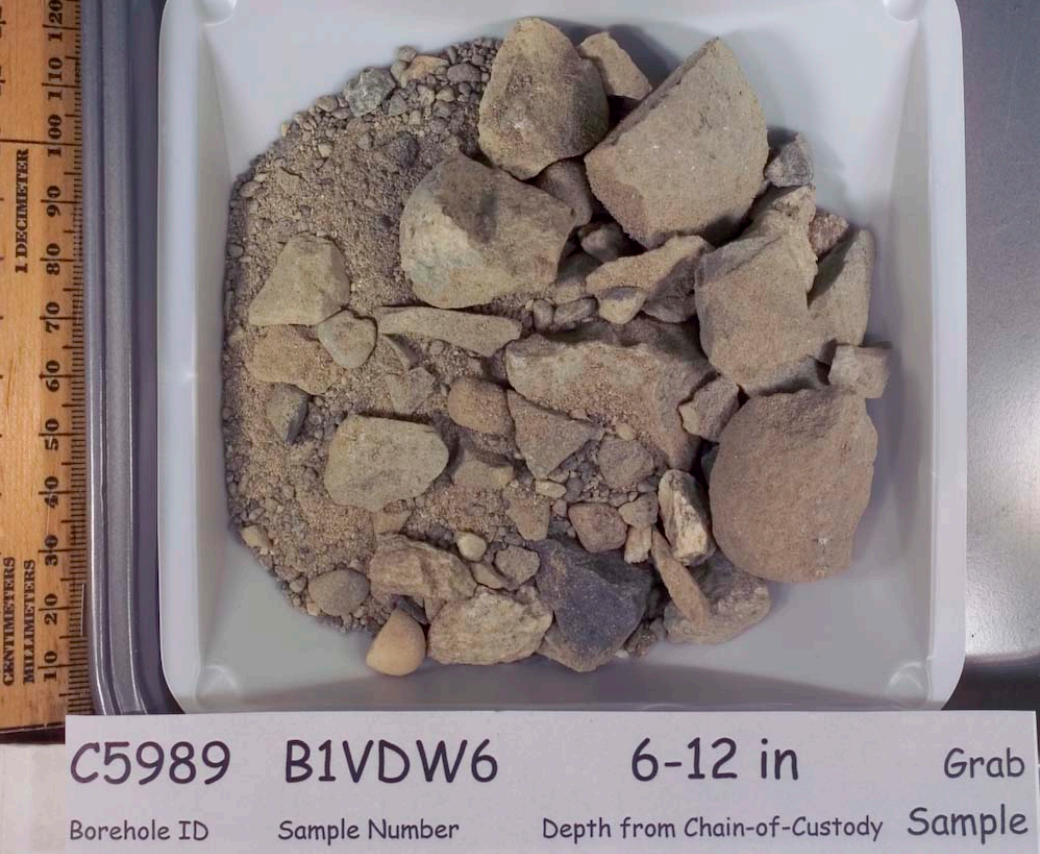




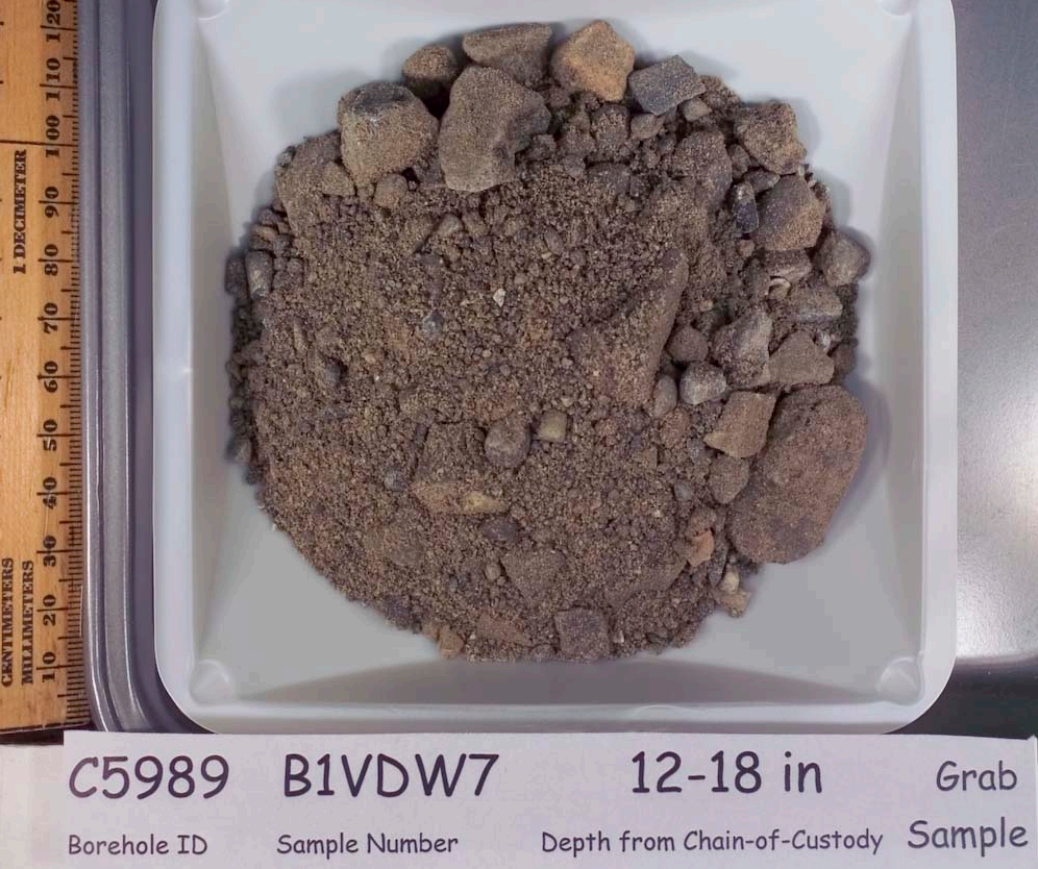




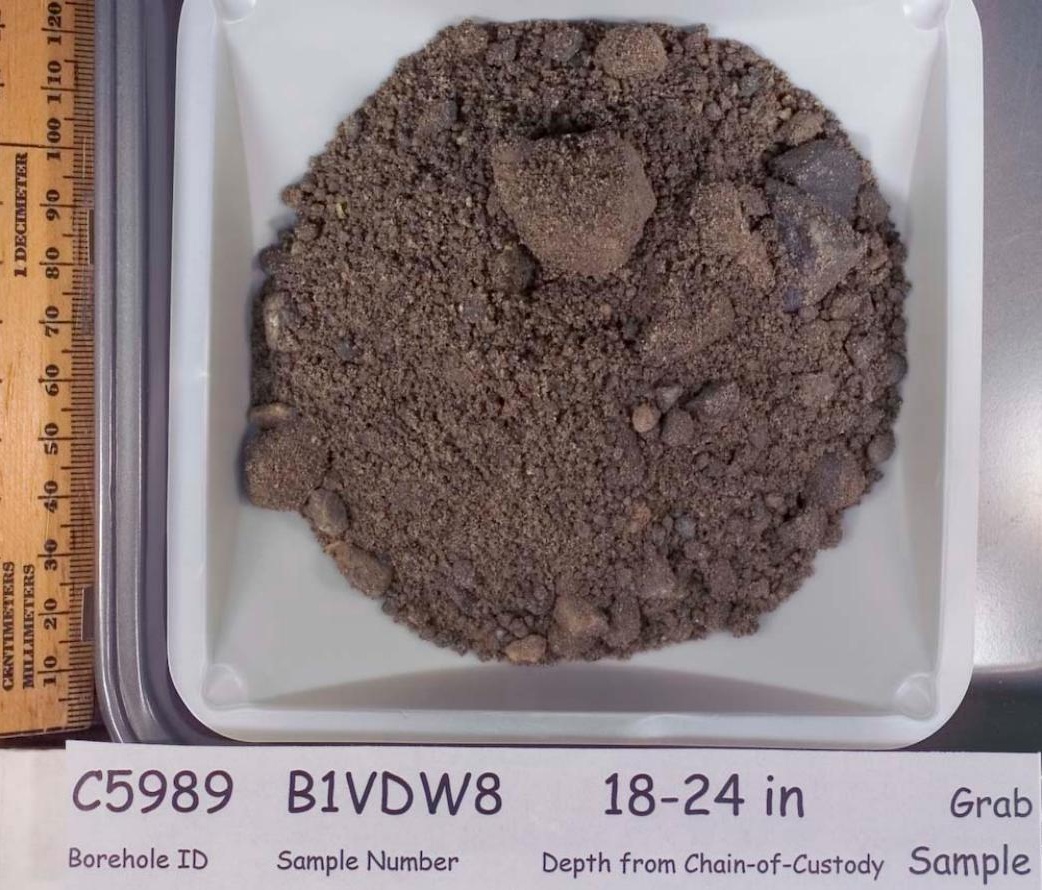




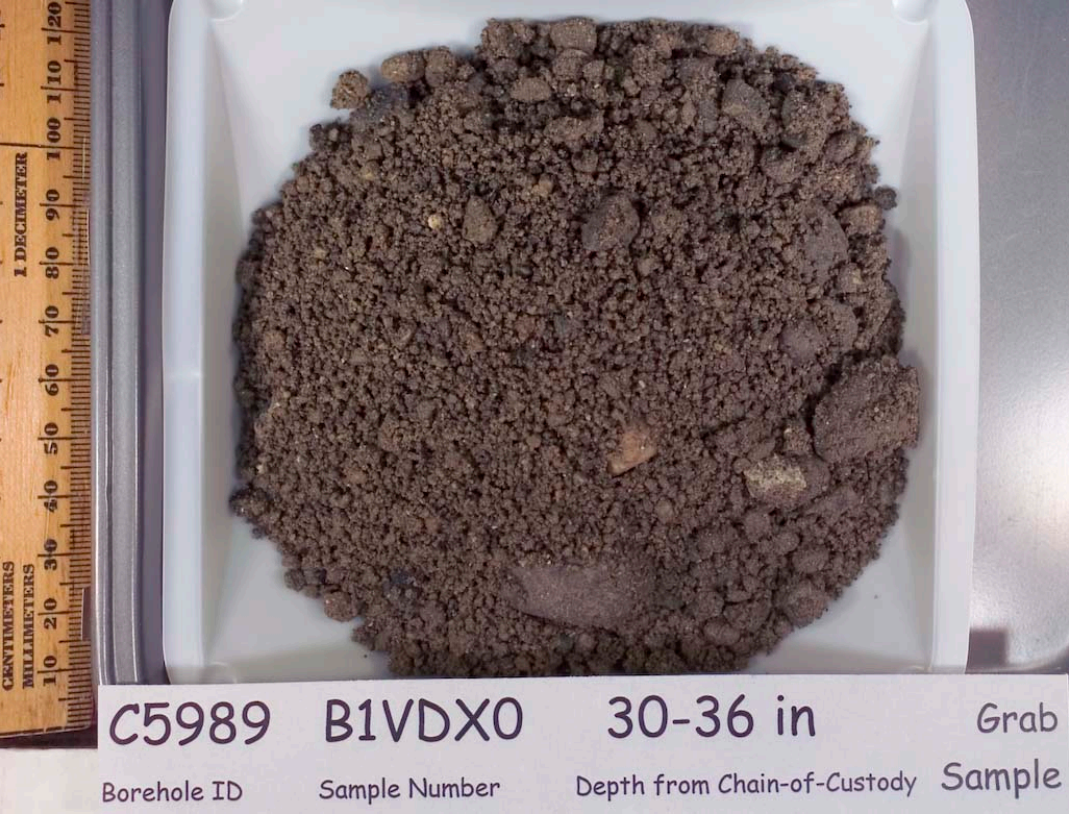




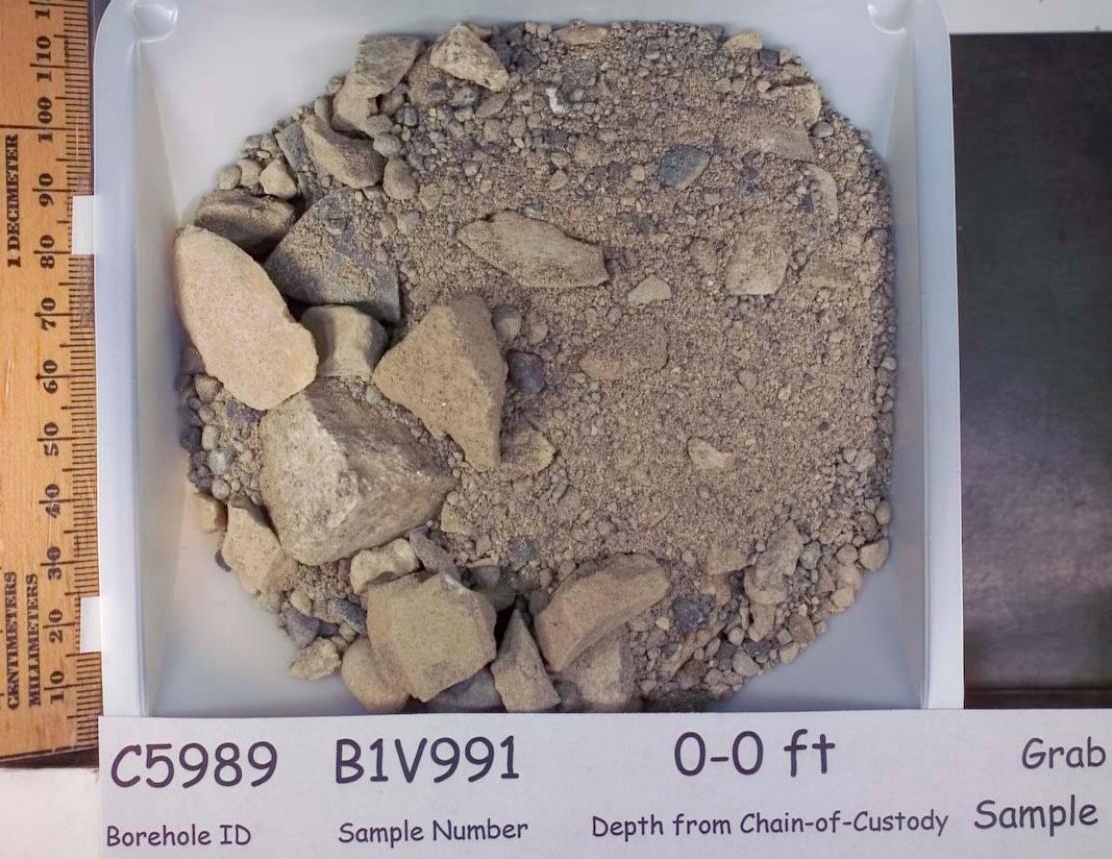




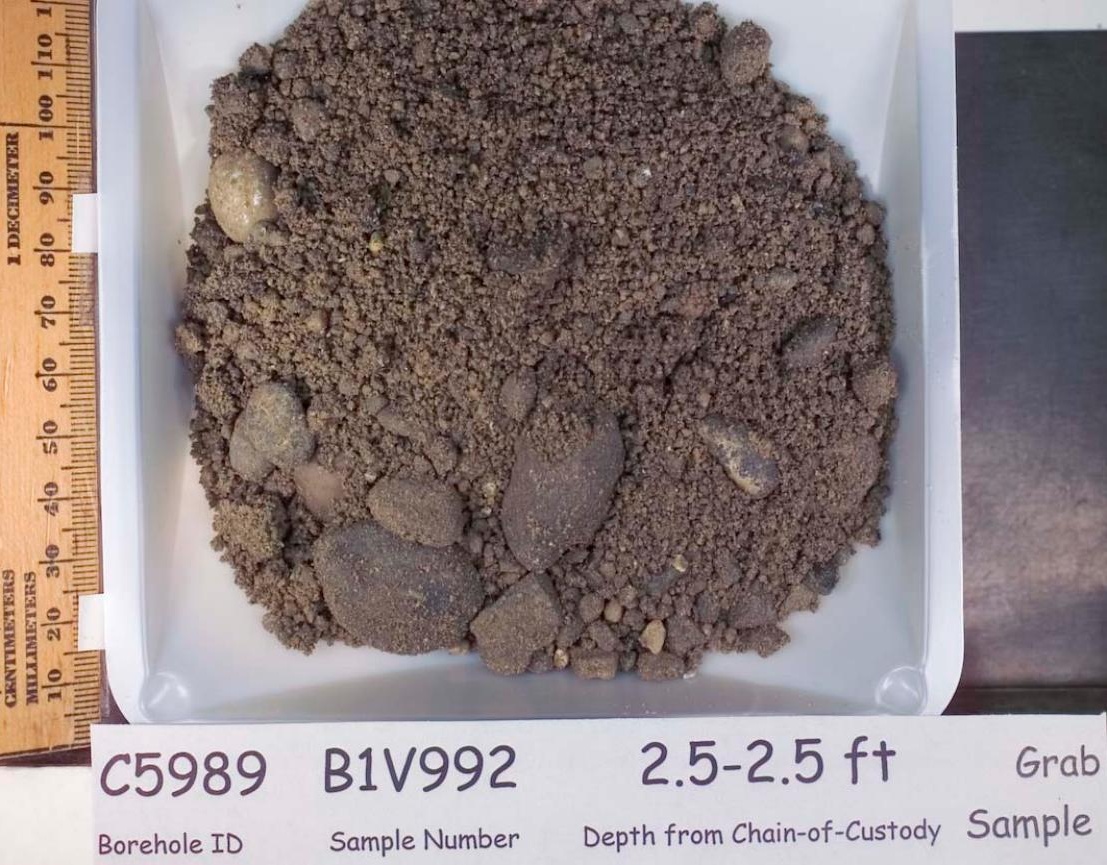




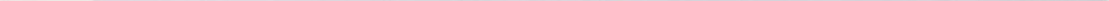




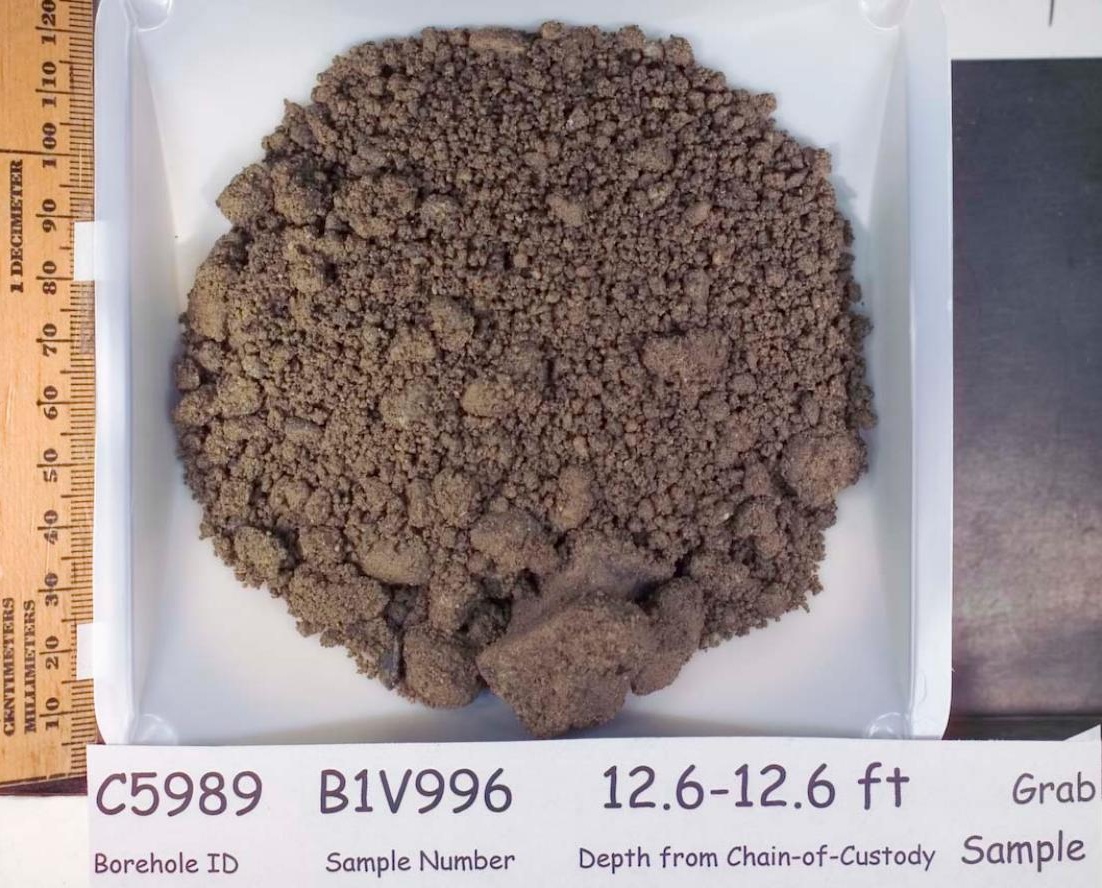




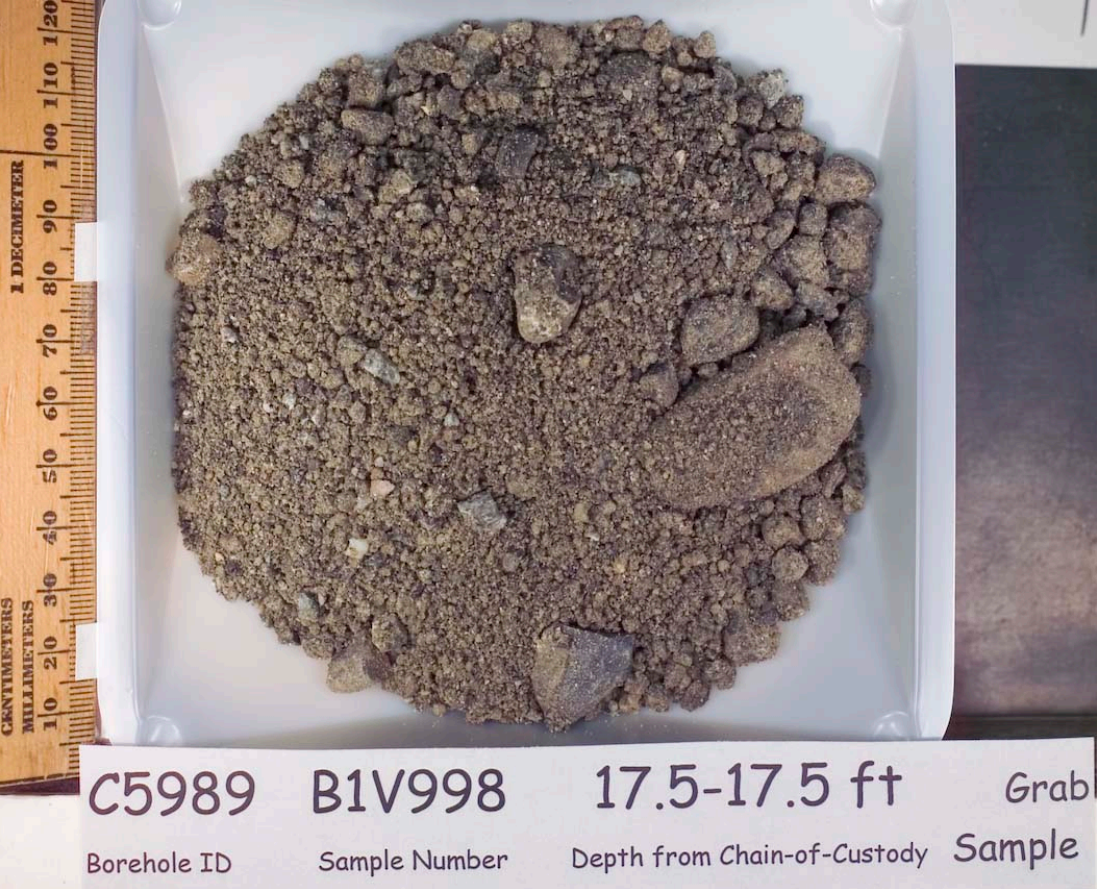




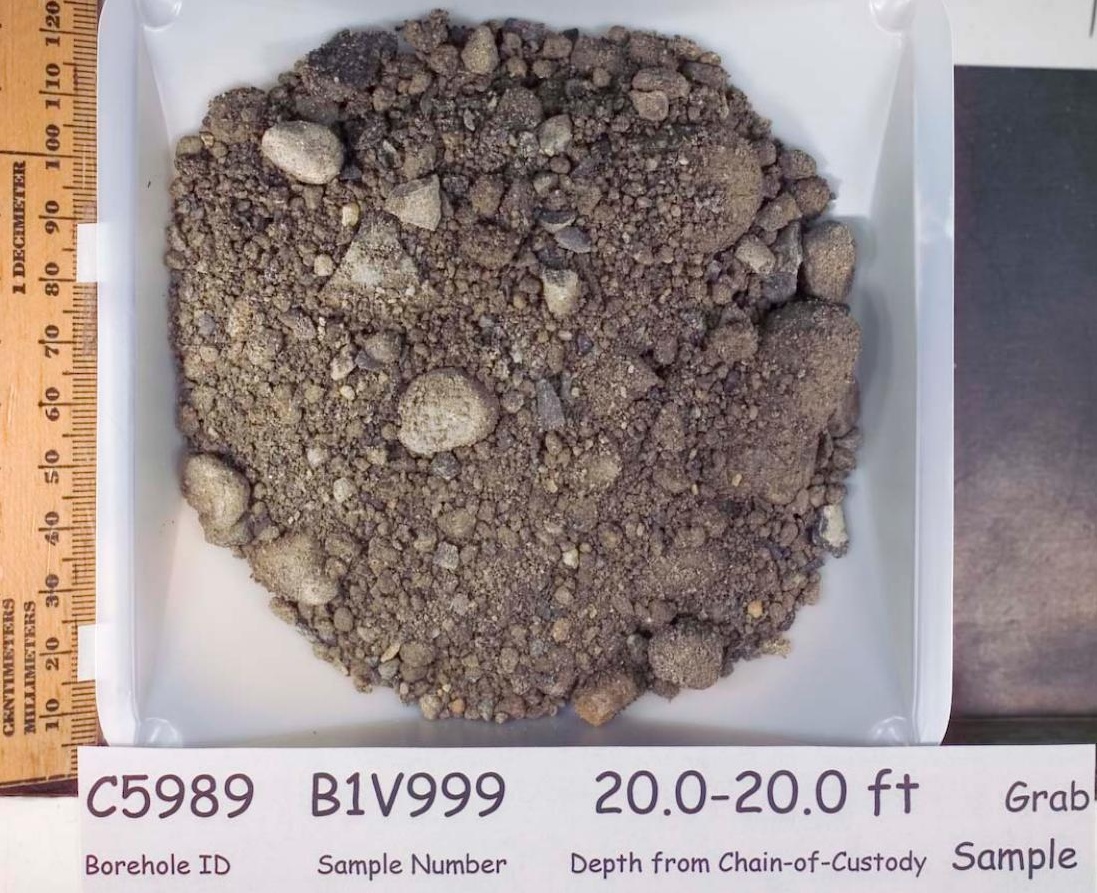




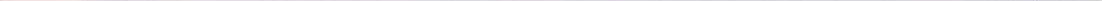




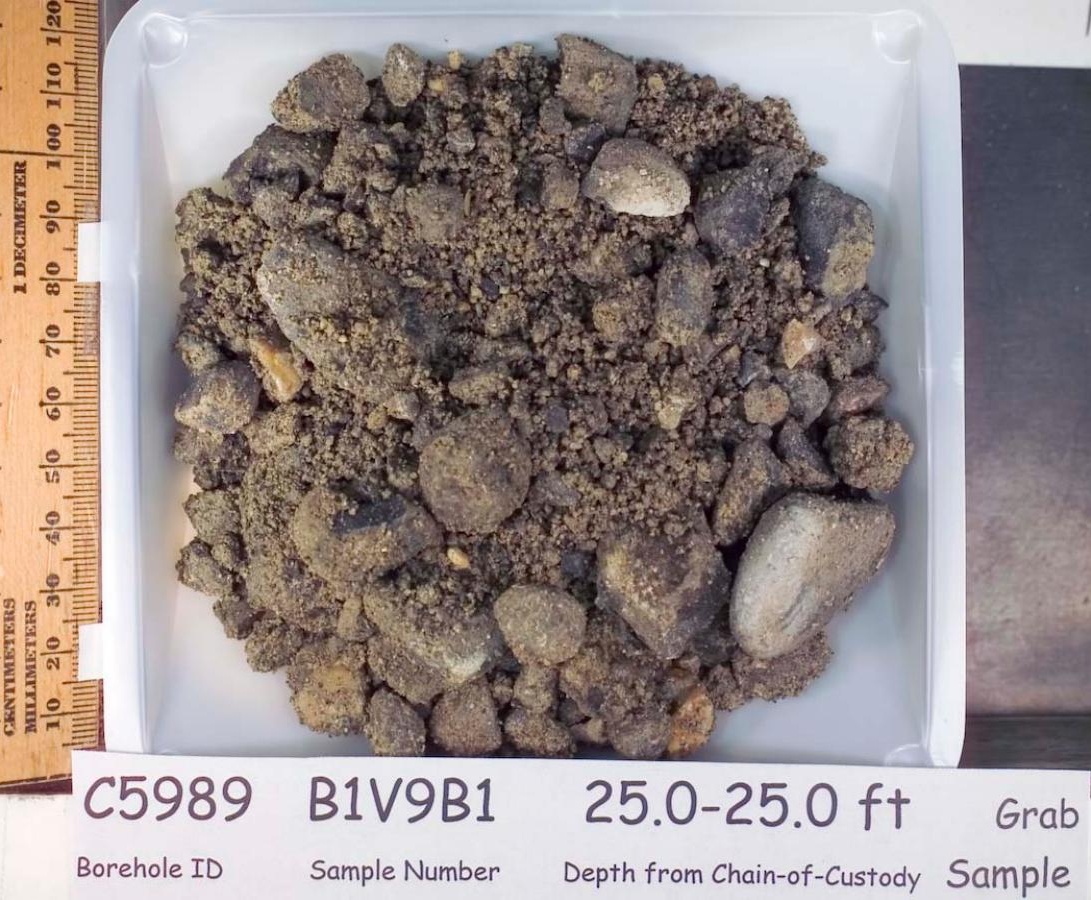




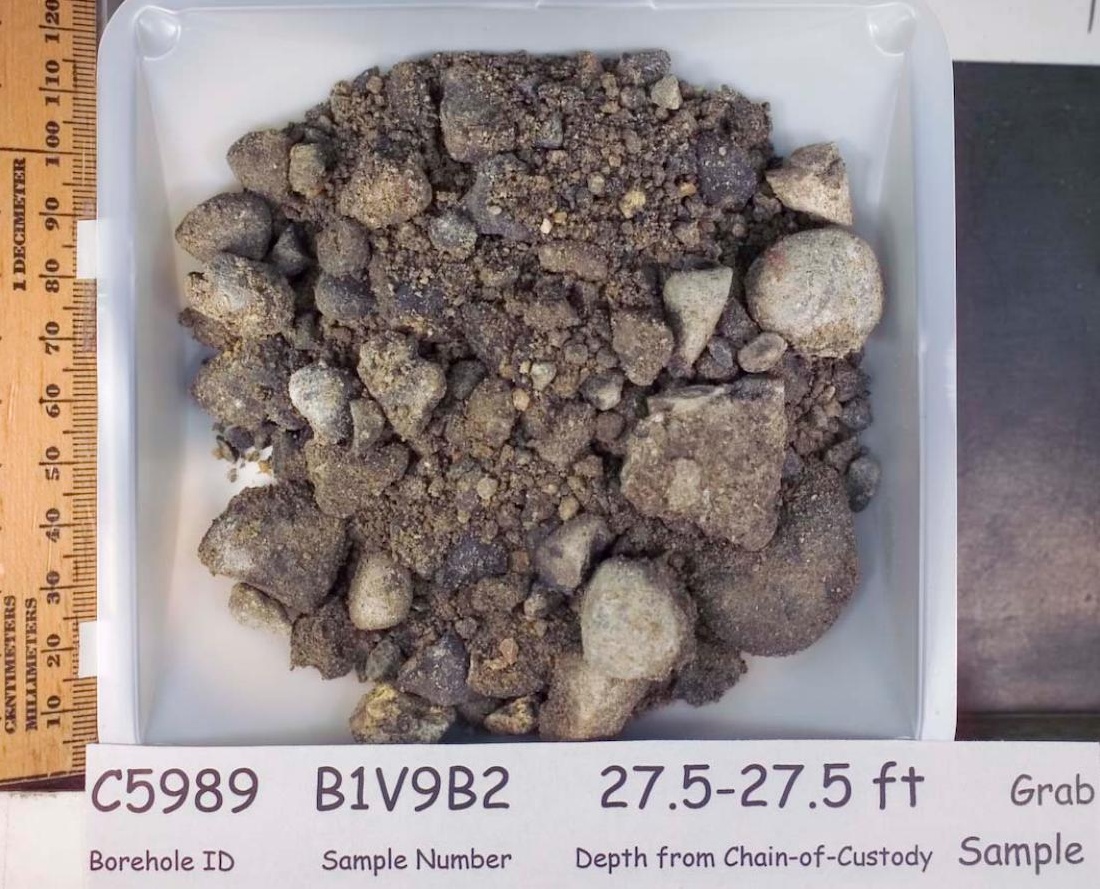




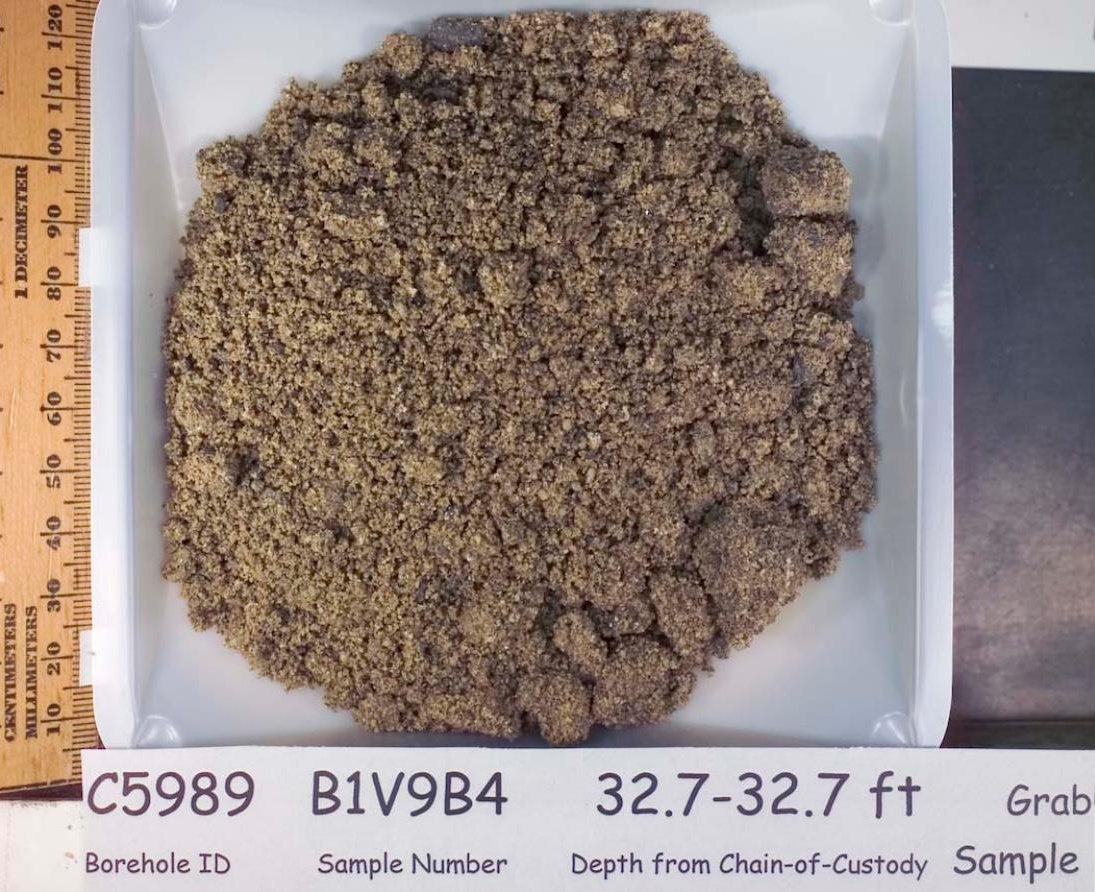




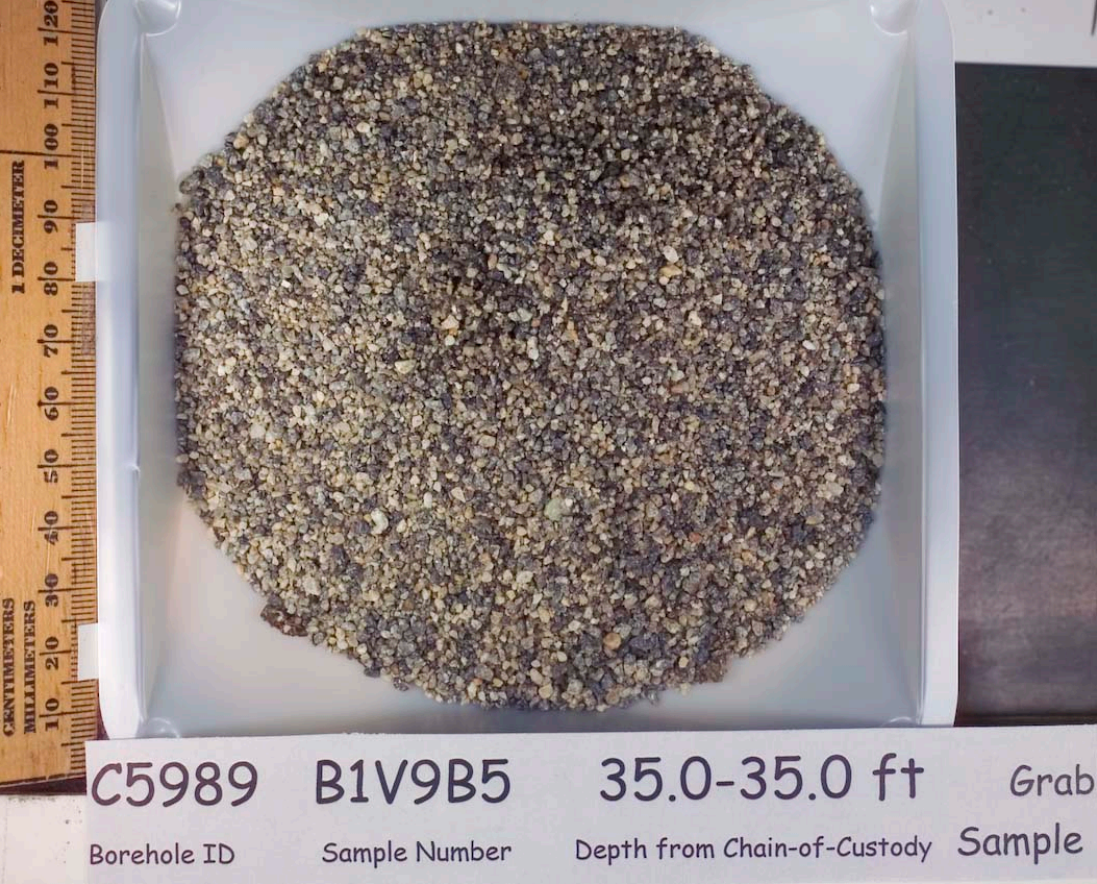




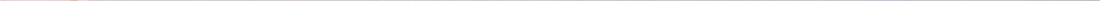




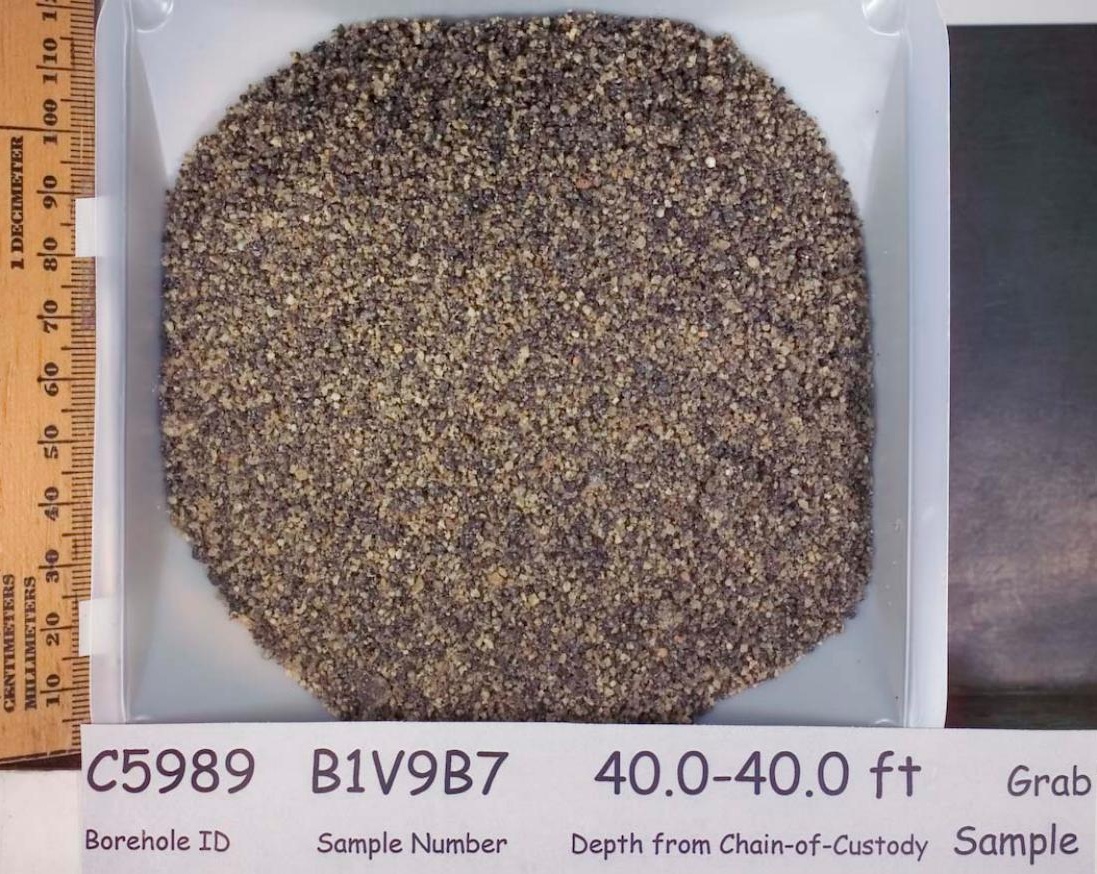




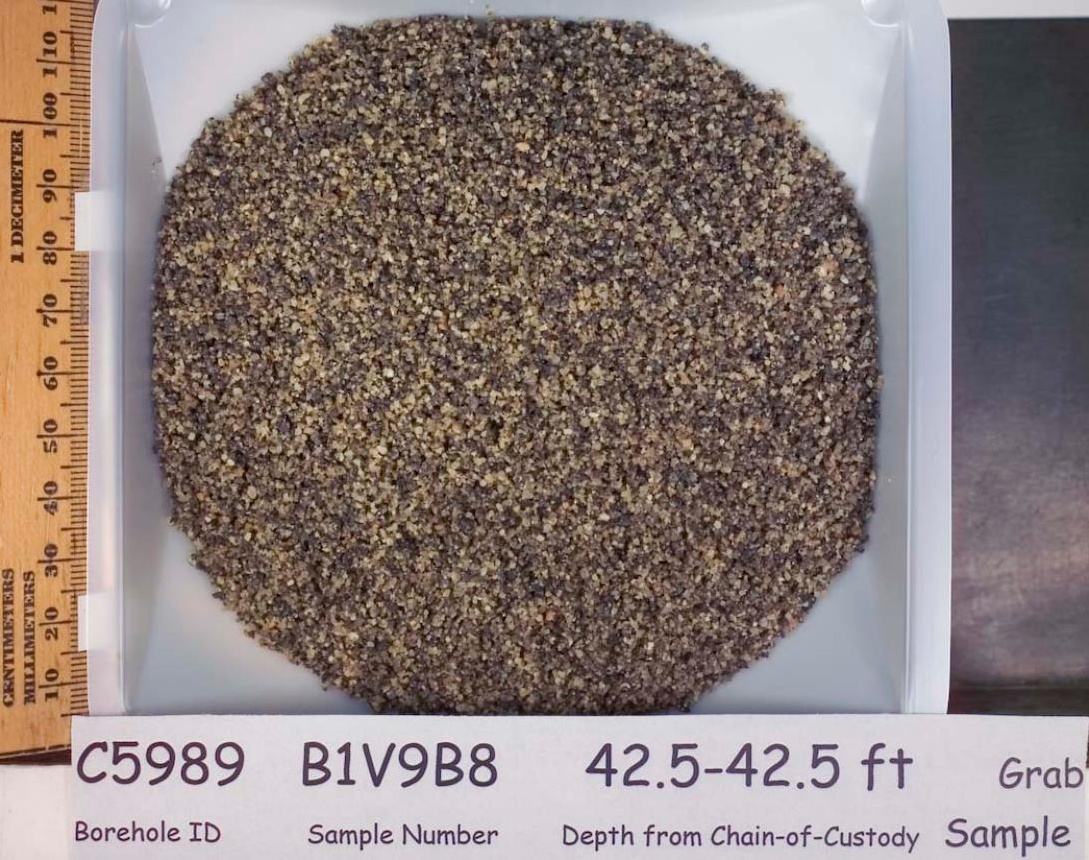




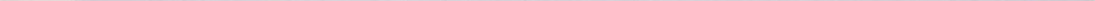




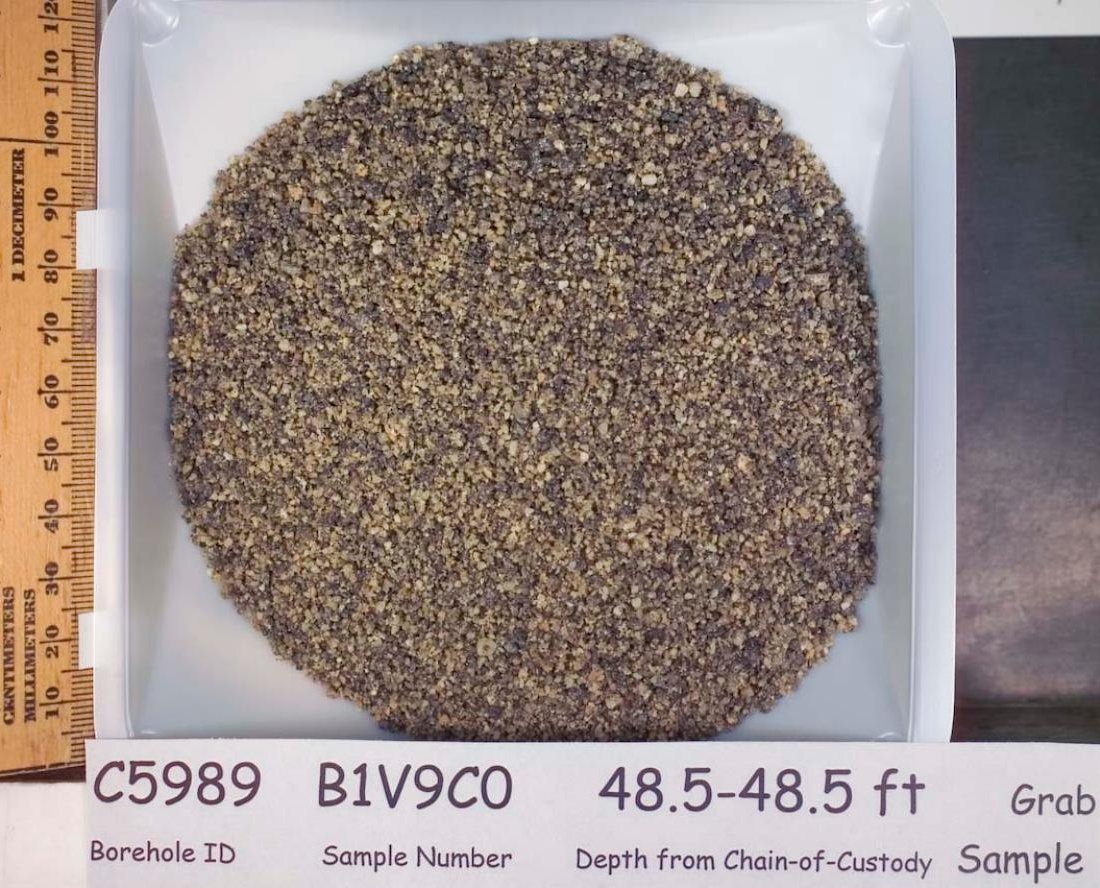




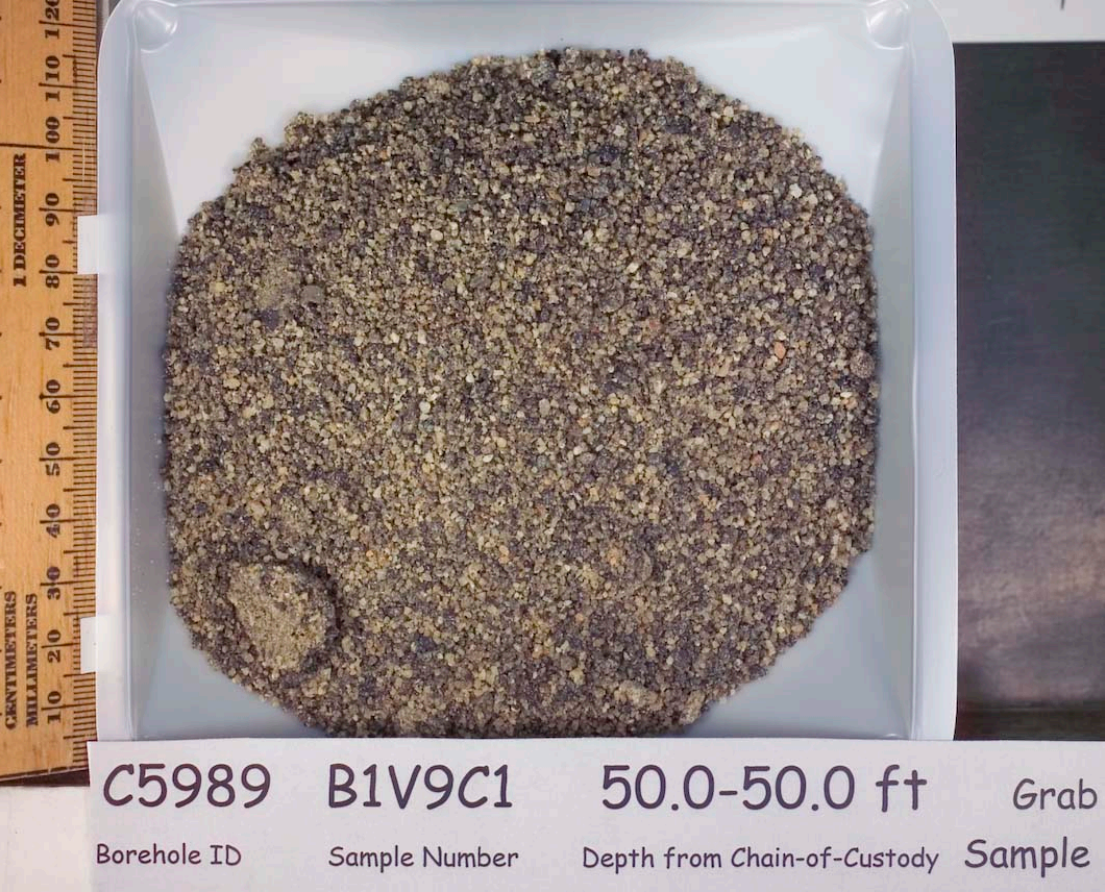




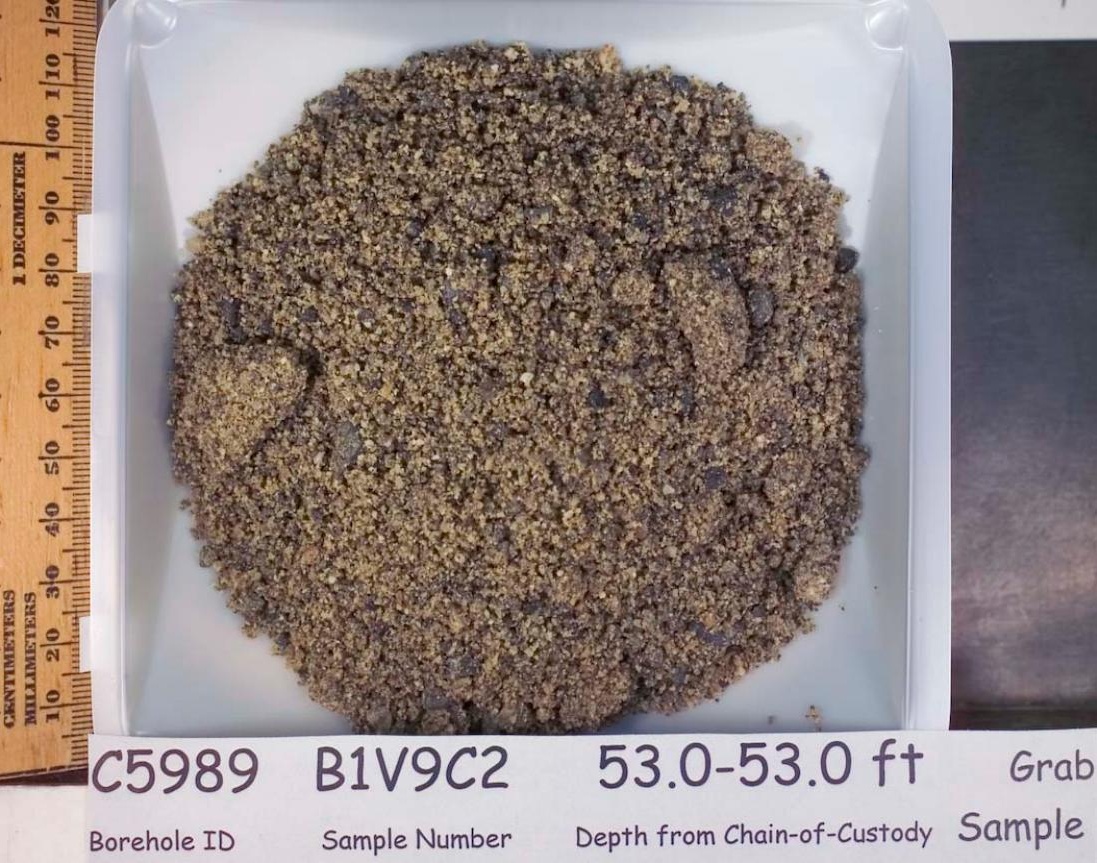




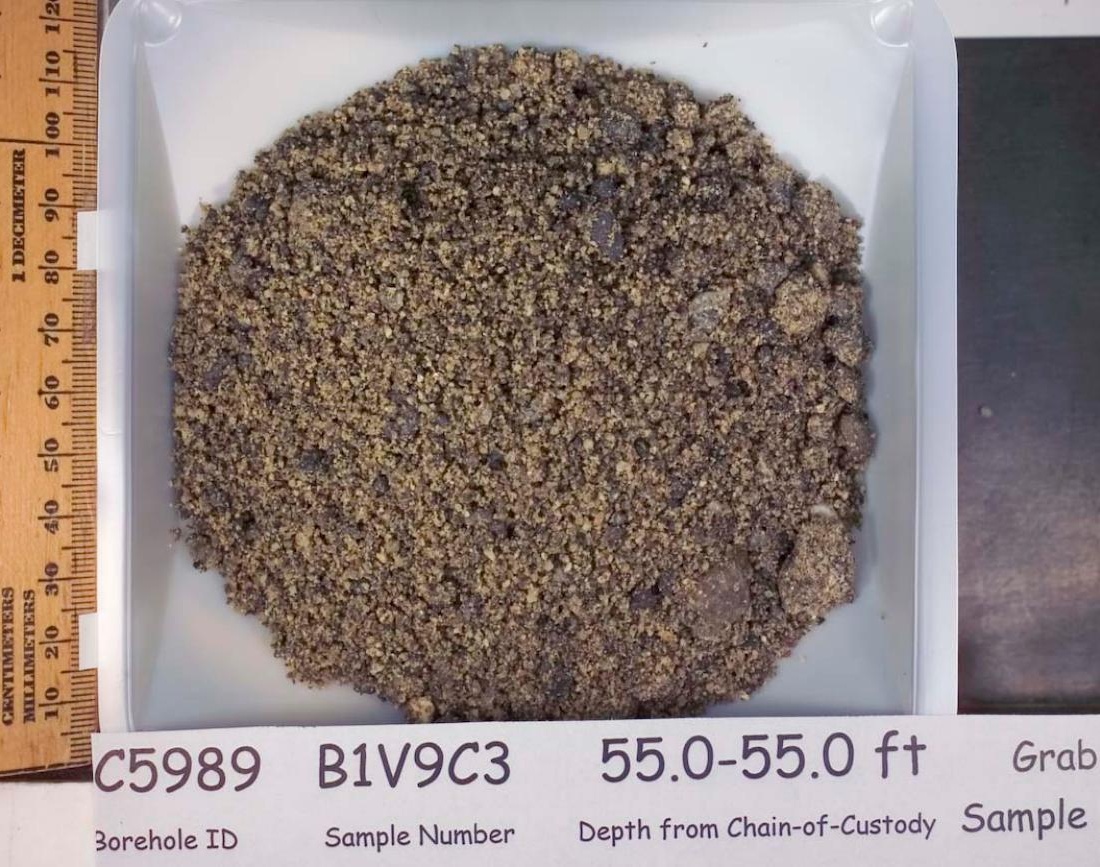




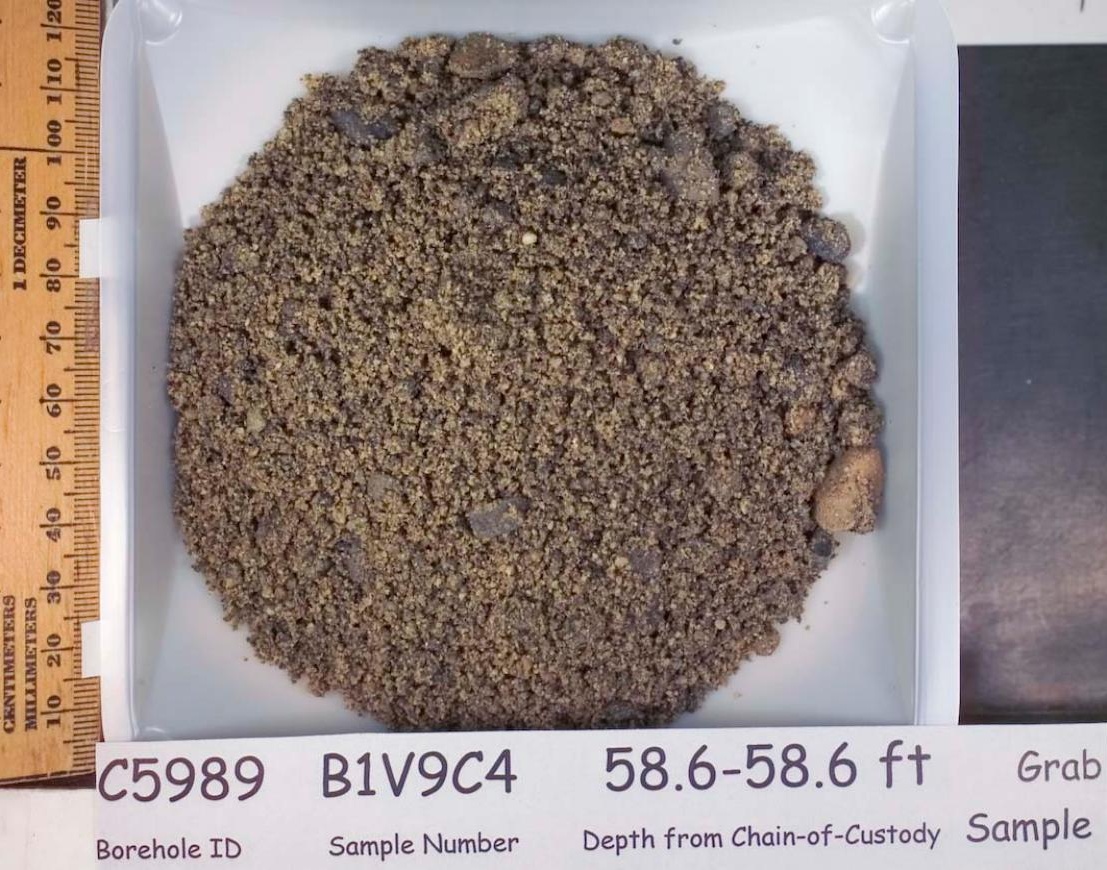




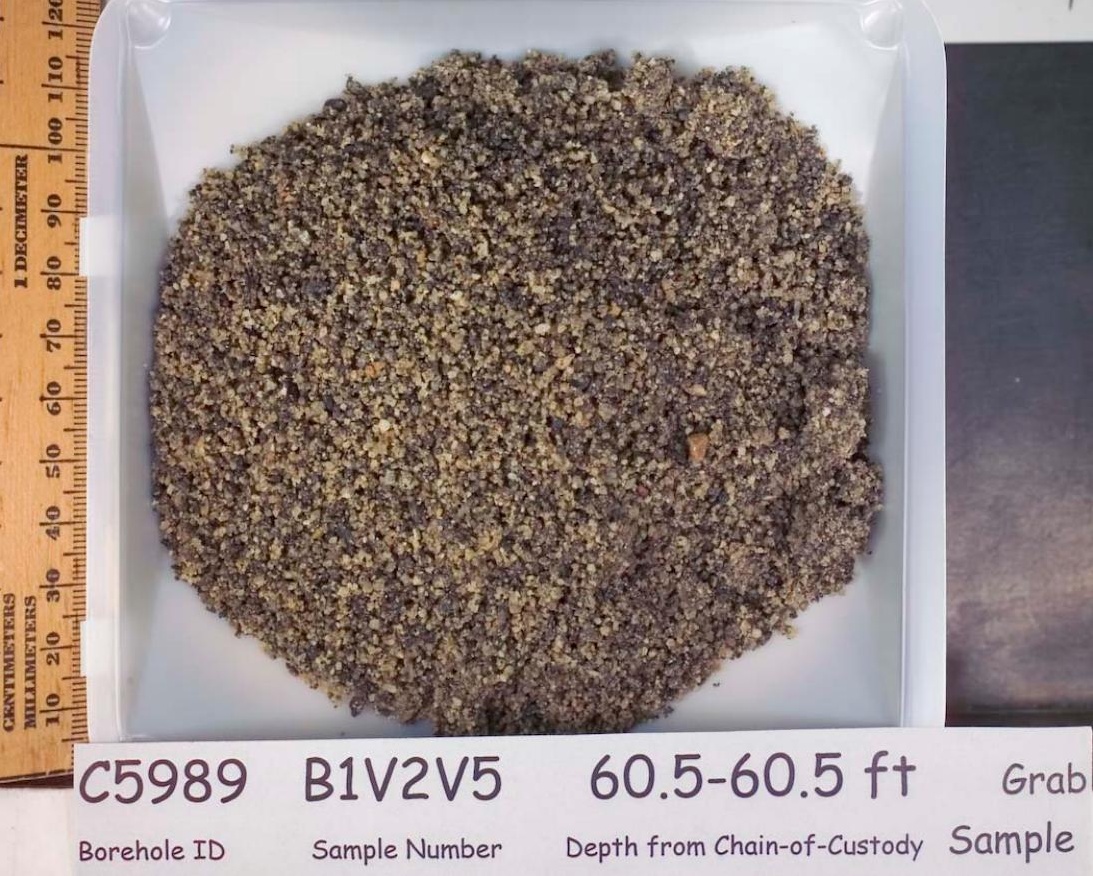




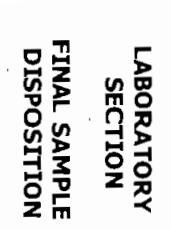

in

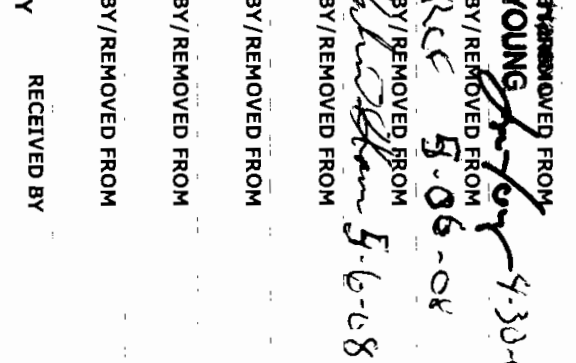

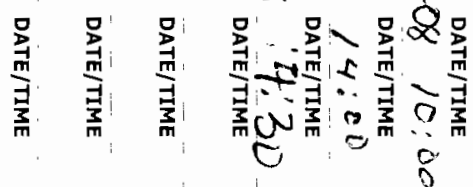

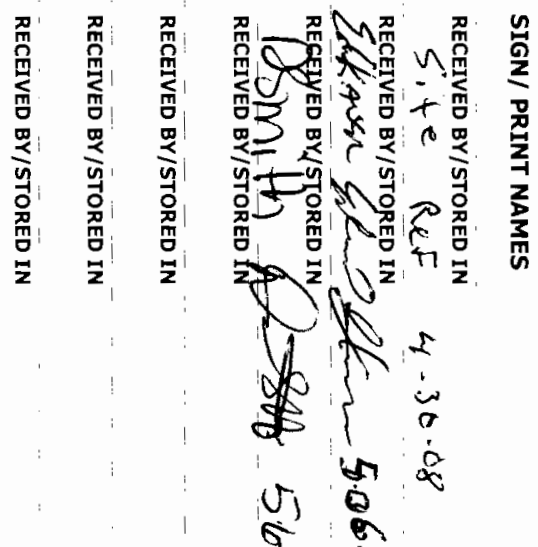

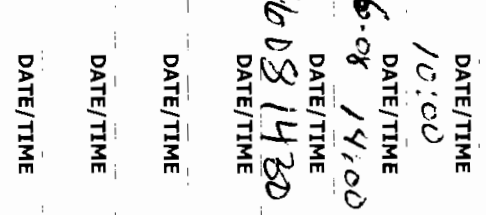
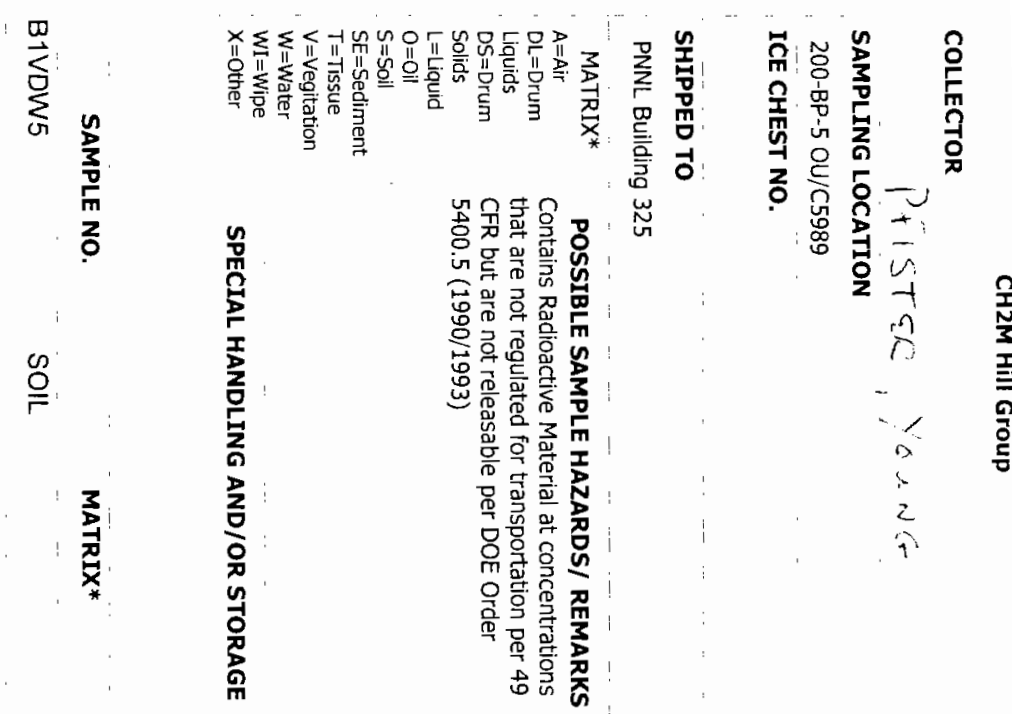

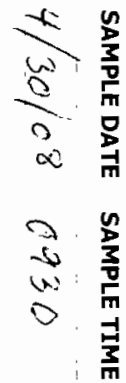

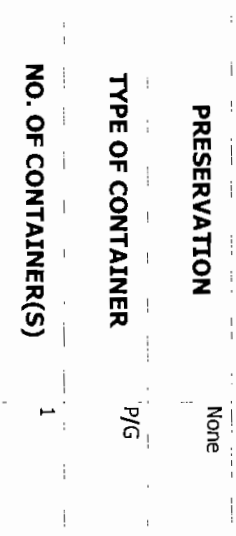

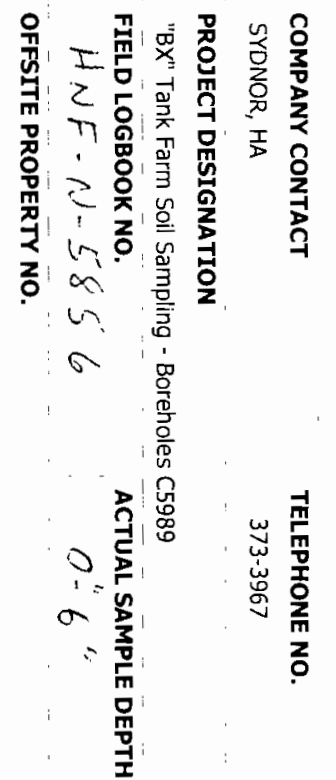

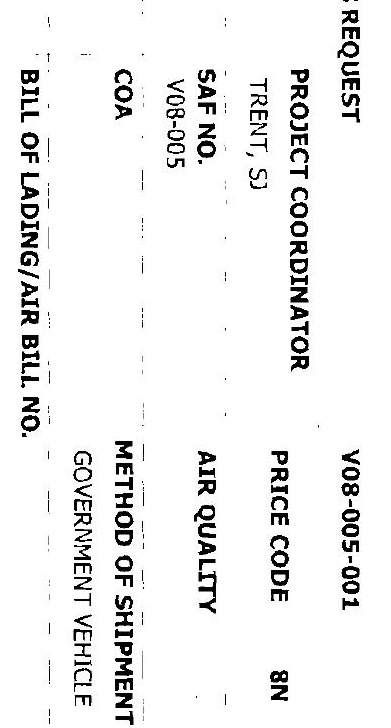

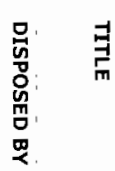




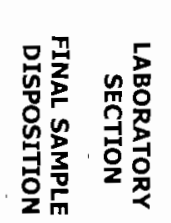

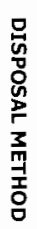

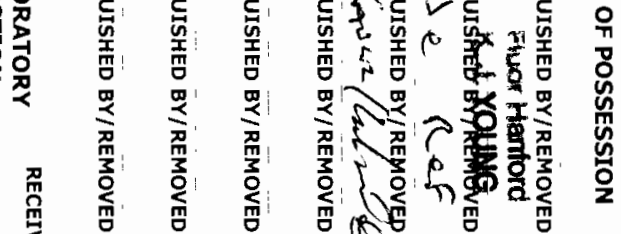

罗

$$
\text { 苟 } \quad \text { गᄁ }
$$

(1)

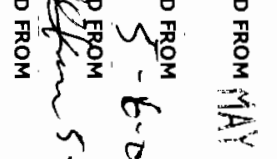

要

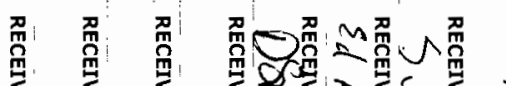

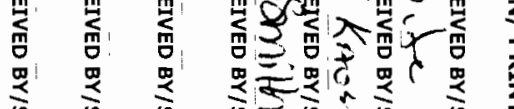

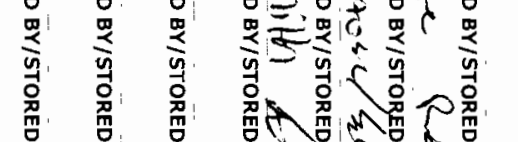

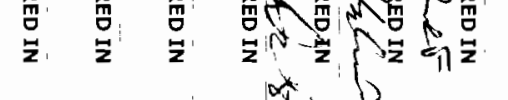

in

总尊

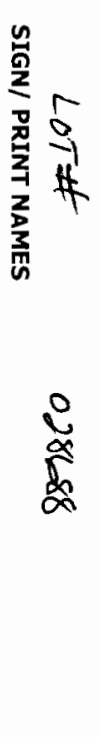

高

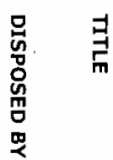

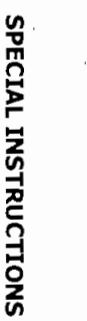

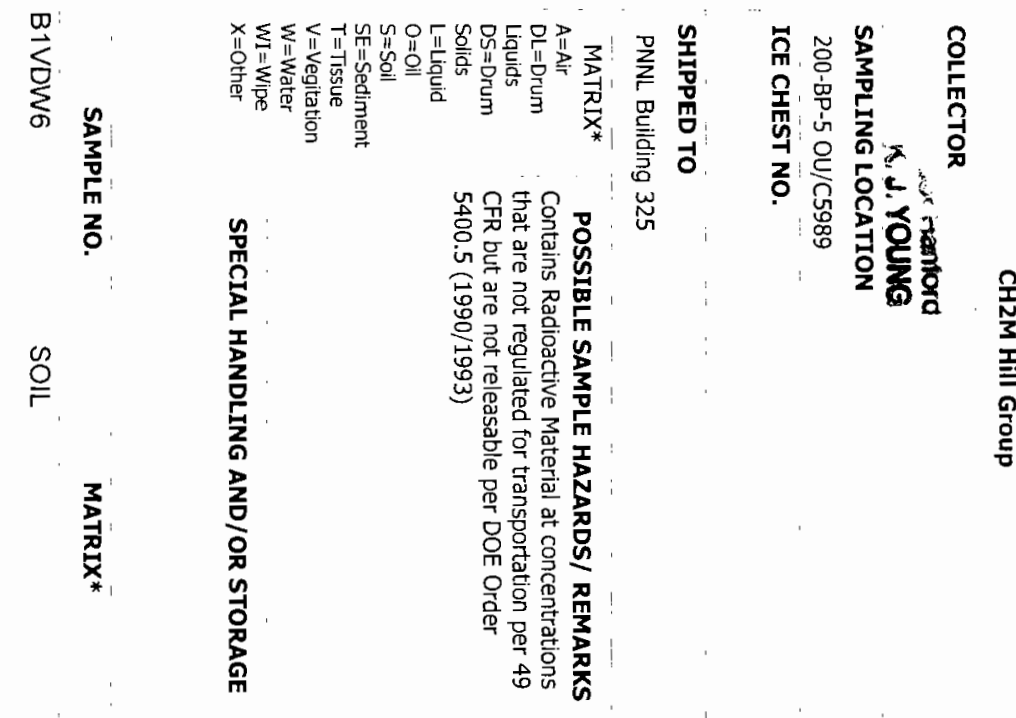

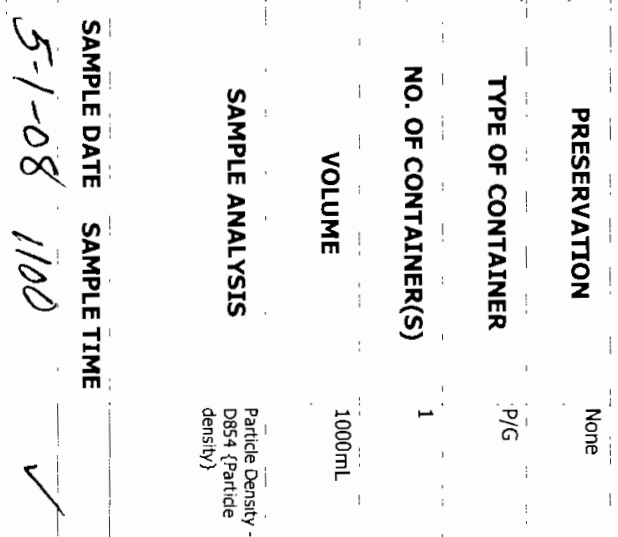

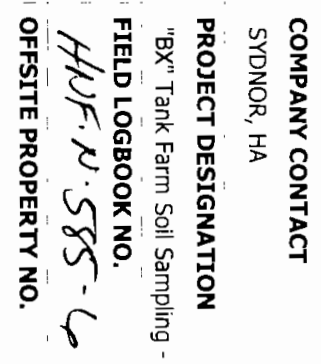
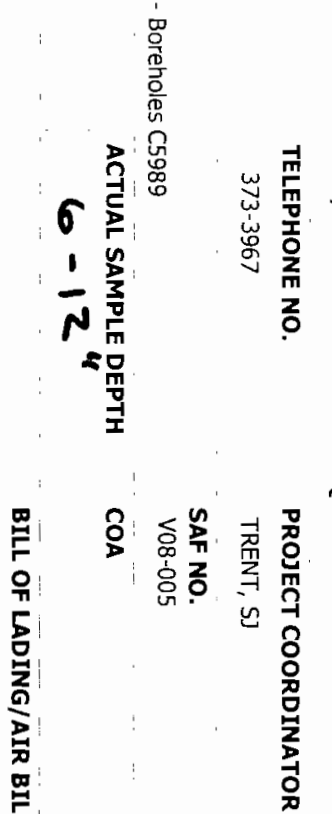

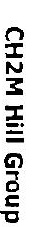
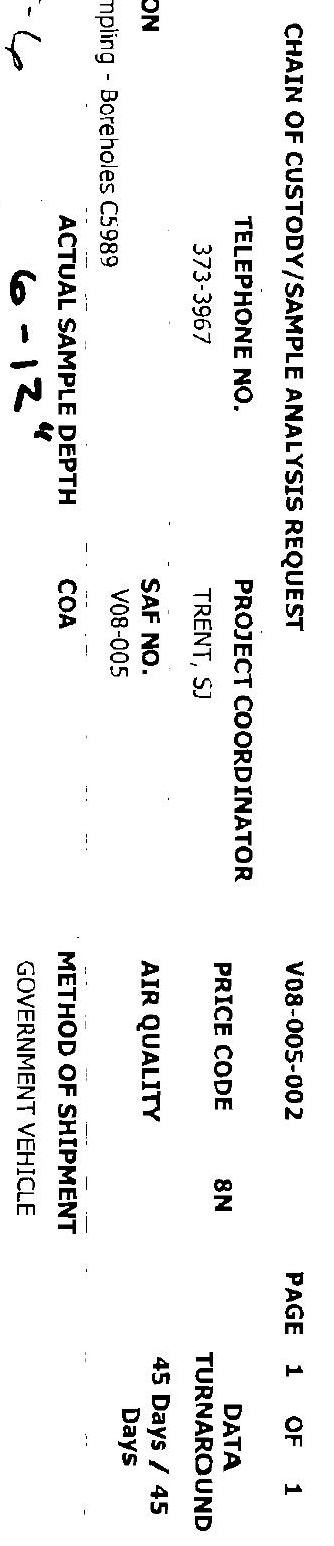


\section{$\|$

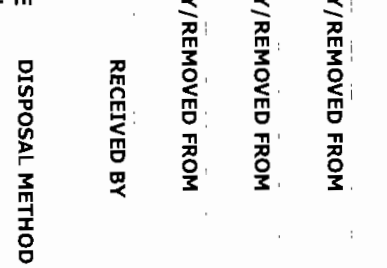

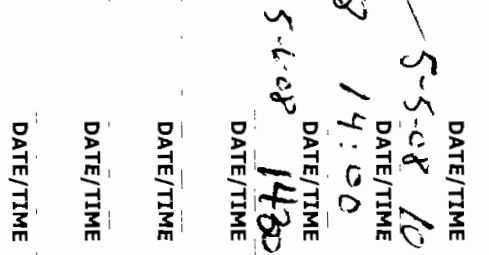
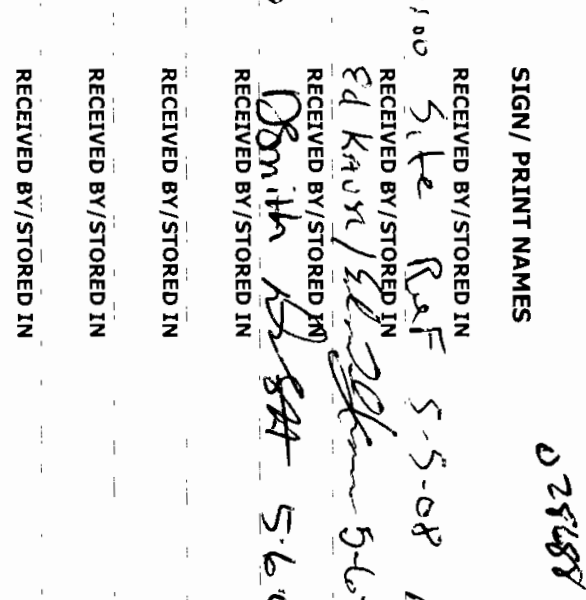

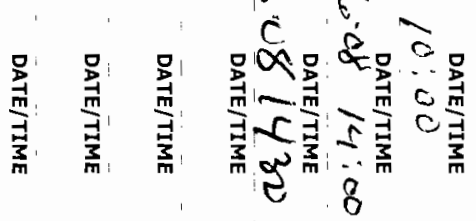

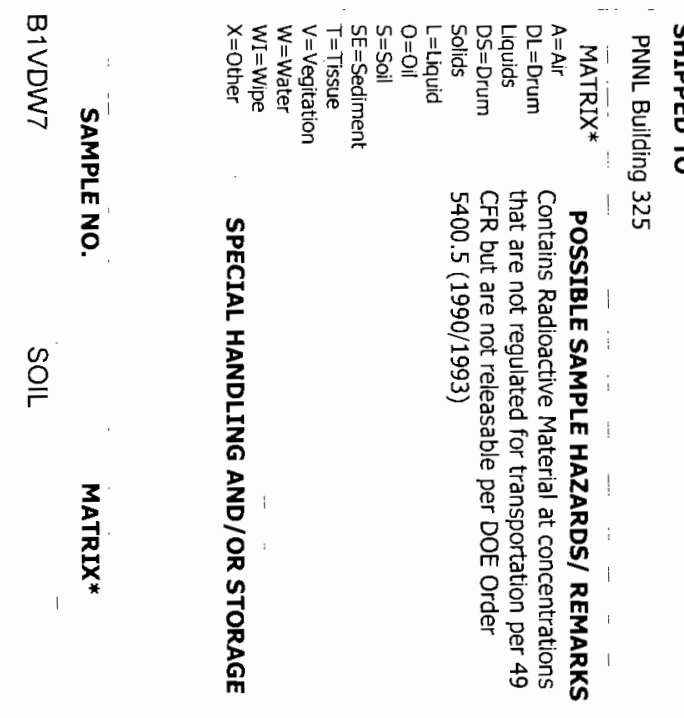

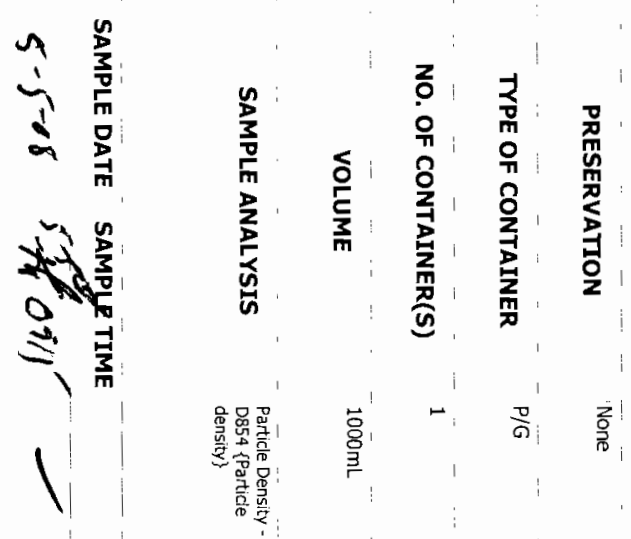

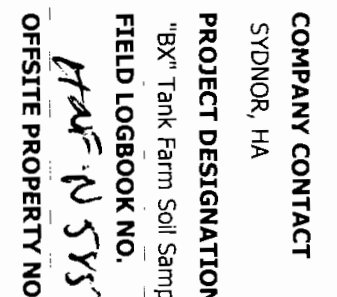

r

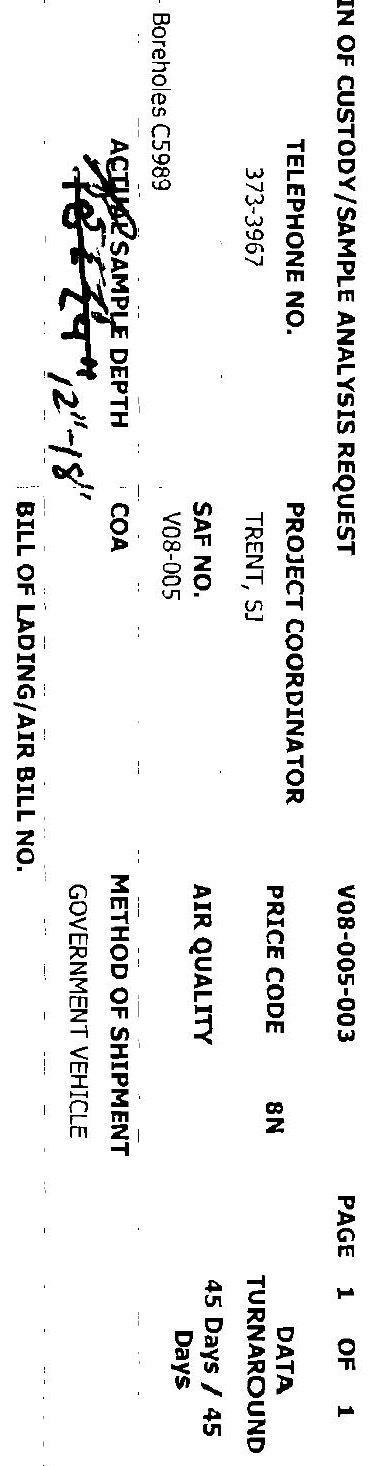




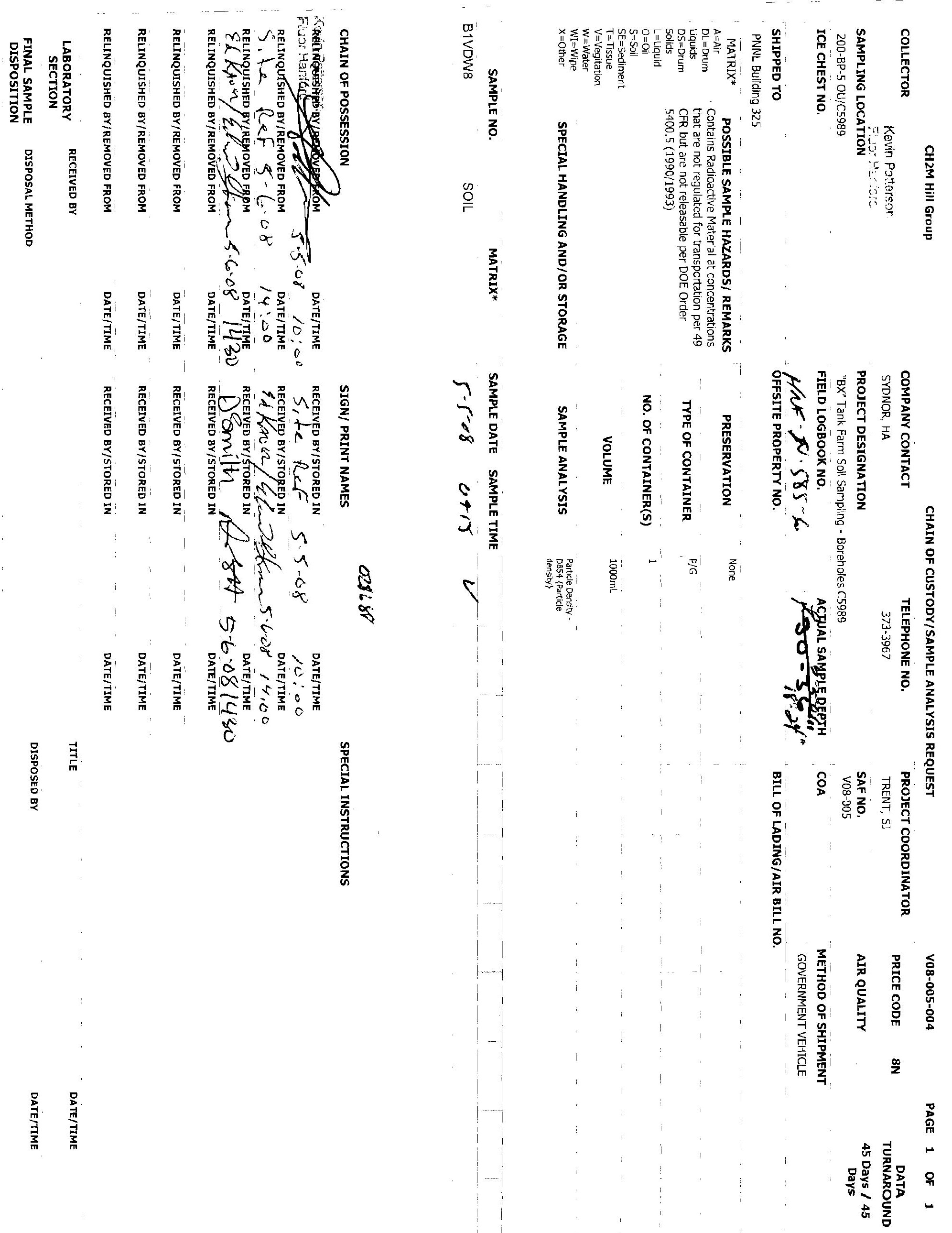


$\|$
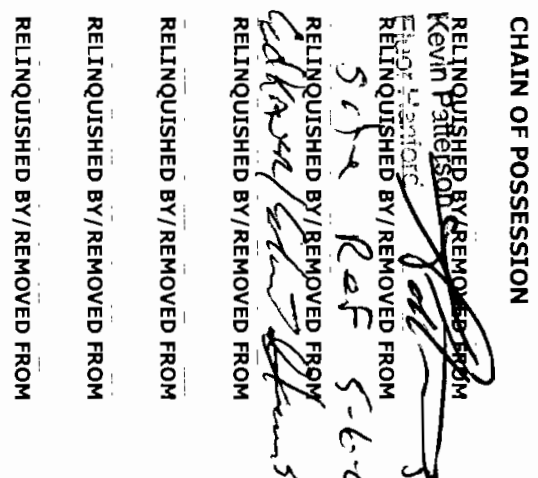

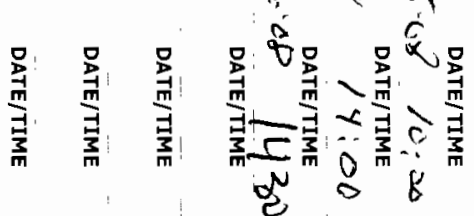

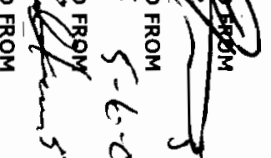

मो 0 i
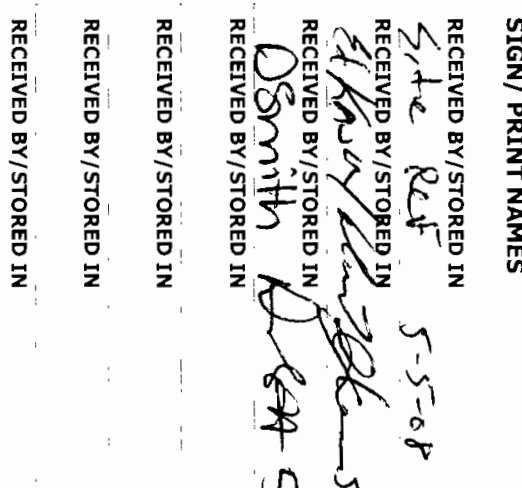

( $)$ iो

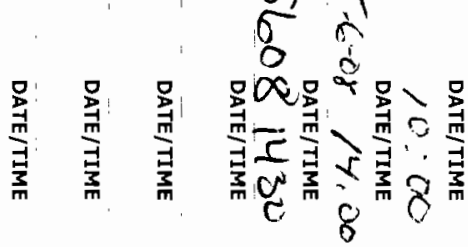

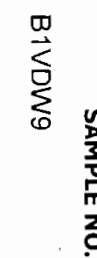

온

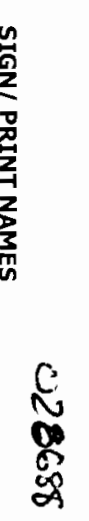

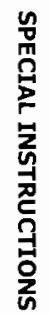

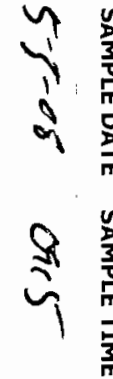

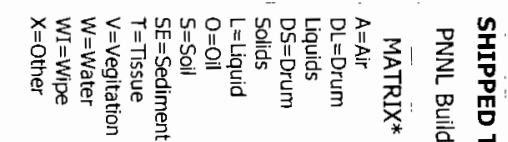

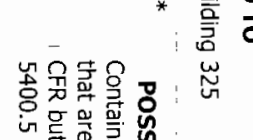

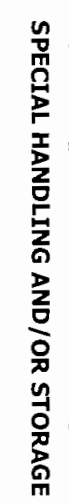

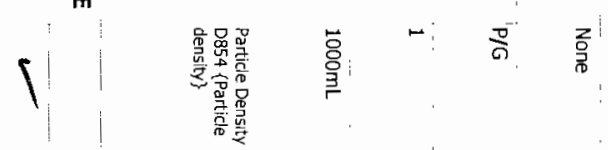

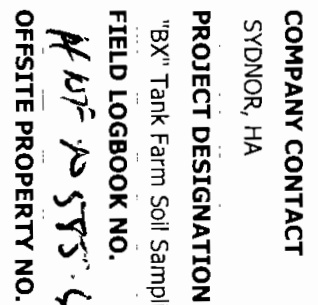

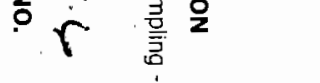

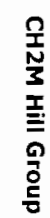

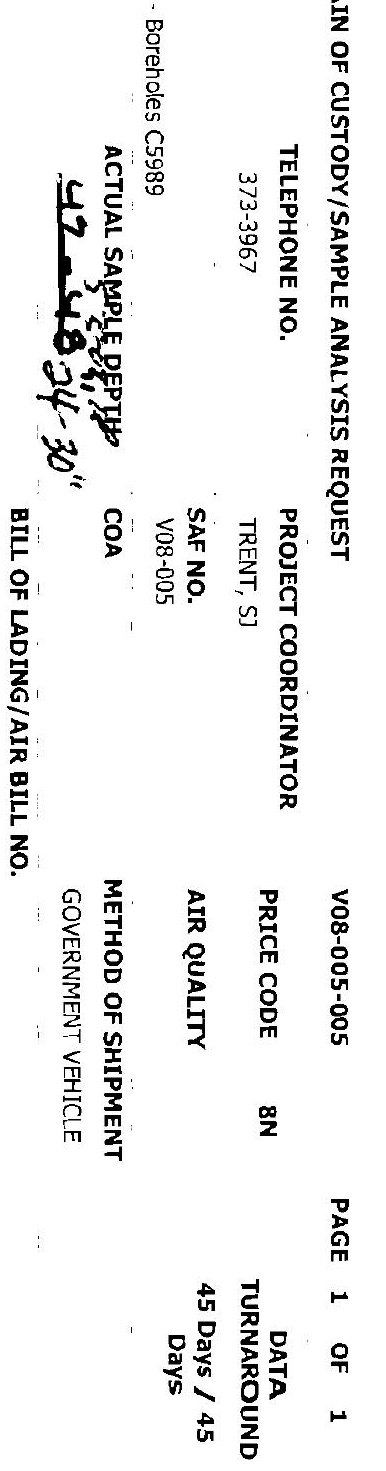


$\|$

,

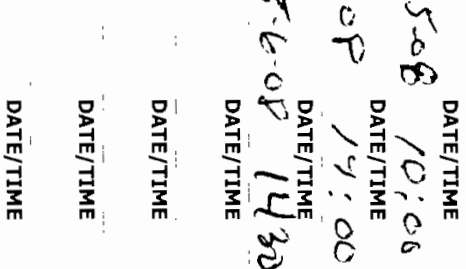

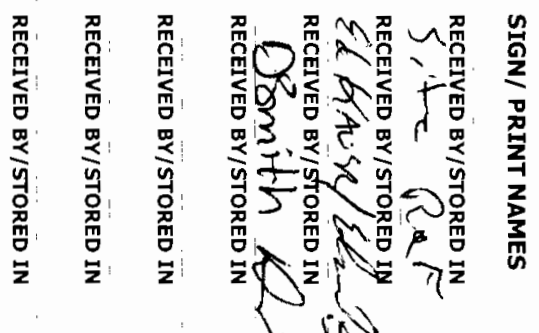

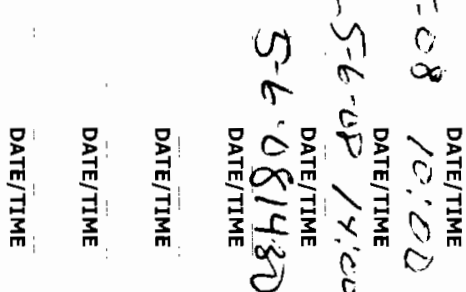

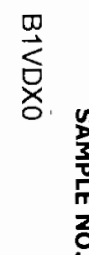

$\stackrel{\mathscr{O}}{\stackrel{O}{F}}$

妾

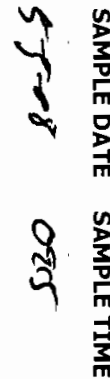

8
0
0

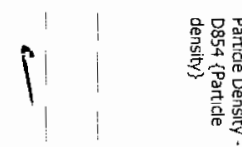

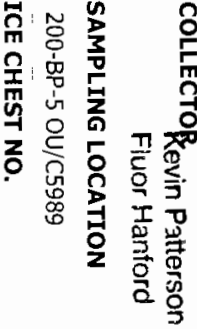

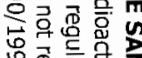

费需需市

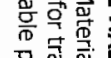

空

品

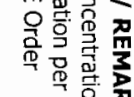

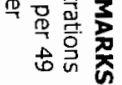

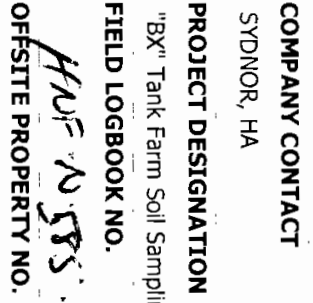

$r$ 蒠

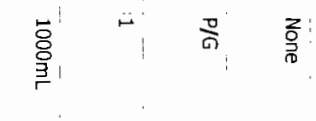

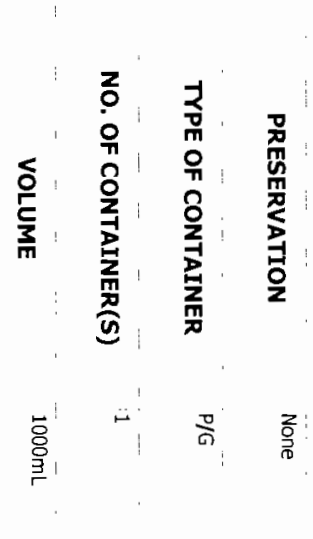

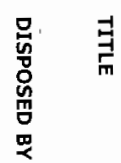

竞

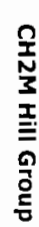

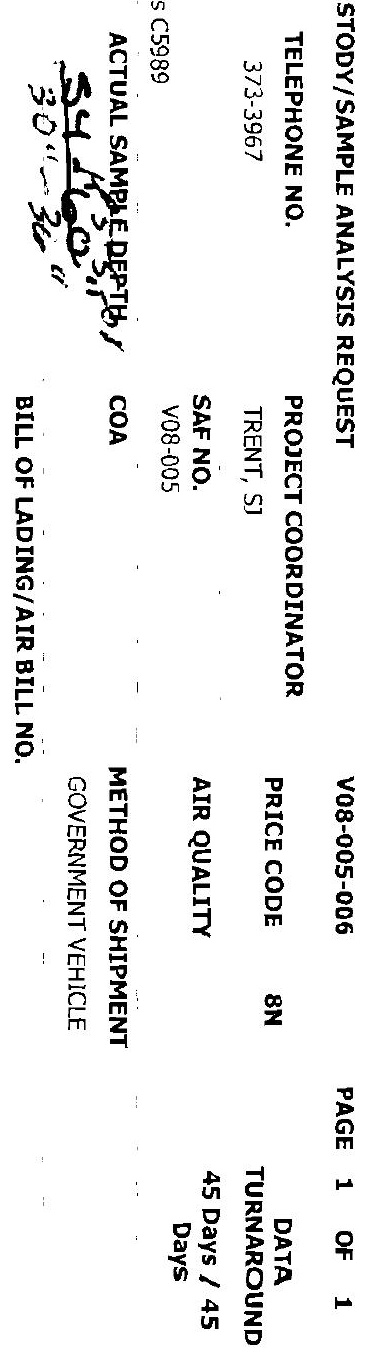




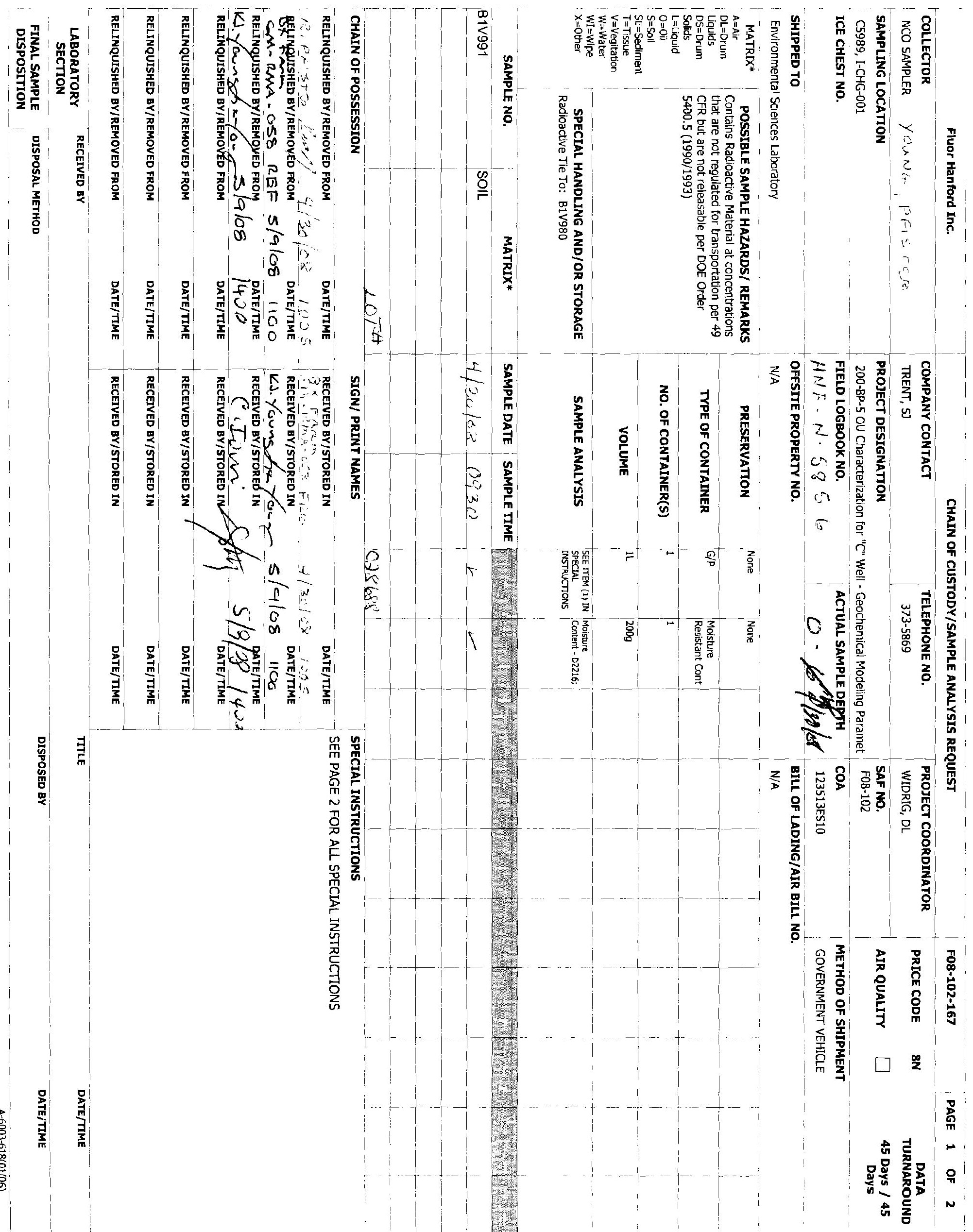




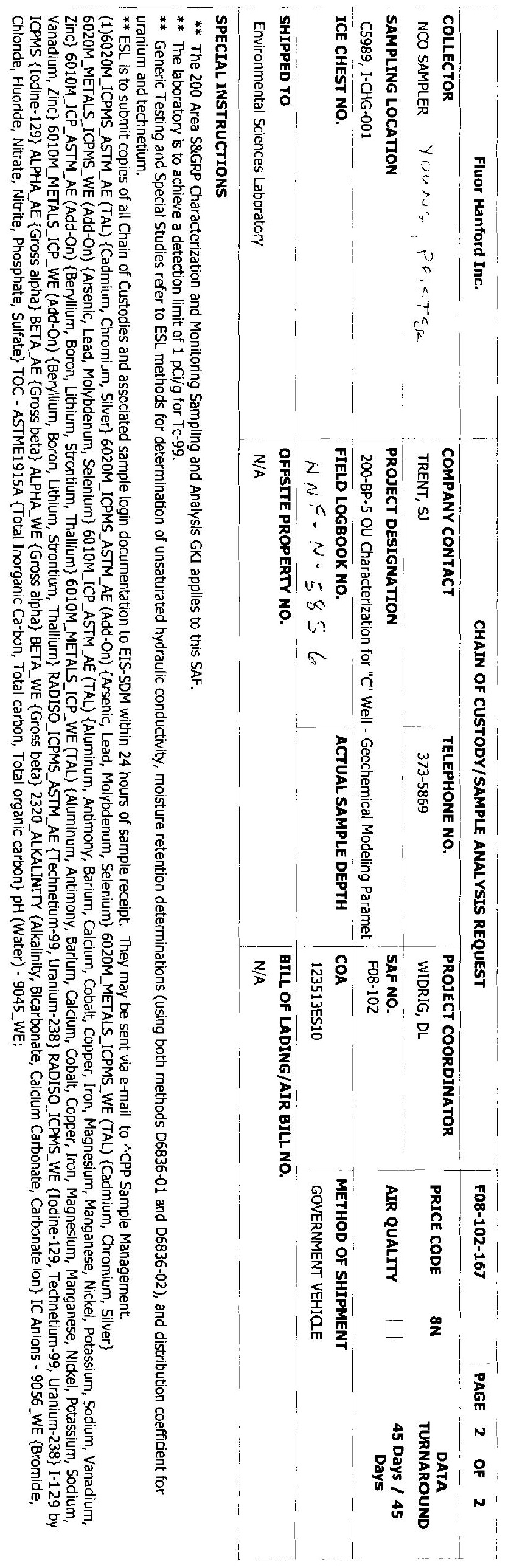




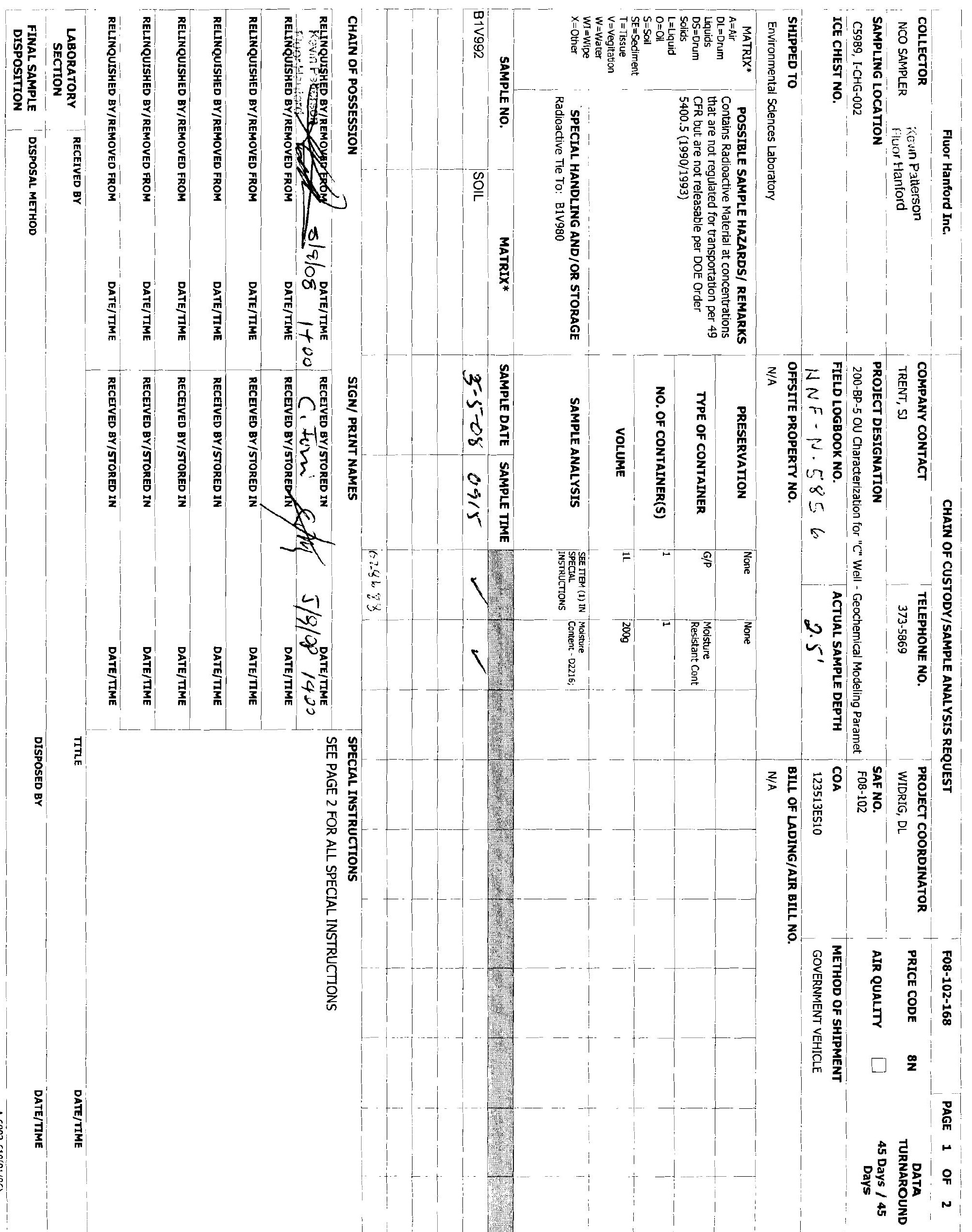



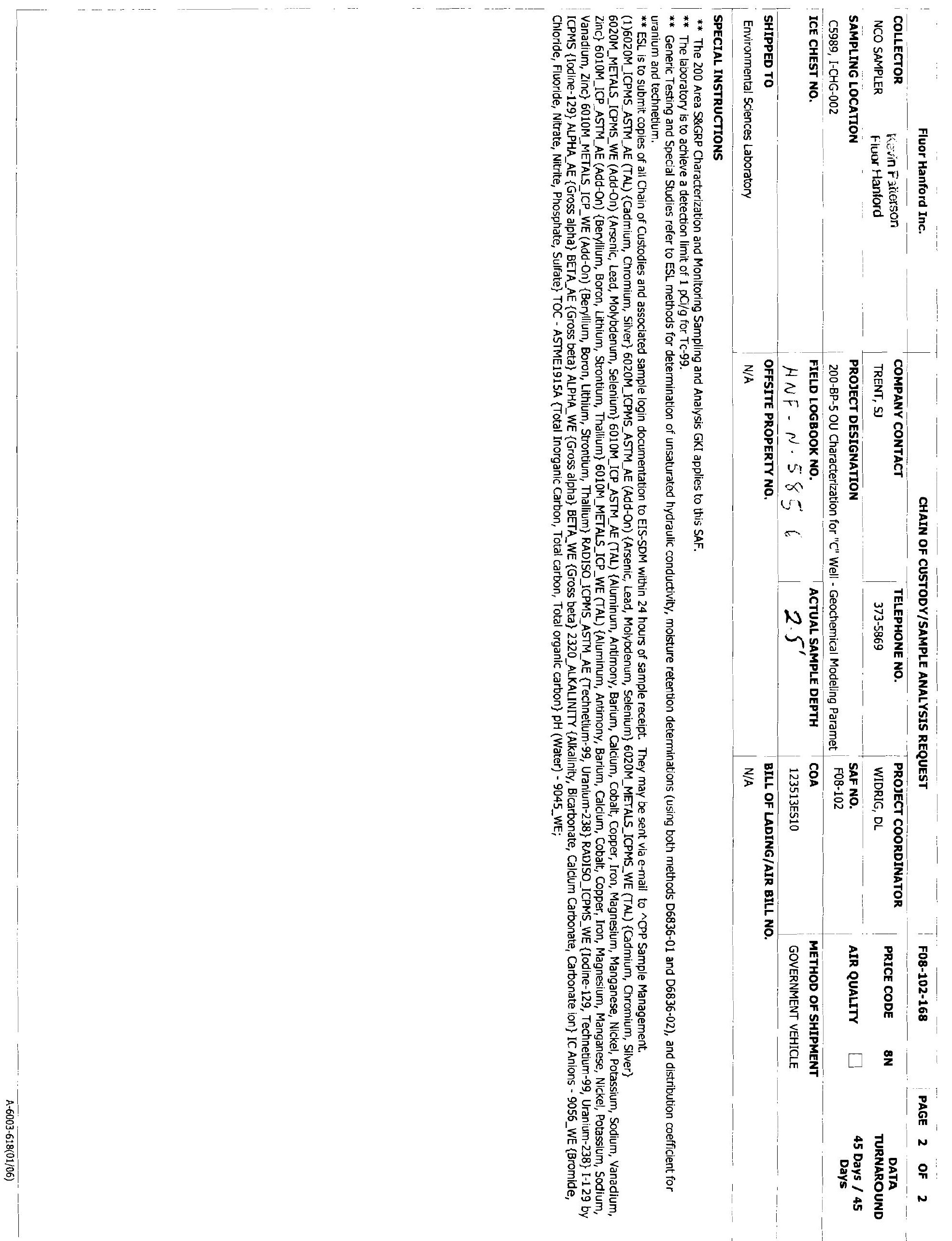


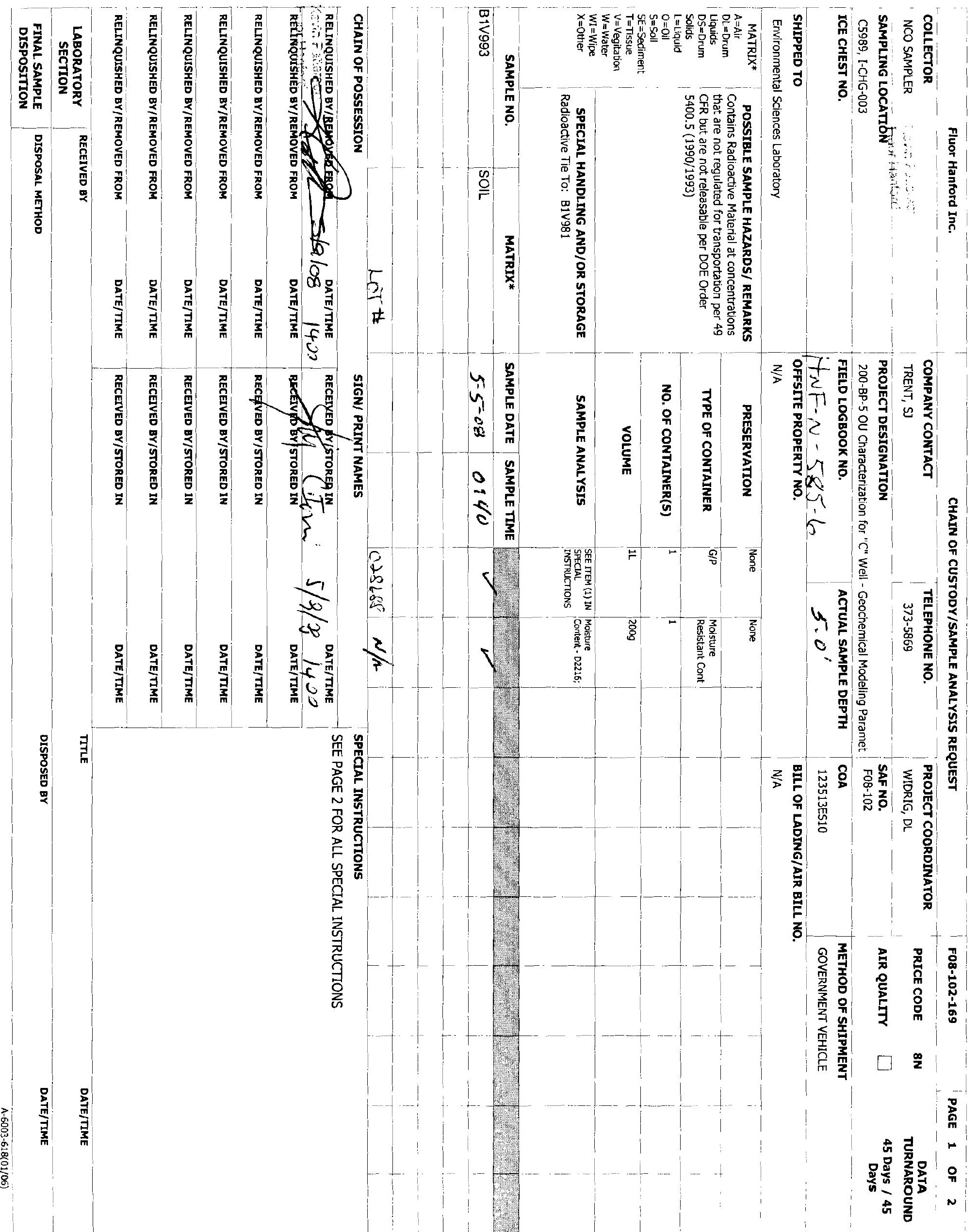




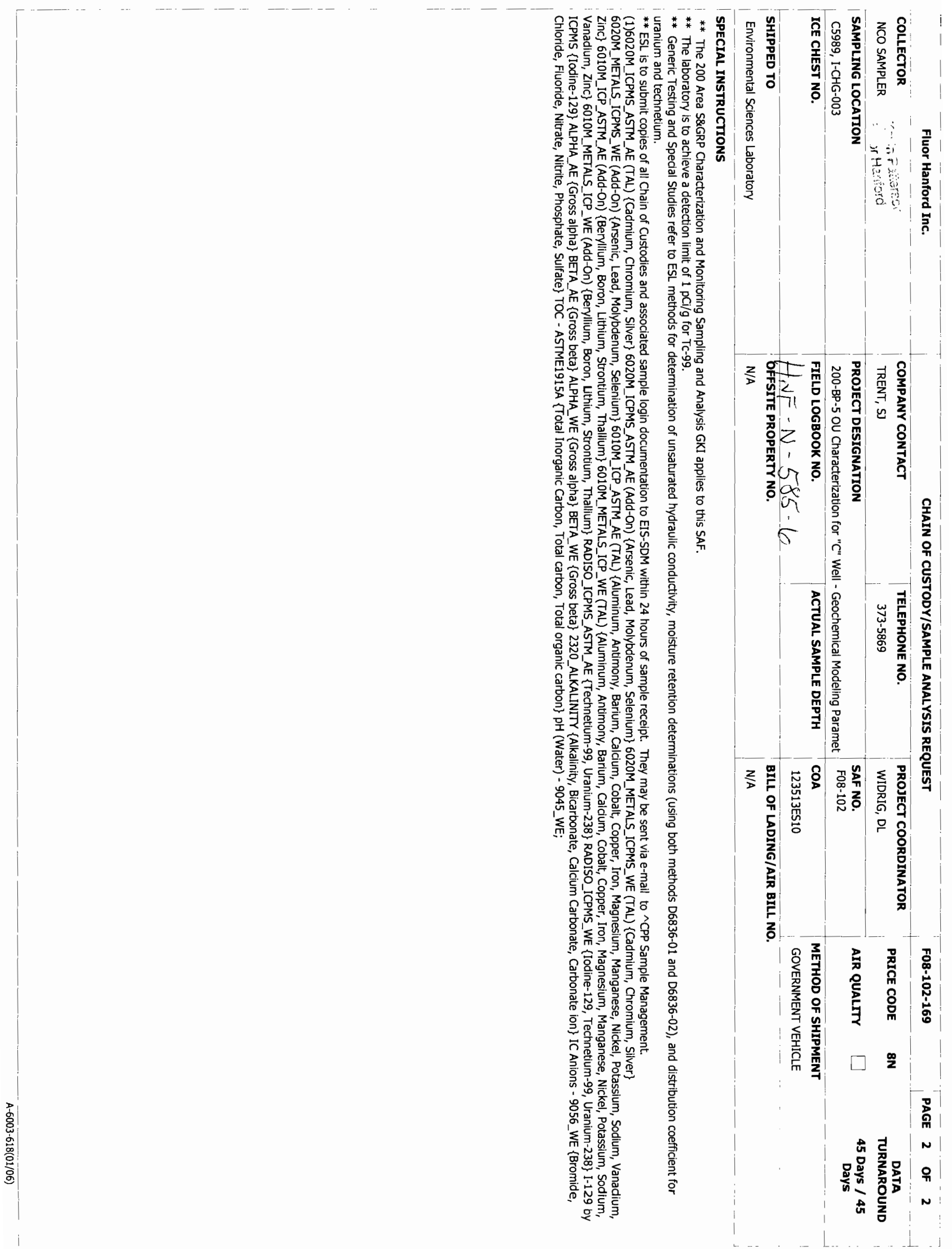




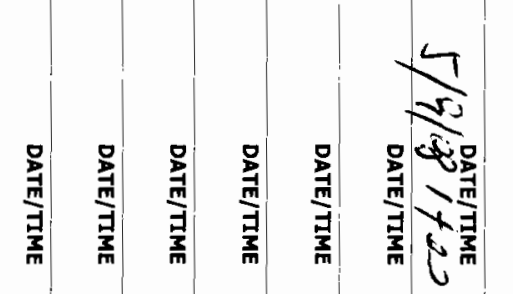

\section{$-$}

40

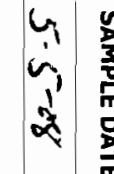

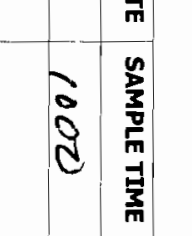

o

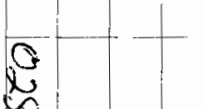

5

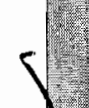

通

5

$+$

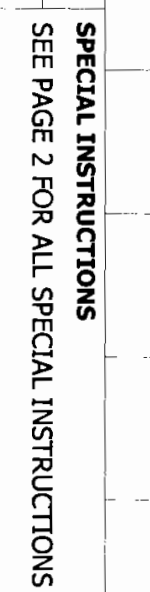

量 


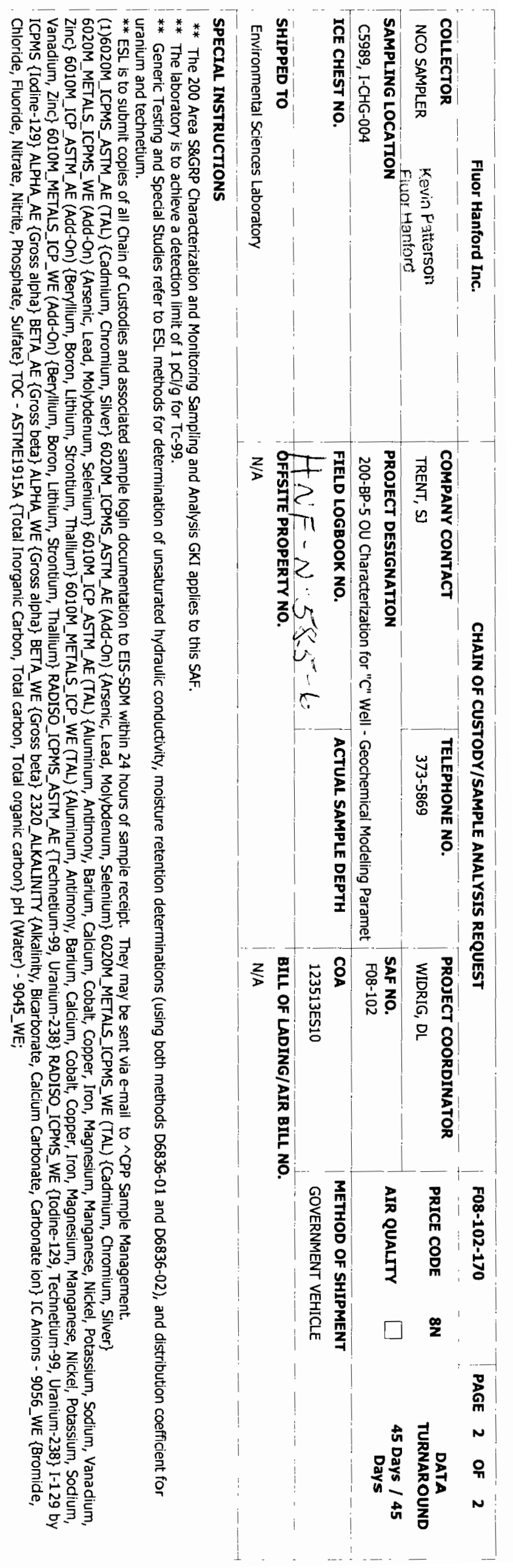




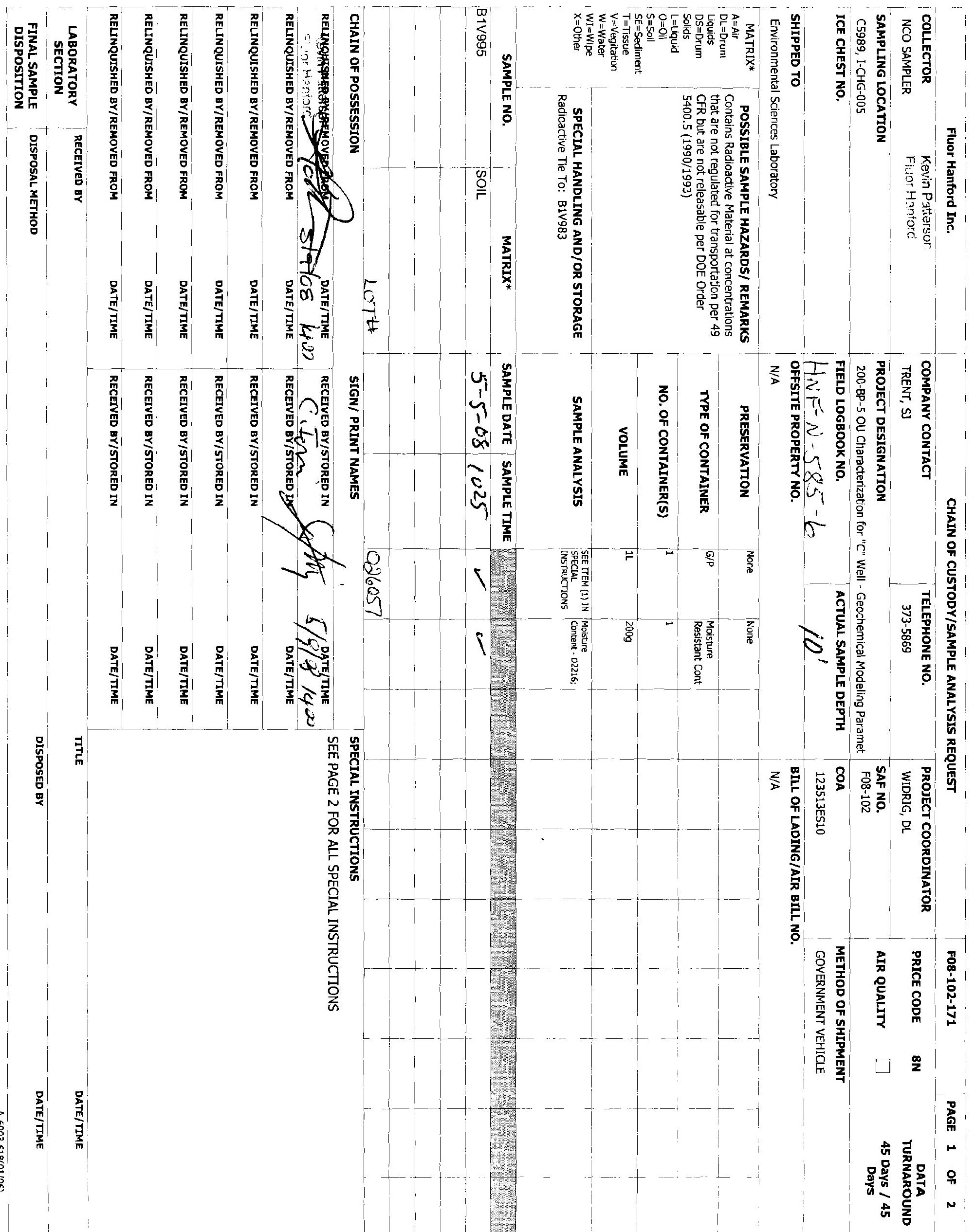




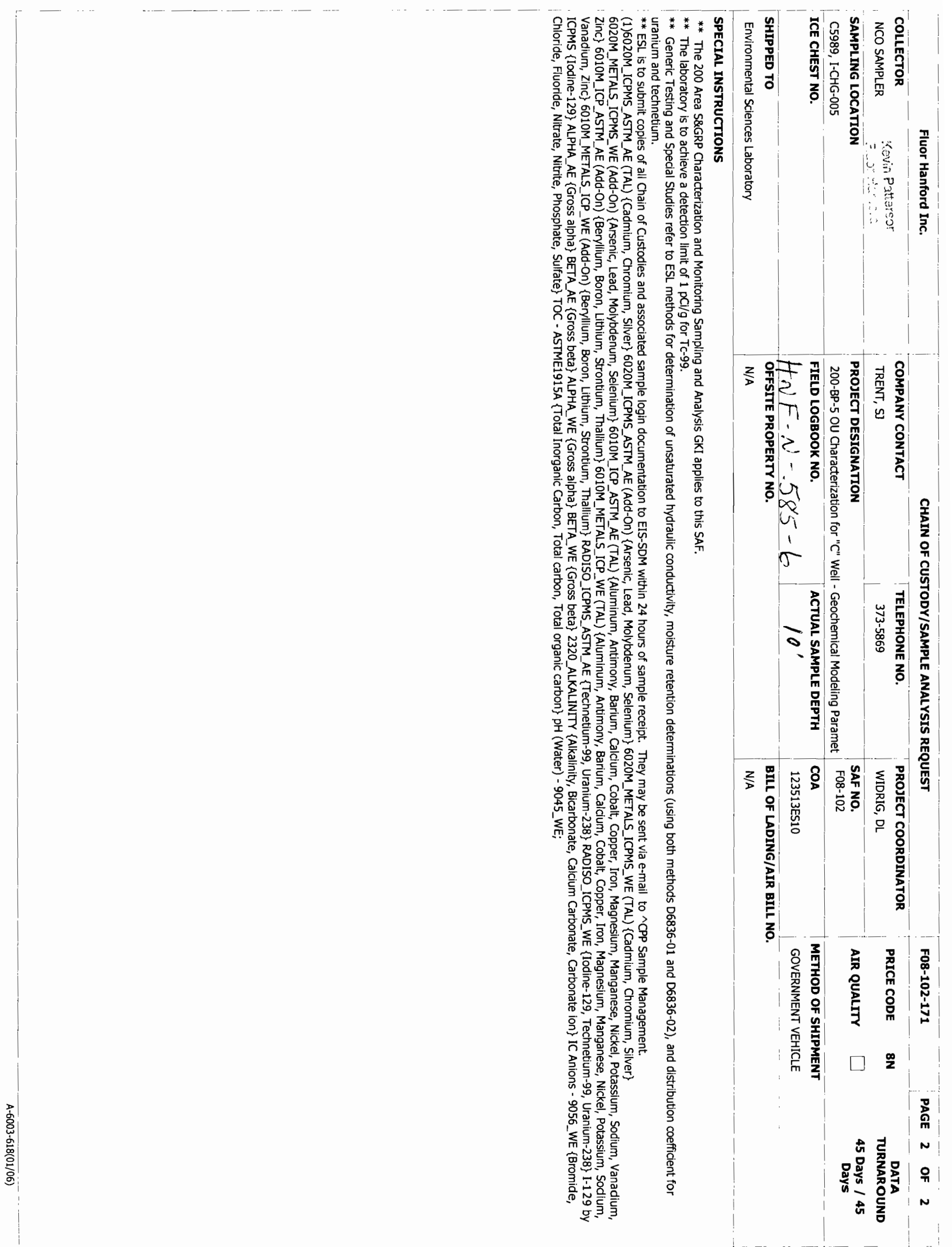




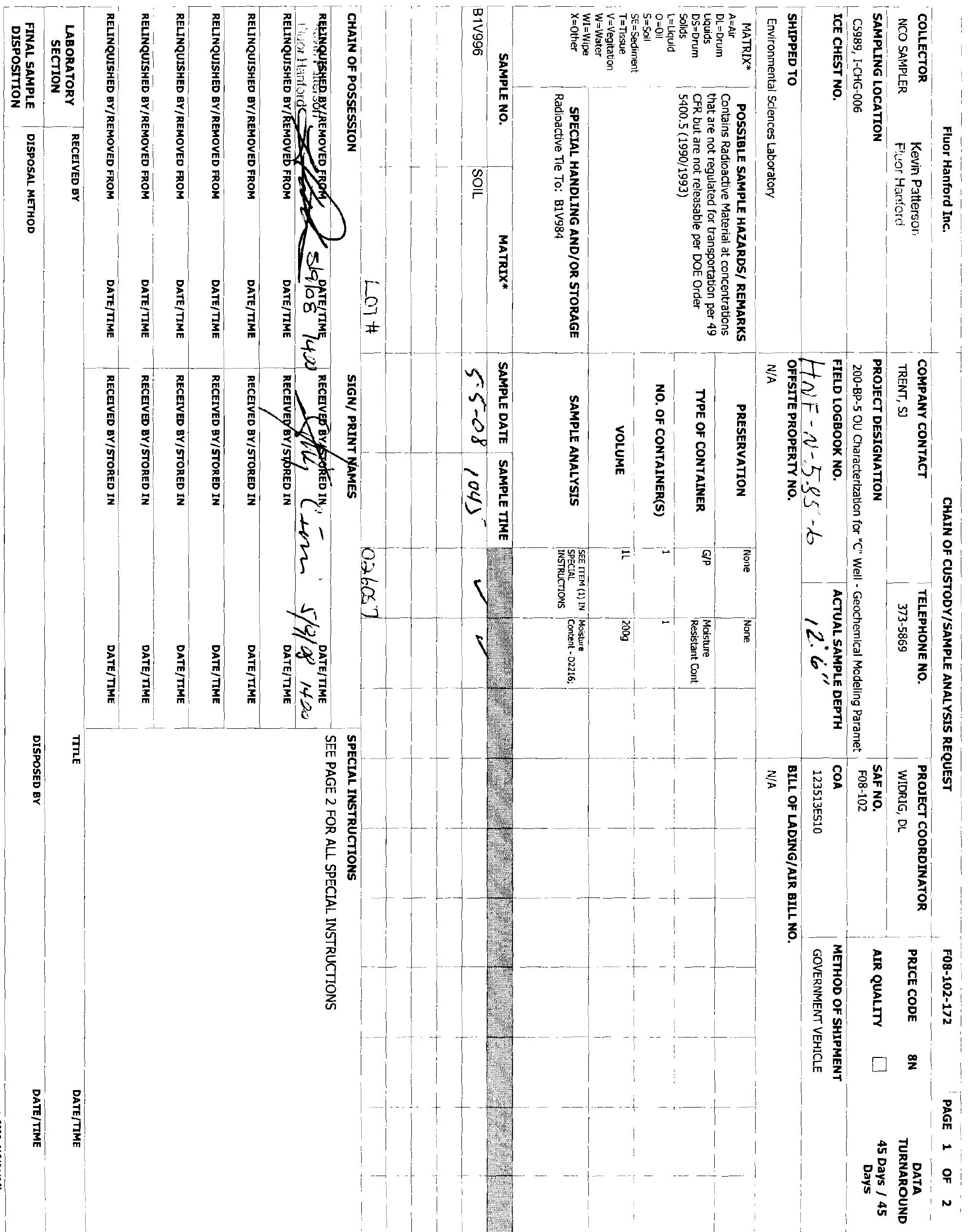



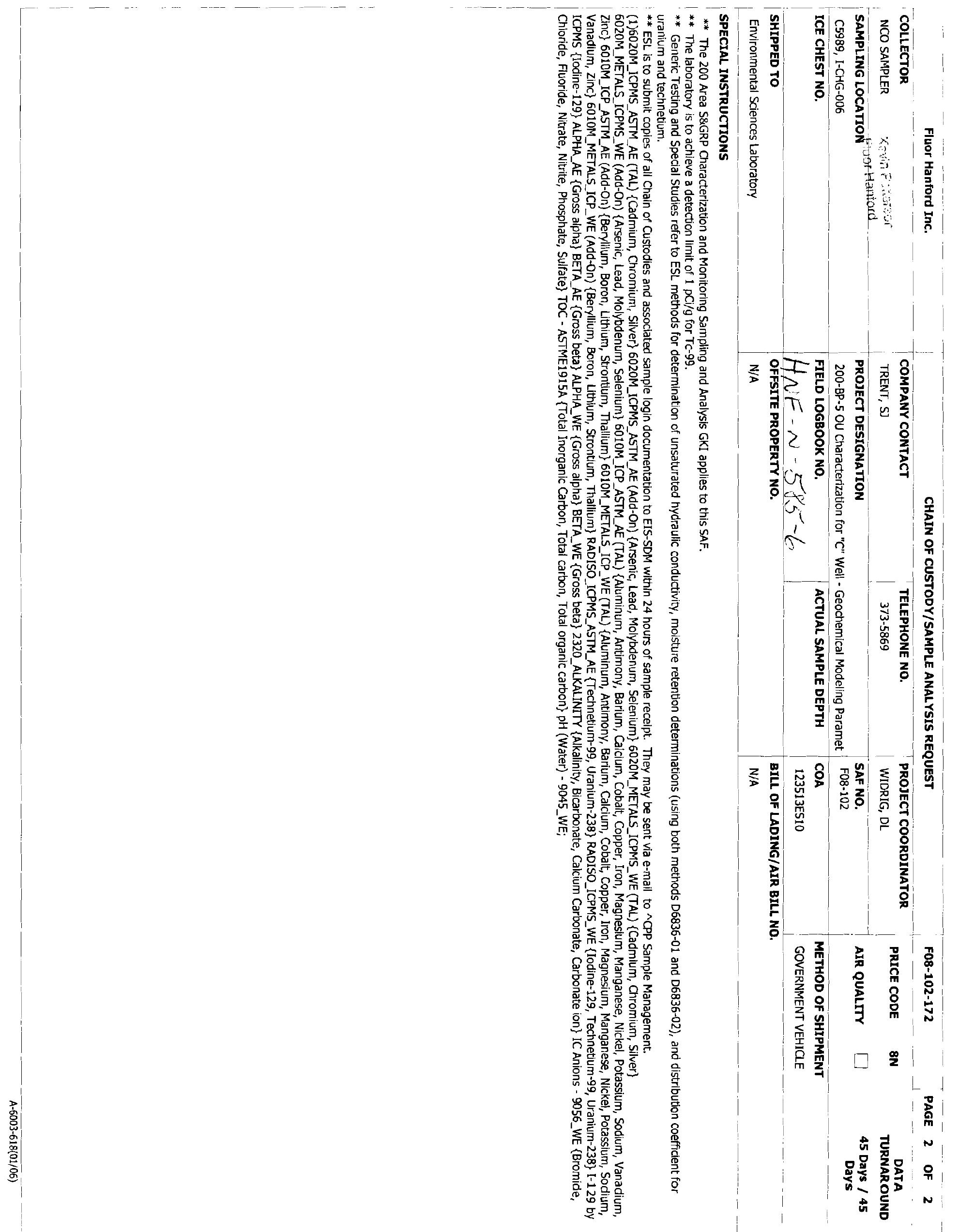


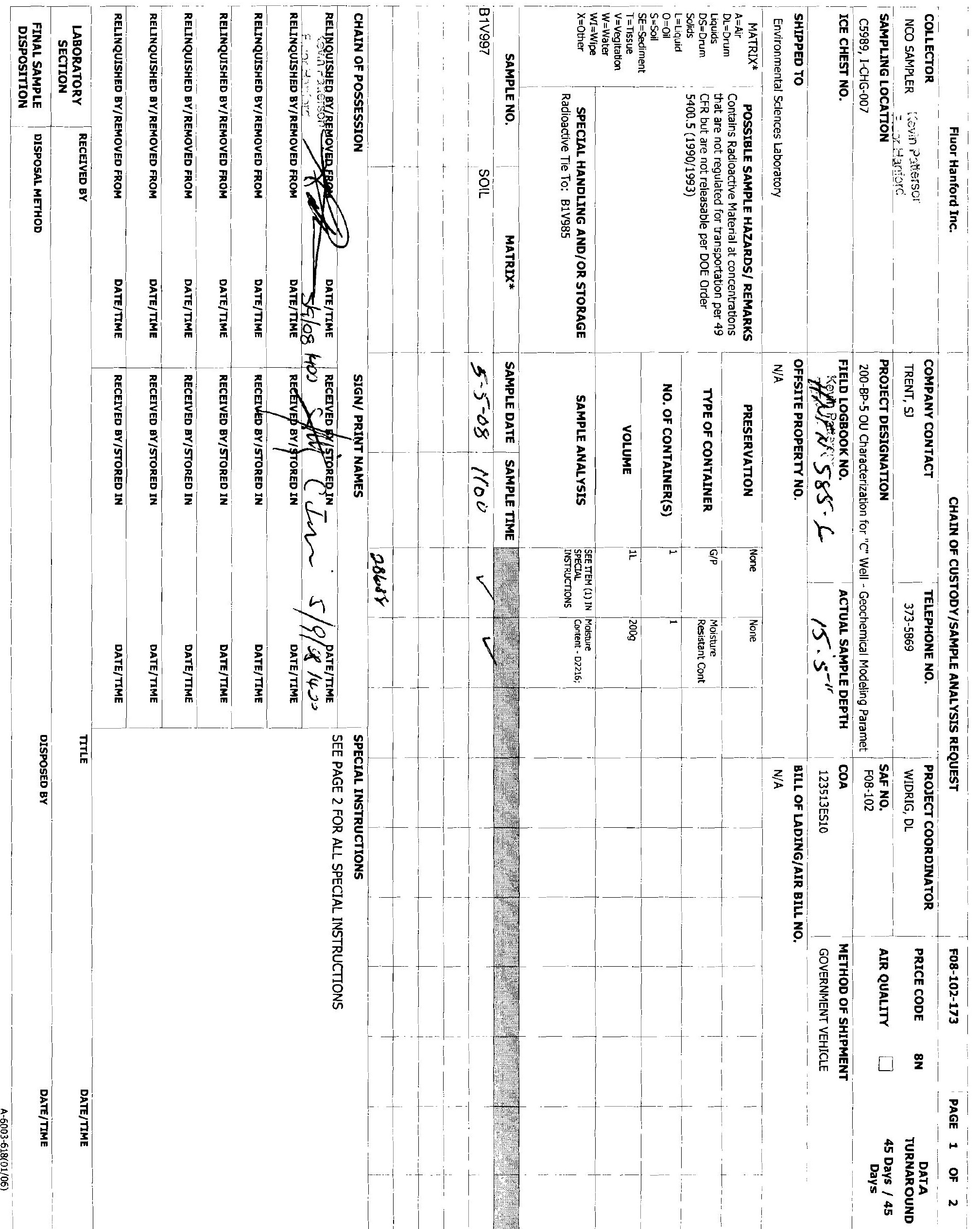




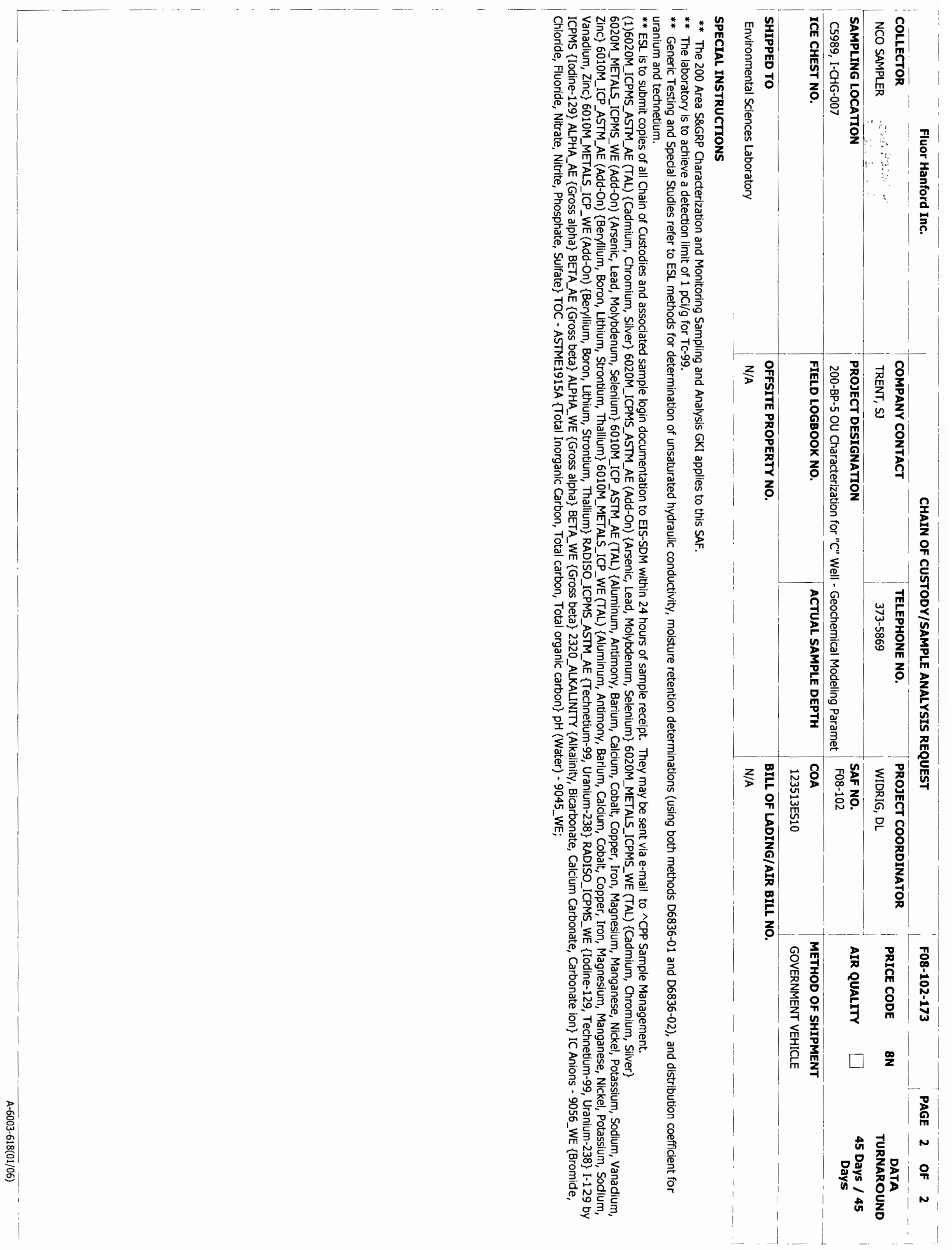




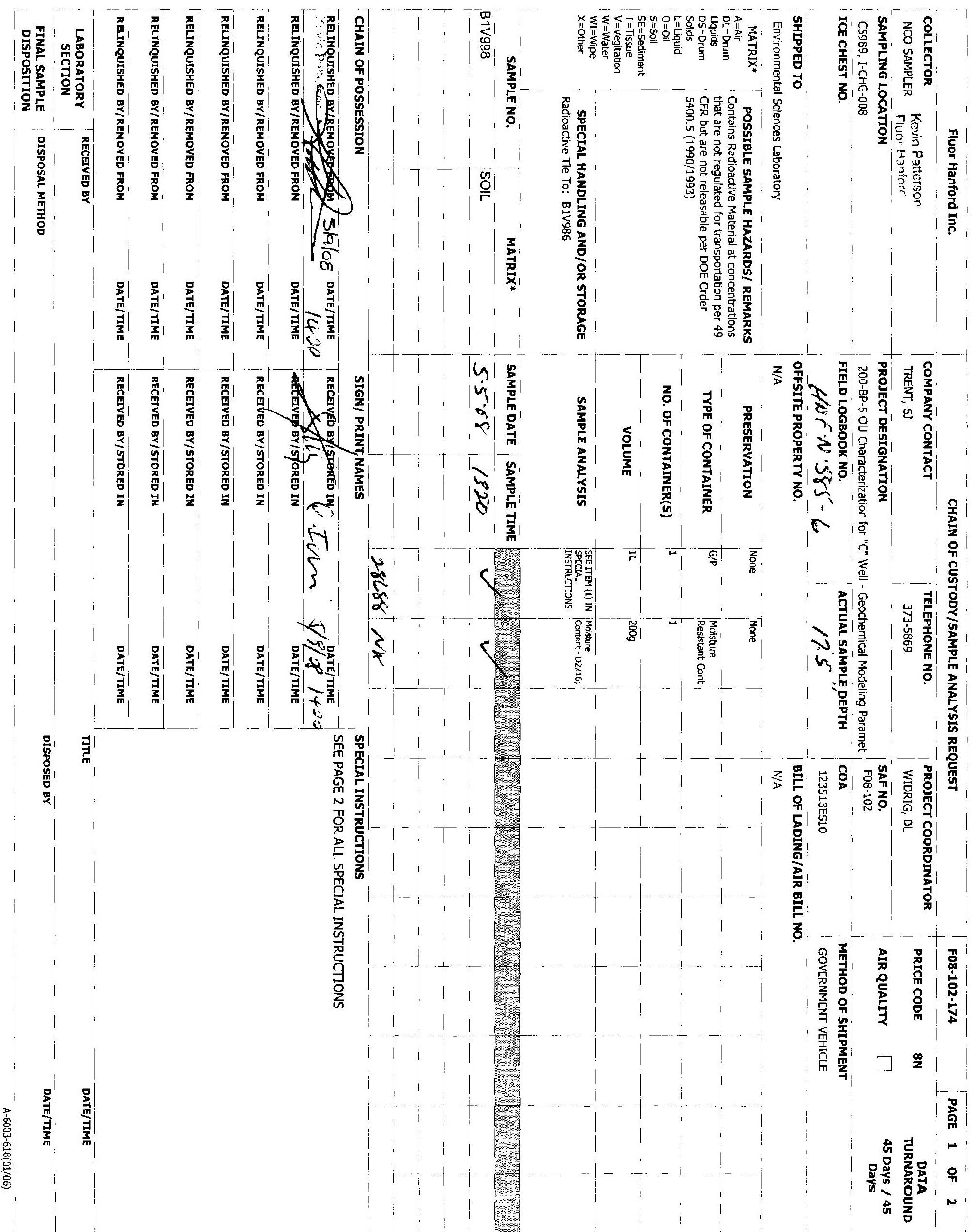




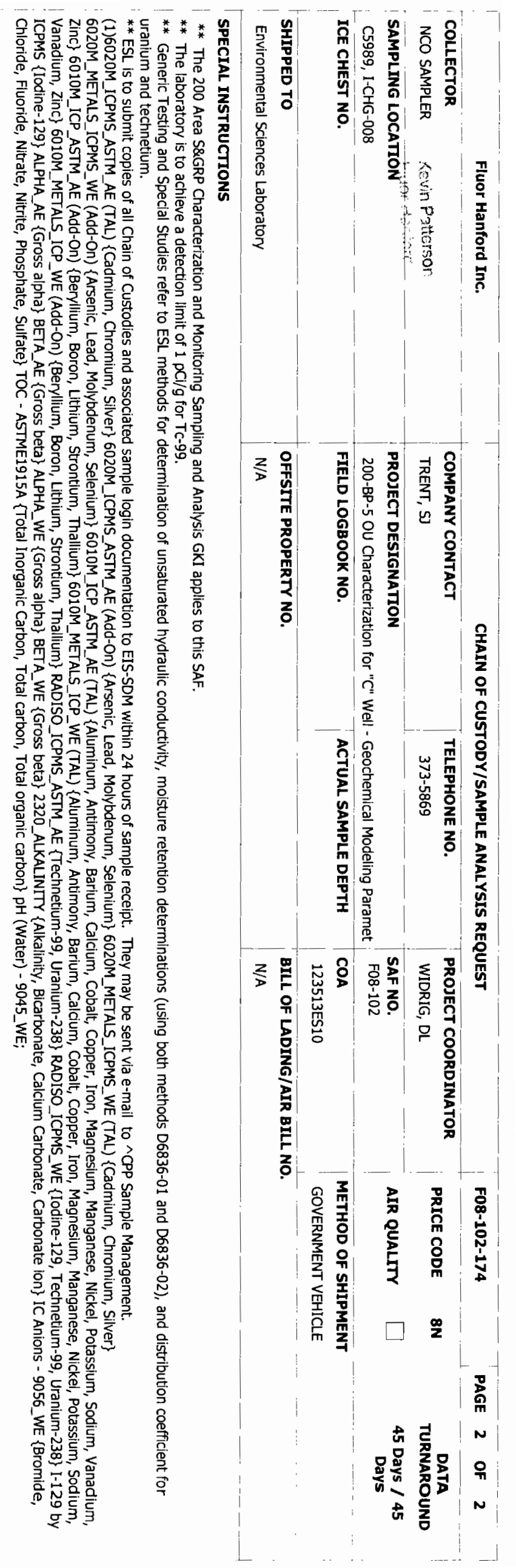




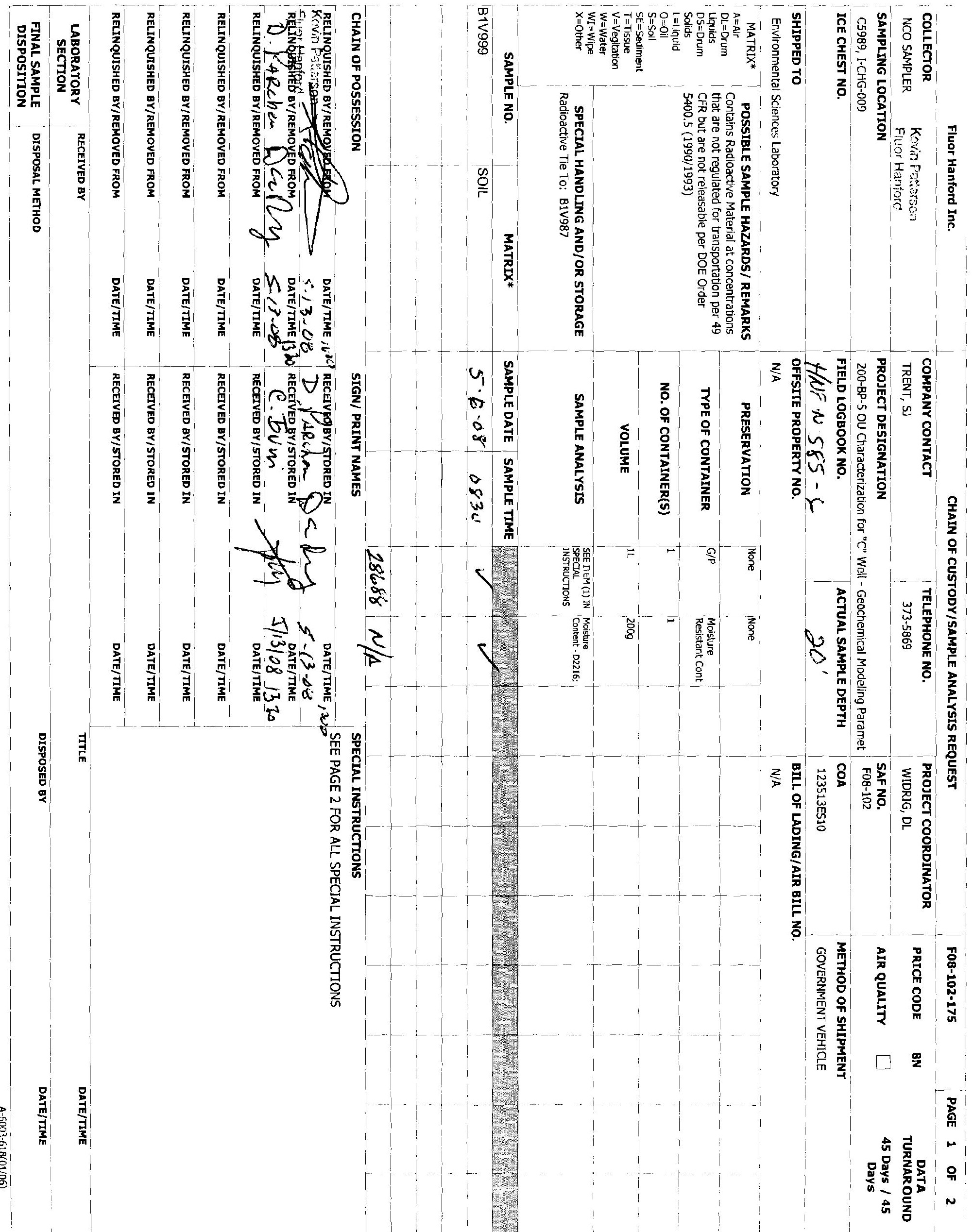




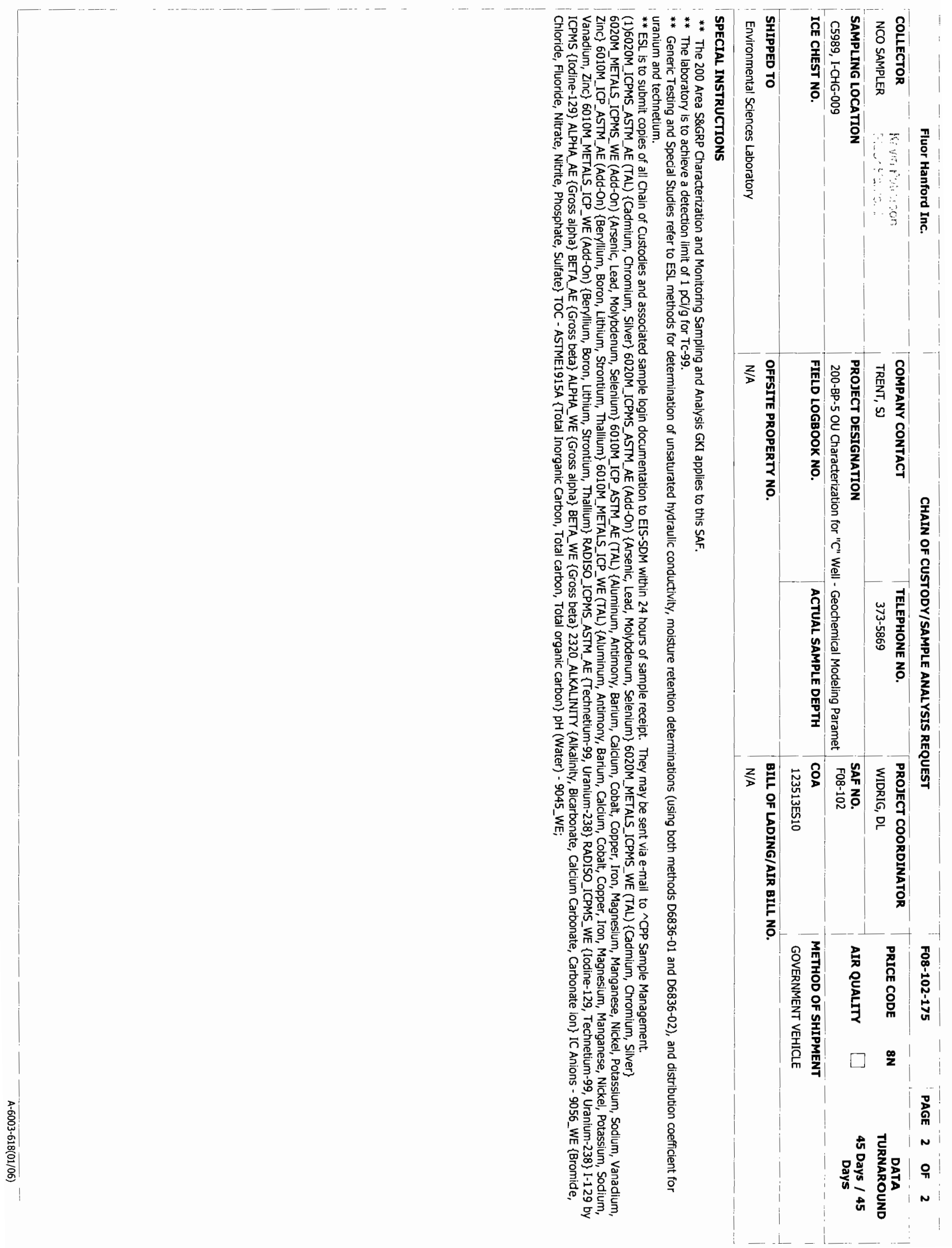




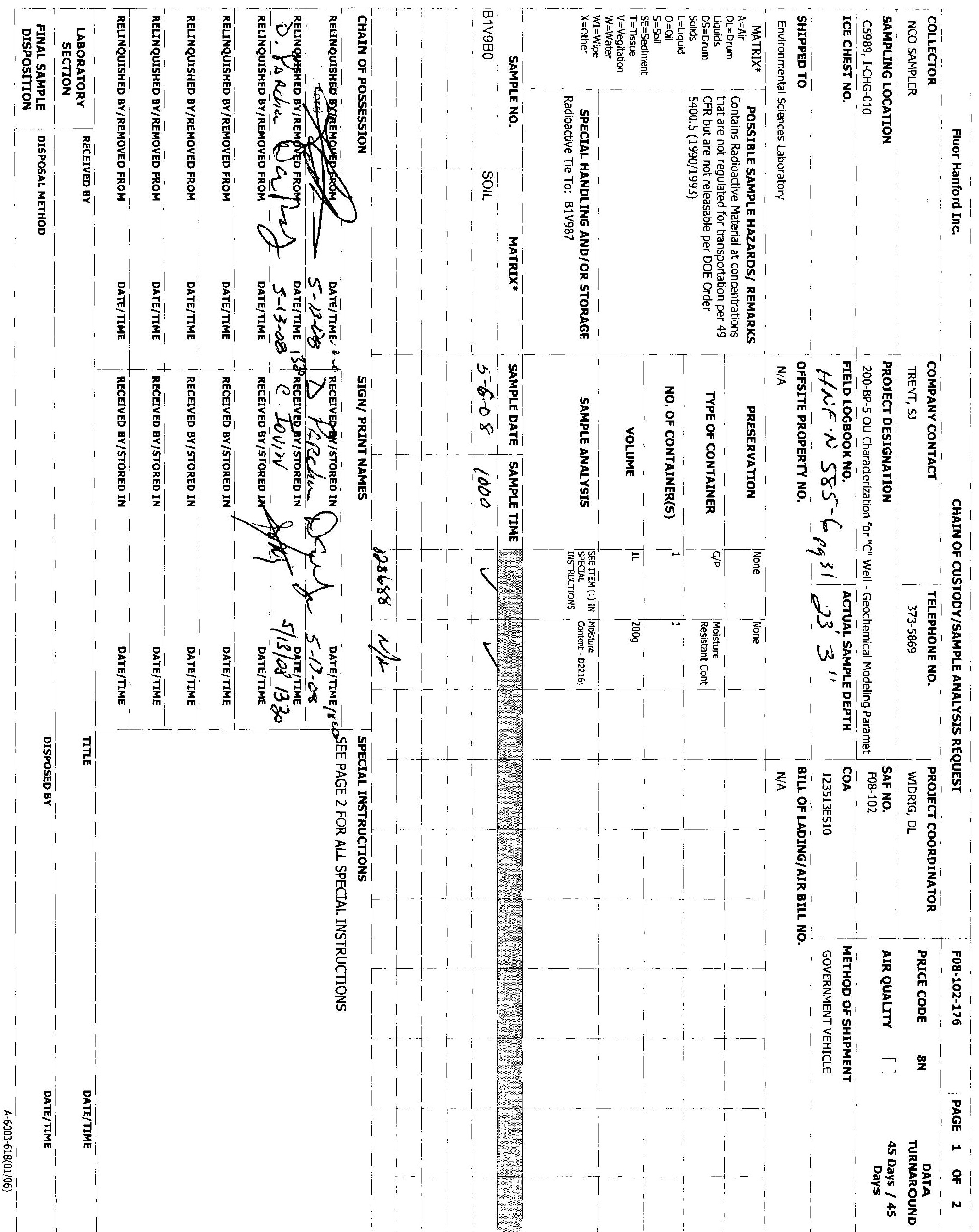



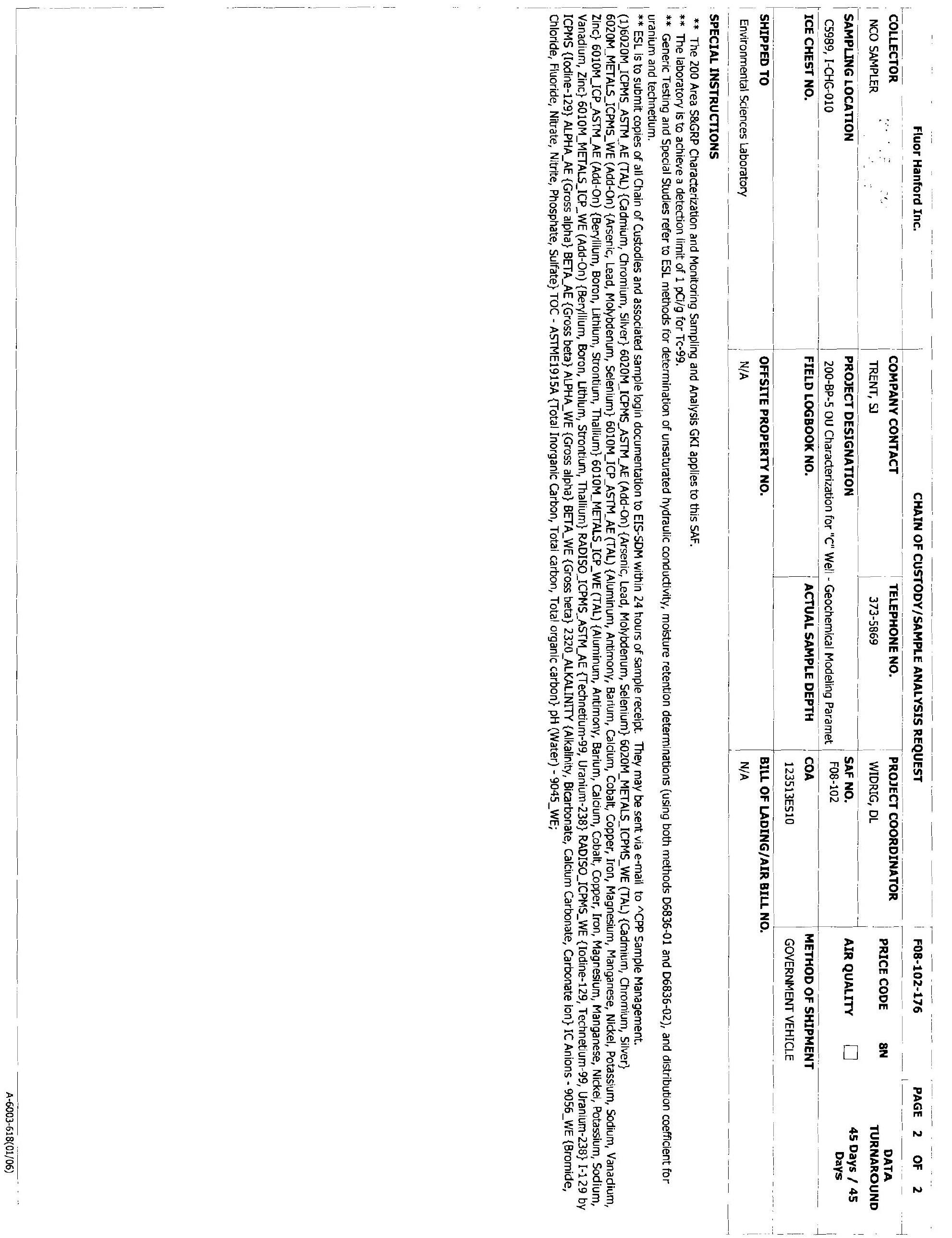


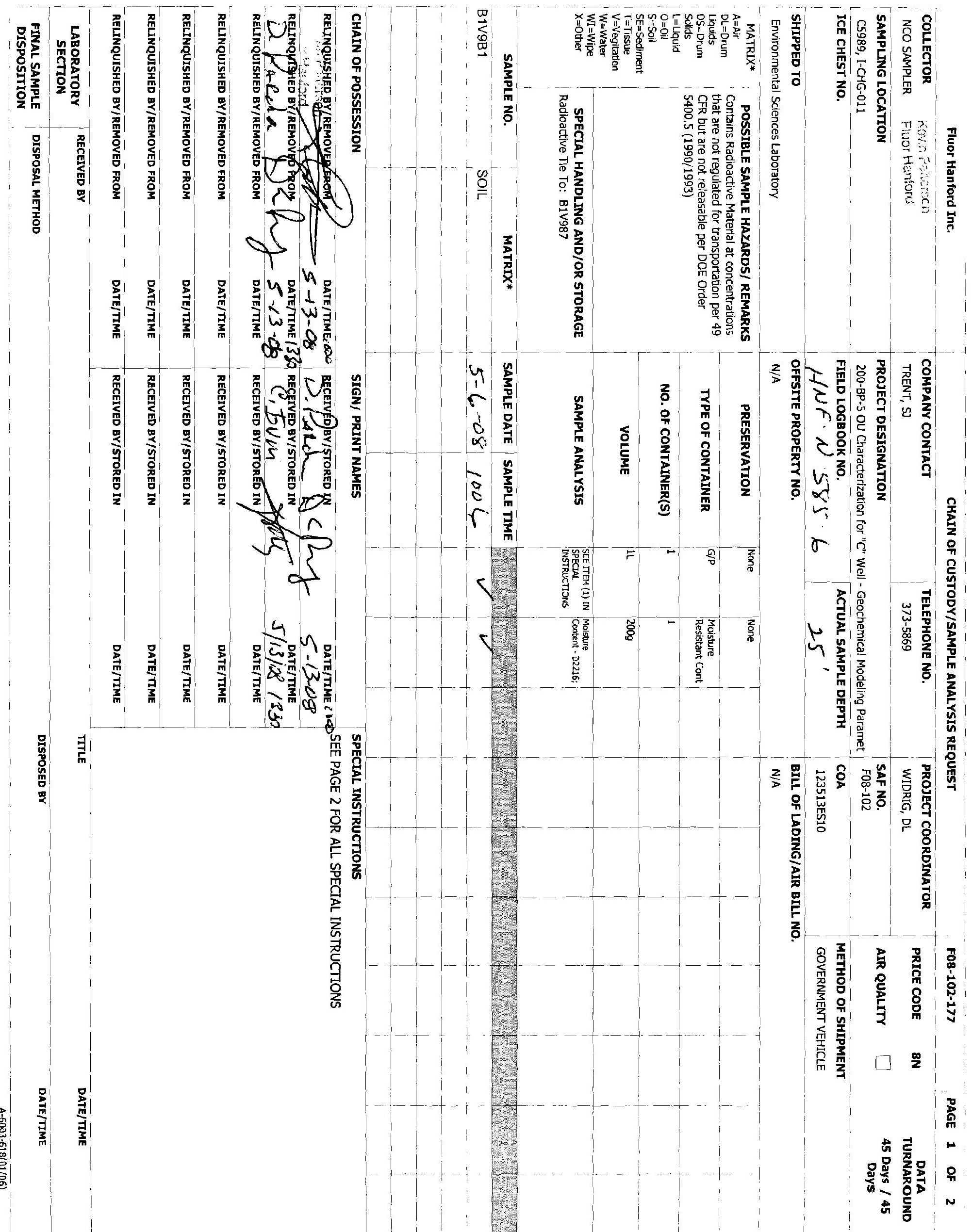




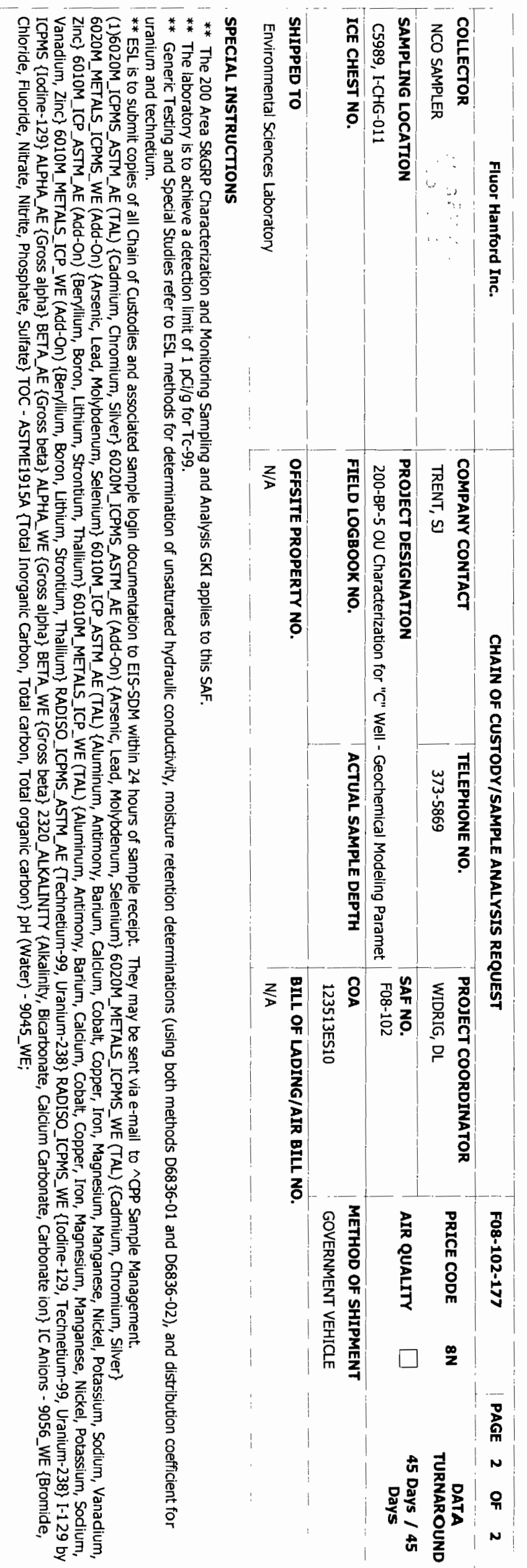




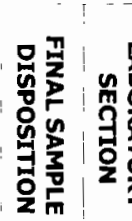
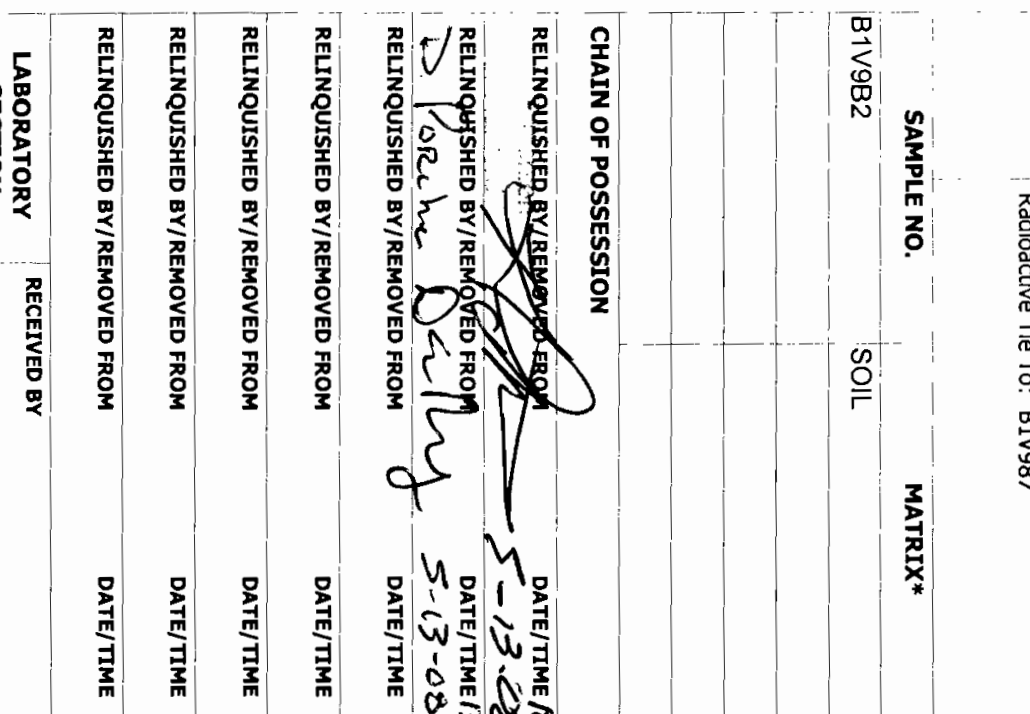

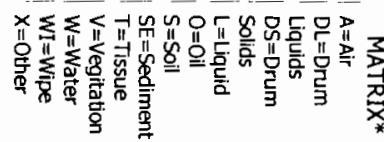

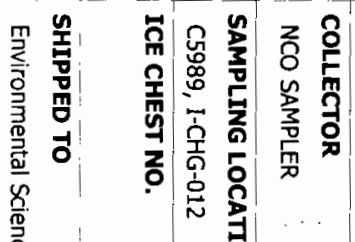

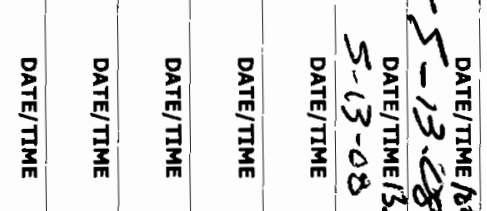
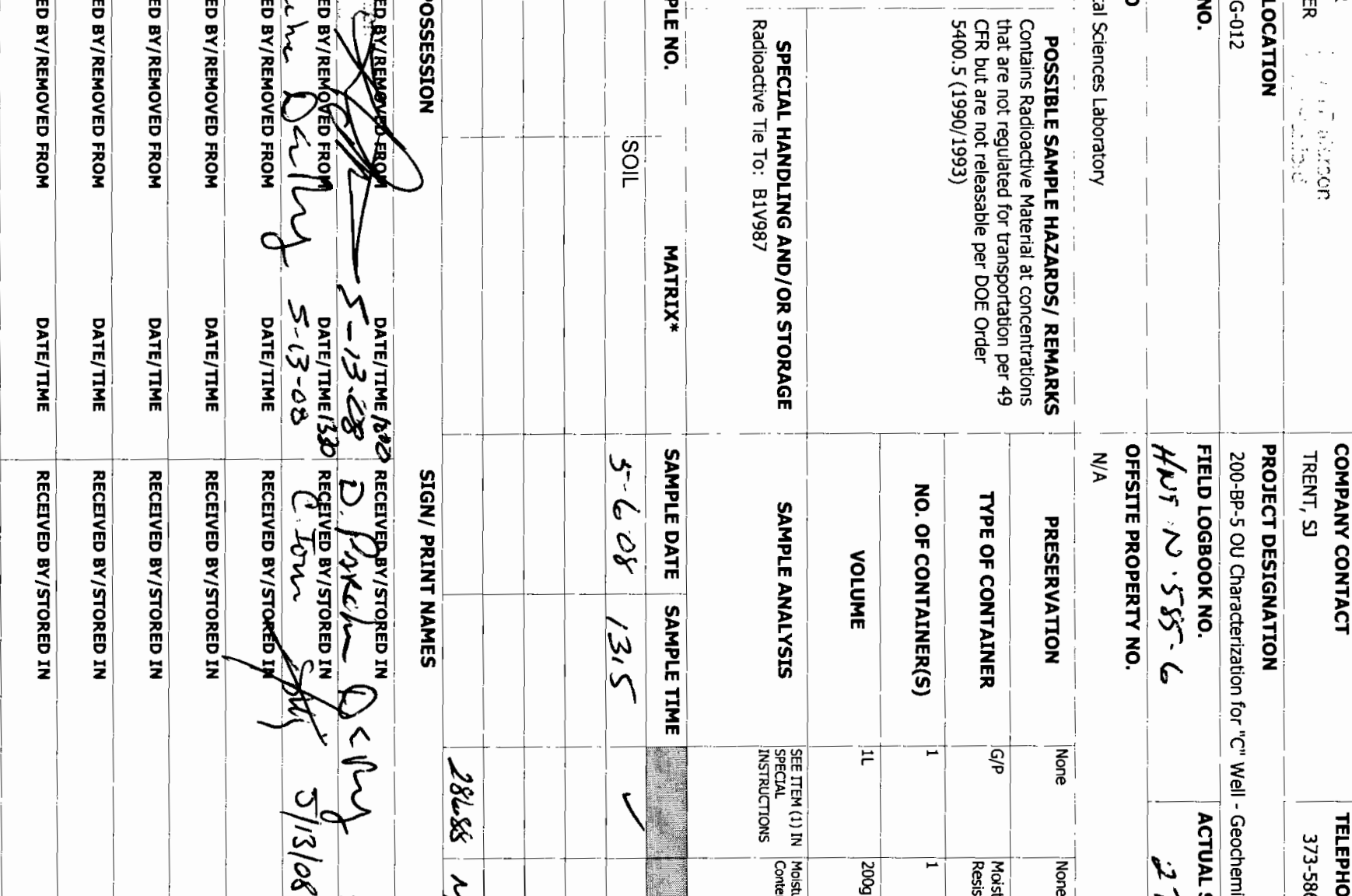

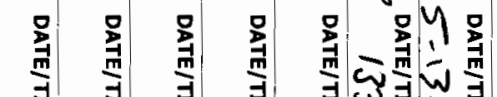
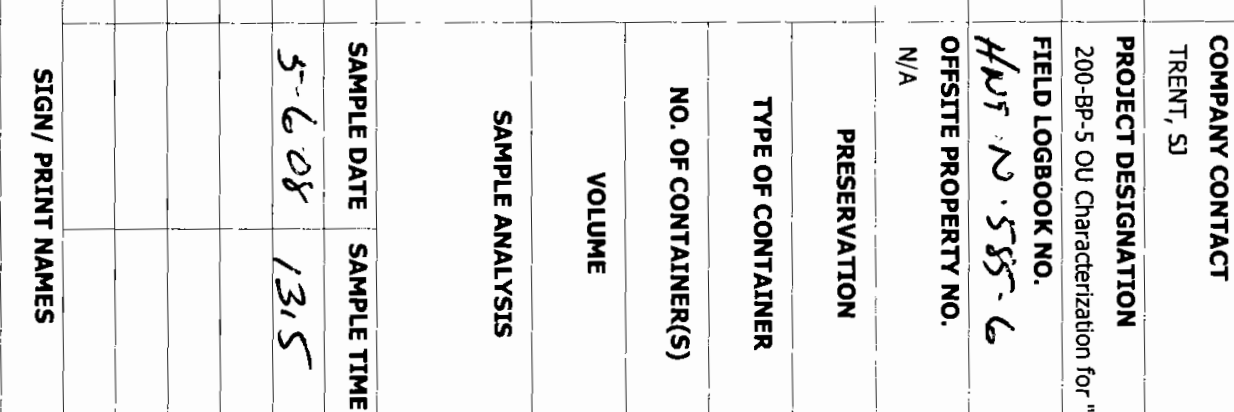

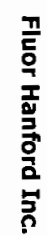

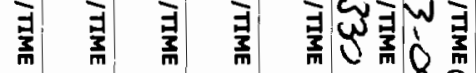

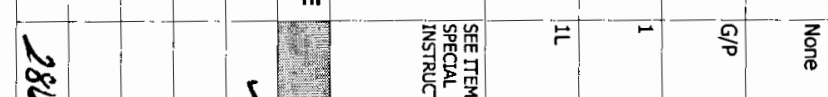

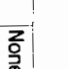

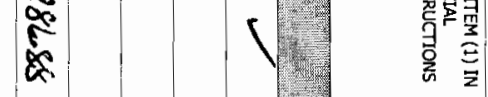

昜

(x)
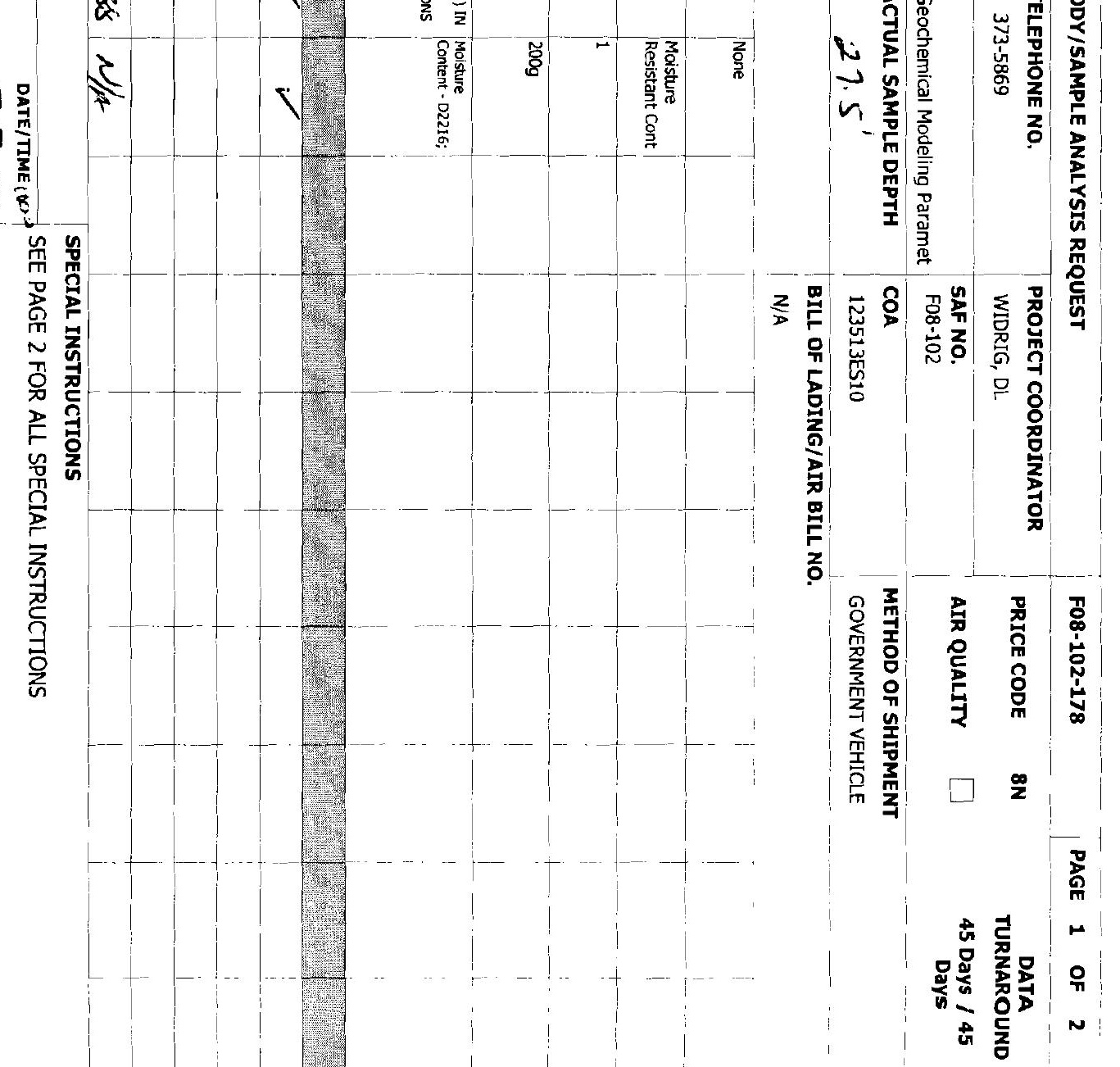


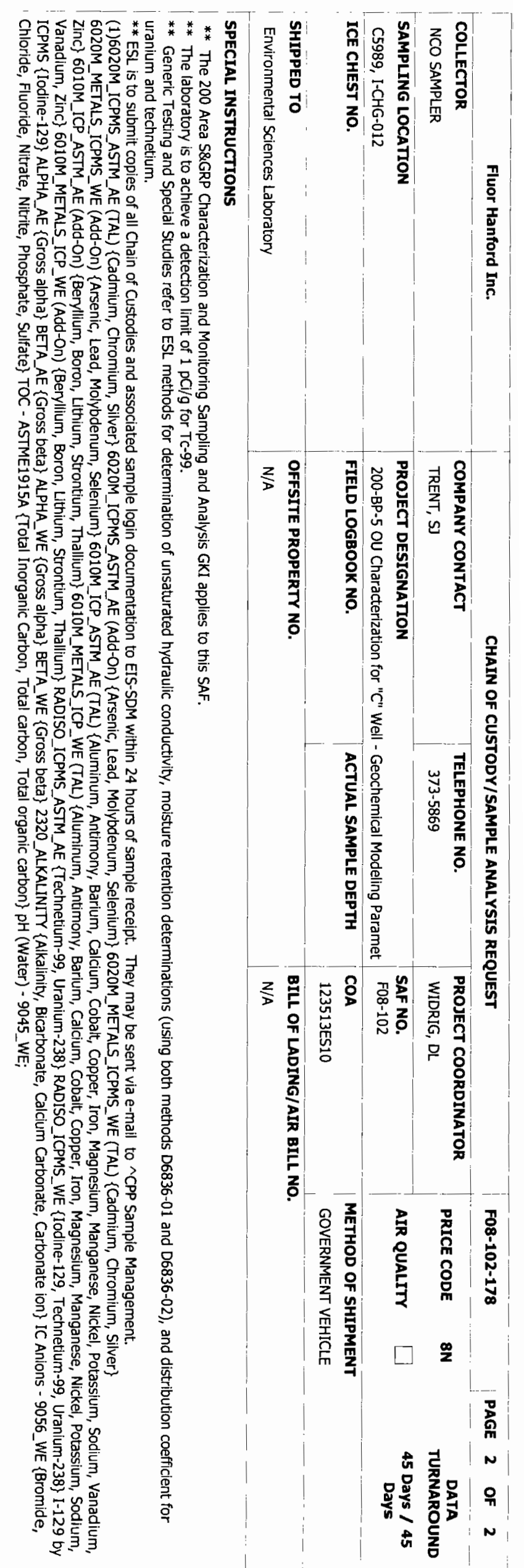




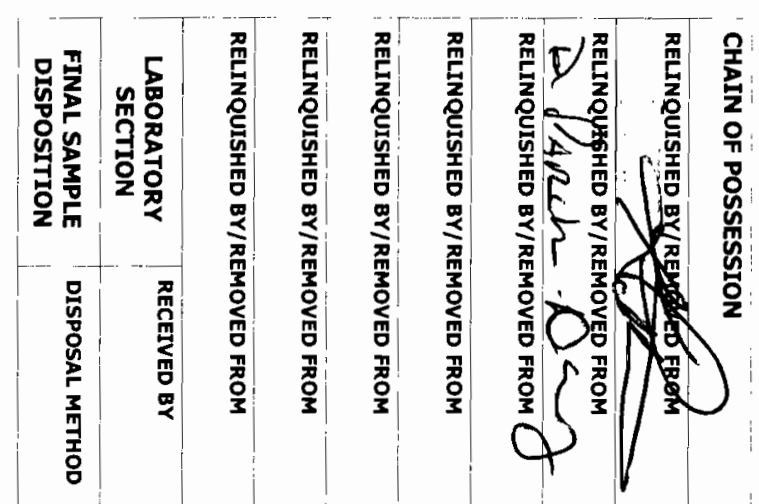

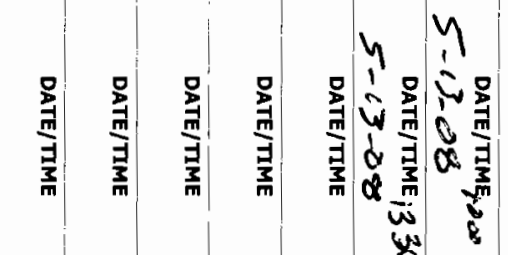
$\frac{1}{1}$

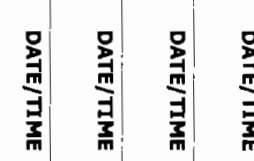

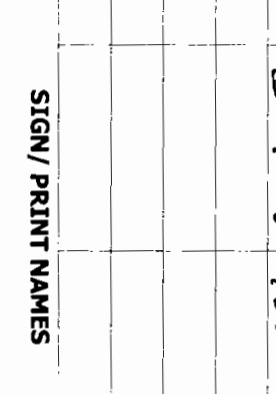

$\left|\begin{array}{l}\vec{w} \\ \mathbf{W} \\ \mathbf{W} \\ \mathbf{\omega}\end{array}\right|$

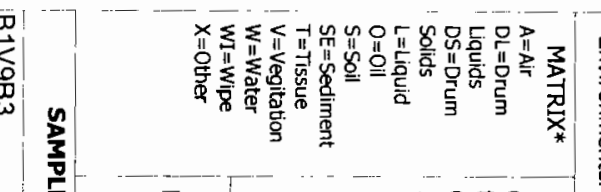

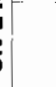

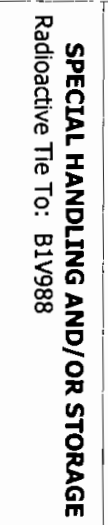
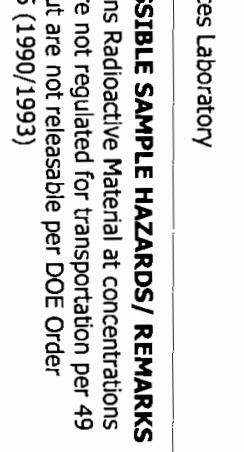

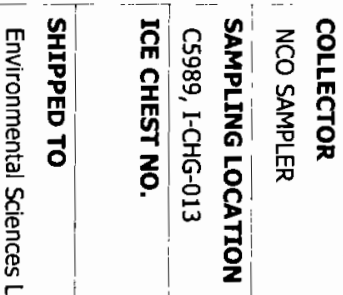

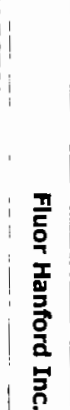

豙

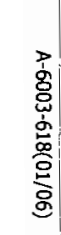

要

包

可重毫

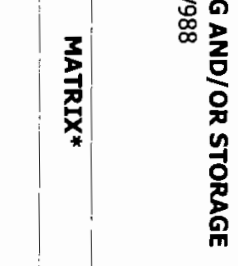

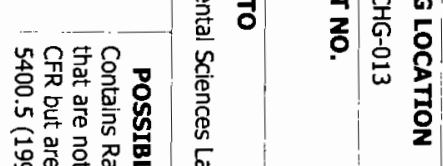

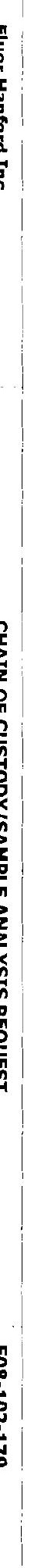

疍

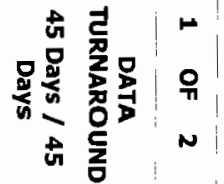




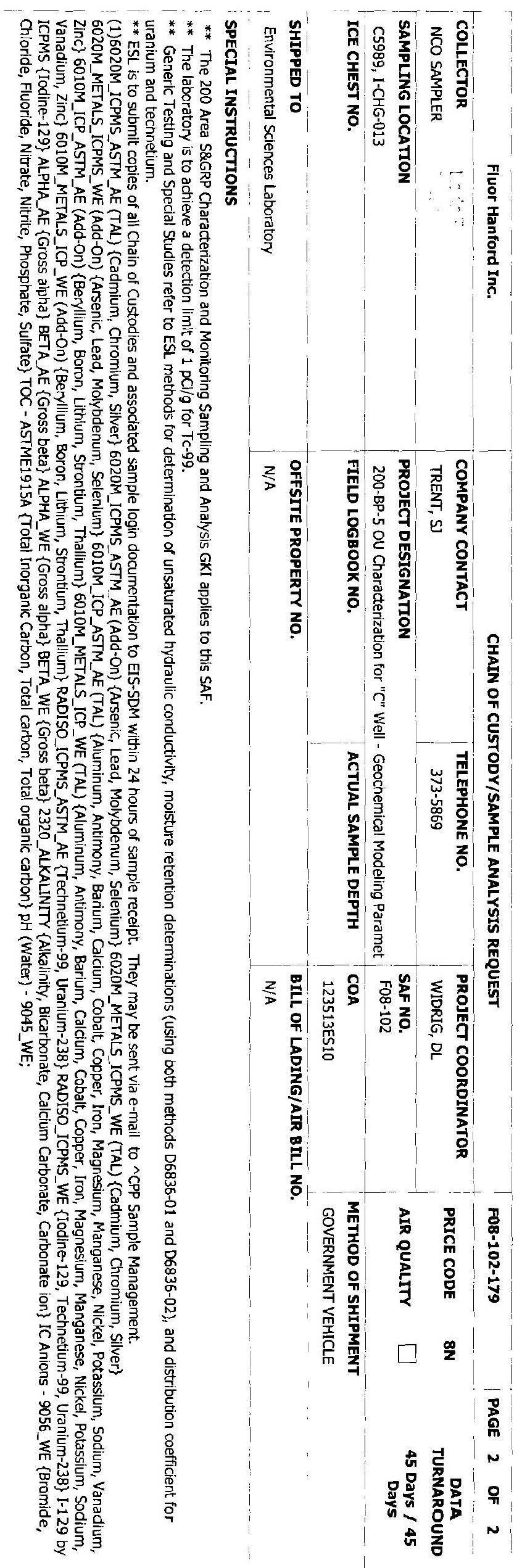




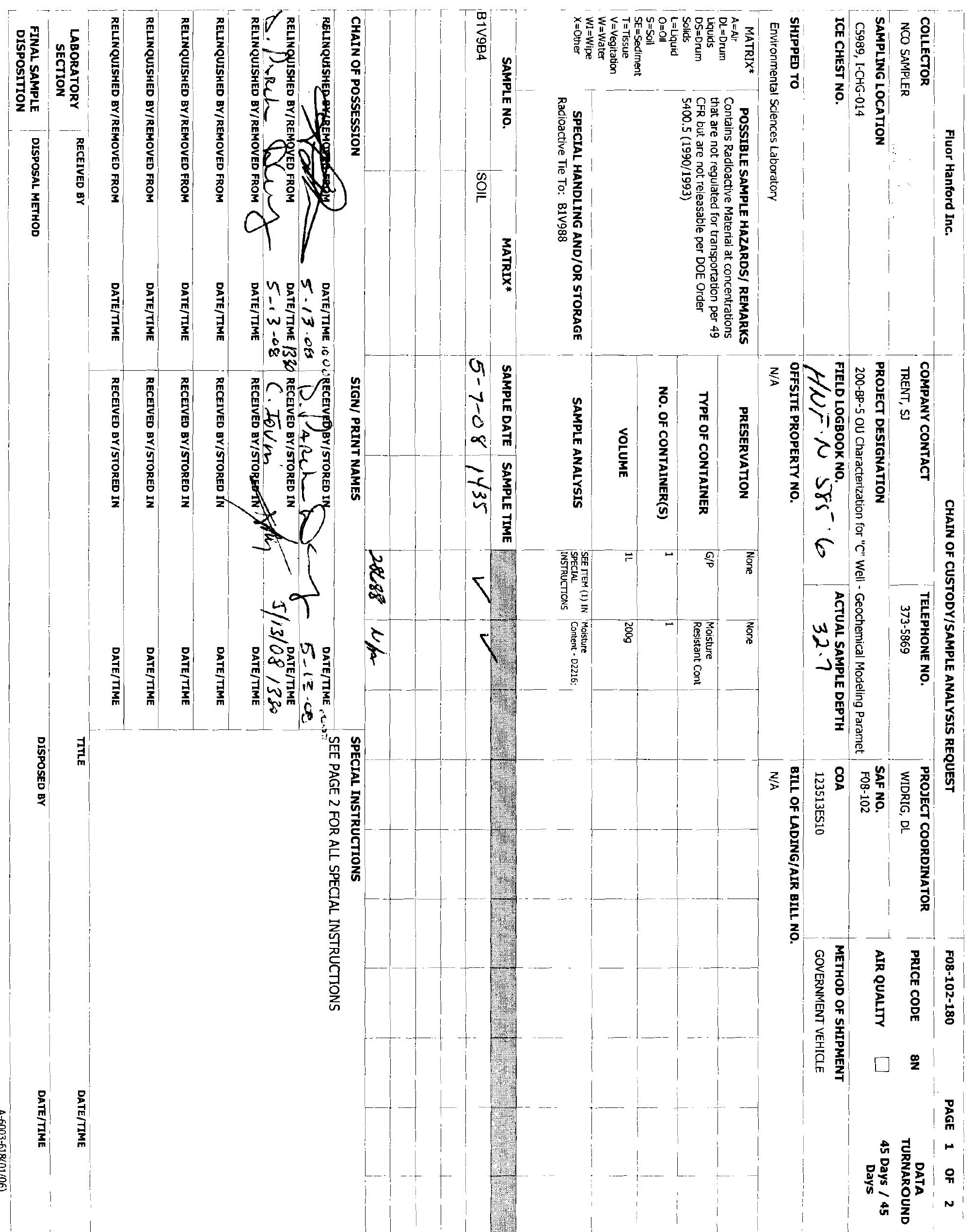




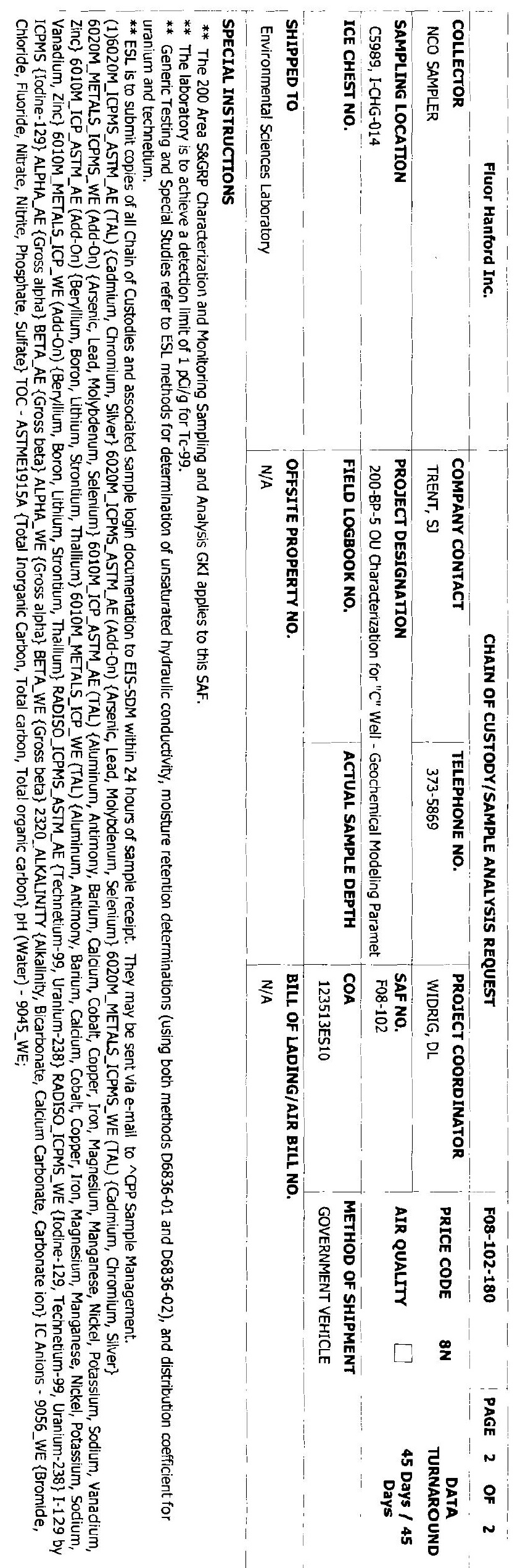




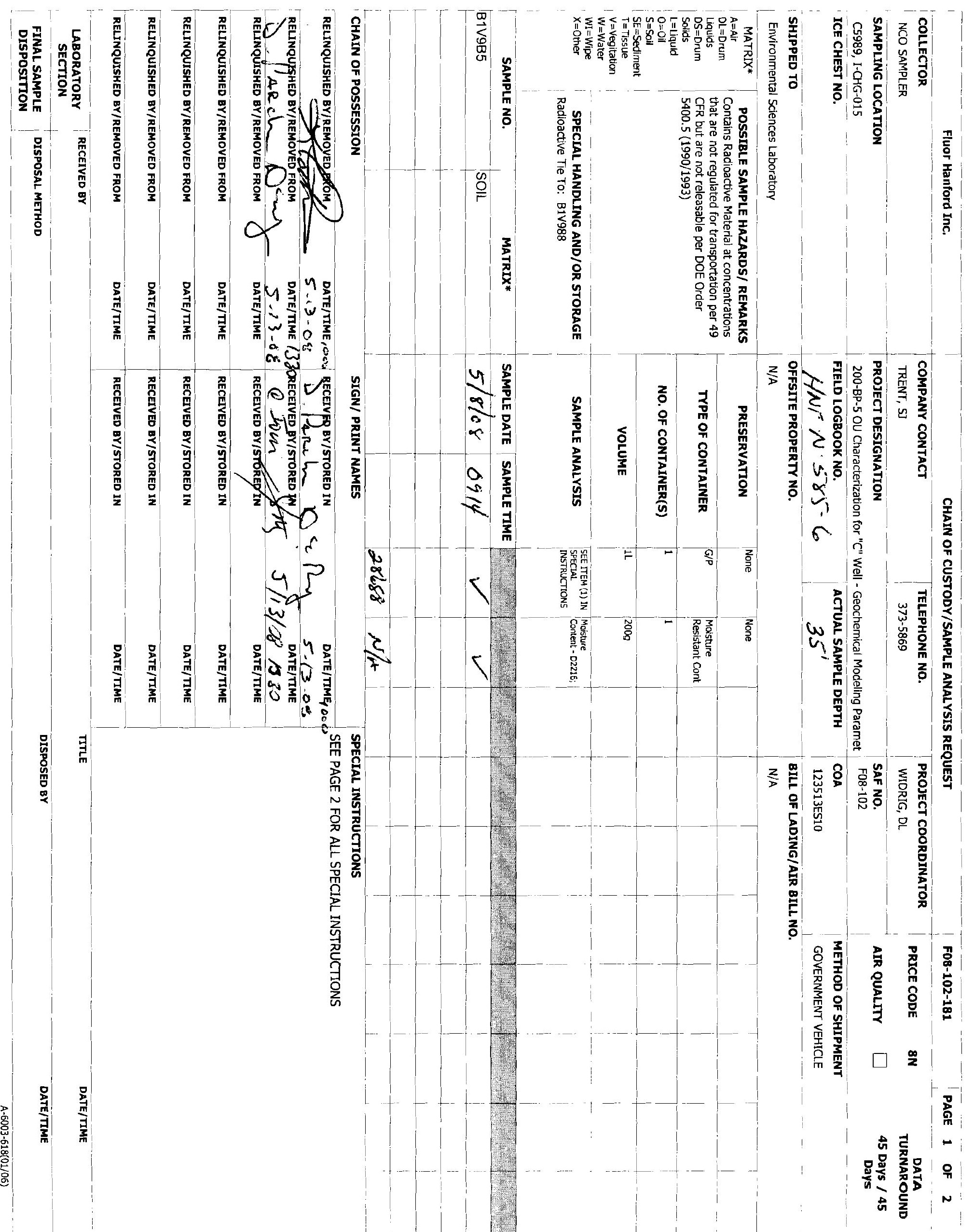




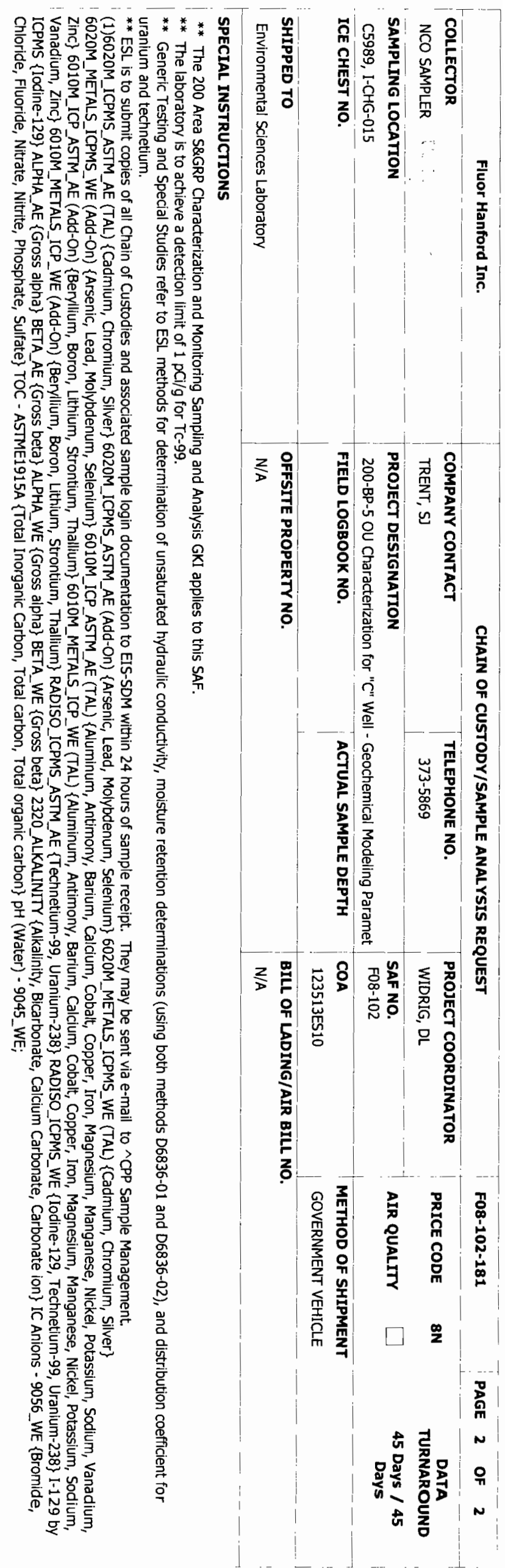




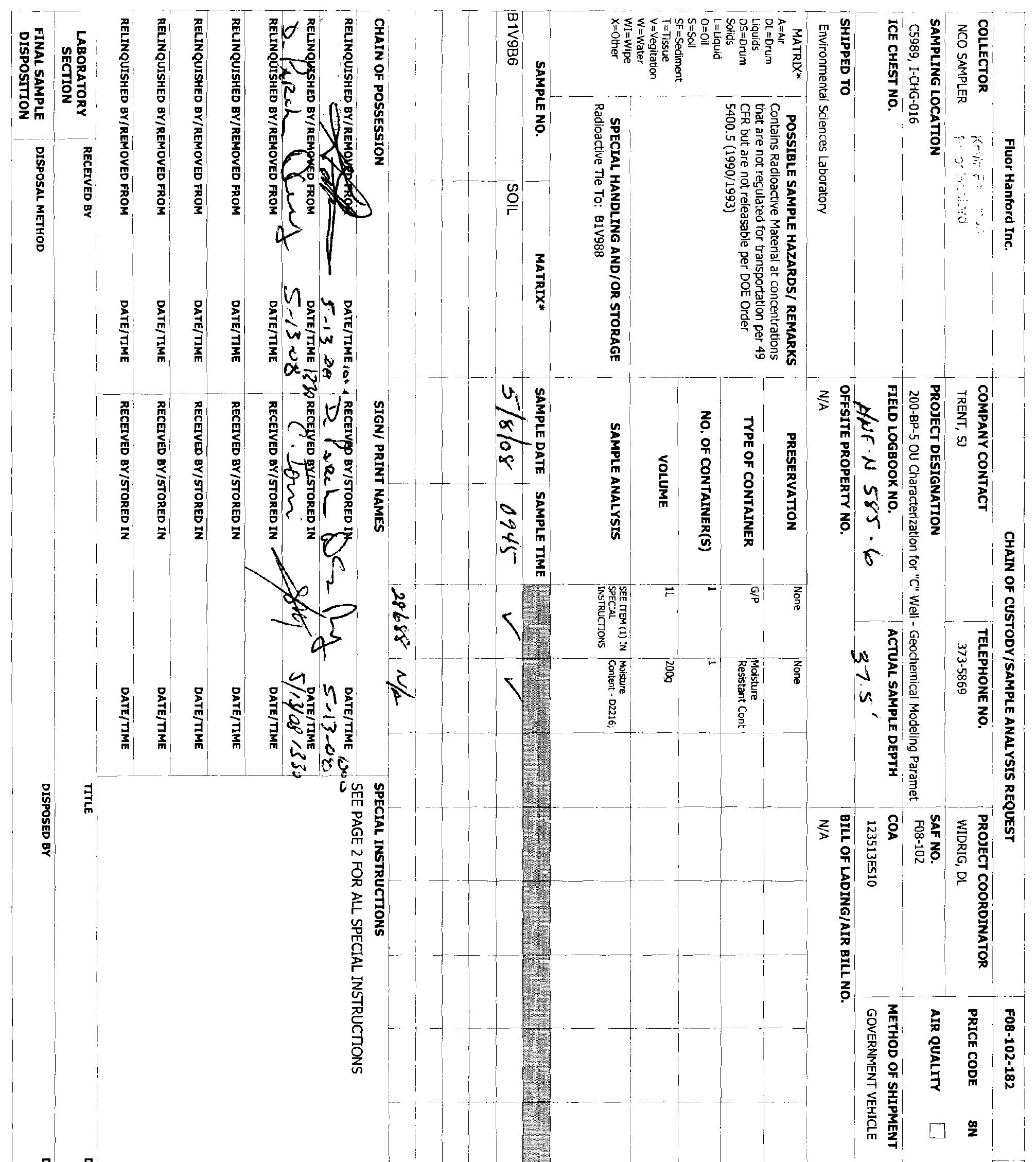

In

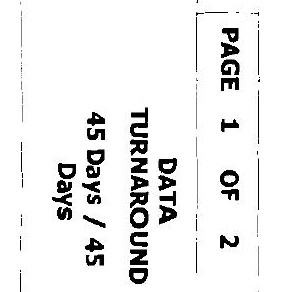




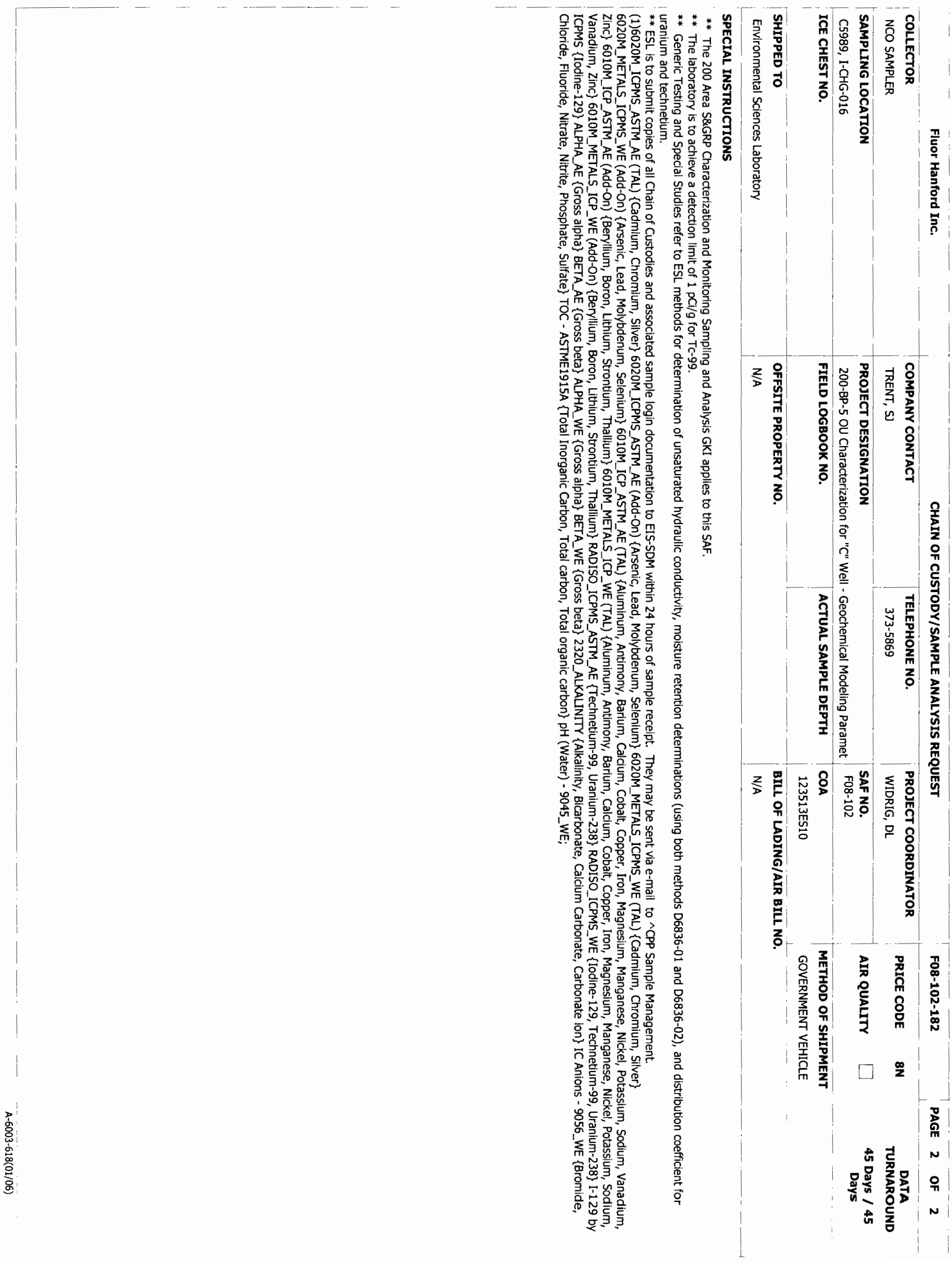




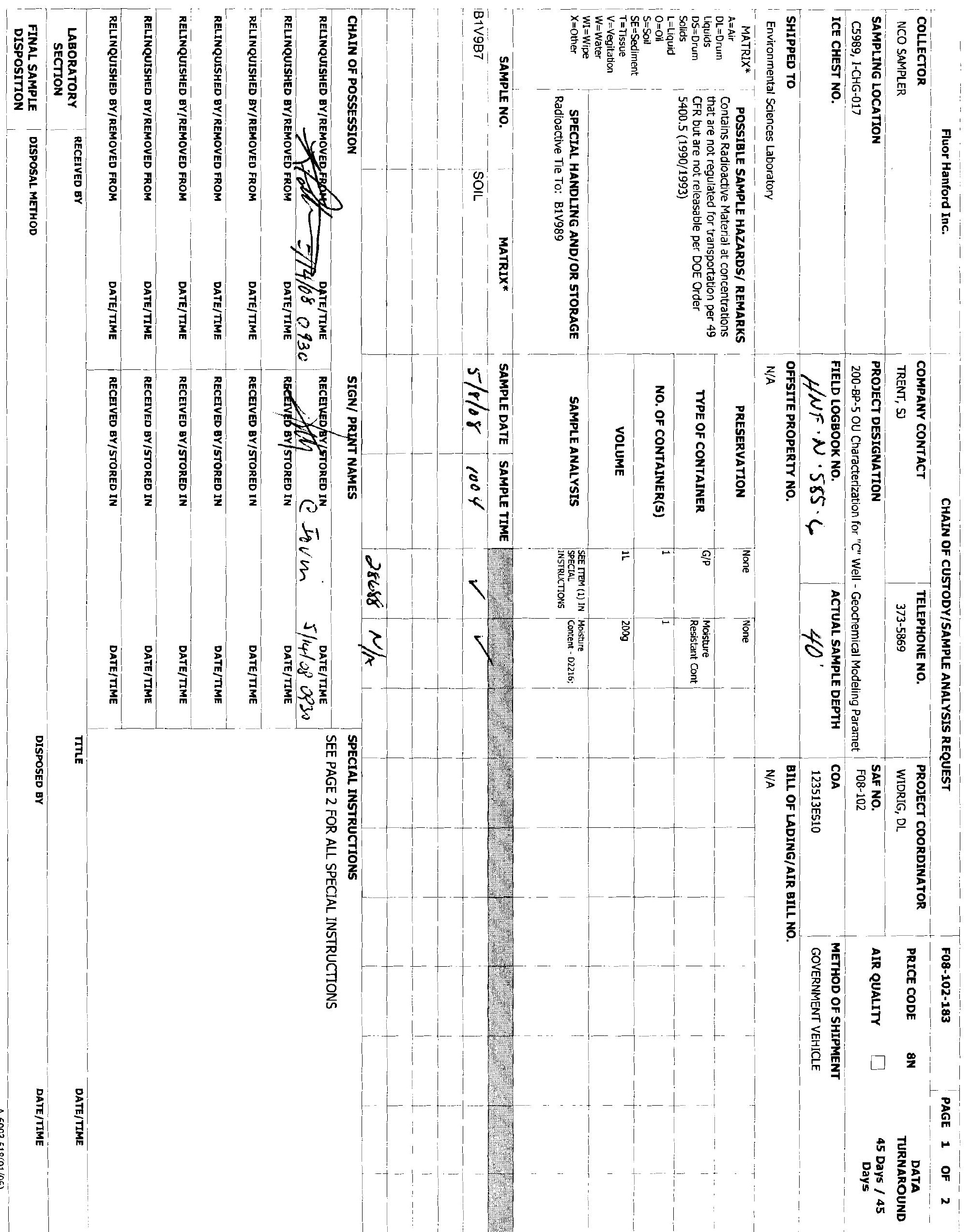




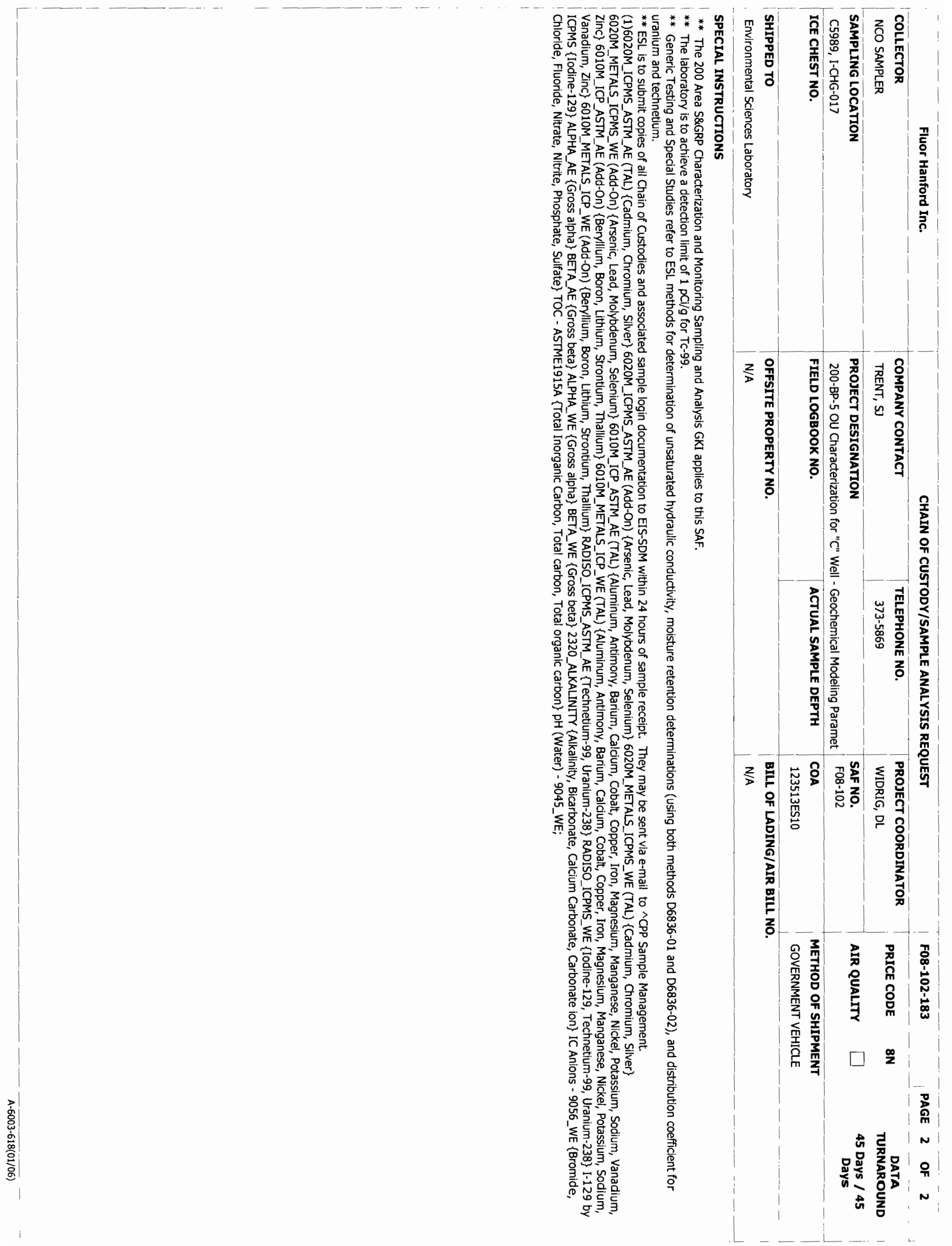



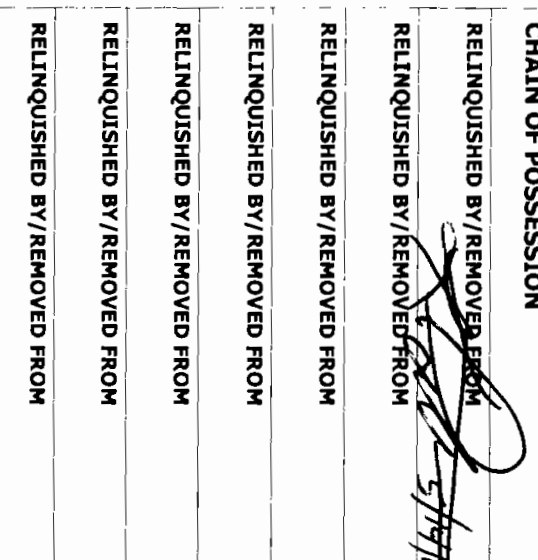

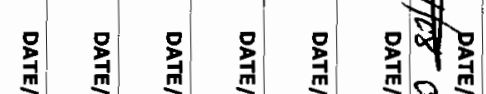

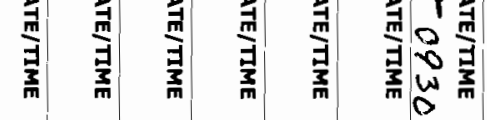

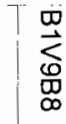

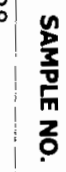

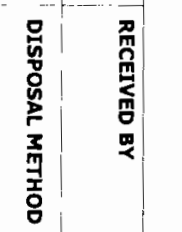

$$
m \text { m m m m m }
$$

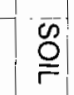

年

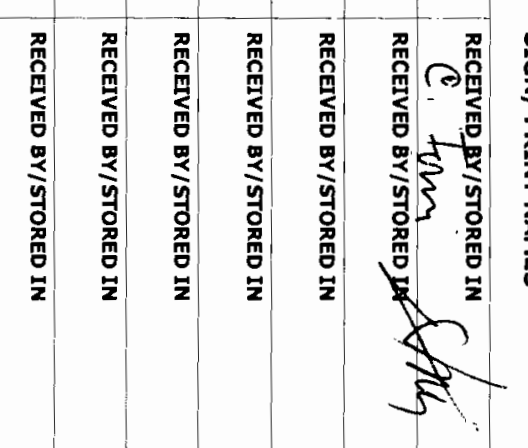

|

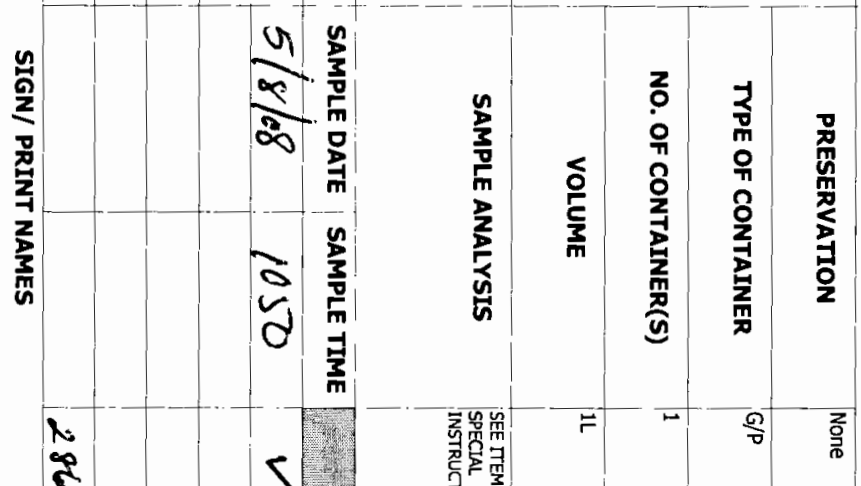

2

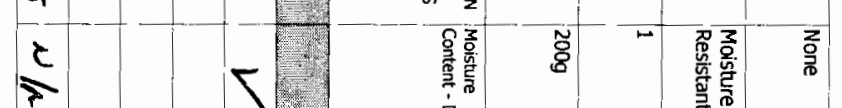

7

i

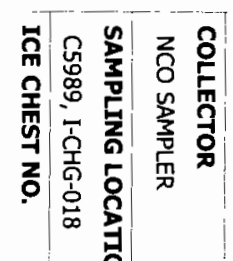

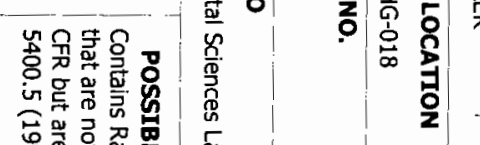

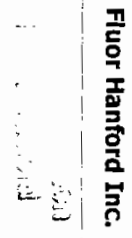

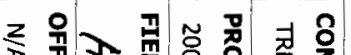

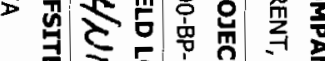

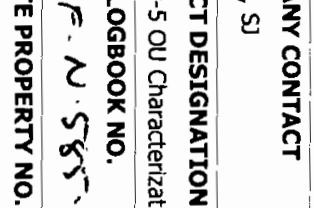
$r \mid$\begin{tabular}{l|l|l|}
\hline \\
$\stackrel{0}{\circ}$
\end{tabular} 올

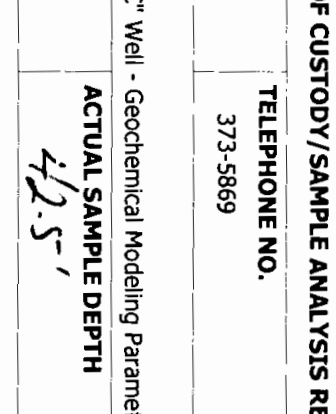

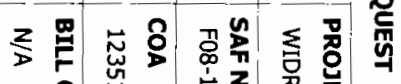

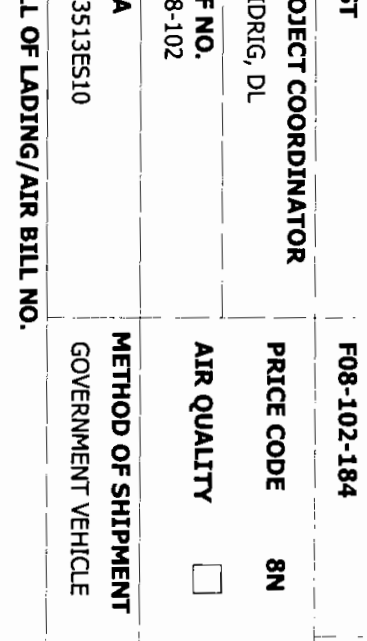
章

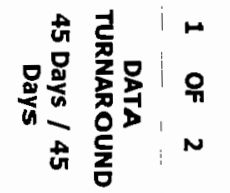




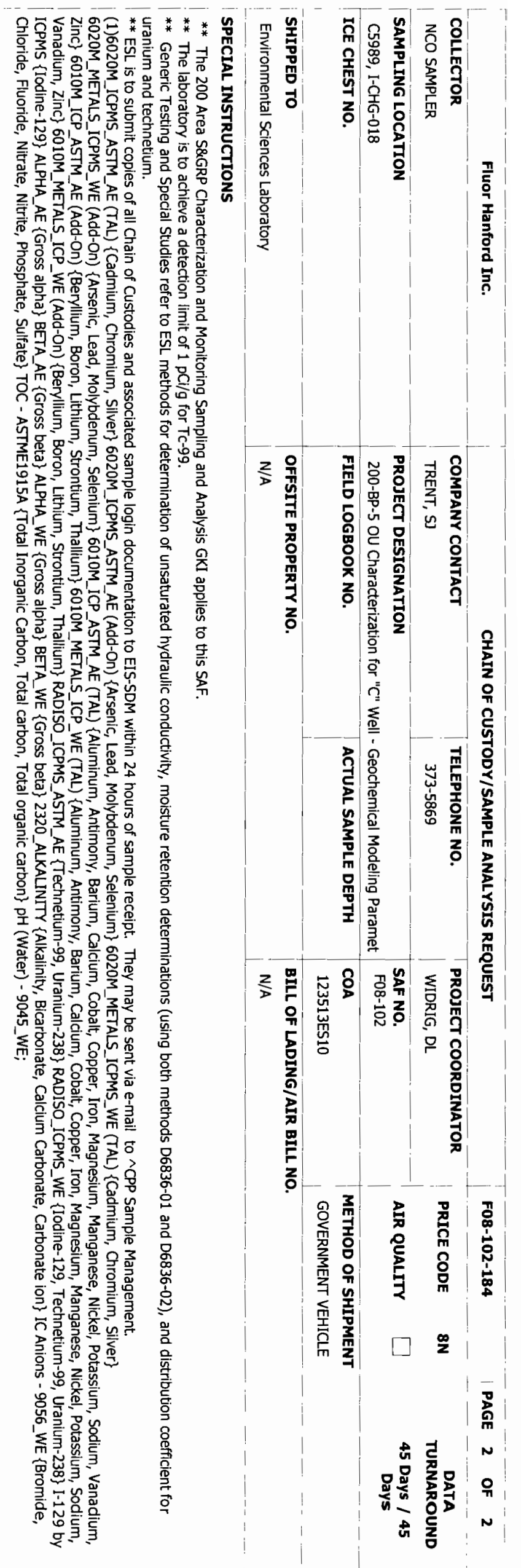




\section{|lin

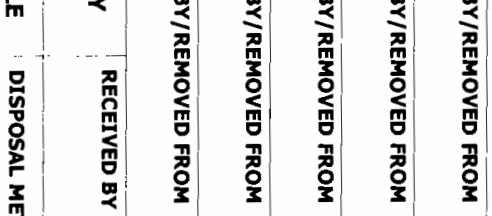 \\ 좀}
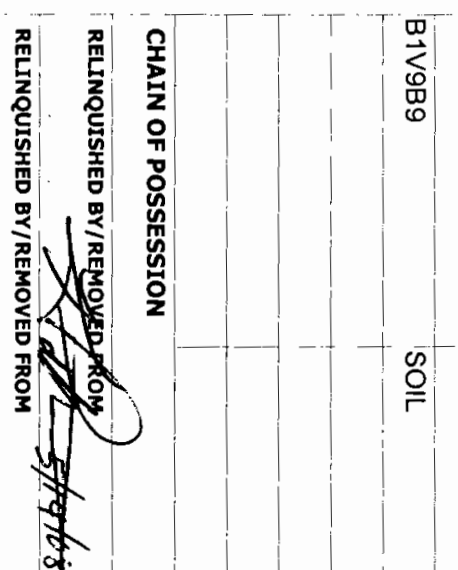

\begin{tabular}{|l}
$\mid$ \\
\\
$n$ \\
$\mathbf{z}$ \\
$\mathbf{z}$ \\
$\mathbf{n}$ \\
$\mathbf{z}$ \\
$\mathbf{n}$
\end{tabular}

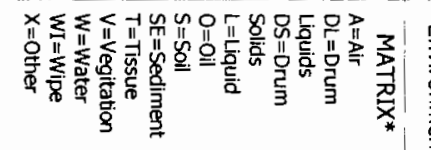

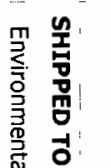

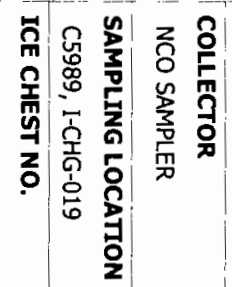

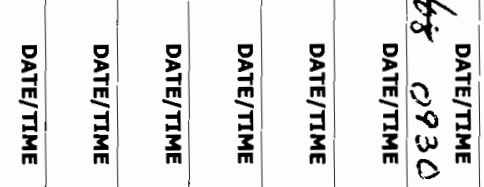

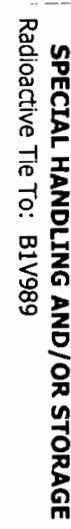

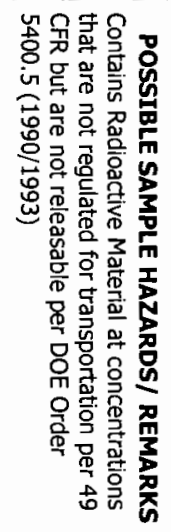

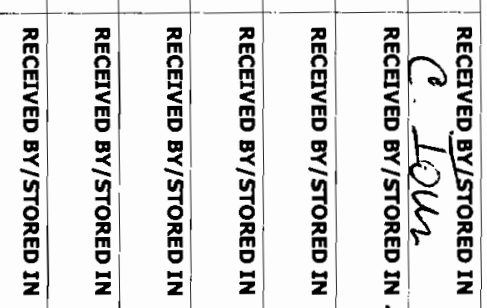
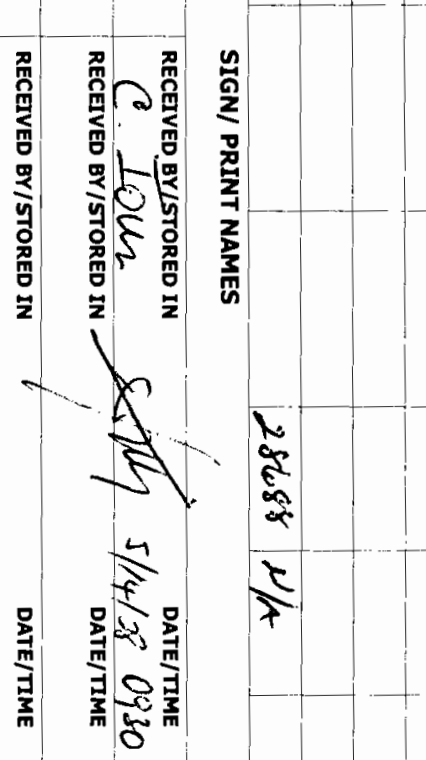

0
0
0
0
0
0

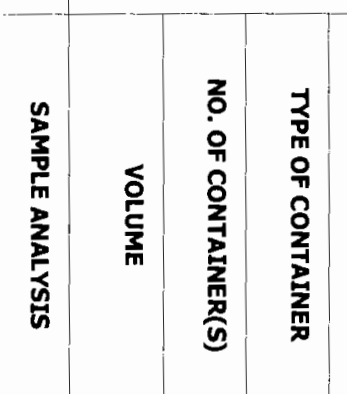

ב.

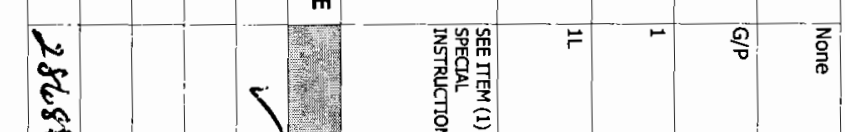

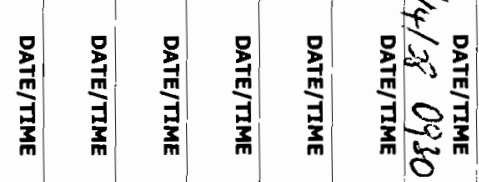
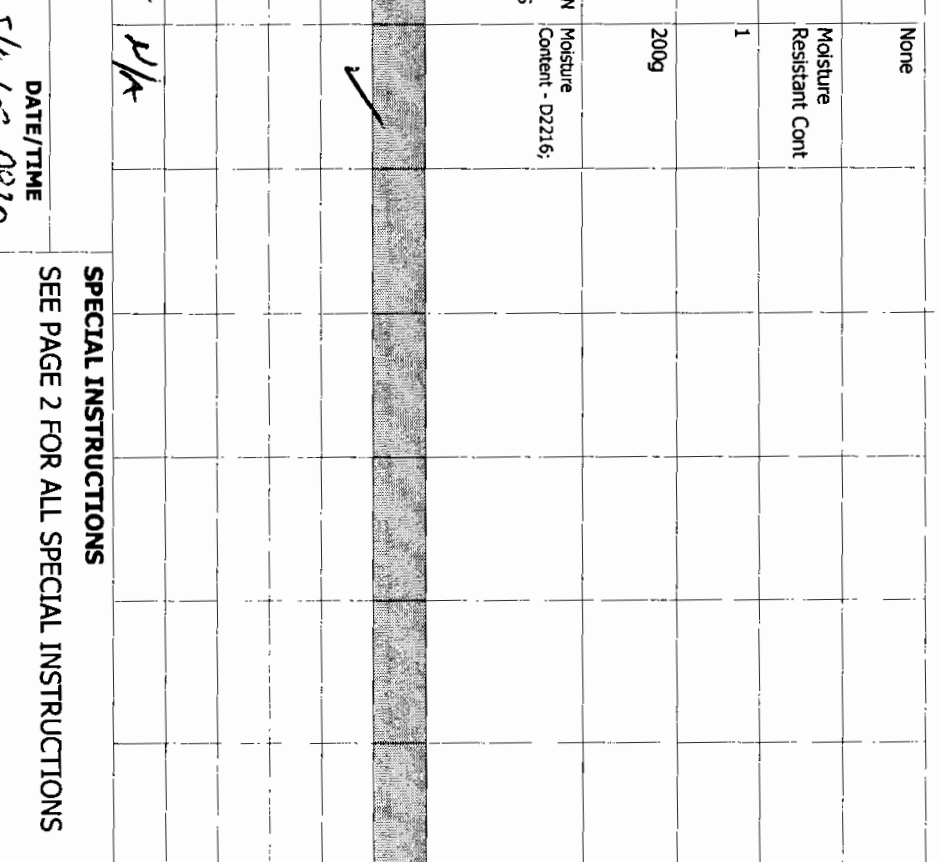

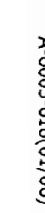

星

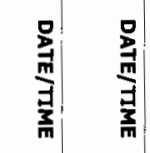

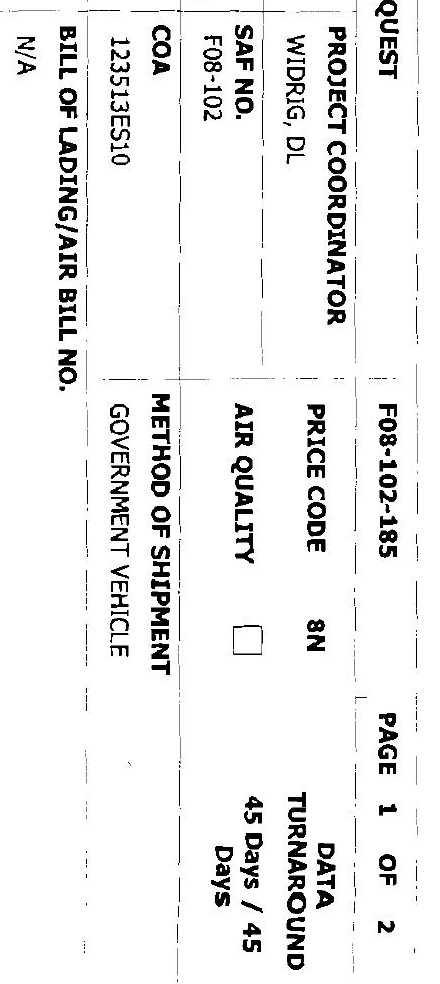




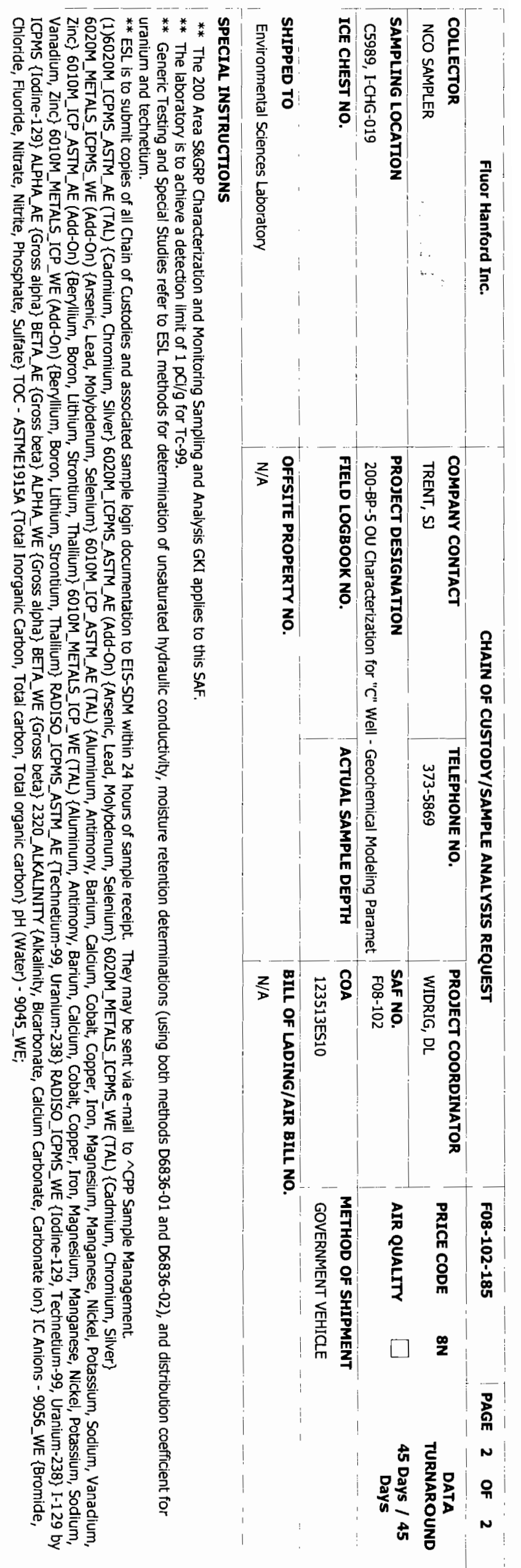




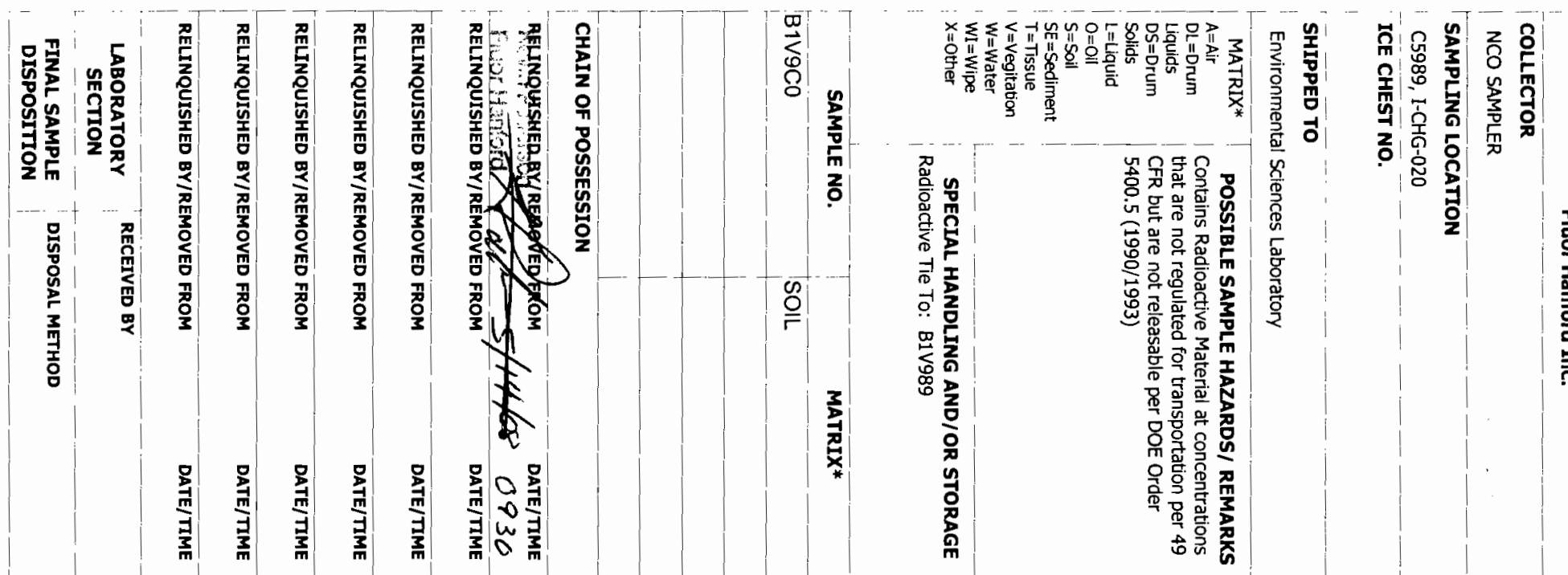

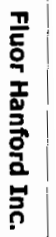

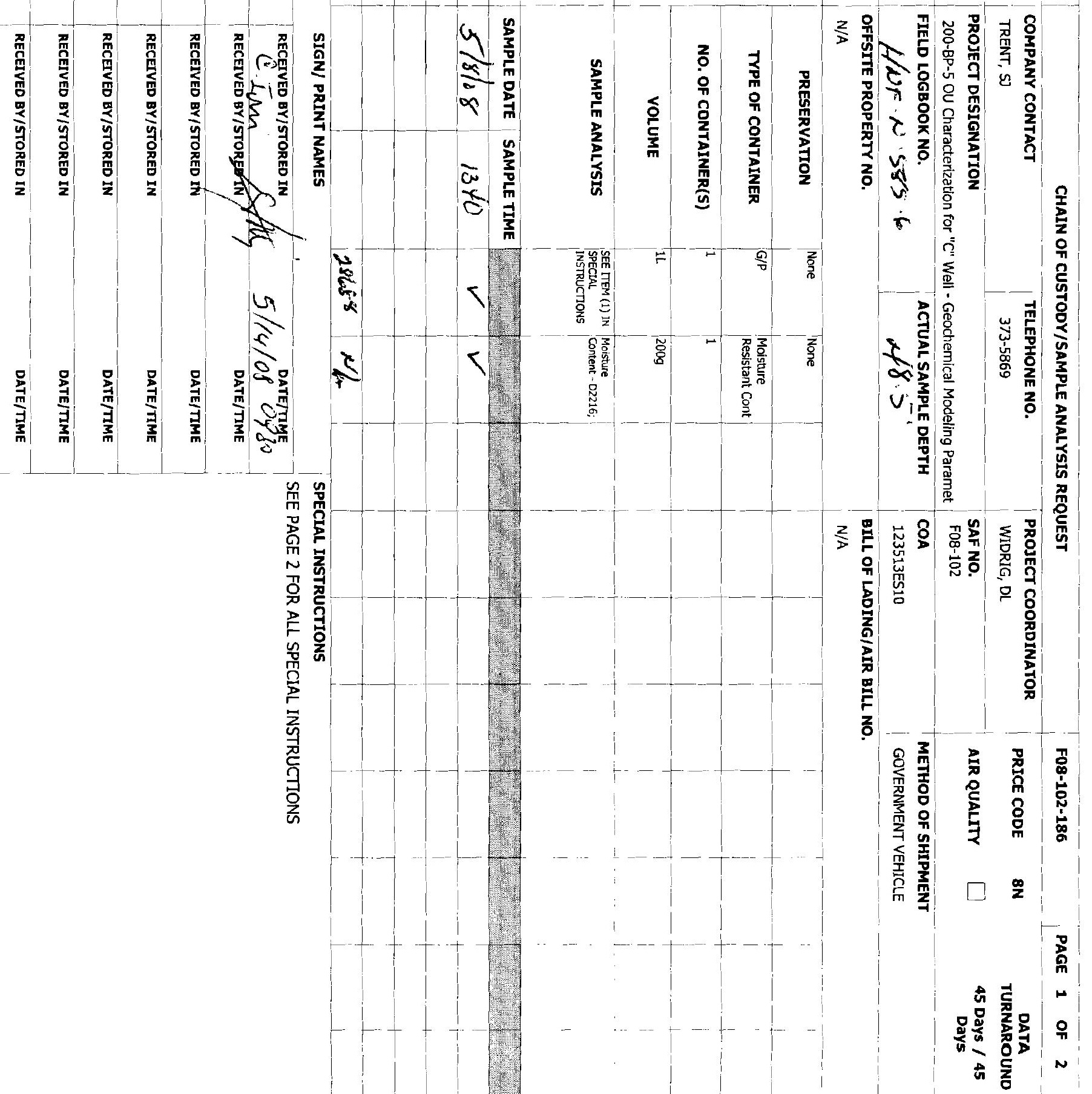




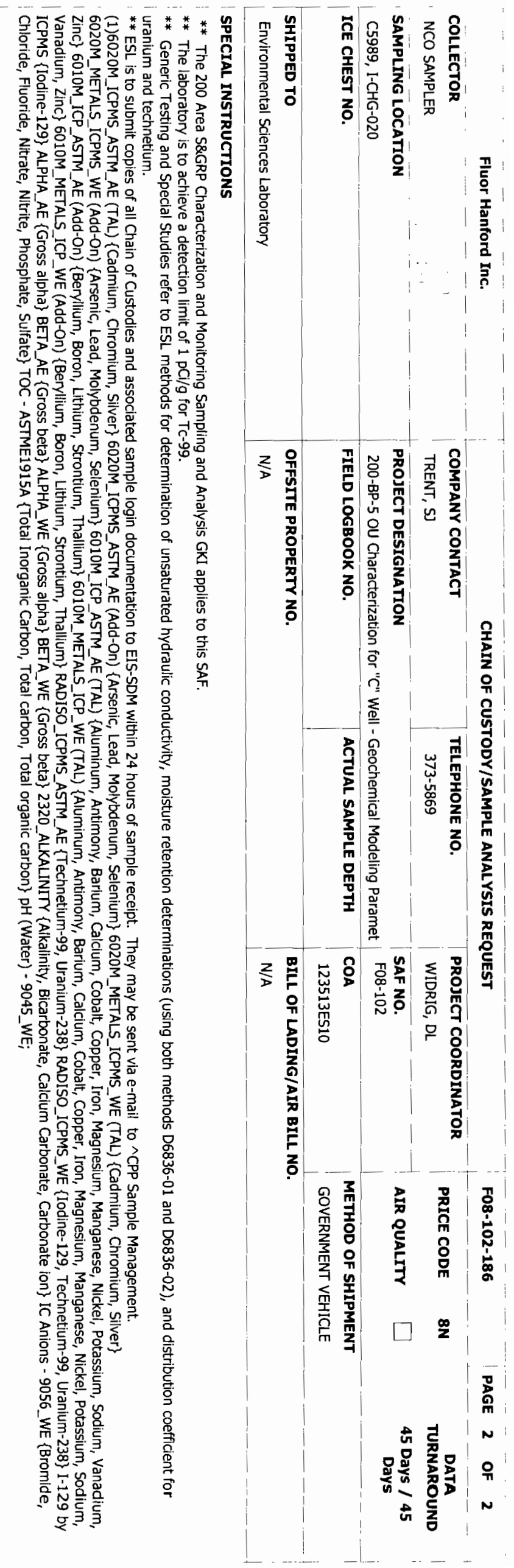




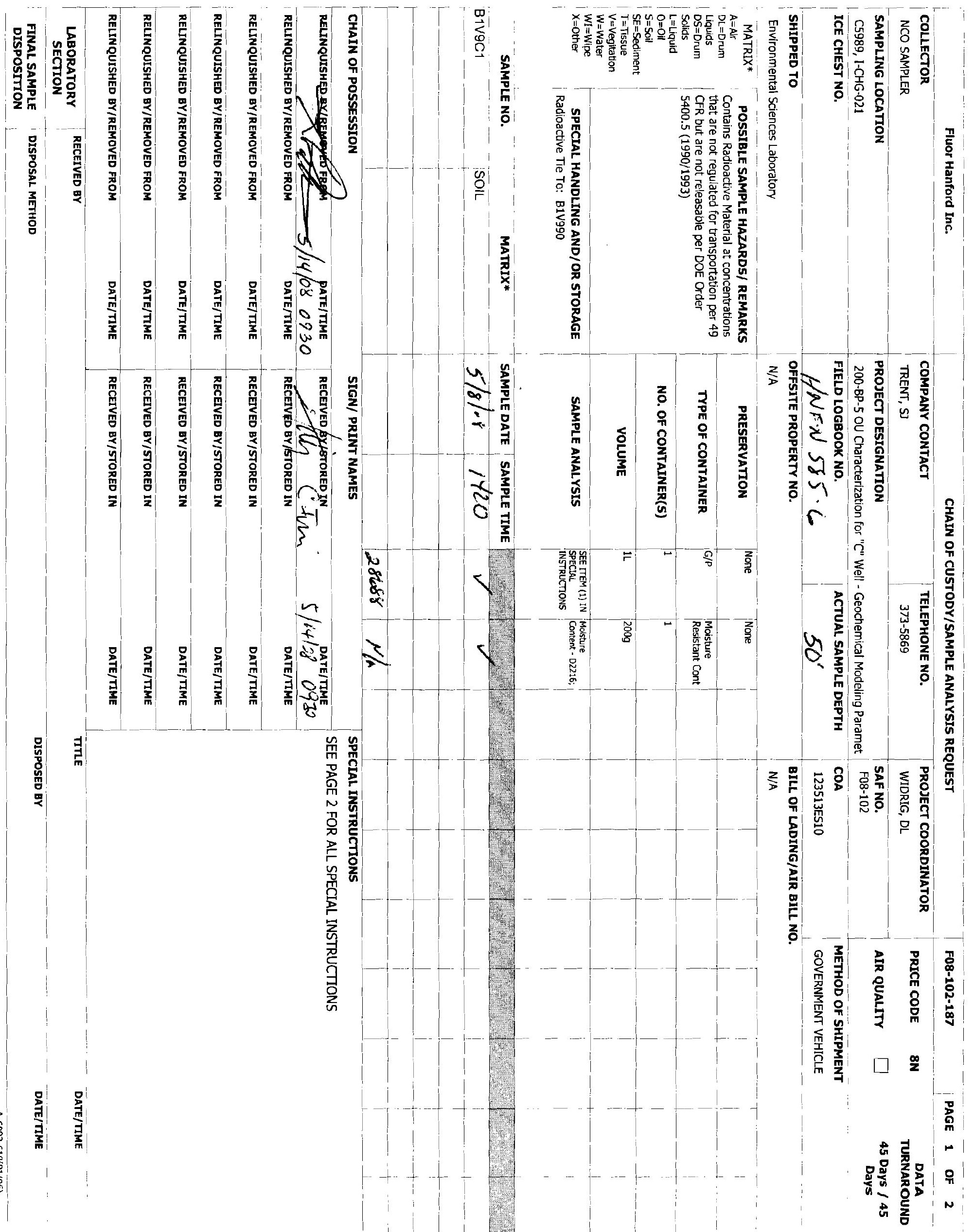




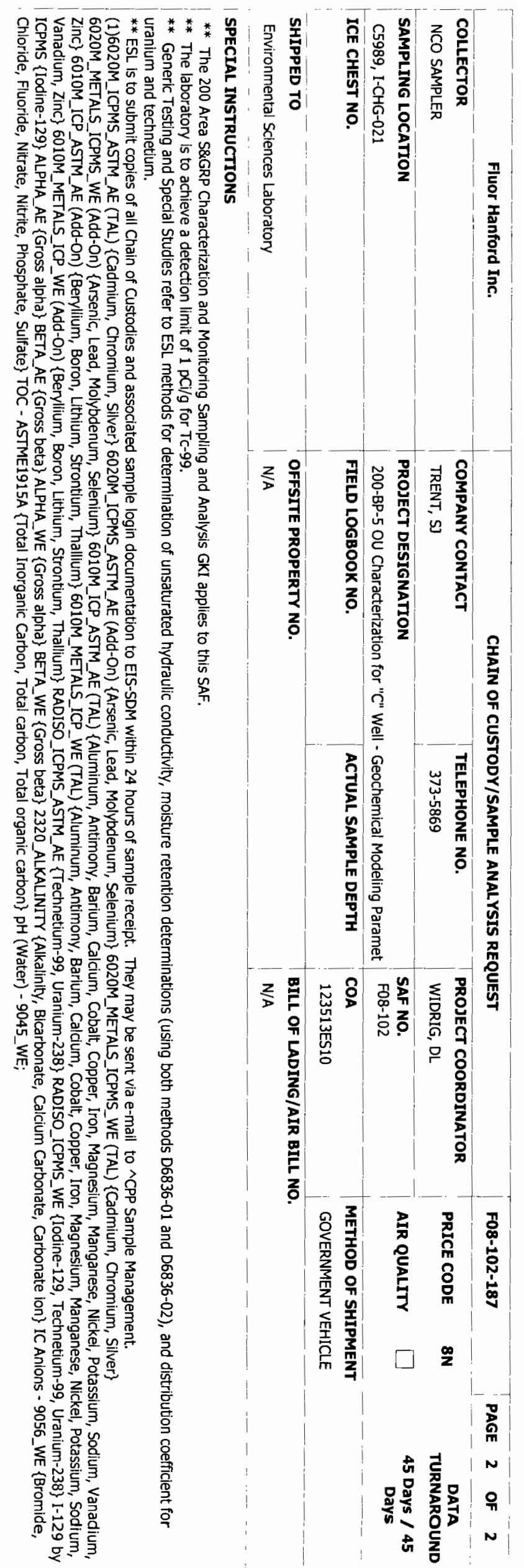




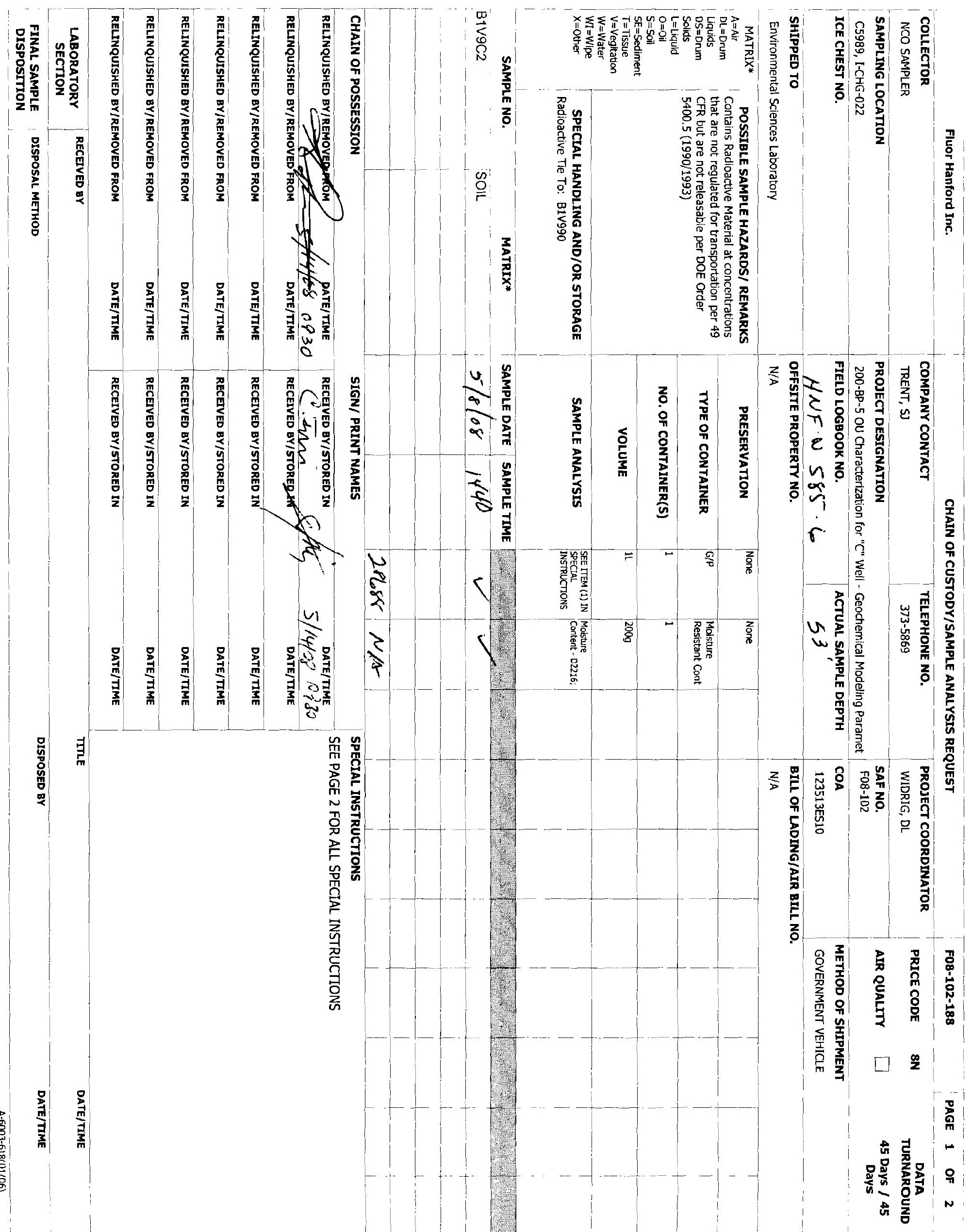




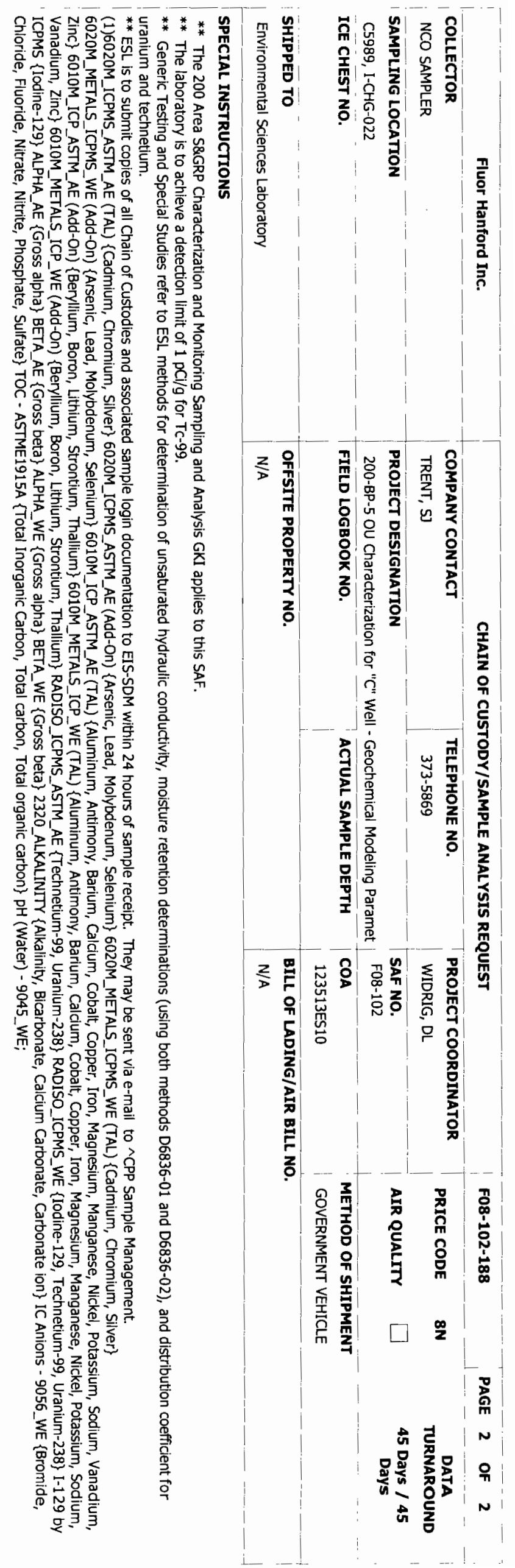




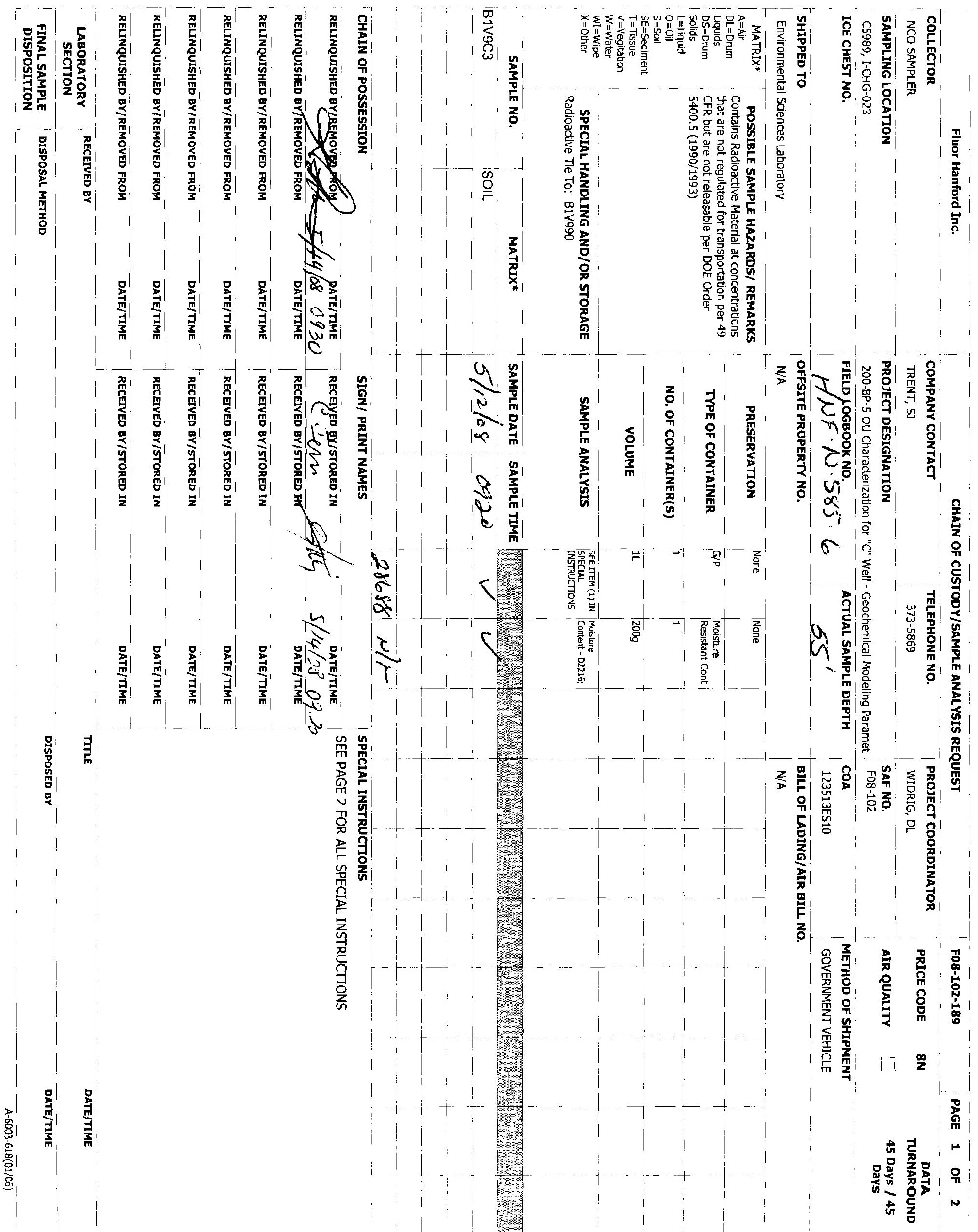




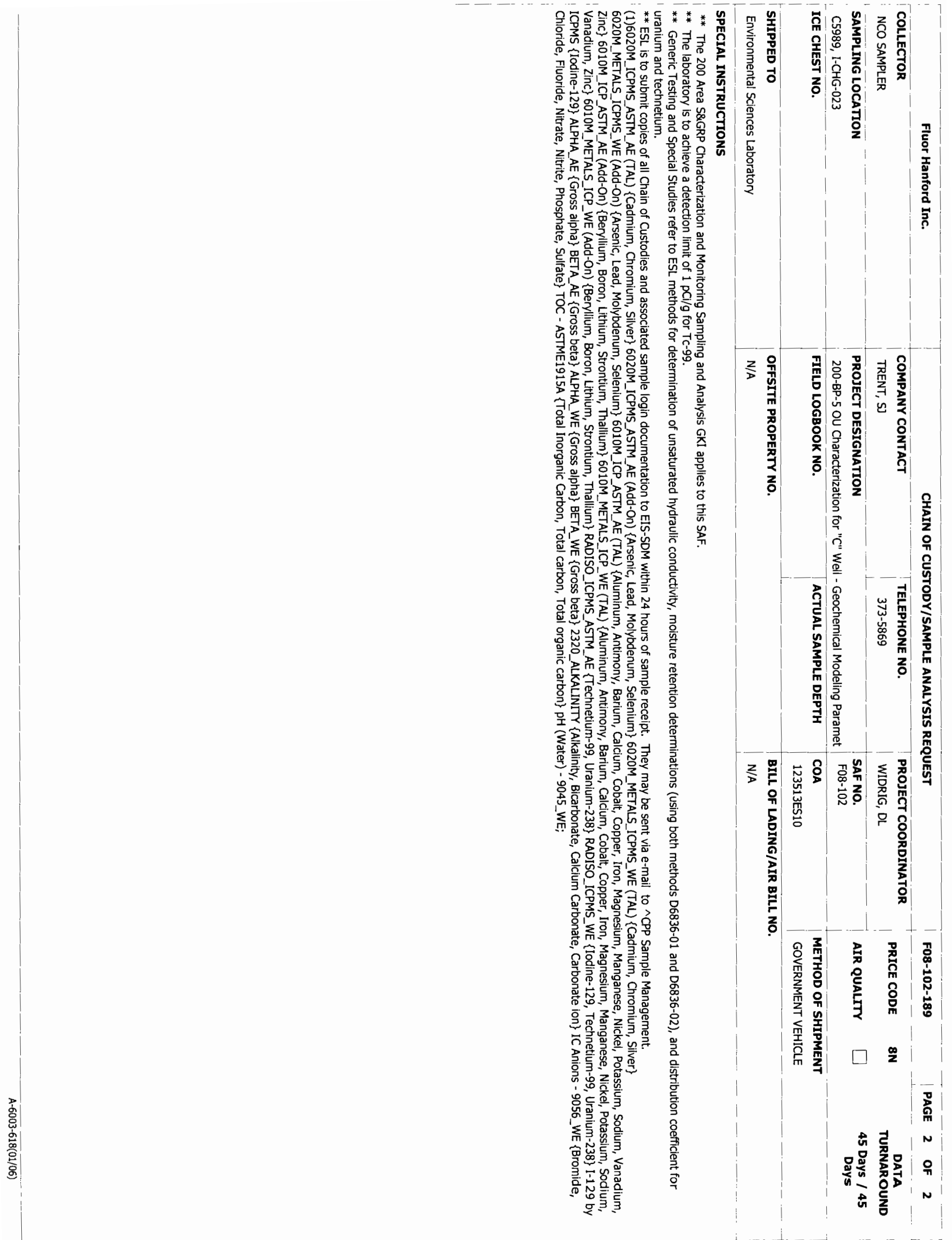




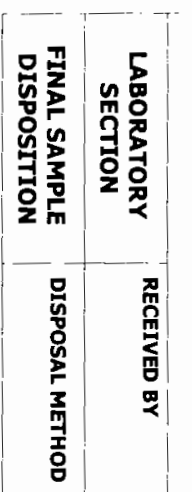

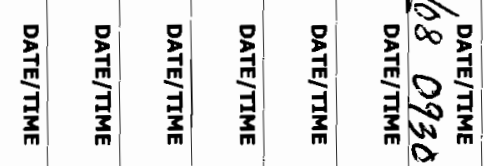

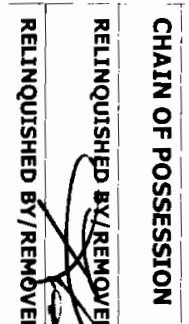

II

IIII!e
辤

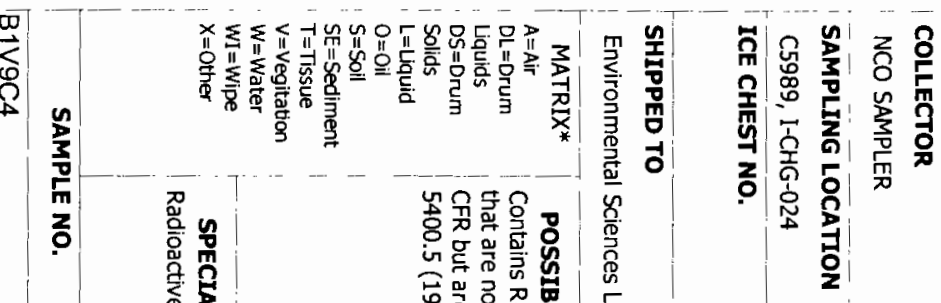

$\stackrel{\circ}{=}$

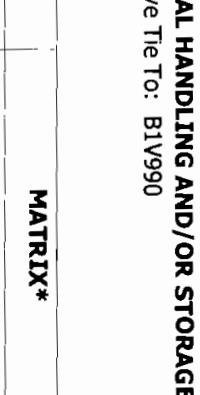

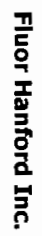

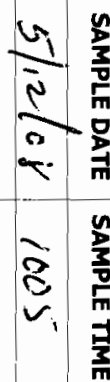

然

일

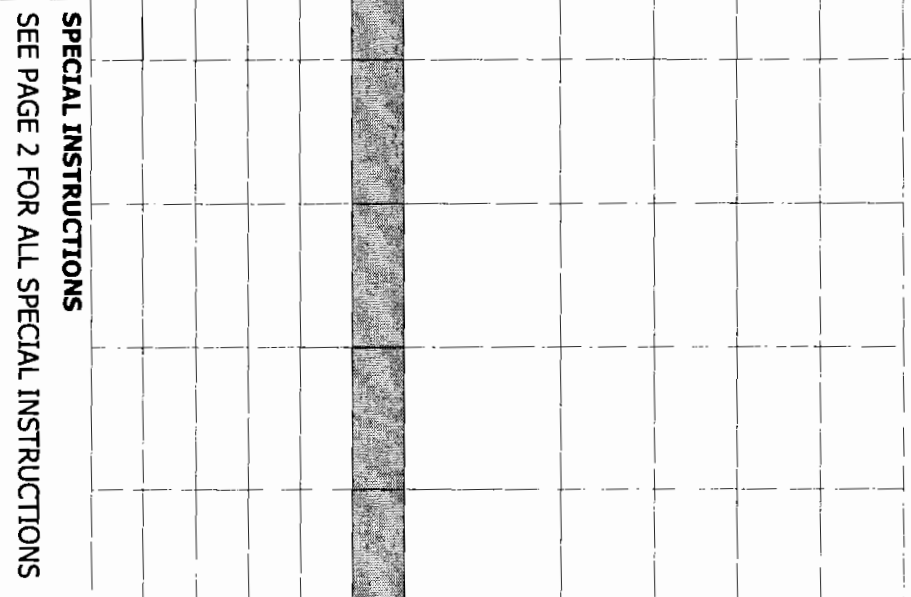

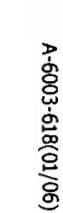

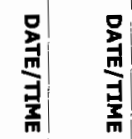

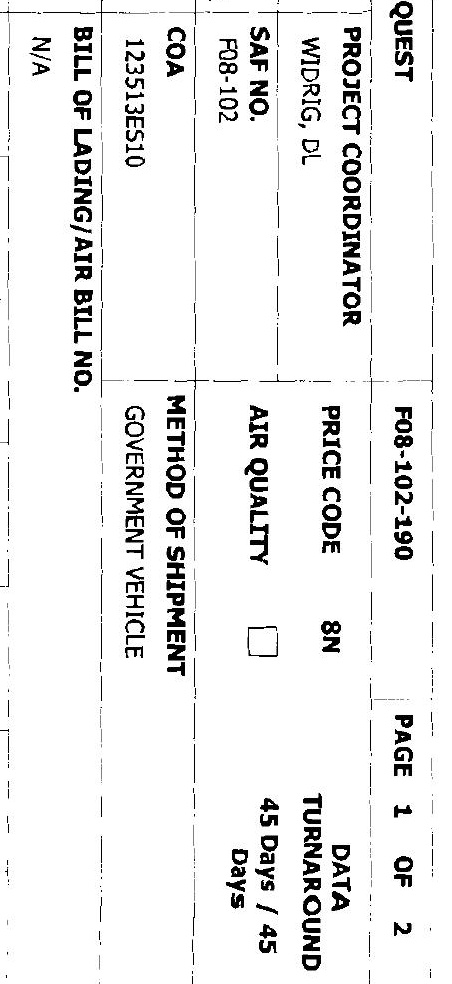




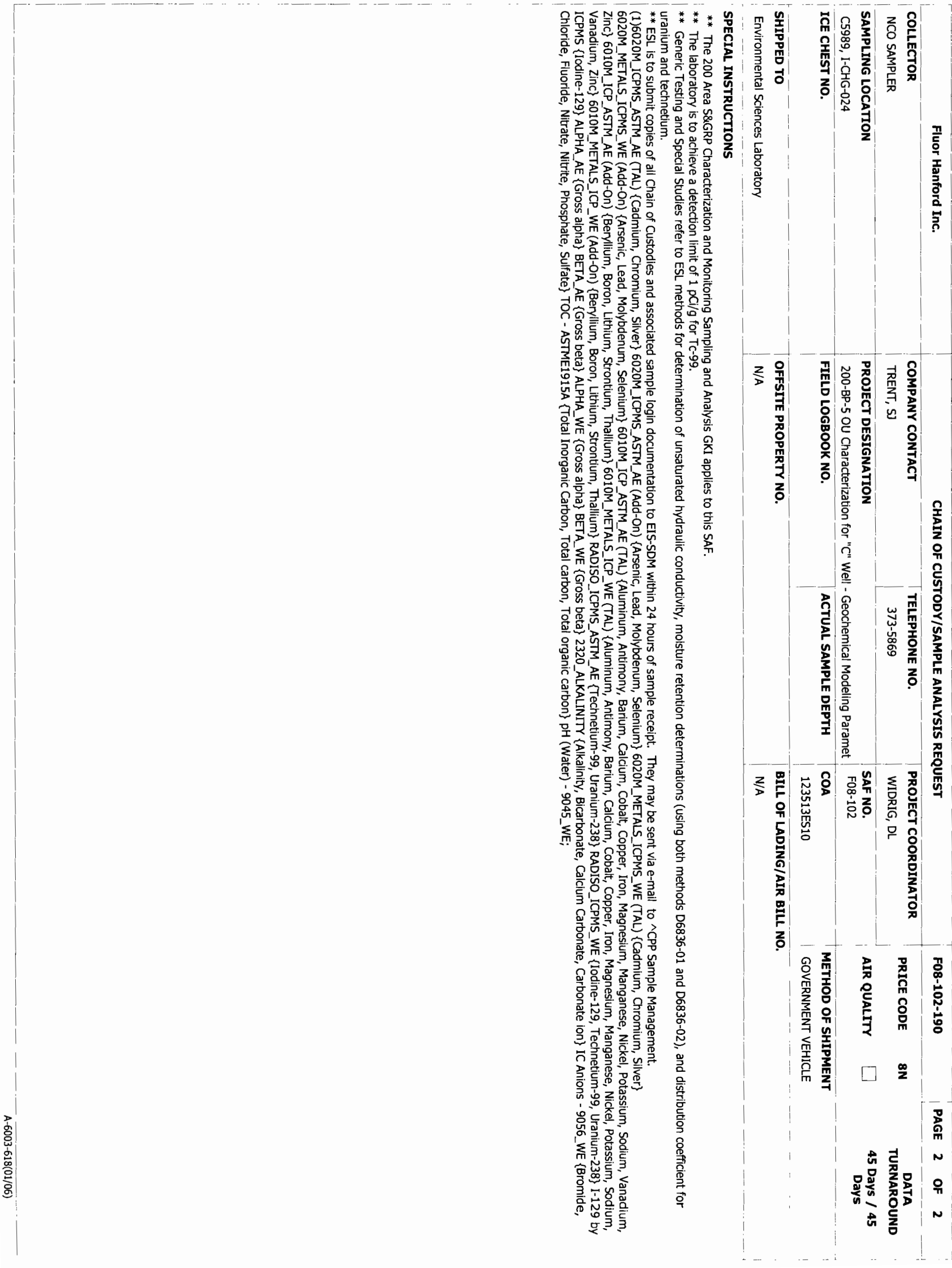




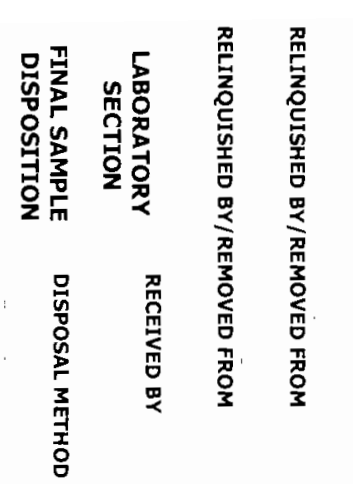

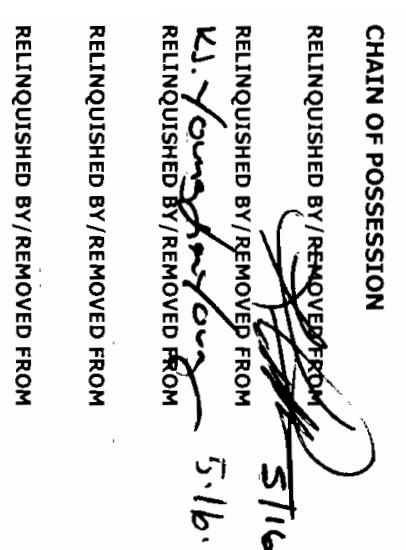

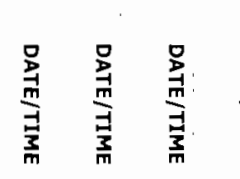

高富富
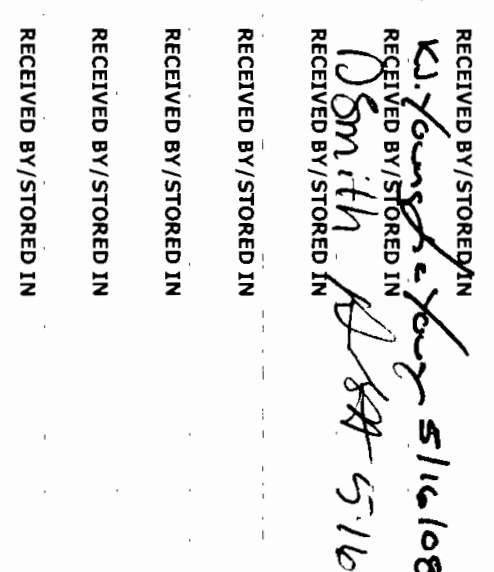

总

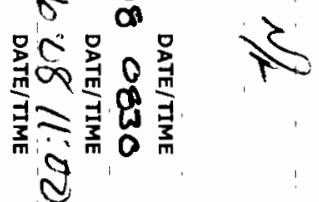

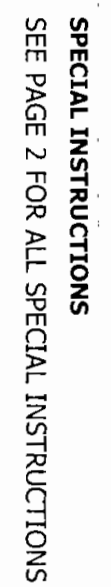

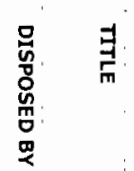

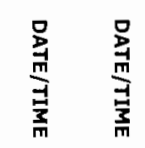

$\sum_{\text {जे }}^{\text {एu }}$

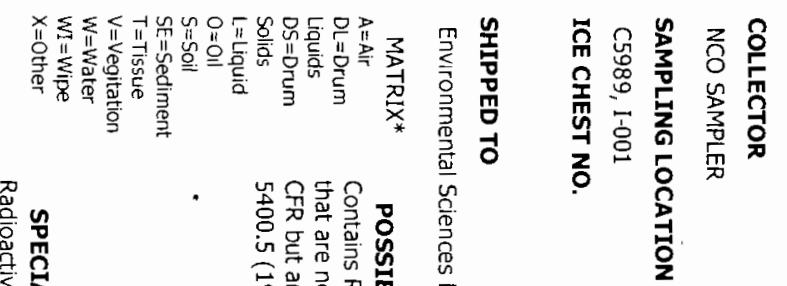

$\frac{2}{2}$

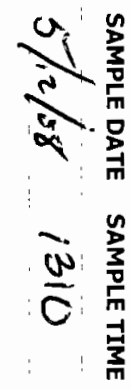

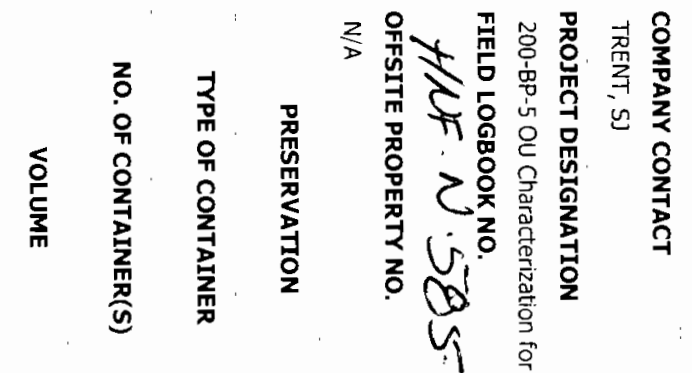

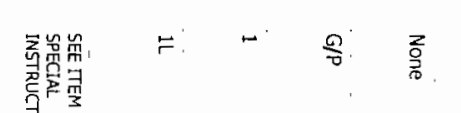

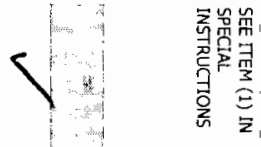

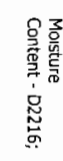

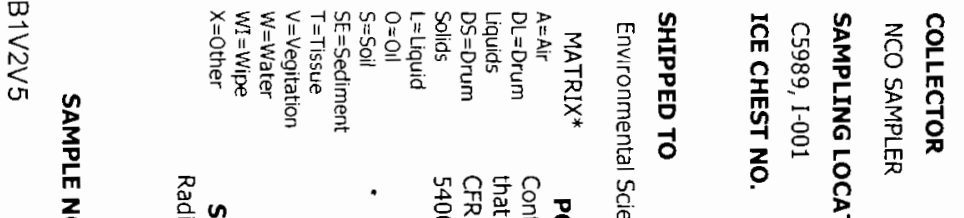

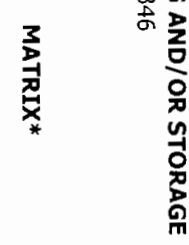

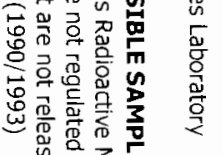

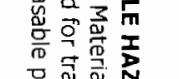

等

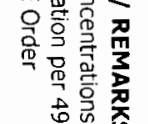

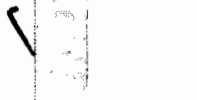

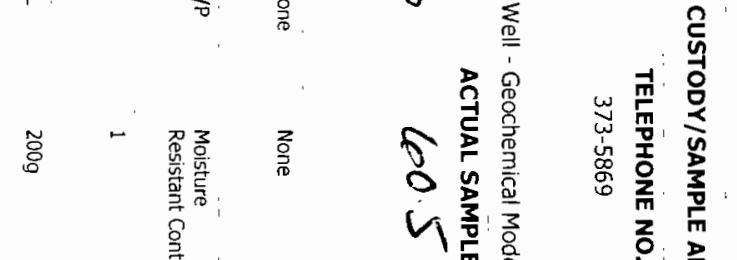
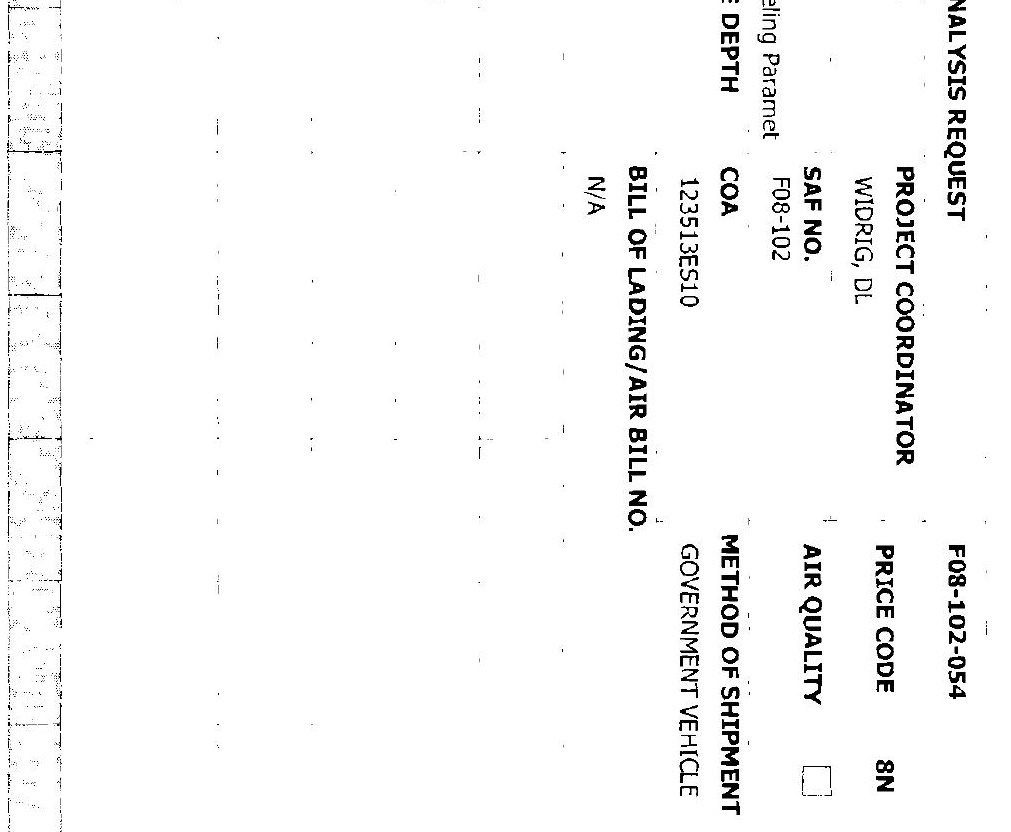


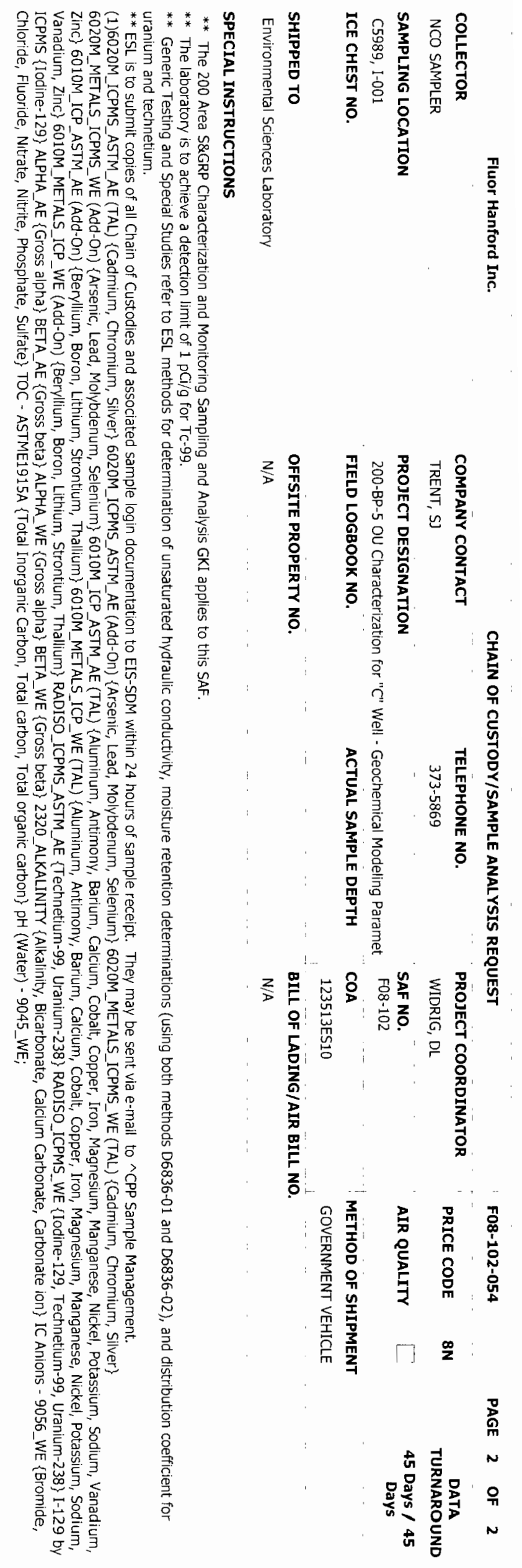

